VICTORIA UNIVERSITY OF WELLINGTON

\title{
Decoding Kashgar: A Digital Design Approach to Steer and Diversify Creative Engagement in Digital Heritage
}

\author{
A thesis \\ submitted to the Victoria University of Wellington \\ in fulfilment of the requirements for the degree of \\ DOCTOR OF PHILOSOPHY \\ in \\ ARCHITECTURE \\ by \\ SERDAR AYDIN \\ BArch (KTU), MA (ULiv)
}

2018

Supervision:

Marc Aurel Schnabel, Professor

Tane J. Moleta, Senior Lecturer 


\section{Table of Contents}

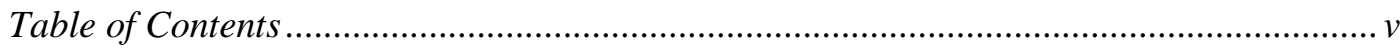

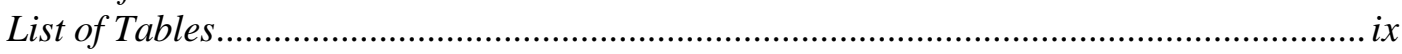

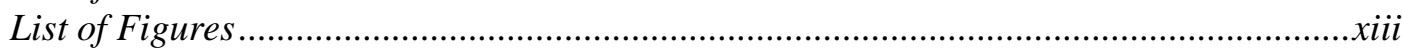

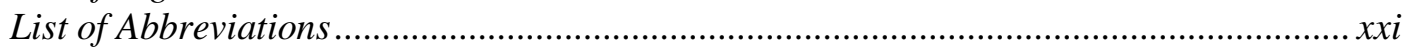

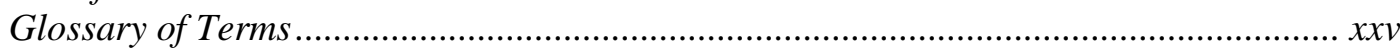

Publications during Candidacy ...............................................................................

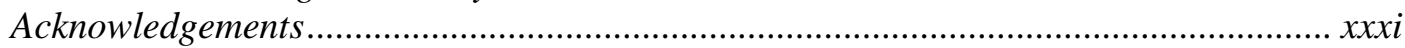

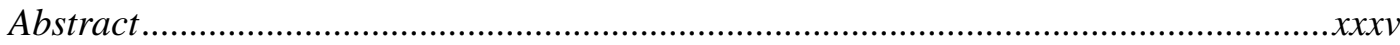

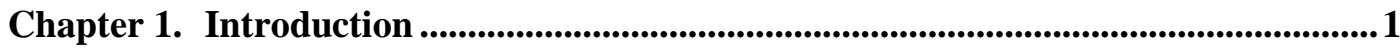

Chapter 2. Overview of Digital Heritage: A History of the Virtual Museum ..............11

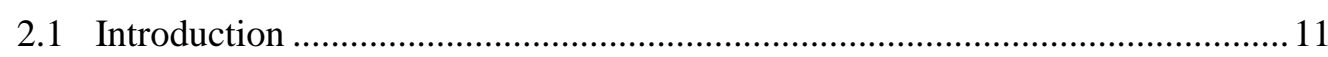

2.2 The Birth of Museum Computing: Binary Codes of Heritage ............................ 14

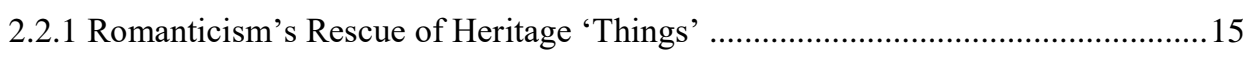

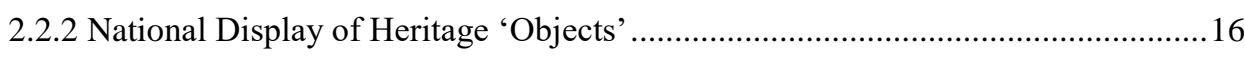

2.2.3 Rise of Information over Knowledge …………................................................19

2.2.4 Automation in Museum Computing ..................................................................20

2.2.5 Role of 'Error' in Information Processing ..............................................................23

2.3 The Birth of the New Museology: Plural Interpretations of Heritage ................ 26

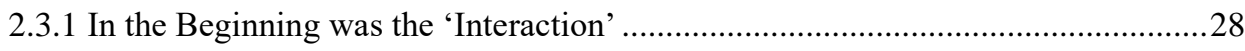

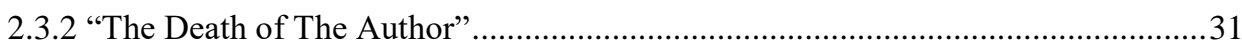

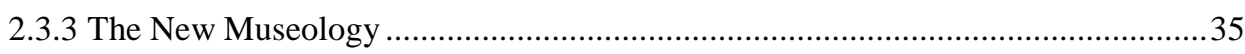

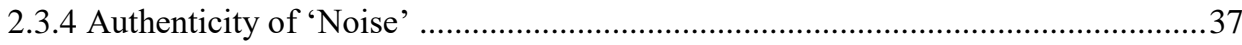

2.4 The Birth of the Virtual Museum: Suspension of Disbelief in Virtual Heritage 41

2.4.1 'The Museum Without Walls' ...............................................................................42

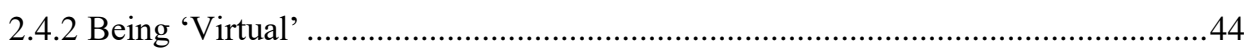

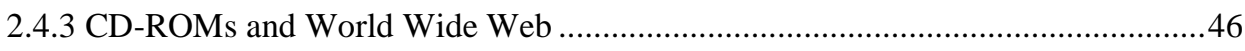

2.4.4 Non-Place, Place-Making and 'Suspension of Disbelief' .......................................48

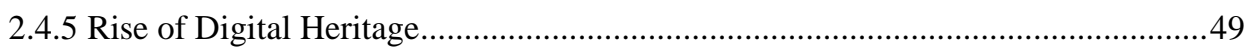

2.4.6 New Principles, Old Concerns …………………….......................................50

2.5 The Death (Deaccessioning) of the Virtual Museum in Information Space:

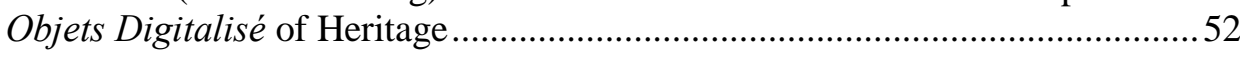

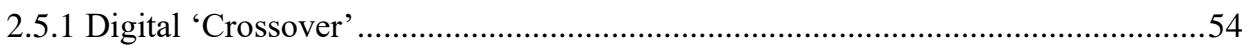




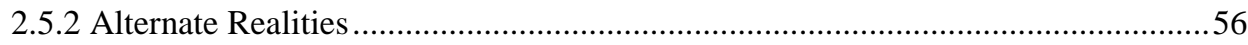

2.5.3 Virtual Communities of Our 'Global Village' ...................................................57

2.5.4 Authenticity of Non-Text-Based Information ...................................................60

Chapter 3. Matters of 'Transcoding' in Digital Heritage ................................................... 67

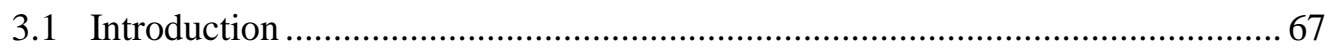

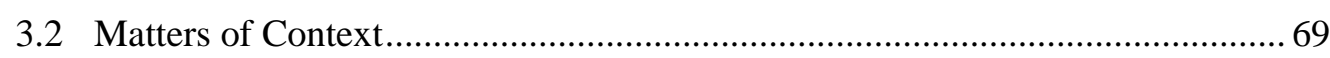

3.2.1 Background: From a 'Farm-State' to Demolition ................................................ 72

3.2.2 Theme: Narrow Roads as Derivation of 'Virtuality' ............................................. 76

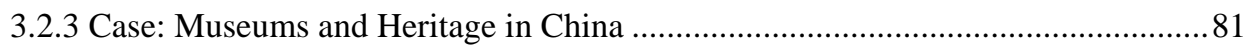

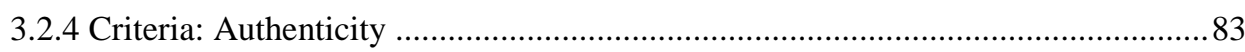

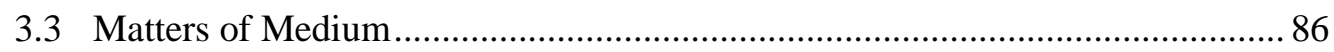

3.3.1 Background: Unmediated Heritage .................................................................. 87

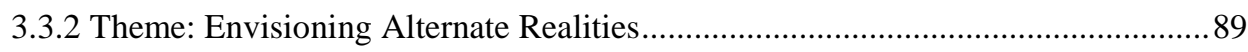

3.3.3 Case: ALiVE Projects by Shaw and Kenderdine ( 1995-2015) .........................93

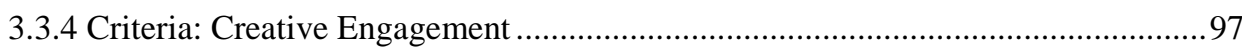

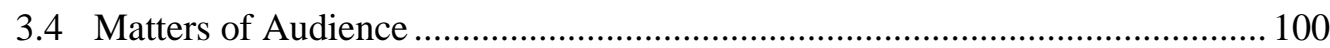

3.4.1 Background: Communication with Emancipated Spectators ............................... 101

3.4.2 Theme: Glitch Aesthetics and User-Generated Content..................................... 104

3.4.3 Case: The Language of New Media, Works of Manovich ( 2000-2010) ............ 107

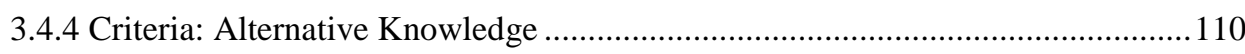

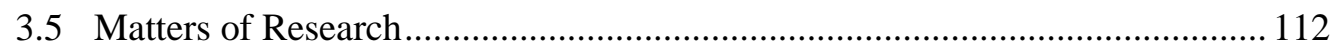

3.5.1 Background: Accuracy versus Information Overload ..................................... 113

3.5.2 Theme: Mapping a Digital Heritage Research ................................................ 121

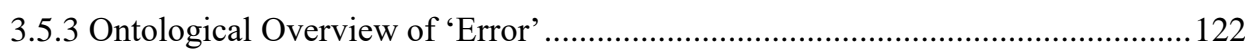

3.5.4 Epistemological Overview of 'Multimethodology' .......................................... 123

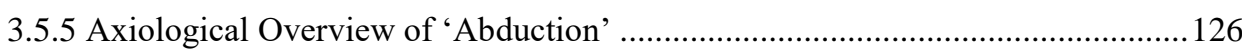

\section{Chapter 4. Digitising Kashgar's Heritage: An Interdisciplinary Methodological} Approach for Documentation, Representation and Dissemination ........ 131

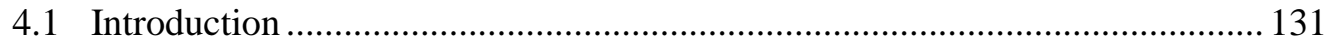

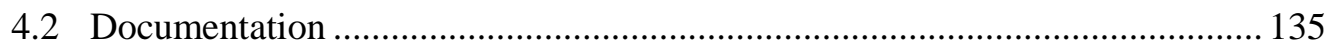

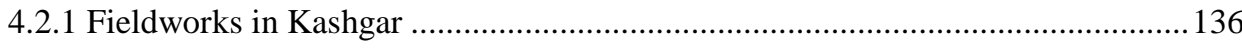

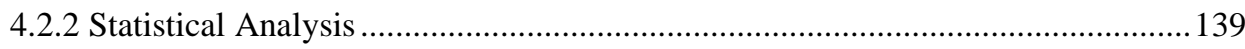

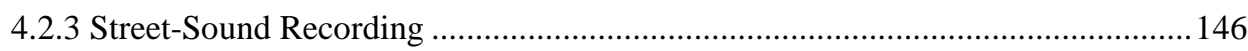

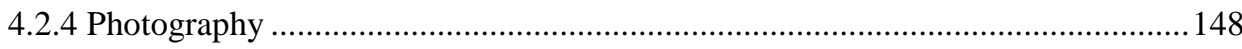

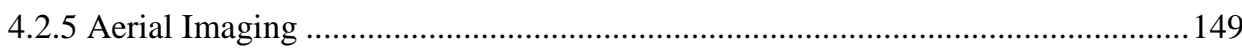

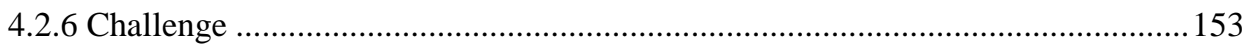




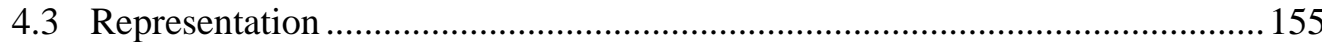

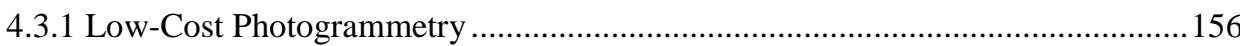

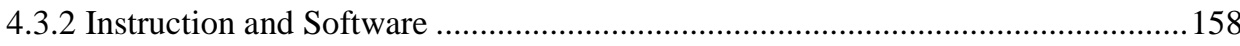

4.3.3 Computational Geometry: Polygon Mesh Processing ..........................................159

4.3.4 Abstract Concepts of Digital Geometry Processing: Surface Representation, Data

Structure, Differential Geometry and Parameterisation ........................................160

4.3.5 Operational Concepts of Digital Geometry Processing: Smoothing, Remeshing,

Simplification and Approximation, Model Repair and Deformation ....................169

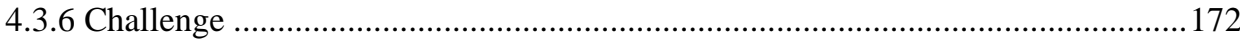

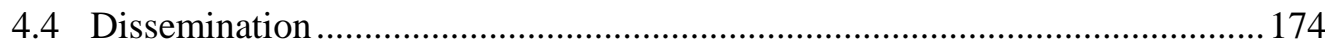

4.4.1 A Hybrid Immersive Environment: Hyve-3D …………………………….......176

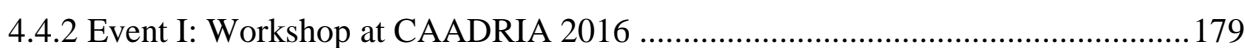

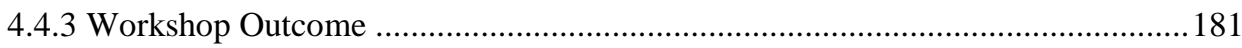

4.4.4 Event II: Public Demo at Te Papa Talks 2017 .......................................................184

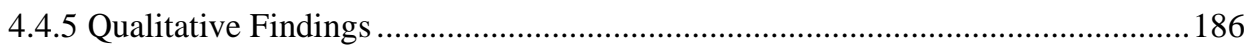

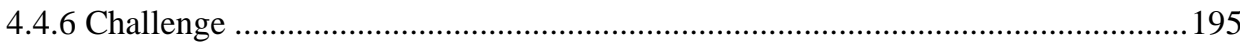

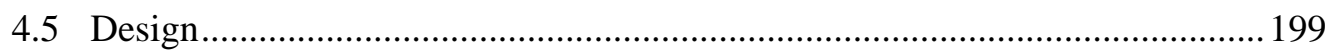

4.5.1 Systematisation of Design as a Novel Procedure in Digital Heritage .....................203

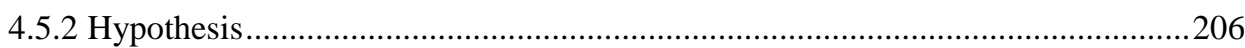

Chapter 5. Decoding Kashgar via User-Generated Content .........................................209

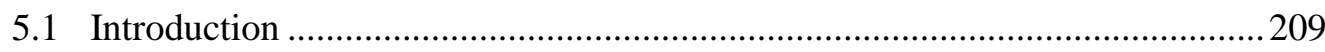

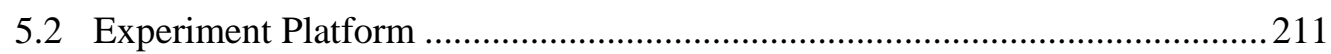

5.2.1 Mechanics and Gameplay Development ............................................................212

5.2.2 Association Rule Finding (ARF) Method .......................................................221

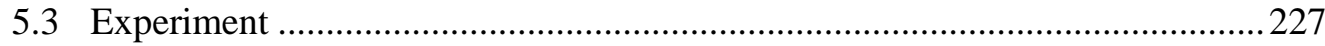

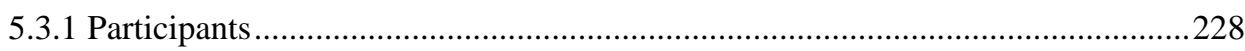

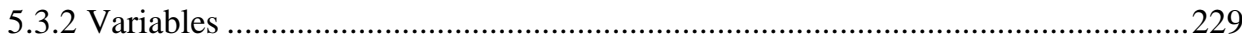

5.3.3 Analysis...................................................................................................231

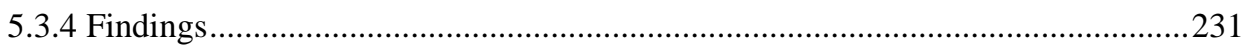

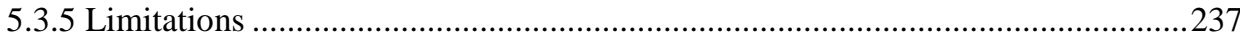

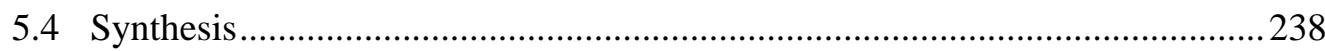

5.4.1 Retrospective Synthesis of Sub-Questions and Theories ....................................239

5.4.2 Prospective (Future) Synthesis...........................................................................24

Chapter 6. Discussion: Design as Virtuality in Digital Heritage .................................249

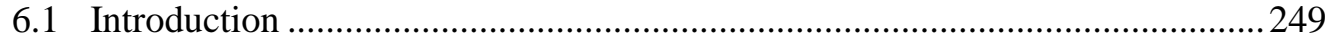

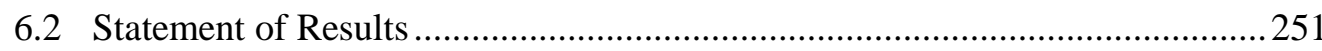


viii

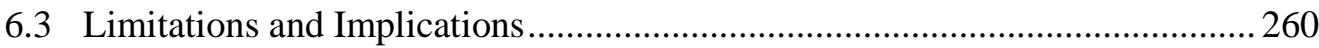

6.4 Future Research: Decoding $X$ in The Museum of Gamers............................... 262

Chapter 7. Conclusions .......................................................................................................... 267

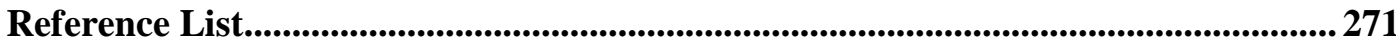

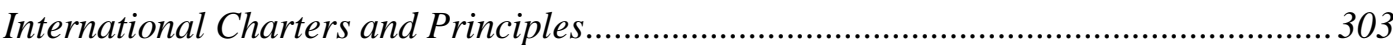

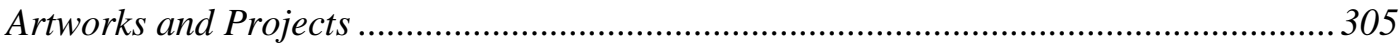

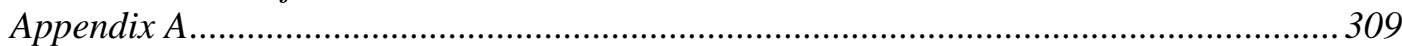

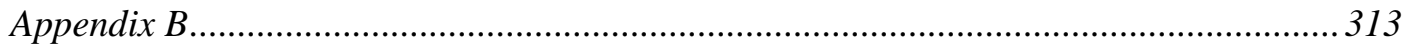

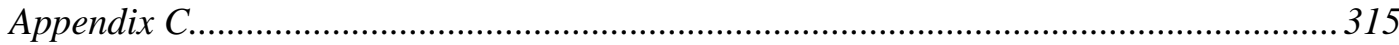

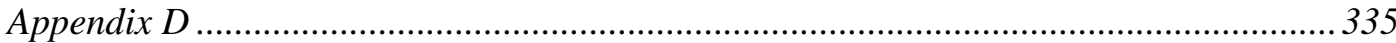




\section{List of Tables}

Table 1. Distribution of computer-assisted research projects in the humanities and the related social science between 1966 and 1972 based on the data adapted from Raben (1977).

Table 2. Select virtual museum concepts and samples until the 2000s (based on medium)............................42

Table 3. Quiccheberg's system of Wunderkammer. Adapted from Quiccheberg ([1565] 2013, 26). .43

Table 4. Crossover processes in the museum of information space. Adapted and modified from Ricardo $(2013,169)$.

Table 5. Challenges and opportunities of digital heritage research in the context of Kashgar in terms of heritage, identity and authenticity.

Table 6. The progress of formulation of research sub-questions

Table 7. Virtuality Matrix (VM) (see further developments of it on p. 97 and 183). Adapted from Richens (2014).

Table 8. Display systems examined at LuxLab.

Table 9. ALiVE's display systems. Adapted and modified from Kenderdine and Shaw $(2015,199)$

Table 10. Positioning the display system of 'Re-Actor' by ALiVE on Richens's VM. Adapted and modified from Richens (2014) (see the original p. 91 and further developed p. 183)

Table 11. The progress of formulation of research sub-questions (further development of Table 6)

Table 12. Selected projects of Lev Manovich between 2010 and 2015. Images and data adapted from Manovich (2018)

Table 13. The progress of formulation of research sub-questions (further development of Table 11)... 111

Table 14. Transformation of Critical Realism (CR) into applied research from the DREIC model to the RRREI schema (Mingers 2006, 23; 2017; Isaksen 2016, 249)

Table 15. Matrix of the RRREI model and the implementation schema in this research.

Table 16. Alignment of matters and themes for a multimethodological approach

Table 17. Ontologically representing the domains of CR. Adapted from Bhaskar $(1978,13)$.....

Table 18. Revised ontological structure of Critical Realism (CR) in the context of digital heritage

Table 19. Summary of the course of the research from Resolution and Redescription to Retroduction and Retrodiction

Table 20. Nine criteria for choosing an appropriate documentation method. Adapted from Pavlidis et al. (2006).

Table 21. Data collection scheme for each fieldwork in 2015 .

Table 22. Transformation of Köziciyerbişi (Gaotai) between 2002 and 2017. There was noticeable change in the density of houses after 2009 when the renewal project was initiated. Satellite images from Google Earth Pro.

Table 23. Area measures in Köziciyerbişi (Gaotai) - calculations were made based on measurements in Wang et al. (2014)

Table 24. Components of courtyard-factor $(C Y b)$ calculation.

Table 25. Points of interest at the convergence of three courtyard integration levels 
Table 26. Audio specification

Table 27. Playlist of street-sound recordings from Köziciyerbişi (Gaotai): (®) Tracks, (•): Playlists (available for listening via Aydin, 2015). A full list of sound recordings available in Appendix A.........148

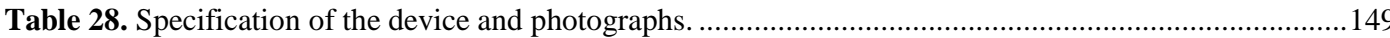

Table 29. Unmanned aerial vehicles used to document the Kashgar old-town from above (specifications available from DJI's website: https://www.dji.com/). .........................................................................150

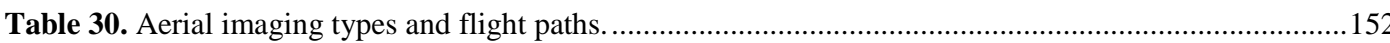

Table 31. Methods, data sources and their planned use.......................................................................... 154

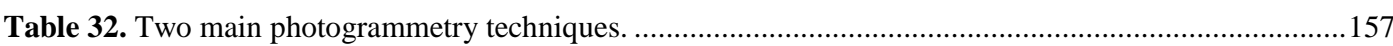

Table 33. Software packages used in this research for 3D digital representation........................................159

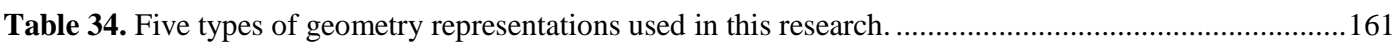

Table 35. Assessment of polygon mesh representation........................................................................... 162

Table 36. Understanding the data structure of polygon mesh processing during photogrammetric modelling in SfM software used in reconstructing Köziciyerbiş̧i (Gaotai). ............................................163

Table 37. Purpose and implications of the abstract concepts of SfM digital geometry processing as happens in the 3D reconstruction of Köziciyerbişi (Gaotai). .................................................................. 166

Table 38. Flaws and defects on polygon mesh diagnosed automatically in Autodesk ReMake during the 3D reconstruction of Köziciyerbişi (Gaotai).

Table 39. Event details to experiment with immerisiveness...................................................................... 175

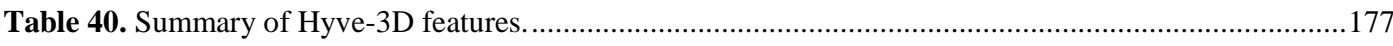

Table 41. Stages of the use of the Hyve-3D system with virtual Kashgar models. .......................................180

Table 42. Workshop outcome and findings classified according to the 'Matters of Research.' ....................183

Table 43. Further development of Richen's (2014) VM (see the earlier development p. 97 and the original p. 91) based on remote collaboration in a hybrid virtual reality environment. ............................ 183

Table 44. Stages of the public event at Te Papa Museum with virtual Kashgar models. ...............................184

Table 45. The shift from a role definition in the immersive experience to the full-fledged agency. ..............186

Table 46. Three types of sketches observed qualitatively. .........................................................................191

Table 47. Summary of findings from the public demo show at Te Papa Museum.......................................191

Table 48. 'Index of representations' in Session A (red lines) and B (orange lines).........................................194

Table 49. The amalgamation of Chapter 4.5 and Chapter 3.5 in the RRREI schema; the vertical connection between chapters and the horizontal combination of subchapters......................................200

Table 50. Revisiting the matters of digital heritage research.....................................................................201

Table 51. Positioning design as a procedure of digital heritage studies in the context of this research. The classification involves overlaps between procedures. But the demonstration of these overlaps is excluded from the table for simplicity.

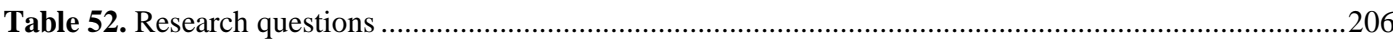

Table 53. Mechanics and gameplay development of the experimental platform...........................................212

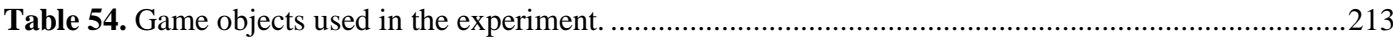

Table 55. Principal gameplay roles in the experimental platform. ..............................................................2218

Table 56. Transactional data for 4 players as an example. ……..................................................................224

Table 57. Pattern items that can be collected from the experiment platform. The size of each sample

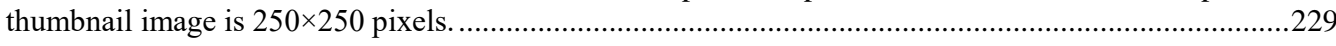

Table 58. A 3-itemset generated by the participant (ID1) .....................................................................22 
Table 59. Association rules found in the database of the experiment, in relation to ID1's 3-itemset transaction of $\{$ Metal Door, Timber Wall Structure, Brick Wall $\}$

Table 60. Synthesis of experiment findings with the three sub-questions of this research.

Table 61. Digital heritage design parameters and their roles in the thesis

Table 62. Macro-level and micro-level design activities observed in the research.

Table 63. The connectivity of museum concepts to the concepts of control for ruling the progress of AHD in different eras

Table 64. The connectivity of museum concepts to the concepts of control for ruling the progress of AHD in different eras

Table 65. From museum computing to digital heritage, the growth of the field.

Table 66. The result of the FP-growth algorithm that searched associations between one million records of artificial participants with the transaction record of the experiment participant whose itemset is $\{$ Metal Door, Timber Wall Structure, Brick Wall $\}$. Mud-Brick Wall=RC; Timber Wall Structure $=T W S ;$ Timber Structure $=T S ;$ Metal Door $=M D ;$ Rec Cobblestone $=R C ;$ Hex Cobblestone $=H C ;$ Red Door $=R D ;$ Blue Door $=B D ;$ Mosque Gate $=M G ;$ Brick Wall $=B W$ 


\section{List of Figures}

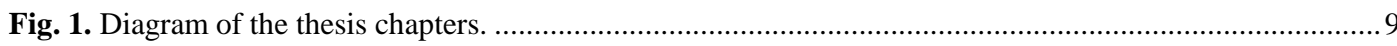

Fig. 2. Ferrante Imperato's museum in Naples. Adapted from Impey and MacGregor (1985, fig.4)............... 17

Fig. 3. Noise source in Shannon's diagram of communication. Adapted from Seising (2010).......................20

Fig. 4. Wiener's edition of Shannon's diagram with two additional semantic boxes. Adapted from Seising (2010)

Fig. 5. The CRT display terminal of IBM 2250. Adapted from Lourie $(1968,225)$

Fig. 6. Light-pen input function of IBM 2250. Adapted from Appel, Dankowski, and Dougherty (1968).

Fig. 7. Sketchpad operated by an 'author' with a light-pen, a box of push buttons a bank of toggle switches. Adapted from Sutherland (1964).

Fig. 8. Left. 3D representation of Glowflow's walls installed with transparent tubes (left). Adapted from Krueger (1969). Right. Zeros-and-ones concept of Psychic Space that shows how a camera-based feedback system senses the 'participant.' Adapted from Krueger (1977).

Fig. 9. Left. Sketch drawings of Telesphere Mask (patent: US2955156). Adapted from Heilig (1960). Right. Sensorama Simulator (patent: US3050870). Adapted from Heiling (1962).

Fig. 10. Left. 'Computer Composition With Lines' by Noll (1964). Middle. 'No Title' by Molnar (1968). Right. 'Random Walk' by Mohr (1969). Adapted from DAM (2018).

Fig. 11. Top. Dufour's Les Sauvages de la Mer Pacifique (1804). Adapted from Dufour (1804). Bottom. Lisa Reihana's in Pursuit of Venus [infected] (2015). Adapted from Reihana (2015). Illustration by Author.

Fig. 12. Measuring visitor characteristics, a heritage management technique for visitor classification. Adapted from Hall and Arthur (1998, 145)

Fig. 13. Room of One's Own by Lynn Hershman Leeson (1990): exterior view of the interactive module (left) and interior view with miniature furnishing and screens. Hershman Leeson's installation induced the viewer's replacement with the female identity staged at a representative position. Adapted from FDL (2017).

Fig. 14. Reinterpretation of Shannon's Choice towards chaos and disorder, or entropy, while the increase in information meant an increase in entropy. Illustration by Author based on a review of Hayles $(1990,59)$.

Fig. 15. Title page of Quiccheberg's Inscriptions. Adapted from Quiccheberg ([1565] 2013, 60)

Fig. 16. A Section of the Rotunda in Leicester Square, in which is exhibited the Panorama of London. Adapted from Open Research Exeter (2009)

Fig. 17. Guggenheim Virtual Museum (GVM). Adapted from Belogolovsky (2016).

Fig. 18. Crossing over between physical, digital and virtual stages during architectural design. Adapted from Schnabel et al. (2004).

Fig. 19. Olafur Eliasson's participatory installation Demented Architecture exhibited at the City Gallery Wellington Te Whare Toi, Wellington, New Zealand. Adapted from Eliasson (2015).............................58

Fig. 20. Foldit interface screenshot. Adapted from (Cooper et al. 2010)

Fig. 21. DreamHack Winter 2004. Adapted from http://www.wikiwand.com/en/DreamHack. 
xiv

Fig. 22. Radial visualisation of MoMA's photography collection based on more than eighteen thousand photographs between 1837 and 2012, with distance from the centre determined by 'year' and radial degree by 'average brightness.' Adapted from Hochman and Manovich (2014).

Fig. 23. Automatic brushstroke extraction for Van Gogh's Cabbages and Onions (1887-1988). Adapted from Li et al. (2012).

Fig. 24. Kingdom Hearts video-game play visualisation. Adapted from Huber (2010).

Fig. 25. Top views of alleys in Kashgar. Photo by Author from Aydin and Schnabel $(2017,104)$.

Fig. 26. The outlook of Kashgar depicting a contrast between heritage and contemporary architecture. Photo by Author from Aydin and Schnabel $(2017,118)$

Fig. 27. Kashgar's location on the world map (without scale) depicted on a red-line sketch of the historical Silk Road. Illustration by Author.

Fig. 28. A section of narrow roads from the Kashgar old-town. Photo by Author from Aydin and Schnabel $(2017,149)$.

Fig. 29. Unique historical architecture is sitting on a hill with terraces and balconies. Photo by Author from Aydin and Schnabel $(2017,168)$

Fig. 30. The relocation from historical architecture into new apartments. Photo by Author from Aydin and Schnabel $(2017,70)$

Fig. 31. Multifunctional use of space that occurs through the penetration of the street life into the courtyard in Kashgar. Photos by Author from Aydin and Schnabel (2017, 142 and 143).

Fig. 32. The Kite Runner movie set in Kashgar, with artificial snow adding an extra layer of hyperreality to represent Kabul, Afghanistan, where the filmed story occurs. Adapted from French (2006)

Fig. 33. Kashgar old-town after renewal. Photo by Author from Aydin and Schnabel $(2017,174)$.................81

Fig. 34. View of the main street in Pingyao. Photo by author. ......................................................................... 82

Fig. 35. Browsing results for 'authenticity of ...' online. Retrieved January 25, 2018, from Google................84

Fig. 36. Screenshot of a Pop-Up Museum set up at NISV in Hilversum, The Netherlands (January 1224, 2017). Visitors login through a browser (without installing an app) to interact with three monitors. Adapted from MuPop (2017a)

Fig. 37. Diagram of the Reality-Virtuality (RV) continuum. Adapted from Milgram et al. (1994).

Fig. 38. 360-degree stereoscopic interactive visualization environments (AVIE and PLACE) and hemispherical projection systems (iDome and MediaDome). Adapted from ALiVE (2017).

Fig. 39. Left: Jeffrey Shaw and Sarah Kenderdine while organising Luxlab workshop participants for a group photo. Right: Introduction to advanced high-definition scanning of heritage artefacts (up to 1,600 DPI). Photos by Author, September 2013, Hong Kong.

Fig. 40. Left: The information sheet of the iTürkiye installation using the iDome display system. Right: iDome's steel hemisphere from behind. Photos by Author, September 2013, Hong Kong.

Fig. 41. UNMAKEABLOVE (2008). Adapted from Kenderdine and Shaw (2012)....

Fig. 42. The dome of Sultan Ahmet Mosque, Istanbul projected in MediaDome, LUXLAB Workshop. Photo by Author, September 2013, Hong Kong.

Fig. 43. Sketches by Price for Fun Palace (circa. 1964). Adapted from Mathews $(2005,74)$ 103

Fig. 44. Screenshots from the posters of the First Prize Winners of the London Internet Museum architecture competition. The winning entry design products ranging from a smartphone app to web interface. It shows how extensive the current mode of thinking is in architecture. Adapted from McCallum and Belitskaja (2016)

Fig. 45. Digital Spontaneity, an algorithmic design-research work of glitch in urban design, produced at AAVS Shanghai 2015, with Author involved (Retsin et al. 2015).

Fig. 46. 3D glitched anaglyph digital form. Illustration by Blaire Haslop, 2017 (Haslop, Schnabel and Aydin 2017b).

Fig. 47. Left. Manga Style Space, interactive exploration via 287-megapixel HIPerSpace. Adapted from Softwarestudies (2010). Middle. The Exceptional and the Everyday: 144 Hours in Kyiv, a child 
visitor facing 13,208 Instagram photos of a revolution in Kyiv in 2014. Adapted from TÊTE-ÀTÊTE (2016). Right. Taipei Phototime, visualisation of big data at The National Taiwan Museum of Fine Arts. Adapted from Softwarestudies (2014)

Fig. 48. The interactive installation, OnBroadway, 'representing the modern city' with non-text-based information extracted from Instagram. From top to bottom, the spectrum of rows of information is street names, street-view facades, façade colours, taxi drop-offs, taxi pick-ups, street-view top, Foursquare check-ins, twitter messages, Instagram photos, median household income, Instagram photo colours and Instagram photos. Adapted from Goddemeyer et al. (2015)

Fig. 49. Analogical Cartesian graphs represent the epistemological understanding of contemporary digital heritage research. Illustration by Author.

Fig. 50. Graphs represent the matters pertaining to digital heritage research that are independent empirically. Illustration by Author.

Fig. 51. Location of Köziciyerbişi (Gaotai) in the Kashgar old-town. Satellite image from Google. 136

Fig. 52. Travelling in a modified three-wheel vehicle across Tuman Road (top-left) from Köziciyerbişi (Gaotai) to other parts of the old-town. Photos by Author from Aydin and Schnabel $(2017,16)$.

Fig. 53. Aerial view of houses with narrow alleys running through from the north of Köziciyerbişi (Gaotai). Photo by Author from Aydin and Schnabel $(2017,117)$.

Fig. 54. Map of Köziciyerbişi (Gaotai) (at right bottom corner) divided from the rest of the old-town by Tuman Road. Illustration of a retrieved plan drawing by Author.

Fig. 55. Köziciyerbişi (Gaotai) in Kashgar. Illustration by Author

Fig. 56. Distribution of the courtyard factor $-C Y b$. The chart shows the courtyard area comparisons in Köziciyerbişi (Gaotai). The full chart is available in Appendix B.

Fig. 57. Map colourised according to the courtyard analysis shows interrelations between courtyards spanning across lanes.

Fig. 58. Map of the tracks in the playlist of field recordings

Fig. 59. Select photographs are documented in a photobook titled "Decoding Kashgar: China's westernmost city on the historical Silk Road" (Aydin and Schnabel 2017).

Fig. 60. Köziciyerbişi (Gaotai) from above (captured with DJI Phantom 2)

Fig. 61. The photogrammetric modelling result of the aerial imaging $\mathrm{C}_{1}$ in Köziciyerbişsi (Gaotai).....

Fig. 62. Screenshot of a piece of raw Kashgar model in Autodesk Recap.

Fig. 63. Polygon mesh photogrammetric model of narrow roads in Köziciyerbişi (Gaotai) (image screenshot from Rhino; software in the production line: ReCap for photogrammetry, ReMake for joining digital geometries, Mudbox for combining files, Rhino for reducing the file size).

Fig. 64. A sketch of the halfedge data structure used in ReCap and ReMake for the implementation of connectivity information between polygons. Content: Section of 3D photogrammetric Köziciyerbişi (Gaotai). Illustration by Author.

Fig. 65. The differential $d f$ of a photographic map $(P M)$ executes corresponding vectors $d f(X)$ and $d f(Y)$ on $f(P M)$. Vectors $X$ and $Y$ are stretched and transformed from one space to another. Content: 3D reconstruction of Köziciyerbişi (Gaotai). Illustration by Author.

Fig. 66. Glitches during the modelling of the tomb of Mahmud Al-Kashgari (1005-1102) in Opal, Kashgar. Software: Autodesk ReCap. Illustration by Author.

Fig. 67. Wireframe view of polygon mesh of the tomb of Mahmud Al-Kashgari (1005-1102) in Opal, Kashgar. Software: Autodesk ReCap. Illustration by Author.

Fig. 68. Delineation of how glitches occur during parameterisation $(S)$ between 2D parameter domain $\left(R^{2}\right)$ and 3D mesh domain $\left(R^{3}\right)$. Software: Autodesk ReCap. Illustration by Author.

Fig. 69. The successive decimation of polygons by $95 \%$ from the left column to the right. Content: 3D reconstruction of Köziciyerbişi (Gaotai). Illustration by Author.

Fig. 70. Screenshot of the texture-mesh post-processing in Mudbox that offers fast solutions for inexpensive models. More complicated mesh models are resolved in Maya and 3ds Max. 
Xvi

Fig. 71. Hyve-3D's concave screen and the laptop station. Photos by Tomás Dorta. Adapted from Schnabel et al. (2016).

Fig. 72. Immersive and non-immersive Hyve-3D installations. In this research, remote collaboration has been tested between Melbourne-Montreal (Event I) and Wellington-Montreal (Event II). Illustration by Author.

Fig. 73. The number of participants involved in different workshop activities during the two-day workshop in Melbourne. It depicts the integration of the immersive platform of Hyve-3D into the production line for digital heritage content-making.

Fig. 74. 3D sketches at the end of remote collaboration between Melbourne and Montreal. Photo by Marc Aurel Schnabel.

Fig. 75. 3D sketches (green) at the end of a remote collaboration session. The first activity of the user is nearly always the imitation of contour lines and edges of mesh surfaces.

Fig. 76. 3D user sketches (green) together with the 3D model of Köziciyerbişi (Gaotai)

Fig. 77. Introducing the research to visitors at Te Papa Museum inside Hyve-3D. Photo by Sasja Mazurkiewicz

Fig. 78. Left. A visitor virtually travels through alleys by using the 'global navigation' which is calibrated by tablet orientation in 3D space. Right. A visitor is sketching while controlling his position through the local navigation of HYve-3D. Visitor motivation in 'public' engagement is more diverse than scholarly engagement at the previous workshop. Photos by Sasja Mazurkiewicz.

Fig. 79. Visitors engage with each other by navigating and sketching on virtual Kashgar models while being remotely connected to partners in Montreal. Photos by Author......

Fig. 80. Most visitors are guided to imitate architectonics (top) and a visitor taking the role of guidance from Montreal to influence more horse cart sketches (bottom). Photos by Author.

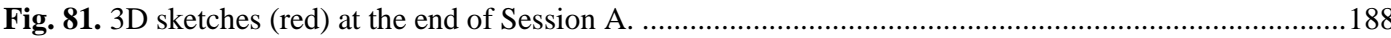

Fig. 82. 3D sketches (red) from Session A displayed on the model of Köziciyerbişi (Gaotai). .......................188

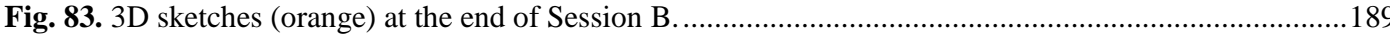

Fig. 84. 3D sketches (orange) from Session B displayed with the model of Köziciyerbişi (Gaotai)................189

Fig. 85. Overlapping Māori patterns are drawn by a New Zealander on the Kashgar model.

Fig. 86. Superimposing drawings (Session A) on polygon mesh model of the west junction in Köziciyerbişi (Gaotai). 1. Cobblestone, 2. Gate, 3. Horse cart, 4. Flowers, 5. Lantern, 6. Human, 7. Cat, 8. Shelter, 9. Text message. Illustration by Author.

Fig. 87. Superimposing drawings (Session B) on a 3D model of the west junction in Köziciyerbişi (Gaotai). 1. Knights, 2. Human, 3. Horse cart, 4. Lanterns, 5. Māori pattern, 6. Shelter, 7. Cat, 8. Flower, 9. Window with an eave, 10. Door with an eave, 11. Tree, 12. Gate. Illustration by Author.

Fig. 88. A visitor wearing a duppa hat, a tradition of Kashgar, captured in a scene after children engaged with the virtual models of the historic town. The visitor looking confused confronting the complexity of user-generated content.

Fig. 89. 3D user sketches together with the 3D model of Köziciyerbişi (Gaotai). Green lines represent the CAADRIA workshop event in Melbourne in March 2016. Red lines represent Session A and orange Session B in the public demo show at Te Papa Museum in March 2017

Fig. 90. Assemblage of 3D user sketches in two events. Green lines represent the CAADRIA workshop event in Melbourne in March 2016. Red lines represent Session A and orange Session B in the public demo show at Te Papa Museum in March 2017.

Fig. 91. Murray's " 3 key pleasures in cyberspace" are shown in relation to the medium-, the audienceand the content-centric factors.

Fig. 92. Systematisation of a process-oriented design procedure in digital heritage. It is called the MAC diagram in this research. Illustration by Author. 
Fig. 93. The focused part of the MAC diagram in this thesis within Chapter 5 that assesses alternate knowledge generation from participatory content-making. Other parts of MAC are greyed out. Illustration by Author.

Fig. 94. The outlook for the game engine software where an experiment platform is constructed.

Fig. 95. SfM photogrammetry models of narrow roads in Köziciyerbişi (Gaotai) are used to construct the experiment platform.

Fig. 96. MDA framework (Hunicke, LeBlanc, and Zubek 2004). Illustration by Author

Fig. 97. The top view of alleys in Köziciyerbişi (Gaotai) transformed into Unity as game objects (compare its alignment with the actual map of the neighbourhood on p. 142).

Fig. 98. Imperfect SfM models (with zoom scales $8 \mathrm{X}, 4 \mathrm{X}$ and $\mathrm{X}$ ) with glitches and holes to stimulate the player to project her/his understanding to complete them.

Fig. 99. Top view of brick collection checkpoints placed on the alleys.

Fig. 100. Brick collection (Type I - Visible): A brick collection checkpoint (cube) before collection (left). It is shrunk into pieces after collection (right). 'Brick Count' gives textual feedback.

Fig. 101. Brick collection (Type II - Hidden): From left to right, a brick collection checkpoint (a door) is guessed, approached, touched and glitched as visual feedback.

Fig. 102. Filling planes of the virtual Köziciyerbişi (Gaotai) model, highlighted with a fluorescent tone of purple to attract the player's attention to interaction.

Fig. 103. Top view of the positions of audio game objects used for generating the ambience of streets in Kashgar's Köziciyerbişi (Gaotai) (see street sound recording in Chapter 4.2.3).

Fig. 104. The 'selection activity' in the experiment platform consists of saving a texture pattern by casting a screenshot within the boundaries of the FP target frame and then placing one of the hitherto saved patterns onto filling planes.

Fig. 105. The flying FP character moves freely within the alleys, looking for patterns to patch on holes indicated with purple filling planes. A brick wall pattern is selected (left) and added in the library (right).

Fig. 106. The flying FP patches the units of a hole with a brick pattern (left) with the visual feedback illustrating the selected pattern from the library. Having called a new pattern, the FP continues the virtual masonry of patching holes with wall patterns (right)

Fig. 107. Mechanics and gameplay summary are showing participant activities in the experiment. Illustration by Author

Fig. 108. Further development of Fig. 93 p. 205: Association Rule Finding positioned in the box of 'Measured by...' as part of the inner feedback loop within the MAC (Medium, Audience and Content) diagram. Illustration by Author.

Fig. 109. Correlation of findings in Chapter 4 and 5 .

Fig. 110. The process of the experiment development in line with chapters and the MAC (Medium, Audience and Content) diagram. Illustration by Author.

Fig. 111. The participant is navigating in the photogrammetric model of Kashgar's narrow alleyways. ........228

Fig. 112. Patterns captured by the participant $(A)$ with pattern IDs, \#ID1.001 to \#ID1.006..........................230

Fig. 113. Confidence graph amongst 1 -itemsets associated with the 3 -itemset of ID1, i.e. \{ Metal Door, Timber Wall Structure, Brick Wall\}

Fig. 114. Confidence graph amongst 2 -itemsets associated with the transaction of ID1, i.e. \{Metal Door, Timber Wall Structure, Brick Wall\}.

Fig. 115. Confidence graph amongst 3 -itemsets associated with the transaction of ID1, i.e. \{Metal Door, Timber Wall Structure, Brick Wall\}.

Fig. 116. Matching the gameplay activities in the experiment with Bloom's Taxonomy used in cognitive and pedagogical studies. In the revised version of the taxonomy, there are remembering (retrieval of relevant knowledge), understanding (determining the meaning of graphic communication), applying (carrying out a procedure), analysing (detecting how the components relate), evaluating 
(making criteria-based judgements) and creating (combining elements to form a novel and original product) (Krathwohl 2002). Illustration by Author.

Fig. 117. Distribution of feedback loops from the inner to the middle and to the outer loop. Conceivably, ARF promotes the generalisation of the MAC diagram by allowing the communication of messages generated in different platforms at various times and remote locations. Illustration by Author.

Fig. 118. Further development of Fig. 57. Location of user-collected patterns (the participant: ID1) drawn on a map of Köziciyerbişi (Gaotai) that depicts the points of interest and courtyard integration levels which are analysed in Chapter 4.2.

Fig. 119. Defining the resolution, the frequency and the range values of user-generated content based on the RGB information visualised three-dimensionally. The open-source software, ImageJ, is used to generate the three-dimensional view of a two-dimensional pattern.

Fig. 120. Participants remotely (from Wellington, New Zealand) visiting the front outer courtyard of Masjid Wazir Khan, Lahore. Photo by Author.

Fig. 121. The cover page of The Museum of Gamers. The website is designed to contain all 'Decoding $X$ ' projects to be conducted in the future. It is an online conceptual museum where user-generated content defines the curation of exhibitions. The online platform is conceived to present the digital heritage of historical towns.

Fig. 122. Decoding Kashgar in The Museum of Gamers

Fig. 123. The responsive website of The Museum of Gamers demonstrate the quality of virtual environments supported by Sketchfab (https://sketchfab.com/), which is an online platform to publish 3D content with different interaction options such as viewing with a VR headset or a cardboard.

Fig. 124. A view of the virtual model of the narrow lanes in Köziciyerbişi (Gaotai)......................................315

Fig. 125. A view of the virtual model of a passage lane in Köziciyerbişi (Gaotai). .........................................316

Fig. 126. A view of glitches seen on the virtual model of a passage lane in Köziciyerbişi (Gaotai)................316

Fig. 127. A view of the virtual model of a timber door in Köziciyerbişi (Gaotai). ..........................................317

Fig. 128. A detail of the virtual model of a pavement section in Köziciyerbişi (Gaotai). Holes appear in the middle of a passage lane where light was limited during taking the photos.

Fig. 129. A detail of the virtual model of a brick wall section being demolished in Köziciyerbişi (Gaotai).

Fig. 130. A view of the virtual model of a narrow lane in Köziciyerbişi (Gaotai), with holes that appeared on the ground because of the shade of the surrounding walls

Fig. 131. A detail of the virtual model of a brick wall section being demolished in Köziciyerbişi (Gaotai).

Fig. 132. A view of the virtual model of a house entrance with timber structure extending from the wall in Köziciyerbişi (Gaotai).

Fig. 133. A view of the virtual model of a junction in Köziciyerbişi (Gaotai)

Fig. 134. A view of the virtual model of a narrow lane in Köziciyerbişi (Gaotai), with glitches that appeared on the texture of the geometry.

Fig. 135. A view of the virtual model of a narrow lane in Köziciyerbiși (Gaotai). .........................................321

Fig. 136. A view of the virtual model of a timber door in Köziciyerbişi (Gaotai). ...........................................321

Fig. 137. A view of the virtual model of a narrow lane in Köziciyerbiși (Gaotai)..........................................322

Fig. 138. A detail of the virtual model of a ground section in Köziciyerbişi (Gaotai), with a texture of the hexagonal pattern

Fig. 139. A detail of the virtual model of a house entrance in Köziciyerbişi (Gaotai)

Fig. 140. An upward view of the virtual model of mud-brick walls and timber doors in Köziciyerbişi (Gaotai).

Fig. 141. A view of the virtual model of a timber door with ground pattern detail in Köziciyerbişi (Gaotai). 
Fig. 142. A view of the virtual model of a timber door on a brick wall in a narrow lane in Köziciyerbişi (Gaotai)

Fig. 143. A detail of the virtual model of a brick arch over a timber wall in Köziciyerbişi (Gaotai)...............325

Fig. 144. A view of the virtual model of a timber door in Köziciyerbişi (Gaotai)...........................................325

Fig. 145. A downward view of the virtual model of a timber door at the end of a cull-de-sac in Köziciyerbişi (Gaotai)

Fig. 146. A view of the virtual model of a cull-de-sac in Köziciyerbişi (Gaotai). ........................................ 326

Fig. 147. A view of the virtual model of a gas meter hung on a brick wall in Köziciyerbişi (Gaotai)..............327

Fig. 148. An upward view of the virtual model of a junction of narrow lanes in Köziciyerbişi (Gaotai)........ 327

Fig. 149. A view of the virtual model of a timber extension overpassing a junction of narrow lanes in Köziciyerbişi (Gaotai)

Fig. 150. A detail of the virtual model of a timber structure that carries an overpassing extension of a house in Köziciyerbişi (Gaotai)

Fig. 151. A top view of the virtual model of a narrow lane in Köziciyerbişi (Gaotai)................................... 329

Fig. 152. A view of the virtual model of a junction of narrow lanes in Köziciyerbişi (Gaotai). ...................... 329

Fig. 153. A detail of the virtual model of a junction of narrow lanes in Köziciyerbişi (Gaotai)......................330

Fig. 154. A view of the virtual model of a timber door at the end of a cull-de-sac in Köziciyerbişi (Gaotai).

Fig. 155. A view of the virtual model of house extension at the end of a cull-de-sac in Köziciyerbişi (Gaotai).

Fig. 156. A view of the virtual model of a cull-de-sac in Köziciyerbişi (Gaotai). ........................................... 331

Fig. 157. A detail of the virtual model of brick walls in Köziciyerbişi (Gaotai)............................................332

Fig. 158. A view of the virtual model of a brick wall with a crack in Köziciyerbişi (Gaotai)..........................332

Fig. 159. A view of the virtual model of a mud-brick wall with glitches in Köziciyerbişi (Gaotai)................ 333

Fig. 160. A detail of the virtual model of rectangular cobblestones in Köziciyerbişi (Gaotai). .......................333 


\section{List of Abbreviations}

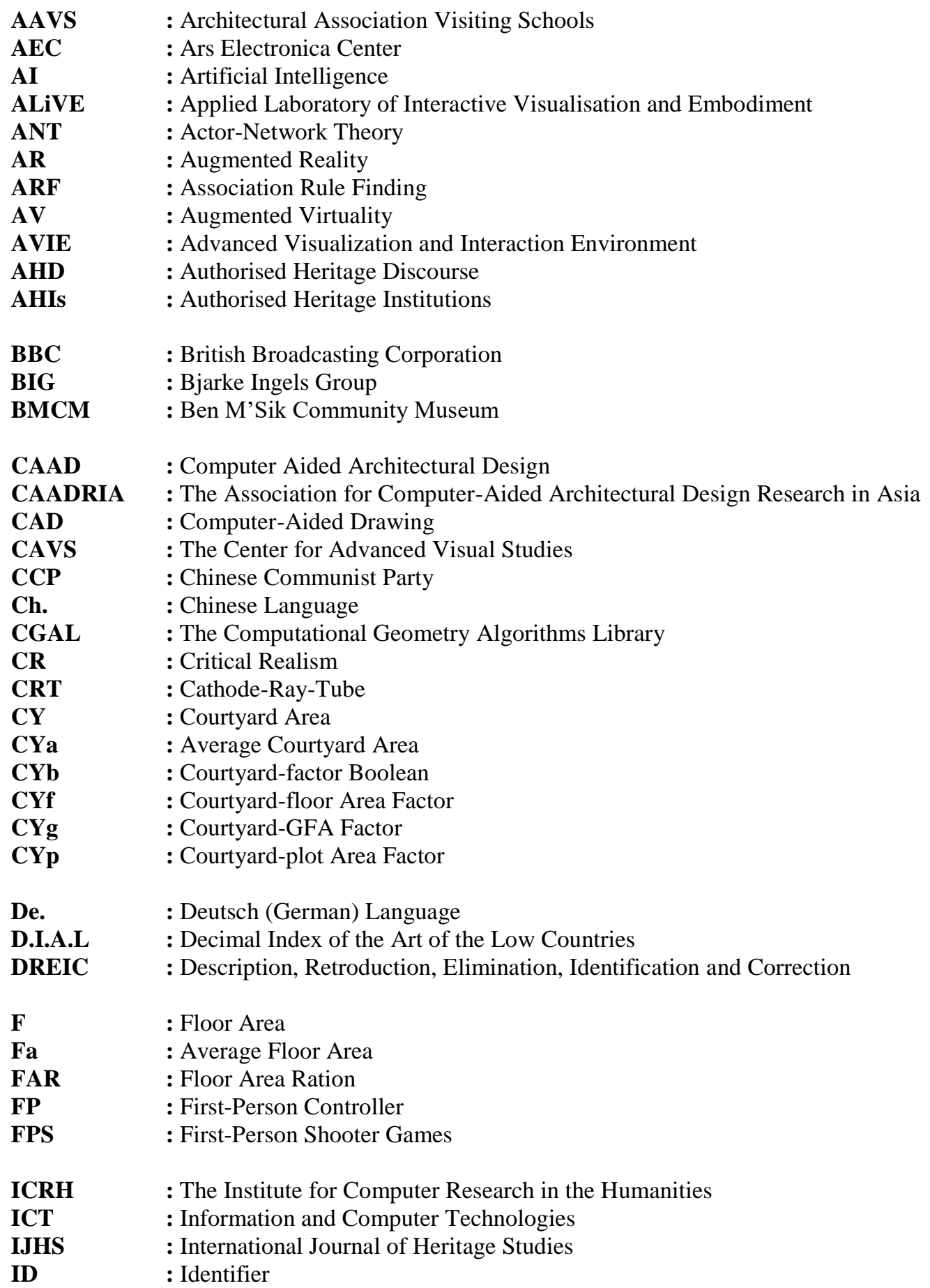


GAC : Google Arts \& Culture

GFA : Gross Floor Area

GFAa : Average Gross Floor Area

GIS : Geographic Information Systems

GLAMs : Galleries, Libraries, Archives and Museums

GPS : Global Positioning System

GRIPHOS : General Retrieval and Information Processor for Humanities Oriented Studies

GUI : Graphical User Interface

GVM : Guggenheim Virtual Museum

HBIM : Heritage Building Information Modelling

HCI : Human-Computer Interaction

HD : High-definition display

Hyve-3D : Hybrid Virtual Environment 3D

LAN : Local Area Network

MAC : Medium, Audience and Content

MCN : Museum Computer Network

MDA : Mechanics, Dynamics and Aesthetics

Mi. : Māori Language

MIT : Massachusetts Institute of Technology

MR : Mixed Reality

MoMA : The Museum of Modern Art

MuPop : The Pop-Up Museum

MUVA : Museo Virtual de Artes El Pais (El Pais Virtual Museum of Arts)
NISV : The Netherlands Institute for Sound and Vision
NTUA : National Technical University of Athens
NURBS : Non-Uniform Rational B-Spline

$\begin{array}{ll}\text { P } & \text { : Plot Area } \\ \text { Pa } & \text { : Average Plot Area } \\ \text { PARM } & \text { : Participatory Action Research Methodologies } \\ \text { PM } & \text { : Photographic Map }\end{array}$

RICHES : Renewal, Innovation and Change: Heritage and European Society

RNN : Recursive Neural Network

RRRE : Resolution, Redescription, Retrodiction and Elimination

RRREI : Resolution, Redescription, Retrodiction (and/or Retroduction), Elimination, Identification

RRREI(C) : Resolution, Redescription, Retrodiction (and/or Retroduction), Elimination, Identification and Correction

RV : Reality-Virtuality Continuum

SELGEM : SELf GEnerating Master

SfM $\quad$ : Structure from Motion

SPAB : The Society for the Protection of Ancient Buildings

U. $\quad$ : Uyghur Language

UAV : Unmanned Aerial Vehicle

UdeM : University of Montreal

UK : United Kingdom

USA : United States of America

UX : User Experience 
VASE : Virtual and Augmented Studio Environment

VM : Virtuality Matrix

VR : Virtual Reality

VROOM : Virtual Room

VUW : Victoria University of Wellington

V\&A : The Victoria and Albert Museum

WELL : Whole Earth 'Lectronic Link

XUAR : Xinjiang-Uyghur Autonomous Region 


\section{Glossary of Terms}

Actuality. The primitive form of the authenticity concept in heritage.

Alternate realities. A novel definition made for the TVX 2017 Conference. It refers to a combination of technologies "that can better guide the user to find its preferred alternate reality, offering appealing, personalized, context aware content selected or recommended from the multitude of the available material online" (https://tvx.acm.org/2017/participation/full-and-short-papersubmissions/).

Alternative knowledge. Undiscovered relations in a database of user-generated content produced as a result of creative engagement. For example, the international news about the protests in Kyiv in 2014 showed the people of the Ukrainian capital under extreme violence and miserable conditions. However, the new media theorist Lev Manovich's investigation of Instagram pictures, which were tagged with where the reported violence took place, argued 'alternative knowledge' that a vast majority had joyful time at the same time and the conditions were not as adverse as displayed in media (see Chapter 3.4.3). In this thesis, it refers to the alternative knowledge of heritage that goes beyond appearances.

Association Rule Finding (ARF). A well-known method in data mining. Two other equivalent terms are association rule mining in finance and association rule learning in machine learning and artificial intelligence (AI). In this thesis, 'finding' means to distinguish its novel usage in digital heritage from financial analysis and AI research.

Authenticity. A disputed term for describing undisputed credibility. This thesis holds the view that today it is less important to come to an ultimate and immediate agreement on the authenticity issue in heritage than it is to reframe it as a process of construction and agreement between conflicting views.

Creative; content making / engagement. The act of visual content-making via public engagement within virtual environments.

Critical Realism. A philosophical movement that derives from a "Realist Theory of Science" by Roy Bhaskar (Bhaskar 1978; Bhaskar 1998; Hartwig 2007, 96). It criticises the positivist empirical realism and the constructivist transcendental idealism, developing a philosophy of 'transcendental realism' that gives an ontological and epistemological background to Critical Realism. Although Bhaskar's philosophy initially focused on open-systemic social phenomena in theoretical studies, other researchers transformed its principles for use in applied research. The critical realist division of domains into the real, the actual and the empirical equips this research with a scientific approach to study the progression from actuality and to virtuality.

Decoding. A dynamic, reciprocal and creative process. In semiotics, 'decoding' involves more than basic recognition and comprehension of what a text says; it includes the interpretation and evaluation of its meaning concerning relevant codes (Chandler 2002, 175). The British sociologist Stuart Hall defines the moment of decoding as "the moment of reception [or] consumption... by... the reader/hearer/viewer", which is regarded by most theorists as "closer to a form of "construction"" than to "the passivity... suggested by the term "reception"" (Chandler 2017).

Digital. Noun (uncountable). Refers to 'the' digital instead of being used as an adjective. Here, it represents a research approach that focuses on the 'digital' and the 'heritage' respectively so that the digital value is not entirely dominated by the heritage value.

Event I. First experiment in the thesis, referring to the first part of a workshop at CAADRIA 2016 Conference in Melbourne. See also EVENT III. 
xxvi

Event II. Second experiment of the thesis. It is a public demo show at Te Papa Museum in Wellington in 2017.

Event III. The third experiment in this thesis, referring to the second part of a workshop at CAADRIA 2016 Conference in Melbourne. So, Event III was a part of the same program as Event I.

Heritage, mediated/unmediated. In the RICHES Taxonomy (2014), mediated heritage is "selected, cared for and interpreted (curated) by designated experts within authorised heritage institutions," whereas unmediated heritage "is understood as heritage curated by individuals or groups of individuals (communities) not attached to authorised heritage institutions." Based on the use of new media technologies, it refers to "more democratic practices of designation and utilisation."

Köziciyerbişi. [Ch. 高台; pinyin. Gaotai; U. كوزنجيار ببشى]. The research focuses on a historical neighbourhood in Kashgar that is called Köziciyerbişi (Gaotai).

Participant. Used interchangeably and contextually with players, users and visitors to give the same meaning except referencing other research.

Player. See PARTICIPANT. Used particularly in the context of Event III that is about testing a gamelike experiment in Chapter 5.

Te Papa. The Museum of New Zealand [Mi. Te Papa Tongarewa]

Transcoding. A conceptual definition taken for granted from a definition made by Manovich (2001) in The Language of New Media. In this thesis, it enhances discussion while diagnosing the matters of digital heritage in Chapter 3. Given that, it refers to the exchange of norms and standards between the cultural layer and the computer layer.

User. See PARTICIPANT.

User-generated content. The creative visual outcome of participatory content-making.

Virtuality. An ancient philosophical term that here stands for the creative assemblage of individuals' imagination.

Visitor. See PARTICIPANT. 


\section{Publications during Candidacy}

** Fully double-blind peer-reviewed /* Peer-reviewed

The following is a list of publications having the work that is substantially modified to include in the thesis, except when otherwise is noted.

\section{Book}

Aydin, Serdar, and Marc Aurel Schnabel. 2017. Decoding Kashgar: China's Westernmost City on the Historical Silk Road. Wellington: DARA. ISBN: 978-0-473-39996-2.

Contribution, > $80 \%$

\section{Book Chapters}

** Aydin, Serdar, Marc Aurel Schnabel, and Iman Sayah. 2017. "Association Rule Mining to Assess User-Generated Content in Digital Heritage." In Future Trajectories. CAADFutures 2017, edited by Gülen Çağdaş, Mine Özkar, Leman Figen Gül and Ethem Gürer, vol. 724, Singapore: Springer. 231-251. doi: 10.1007/978-981-10-5197-5_13

Contribution, > 85\%,

Experiment results in this publication relate to Chapter 5.3 and 5.4 in this thesis.

** Aydin, Serdar, and Marc Aurel Schnabel. 2016. "The Museum of Gamers: Unmediated Cultural Heritage Through Gaming." In Cultural Heritage in a Changing World, edited by Jan Karol Borowiecki, Neil Forbes and Antonella Fresa. Cham: Springer. 125-141. doi: 10.1007/978-3-319-29544-2_8

Contribution, > $90 \%$

\section{Journal}

** Haslop, Blaire, Marc Aurel Schnabel, and Serdar Aydin. 2017. "Digital Decay." International Journal of Parallel, Emergent and Distributed Systems. 1-7. doi: 10.1080/17445760.2017.1390092.

Contribution, $<15 \%$

\section{Conference Proceedings}

** Abid, Mehwish, Serdar Aydin and Marc Aurel Schnabel. 2017. "Reclaiming Heritage by Retelling 'The Thing' in Virtual Reality: Decoding Walled City of Lahore." In Back to the Future: The Next 50 Years, Proceedings of the 51st International Conference of the Architectural Science Association (ANZAScA), Wellington, New Zealand, November 29December 2, 2017. 15-24

Contribution, $>40 \%$ 
xxviii

** Aydin, Serdar, and Marc Aurel Schnabel. 2017. "Glitch in the Museum: A Digital Heritage Project Rejecting Authorised Heritage Institutions.” In Rejecting/Reversing Architecture, Proceedings of LIVENARCH V, Trabzon, Turkey, September 28-30, 2017.

Contribution, > $90 \%$

** Aydin, Serdar. 2017. "Decoding Kashgar: Participatory Digital Heritage Making via Digital Online Interaction and Gamification." In Adjunct Publication of the 2017 ACM International Conference on Interactive Experiences for TV and Online Video (ACM TVX 2017), Hilversum, The Netherlands, June 14-16, 2017. 93-97. doi: $10.1145 / 3084289.3084291$

Contribution, $100 \%$

* Aydin, Serdar, and Marc Aurel Schnabel. 2017. "A Collective Intelligence Assessment Approach through Alternate Realities in 'The Museum of Gamers.' In Proceedings of the In-Programme Personalisation for Broadcast (IPP4B) Workshop, ACM TVX 2017, Hilversum, The Netherlands, June 14-16, 2017. doi: 10.6084/m9.figshare.c.3793435.v1.

Contribution, > $90 \%$

** Haslop, Blaire, Marc Aurel Schnabel, and Serdar Aydin. 2017. "Glitch Space: Experiments on Digital Decay to Remap the Anatomy of Glitch in 3D." In Protocols, Flows and Glitches, Proceedings of the 22nd International Conference on Computer-Aided Architectural Design Research in Asia (CAADRIA 2017), Suzhou, China, April 5-8, 2017. 591-600.

Contribution, $<20 \%$

** Schnabel, Marc Aurel, Serdar Aydin, Tane Moleta, Davide Pierini, and Tomás Dorta. 2016. "Unmediated cultural heritage via Hyve-3D: Collecting individual and collective narratives with 3D sketching." In Living Systems and Micro-Utopias: Towards Continuous Designing, Proceedings of the 21 st International Conference on Computer-Aided Architectural Design Research in Asia (CAADRIA 2016), Melbourne, Australia, March 30-April 2, 2016. 683692.

Contribution, > $35 \%$

** Aydin, Serdar, Marc Aurel Schnabel, and Tane Moleta. 2016. "Transcoding of Game Design into Museology: An Object-Oriented Perspective." In Inclusiveness in Design, Proceedings of 2016 Design Communication European Conference, Istanbul, Turkey, May 11-14, 2016. 375-384

Contribution, > $85 \%$

** Aydin, Serdar, and Marc Aurel Schnabel. 2015. "Decoding Kashgar." In 2015 Digital Heritage, Proceedings of the 2nd International Congress on Digital Heritage 2015, VSMM, EUROGRAPHICS, CIPA ICOMOS/ISPRS, Granada, Spain September 28-October 2, 2015. 471-472. doi: 10.1109/DigitalHeritage.2015.7419553

Contribution, $>90 \%$

** Schnabel, Marc Aurel, and Serdar Aydin. 2015. "Amphiboly of Digital Heritage." In 2015 Digital Heritage, Proceedings of the 2nd International Congress on Digital Heritage 2015, VSMM, EUROGRAPHICS, CIPA ICOMOS/ISPRS, Granada, Spain September 28-October 2, 2015. 619-622. doi: 10.1109/DigitalHeritage.2015.7419582

Contribution, $50 \%$ 
** Aydin, Serdar, and Marc Aurel Schnabel. 2015. "Fusing Conflicts within Digital Heritage through the Ambivalence of Gaming: Research through design for a digital heritage project." In Emerging Experience in Past, Present and Future of Digital Architecture. Proceedings of the 20th International Conference of the Association for Computer-Aided Architectural Design Research in Asia (CAADRIA 2015), Daegu, South Korea, May 2022, 2015. 839-848.

Contribution, $>80 \%$

** Aydin, Serdar, and Marc Aurel Schnabel. 2014. "Augmenting Kashgar." In Image \& Archive, Proceedings of Virtual Systems \& Multimedia (VSMM) 2014 International Conference, Hong Kong, China, December 9-12, 2014, 12-15. doi: 10.1109/VSMM.2014.7136661

Contribution, > $90 \%$

** Schnabel, Marc Aurel, Tian Tian Lo, and Serdar Aydin. 2014. "Gamification and Rule Based Design Strategies in Architecture Education." In ACTION!-Doing Design Education. Proceedings of the 10th DesignEd Asia Conference 2014, Hong Kong, China, December 2-3, 2014. 1-11. doi: 10.13140/2.1.5150.3689

Contribution, > $30 \%$

** Aydin, Serdar, Tian Tian Lo, and Marc Aurel Schnabel. 2014. "Gamification of Shape Grammars - Collaborative and Participatory Mass-Housing Design for Kashgar Old Town." In Fusion, Proceedings of the 32nd International Conference on Education and research in Computer Aided Architectural Design in Europe (eCAADe 2014), Volume 1, Newcastle, UK, September 10-12, 2014. 603-612.

Contribution, $>40 \%$ 


\section{Acknowledgements}

It was in 2013 and the School of Architecture, The Chinese University of Hong Kong (CUHK) offered me a scholarship to study a $\mathrm{PhD}$, with the support of my primary supervisor Prof Marc Aurel Schnabel, who was teaching there, Prof Ho Puay Peng (何培斌), who was the head of school and Prof Tsou Jin Yeu (郎經宇), who was also supervisor. My gratitude to these three excellent academics is immense and their support gave me the opportunity to undertake a long journey from Turkey to Hong Kong, SAR (China).

My motivation came from Mike Knight who supervised me for a Master's degree at the University of Liverpool (ULiv), UK. Mike encouraged me to develop further my thesis which was about "A Parametric Quasi-Grammar for Generating a Flexible Urban Design Tool." I am grateful for his advice to continue with a PhD. To do so, I had to find a useful context since design-related works without a context are ill-defined. I had two historical cities in mind that reflected my keen interest in Islamic patterns and architecture. In fact, this personal interest is the admiration of my father's work that is to make traditional timber furniture for mosques in Turkey. The two historical cities were Kashgar in China and Mostar in Bosnia-Herzegovina. Considering that Kashgar is populated by a Muslim ethnic group in China who speak a Turkic language called Uyghur (U.) that I can understand, and that I wanted to do my PhD in Hong Kong, Kashgar was more appealing. Presented with this idea in the proposal, the architectural significance of Kashgar also intrigued Marc Aurel to supervise my work at CUHK.

Having started with shape grammar research to design a 'great' variety of Kashgar's architectural language based on computational methods, I initially had little interest in digital heritage. However, the context of Kashgar with unique circumstances and opportunities moved the course of this research from a lab-based computational design approach with a problem at hand, to a dirty explorative style of meandering around the objective of its digital preservation. In this case, adventurist 'designerly way of knowing,' once again, won over scientific and rational decision-making.

September 2013, just a month later than I started my $\mathrm{PhD}$ at CUHK, was the first time when I met a digital heritage community on an academic platform. It was the LuxLab 
xxxii

workshop of Sarah Kenderdine and Jeffrey Shaw, held at their Applied Laboratory of Interactive Visualisation and Embodiment (ALiVE) in the Hong Kong Science Park premises of the City University of Hong Kong. It was a half-hour walk distance from the CUHK campus and at that time I, as an architect only interested in computational design, could never imagine to finally write a $\mathrm{PhD}$ thesis that is much related to the rigorous research of Sarah and Jeffrey. Many thanks for their inspiring works of art and digital heritage that provided me the substantial advantage to view the field of digital heritage at a close range. In this connection, I owe thanks to researchers of digital heritage from a background of computer-aided architectural design and research, including Prof Sambit Datta, Prof Erik Champion, Prof Andre Brown, Prof Thomas Kvan, Prof Bharat Dave and Prof Yehuda Kalay whose work influenced me and this thesis.

Eventually, I decided to leave Hong Kong for New Zealand to follow Marc Aurel who got a new position at the School of Architecture, Victoria University of Wellington. For this opportunity, I would like to thank the Pro Vice-Chancellor Prof Mike Wilson who contracted with Marc Aurel for two transferring $\mathrm{PhD}$ candidates from Hong Kong to Wellington (the second one being Sky Lo, Tian Tian). Without this, it may not have been possible to follow our supervisor all the way to the 'Middle of Middle-Earth.'

Weeks after moving to Wellington, Marc Aurel and I attended the 2015 Digital Heritage Congress in Granada, Spain the exact antipode to New Zealand. Along the way, I travelled through one of the hottest places in the world in autumn (Kashgar) and the coldest (Astana, Kazakhstan). The sudden temperature change and long-distance travelling weakened me before attending the congress. However, it was no encumbrance; I was thrilled by the diversity of scholarship surrounding digital heritage. We presented two papers that received excellent feedback and the following interest that I became sure to continue with what I was about to do in New Zealand.

Moving to a new place I am blessed to be supervised (secondary) by Tane J. Moleta whose patience for listening to me and my research ideas all the time, and for bread baked and brought from home to feed me while writing this thesis. His support provided great confidence to spell out my designerly capabilities in research.

Of three field trips, I conducted the first one with the support of Design Research Society's (DRS) Student Bursary granted in 2015. I would like to thank Pazlat Mutallip (帕 孜来提・木特里甫) and Yakupjan Ismayil (亚合甫江・司马义) for their help during my field trips. I managed to go to Kashgar for the second and third field trips thanks to the Faculty Strategic Grant, SEAD, Victoria University of Wellington. During these fieldworks, I met so many great people and made terrific friends. Without their assistance and guidance in 
Kashgar, I would have been able to achieve obtaining the necessary documentation done in a short time.

Scott Meekings helped in coding for interactive relations between game objects in Unity (Chapter 5.2). Iman Sayah did the data processing in MATLAB (Chapter 5.3). I thank both of them for their collaboration. I also thank Blaire Haslop for pondering together about 'glitch' in digital architectural design during her master's degree at VUW. After all, she was the 'glitch girl' of the school with her inspiring visual work that inspired aspects of this thesis (Chapter 3.4.2). Blaire and Scott were part of Marc Aurel's 2016 master's thesis group together with Claudia van Velthooven and James Holth. I shall thank them for their thoughtprovoking use of digital design methods.

There are colleague-friends I will thank for their intellectual support and friendship from CUHK: Sky Lo Tian Tian, Carol Dai Qun; and from VUW: Zhang Yingyi, Mint Penpisuth Wallace, Harry He Yi, Shuva Chowdhury, Jasim Azhar, Rehan Shaikh.

I would like to thank the Ministry of Education in Turkey for first providing me the opportunity to study a master's degree in the UK. Without their YLSY scholarship, I might not have had any other financial means to start the journey of my postgraduate research in the first place.

It cannot be stressed enough that I am in debt to Marc Aurel for a great experience of doing research together and for everything I learned from him. He was a friend, colleague and supervisor. I cannot imagine any better candidate-supervisor boundary than what he drew between us. If the future students of the School of Architecture at Mardin Artuklu University in Turkey will think that I am a good teacher, it is because I learned almost everything from Marc Aurel. If not, then I have not translated his guidance correctly. Thank you, Marc!

Lastly, there are my people who do not need my formal acknowledgement. However, it is such an honour to thank them here. I thank my mother, Tülin, for never hanging up the phone before I do when I call her from far away because her heart does not let her. Such a simple gesture makes me the most energetic person on this planet. I thank my father, Osman, for trusting me without limits. He is my wildcard character and most valuable consultant when it comes to critical decision-making. I thank my sister Elanur for WhatsApp-ing me the pictures of her math questions and cakes that she bakes at home. They never distracted me while writing this thesis but somewhat filled my mind with the feeling of being at home. I thank my brother İlker for looking after the above members of the family. When I am back, it will be his turn to go out and explore. Finally, I thank my aunt Seyhan, my cousins Enes and Aysunur, and my dear Şükran for loving me. I love you too. 


\begin{abstract}
Digital tools have become critical instruments in preserving and communicating the value of heritage as important cultural expressions of the past. A consequence of digitalisation is the democratisation of heritage institutions, such as museums, which are found to value increasingly new types of content and new profiles of audiences. Digitisation plays a vital role in the alteration of the convictions of the heritage field to 'materiality' and 'actuality.' Although researchers acknowledge the significance of digital heritage in leading us into new ways of expressing 'authenticity' and 'virtuality,' studies have been confined to heritage activities comprised of digital documentation, representation and dissemination. Previous studies have reported on the role of public engagement in digital heritage which is criticised as consumptive, passive, guided and descriptive. Instead, the motivation of this research is to explore a new role that is 'generative,' 'active' and 'creative' for the production of heritage knowledge.

This dissertation demonstrates an innovative digital design approach to creative and participatory content-making in digital heritage. The research investigated the use of creative content generated collaboratively for knowledge production and acquisition in architectural heritage and tested in Kashgar, the westernmost city in China. The research conceives an interdisciplinary methodology, integrating design with the standard activities involved in digital heritage.

This dissertation is undertaken using different digital media equipment for collaboratively expressing authenticity and virtuality of heritage information. The research examines the role of creative engagement for constructing digital heritage. Creative engagement in a hybrid immersive virtual reality environment is experimented with and findings are analysed qualitatively. Then, to measure the outcome of creative engagement quantitatively, a well-known technique in data mining is used to expose undisclosed patterns. It is the first time in digital heritage that a study employs association rule mining to interpret user-generated content. The qualitative findings of two initial experiments are synthesised with quantitative results of the third experiment to investigate how the creative contribution of people in content-making is generalizable.
\end{abstract}


xxxvi

The investigations foster the advancement of research and practice in digital heritage beyond the frontiers of current knowledge. There is a number of fields that can apply the results of this research, including cultural heritage, computer-aided architectural design, museology, new media, TV, education, MOOC, streaming, as well as culture and game studies. 




\section{Chapter 1. Introduction}

In my household, well-prepared tea is an essential element of our typical breakfast table. My father would drink his tea from the same glass teacup since as early as I could remember. For a long time, it bore the weight of use, with scratches growing by the year, and as such my mother felt slightly embarrassed when a guest visiting her home were to notice the old glass on the cupboard. Meanwhile, she bought a new breakfast cup for her husband as a gift and as a replacement for the old one. Left idle in the cupboard, the old glass started disturbing my mother's plans from getting more new utensils, so she wanted to be rid of it entirely. Having become aware of this idea, my father vehemently opposed it because the old cup was an authentic object that was given by his mother a long time ago. Then started long days of conversation and debate around the issue of heritage in our house. To cut a long story short, the old glass teacup no longer occupies a space in our kitchen. Though the teacup has not become lost either, it took a very long while for my parents to relinquish discussion on this matter. Since then, the quarrel of how to preserve a domestic piece of heritage has remained unresolved. Meanwhile, I have studied digital heritage to build this thesis. Our case of a domestic teacup provided me with my first insights on the role of heritage in our lives, situated in a very 'authentic' experience.

In Heritage: Management, Interpretation, Identity, Howard (2003) asks "What is Heritage?" The question yearns to bring coherence to the theoretical landscape of heritage. To illustrate his point, Howard calls for an applied context:

To act as a clear reminder that heritage is about people, write a list of the things which you regard as your most precious heritage - the things which you are keen to pass on to your legatees. These things may be as universal as clean air, or as personal as your stamp collection, as concrete as a house or as abstract as a philosophy. What matters so much you take active steps to conserve it? (13)

Central to research in heritage is the contextualisation of 'theoretical' objectives, which specifies 'what matters.' As a field that has evolved from heritage, the scholarship of 'digital 
heritage' presents multiple goals and definitions. For the past two decades, a number of researchers have worked to define what digital heritage is. Cameron and Kenderdine (2007) bring together articles that present a theoretical appraisal of digital media applications used in authorised heritage instutitions (AHIs), such as museums. Parry (2007) covers an extensive overview of digital heritage that has roots in early museum computing activities in the 1960s. Addison (2000) defines digital heritage as an "emergent" discipline made of three key processes, namely documentation, representation and dissemination. UNESCO (2003) defines digital heritage as a rapidly growing "new legacy" that recognises the world's heritage as "resources of information and creative expression" [emphasis added]. To summarise, digital heritage is an evolving scientific phenomenon that positions the fusion of 'discursive novelty' and 'effective innovation' central to the appraisal of cultural heritage. In this regard, Howard's question of "what matters so much?" for novelty and innovation has a role of guidance regarding its emphasis on the contextualisation of matters in applied research.

Digital heritage has proved an essential literary genre in computer-aided architectural design and research (CAAD). Recently, there has been an increasing interest in involving digital heritage as one of the main topics in CAAD conferences, such as eCAADe (Education and research in Computer Aided Architectural Design in Europe), CAADRIA (The Association for Computer-Aided Architectural Design Research in Asia) and CAADfutures (Computer-Aided Architectural Design Futures). Research about the architectural interpretation of digital heritage concentrates on different aspects including 3D documentation and visualisation. Representing the CAAD community, Affleck and Kvan (2005) and Kalay, Affleck and Kvan (2007) contribute to the theorisation of digital heritage. Datta and Beynon (2014) discuss the role of 3D reconstructions in understanding the value of tangible architectural heritage. Webb and Brown (2011a; 2011b;2016) coin the expression of "digital forensics" as a tool for augmenting architectural analysis. In Playing with the Past, Champion (2011) relates digital heritage to the unified concepts of architectural place-making and serious games.

Although these scholars recognise the value of transferring architectural methods into digital heritage, the effort remains indistinct as to what degree the three key components of digital engagement are involved, which are immersiveness, agency and transformation. The current research and practice in digital heritage focuses on the realist perfection of imagery that leads to technical improvement rather than understanding the value of digital heritage content. A design approach is proposed in this research to close the gaps between the use of medium, the role of audience and the production of content. The role of design is 
investigated as to what extent creativity of users can be integrated with the general purposes of digital heritage, which are documentation, representation and dissemination. Previous CAAD studies of digital heritage have insufficiently concentrated on design despite the fact that it is a fundamental activity of architects. Few studies have proposed design in digital heritage. Schnabel et al. (2016) use virtual reality (VR) to examine creativity and intuition that are derivates of design. In general, the existing accounts fail to resolve the connection between design and research. However, several studies have focused on the visionary role of design in research about architecture (Glanville 2007; Fischer 2017a). In 2000, the Faculty of Architecture Delft University of Technology organised an international conference that positioned the concept of "research by design" central in the production of new knowledge through design (Van Ouwerkerk and Rosemann 2001).

Given the importance of design, this research sought to understand, in Howard's (2003) words, "what matters so much?" in studying digital heritage from an architectural perspective. This research appended design as a novel procedure to the standard activities of digital heritage. The design challenge in this research was to make use of creative engagement as a knowledge generation tool and test it in the context of Kashgar, the westernmost city in China. Due to specific circumstances of the context, the digitisation of Kashgar's heritage incorporated an additional layer of challenges for the generalisation of knowledge. By testing the claim that there is exceptional knowledge implicit in the creative outcome of user engagement, the research aimed to address the following questions:

\section{Q: How does a digital design approach steer and diversify creative} engagement in digital heritage?

Subsequently, the thesis addresses three sub-questions:

sQ1: How can architectural heritage be translated into creative digital forms?

sQ2: How can an immersive digital heritage application convey virtuality to the public via creative engagement?

sQ3: What alternative knowledge of virtuality lies in participatory contentmaking?

The main question $(\boldsymbol{Q})$ aims at understanding 'what matters so much' in translating heritage information into digital. In what ways does digital design depart from the traditional logic of heritage research? What happens when a design role is granted to people to generate creative content in digital heritage? Furthermore, how does digital design intensify the 
experience of people with digital heritage? With the main question, this research challenges the notion of treating user engagement with heritage information from a consumptive perspective.

The sub-question $1(s \mathbf{Q 1})$ relates the specific context of Kashgar to the matters of digital representation. It is precisely aimed at understanding how creativity can be resourced from the limitations of heritage embeded in its actuality when it is translated into the domain of virtuality. With this question, this research is precisely interested in the assemblage processes of digital reproductions. The aim of the sub-question $2(s Q 2)$ is understanding the development of virtuality when people intuitively collaborate for content-making in digital heritage. What are the advantages of inviting people to take part in alternate realities for content production in heritage? The primary research interest for asking this question is to know the impact of immersiveness on creativity and to qualitatively measure the role of creativity for developing a sense of virtuality. Finally, the sub-question 3 (sQ3) is about deciphering the relational codes of creative content produced by people. In a more democratic view of content-making, what is the originality of accepting the involvement of people and their creative activities in digital heritage? In this logic, what layers of knowledge exist in user-generated content and provide an alternative understanding of what heritage tells us?

In this research, creative engagement of people is analysed and synthesised through different modes: (1) immersiveness, (2) agency and (3) transformation. Data are collected from three events of experiments, examined with regards to alternative knowledge production of virtuality:

- Event I: The experiment of Event I was part of a workshop at the CAADRIA 2016 Conference in Melbourne. Workshop participants engaged with Kashgar's digital reproduction in a hybrid VR environment. Data was retrieved from usergenerated content during the workshop.

- Event II: The experiment of Event II was part of a public demo show at the Museum of New Zealand [Mi. Te Papa Tongarewa] during Te Papa Talks 2017. Museum visitors expressed their creativity in collaborating in a scenario of content-making in a hybrid VR environment. Data consist of the records of usergenerated content.

- Event III: The experiment shown in Event III took place during the workshop at CAADRIA 2016 of which Event I was also a part. This experiment did not employ the hybrid VR environment in contrast to Event I and II. Instead, an adhoc experiment platform designed for this research was used to retrieve further data about creative engagement. 
All experiments focused on the outcome of user participation within different modalities of digital engagement. 3D reconstructions of narrow roads in Kashgar were a source for the original content with which participants engaged through intuitive processes of content-making. Data that sprang from Events I and II were 3D sketches drawn in a hybrid VR environment that was one of two experiment platforms in this research. Data in Event III were image-based, made of $250 \times 250$ pixel thumbnails that were generated by the participant during the creative activities on the other experiment platform. These data from Events I, II and III refer to 'user-generated content' and 'the outcome of creative engagement' throughout the thesis. Unstructured interviews and observations are used to support these datasets.

No previous study has investigated 'creativity' in digital heritage. Much research has registered knowledge production in terms of 'actuality,' 'materiality' and 'accuracy.' The existing accounts fail to resolve the contradiction between reality and virtuality in the dissemination of digital heritage. The research to date has tended to focus on 'heritage' rather than 'digital,' reality rather than virtuality and learning rather than creating. With data collected from Events I, II and III, this research investigates a progression of levels of digital engagement that lead to creativity. Linking reality to virtuality, the outcome of creative engagement is analysed via qualitative assessments in Events I and II. The research synthesises the quantitative analysis of the results obtained from Event III with the qualitative findings of previous events. The primary motivation was to explore thresholds of a progression from 'immersiveness' to 'agency' and from 'agency' to 'transformation' during virtual journeys inside the replicas of narrow roads in Kashgar. With this, the research explores a new role for user-generated content in digital heritage platforms. Instead of being consumptive, passive, guided and descriptive, this role is generative, active, creative and guiding the production of heritage knowledge. For this, the research concentrates on the transformation of content.

Studying the creative outcome of engagement in digital heritage is a relatively recent line of research. The interdisciplinary digital design approach of the research blends methods from computer-aided architectural design, digital heritage, new media and game design. The thesis uses diagrams to explicate the processes, the methods and their connections, supplementing with tables that summarise finding. Fig. 1 provides an overview of the flow of chapters, with inter- and intra-connection between chapters and sub-questions. This dissertation is composed of five themed chapters, in addition to this introduction and the last chapter of conclusions. 
Chapter 2 gives an overview of a non-linear history of digital heritage. Preceding this research, different authors have studied the history of digital heritage (e.g. Jones-Garmil 1997; Williams 2010; Parry 2007). Traditionally, the early museum computing activities in the 1960s are vital to the history of digital heritage. For this thesis, 'The Birth of Museum Computing' (Subchapter 2.2) is extended to the early nineteenth century developments in AHIs, including the themes of the binary codes and the role of plurality in heritage. This chapter reviews the influence of information theory on the rise of museum computing activities alongside 'The Birth of the New Museology' (Subchapter 2.3) movement that stimulated the inclusion of new media tools in museums as part of their newly raised responsibility for serving the society. 'The Birth of the Virtual Museum' (Subchapter 2.4) demonstrates the crisis years of VR in AHIs, whereas 'The Death (Deaccessioning) of the Virtual Museum' (Subchapter 2.5) depicts several topics in the course of the development that digital heritage is taking, or about to take.

Referring to a correlation between the "computer layer" and the "culture layer" made by Manovich (2001), Chapter 3 is about the resolution of the two layers blended in digital heritage. The analysis breaks the abstract concept of digital heritage into its formative components with the present 'Matters of 'Transcoding' in Digital Heritage.' The main matters in a digital heritage study include that of context, medium and audience, with each given in a separate subchapter. Each of these subchapters contains the sections of (1) 'Background,' (2) 'Theme,' (3) 'Case' and (4) 'Criteria.' The last one critically engages with other sections and appends a sub-question. In the subchapter 'Matters of Context,' architectural heritage of Kashgar is introduced with a qualitative review of cases from China. The criteria driven by the matters of 'context' help redefine the sub-question 1 ( $\boldsymbol{Q} \mathbf{Q}$ ). Subsequently, the subchapter 'Matters of Medium' makes a qualitative assessment of the works by two prominent scholars of digital heritage and new media, Sarah Kenderdine and Jeffrey Shaw. From this qualitative case study of matters related to 'medium,' sub-question $2(s Q 2)$ restates an enquiry of a relationship between immersiveness and virtuality. With regards to the subchapter 'Matters of Audience,' the works of Lev Manovich are assessed to show how digital content made by people can reveal alternative knowledge. Rendering the relationship between creative content-making and knowledge production about virtuality, this subchapter of 'audience' matters relates to sub-question 3 (sQ3). In the last subchapter, the 'Matters of Research' synthesises the previous subchapters and provides connection to the themes of the forthcoming chapters. It theoretically reformulates the critical matters for meaningful explanation specific to this research. It provides a scheme of the research approach that helped the thesis critically engage with digital heritage discourse 
while reinforcing it with methodological principles to be followed in the rest of the work. Showing an evolution of Critical Realism from theoretical studies to applied research, the chapter presents the followed principles of RRREI (Resolution, Redescription, Retrodiction, Elimination, Identification).

In Chapter 4, three sets of methods are explored. The alleyways of Kashgar are digitally 'documented,' 'visualised' and 'disseminated' with a scope of interest in a specific neighbourhood. With 'Documentation' (Subchapter 4.2), three fieldworks are explained as to how the data recorded in Kashgar has been documented and used in this research. In the subchapter of 'Representation,' the assembly line of digital geometry processing is laid out in details, making a direct relation to sub-question 1 ( $\mathbf{Q Q 1})$. Afterwards, 'Dissemination' (Subchapter 4.2) gives the details of Event I and Event II where two experiments address an enquiry of sub-question 2 (sQ2). Event I was carried out during a workshop at the CAADRIA 2016 Conference in Melbourne, whereas Event II was part of a public demo made at the Museum of New Zealand Te Papa Tongarewa in 2017. This subchapter pertains to exploring a relationship in-between and progression from immersiveness to agency, i.e. playing a meaningful role, fundamental to creative engagement. Proceeding in parallel with the structure of Chapter 3, Chapter 4 closes with a final subchapter that synthesises the previous subchapters as well as relating to the subsequent chapter. With this, 'Design' is appended to the standard procedures of digital heritage, i.e. documentation, representation and dissemination. The subchapter 'Design' diagrammatically explicates a relationship between medium, audience and content, pertaining to immersiveness, agency and transformation, respectively. It highlights the deficiency of the field to dwell on creativity by relating an answer to sub-question $3(s Q 3)$ to be addressed in the following chapter. Therefore, the final subchapter summons background information for the experiment of Event III in Chapter 5 while synthesising the qualitative findings of Events I and II in Chapter 4.

Chapter 5 starts with the construction of a second experiment platform in Event III. 3D assets presented in Chapter 4 are a resource for building the experiment platform. The experiment makes use of gameplay that urges the participant to engage creatively with digital replicas of alleyways in Kashgar. The experiment is conducted with a participant from the workshop at CAADRIA 2016. A well-known technique in data mining is used to disclose patterns through quantitative findings. It is the first time in digital heritage that research has employed association rule mining to interpret user-generated content. Quantitative findings usefully supplement and extend the qualitative findings of the experiments conducted in the previous chapter. Quantitative experiment results are then 
synthesised with qualitative findings from experiments to investigate how the creative contribution of people in content-making is generalizable. The subchapter 'Synthesis' utilises the findings from the experiment to respond to the three sub-questions of this research. The synthesis consists of two divisions. The first is a retrospective synthesis of sub-questions ( $s Q 1, s Q 2$ and $s Q 3)$. The second synthesis is prospective and proposes opportunities for improvements of the experiment and the analysis techniques of Association Rule Finding. The synthesis of qualitative and quantitative findings and subquestions paves the way for how the subsequent chapter discusses the main question.

Chapter 6 discusses the results of this study as to how a digital design approach contributes to a fusion of 'discursive novelty' and 'effective innovation' in digital heritage. Searching both goals in a scenario of a real context promotes the generation of principles for 'creative engagement' in digital heritage. The quality of a contribution on 'discursive novelty' is assessed in connection to 'Matters of Research' in Subchapter 3.5. The function of the design approach is discussed. By synthesising the learnings from Subchapters 3.5 and 4.5 in experiment design, Chapter 5 summarises the role of design in the outcome of creative engagement in digital heritage. Furthermore, Chapter 6 explains the limitations and implications of this study.

Overall, the structure of this dissertation reflects an effort to distil succinct knowledge from a wide-spanning field of digital heritage. Fig. 1 renders the chapters constructed through logical connections. In addition, each subchapter in this dissertation is given a 'Subchapter Outline' to introduce the incoming content briefly. The sub-subchapters are called 'sections' throughout the thesis, e.g. 3.3.4 Criteria: Creative Engagement. Chapters 2, 3 and 4 are each divided into five subchapters that are aligned vertically, e.g. the content in 3.4 Matters of Audience and 4.4 Dissemination have parallels with each other. There are qualitative findings from two experiments given in 4.4.3 Workshop Outcome, 4.4.5 Qualitative Findings, 4.4.6 Challenge and quantitative findings from the third experiment in 5.3.4 Findings. The final subchapters 3.5 Matters of Research, 4.5 Design and 5.4 Synthesis comprise the backbone of the thesis, making vertical connections between chapters.

To summarise, the research goes beyond the frontier of current knowledge in digital heritage that is restricted to materiality and actuality, hence methodologies overconcerned with accuracy. This study fosters the advancement of research and practice in digital heritage by using an innovative interdisciplinary methodology, which bridges a number of fields including cultural heritage, computer-aided architectural design, museology, new media, TV, education, MOOC, streaming, as well as cultural and game studies. 


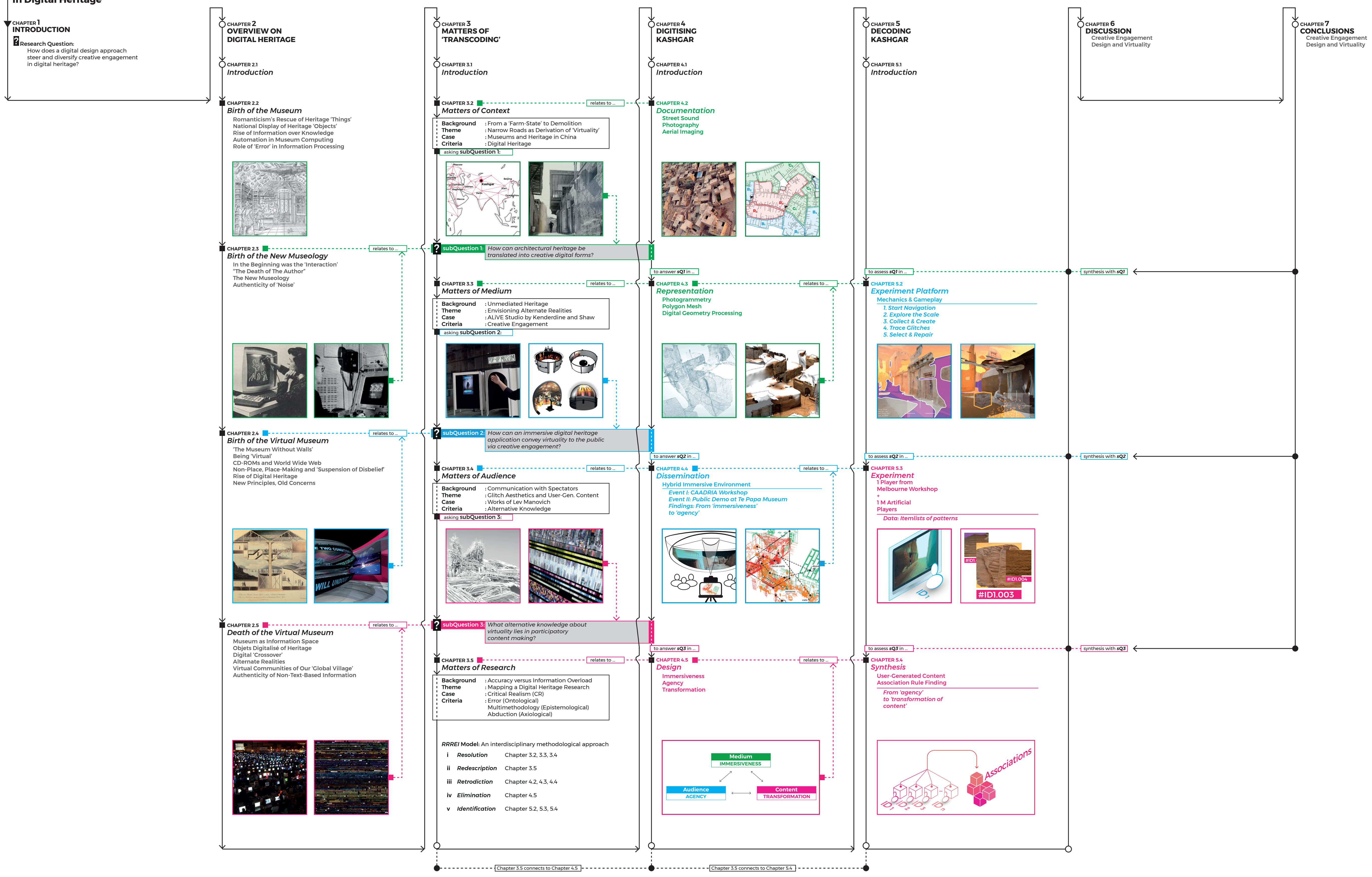





\section{Chapter 2. Overview of Digital Heritage: A History of the Virtual Museum}

We therefore need a different theatre, a theatre without spectators: not a theatre played out in front of empty seats, but a theatre where the passive optical relationship [...] is subjected to a different relationship.

- Jacques Ranciére, The Emancipated Spectator $(2009,3)$

\subsection{Introduction}

As introduced earlier, the term digital heritage embodies a multitude of concepts that correlate heritage resources and digital technologies. The primary operation of a digital heritage application is to recognise historical information by gathering data from real complex environments. The type of historical information varies from artefacts (tangible) to traditional practices (intangible). By examining various types of resources, operations of digital heritage combine the value standards of two distinct fields; however, they are prone to generating a point of conflict between the 'old' and the 'new,' the 'historical' and the 'modern' and the 'cultural' and the 'computational.' In the context of museums, Marchand $(2000,179)$ traces this struggle in a seventeenth-century 'quarrel' between the defenders of the ancient and the advocates of the modern. Her account documents the slow evolution of the modern in museums to achieve the appeal of antiquity's longevity. Likewise, Waterton and Watson (2013) identify a theoretical delay to grow a "critical imagination" [italics in original] in heritage discourse despite the novel means of dialogue brought about by new technologies.

A review of the literature reveals that heritage studies evolved from material-based views to politically-engaged social aspects of visualisation and authenticity. Waterton and Watson (2013) divide the theorisation of heritage studies into three groups; theories 'in,' 
'of' and 'for' heritage. Their classification is more of a chronological account for the last four decades of light theoretical debate than an evolution of a holistic understanding of the past, present and future of heritage. This background chapter critically summarises the history of AHIs through the progression of museums and other fields of influence.

Curiously, museums were amongst the first organisations for employing computers for their purposes. However, the early museum computing activities in the 1960s primarily concentrated on cataloguing. In the following two decades, museum computerisation was acknowledged as part of digital humanities. With the emergence of personal computers and virtual reality technologies in the 1990s and 2000s, new definitions replaced 'museum computing,' such as 'visual heritage' and 'virtual heritage.' Later on, even more profound engagement with heritage information enabled the transformation of virtual heritage into digital.

Digital heritage is a diverse field that includes researchers from archaeology, who rely on strict preservation guides, to game designers, who have been found to recompose heritage elements in virtual environments. This diversity of expertise, approach and opinion surrounding the field leads to confusion on the definition of 'digital heritage.' The lack of consensus on the definition of digital heritage could be an indicator as to why the biannual digital heritage congress has failed to meet for the third time in 2017 following the congresses in Marseille (2013) and Granada (2015).

Chronicling an overview history of digital heritage is unlike a linear timeline. Unfortunately, there is not a single inventor of a mathematical formula whose biography would yield a full picture. Many threads are related to the history of digital heritage. On the one hand, there are accounts from science fields that include computer history (Sutherland 1964), computer graphics (Burton 1967) and communication theory (Shannon 1948; Teyssot 2013). On the other hand, studies from the Humanities include heritage discourse (Waterton 2010), heritage management (Uzzell 1998; Howard 2003), museology (HooperGreenhill 1992; Witcomb 2003), museum computing (Parry 2007), museum history (Quiccheberg [1565] 2013); Impey and MacGregor 1985; Bennett 1995), and participatory museum (Weil 1999). Finally there is scholarship that links two sides, ranging from digital design (Schnabel et al. 2004), to exhibition design (Huhtamo 2002), game design (Borowiecki and Prieto-Rodriguez 2014; Champion 2015; 2016) and human-computer interaction (Kenderdine 2015), new media theory (Hayles 1984; Murray 1998; Weibel 1999; Manovich 2001), philosophy (Barthes 1988; Baudrillard 1994; Rancière 2007), virtual space design (Champion 2010) and cyberspace (Lévy 1997). 
The domain of digital heritage displays an evolutionary development in parallel with theoretical developments in science. An early attempt to provide a theoretical background is Theorising Digital Cultural Heritage (Cameron and Kenderdine 2007). Its introduction by Cameron and Kenderdine $(2007,1)$, who are both prominent authors in the field, relates transformation in culture to new media and vice versa. One of the advents of this symbiotic relationship is Manovich (2001) who correlates the "computer layer" to the "culture layer." Champion's $(2010 ; 2015 ; 2016)$ research links the two layers with a background in spatial understanding grounded in an architectural view.

Documentation, representation and dissemination are fundamental to heritage, digital and museum activities, respectively. The following sections in this chapter detail the intersecting correlations between them. It associates theories from heritage and digital. It joins heritage studies, information and computer technologies (ICT) and museology (or museum studies). This chapter will give a theoretical perspective that is organised using subheadings, i.e. the birth of 'museum computing' (2.2); 'the new museology' (2.3); 'the virtual museum' (2.4); and finally the death of 'the virtual museum' and its dissolution into information space. 


\subsection{The Birth of Museum Computing: Binary Codes of Heritage}

Subchapter Outline

Purpose: Several attempts have been made to give an overview of digitisation in authorised heritage institutions (AHIs). The museum computing activities in the 1960s are considered the opening remarks of digital heritage. This subchapter aims to extend the history of digital heritage beyond the technological affluence of the post-war era and to give an overview of binary perspectives in heritage and related fields.

Design/Approach: The subchapter is a comprehensive literature review on how digitisation entered heritage as a new perspective from the first manifesto of architectural heritage and the first museum in Europe to the rise of information over knowledge and the early museum computing activities.

Findings: The review shows that the rise of information over knowledge had a significant impact on the beginning of computational activities in AHIs. Despite the early enthusiasm for computer-led projects, the digitisation progress in AHIs, in effect, remained computer-assisted. The positioning of 'error' appears to be a primary concern of scientists who embark on solving realistic problems.

Limitations/Implications: This section discloses the early understanding of 'inclusivity' in heritage while research conducted in chronological order remains to be established. It completes the gaps in knowledge by overlapping historical events in different fields, but more direct correlations require further studies.

Originality/Value: This section extends the focus of writing a history of digital heritage, which has been confined to the early museum activities, to developments in different areas, such as research on information science, cybernetics, semiotics and early museum history.

Keywords. Romanticism; authorised heritage institutions; museums; museum computing; computer-assisted; error.

Bennett's (1995) seminal book The Birth of the Museum explores the ideological background of museums as AHIs. The book is widely cited across heritage scholarship, which seeks to assess the symbolic role of museums. Bennett's discussion is contingent on Foucault's notion of 'disciplinary societies.' He relates museum institutions to their political rationality by engaging critically with an overfilled history of their formation. This political entanglement is widely employed in the field and is about the present rather than the past of museums. It makes museum studies contingent on heritage discourse that involves theoretical discussions about the representational meaning of display. This 'meaningseeking' approach is related to our communication with heritage. In this framework, this communication is frequently analysed through phenomenology on the theoretical side, whereas museum computing can be best studied by reading the developments in information science, semiology and structuralism. The evolution of computerisation in heritage can be tracked down by reading the roots of structuralism through the works of early semioticians. 
The present section outlines a modest account of the history of architectural heritage, museums and museum computing, which is known today as digital heritage.

\subsubsection{Romanticism's Rescue of Heritage 'Things'}

There is little evidence that architectural conservationists of the nineteenth century had anything common with influential semioticians, Saussure (1857-1913) and Peirce (18391914). Nonetheless, historically, architects and their counterpart semioticians were motivated by the articulation of 'fixed principles' to analyse complex phenomena. On the one hand, Le Corbusier's five points of architecture were a system of 'spatial' configuration. On the other hand, Saussure's semiology was a linguistic organisation of 'signs.'

In the nineteenth century, the practice of architectural heritage relied on fixed codes that were assumed to organise meaningful encounter between a specific place ('spatial') and its meaning ('sign'). The manifesto issued by the Society for the Protection of Ancient Buildings (SPAB) in 1877 is acknowledged to be the first of its kind in heritage (Miele 2005). It sets up several conservation principles that have existed unchanged until today. Applicants for SPAB membership are required to sign an agreement with the conservation principles of the SPAB manifesto. The society explains that "the manifesto extends protection to 'all times and styles' and remains to this day the philosophical basis for the Society's work" [quotation marks in the original] (SPAB Manifesto [1877] 2018).

The SPAB manifesto is rooted in John Ruskin's (1819-1900) fascination with preservation of historical buildings, responding Viollet-le-Duc's (1814-1879) preference for restoration. There are methodological differences between the former representing the Victorian English approach for preservation and the latter the French practice favouring restoration (Emerick 2014; Howard 2003, 34). However, both architectural historians shared an objective that was to universalise a set of standards in heritage practice. They were prominent figures of Romanticism, Gothic Revival and universalism, yet nationalists in politics who exclusively addressed the taste of bourgeoisie (Loukaki 2008, 89).

Co-founded by William Morris, the SPAB preserved its stable position in heritage practice and theory for many years. The romanticism of John Ruskin, a friend of Morris, influenced the SPAB (Wells 2007). Ruskin was an advocate of phenomenology (Morgan 1988, 301). His concept of architectural conservation romanticises the preservation of literally every 'thing' found in architectural heritage sites (Ruskin 1849; Donovan 2008, 102; Ballantyne 2015, 211). Ruskin's “romantic rescue" of architectural heritage shares with Heidegger's phenomenology (Morgan 1988). To give a glimpse of his phenomenological 
16

portrayal of every 'thing,' an object is understood as a 'natural' subjugation of our experiences according to Heidegger $(1967,38)$. A 'thing' becomes an 'object' immediately after having achieved the function of "demonstration" (Heidegger, 1967, 26). Therefore, there is an authentic moment when a thing transforms into an object. This notion of authenticity generates 'aura,' a term coined by Walter Benjamin (1892-1942), whose thoughts are aligned with German Romanticism (Hanssen and Benjamin 2002).

The longstanding continuity of the SPAB manifesto lies in an enquiry for an artistic engagement evocative of the materiality. It reflects a desire to reveal deep historical structures of architectural heritage as universal knowledge for public and future generations. Later on, more principles, such as the Athens Charter (1931) and the Venice Charter (1964), ensured the continuity of the SPAB's doctrine. Both charters appeared in parallel with the introduction of new technological media. Following short-lived nickelodeons, the former was contemporary to the rise of the cinema, and the latter to the computer. Despite unceasing challenges posed by new media, architectural heritage seemed to have always trailed behind conventional appreciation of cultural conservationists, such as Ruskin.

Photography entered the museum space at the beginning of the nineteenth century, with a central concern in the recording. It was celebrated to capture the 'truth' more objectively than a Renaissance perspective painting (Walsh 2007, 22). The Victoria and Albert Museum (formerly the South Kensington Museum) in London photographed its collections for preservation as well as reproduction. The Victoria and Albert overcame traditional challenges against photography. The Victoria and Albert later inspired the museums in the USA (27). However, the pioneers of displaying reproductions instead of reals were German art historians urging museums to cast Greek and Roman sculptures "whiter" than their originals (Marchand 2000, 182; Borbein 2013). As it was almost impossible to bring the original pieces to Berlin, replicas were used to reflect the imperial/political purpose of dominance over far regions. It is these replicas that indicate the start of the growing political role of museums in parallel with the increased importance of public opinion for the credibility of power. As a result, there was a need for 'binary codes of heritage' that were sufficiently universal to bestow museums with the right to speak on behalf of a nation.

\subsubsection{National Display of Heritage 'Objects'}

During the Age of Enlightenment, the wealthy elite living in the European capitals of Venice, Vienna, Paris, and London experienced a rapid increase in living standards. Bourgeoisie exhibitions of the affluent were envisioned to display the finest objects in 
private collections. They were devoted to the cult of the ruling families of Europe. The Medici Palace is acknowledged to be the first museum of Europe, which exhibited alreadyacquired objects of the Medici family from fifteenth-century Florence (Hooper-Greenhill 1992, 11). Early 'cabinets of curiosity' were mostly exhibited in private rooms with paintings, taxidermy and so on, which were hung even from the ceiling (Fig. 2). These exhibition spaces had some ambiguity by definition. Before the modern institutionalisation of the 'public' museum, several other terms emerged to include 'cabinet of curiosities,' Wunderkammer, Kunstkammer, Guardaroba and even 'library' (Carbonel 2004, 3). In the nineteenth century, the public sphere received increased priority in politics. Moving from an imperial statehood to a national, the political climate changed the role of museums from being about the elite to being for the nation. The museum was reimagined through the lenses of the public instead of the royalty. Museums became "governmental instruments" representing the whole nation (Bennett 1995, 28). The proponents reasoned that museums would help cultivate public coherence within the national state forms (Marchand 2000, 182) while transforming "things into objects" (Desvallées and Mairesse 2009, 61).

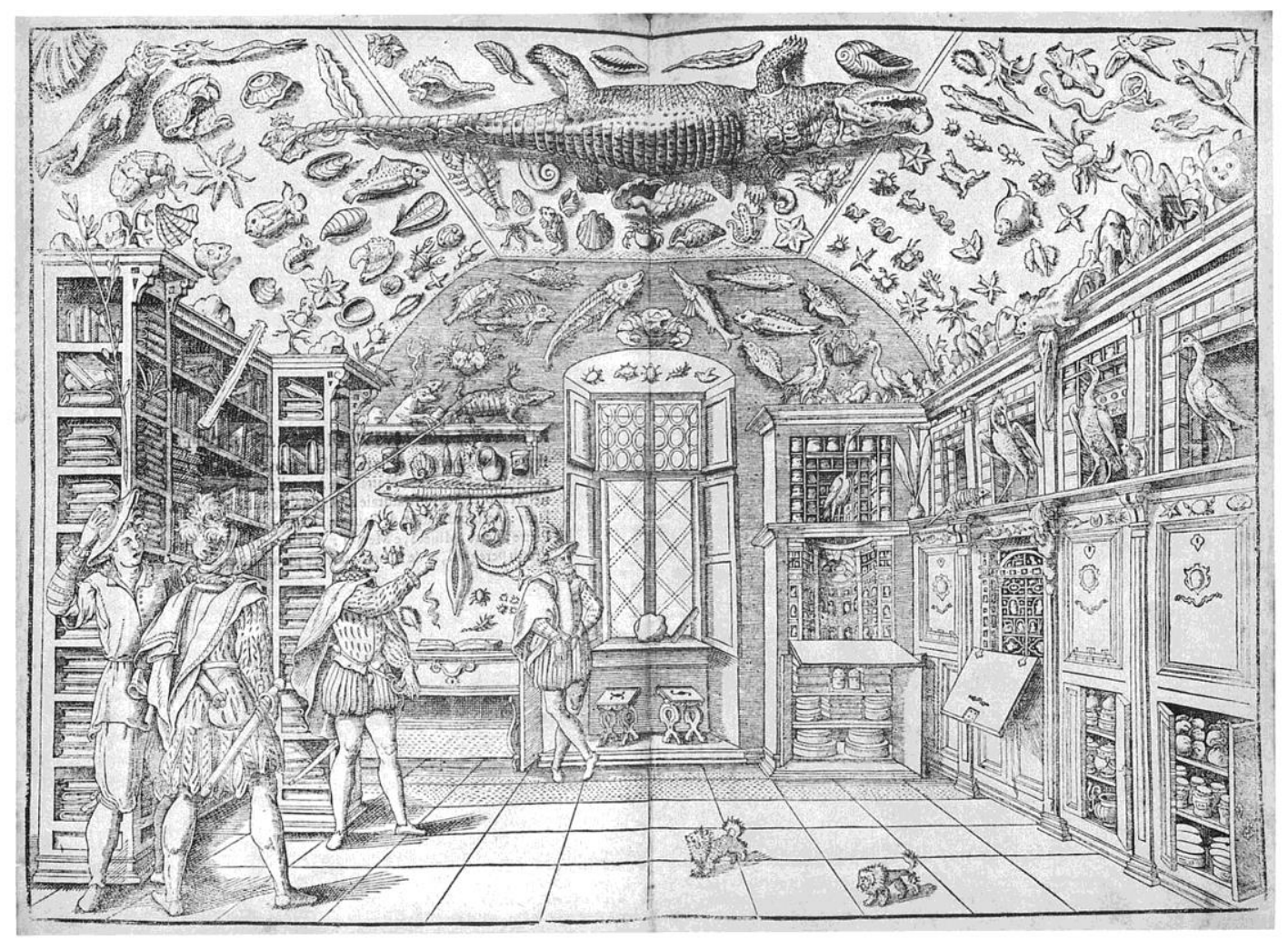

Fig. 2. Ferrante Imperato's museum in Naples. Adapted from Impey and MacGregor (1985, fig.4). 
Museums had a role in the institutionalised nationhood while displaying authentic objects to inform the public. They represented a unified memory of society as a means to stake a collective claim on the possession and revival of the past (Findlen 2000, 161-162). Bringing together the domains of arts, history and science, museums became a place for displaying the 'objects' of national solidarity, identity and supremacy. Bennett (1995, 89105) considers this as "the political rationality of the museum" that led to "the order of things" and "the birth of the museum."

The relationship between the national patronage and museum collections appears in the establishment of the Altes Museum (De: Old Museum) in Berlin (1822-30) designed by Karl Friedrich Schinkel (Moyano 1990, 585). The antiquarian Alois Hirt, who was the author of the first proposal (1797) of the Altes Museum, envisioned a "systematic organisation" [emphasis added] for an all-inclusive collection that would "serve several audiences" (598). However, after the defeat of Napoléon in the Wars of Liberation (18131815), which gave rise to patriotism, the Altes Museum was conceived to demonstrate the objects of royalty primarily for the reassurance of the nation (586). The director Wilhelm von Humboldt insisted on the display of only classical works of art (Marchand 2000, 182). Consequently, "absolute aesthetic standards replaced the systematic, historical organization of the collections" (Moyano 1990, 585).

Hooper-Greenhill ascribes the current political message of modern museum institutions to the role of periodisation, which distinguishes new objects from older ones $(1992,196)$. Until the nineteenth century, periodisation was not a practice followed by museums. Regardless of the date of creation, all objects were exhibited in sequence to valorise the past. The political motivation of this practice paved the way for Viollet-le-Duc's ethics of restoration. However, authenticity is later recognised differently in a more integrated function with the 'real thing' (197). This historic shift in values is related to Foucault's definition of 'disciplinary societies' (Batkin 1997). A review of the literature suggests that the changing role of museums in the nineteenth century shifted the practice from solely serving royalties to supporting national identities in Europe. Therefore, in modern museums, the practice of periodisation could be seen to deny the royal history by exclusively distinguishing the present from the past. Museums became means of reclaiming dominion over the values of old systems by rationalising and reducing them into periodically frozen forms, which are a-historical. This periodisation would need sophisticated recording techniques, ensued by what was known as museum computing.

The early museum computing activities were contingent upon the developments in information science in the USA. Advances in information sciences influenced the museum 
community at the MCN conferences in the USA. The majority of museum computing projects in the late 1960s were from the USA and Canada with collaborations in other countries such as England. In this case, the funding structures and sponsorship in museum computing were shaped mainly in the USA. The reliance on the developments in the USA had a crucial impact on the course of museum computerisation (Parry 2007, 15-19). The relationship between politics and museums was once again brought into consonance, this time, with computer-based pragmatic solutions such as SELGEM and GRIPHOS. These systems were distributed across the museums in the USA because of increased demand for information (Williams, 2010).

\subsubsection{Rise of Information over Knowledge}

At the end of the nineteenth century, there was a rise of information over knowledge. Systematic collection of information was the primary concern of science. Semiotics, literary theory, culture theory, cybernetics and information theory examined the structure of information to produce generalised and universal knowledge systematically. Semioticians Ferdinand Saussure (1857-1913) and Charles Sanders Peirce (1839-1914) studied the structure of sign and literary codes ruling meaning. Their studies stimulated ensuing developments in structuralism that enabled semioticians for analytical studies (Chandler 2002, 21).

The notion of différence was elemental to analytical methods of structuralism. The theory of différence was a matter of 'exchange and communication' that had been interpreted mathematically by Claude E. Shannon (1916-2001) (Teyssot 2013, 172). Shannon's formulation of communication took noise source into account between information source and receiver (Fig. 3). During World War II, cybernetic research transferred Shannon's information theory into feedback control systems deployed for military purposes (Johnston 2008, 26). Later, the common understanding of information for developing control systems was to encapsulate meaning into "formal, quantifiable and context-independent" interpretation (Kockelman 2013, 115). This overt "enclosure of meaning' somewhat diverted from the original ideas of Shannon and Peirce, who had envisioned 'error' as to how it reveals the truth. In cybernetics, however, 'error' was repositioned as a "negation of intent" (Nunes 2011, 12). In other words, the war affected the course of science remarkably because a small ambiguity in systems might cause unrecoverable damage. 


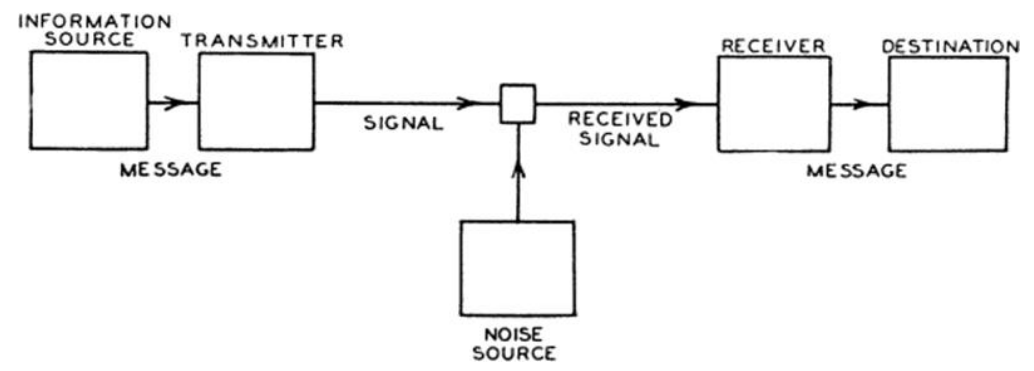

Fig. 3. Noise source in Shannon's diagram of communication. Adapted from Seising (2010).

Following World War II, the need for mass-production to meet public demand in the West required efficient information management. With the influence of faster communication and worldwide safety, the mobilisation of museum staff became a common practice. This led to loss of knowledge about museum collections that individuals used to record in their memories (Williams 2010, 16). They carried away their knowledge that made museums think about systematic solutions. In this case, mathematicians and computer scientists who became used to developing systems during the war did not struggle to extend their work to the emerging demands of museums. In the 1950s, the works committed to the automatic cataloguing of collections solidified the structuralist influence in early museum computing. Meanwhile, the New Archaeology movement, which is now known as Processual Archaeology, appropriated early structuralist notions to heritage (Parry 2007, 29; Di Giuseppantonio Di Franco 2014, 10). Its scholarship focused on the categorisation of artefacts to formulate "evolutionary culture change" (Binford and Binford 1966, 293), in other words, "processual change" (Binford 1962, 217).

\subsubsection{Automation in Museum Computing}

The functional arrangement of objects in museums is traced back to the Medici family's collection, which augments the communication of information through a "complicated" indexical system (Olmi 1985, 5-7). In the 1960s, museums were one of the first organisations to test computers. The primary purpose was automation in cataloguing, recording and retrieval. Museums in the USA were leading at the beginning (Jones-Garmil 1997, Williams 2010; Parry 2007, 15). Museum professionals conceived a conceptual network of museum data banks in New York. After a few years, a steering committee established the Museum Computer Network (MCN) under the directorship of Everett Ellin (Wilkinson 1968, 305-306). MCN sought to build a global museum information network across the USA, growing from New York museums (Ellin 1968). It was one of the first implementations of computer-based information networks. MCN was initially a physical 
computer network controlled by a centralised 'data bank.' It was meant to share information between museums and to overcome the challenges of recording collections in the USA (Ellin 1971). MCN provided its data-structure built on a suite of programmes called the General Retrieval and Information Processor for Humanities Oriented Studies (GRIPHOS). It was created by Jack Heller who was the lead computer scientist at the Institute for Computer Research in the Humanities (ICRH) at the New York University (Misunas and Urban 2007).

Developed initially as a set of automation tools for library cataloguing, GRIPHOS became central to the design and development of computer techniques for museums (Wilkinson 1968, 306). Initially, MCN used it for a collaboration with the Metropolitan Museum of Art where Carl Dauterman of the Western European Arts Department analysed enigmatic incised marks found on Sévres porcelain in extensive collections (Burton 1967, 20). Heller's system quickly became an early success for MCN, franchised to several computer-assisted projects at other museums of the network. According to the survey edited by Raben (1977), five major projects were deploying the systems and programmes of MCN. Based on the data collected from published articles in the Computer and Humanities journal between November 1966 and May 1972, there were more than nine hundred computerassisted research projects in the humanities and the related social science. Amongst the entries, museum-related projects comprised around 0.6 percent and archaeology projects about 2 percent, whereas most of them were about processing data for language analysis and literature-related studies such as vocabulary, syntax and usage (Table 1).

Table 1. Distribution of computer-assisted research projects in the humanities and the related social science between 1966 and 1972 based on the data adapted from Raben (1977).

\begin{tabular}{rrr}
\hline Area & Number of projects & Percentage \\
\hline Education & 2 & $0.22 \%$ \\
\hline General & 67 & $7.27 \%$ \\
\hline Language and Literature & 551 & $59.76 \%$ \\
\hline Music & 79 & $8.57 \%$ \\
\hline Philosophy & 17 & $1.84 \%$ \\
\hline Social Sciences & 180 & $19.52 \%$ \\
\hline Visual Arts & 26 & $2.82 \%$ \\
\hline Total & $\mathbf{9 2 2}$ &
\end{tabular}

As Table 1 shows, the share of visual arts projects amongst museum initiatives is relatively lower than other fields, whereas more than half of the computer-assisted projects in the humanities process language data. It was primarily semioticians in linguistics who, 
deploying the analytical methods of structuralism, augmented the computer-assisted research in the humanities. The technical reason behind Visual Arts remaining low is that the cathode-ray-tube (CRT) was still under development. CRT is the technology that extended the use of computers from only text display to images. The first museum project that celebrated the CRT technology was Burton's (1967) stylistic analysis of Egyptian pottery.

Eventually, the first visual reconstruction came from the museum of the University of Pennsylvania. Ray Winfield Smith's team computer-analysed over 35,000 photographs of stone blocks from the temples of Karnak in Egypt (Ellin 1968). The visual reconstruction was made by overlapping the black-and-white photographs of thumbnail size. They studied polychrome relief-cut blocks from the Aten Temple (Smith 1967). Therefore, the pictorial reassembly enabled a composition of "historical, descriptive, religious and artistic" knowledge about the temple at Karnak (Friendly 1973).

In 1968, Smith's project was one of more than fifty major museum projects in the world that used computers. To show there was an urgent need for more museum computing to deal with the information crisis of the post-war era, Everett Ellin prepared a report of museum computer activities around the world (Ellin 1969, 25; Parry 2007, 26). In the 1960s, museum computing projects were mostly about automating the documentation of museum collections, especially in natural sciences. However, two projects amongst them demand attention; one is Smith's visual reconstruction project and the other is Chephren Pyramid directed by Luis Alvarez from the University of California (71). Based on cosmic ray casting methods, Alvarez et al. (1970) performed a computerised pattern analysis to search the hidden chimneys of pyramids in Egypt.

Both projects of Smith and Alvarez were supported by the Smithsonian Institution, which had made an earlier attempt in museum computing than MCN (Parry 2007, 15). The output of the early Smithsonian initiative was an information management computer system called SELGEM for SELf-GEnerating Master (Bridge 2016b). SELGEM was designed primarily for natural sciences where developers Reginal A. Creighton and James J. Crocket from the Information Systems Division had a mutual understanding about cataloguing with the end-users as it was their traditional work at the Museum of Natural History at Smithsonian Institute. 


\subsubsection{Role of 'Error' in Information Processing}

In the 1970s, mini and microcomputers changed funding patterns because early systems, such as SELGEM, developed at the Smithsonian, and GRIPHOS, marketed by MCN, were not compatible with new computer technologies (Williams 2010, 19). Under these circumstances, MCN ended up with a mere, yet eminent, 'professional' network despite their initial ambition to create a cybernetic communication system based on physical computer networks (Misunas and Urban 2007). These consequences slowed down the speed of computerisation in museums, causing hesitation in other museums (Williams 2010, 20). The focus of computer science, shifted from knowledge transfer in social disciplines to more 'grandeur' areas of aerospace and artificial intelligence, left the advocates of museum computing without sufficient technical support. The initial enthusiasm faded away. Nonetheless, the early museum computing played a revolutionary role in the history of what has become to be known today as 'digital heritage.'

SELGEM and MCN were the locomotives of museums' computing needs for at least two decades (Williams 2010). Despite a very 'specific output' desired by the museum staff, the 'error' concern played a crucial role in the flexibility of SELGEM. In a technical report of SELGEM, Bridge (2016a) writes "it was generally believed that a more flexible design was achieved by providing gaps when assigning Category Numbers." The data structure of SELGEM consisted of a serial number, a category number, a line number and content information data. Only data type was characters, i.e. no image data, currency or date. Of 77 characters, 64 characters of data were for the content of a single record. SELGEM became functional for a range of institutional purposes in the USA, e.g. recording museum objects, bibliographic applications, systematic checklists, specimen catalogues and inventories of art pieces (Bridge, 2016c). Data was to be typewritten and recorded on tape drives. The files were in prototype format as "a proof of concept." As the system targeted multiple purposes, the prototype design included the allocation of gaps for flexibility in use. For example, the input data might look like:

- 071 genus

- 075 species

although the data structure is:

- 071 genus

- 073 subgenus

- 075 species

- 077 subspecies (Bridge, 2016a). 
It remains the case that the flexible design of SELGEM may align with the underlying concept of "Shannon's Choice" (Hayles 1990, 32) and this is an under-studied area. This idea refers to Shannon's inclination toward 'chaos and disorder' as a 'new attitude' in science. Shannon's concept equates information with entropy. 'Error,' or 'noise,' becomes an indispensable element of communication in Shannon's choice of disorder (49). The sublime quality of Shannon's choice is comparable to Wiener's interpretation of entropy in cybernetics:

"...for Wiener, increases in information signal a decrease in entropy and an increase in order. We can contrast this view with Claude Shannon's statistical analysis of communication as a system, which pairs an increase in information with an increase in entropy" [emphasis in original] (Nunes 2011, 12).

The stem of communication theory developed by Wiener is differentiated from Shannon through the exactness pursued in observation of probabilistic statistics (Seising 2010, 4467) (Fig. 4). The introduction in Error: Glitch, Noise, and Jam in New Media Cultures (Nunes 2011, 2-23) traces probabilistic approaches from Gaussian mathematics to the Peircean theory of error and Shannon's mathematical information theory. Apart from Peirce, Shannon and Wiener, the role of 'error' in information processing was also studied by others from different fields. Seising (2010) makes an epistemological analysis of its contribution to the development of fuzzy concepts in science. Having identified the need for an epistemological foundation of fuzzy concepts, he states that "fuzzy objects require fuzzy concepts" [emphasis in original] (4475).

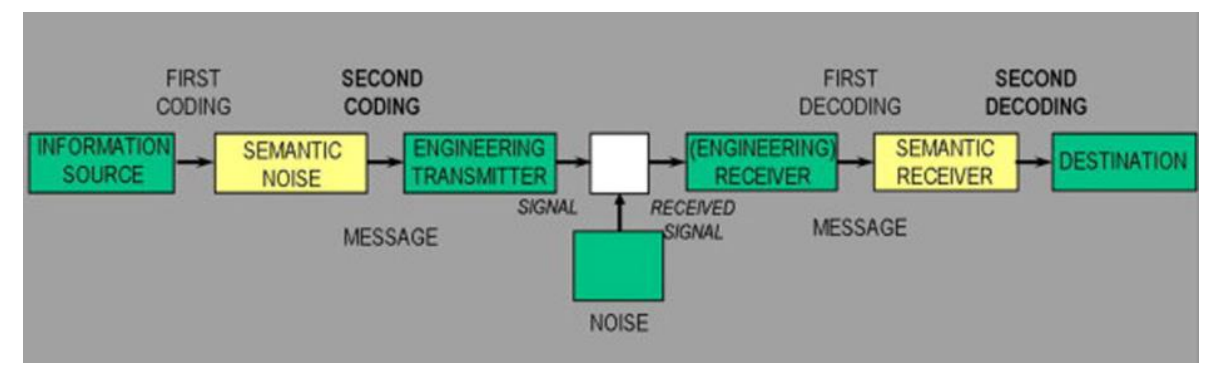

Fig. 4. Wiener's edition of Shannon's diagram with two additional semantic boxes. Adapted from Seising (2010).

The creativity involved in configurability made the structuralist theory of Claude LéviStrauss stimulating to engineers. The design of SELGEM demonstrates a conceptual understanding of the original version of the probability distribution. This repositioning of error values "inexact reasoning and processing knowledge that is imprecise, incomplete, or totally reliable," Lotfi Zadeh claims (quoted in Seising 2010, 4475). In the context of museums as AHIs, automatic systems of computing following the same concept of error 
would be required to accentuate impression and incompleteness while transforming 'things' into 'objects' for display. However, museum professional has always sought error-prone applications for their fuzzy concepts that involve semantic ambiguities. The demand for exceptional functionality resulted in the slow pace of implementation in museum computerisation and financial hesitation amongst fund suppliers. The "absence of theoretical noise" (Waterton and Watson 2013) in heritage discourse also prevented the field from new interpretations and alternative ways of knowledge generation. The overconcern with the materialistic interpretation of authenticity in discourse and theory led to small applications. For instance, Christian Wolter's use of computing was to merely report whether the Stuttgart Self-Portrait is attributable to Rembrandt based on highly technical data of the seventeenthcentury painting (Van der Wetering 2005, 493). A further study of records might reveal a role of error in the possible probabilistic distribution of their observations. 


\subsection{The Birth of the New Museology: Plural Interpretations of Heritage}

\section{Subchapter Outline}

Purpose: Heritage interpretation has been topical since the computerisation of AHIs started in the middle of the twentieth century. This subchapter aims to reveal the influence of other fields and poststructuralism in the rise of heritage interpretation.

Design/Approach: The subchapter positions the influence of poststructuralism as central in reviewing plurality in heritage interpretation.

Findings: It is found that there is a relationship between the early advances in computer graphics and the rise of plurality in heritage interpretation. The rise of plurality in heritage leads to the New Museology that encouraged more inclusive and socially responsive platforms.

Limitations/Implications: The theoretical background of plurality in heritage has connections with several domains, such as poststructuralism, digital art, interactive media and heritage management. Reviewing plurality in heritage requires an extensive and multidisciplinary perspective.

Originality/Value: Given the early admission of computers to museums in the 1960 s, the rise of virtual heritage at the end of the 1990s is a notably late development. However, its examination remains considerably under-reported. This review gives an overview of the influence of computers on AHIs.

Keywords. The New Museology; virtual heritage; computer graphics; interaction; poststructuralism; heritage interpretation.

Many computer innovations appeared during the 1960s. With the introduction of vacuum tubes, the interaction between the human and the computer was emancipated from the holes of punch cards into the holodeck of cyberspace. It was a conditional release, depending on cost, time and memory, i.e. available technology. However, the ingredients for a great leap toward the computer age were abundant. Museums were one of the first institutions to employ visual applications that were one of many great appeals of computers.

Computer graphics is of a central position in the progression of virtual heritage. To situate its exact role would require us to explore many early innovations in computer graphics. They could serve a myriad of purposes for use in museum computing such as executing aesthetic decisions. Museums' first experiments with the newly developed cathode-ray-tube (CRT) were about different visual input methods (Fig. 5). It was a collaboration between the researchers at IBM's New York Scientific Centre and the curators from The Metropolitan Museum of Art (Lourie 1968). The project director Virginia Burton monitored the stylistic analysis of about 2000 Egyptian vessels by comparing their contour characteristics (Burton 1967; Ellin 1968, 78). 


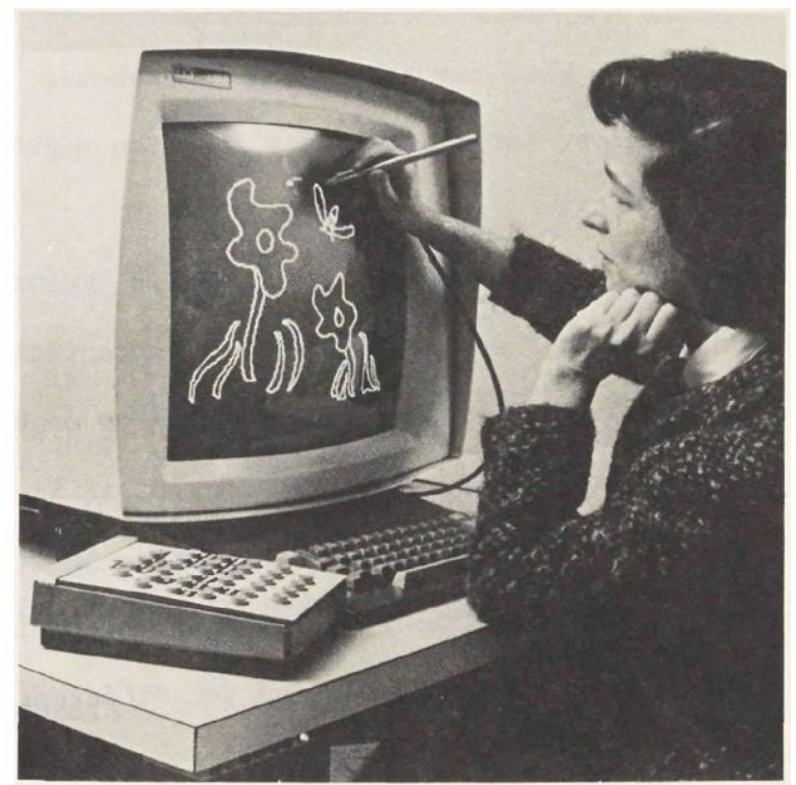

Fig. 5. The CRT display terminal of IBM 2250. Adapted from Lourie $(1968,225)$.

The computer solution aided by the CRT display terminal of IBM 2250 allowed the curator to input visual data with a light-pen in a similar way to interaction with today's touchscreens (Fig. 6). Recursive operations such as move, enlarge and delete were executed on a table of commands. The keyboard was operational to inputting written and coded information (Burton 1967). While the CRT technology permitted image-scanning, it was, however, not preferable because of concerns about the originality of the artefact. In most cases, researchers preferred light-pen re-drawing to photo-scanning as it was not seen "necessary to digest the masses of information from the optical scanning of a photograph process" (Lourie 1968, 228).

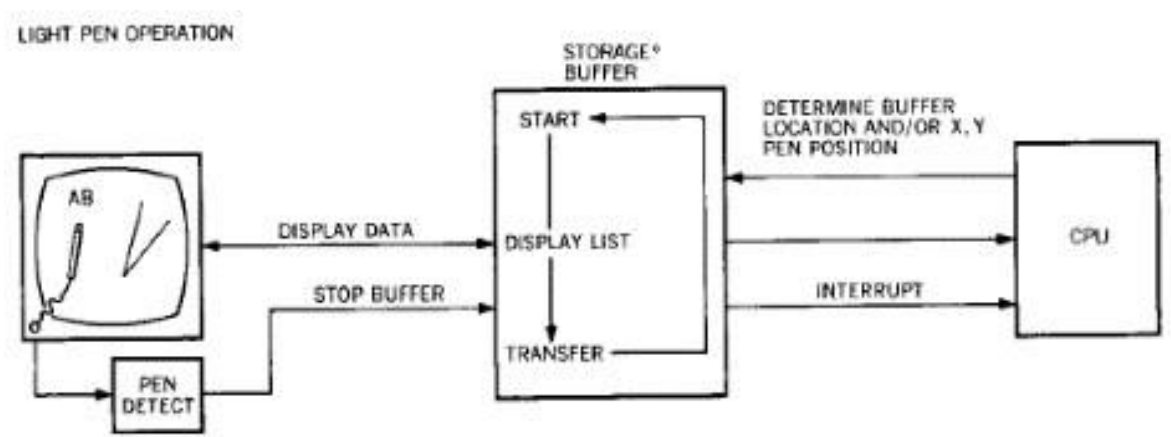

Fig. 6. Light-pen input function of IBM 2250. Adapted from Appel, Dankowski, and Dougherty (1968). 


\subsubsection{In the Beginning was the 'Interaction'}

When the light-pen interaction was integrated to Sketchpad, developed by Ivan Sutherland (b. 1938) in 1963, it was the beginning of a new era. Research and development in computing was often driven by military needs and it still is the case. For example, Sutherland's (1964) invention was built on a TX-2 for his PhD at Lincoln Laboratory that the US Air Force supported. This machine of the 1950s was originally engineered to track and prevent a possible Soviet nuclear attack (Sito 2013, 1) (Fig. 7). However, the invention of Sketchpad indicates an ensuing liberation of computers from military labs. It is a pioneering invention in the field of human-computer interaction systems.

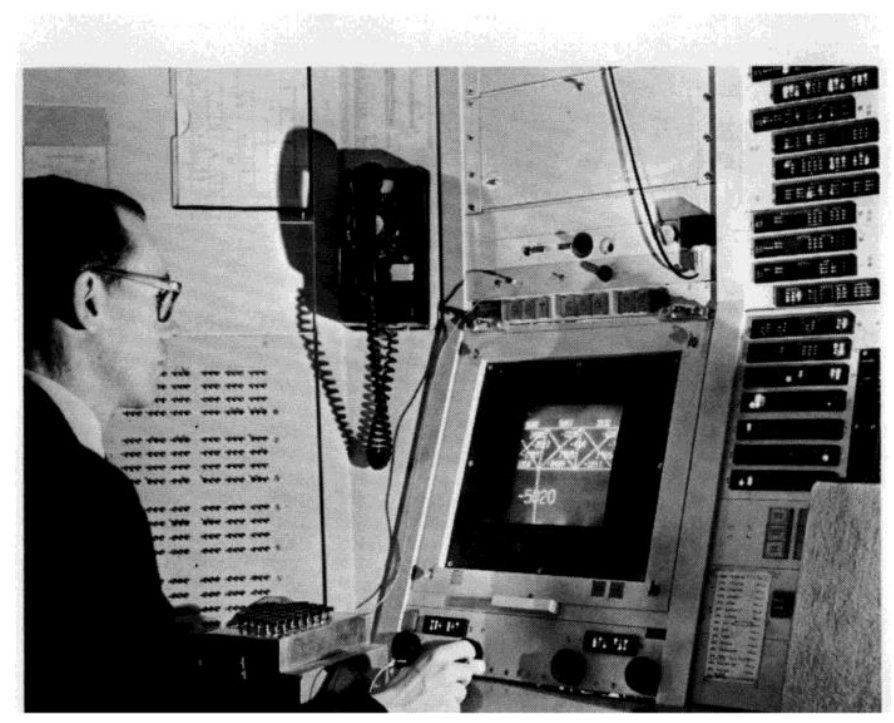

Fig. 7. Sketchpad operated by an 'author' with a light-pen, a box of push buttons a bank of toggle switches. Adapted from Sutherland (1964).

One can still argue that the war effect demonstrates itself in Sketchpad as it was designed to generate computational geometries based on specific parametric dependencies and recursive operations (Davis 2013, 1). It was the first Graphical User Interface (GUI), which promoted the combinatory initiatives between arts and computers, such as the Center for Advanced Visual Studies (CAVS) founded in 1967 by MIT professors with an aim to combine art and science. For many other advances, Sketchpad was one of the two most significant devices to interact with computers. The other key invention was the first iteration of the 'mouse' by Douglas Engelbart (1925-2013).

In 1969, the first usage of the term 'interactive art' appeared with Glowflow, which was a "computer-controlled light-sound environment" (Kwastek 2014, 8). A group of artists and scientists at the University of Wisconson worked collaboratively to design Glowflow. Composed of 'phosphorescent particles' running through transparent tubes, Glowflow was 
defining an 'illusory space' with glowing lines on the walls of a dark room (Fig. 8, left) (Krueger 1991, 12). Krueger's experiments continued with Psychic Space (1971) that could track individuals' positions in a room via a camera-based feedback system (Fig. 8, right) (24). In their prologue, Sensorama patented by Morton Heilig (1926-1997) in 1962 was the first digital application conceived to evoke multiple human senses (Seiler, Koch and Both 2015) (Fig. 9).
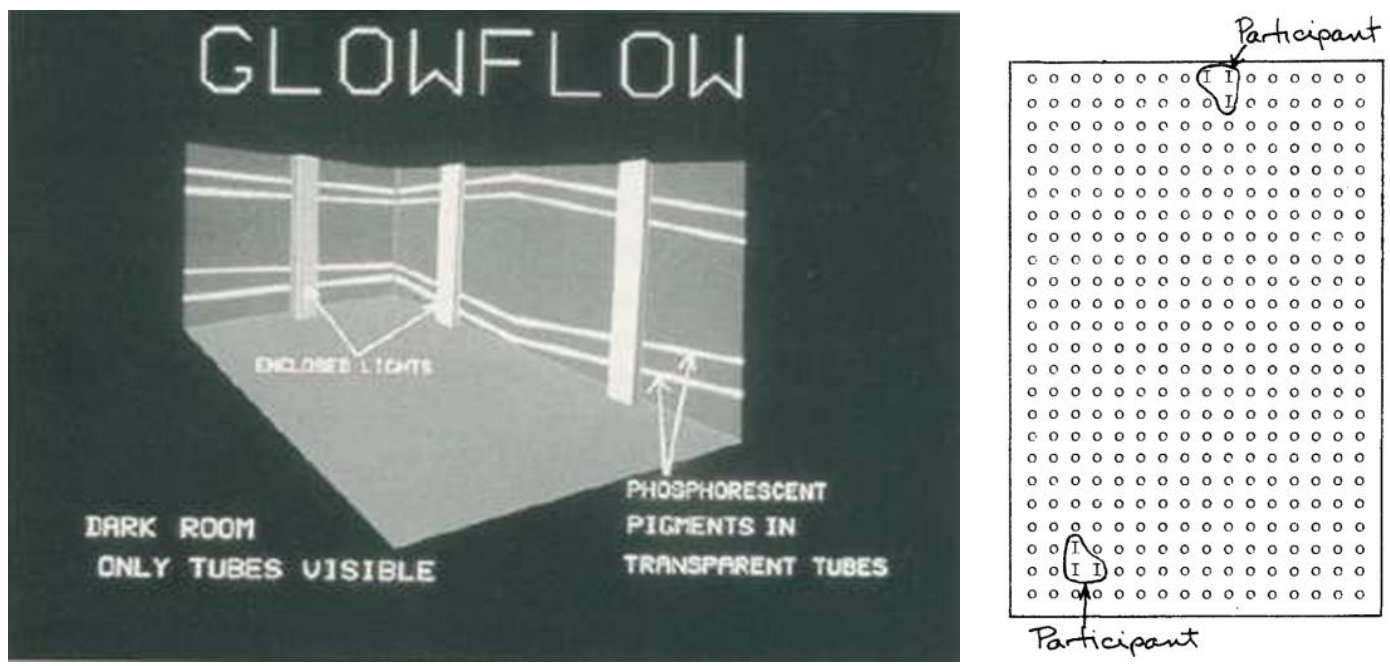

Fig. 8. Left. 3D representation of Glowflow's walls installed with transparent tubes (left). Adapted from Krueger (1969). Right. Zeros-and-ones concept of Psychic Space that shows how a camerabased feedback system senses the 'participant.' Adapted from Krueger (1977).
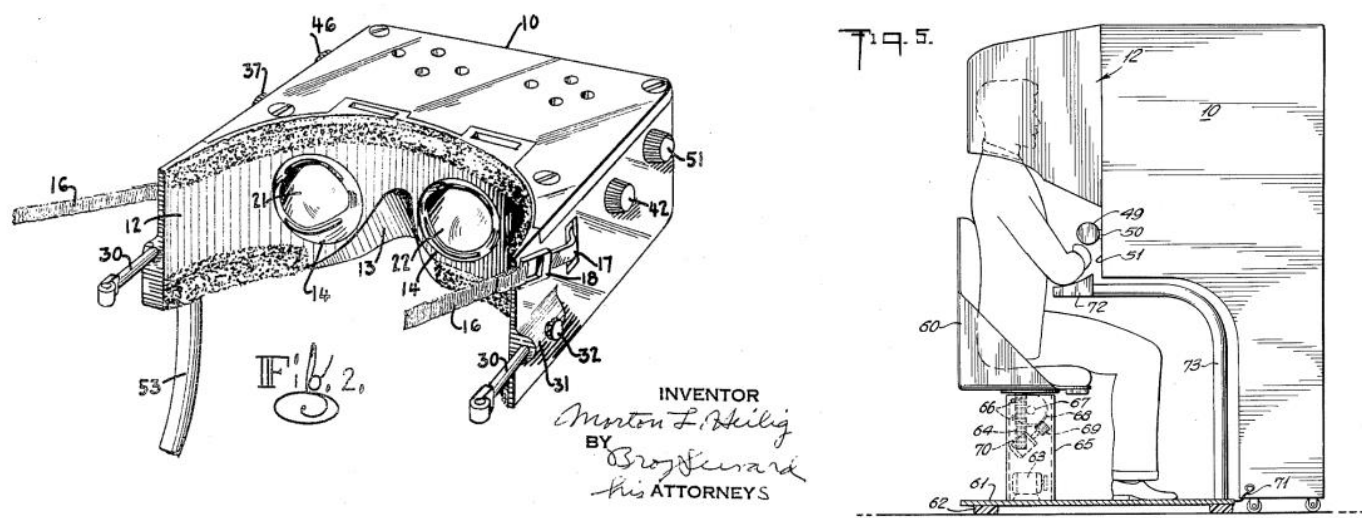

Fig. 9. Left. Sketch drawings of Telesphere Mask (patent: US2955156). Adapted from Heilig (1960). Right. Sensorama Simulator (patent: US3050870). Adapted from Heiling (1962).

Subsequently, these developments gave rise to early pioneers of computer-generated digital art. Scientist-artists of that period included A. Michael Noll, Vera Molnar and Manfred Mohr (Fig. 10). Techno-artistic contributions included those from the Bell 
30

Telephone Labs, where Shannon (1948) wrote his influential paper titled "A Mathematical Theory of Communication." In the context of museum computing, Burton (1967) called it "Computers Confront the Curator." Eventually, in the 1960s, the emancipation of computers meant more than the evolutionary development of technology. It promoted new ways of thinking about the technological artefact and contemporary culture. On the one hand, innovations, such as CRT (see Fig. 5, p. 27), Sketchpad and Sensorama, paved the way for 'man-machine interaction' and collaboration. On the other hand, this revolutionary era somewhat urged the alteration of singularity in authorship. In 1967, the French literary critic and theorist Roland Barthes (1915-1980) called it "the death of the author" (Barthes 1988).
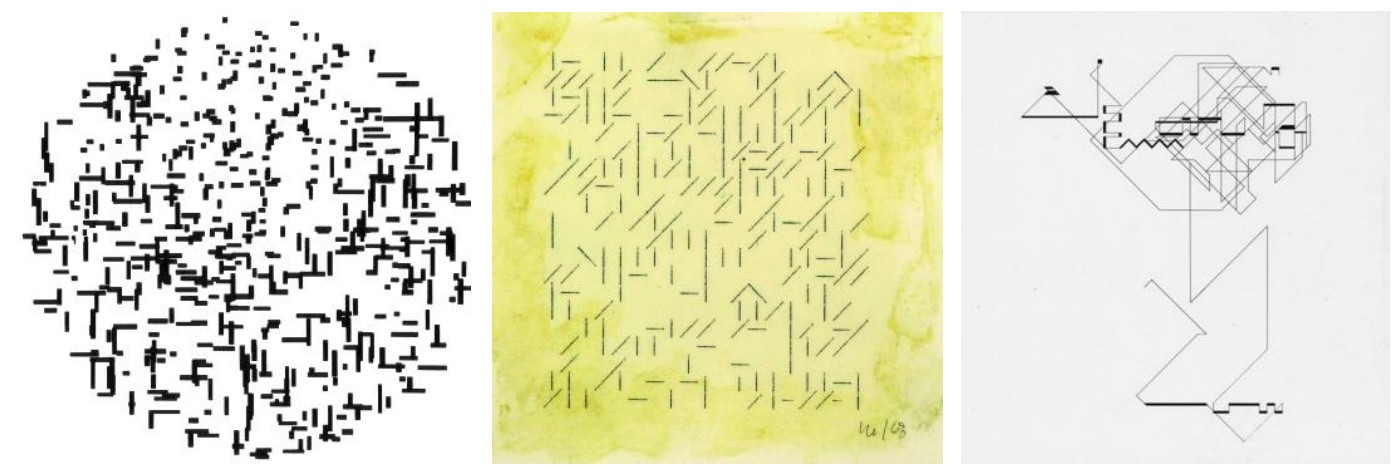

Fig. 10. Left. 'Computer Composition With Lines' by Noll (1964). Middle. 'No Title' by Molnar (1968). Right. 'Random Walk’ by Mohr (1969). Adapted from DAM (2018).

Today, immersiveness via panoramic displays is topical in heritage studies. Kenderdine's $(2007 ; 2015)$ research and practice deal with embodiment theory. Kenderdine (2003) associates panoramic embodiment with the democratisation of the museum space, with an example of a "peep-hole" display machine called the Kaiserpanorama (1883) that fragments perception and separates individuals. Since the Kaiserpanorama, the concept of museum space has been liberated from the boundaries of architectural space (see GVM in Chapter 2.4.3). The contemporary museums allocate space for panoramic interactive exhibitions, such as New Zealand's Māori artist Lisa Reihana's (b. 1964) in Pursuit of Venus [infected] (2015) exhibited at Auckland Art Gallery Toi o Tāmaki. It is a computational museum work that reinterprets the nineteenth-century panorama painting Les Sauvages de la Mer Pacifique (1804) (The Savages of the South Pacific) by Joseph Dufour combining 20 panels (Fig. 11). Transforming fragmentation into a continuum, other interactive and embodying panorama examples include The Qianlong Emperor's Southern Inspection Tour (2010) (Din, Bailey and Lin, 2015) and We are like the Vapors (2013) (Kenderdine and Shaw, 2015). These projects are different from still panoramic images with moving characters embedded for 'interactive' viewing that adds 'continuum' to perception. 

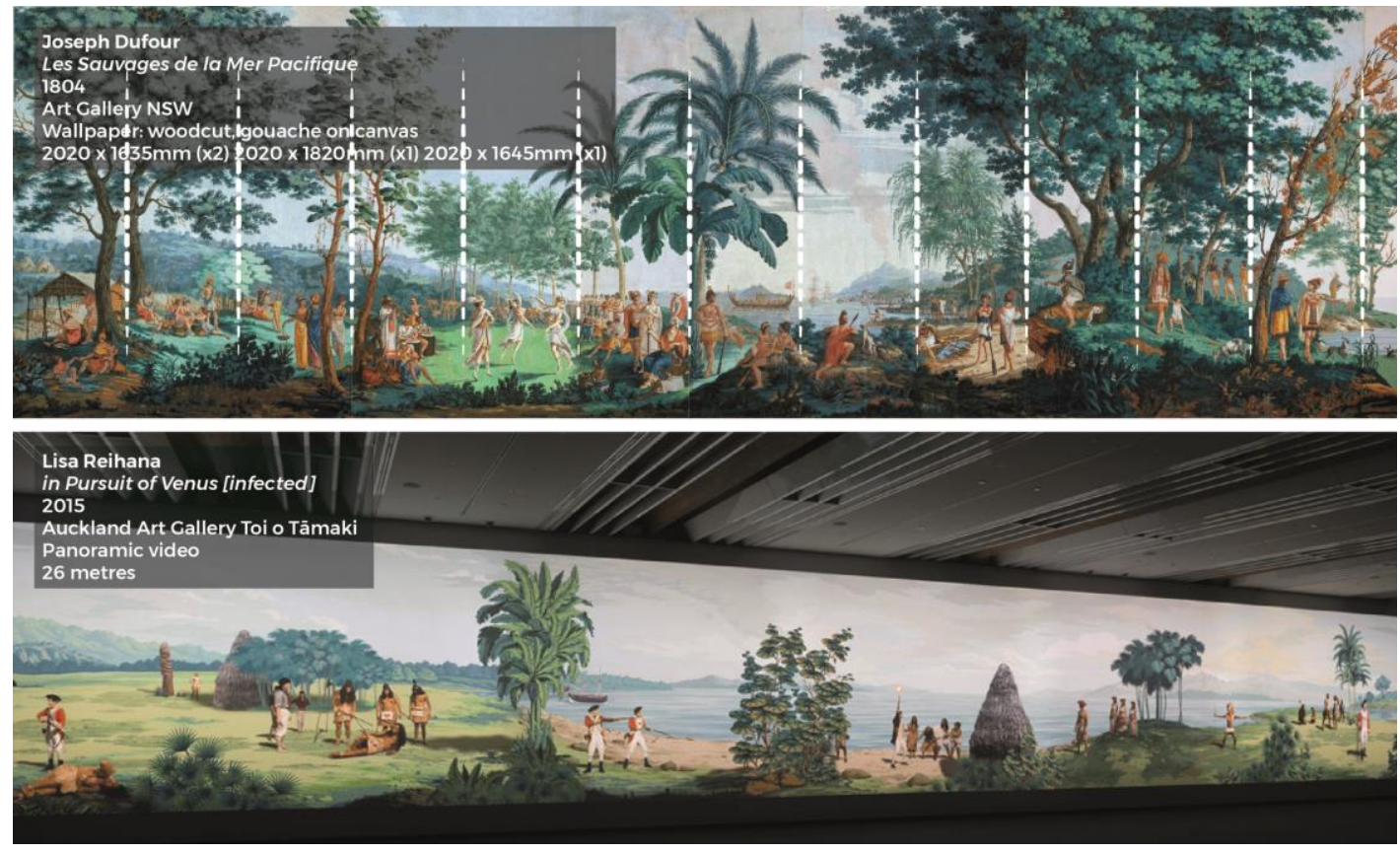

Fig. 11. Top. Dufour's Les Sauvages de la Mer Pacifique (1804). Adapted from Dufour (1804). Bottom. Lisa Reihana's in Pursuit of Venus [infected] (2015). Adapted from Reihana (2015). Illustration by Author.

\subsection{2 "The Death of The Author"}

Although the 1960s' innovations were so overwhelming, museum staff were mostly unable to explore various amplitudes of computer graphics. Researchers who worked with computer graphics had to make rather simple analogies, for example, between the CRT display terminal and television (Burton 1967, 20; Lourie 1968, 225). Television was not only a familiar concept for non-computational museum staff, but it was also the representative medium of their contemporary culture, i.e. the television generation, whose demand of more information was a major shift that initiated museum computerisation (Williams 2010, 16). On this basis, the introduction of computer graphics to the museum delivered an incomplete spectrum of underlying messages. The innovation was that computers were no longer isolated in military labs, with novel user-friendly interfaces that went about improving communication with their code-based understanding. However, televisions were authoritarian, to which early innovations in computer graphics alluded to an interactive media future. Sutherland's Sketchpad and Engelbart's mouse ended the assumption that computers were antagonistic to basic human intelligence. Museums remained institutionally entangled with the cold side of computers, i.e. lacking interactive engagement. 
Therefore, for a long time, museums have remained exclusive and been restricted to traditional archival workflows despite the communicative and interactive role of computers. In fact, museums in the 1970s were still more concerned with the completion of their "role specialisation in the public sector" with a "main emphasis on the use of manual cataloguing systems" (Roberts 2001, 15). Lack of specialisation means that there was no 'author,' yet, who could account for weighty criticism of Barthes's poststructuralist analysis. In his essay, “The Death of The Author" Barthes (1988) gave a critique of the ancient figure of the author (White 2012, 112). He states that a literature product, such as Balzac's Sarrasine, can be interpreted from different perspectives, i.e. "indiscernible voices" (Barthes 1988, 142).

In general, the 'heritage discourse' seemed to follow these theoretical discussions more closely than museum professionals. In heritage discourse (Waterton and Watson 2013), the first explicit attack on the undisclosed role of authority was made by Tilden (2007) in his seminal book Interpreting Our Heritage. Originally published in 1957, the book remarks on the beginning of talks about plurality in heritage interpretation (Howard 2003, 245). In extension, Robertshaw $(2006,52)$ notes that Tilden positioned personal interpretation in a higher role "before the computer age dawned for most museums." However, Tilden's demonstration of an interpretivist stance in heritage was a managerial perspective (Waterton and Watson 2013). From the 1950s, the scholars of cultural heritage management discussed how to understand and improve heritage-visitor relations. As a new phenomenon, tourism was very influential in these discussions, leading to more emphasis on heritage marketing and commodification. These managerial perspectives on tourism, museum and heritage took advantage of the structuralist theories that prioritised structure over function. Eventually, the ancients who favoured exclusivity were more influential in decision-making mechanisms of museums than the moderns who were candid with innovations. Additionally, the specialisation of museums was incidental to international heritage charters.

In 1964, the Venice Charter studied architectural heritage as a singular concept, succeeding the seven-article manifesto of the Athens Charter (1931) (Wells 2007). The Venice Charter continued to extend on its antecedent for overlooking the plurality of authenticity. However, it was the first formal declaration of heritage to outline the myth of authenticity. Universal codes, practices and principles defined methods for interpreting authenticity, which was incommensurable to developing an understanding beyond materiality. The overconcern with materiality and management within a structuralist view of heritage led to charges of inauthenticity because of articulating "purposiveness for order" (Waterton and Watson 2013) for a dynamic and fluid phenomenon. Authenticity was understood as a universal value to be guarded by the institutionalised formats of the cultural 
heritage field, such as museums. Therefore, despite the signified importance of publicity, participation remained without interaction and deep questioning in the doctrine of the 1960s and the 1970s.

Later in the 1980s, museum informatics continued to evolve with the availability of inhouse IT systems and microcomputers (Roberts 2001, 16). The early museum computing proved that bringing museum collections automatically under control was formidably challenging (Chenhall and Vance 1988, 3). The primary concern of heritage management and museum cataloguing activities was mainly about the informational content (or metadata) (Chenhall 1978, 4). There was a lack of interest in the computing techniques of the 1960s, e.g. the CRT display by Burton (1967), pictorial reassembly by Smith (1967) and cosmic ray casting by Alvarez et al. (1970). Therefore, the evolution of computing heritage from documentation and cataloguing techniques to that of representation and interaction was a slow event.

Heritage management techniques used by interpreters were advanced mostly in the USA until the 1980s (Hall and Arthur 1998, 167). Transferred from there, they made various impacts in continental Europe. The New Museology (Fr: La Nouvelle Muséologie) was born as a French movement rooted in theoretical discussions of the 1970s. It was devoted to societal functions of museums and interdisciplinary methods such as pedagogical interaction (McCall and Gray 2014; Desvallées and Mairesse 2010, 55). It would eventually become internationally renowned, i.e. in English literature, only towards the end of the next decade (Vergo 1989a). Towards the end of the 1980s, visitor-oriented approaches were denoted "productive" (Uzzell 1989b) for heritage marketing and interpretation. In those years, the audience was treated as part of an issue of market segmentation systems, which were appropriated from "national tourism agencies" (Hall and Arthur 1998, 171) and "theme parks" (Swarbrooke 1995, 50) (Fig. 12). 


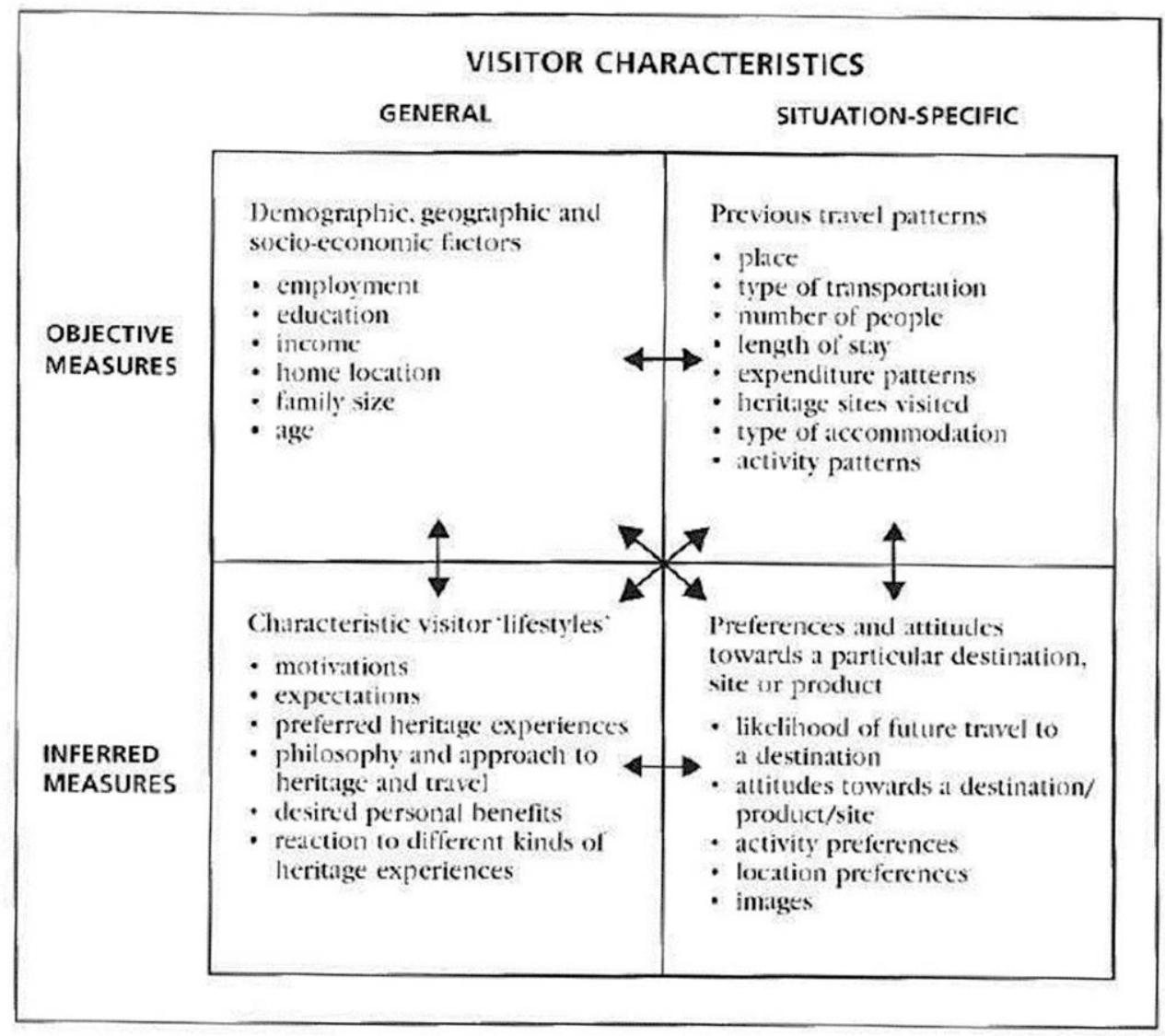

Fig. 12. Measuring visitor characteristics, a heritage management technique for visitor classification. Adapted from Hall and Arthur (1998, 145).

In the 1990s, managerial accounts of heritage focused on the integration of development, management and classification of heritage-visitor attractions based on old and new international charters and principles (Swarbrooke 1995; Hall and Arthur 1998; Leask and Yeoman 1999). Aligning to these charter-based managerial perspectives, heritage discourse and museology remained 'rather evolutionary than revolutionary.' The "heritage industry" (Uzzell 1998), notably as a productive alliance between conservation, tourism and education, resisted new forms of interpretation and computer interaction. Instead of complying with upcoming challenges of changing demands, they focused on systematisation of openly challengeable principles in conservation, engagement and interpretation (Wells 2007, 9).

Meanwhile, the growing role of technology in the 1990s was still challenging to admit for heritage management professionals. Instead of assisting in full integration with technology, they focused on rhetorical assumptions. Bennett $(1999,87-91)$ outlined several emerging issues ranging from "the media becomes the message" to "visitors do not learn" and to "virtual reality substituting the experience." Another note from the same paper is that 
"stirred" by technology, the issue of authenticity was noted to become more "contentious" (90). Even more hazardous, Stevens $(1989,103)$ wrote that "interpretation is, today, in greater danger of being hijacked by the designers and media technocrats than ever before."

\subsubsection{The New Museology}

Exhibition modes of the early modern museum were divisive (Ross 2004). From the 1950s onwards, interpretivist accounts of heritage encouraged public engagement. They were managerial contributions surrounded by issues spotted by heritage scholarship of critical theory. These critics catered to the poststructuralist analysis of museums and heritage. The most well-known examples are Lowenthal (1985), Hooper-Greenhill (1995) and Kirshenblatt-Gimblett (1998). They purveyed the influence of the inclusive museum concept, such as community-led eco-museums.

The New Museology thinking provided an overall structure to communicate the social role of museums. The New Museology was born to be "a state of widespread dissatisfaction with the 'old' museology," for whom the social relationship with communities did not matter (Vergo 1989a, 3). It was rooted in subjectivist, constructivist, critical and naturalistic thinkers whose influences were not neatly overlapping but amalgamated into each other. The main argument was about visitor involvement as part of museums' social role, thus collection standards.

To discuss standards of visitor types and collections in museums is contingent on an ancient dialectic of presenting a comparison of two opposite sides. It has appeared in different couplings, such as the national and individual, exclusion and inclusion and the ancient and the modern. To review New Museology, this section studies the repercussions of this duality under two primary schools of thought: phenomenology and poststructuralism. The primary purpose is to see 'how' idealised accounts of phenomenology and systemoriented poststructuralism realise a third realm between the dialectical notions of the real and the virtual.

In the schematisation of phenomenology (e.g. Bergson, Heidegger, Merleau-Ponty and Lefebvre), an orthodox duality of the western philosophy occurs between Lebenswelt (Lifeworld) and Gesellschaft (Society) (Corsane 2005, 186). In Heidegger, the former contains a bringing-forth (i.e. poiesis), whereas the latter contains a standing-reserve [i.e. Bestand], which is concealed by Ge-stell, or Enframing (Heidegger 1977, 21).

In this sense, the 'old' museology restricted the use of technology to the Enframing of managerial perspectives. Their totalising structure somewhat misconstrued the remarks of 
early computer graphics. However, the aspiration of new media artists supported novelty via new media installations in museums, such as Legible City (1988-1991) by Jeffrey Shaw (b. 1944) and Room of One's Own (1990-1993) by Lynn Hershman Leeson (b. 1941) (Fig. 13). These interactive works required corporeal engagement by design, leaning towards phenomenology.
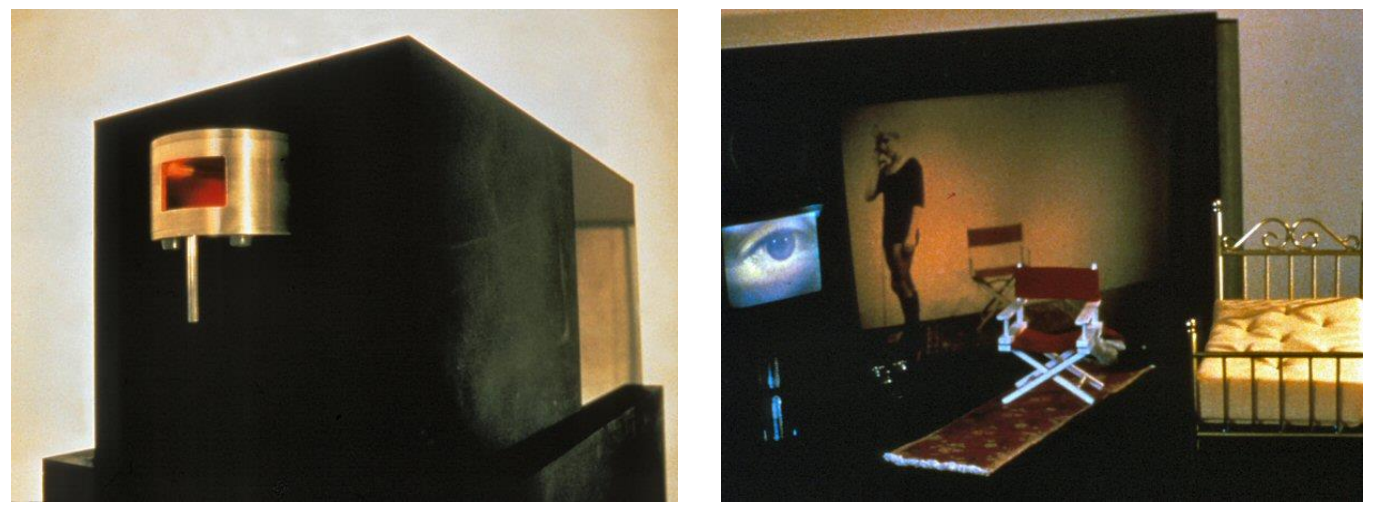

Fig. 13. Room of One's Own by Lynn Hershman Leeson (1990): exterior view of the interactive module (left) and interior view with miniature furnishing and screens. Hershman Leeson's installation induced the viewer's replacement with the female identity staged at a representative position. Adapted from FDL (2017).

Meanwhile, the pioneers of interactive art used poststructuralist interpretations such as the city blocks in text forms in Legible City. These works pointed out the discovery of reconfigurable relations between the units of the Enframing. With his 1991 installation, The Moment of The Truth, Daniel Dion (1958-2016) stated, as summarised from Kwastek (2014, 144), that even standard media configurations (mouse/keyboard and projector/screen) "convey a subtext that enters into the overall configuration of the aesthetic experience." Vergo's (1989b, 41) "reticent object" is enticed into embodied experiences, turned into "obliging" (Robins 2007, 23). In this sense, visitor-oriented arguments of the New Museology are persuaded by the existentialist phenomenology of Maurice Merleau-Ponty (1908-1961), with the body beguiled by 'transcendental' orders of the mind.

On the one hand, the existentialist phenomenology was instrumental to the New Museology discourses for diversity and authenticity. It was about the endless configurations of the viewer moving through compartmentalised elements. Conversely, a poststructuralist celebration of the discretion and disassociation of these configurations from movement equipped the discourse with system-oriented and network thinking. For example, the Croatian museologist Tomislav Šola's (b. 1948) notion of heritology and cybernetic museum explores a systematic approach to increase the social role of museums (1997). 
Poststructuralists, such as Roland Barthes (1915-1980), Michel Foucault (1926-1984), Gilles Deleuze (1925-1995) and Jacques Derrida (1930-2004), were pleased with gaps, failure and breakdown. In contrast to the thinkers of phenomenology who analysed the continuity of movement, poststructuralists contended with the discretion and disassociation of time from movement (Reynolds 2015, 102; Peters 2015, 33). The birth of the digital phenomenon was rooted in this thinking while leading to Heidegger's concern with Enframing.

The New Museology thinking promoted interactivity, such as hypertext. Coined by Ted Nelson, the invention of hypertext enabled non-linear reading experiences on the web, which was related to Barthes and thought to be the beginning of its interactivity. In the 1990s, Derridean intertextuality was influential (Kwastek 2014, 56). The criticism was that hypertext was merely instrumental to the 'archivist' purposes of search engines, such as Google (Coyne 2011, 94). Cited from the German digital media critic, Roberto Simanowski, the use of hypertext helped the author enjoy dominance over the viewer engulfed with "combinatorial activity" within a "hierarchy of associations," confining the freedom of “connotative interpretation" (Kwastek 2014, 56).

\subsubsection{Authenticity of 'Noise'}

In the context of New Museology, digitalisation had to resolve struggles between dualities, such as place and space and culture and society (Bautista 2014). Since 1979, Ars Electronica Center (AEC) has promoted artistic ideas with a concept of 'hybrid museum' between science and art, in general; and new media and society, in particular (Rectanus 2002, 8; Drioli 2006). The participatory and interactive installations exhibited at the AEC had an influence on the New Museology thinking. Artists of Ars Electronica showed how to exploit new information infrastructures, i.e. Heidegger's Enframing. Exhibition instruments included television broadcasting in different creative forms that enabled people to participate actively, e.g. Hole in Space (1980), The World in 24 Hours (1982), Hotel Pompino (1990) by Van Gogh TV, Nobody Is Safe (1991) by Stadtwerkstatt-TV (Druckrey 1999). These exhibitions contributed to generating a new spatial understanding of the digital age. It was 'locality' that gave momentum to the New Museology thinking where public involvement had a role in questioning contradictions such as space and place (Bautista 2014, $8)$.

In heritage discourse, these contradictions aligned with public participation were discussed noticeably belatedly. In the 1990s, some research studied the locality. It was a 
self-criticism of heritage management scholarship that without challenging people to reconsider the value of heritage, they would be no more than consumers, tourists or visitors (Uzzell 1998). The central rhetoric was a prevailing view concerned with materiality as well as publicity through universal codes (Waterton and Watson 2013). In this period, the Nara document was the first international manifesto to recognise regional differences on interpreting authenticity. The Nara Document on Authenticity (1994) submerged its antecedents such as the Venice Charter (Wells 2007). Its consideration of diversity amalgamated plurality into the heritage discussion. In Nara, the example of Japanese temples, which were traditionally dismantled and subsequently reassembled periodically, engendered a supportive approach to plurality in authenticity discourse, which used to be a criterion defined by material aspects instead of processual. It was the end of the isolation of principles that treated diversity in collection and audience as 'noise.' Early modalities of heritage management and its operational quality were defined by standards, codes and charters, which declined the positioning of 'error' for communicating museum information to the public. The critique of heritage could oscillate on the surface of phenomenology and poststructuralism. The emphasis on the postmodern rhetoric of representation caused an "absence of theoretical 'noise' surrounding the field" with restricted "critical imagination" [italics in original] (Waterton and Watson 2013).

The New Museology was an attempt to change the structure of museums. As an institutional practice in alliance with managerial accounts of heritage and tourism, museum informatics survived the challenges of information theory. New media exhibitions in Ars Electronica pursued loopholes in the encounter between technology and society. Artists cultivated the exhibitions at ACM as a dichotomy between information and entropy. The disagreement was about the definition of the public participation. There was the "noise of the observer' involved in interactive exhibitions. Entropy had roots in the nineteenth-century thermodynamics that mulled over the idea of "missing information" (Weibel 1999, 140). Information, entropy, noise, error, interactivity and feedback were no longer mere terminologies of science as studied in Wiener's cybernetics, Shannon's information theory and even Albert Einstein's quantum theory.

Ars Electronica embedded them directly into arts and digital humanities and indirectly into museums, heritage and architecture. The scholarship was then influenced by the theoretical developments in the second half of the twentieth century when everything was a subject of decoding to understand the concealed structure of sign (Plummer 1996, 241). In semiotics, the basis of Shannon's communication was thought to make "no allowance for the importance of social contexts and codes" (Chandler 2002, 176). However, this 
observation omitted the fact that the reinterpretation of Shannon's work by his commentator and co-author, Warren Weaver (1894-1978), revealed quite the contrary. Weaver recognised an 'ambiguous quantity' in Shannon's equations that he labelled 'equivocation.' For Weaver and Shannon, “a 'noisy' message [was] more surprising [while carrying] more information" (Hayles 1990, 55) (Fig. 14).

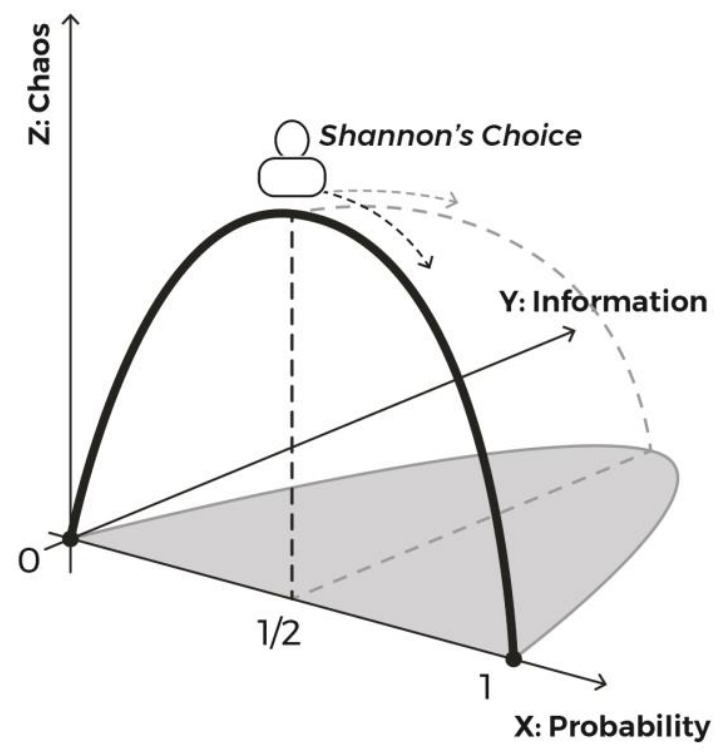

Fig. 14. Reinterpretation of Shannon's Choice towards chaos and disorder, or entropy, while the increase in information meant an increase in entropy. Illustration by Author based on a review of Hayles (1990, 59).

Hayles's (1990) interpretation of Shannon's theory suggests "orderly disorder in contemporary literature and science." It seems that Shannon's quantification of 'noise' was repellent to museum and heritage scholarship trying to expand on interpretation and meaning. Despite the warning made by Shannon and Weaver that "information must not be confused with meaning" [emphasis in original] (McCarty 2005, 110), some research misinterpreted their emphasis on entropy. It was a probabilistic measurement in which uncertainty was an ally of information (Hayles 1990, 59) (see yukarıda Fig. 14). The Ars Electronica Festival in 1995 "lift[ed] the curtain worldwide for the first time to allow a glimpse at this horizon of the digital data highways" where "giant cataclysms of exploding errors" would emerge from "prematurely incinerated information dust" (Weibel 1999, 147).

It was a matter of inclusivity that the recognition of 'error' underwent a series of interpretations of interaction ranging from information science to new media and the New Museology. With Tilden's 1957 book, subjectivity in heritage interpretation grew out of interest in visitor-oriented management strategies in tourism (Bærenholdt et al. 2004). The 
40

rhetoric of heritage interpretation followed a less contested line of postmodernism, with analysis of representation being used to stress political discussions.

The New Museology thinking departed from this with a keener interest in new media, which was driven by poststructuralist methods and phenomenological underpinnings. New media exhibitions such as those at the AEC assisted the New Museology thinking to develop appetites for new standards of collections and visitors. Empirical and objective approaches of the early museums became less appealing than contextuality and subjectivity in phenomenology, poststructuralism and postmodernity, from which constructivism made the highest impact.

The increasing social role of museums and heritage interpretation obliged visitororiented active learning programmes. Because funding for education was valued more, constructivism received more attention. Despite computer graphics offering visual interaction, the emergence of the virtual museum was dependent on managerial and statistical views, so the growing use of new media facilitated instead deskilling museum staff and heritage researchers. Heritage professionals pursued the procedural norms of the social role that memory institution could play. Nevertheless, the novelty brought by the New Museology thinking speculated powerfully to make museums self-critical to follow the novelties of new media. 


\subsection{The Birth of the Virtual Museum: Suspension of Disbelief in Virtual} Heritage

Subchapter Outline

Purpose: This subchapter aims to give an overview of the notion of 'virtuality' in museums and to reveal how the understanding of 'virtual' has evolved in AHIs.

Design/Approach: This section is built on the evolution of the virtual museum from Quiccheberg's Inscriptions (1565) to Malraux's imaginary museum (1947) to Guggenheim Virtual Museum (GVM) (1999).

Findings: It is discovered that early virtual heritage research was replete with misconceptions about 'virtuality.' Old concerns of AHIs are found persistent until recently, despite novel technologies.

Limitations/Implications: The review is confined to the samples of visual media for the realisation of 'virtuality' in AHIs.

Originality/Value: The subchapter is one of the first attempts at writing the history of digital heritage based on the notion of 'virtuality.'

Keywords. Virtual museum; digital heritage; virtuality; virtual reality; cybermuseum; web-museums; non-place; place-making; London Charter.

The word, 'virtual,' used to be confused with the exact opposite of 'real' in meaning, thus non-existent, which was spotted as misdirection by many thinkers. Charles S. Peirce's account of 'virtual' was the opposite of 'potential' instead of 'real.' For Peirce, "a virtual X (where $\mathrm{X}$ is a common noun) is something, not an $\mathrm{X}$, which has the efficiency (virtus) of an X" (Esposito 2017). It was not meant to be a "mental copy of its real object, but a portrayal of its practical applications, predicting what and how it would produce other real objects" (Thellefsen and Sorensen 2014, 495).

The Deleuzian concept of 'virtuality' distinguished it from 'intensity.' For Deleuze, in Hughes's $(2009,170)$ interpretation, the former is about "the future whereas intensity expresses the immediate present of affection.” The British artist Roy Ascott (b. 1934), who worked on cognition, cybernetics and telematics, attacked the reality instead of defending the virtual in a 1989 essay for Ars Electronica. He pointed to the suspension of disbelief as a condition of not only constructing virtual realities, but also the reality itself to perceive our observation (Ascott 1999, 87).

In 1995, Wired magazine published Nicholas Negroponte's (b. 1943) infamous comparison between bits and atoms (Negroponte 1996). The founder of the MIT Media Lab pointed out that being digital has its special domain of value. In Becoming Virtual, Pierre Lévy (b. 1956) called attention to a pre-Manovich deduction of 'transcoding' between the 'computer layer' and the 'culture layer.' Lévy $(1998,15)$ wrote that "virtualisation has 
begun to affect not only the fields of information and communication but also our physical presence and economic activities." In an analysis of its 'economic foundations,' Miller (2011) explained that digital media could have concrete impacts on the exchange value despite being ephemeral.

Moreover, a final note should be granted to mention the contemporary concepts of 'virtual' ascribed to computers. Marie-Laure Ryan $(2015,18)$ (b.1946) identified a range of aspects, including anything experienced in cyberspace as virtual to real experience; zerosand-ones operations executed in the machine as virtual to human; memory storage disks external as virtual to the computer; and versatile software such as a virtual calculator. Her book, Narrative as Virtual Reality, provides a lengthy theoretical background, following the etymology of 'virtual,' which comes from virtus (Latin: strength, manliness, virtue) with a "concept of [...] force or power" (Ryan 2015, 18). From the multi-vocal 'virtual' there appeared different museum concepts as summarised in Table 2 below. The table is limited to samples that inform the content in the rest of this chapter.

Table 2. Select virtual museum concepts and samples until the 2000s (based on medium).

\begin{tabular}{llll}
\hline Medium & by & Concept work & Year \\
\hline Text & Samuel v. Quiccheberg (1529-1567) & Inscriptions & 1565 \\
\hline Panorama painting & Robert Barker & Panorama of London & 1801 \\
\hline Photography & André Malraux (1901-1976) & Le Musée Imaginaire & 1947 \\
\hline 3D still digital & John Sanborn and Dean Winkler & Luminare & 1986 \\
\hline $\begin{array}{l}\text { Digital image } \\
\text { (in CD-ROMs) }\end{array}$ & Louvre Museum & Le Louvre & $1990 \mathrm{~s}$ \\
\hline 3D interactive & Apple Computer & The Virtual Museum & 1992 \\
\hline $\begin{array}{l}\text { 3D web-museum } \\
\text { (skeuomorphic) }\end{array}$ & Alicia Haber & $\begin{array}{l}\text { Museo Virtual de Artes El } \\
\text { Pais (MUVA) }\end{array}$ & 1997 \\
\hline $\begin{array}{l}\text { 3D web-museum } \\
\text { (abstract) }\end{array}$ & Asymptote Architecture & $\begin{array}{l}\text { Guggenheim Virtual } \\
\text { Museum (GVM) }\end{array}$ & 1999 \\
\hline
\end{tabular}

\subsection{1 'The Museum Without Walls'}

From the sixteenth century to the twenty-first, a virtual museum was in the agenda of thinkers and museologists, such as Samuel van Quiccheberg (1529-1567) and André Malraux (1901-1976). Coincidently, they both had deep connections with the government; 
in fact, the latter art historian Malraux was a prominent figure in politics. However, Malraux's career demonstrates his interest in multiple areas. Earlier to this position as the first Minister of Cultural Affairs for France between 1958 and 1969, he was the Minister of Information (1945-1946). In its aftermath, he combined concepts of information and science with culture and arts. Malraux (1953) wrote a seminal book with its famous first chapter on musée imaginaire, i.e. 'the museum without walls' or 'the imaginary museum' first published in 1947.

Malraux's (1953) analysis of the museum without walls stressed the changing significance of collections in modern museum institutions (Allan 2009, 264). He visualised what museums would have to confront in the verge of digital reproduction. His appraisal of photography attained the object's plurality in interpretation, with the visitor invited to partake in performance, liberated in time and space. He was conversant with the mobilisation of the content reproduced by 'printing' (Malraux 1953). At that time, it was rebuked as heretical for alienation of authenticity. However, from his point of view, the imaginary museum was in the constant formation of new contexts for the content. For example, his use of 'aura' attains an effect to "metamorphoses" instead of Benjamin's to the 'original' (Allan 2009, 249n37).

An equally revolutionary positioning of the object belongs to Samuel Quiccheberg who wrote the first text on museology in the sixteenth century (Quiccheberg [1565] 2013). His inscriptions formulated a system of classification in three major categories, which are art collection, natural history collection and the "rarity cabinet" for others (Dreier 1985, 106) (Table 3) (Fig. 15).

Table 3. Quiccheberg's system of Wunderkammer. Adapted from Quiccheberg ([1565] 2013, 26).

\begin{tabular}{cc|c}
\hline \multicolumn{3}{c}{ Wunderkammer } \\
\hline $\begin{array}{c}\text { 1. Founder and creator } \\
\text { (representation) }\end{array}$ & \multicolumn{1}{c}{$\begin{array}{c}\text { 4. Enacted knowledge } \\
\text { (representation) }\end{array}$} \\
\hline $\begin{array}{c}\text { 2. Artificialia } \\
\text { (crafted objects) }\end{array}$ & $\begin{array}{c}\text { 5. Tools of artifice } \\
\text { (means of craft) }\end{array}$ \\
\hline & 3. Naturalia \\
(world of nature)
\end{tabular}




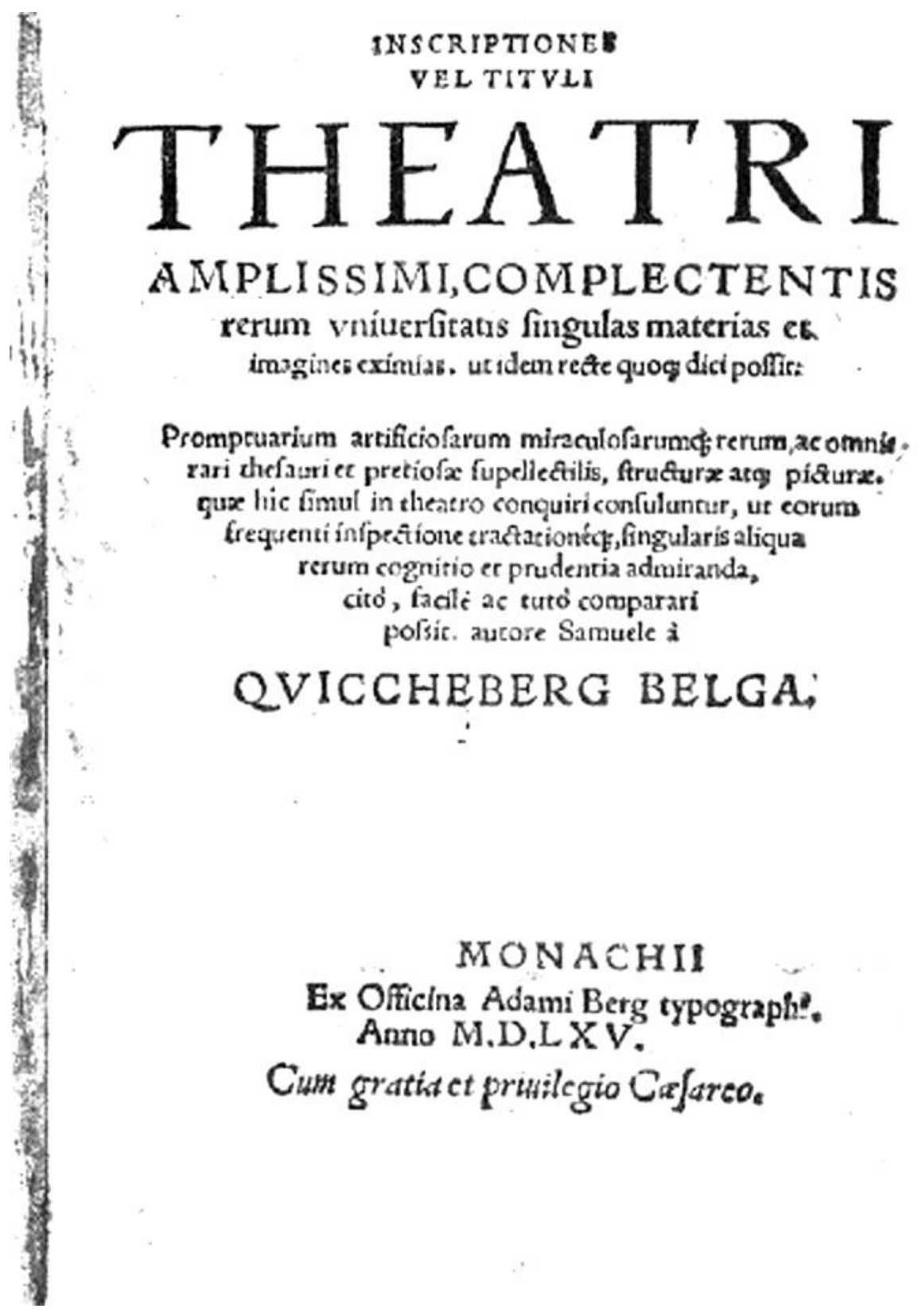

Fig. 15. Title page of Quiccheberg's Inscriptions. Adapted from Quiccheberg ([1565] 2013, 60).

\subsubsection{Being 'Virtual'}

Two panorama works from the nineteenth century are mentioned earlier to show an evolution of the concept of embodiment in museums from still panorama paintings (Les Sauvages de la Mer Pacifique, 1804) to machines (Kaiserpanorama, 1883). Exhibited in the panoramic architecture of Rotunda in Leicester Square, Robert Barker's Panorama of London is one of the first panoramas that contributed to the "emergence of virtual reality" (Otto 2011, 27) (Fig. 16). 


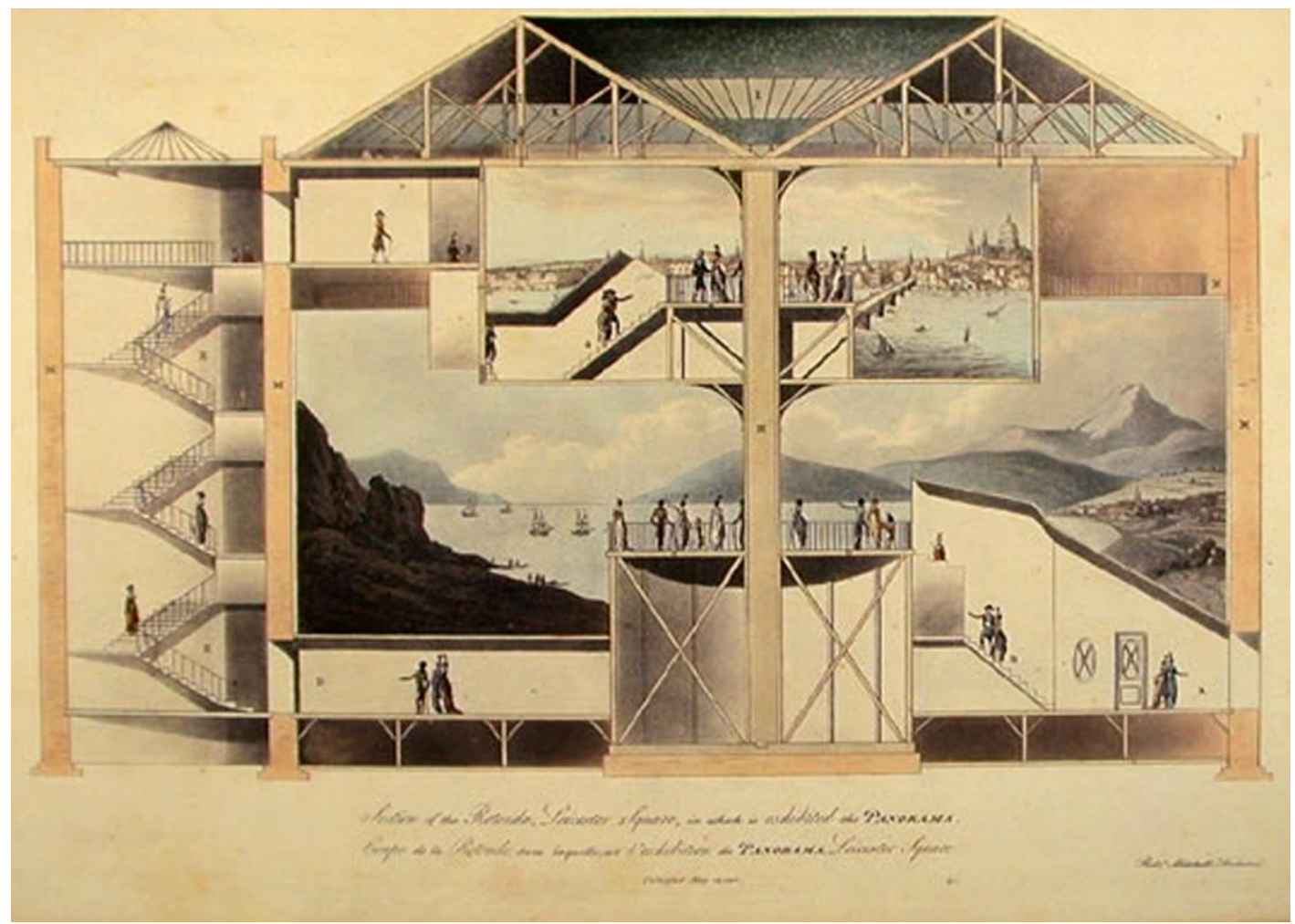

Fig. 16. A Section of the Rotunda in Leicester Square, in which is exhibited the Panorama of London. Adapted from Open Research Exeter (2009).

In Deloche's $(2001,254)$ words, the virtual museum is a "globally designated concept of the muséal issues, which resolves the processes of decontextualisation / recontextualisation" [emphasis in original]. Contingently, Desvallées and Mairesse (2010, 59-60) argue that 'cyber museum, often incorrectly called 'virtual,' is a package of solutions" [emphasis in original] that has not existed before. In this broad sense, Quiccheberg's invention of a classification system injected the first notion of the virtual museum (Aquilina 2017).

Quiccheberg's system thinking bestowed the leading creative role upon the 'object' instead of considering it as a mere source of inspiration (Aquilina 2011). Likewise, the main stimulus that Malraux's prospect asserted was the "prodigious evolution" of a new role and function of the "work of art" (Battro 2010, 137). The mythical 'style' of the author was to be decomposed by photographic reproduction to reveal the object in a new context, i.e. the stylistic power of the author (Allan 2009, 266).

In that year, it was a favorite dialectic centred on the position of the artist. Artists like Dadaist Marcel Duchamp (1887-1968) made use of objets trouvés [found objects] to highlight a sense of 'authentic noise' in content-making while debasing mythical bourgeoisie of artistic composition. Similar 'randomised' operations were used by Jackson 
Pollock (1912-1956), however, "enhancing his artistic expressivity” Kwastek (2014, 11). Malraux's definition of the imaginary museum was equally contradictory. On the one hand, every recipient should have their imaginary museum, and on the other, the reproduction of the work promotes the position of the generator in the museum institution (Allan 2009, 266).

In the 1990s, the concept of the museum without walls evolved into new forms interactive virtual communities built across personal computers. The virtual museum's pathway crisscrossed from being a spatially confined authority to a global facilitator for many authors. In Weil's (1999) words, it was an ongoing campaign "from being about something to being for somebody" [emphasis in original]. Visitor expectations for "highquality services" increased the significance of computer systems and inaugurated the deployment of external networks (Roberts 2001, 17). One of the pioneers of this transformation was The Museum inside the Telephone Network, which was a 1991 "experimental" art exhibition organised by a Japanese telecommunication company (Huhtamo, 2002). A pre-web communication system of telephone, fax and computer modem were the constituents. It gave the public access to numerous artworks in a range of genres of voice, sound and facsimile collections. Activated by pushing speed-dial buttons, its genre structure was composed of dialogues, recitations, music, novels and comics (ICC 1991).

At the same time, 3D simulation techniques became a new research instrument in heritage studies. In the second half of the 1980s, the IBM United Kingdom Scientific Centre's software system, WINSOM, was employed to produce one of the earliest 3D computer modelling and animation projects in archaeology. Computer scientists digitally reconstructed an eleventh-century cathedral based on historical archives and archaeological evidence (Burridge et al. 1989). In 1985, John Sanborn and Dean Winkler's Luminare was commissioned to computer-generate a seven-minute video for Expo '86 in Vancouver, Canada. It depicted a digital virtual museum space with works of legendary artists such as Picasso and Mondrian (Sanborn and Winkler 1985, mins 3:04-3:48).

\subsubsection{CD-ROMs and World Wide Web}

The pervasive use of in-house display technologies and personal computers gave momentum to commercial museum products such as CD-ROMs. They depicted scale-free exhibitions in 3D virtual space from museum collections, e.g. Le Louvre and Musée d'Orsay in Paris, Hermitage in Saint Petersburg. These examples were far from replacing or even speculating on museums' physical walls. Having been visualised in then-sophisticated software such as VRML, Paintbox and QuickTime VR, the early 3D models were distributed in CD-ROMs 
and the World Wide Web (Huhtamo 2002). These examples later included games sold in CD-ROMs to teach the public. The pedagogical purpose was more prioritised in those games, whereas others were only informative as to what is on display in the real museum space (Roussou and Efraimoglou 1999).

Participation in the early digital heritage applications was limited to display technologies. The use of cyberspace was skeuomorphic to the real museum spaces where the display of collections was in specific orders. Some of them were 'web-only' museums, e.g. MUVA for "Museo Virtual de Artes El País" (El País Virtual Museum of Arts). The outcome was an imitation of actual museum spaces (Battro 2010, 144). MUVA became online in 1997 as the first virtual museum of Uruguay (Haber 2000, 26). Designed by architects using DataCAD, which used to be one of the most widely used computer-aided design software, MUVA's cyberspace was idiosyncratic to physical museum spaces, with a 'building area' equivalent to six-thousand square meters of space (28).

Another virtual museum of that period was the Guggenheim Virtual Museum (GVM). Asymptote Architects, founded by Hani Rashid (b. 1958) and Lise Anne Couture (b. 1959) were commissioned to design an inspirational virtual museum in 1999. It was planned to be one of the branches of the Guggenheim chain all over the world. GVM was the first in cyberspace "to contain art generated exclusively within and for the Internet" (Rashid 1999, 31). The virtual museum had an interactive web-based interface (Fig. 17). Before its launch, Alexander Galloway, a new media critic, commented on the project in an interview in Wired magazine, comparing it to video games. Galloway expressed that "it is exciting because 3D is a whole new realm, ready to be explored [...] If (the museum) is as good as Half Life, it will be a winner" (Spingarn-Koff 2000). Within the confines of then technologies, GVM underlined a crucial social role to connect the public with the cutting-edge technology.
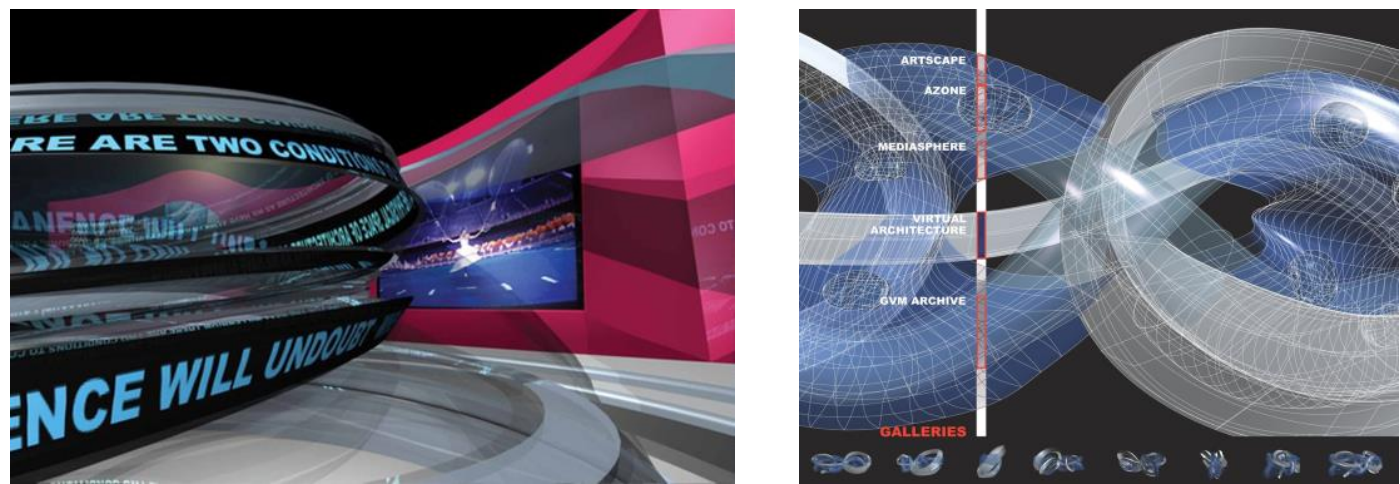

Fig. 17. Guggenheim Virtual Museum (GVM). Adapted from Belogolovsky (2016). 
Launched in 2011, the web-based Google Arts \& Culture (GAC) (formerly Google Art Project) has focused on the educational role of museums. In this project, online interaction means, such as scrolling and browsing through multimedia connections, construct nonlinear narration. GAC published online exhibitions of artworks with the support of the Google Street View providing virtual tours. For example, one can tour inside the British Museum, wandering around like a single visitor (Google Cultural Institute 2015). In a way, it creates a 'non-place' museum accessible from anywhere and anytime.

\subsubsection{Non-Place, Place-Making and 'Suspension of Disbelief'}

According to Merleau-Pontian phenomenology, web-museums have been an empirically "non-place" as coined by the anthropologist Marc Augé (b. 1935) in the context of “supermodernity” (Augé 2008). These non-anthropological spaces are, in Merleau-Ponty's words, confined by "morose walls for the pleasure of Sunday strollers or Monday 'intellectuals," yet to be "wrongly" misinterpreted as antithetical to Malraux's imaginary museum (Allan 2009, 265). In this regard, it was discernible that real museums, which were designed by architects of 'hypersurface,' became subject to Augé's critique of nonanthropological non-places of supermodernity. As examples of this, the Guggenheim Museum Bilbao (1997) by Frank Gehry (1929) and the MAXXI Museum (2010) by Zaha Hadid (1950-2016) interpreted spatial 'effects' in decontextualized forms concerning Deleuze's "affect" (Andreotti and Lahiji 2016, 157). To demonstrate the impacts of digital media use, popular museums, such as The Museum of Modern Art (MoMA) in New York, were examined through a critique of "non-place" (Bautista 2014). In early accounts, existential shortcomings of cyberspace were pointed out by politically-motivated postmodern critiques, e.g. Kitchin (1998) and solution-based designer advocates, e.g. Anders (1999). These studies relied on theoretical, socio-cultural, techno-cultural and literary works (Baudrillard 1981; Benedikt 1991; Castells 1996; Haraway 1985; Hayles 1984; Gibson 1984).

One of the implications of early virtual reality (VR) applications was to admit essential imperatives of feeling immersed in virtual environments. The two prerequisites were "suspension of disbelief" and "viewer-centered perspective." Neira et al. (1992), who presented the CAVE project at SIGGRAPH '92, candidly ask viewers to "ignore the medium" and to "give in to simulation," defining it as an "ability." Many pioneering studies in the area were mostly conducted by computer scientists who were not familiar with abstract concepts of visualisation and representation; so viewer-centred perspective was 
taken for granted as an indisputable advantage for "suspension of disbelief" in VR (Neira et al. 1992). As early as the 1970s, navigation, i.e. 'virtual travel' was also one of the first aspects of virtual reality, employing viewer-centred perspectives (Naimark 2006).

\subsubsection{Rise of Digital Heritage}

The field of virtual museum/heritage demonstrated itself in theoretical discourses, methodological papers and practical applications. From Benjamin (1936) to Manovich (2001), theoretical contributions have constructed a firm foundation. Likewise, from Malraux's (1953) “imaginary museum" to Lévy's (2010) Digitongs, many methods and methodologies have made improvements in understanding its epistemology. In the same vein, from the photography documentation at the V\&A to Burton's (1967) introduction of the CRT display, and to Kenderdine's (2015) integrated immersive multisensory installations, each praxis of digital heritage has provided substantial assistance in the development of a multi-disciplinary scholarship.

In digital architectural heritage, Champion (2006), Chen and Yehuda (2007) and Tan and Rahaman (2009) study 'place-making.' They address the challenge of integrating virtual environments with activities. Earlier research in architectural design had focused on activities in virtual environments (Schnabel 2004; Dorta 2001). Alongside these works, virtualisation of computer-generated geometry has attained its popularity in digital heritage via the pioneering works of Barcel'o, Forte and Sanders (2000) in various computer applications in archaeology; Frischer et al. (2000) in 3D modelling and visualisation of Rome; Pletinckx et al. (2000) in virtual reality and multimedia application; Ioannides (1993) in 3D reconstruction via laser scanning; Wahba, Bahgat and Saleh (2001) in satellite imaging via GIS; Borgo, Cignoni and Scopigno (2001) in 3D mesh visualisation and geometry computing; Santana Quintero (2003) in 3D documentation and dissemination; Rousso (2004) in engagement and constructivist learning in virtual environments; Kenderdine (1998) in interactive virtual reality and online multimedia applications; Debevec, Taylor and Malik (1996) in photogrammetry and view-dependent texture mapping; Refsland et al. (1998) in virtual dome (cave) applications; DeLeon and Berry (2000) in interactive video-game applications; and Mania and Chalmers (2001) in multisensory perception and memory impacts in simulated heritage environments.

Although the fields of archaeology, heritage, history and computer science are dominant, architecture scholarship has made a significant contribution to digital heritage by hosting events, publishing at high-end venues and promoting research. In 2007, the first 
50

multidisciplinary attempt was made to bring scholars together to discuss how to preserve cultural heritage through new media. In New Heritage: New Media and Cultural Heritage, Kalay, Kvan and Affleck (2007) edited papers on 'virtual heritage' around a range of discussions. These topics have been valid since then, representing the overall structure of the digital heritage field, by answering 'Why?' 'What?' and 'How?' is digital heritage made.

\subsubsection{New Principles, Old Concerns}

Addison's (2000) paper is possibly the first of its kind in recognising the emerging rise of "believable" technologies as a new phenomenon in heritage. "Accuracy' and 'immersiveness' were two common technical concerns in the 1990s. The efforts were experimental in the technical sense and the following decade was interested in developing accountable methods as the number of adversaries was not insignificant. In the 2000s, new display technologies replaced the old ones such as cathode-ray-tube (CRT). New screens with better accuracy, faster viewing and more efficient functionality gave rise to attention in the virtual heritage field.

Computer-generated museums and digital heritage techniques of the 1990s and the 2000s succumbed to fast changes in computer graphics and new media. Their ephemeral impact and fragile quality provided new concepts for thinkers to consider in heritage. On the one hand, it was the preservation of digital heritage itself (Lusenet and Wintermans 2007; Bentkowska-Kafel 2009). On the other hand, digital works were criticised as being somewhat implicit and self-credited, so provenance of documentation processes gained significance (Bentkowska-Kafel, Denard and Baker 2012). Other critiques involved authenticity, interpretation, meaning-making, user-engagement, place-making and technological limitations such the lack of virtual realism. New charters and principles were informed to keep the sincerity of the acquired knowledge through digital models.

Beginning with SPAB Manifesto ([1877] 2018) that was followed by Athens Charter (1931), Venice Charter (1964), Burra Charter ([1979] 2013) and Nara Document on Authenticity (1994), international scholarship has introduced many principles to follow in heritage conservation, preservation and management. In the later parts of the $20^{\text {th }}$ century, the adoption of these principles has revolved gradually. They encountered the use of new technologies for 'interpretation' and 'interaction,' which were theorised by accounts in tourism. Pervasive use of new methods and the cultural impacts of technology required amendments in the scholarship of such principles. The rise of digital methods triggered new concerns. The London Charter ([2006] 2009) and the Seville Principles (2011) were released 
to ensure consistency in the field. They primarily sought accuracy, provenance and paradata in digital heritage applications.

The aim of the London Charter for the computer-based visualisation of cultural heritage is set "to ensure the ever-increasing expressive power of computer graphics become [sic] accountable to the rigorous standards of historical research" (Denard 2012). By that, the charter deals with a contradiction generated by popular misconceptions about virtual reality. The old 'dream' was that "we might create complete experiences of long-lost places and periods that would be virtually indistinguishable in appearance from actuality" [emphasis added] (Denard 2012). With the impact of the London Charter on heritage visualisation, virtuality, in Peircean terms, was reinterpreted as more than a "mental copy of its real object" (Thellefsen and Sorensen 2014, 495).

The Seville Principles of archaeology (2011) confirms the London Charter as the hitherto "most advanced international document." It makes additional comments with eight principles ranging from interdisciplinarity to authenticity. With these two international documents, new research directions appeared based on scholar acknowledgement. The boom of virtual reality technologies after 2010 and the pressure of previous works to go beyond made it compelling for digital heritage scholars to explore research directions. They range from crowdsourcing heritage via online and multimedia applications to data mining and machine-learning strategies to extract meaningful information.

From the 1990s onwards, virtual heritage became one of the primary interests of heritage and museum scholars. The initial inspiration was the possibility of virtual-realism in cyberspace. The evolution of the virtual museum (i.e. cybermuseum or digital museum) demonstrated inconsistencies with 'virtuality.' In line with theories interpreting 'virtuality,' pioneering digital heritage studies in the 1990s and the 2000s pushed the boundaries of the field. To account for academic rigour, new principles emerged along with new research directions. Therefore, the somewhat painful and glorious history of the 'virtual museum' dissolved our interests into the information space. By that, it has proven an old hypothesis that no museum does not operate the terminal activity of "deaccessioning" (Chenhall 1978, $4)$. 


\subsection{The Death (Deaccessioning) of the Virtual Museum in Information Space: Objets Digitalisé of Heritage}

Subchapter Outline

Purpose: Digital platforms enable easier access for public participation. The contemporary media allow individuals to produce content. The subchapter aims to mark non-text-based information resulting in visual participation in digital platforms.

Design/Approach: The subchapter first looks at digital workflows and the new role of digital content. Then, the evolution of virtual communities is reviewed in terms of engagement in alternate realities.

Findings: The samples of digital engagement reveal that user-generated and non-text-based information has authentic value to be discovered with special methods.

Limitations/Implications: The rise of information over knowledge was shown in the first subchapter. There are still issues around the use and meaning of information. The subchapter gives an overview of how important it is to touch on the use of information in AHIs that embark on the digitalisation of content.

Originality/Value: The subchapter is one of only a few studies that review user-generated information to build meaningful and authentic outcome in AHIs.

Keywords. Crowdsourcing; alternate realities; crossover; objets digitalisés; information space; user-generated content.

Museums occupy an equivocal territory. On the one hand, they share the same funding opportunities with other memory institutions such as galleries, libraries and archives. On the other hand, they share the same interests with creative industries such as design, architecture, arts and even video games. However, museums have to deal with dynamic sets of information, unlike libraries (Parry 2007, 23), and they have to explicitly communicate information, unlike arts.

When museums confronted computing technologies from the 1960s to 1990 s, there was a compatibility issue between museum staff and information technologies. The problem was that new media tools and computing technologies were alienating (Jones 2008). For many years, museums touched the surface of cyberspace via CD-ROMs, which were souvenirs in museum shops. Hiring ICT experts who could manage sophisticated computing and new media technologies has been a costly business for museums (Marty 2008). They relied on funding from institutions that were not aware of an upcoming end for museums in the digital age. Instead, digital applications were expected to rely on simple operations like turning on and off. Many virtual museums were born in the 1990s and the 2000s. They were 
far from attributing a new role to museums in the information age, dreaming of being indistinguishable from their actual counterparts.

However, throughout the digital past of museums, there has been an exchange, or rather "transcoding" (Manovich 2001), between 'cultural' institutions, which are managed by people, and 'computers,' which are also managed by the same species. Therefore, the communication between the two sides somewhat enabled/disabled and skilled/deskilled each other. Despite the increasing amplitude of sympathy on each side, they, as Naimark (2006, 334) reflects, "drink in different bars."

Eventually, digital heritage arose as an area to bring closer these two different domains of value. This thesis interprets the shift from virtual heritage to digital heritage as a disentanglement from conventional modes of thinking. Conceptualising the crisscrossing in values as the death of the old virtual 'museology' designates digital transformations for the examination of digital heritage. Thereby, the digital (or cyber) museum in information space is distinct from its precedents, namely the virtual/visual heritage of the last two-three decades.

The death of the virtual museum is the 'deaccessioning' of the past institutional formats that became available in the web at the disposal of virtual communities. The components of the museum as information space include dynamic sets of information ranging from metadata, paradata and museum itself to content and users (Navarrete and Owen 2016). Instead of products, they are processes of crossing over between information about the constituents. The objects of these processes are defined rather than relational. Processual and discrete explanations (Cameron 2007; Parry 2007; Witcomb 2007) replaced the binary interpretation of the digital and the real object. As a subjective form of crossovers between processes, user-generated content is required to be indexical in the 'post-literary' (or posttextual) mechanism of engagement (2013) (Table 4).

Table 4. Crossover processes in the museum of information space. Adapted and modified from Ricardo (2013, 169).

\begin{tabular}{lll}
\hline Crossover & Character & Purpose \\
\hline Exposition & Propositional logic & Establishment of order \\
\hline Transgression & Impulse to freedom & Escape from structure \\
\hline Reflexivity & Perceptual circularity & Indexical self-reference \\
\hline
\end{tabular}




\subsubsection{Digital 'Crossover'}

As outlined above, it is well-known that there is a paradox of virtual reality. It is a subject that has remained constant throughout the history of philosophy in, to name a few, Plato's knowledge (episteme) and opinion (doxa); Aristotle's form and matter; Avicenna's existence and essence; Descartes's mind and body; Kant's phenomena and noumena. The primary condition of the 'virtual' is to project accurately what is subject to being virtualised. There appears a paradox between remining original and altering the original. The distinction between these philosophers lay behind their resolution to understand these dualities, which form unique dialectics of difference. Crossing over between two sides, their theories proposed mechanisms, such as mimesis by Plato and schematism by Kant (Bell 1998, 3). These theories of 'media' are reproduced in other contexts in arts, literature, semiology, information theory, general system theory, cybernetics, new media, and virtual heritage and so on. In Peircean semiology, meaning transpires as reality when the sign, or the virtual, alters the object that it signifies. Peirce introduces the role of an "interpretant" that conditions the crossover of the sign to the object (Eco 1976; Kockelman 2013). The recognition of crossover enables process-thinking in contrast to a-historical and sterile theories. Manovich (2001) reads a similar process-oriented crossover, namely transcoding, between culture-layer and computer layer.

Shortfalls of early virtual heritage works included a lack of understanding of the process as generative systems that can bring forth new forms of imagination in each iteration. There were technological limitations as well as a stubbornness of traditional sciences that were only familiar with closed-systems that preserve the role of the museum as places where the visitor enters and exits. Roy Bhaskar's (1978) (1944-2014) philosophy of "Critical Realism" (CR) almost condemns such empirical determinacy in science seeking knowledge from closed-systems. Although there appears a visionary reflection on the heritage field and museum studies, methodological inaccuracies hindered the development of the 'transcoding' in applied research until the 2000s. It was then realised that crossing over between heritage and virtual realities makes possible the transcendence and immanence. Accordingly, new principles were introduced. New voices emerged to theorise digital heritage and the role of what can be called its 'crossover' function (Cameron and Kenderdine 2007; Parry 2007). As intermediation processes, crossover methods are also multiplied and crossed over within themselves. Examples are given in digital design research. 
Schnabel and Kvan's digital architecture research is an explicit example of 'crossover.' Conducted at The University of Hong Kong, students experiment with 'found' tools, i.e. "objets trouvés," in their design studio. They are used to translate the object from physical to digital to virtual. Using a variety of "objets trouvés" (e.g. 3D scanners and light pens) and "objets digitalisés" (e.g. glitches), students constantly switch between physical manipulation, translation, digital transformation, virtual manipulation and physical realisation during the design process (Fig. 18). Peeping through the "gap between the imagination of design and its representation," they show how a series of solutions from both the digital and physical enable a richer understanding of spatial qualities (Schnabel et al. 2004).

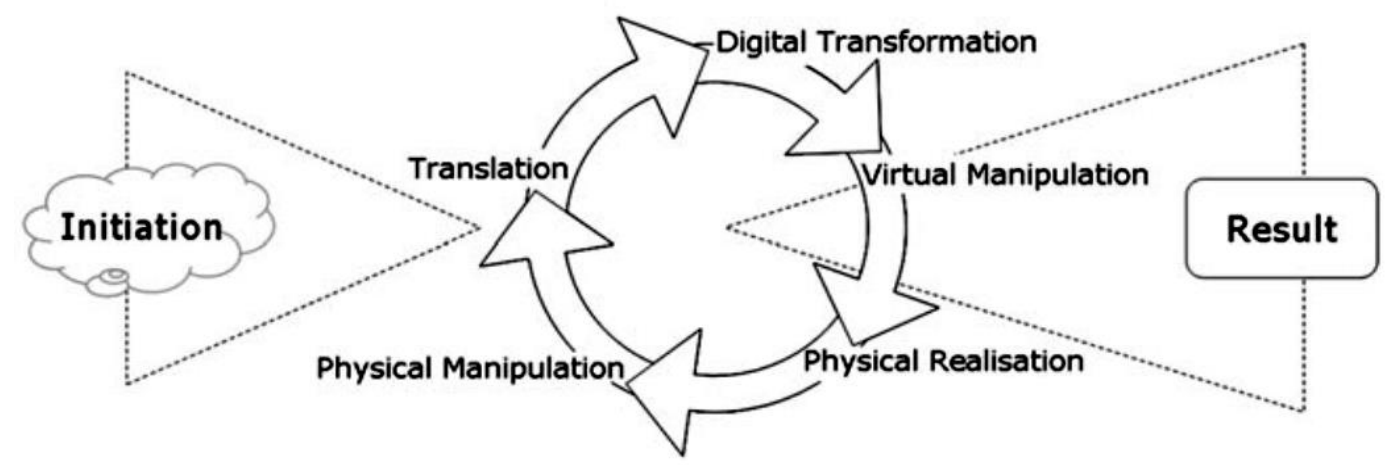

Fig. 18. Crossing over between physical, digital and virtual stages during architectural design. Adapted from Schnabel et al. (2004).

This kind of non-linearity of the heritage digitisation has not been fully realised yet. It is partly because of the understanding of digital heritage as a closed-system that operates through accuracy (see further details in Chapter 3.5). Digital heritage requires the use of highly advanced technologies. But current research remains reluctant to test interoperational methods, and technology suppliers are likely to step back when it is a matter of interoperability. It requires a large investment. Their closed-system is being enforced by theoretical underpinnings that are also blamed for being too 'postmodern' to listen. In this case, as a process, crossover in the digital transformation of heritage requires a simplistic approach to highlight its primary and social usability.

These research has overtly been focused on educational aspects of digital heritage. Parry and Arbach (2007) unfold educational objectives with three interconnected aspects: localisation, personalisation and constructivism. Their theorisation of online learning somewhat ensures the old role of museums as being a guardian of authentic knowledge. In process-oriented digital production, it is evidenced that laypersons could teach the authority. 
Yuan's construction of Ripple Wall project in Chengdu, China prepared parametric solutions to teach workers how to lay bricks with particular angles. Ironically, they, in return, taught the architect how to build a wall parametrically (Yuan, Zhang and Han 2013). There is a myriad of cases depicting the power of laypersons. Chapters 2.5.2 and 3.3 contextualise more on this, with the themes of crowdsourcing and unmediated cultural heritage, respectively.

\subsubsection{Alternate Realities}

The digital heritage field used to be recognised as 'virtual heritage' until the 2000s (Addison 2000). From early computers used in museums to 360 degree panoramic and stereoscopic media screenings, the understanding of the 'virtual' has changed and transformed into the 'digital.' The former systems were 'virtual' in operational terms, whereas the virtuality of following applications is manifold. In the 1990s, some leading museums such as Le Louvre started to sell CD-ROMs, which are acknowledged as early examples of digital heritage applications (Huhtamo 2002). The Virtual Museum, which was presented by Apple Computer at SIGGRAPH '92, was an interactive electronic museum divided into simulation rooms (Miller 1992). With the advent of virtual reality (VR) technologies, the scope of digital heritage applications was enlarged. The virtual museum evolved manifold and formed many alternate realities through a range of interactive new media.

Recent practice has been focused on how alternate realities of new media enrich our experience in participatory heritage (Adair, Filene and Koloski 2011), in heritage and social media (Giaccardi 2012), and in digital visual culture (Bentkowska-Kafel, Cashen and Gardiner 2009). Theoretical underpinnings of alternate realities underlined the necessity of new dimensions in understanding old heritage topics, with authenticity discussed in detail (Parry 2007; Witcomb 2007; see also Colley 2015). It has also been focused on how to extract knowledge from these experiences in alternate realities of multimedia (MujooMunshi and Chaudhuri 2014). The contribution of other research remains relatively unnoticed, such as alternate realities in TV and online media (Van Hoff 2017). These system-based approaches leave nearly no trace in digital heritage, which struggles with an "intellectually stubborn" (Winter 2013, 540) divide between science-based and humanitiesbased approaches. The early digital museum technology had to be managed by the viewer (switch on and off). This lack of expertise further hardened the position of museums as "Authorised Heritage Institutions" (Smith 2006, 87). The role of these "authenticators as guardians of knowledge" is challenged by contemporary digital platforms, where individual 
story making captures more attention (Howell and Chilcott 2013). This type heritage making as a collective memory is deeply connected to early virtual communities.

\subsubsection{Virtual Communities of Our 'Global Village'}

Marshall McLuhan (1964) (1911-1980) coined the term "global village" as an electronically wired world that would result in ultimate Simulacra (Baudrillard 1994) in which there is no longer illusion. In accord with these postmodern theories, virtuality has been conditional to reality, whereas 'virtual communities' started to emerge and occupy cyberspace. Prior to the SecondLife, one of the earliest virtual communities took shape in the WELL standing for Whole Earth 'Lectronic Link, which accommodated a few thousand people in the late 1980s. Rheingold (2000) explains how its users generated textual content about various topics to end up with one of the first successful 'virtual communities' of cyberspace. Since then, a variety of overlapping definitions arose with regards to virtual communities, for example, collective intelligence (Lévy 1997), crowdsourcing (Howe 1996) and gamification (Deterding et al. 2011). They are part of the new web jargon but old in practice, which dates as early as 'citizen science' initiatives in the 1880 s (Simon 2010, 185).

Recent research has embarked on conceptualising crowdsourcing and participation for heritage and museums integrated with online and digital platforms (Ridge 2014a; see also Roued-Cunliffe and Copeland 2017; Adair, Filene and Koloski 2011; Giaccardi 2012). Crowdsourcing is defined as an activity that mutually "benefits both audiences and institutions" (Ridge 2014b). Some of the crowd activities in recent crowdsourcing projects in heritage, museums and galleries include tagging, e.g. Waisda? (Hildebrand et al. 2013), assessing proposed exhibition content, e.g. Click! (Bernstein 2008), playing with Lego pieces, e.g. Demented Architecture (Eliasson 2015) (Fig. 19), uploading photos for 3D reconstructions of war-torn sites, e.g. \#Newpalmyra (Khartabil 2017) and Project Mosul (Rekrei 2015). 
58

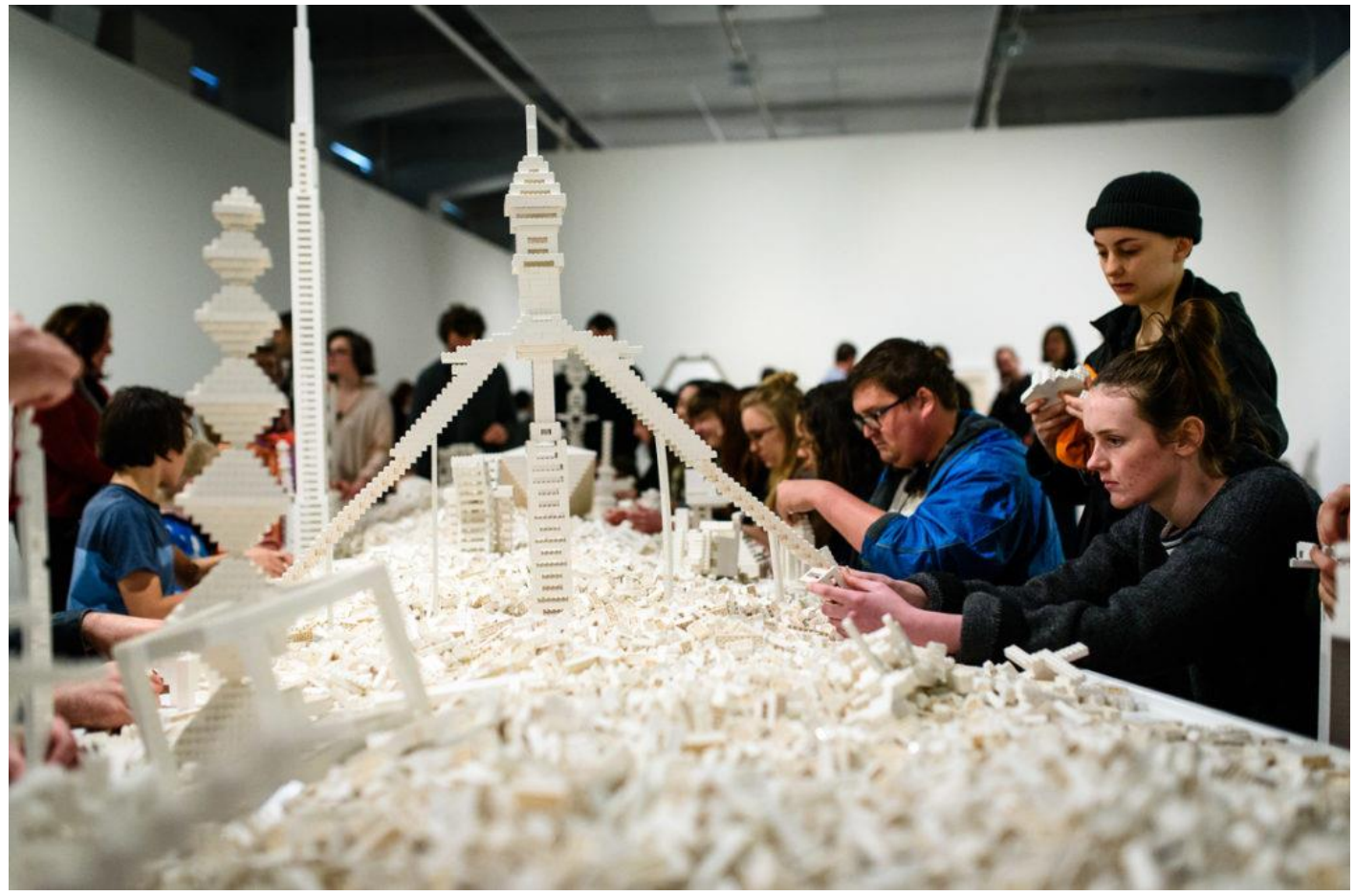

Fig. 19. Olafur Eliasson's participatory installation Demented Architecture exhibited at the City Gallery Wellington Te Whare Toi, Wellington, New Zealand. Adapted from Eliasson (2015).

There appear two types of crowdsourcing research projects in the broad context of heritage. Research has been focused on, on the one hand, individual roles and activities; on the other, 'knowledge ecosystems.' With regards to the former, Ridge (2004) accumulates eight projects and four theoretical discussions about crowdsourcing in heritage. In the context of the latter, Szoniecky and Bouhai (2017) bring together seven articles in Collective Intelligence and Digital Archives: Towards Knowledge Ecosystems.

There is a challenge to answer who is the expert in this participatory web-culture (Westberg Gabriel and Jensen 2017). Andro and Saleh $(2017,136)$ define two types of crowdsourcing, explicit and implicit. The former refers to voluntary user participation, such as Wikipedia, and the latter, involuntary, such as reCAPTCHA (137). In their bold generalisation, 'playing games' and 'gamification' belong to the former. There are also two examples from bio-chemistry.

Foldit is a sci-game designed by using gamification techniques to outsource the prediction of possible protein combinations. The goal of Foldit is to solve new protein chains that can help prevent diseases (Cooper et al. 2010). Likewise, in Phylo, online players contribute to the discovery of novel DNA sequences that can be used for research in genetic disorders like cancer (Kawrykow et al. 2012). By crowdsourcing, both examples of research 
get away with designing computationally expensive algorithms. They use game design elements such as rewards, chat rooms and visual feedback (Fig. 20).

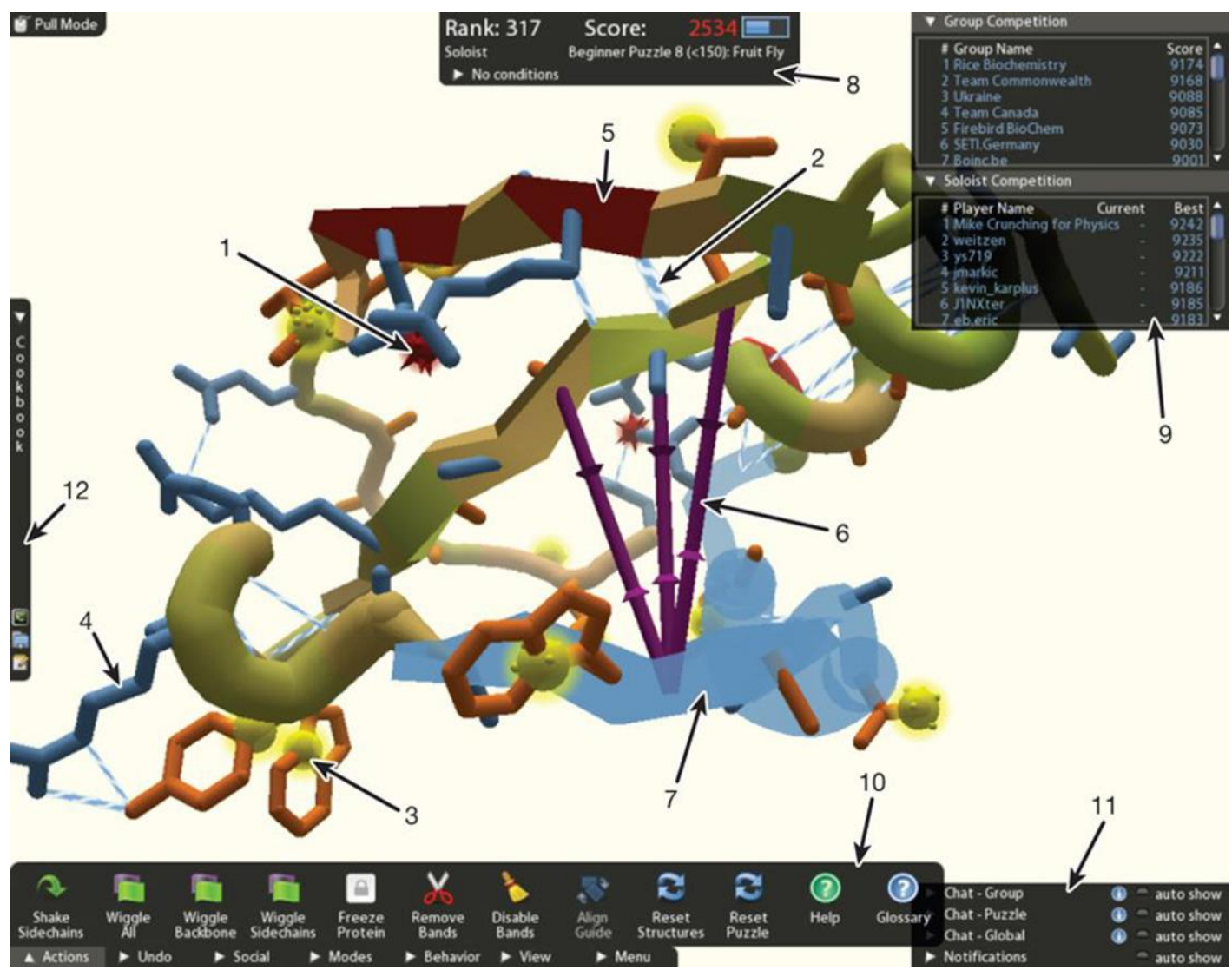

Fig. 20. Foldit interface screenshot. Adapted from (Cooper et al. 2010).

The first computer game was Spacewar developed at MIT in 1961. Later with cathoderay-tube display terminals plugged in as a computer interface, its developers connected game playing with TV screens. They called it Atari, which hinted at the beginning of videogame player communities of overwhelming size. Today huge digital festivals, e.g. DreamHack and eSportsPool, are organized, bringing together virtual communities from all over the world (Fig. 21). By 2015, the world's largest LAN (Local Area Network) party, which hosted 22,180 game players, was held at the DreamHack Winter 2013 in Jönköping, Sweden (Guinness 2013). In fact, the event has beaten its own record repeatedly since the first gathering in 1994. 
60

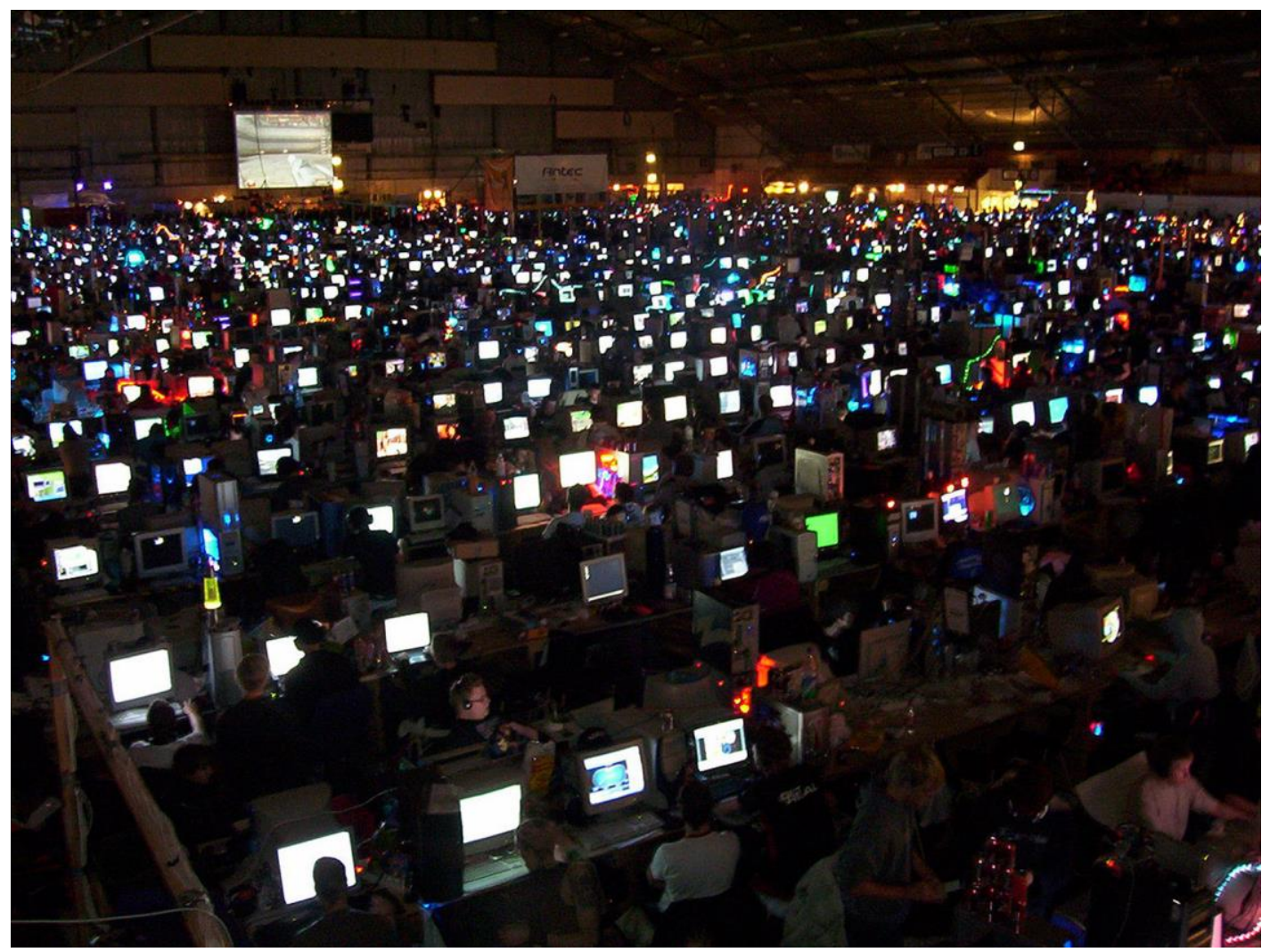

Fig. 21. DreamHack Winter 2004. Adapted from http://www.wikiwand.com/en/DreamHack.

Figures from the USA show that the average video game player is 35 years old. Another grouping of gamers may be defined by gender (ESA 2017). But these statistics have been likely to generate misconceptions about virtual communities. The reason why game playing frequency decreases with age is found to be dependent on a myriad of factors. Borowiecki and Prieto-Rodriguez (2014) investigate video game playing as a cultural consumption like other art activities by considering socioeconomic variables as well as demographic and geographic factors. They divide gamers into two groups: those who never play and those who are likely to play, adding that they are both "heterogeneous populations" (Borowiecki and Prieto-Rodriguez 2014). The heterogeneity of the video game culture has grown so long that it has overcome temporality. Stuckey et al. (2015) analyse what museums can learn from retrogamer activities, suggesting a new historisation of the video game culture (see also Stuckey and Swalwell 2014).

\subsubsection{Authenticity of Non-Text-Based Information}

Contemporary understanding of authenticity is no longer based on definitive models (Lowenthal 1999). Today, it is less important to come to an ultimate and immediate 
agreement on the authenticity issue in heritage practice, than it is to reframe it as a process of construction and agreement between conflicting views. This is examined by research focusing on digital museum object (Cameron 2010), cultural heritage management (Fairclough 2008), digital heritage (Affleck and Kvan 2005), participatory museology (Adair Filene and Koloski 2011), and heritage theory (Waterton and Watson 2013; Harrison 2013). Even though it is a new scientific domain with less than 50 years of history (Münster and Ioannides 2015), digital heritage is extending knowledge about authenticity in the information age (Colley 2015).

"What is information in the museum context?" ask Orna and Pettitt (2010), who define it as the "essential food of knowledge" (28). At the bottom line of their definition lies a combination of theories from information science (Ingwersen 1992) and cybernetics (Argyris and Schön 1996; Checkland and Scholes 1990). At its centre are 'collections' on which all the information a museum would require is based (Orna and Pettitt 2010, 33). System perspectives on museum information tend to assign museums to be the 'guardian of knowledge.' Systems help museums "strive to articulate their knowledge" in various levels of detail (Bearman 2008). Developed by the New Museology thinking, participatory agendas, social media and digital interaction, museums seek a solution to manage the dynamic production of user-generated collections. Today, "the emancipated spectator" (Rancière 2009) of alternate realities is given the role of the 'guardian of knowledge,' whereas museums facilitate it (Hooper-Greenhill 2000; Howell and Chilcott 2013).

Virtual communities leave limitless and manifold data traces. With reference to Rancière's (2007, 109-138) essay titled “Are Some Things Unrepresentable?” Galloway (2012) argues that "data have no necessary visual form" on the large surface of the information network. There is "only one visualization" in the information space. Nevertheless, visualisation of big-data has been a topical area of new media studies. To make sense of virtual communities in social media, researchers produce visually appealing analysis (Yazdani, Chow and Manovich 2017). Manovich admits that "every item [of new media collections] has the same significance as any other" (Manovich 1999, 80) (Fig. 22). In fact, new media thinkers give a message that "processhood" in information space frees function from "objecthood" in form, material and structure. Emancipated across space and time, engagement with new media embraces aesthetics and authenticity of the process (Ricardo 2013). 


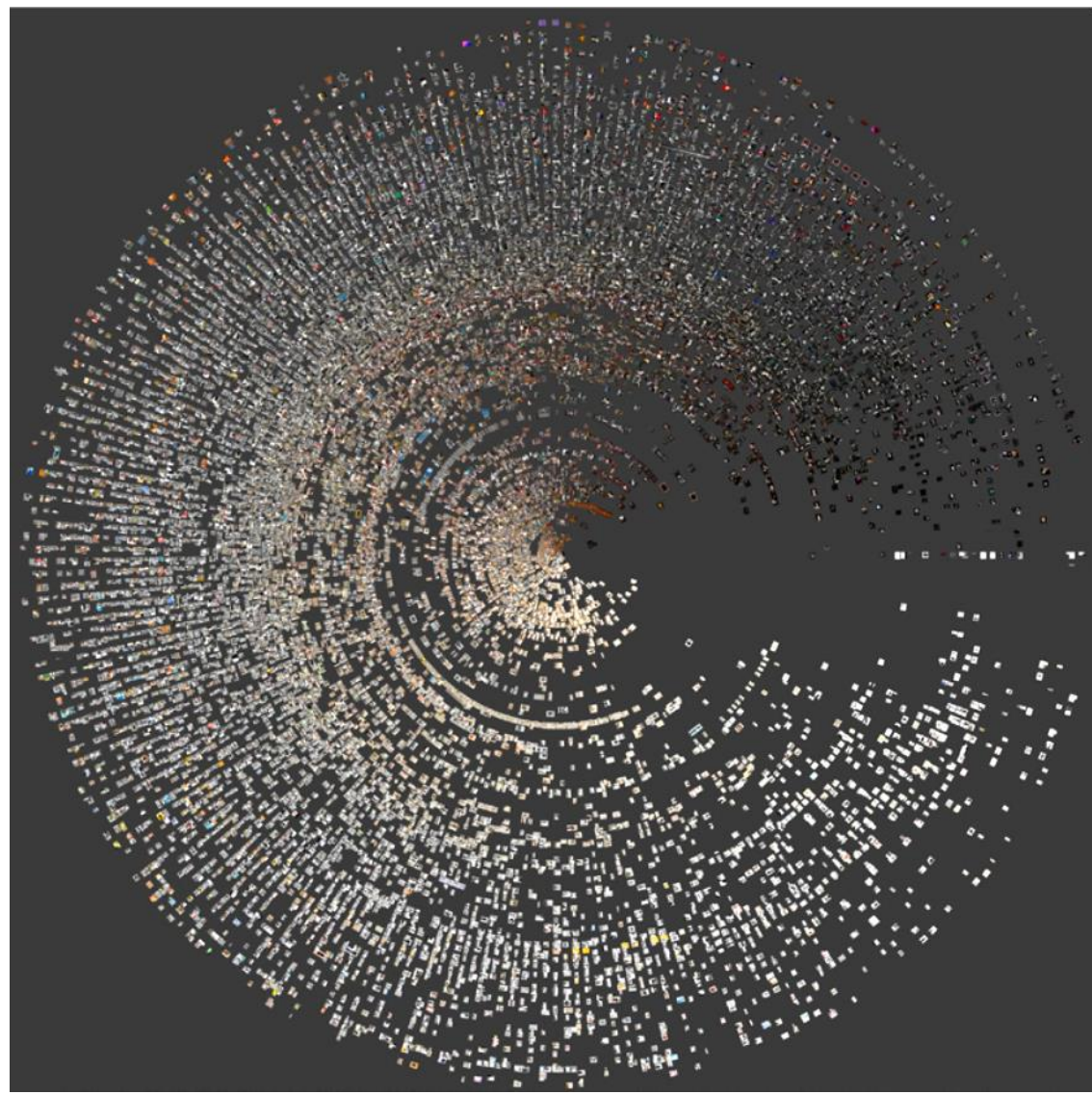

Fig. 22. Radial visualisation of MoMA's photography collection based on more than eighteen thousand photographs between 1837 and 2012, with distance from the centre determined by 'year' and radial degree by 'average brightness.' Adapted from Hochman and Manovich (2014).

The persistent evolution from object-centred thinking into the process-centred has transformed philosophy (Deleuze and Guattari 1980; Latour and Weibel 2005; see also DeLanda 1997), architecture (Schumacher 2009), design (Schön 1983; 1992), archaeology (Binford 1962), new media (Hayles 2012) heritage (Waterton 2010), museology (Witcomb 2003) and museum informatics (Bearman, 2008). In literature, "the death of the author" in Barthes's (1988) postmodern dis/utopia referred to the emergence of many authors. In new media, this change was expressed as a shift from broadcasting model to web 2.0. In heritage, the 'deaccessioning' of the 'virtual museum' is the emergence of virtual communities and their muséal activities in information space. At the end of the shift from one-to-many (broadcast) to many-to-many (multimodal) interfaces, Ricardo (2013, 29) reads a "transmodal" engagement. It is an ultimate "mirror phenomenology" [emphasis in original] as the "demonstrable evidence" of subjectivity (172). By that, it answers the question of Rancière "Are Somethings Unrepresentable?" The personal content crosses over between mechanisms and structures by means of "indexical self-reference" (169) (Fig. 23). 

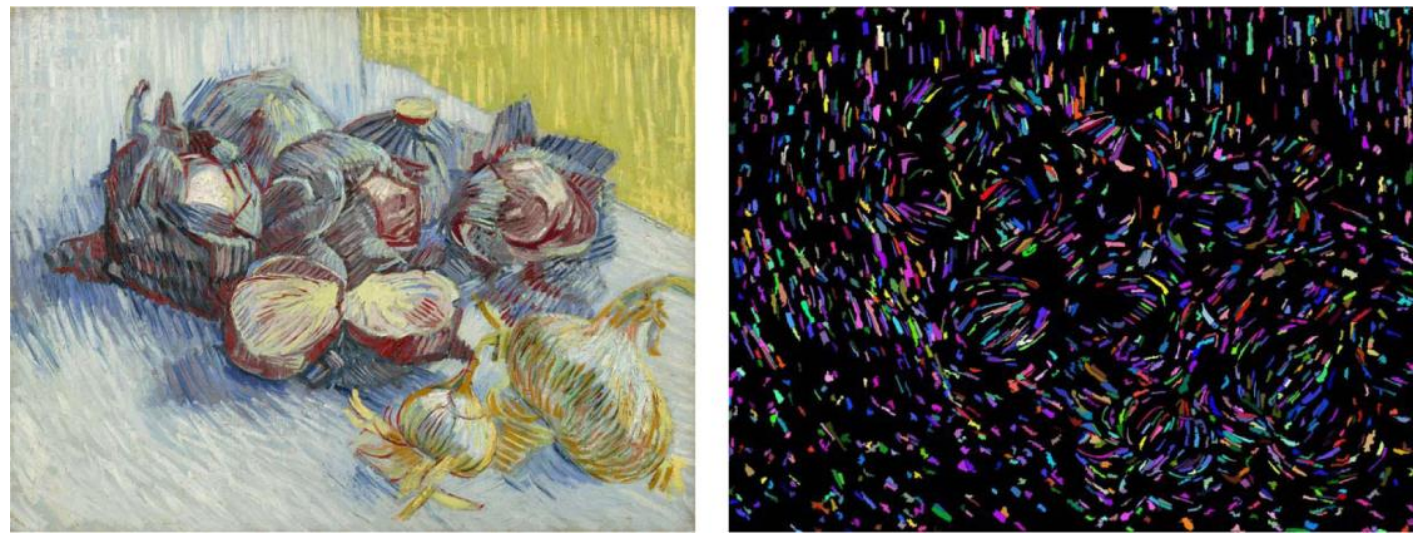

Fig. 23. Automatic brushstroke extraction for Van Gogh's Cabbages and Onions (1887-1988). Adapted from Li et al. (2012).

Examples of indexicality demonstrate an evolutionary development from Peirce's attribution to photography art to Manovich's mapping of online media. For the former, chemical processes conditioned the virtual product of photography to physical reality. Conversely, for the latter, activities of virtual communities condition the translation of physical space to cyberspace (Fig. 24). Museums in information space are the unfolding of the latter. It highlights the "polysemic nature of objects [...] for personalised systems of communication" [emphasis added] (Navarrate and Owen 2016, 118).

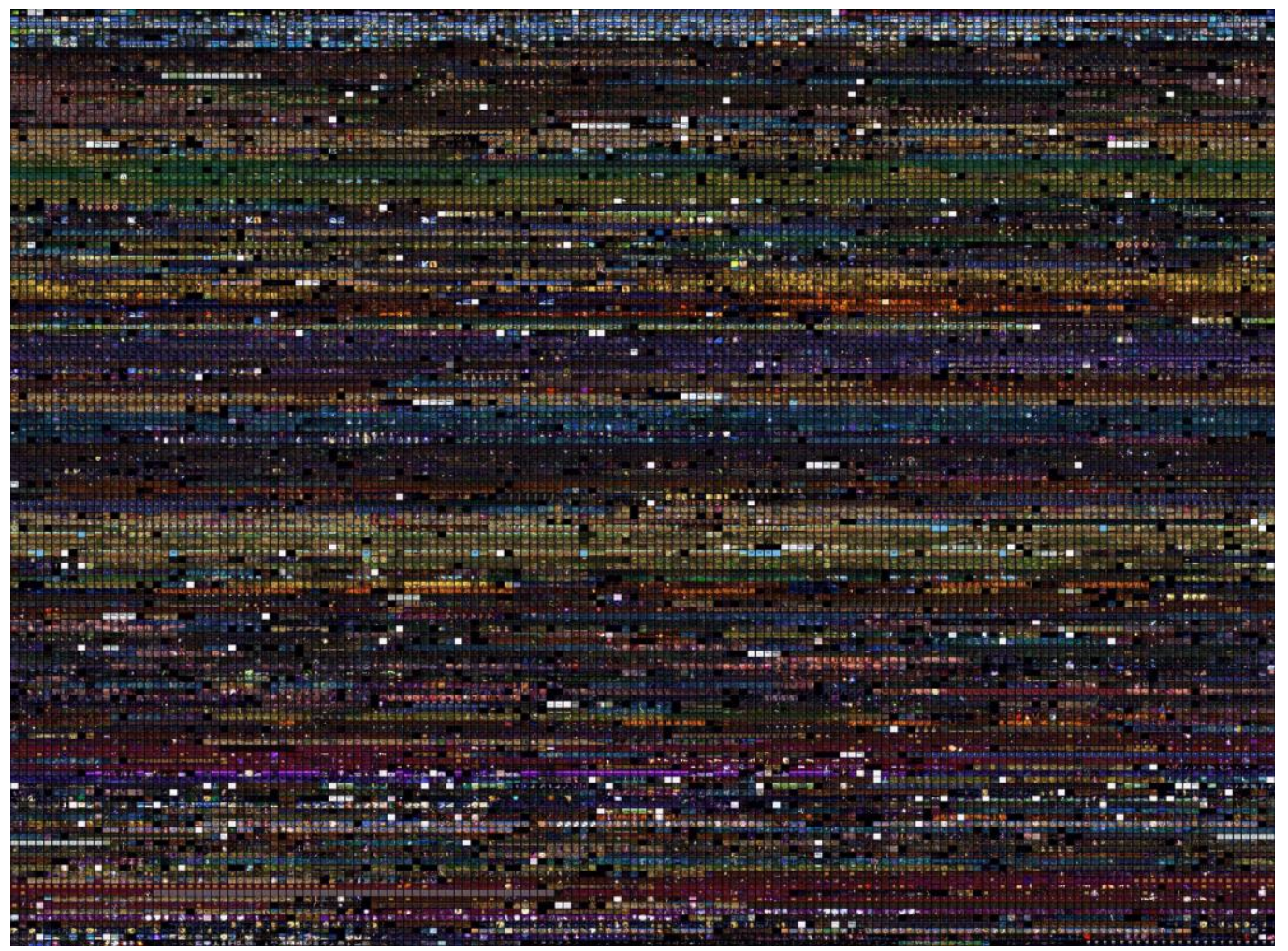

Fig. 24. Kingdom Hearts video-game play visualisation. Adapted from Huber (2010). 
With the transformation of values in the multidimensional information space, e.g. hyperlinks, the participatory content-making challenges the perceived view of objects in museums. The correlations that generate value are multiplied by new attributes of the basic visual units such as pixels instead of brush strokes on paintings. New correlations, which are often unnoticed until analysed, are generated. Feature detection and extraction algorithms are used in computer science to unveil undiscovered associations, such as "analysis of culture using millions of digitised books" (Michel et al. 2011). As such, novel multidisciplinary methods are applied in digital humanities for retrieval of digital cultural objects by using image databases (Foster and Rafferty 2016). King, Stark and Cooke (2016) examine museums in terms of user-generated content and collective intelligence on digital applications. Their research unveils subjective multiplicity in the interpretation of objects via personalised systems of communication. In this regard, it becomes today less important to come to an ultimate and immediate agreement on the authenticity issue in heritage practice, than it is to reframe it as a process of construction and agreement between conflicting views. 



\section{Chapter 3. Matters of 'Transcoding' in Digital Heritage}

Taş, toprak ya da çelik ve betondan değil, beyazı kâğıt, siyahı mürekkeple yapılmış bir kubbe de, herhangi bir firtına ve tipiye karşılık verip, nesilden nesile kalacak güzel bir kubbe olabilirdi

Not built with stone, steel or concrete, but, a structure made of white paper and black ink so that it can survive generations after generations enduring any storm. [Quote from a masterpiece novel in Uyghur literature. Translated from Turkish by Author]

- Abdürrahim Ötkür, İz [Trace; U. ئز] ([1985] 2017)

\subsection{Introduction}

The previous chapter introduced an overview of heritage research and development plus application through museums regarding the use of digital means. With regards to digitising heritage information, this chapter defines four layers of matters of context (3.2), medium (3.3), audience (3.4) and research (3.5). They are the theoretic and abstract components with which any digital heritage study has to deal. Each of them corresponds to the subsequent chapter's documentation (4.2), representation (4.3), dissemination (4.4) and design (4.5), respectively.

The previous chapter underlined the evolution of inclusivity in museum practices and theories that have influenced the heritage field. It showed that the field relates overlapping domains to each other that may have conflicting views about the value of novelty in the digitalisation of heritage. On the one hand, there are the 'binary codes of heritage' and on the other hand, the 'plural interpretations' are increasingly more valued. The concept of the virtual museum has been convincing about the 'suspension of disbelief' in virtual reality, but also it transfers new concepts into the field of heritage, such as 'museum as information 
space.' Proceeding from an extensive overview of digital heritage, this chapter draws three criteria from matters of context, medium and audience. They are extracted from 'resolving matters' into abstract terms to set up a research foundation. The first one, 'authenticity' is about finding ordinary things and the immateriality of tangible heritage. The second, the virtuality of 'creative engagement,' derives from a retrospect of immersive digital heritage applications. The third one, 'alternative knowledge' in user-generated content, is motivated by a synopsis of contemporary means of participation via new media.

By demonstrating matters related to context, medium, audience and research, this chapter uses the concept of "transcoding," which is one of "five principles of new media" asserted by Manovich (2001). It makes a natural connection between the dichotomies of digital and heritage that require an understanding of the interdependence between the 'computer layer' and the 'culture layer.' "Mapping new media" in a persuasive theoretical textbook titled The Language of New Media, Manovich discusses "the impact of digitalization itself' on cultural phenomena (Bogost 2006, 27). His mapping of new terminology for the new media projects a paradoxical dilemma between culture and the computer technology. Analogous to this, this chapter investigates the reciprocal relationship between the values of the 'digital' and 'heritage' while examining matters about context, medium, audience and research. 


\subsection{Matters of Context}

Subchapter Outline

Purpose: The subchapter introduces the context of Kashgar.

Design/Approach: The background subchapter is informative on Kashgar's geography, location, history and architecture. The theme of alleyways is depicted in the context of heritage, identity and authenticity in Kashgar. The first sub-question $(s Q 1)$ is formulated based on knowledge about the progress of Kashgar old-town from being a farm-state to demolished heritage and about the theme of narrow roads as the food of 'virtuality.'

Findings: The introduction of Kashgar reveals the importance of alleyways in the old-town. The historical formation of narrow roads has a strong relationship with the sense of neighbourhood and household.

Limitations/Implications: The findings of the subchapter rely on the overview of the history of Kashgar from which the generalisation of the matters of the context are generated.

Originality/Value: This is the first attempt to embed narrow roads in the Kashgar old-town with an explication of heritage, identity and authenticity. This depicts the basis of their muséal quality to be examined.

Keywords. Kashgar; immateriality of tangible heritage; Xinjiang; heritage in China; neighbourhood; heritage; identity; authenticity.

For various reasons, Kashgar offers an exemplary case for research. To understand the circumstances of the Kashgar old-town is instrumental in recognising the need for it being selected as context. In this section, the 'Matters of Context' are examined. The most relevant matter is about the architectural heritage in Kashgar and its renewal.

A large-scale renewal project subjected nearly eighty percent of the houses in the Kashgar old-town with demolition and reconstruction. Local people were arguably disengaged from decision-making (Bellér-Hann 2014, 178; Pawan and Niyazi 2016, 123; see also Holdstock 2015, 203-204). The demolition of the Kashgar old-town represents a worldwide problem of state-led mechanisms to "manipulate" minority heritage (Aygen 2012, 28).

In this context, matters can be grouped into three interrelated concepts: heritage, identity and authenticity. Choosing Kashgar as context also contains challenges and opportunities for documentation. They arise from a subjective interpretation of three overlapping concepts (Table 5). Some of them, such as environment and literature resources, might have a marginal impact on research objectives. Some others, such as architectural and historical facts, play a central role. And some challenges and opportunities draw a median line in-between. 
Table 5. Challenges and opportunities of digital heritage research in the context of Kashgar in terms of heritage, identity and authenticity.

\begin{tabular}{|c|c|}
\hline Kashgar's & Challenges and opportunities (in the order of priority) \\
\hline Heritage & $\begin{array}{l}\text { Historical } \\
\text { Ancient history } \\
\text { Intangible heritage } \\
\text { Tangible heritage } \\
\text { Literature } \\
\text { Historical resources (in Chinese, Uyghur, Turkish, Persian, Russian } \\
\quad \text { and Arabic) } \\
\text { Under-recognition in English } \\
\text { Tourism } \\
\text { Commodified heritage } \\
\text { Exchange value } \\
\text { Environment } \\
\text { Climate change } \\
\text { Dust storms (from Taklimakan Desert) }\end{array}$ \\
\hline Identity & $\begin{array}{l}\text { Individual } \\
\text { Topophilia (place-love) } \\
\text { Topophobia (place-dislike) } \\
\text { Tourism } \\
\text { Staged authenticity } \\
\text { Societal } \\
\text { Income gap } \\
\text { Minority vs. majority } \\
\text { Political } \\
\text { Political conflicts (domestic and international) } \\
\text { Security concerns } \\
\text { Separatism }\end{array}$ \\
\hline Authenticity & $\begin{array}{l}\text { Architectural } \\
\text { Configurational typology } \\
\text { Gentrification } \\
\text { Inauthentic renovation } \\
\text { Multifunctional spaces } \\
\text { Narrow roads } \\
\text { Local neglect } \\
\text { Old-town renewal project } \\
\text { Tourism } \\
\text { Paid-only entrance }\end{array}$ \\
\hline
\end{tabular}

Once a major city along the Silk Road, Kashgar is of limited accessibility. As the westernmost city in China, Kashgar borders with Tajikistan, Pakistan, India and Kyrgyzstan. It is situated on the convergence of Central Asia to the West, the Indian subcontinent to the 
South and China to the East. In its long history, old peoples (e.g. the Chinese, the Tibetans, the Mongols, the Indians and the Turks), religions (e.g. shamanism, Buddhism and Islam) as well as modern nations (e.g. Russia, England, Japan, China and the discontinued Uyghur state) vied with one another for Kashgar. Likewise, it is presently a place of conflict between Uyghur separatists and the Chinese security forces. This limits access to the city, as a significant obstacle for fieldwork. Nevertheless, Kashgar is a place of growing attraction in China with a so-called government campaign of Go-West.

The architectural heritage in the Kashgar old-town is remarkably distinct. Its distinct features will be pointed out in the next two subsections. The distinction is jeopardised by neglect and transformation caused by neo-liberal urban development. In the maze-like oldtown, there are mind-boggling vistas that perpetually grow and unfold in a fractal manner. Today, this spatial quality is mitigated by urban renewal plans, even while the vernacular organisation of houses alongside alleys demonstrates distinct creativity.

The narrow roads of the old-town in Kashgar sustain a strong sense of community. Houses are domestically compact (i.e. courtyard typology) and inter-domestically attached to each other (i.e. alleys). But it remains unclear what associations between material features (e.g. textural, orange peel effect, brick thickness) and functional connections (e.g. between narrow roads and courtyards) have contributed to the sense of neighbourhood.

To encode the unique aspect of these alleys into digital formats requires a surprising amount of effort and a reasonably careful study of their formation. Such research would be hardly feasible with physical traditional preservation techniques since it would require a massive investment of time and budget. Therefore, Kashgar is a good challenge for contribution to digital heritage. The documentation, representation as well as dissemination of narrow roads convey interconnected challenges and opportunities to shed some light on the complexity of Kashgar's architectural spatial heritage.

A photobook was published by Author during candidacy (Aydin and Schnabel 2017). The book demonstrates thirteen sections, each with their own stories and themes based on specific content ranging from historical architecture to people of Kashgar and from blackand-white urban scenes to 3D digital reconstructions. In this thesis, some figures are from the photobook and accordingly credited in the captions (Fig. 25). 
72
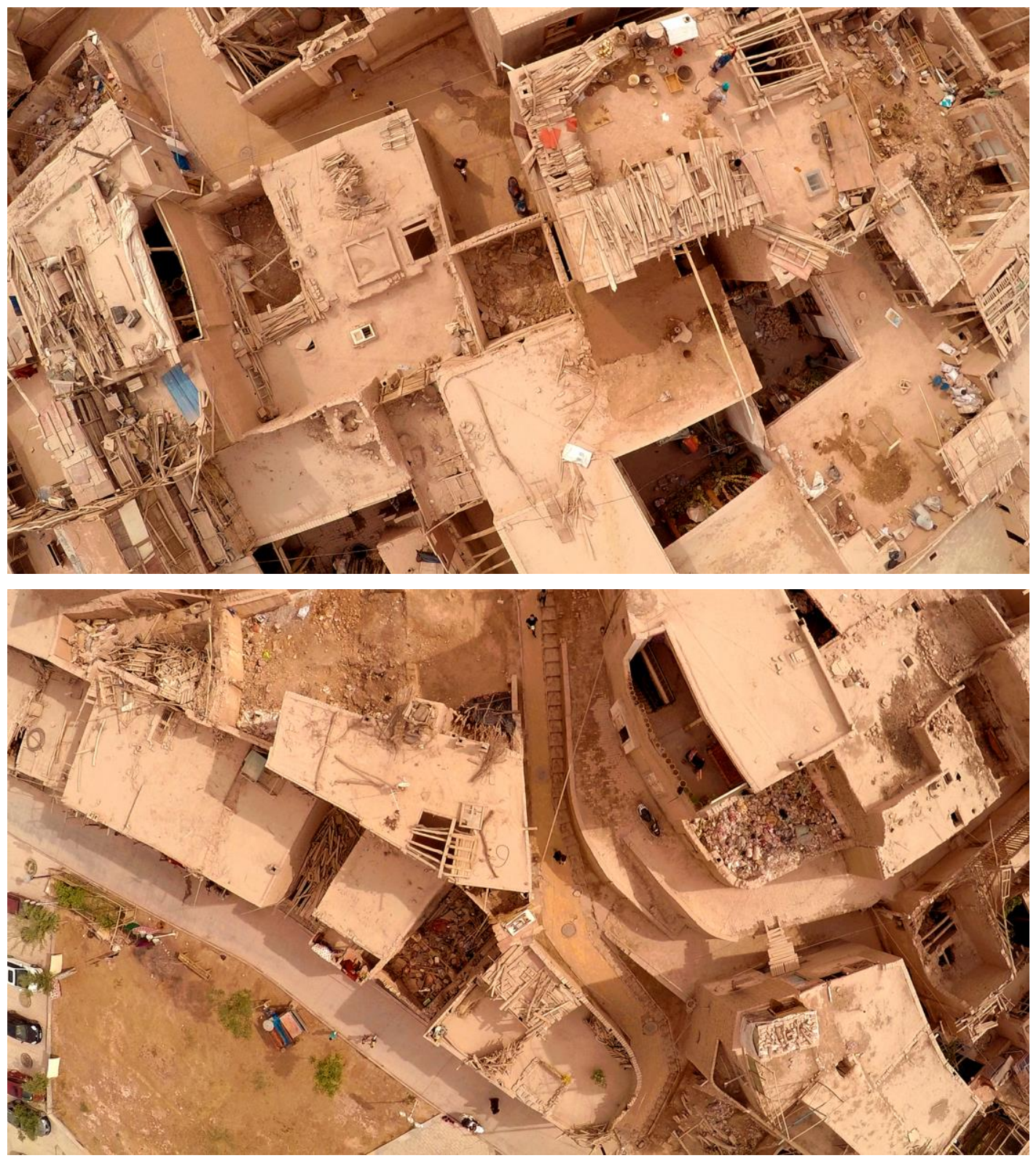

Fig. 25. Top views of alleys in Kashgar. Photo by Author from Aydin and Schnabel (2017, 104).

\subsubsection{Background: From a 'Farm-State' to Demolition}

\section{- Geography: Xinjiang [New-Territories]}

Kashgar [Ch. 喀什; pinyin. Kashi; U. قششقر] is the westernmost city in China (Fig. 26). It is located where many different cultures and religions have juxtaposed thanks to its geographical position. Its splendour is embedded in its distinct Uygur identity which is a multifaceted composition of Islamic, Central Asian, Middle Eastern, Chinese, Mongol, Tibetan, Persian and even Indian influences. The ancient city is described as "the heart of one of the most lovely and bountiful oases in all Central Asia" (Starr 2013, 307). 


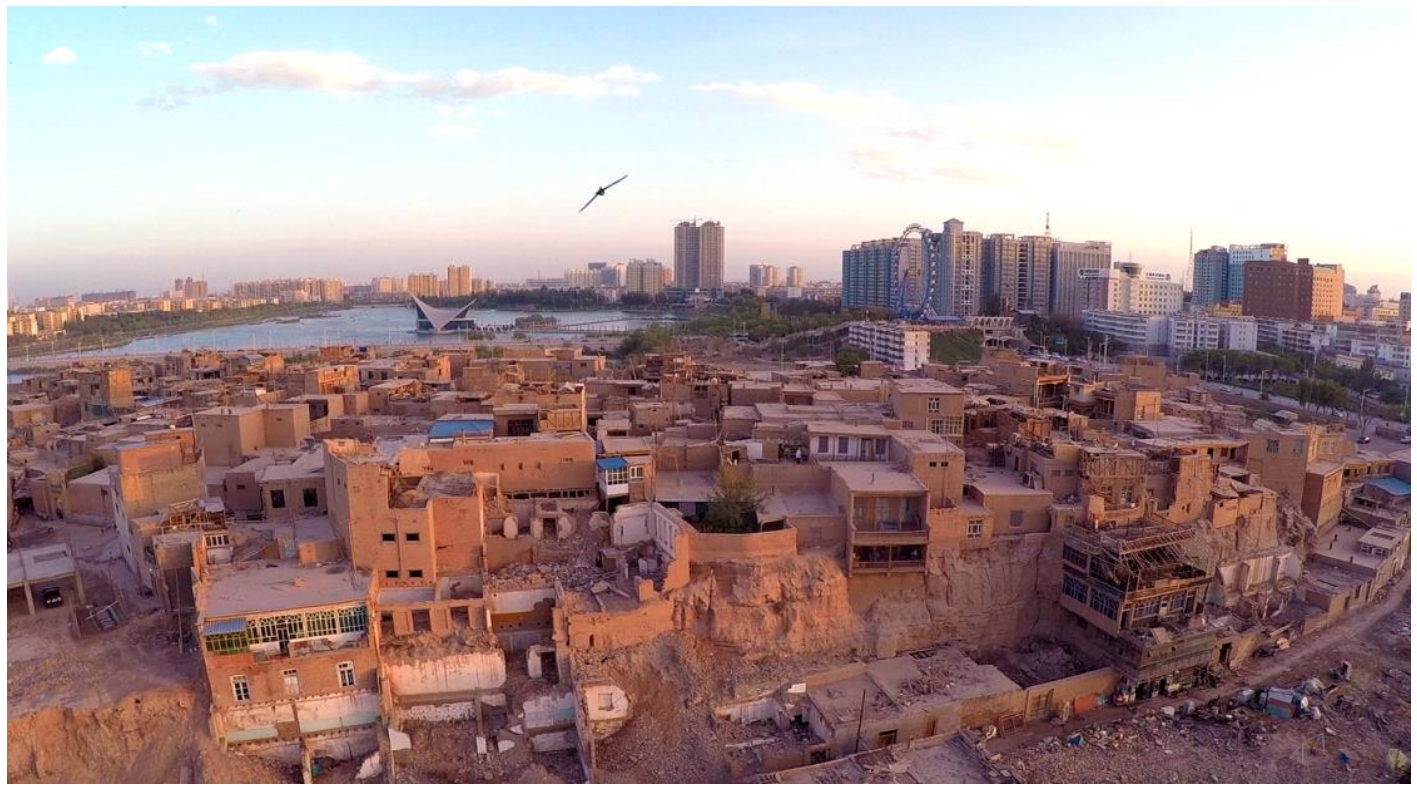

Fig. 26. The outlook of Kashgar depicting a contrast between heritage and contemporary architecture. Photo by Author from Aydin and Schnabel $(2017,118)$

The administration of Kashgar prefecture belongs to the Xinjiang-Uygur Autonomous Region (XUAR) [Ch. 新疆维吾尔自治区; pinyin. Xīnjiāng Wéiwú'ěr Zìzhìqū; U. شنجالكُ ئويغؤر [نايتونومر ايونى It is the westernmost and largest province in China. Along with other ethnic groups, including Kazakhs, Tajiks, Kyrgyz, Mongols and Huis, the majority of XUAR are Han Chinese and Uyghurs who are a Turkic-speaking Muslim minority. Uyghurs widely acknowledge Kashgar as a cultural capital of identity. To the north of Kashgar is located Ürümqi [Ch. 乌鲁木齐; pinyin. Wūlǔmùqí; U. ئورؤمجى], which is the official capital of XUAR and the world's most landlocked city (Dong and Zhang 2011).

Xinjiang is home to the second highest and the second lowest places on earth, Qiaogeli on the Kunlun range (altitude: $+8,611 \mathrm{~m}$ ) and Ayding Köl [Lake Ayding] (altitude: $-154 \mathrm{~m}$ ), respectively (Millward 2007, 5). Despite its land area being enormous in size, Xinjiang is mostly uninhabited since it is mostly covered by the Taklimakan Desert. Because of distinct geographical features in the region, climate conditions and distance to seas, vernacular architecture in the region displays a unique character that is invariably adobe and mud-brick.

\section{- Region: 'Tarim' [agriculture] Basin}

Kashgar is located to the west of Taklimakan. The desert is surrounded by the Tarim Basin that is crescent in shape and rich in water resources. To the east of Kashgar, there is Ferghana Valley which is another water-rich area. Its fertile land used to bridge the trade route from the Tarim Basin to Samarkand and Bukhara further west. In this geographical context, Kashgar used to be pivotal to the trade along the historical Silk Road (Fig. 27). 


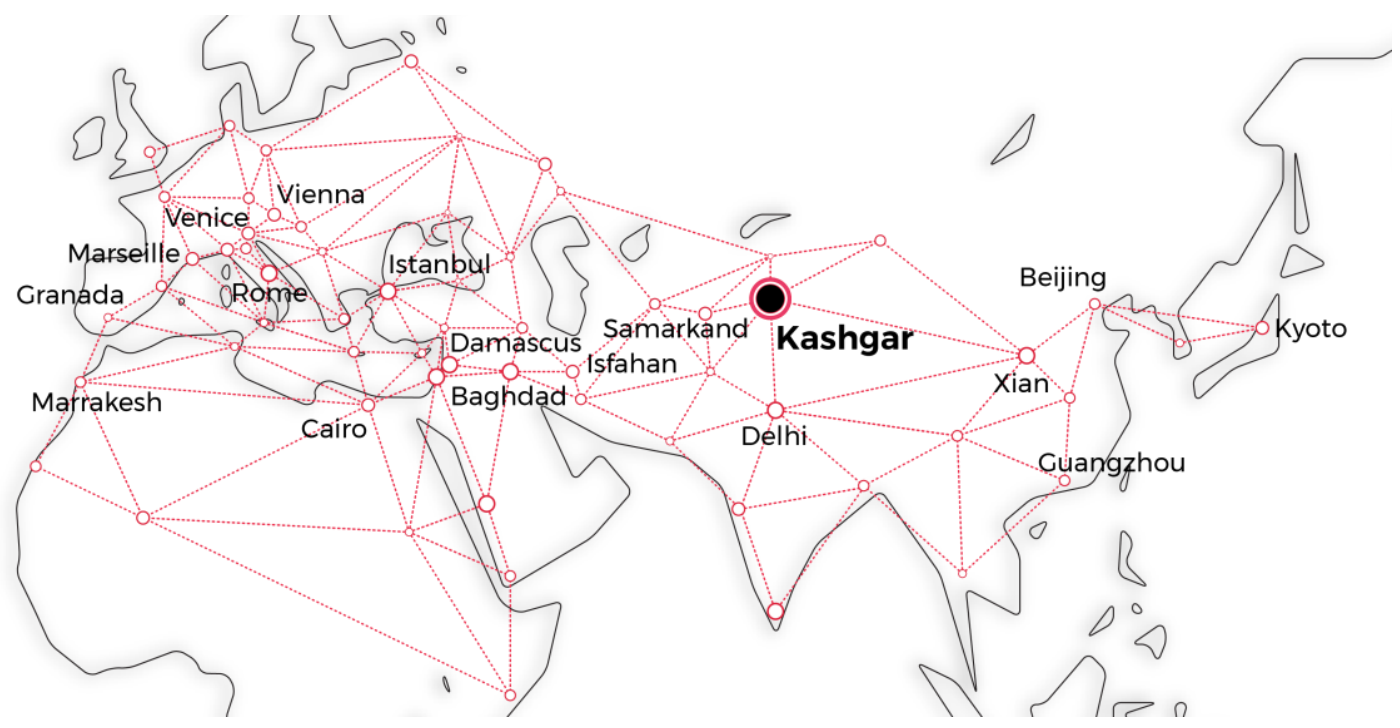

Fig. 27. Kashgar's location on the world map (without scale) depicted on a red-line sketch of the historical Silk Road. Illustration by Author.

The name Tarim [farming] defines the southern part of Xinjiang. Living along the rivers of the Tarim Basin, Uyghurs became affluent via agriculture. They were the first to adapt to permanent settlement amongst other nomadic Turkic dynasties. Their name 'Uyghur' drives from this fact that literally means 'the civilised' or 'the cultivated.' Today, Kashgar is widely acknowledged as the cultural capital of Uyghurs because of its historical and cultural importance. Agriculture is a significant income for Kashgar people. It is estimated that, by 2020, more than half of the population in Kashgar will be still surviving on agricultural income while developing on a relatively slower pace of urbanisation (Zhang and Lei 2006, 187).

\section{- Farm-States: 'Altishahr' [Six-Cities]}

Many more definitions are also used in literature such as Little Bukharia and Kashgaria in western travellers' diaries (Beller-Hann 2008, 39) and Huijang (Muslim frontier) and Bacheng (eight-cities) as in the Chinese sources from the Qing era (Newby 2005, 4 n.10). Another common term to define the Tarim Basin is Altishahr which means 'six cities' (Newby 2005, 4 n.10). Today's most prevalent term, Xinjiang, refers to the nineteenthcentury definition of the Qing, meaning 'new territories.' The context of each name provides hints about the heritage, identity and authenticity of Kashgar.

In the eighteenth and nineteenth centuries, the city was a polis-state in alliance with other surrounding cities along the rim of the Tarim Basin. The relationship between the alliance of the Tarim cities and the Qing has attracted scholarly interest on Central Asia, China and Turkish studies from the USA (Millward 2007; 1998; Starr 2013), England 
(Newby 2005) and Denmark (Beller-Hann 2008). Combined with the scholarship that elaborates on the socio-cultural aspects (Steenberg 2014; Pawan and Niyazi 2016), these works render a relationship between the social institutions of historic neighbourhoods and the tradition of semi-autonomous administration. In other words, a close review reveals a deep connection between the architectural formation and political status of Kashgar.

\section{- Households of a State-Farm}

The alliance known as Altishahr from the eighteenth century was forerun by the cities of Kashgar and Yarkant with strong 'organisations of households' transformed into 'statefarms' (Millward 1998, 50). As independent agencies forming Altishahr together, the landlocked allies relished the looseness of being inconceivably far from the sea. The strong sense of neighbourhood dwelled upon special configurations of $\ddot{y} y$ [house]. In the eighteenth century, they were fundamental for founding the farm-state of Kashgar. The concept of $\ddot{o} y$ [house] was a concept of power, virtue and 'virtuality' (Fig. 28).

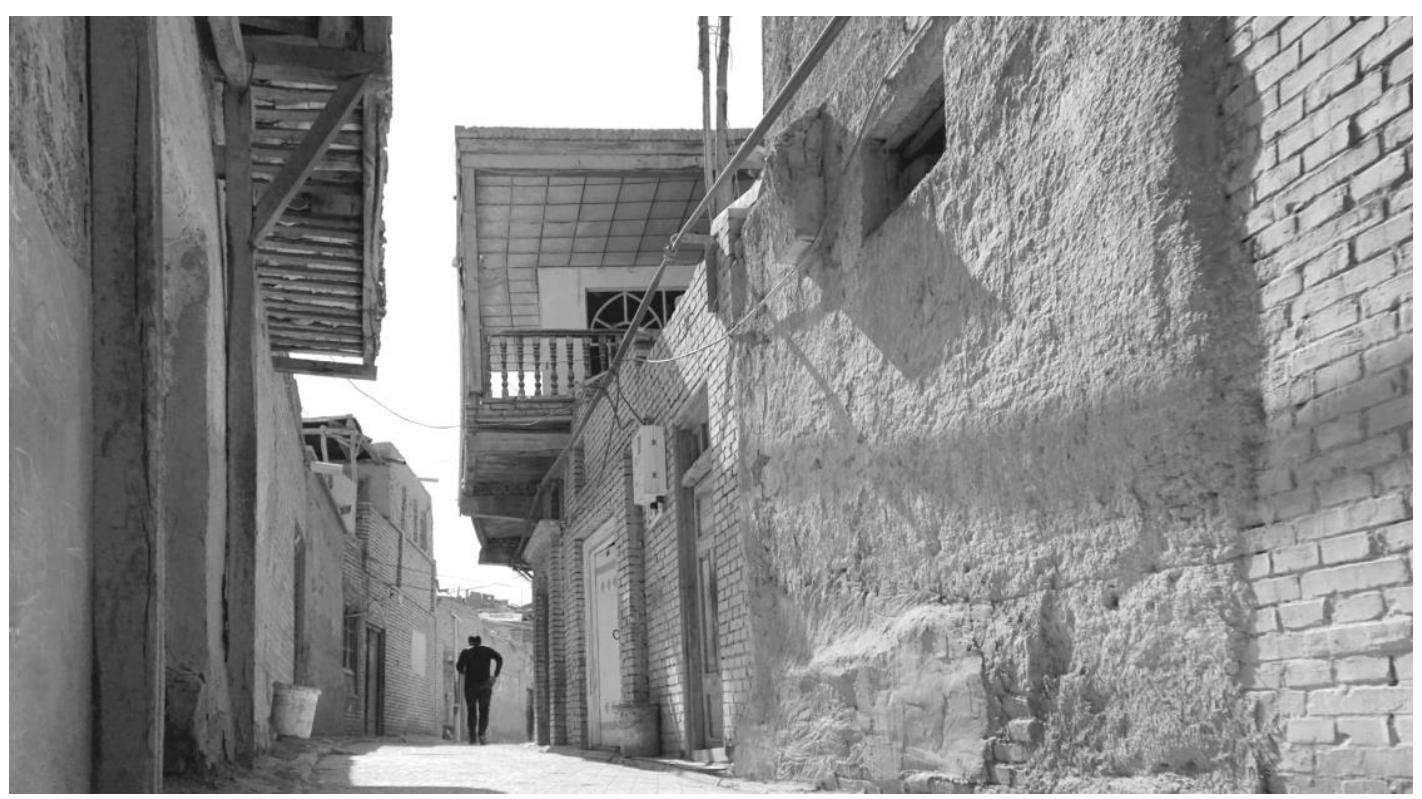

Fig. 28. A section of narrow roads from the Kashgar old-town. Photo by Author from Aydin and Schnabel $(2017,149)$.

Architecturally, this self-autonomy in organisation appears to have yielded unique characteristics to Kashgar architecture, whereas the arid climate became an external force in their structural and functional formulation. Representing the old concept of the farm-state, underground passages and storage areas evidence another layer of complexity onto the entire agglomeration of the vernacular housing. 
At the societal level, this dual concern of inner coherence and outer resistance has significant impacts on the definition of family. Elsewhere, it is comprehensively analysed that the concept of household in Kashgar old-town is a broader definition of family that extends beyond kinship (Steenberg 2014). Other works underlie narrower relations, such as, between neighbourhood and market (Pawan and Niyazi 2016).

Most of the houses have courtyards regardless of the householder's social and financial status. It is a cultural and religious condition that the balance between privacy and sister/brotherhood defines the level of spatial permeability in the old-town. Accessed from alleyways, the courtyard typology serves as a composition of vertical and horizontal movements. The allocation of different functions around the courtyard proves a connection to water and thermal comfort in the formation of houses (Florenzano, Courel and De Domenico 2010). Outdoor spaces are invariably rooted in the management of water resources for irrigation as well as for living.

However, the heritage of neighbourhood quality has been debased mainly in the last two decades, while inhabitants have been relocated to second-class spaces in rural areas. The authorities are blamed for the degradation of the heritage value as is evidenced by with many violation cases of minority rights (Bellér-Hann 2013). It has been detrimental to the role of neighbourhood communities (Steenberg 2014; Pawan and Niyazi 2016).

\subsubsection{Theme: Narrow Roads as Derivation of 'Virtuality'}

The maze-like pattern of roads in Kashgar evokes a constant sense of pleasure, wonder and imagination. Moving through the old-town begins with depicting ostensibly predictable pathways. And then embedded alongside are abrupt diversions and intersections. Repeatedly navigated throughout, alleys unfold into arrays of spatial cognition. Social cohesion between occupants is enabled by configurational elements, such as tunnels, arcades, niches and corridors. They are primarily made of mud-brick for a range of functions such as houses, religious buildings and towers. In this environment, one is tempted to make virtue out of personal narratives.

\section{- Heritage}

The unique architectural heritage of Kashgar (Aydin and Schnabel 2015; 2014) is a product of interwoven arrangements, where strong social relationships are fundamental to its agglomeration. Labyrinthine agglomeration of mud-brick houses, the outdoor space in OldTown Kashgar resembles Cedric Price's ‘Fun Palace' designed for social interaction (see p. 
103). Mechanical qualities of the Fun Palace appear in a native format in Kashgar. Tangible and intangible heritage complement each other in its multifunctionality. All these elements propagate a specific 'Kashgar' language on a visual layer. The concept of mahalla (neighbourhood) is core to the social structure of Uyghurs in Kashgar. It has played a key role in building a unique identity. The historical urban fabric in Kashgar was once 'the best preserved example of a traditional Islamic city to be found anywhere in Central Asia' (Michell et al. 2008, 79). It has recently been one of the most-endangered too (Fig. 29).

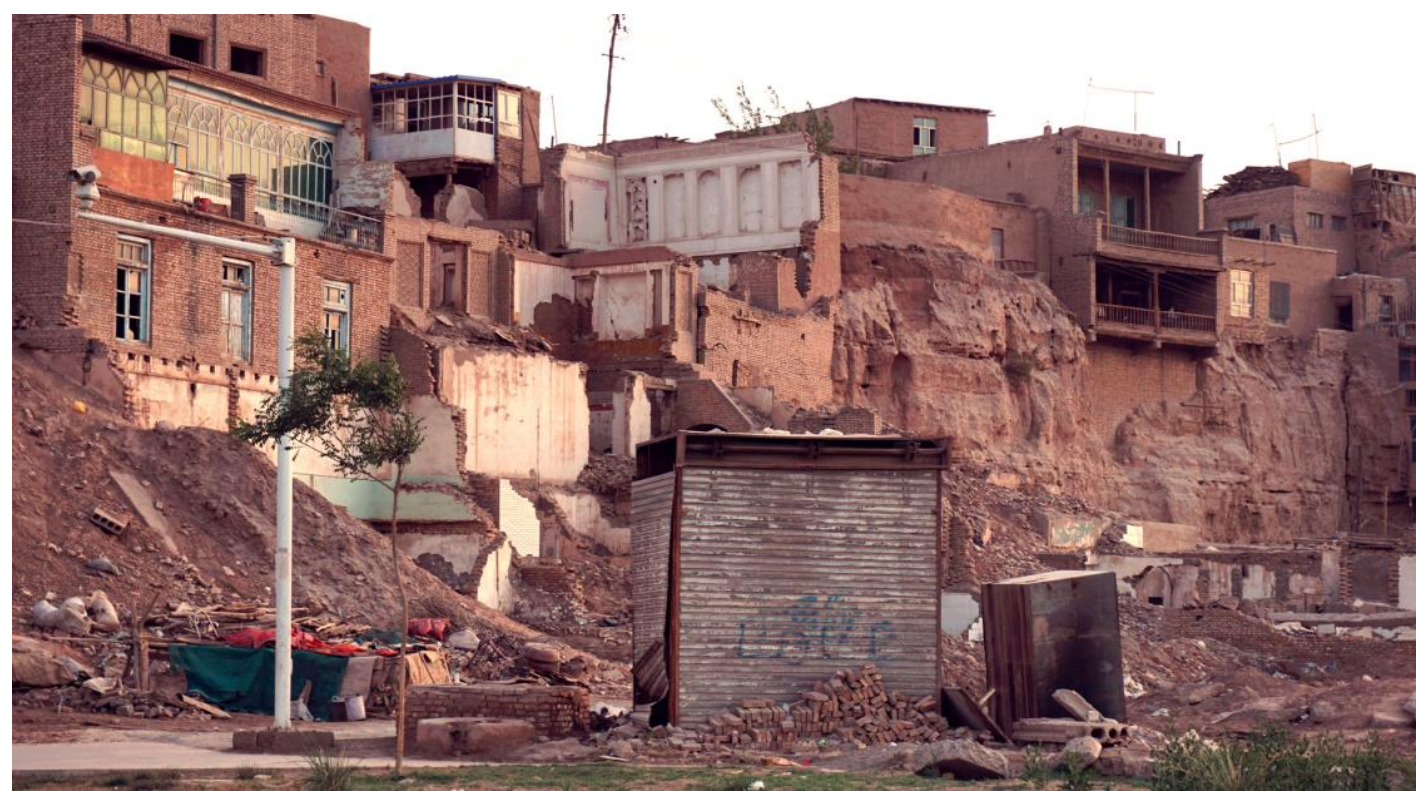

Fig. 29. Unique historical architecture is sitting on a hill with terraces and balconies. Photo by Author from Aydin and Schnabel $(2017,168)$.

Despite international and national critical reviews of the multibillion-dollar plans, demolition began in 2009 (RFA 2010). Around fifty thousand households (approximately eighty percent of the old-town) that accommodated about two hundred thousand people were resettled eventually (Pawan and Niyazi 2016, 124) (Fig. 30). The old houses of Kashgar were identified as mostly 'dilapidated' and to demolish, around half a square kilometre (122). The state-run renewal of the old-town tore the houses down and replaced them with new replicas that look sufficiently old. 
78

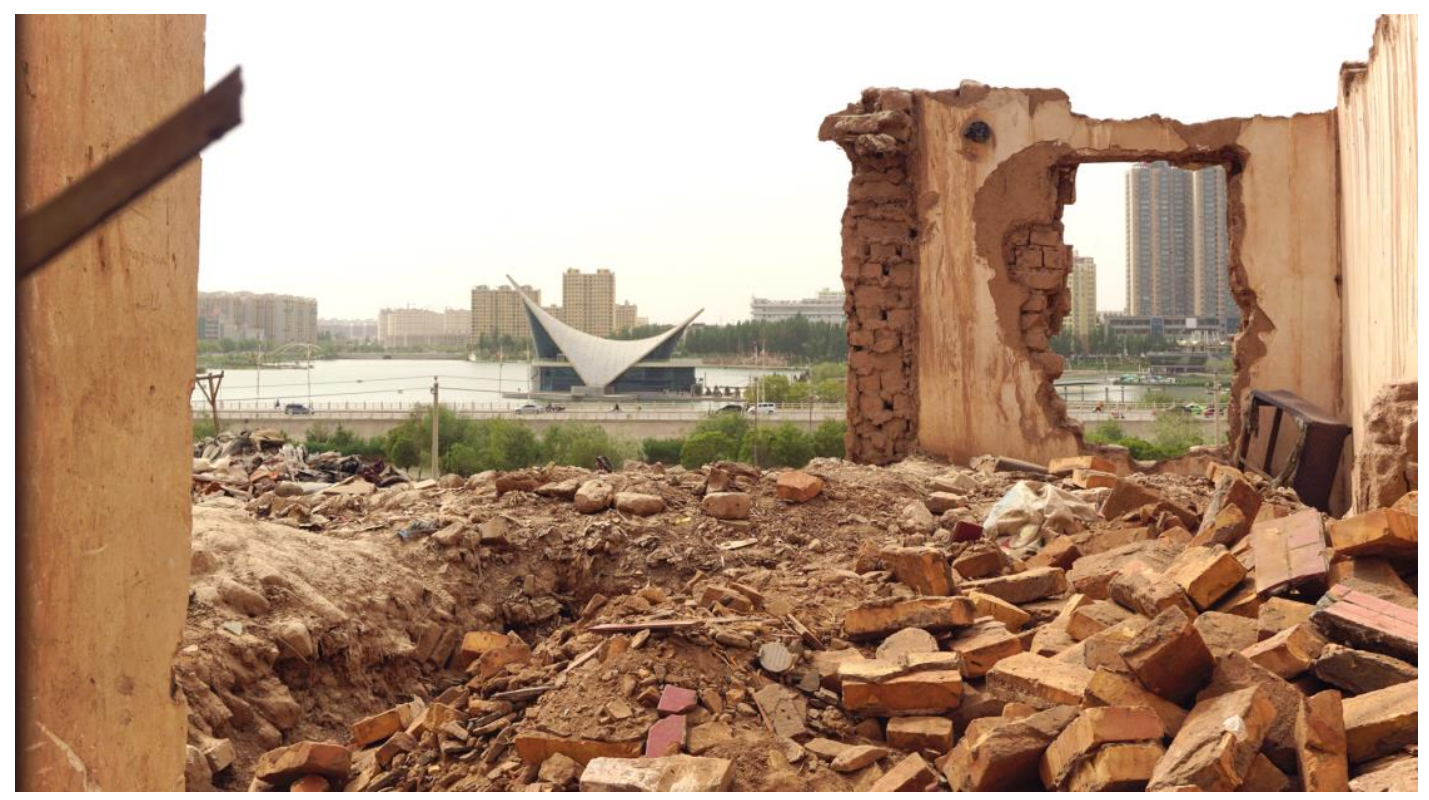

Fig. 30. The relocation from historical architecture into new apartments. Photo by Author from Aydin and Schnabel $(2017,70)$.

The renewal project was defended on the basis that old houses are not earthquake-proof and therefore not safe. However, the critics of the project represent three significant streams of politics, heritage and anthropology. The first group cites politically-motivated accusations on the violation of ethnic minority rights (e.g. Holdstock 2015; Bellér-Hann 2013). The second investigates the implemented programme of heritage management (e.g. Aygen 2012). The third type of critical reviews is based on ethnically-informed readings of changing cultural aspects (e.g. Bellér-Hann 2015; Pawan and Niyazi 2016).

\section{- Identity}

The renewal of the old-town has accelerated Kashgar's transformation from a 'state-farm' to a place of 'staged' heritage. The architectural form of the old-town largely remains the same. That the entire town is demolished and rebuilt does not mean the local identity is entirely lost. Steenberg (2014) marks that the strong household concept in Kashgar does not disappear but transforms with increased mobility of people. Steenberg (2014) provides an anthropological account of the impacts of the renewal. He studies the transforming concept of the $\ddot{o y}$ (house) in Kashgar. His methodology follows a Lévi-Straussian concept of structuralism for analysing the house as a primary social unit. He suggests that the interactions between houses define a social category. In Kashgar, these interactions used to be fundamental to the firm household organisations. From the eighteenth century onward, neighbourhood communities dwelled upon interactions between houses. As an underlying factor, these interactions enabled Kashgar people to prevent their state-farm from invasion. 
The original concept of the house is more inclusive than kinship-based ties. Spatially, these interactions are constructed on a relationship between inner courtyards and outer alleyways in Kashgar old-town (Fig. 31). The isolation of these interactions into smaller boundaries of kinship is the real transformation that will have an impact on the identity of the old-town (Steenberg 2014). The transforming social unit of the family will also transform the unique identity of narrow roads in relation to courtyards.
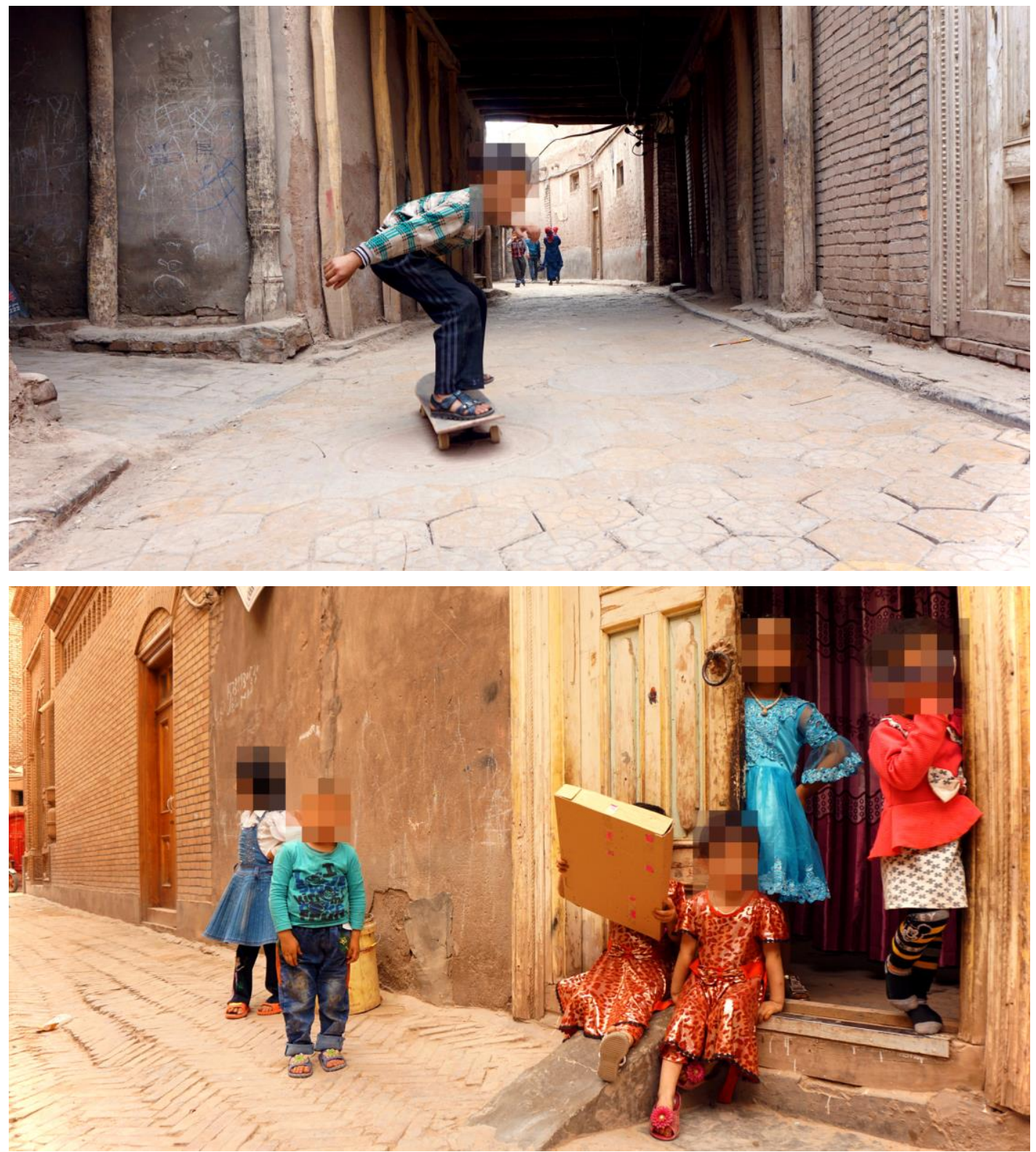

Fig. 31. Multifunctional use of space that occurs through the penetration of the street life into the courtyard in Kashgar. Photos by Author from Aydin and Schnabel (2017, 142 and 143). 


\section{- Authenticity}

The only part of the Kashgar old-town that has not been demolished is the section called Köziciyerbişi [Ch. 高台; pinyin. Gaotai; U. كوزجيار بيشى]. Its size is one-tenth of the size of the entire old-town, which is around nine square kilometres (Pawan and Niyazi 2016, 122).

Köziciyerbişi (Gaotai) is divided from the rest of the old-town area by a four-lane motorway isolating its inhabitants from the rest of the town. It has become the responsibility of the inhabitants to represent the 'authenticity' of old architecture as the area is left as an open museum. This is named as "staged authenticity" by MacCannell $(1992,298)$. He borrows a Baudrillardian term of 'hyperreality' to define the consequences of staged authenticity, i.e. "re-incorporation of the traditional on the condition that it assumes the form of a perfected version of itself" (299).

In the context of Kashgar, there is a contemporary example that can be instrumental to clearly explain "staged authenticity." The alleys of the old-town are featured in The Kite Runner (2008), a Hollywood movie that tells the story of an Afghan boy from Kabul at the end of the 1970s. In 2006, the movie was shot in Kashgar instead of insecure Kabul (French 2006). The digital techniques used to put Kashgar's alleys on stage depict the hyperreality (Kabul) of the hyperreality of another place (Kashgar). As Kashgar was entirely unknown to the Western audience, its substitution for Kabul was unnoticed by the audience (Sandberg 2014, 26). The Kite Runner employs a "superficially visual layer of culture that can be taken on and off like clothing" (25) (Fig. 32).

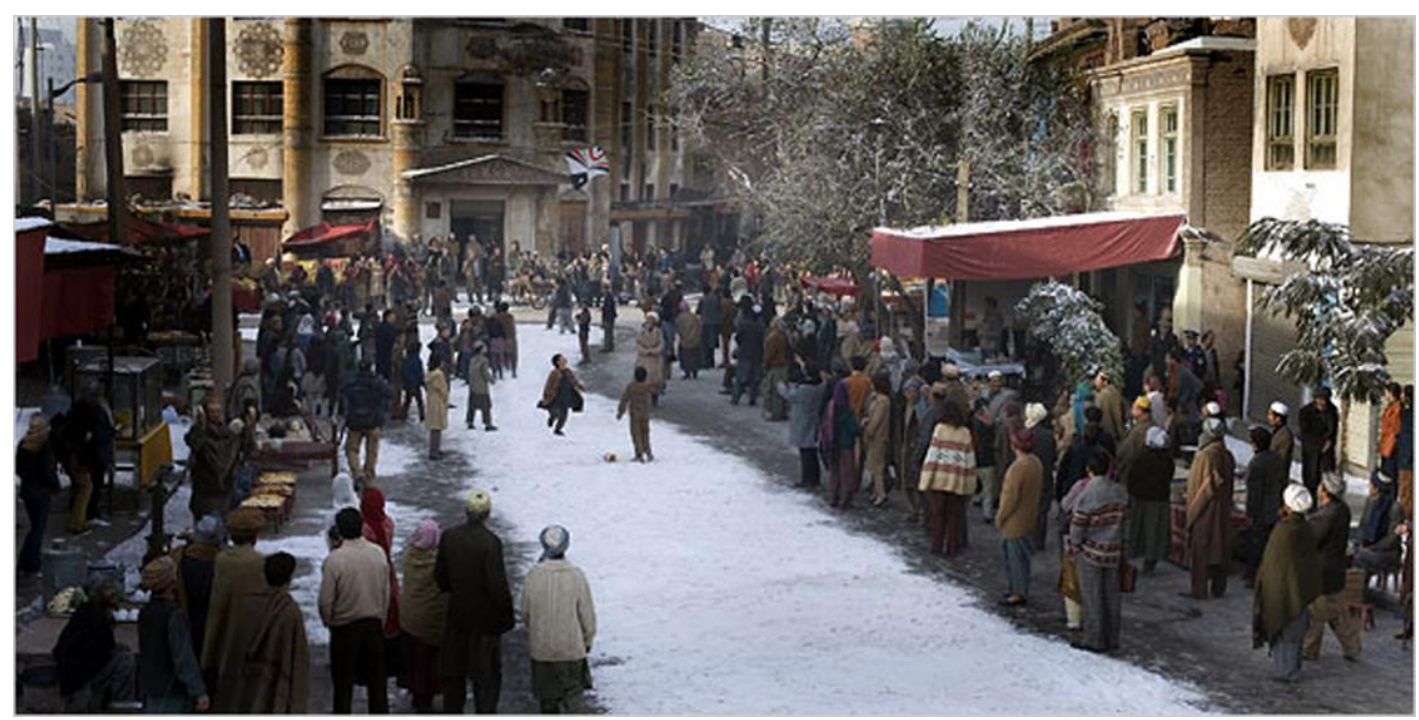

Fig. 32. The Kite Runner movie set in Kashgar, with artificial snow adding an extra layer of hyperreality to represent Kabul, Afghanistan, where the filmed story occurs. Adapted from French (2006). 
Having experienced nearly a decade of renovation and renewal, the old-town in Kashgar is subject to the same issue of authenticity in relation to its real environment. The 'visual layer of culture' that characterised Kashgar's old-town in appearance results in a 'sufficiently' old-looking environment and a fictional sense of being there (Fig. 33).

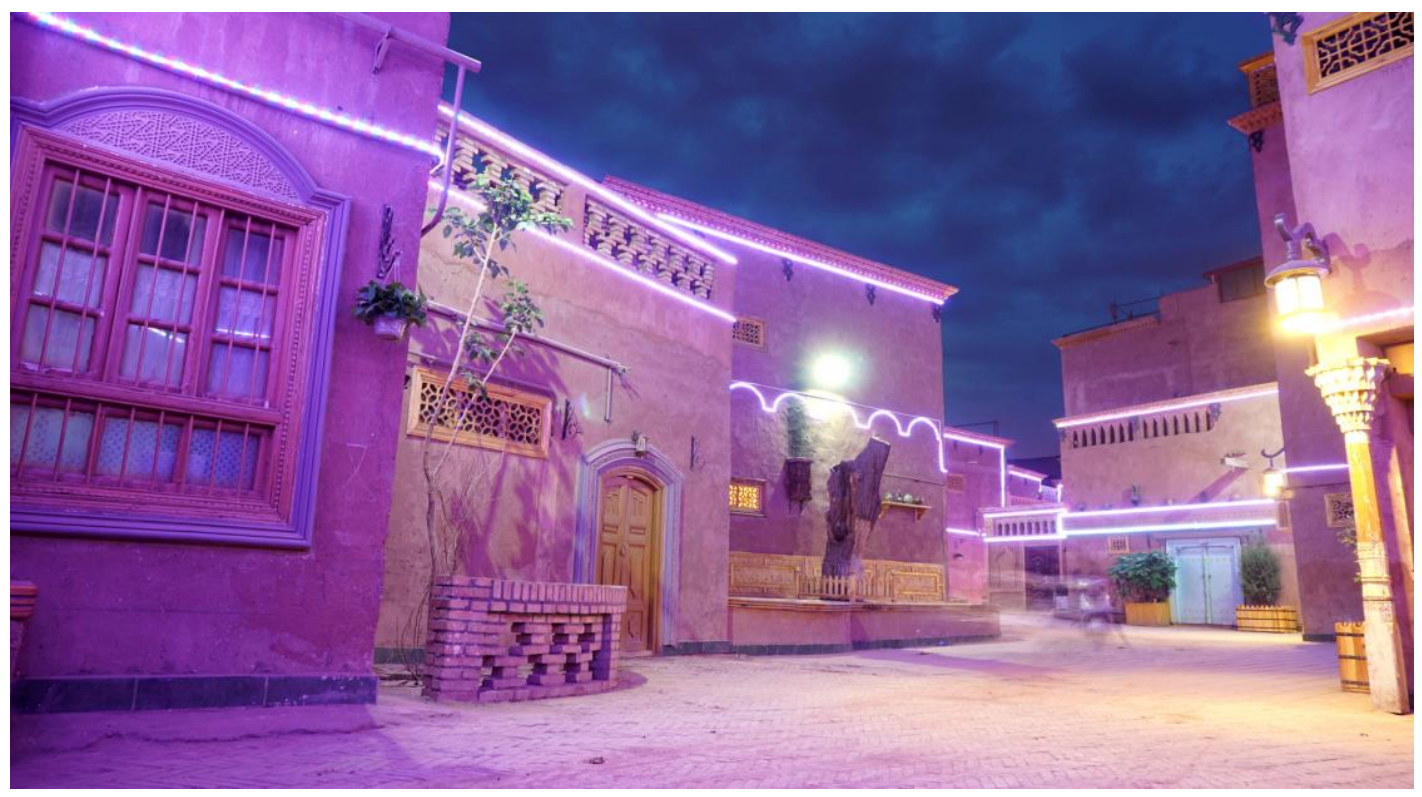

Fig. 33. Kashgar old-town after renewal. Photo by Author from Aydin and Schnabel $(2017,174)$.

\subsubsection{Case: Museums and Heritage in China}

The early museums in China, which were built by missionaries during the colonial era, are ideologically causal to the current heritage practice in China. The earliest museum is the Musee Heude (Zikawei Museum) founded by the French in Shanghai in 1871, followed by museums established by the American and the English (Su 2008, 120; Lu 2014, 19). These missionaries "build up their national and individual pride" that undermined the Chinese civilisation. Their museum propaganda made a permanent impact on the mindset of many generations in China that the West is superior to the East (Lu 2014, 61).

The rest of museum development in China fluctuates because of wars and conflicts. The first museum by the Chinese was established towards the end of the Qing era. To display the Chinese heritage of poetry, calligraphy and paintings, a Chinese entrepreneur founded the Nantong Museum in 1905 (79; Claypool 2005). After the revolution of the Chinese Communist Party (CCP) at the end of the 1940s, museums were reinvigorated by the power of national representation. The overarching theme of these museums is the unity of people. 
The Xinjiang Regional Museum in Ürümqi, which is the first museum of Xinjiang and built in 1953, presents Uyghur women in "exoticised and eroticised" forms "with deliberately noticeable round breasts" and "clearly defined nipples" (Hayes 2016, 57). This presents a contrast to the locals' moral values. Gladney $(1994,98)$ marks this type of sexual minority representation as a common practice in Chinese art, film and media. Gladney assumes that this valorises the view that political hierarchies are mirrored on representations of minorities in China. Bellér-Hann's (2013) analysis renders how, in the context of Xinjiang, the importance of minority identities is degraded and debased in a politicallymotivated systematic way.

In the context of architectural heritage, it may also be argued that the violation of values is evenly distributed all over China (Zhu 2017). The case of Pingyao is relevant in this context and it was visited by the author to make direct observations. Pingyao is "perhaps the best-preserved ancient city in China" (Waldmeir 2014) (Fig. 34). However, its renovation caused the removal of nearly half of the residents; the displacement of the local businesses that once met the daily needs of the residents; and subsequent elimination of everyday life (Wang 2012).

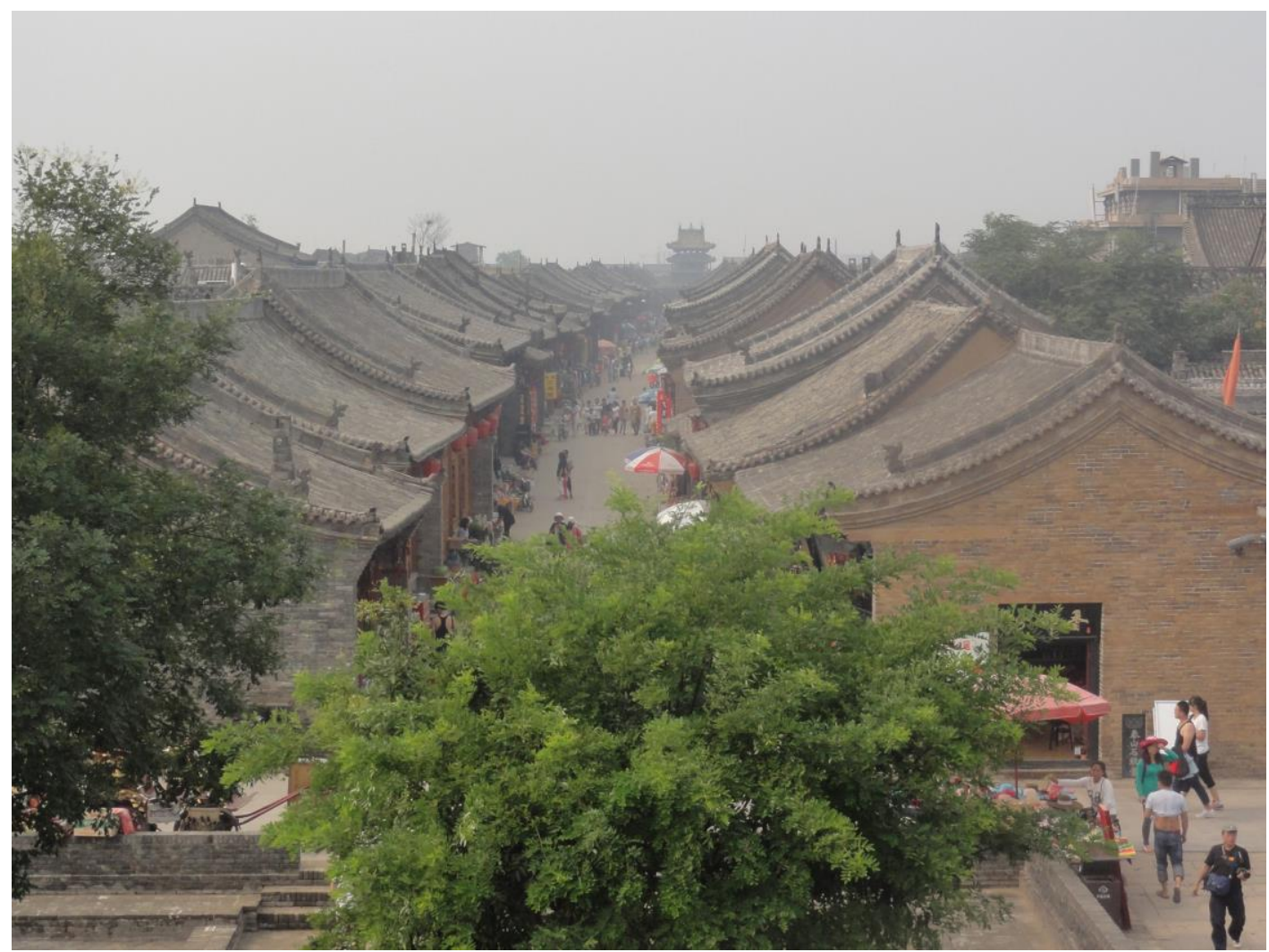

Fig. 34. View of the main street in Pingyao. Photo by author. 
Speculatively, gentrification in ancient Chinese towns is notoriously called "hollowing phenomenon" [Ch. 空心化; pinyin. kongxin hua] (Shepherd and Yu 2013). It describes the departure of original residents and the arrival of business people from outside. Because of heritage marketing, the quality of local life, as well as its uniqueness and authenticity, are affected. This brings the renewal projects to bear critical inauthenticity charges.

\subsubsection{Criteria: Authenticity}

The above sections can be summarised under the matter of authenticity in heritage interpretation. But authenticity is an ambiguous term in general. Googling what comes after browsing 'authenticity of ...' brings up a mixture of results ranging from theological and historical topics to computer-based (Fig. 35). The term is so susceptible to discussion that even its own textual representation can be a domain of enquiry: either 'authenticity' or ' $/: \theta \varepsilon n ' t$ tistil/.' Exchange of views on authenticity is linked with a philosophical underpinning of Stephen Greenblatt who questions "what function of the imagination can erect absolute difference at the point of deepest resemblance" (quoted in Osborne 2004). It is this duality about interpreting authenticity that has been an endeavour of heritage critics (Smith, Messenger and Soderland 2010).

As described by Lowenthal (1999), "each generation views authenticity in a new guise, reflecting its new needs for truth, new standards of evidence, and new faiths in the uses of heritage." So, it may be best understood as a concept mostly dependent on context. In the context of digital heritage, the controversy over authenticity moves into a slightly different level than the material-oriented conversation discourse of heritage. Nevertheless, it is topical to discuss authenticity in digital heritage (Parry 2007; Witcomb 2007; Pujol and Champion 2012; Colley 2015).

Returning to the browsing result on Google, there is another piece information worth raising in this regard. According to Google's metrics, the use of the term 'authenticity' decreases until the 1910s and increases until now. It is noticeable that authenticity gains importance with the rise of computers and information science (see Chapter 2.2). Nearly half of my Google search results for 'authenticity of ...' show that people tend to relate authenticity with new digital concepts, such as 'host can't be established' (a server error), 'information,' 'data' and 'bitcoin' (Fig. 35). In this connection, the authenticity discussion in digital heritage might have a connection to this. It may be rather the immaterial aspects of heritage that can be strongly defended as authentic in digital reinterpretations. The immateriality of heritage does not have to be intangible either. It is a relational concept that 
84

brings about correlations between material aspects of heritage. In this way, the value of heritage can be sought in the basic units of its digital representation rather than the 'grandeur' and holistic meaning of the high-definition quality of its imagery.

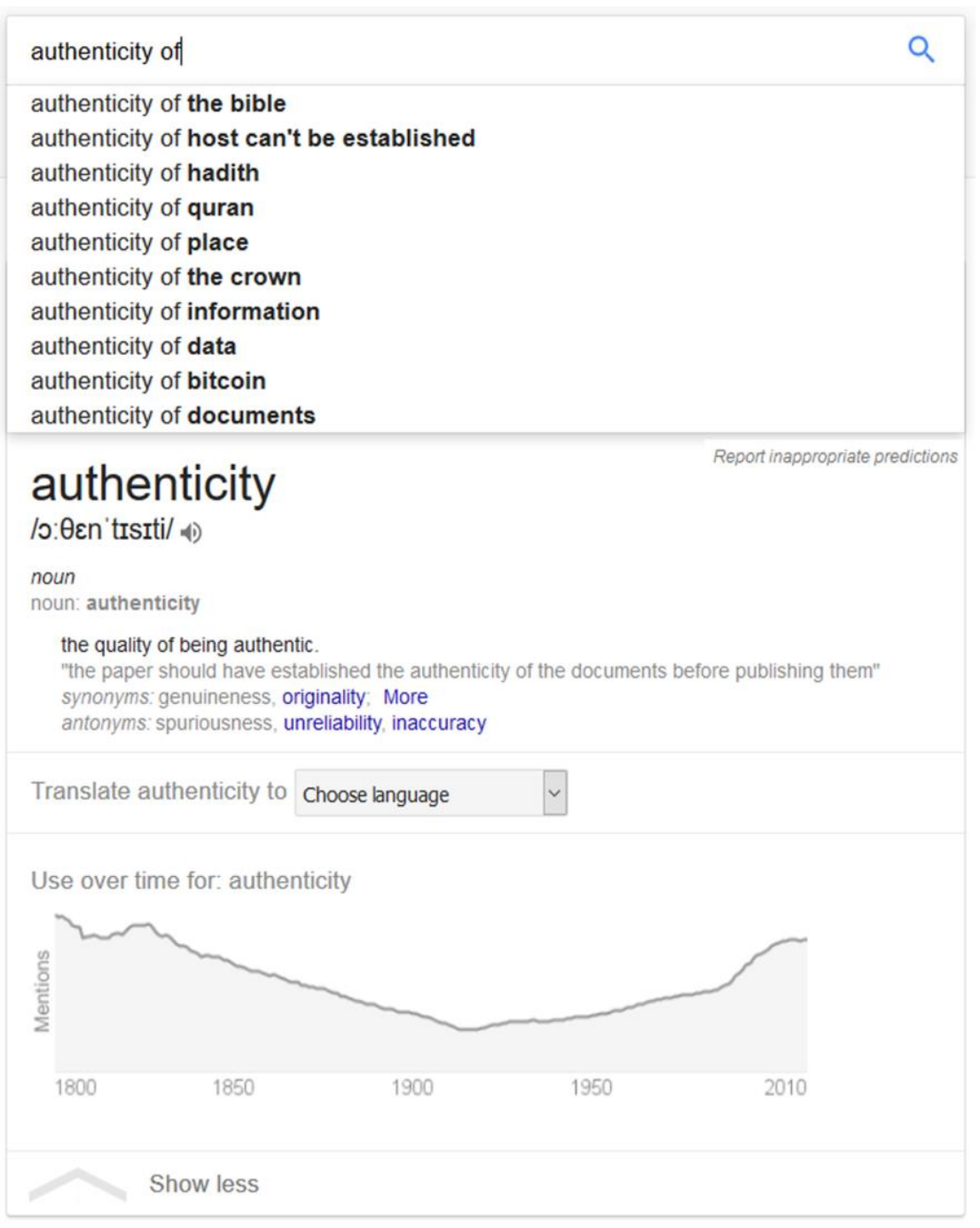

Fig. 35. Browsing results for 'authenticity of ...' online. Retrieved January 25, 2018, from Google.

The Museum of Innocence is an endeavour of Pamuk (2010) who wrote a novel and built a physical museum with the same name (Aydin, Schnabel and Moleta 2016). In favour of a smaller and more joyful museum concept, Orhan Pamuk argues that "museums- just like novels - can also speak for individuals" (Pamuk 2015). Kemal, the main character in the novel, defines two types of collectors: "The Proud Ones" and "The Bashful Ones." The former is correspondent with voluntary memory, whereas the latter talks to involuntary memory (Xing 2013; Ertuna 2010). 
Exhibiting 'proud' objects tends to expose the meaning explicitly, 'predominant in the West' Kemal adds, whereas 'bashful' objects cause inhibition in involuntary memory. Building a museum in the novel for his love, Kemal proves to be carrying some of the characteristics of the author, Orhan Pamuk, who, in contrast to his manifesto for museums, crowns his novel with a real museum. The objects that are imagined by readers are therefore fetishized by the 'proud' act of the author. But this at the same time proves their 'humility.'

Similarly, 'the humility of objects' that can be found in Kashgar is an inherently rich concept abundant with immaterial forms of cultural heritage information. In this research, a digital design approach aims to articulate Kashgar's heritage based, not on the grandeur of the material features of objects, but on correlations of immaterial aspects.

The purpose of reviewing the 'Matters of Context' was to formulate the first subquestion of the thesis. It has been explained that Kashgar is an appealing context for digital heritage research. Narrow roads of Kashgar are found to be elemental to the local identity representing historical events, such as the firm neighbourhood concept that helped form a farm-state in Kashgar in the sixteenth century. Presenting a theme of the narrow alleys of Kashgar with examples to explain the concept of 'staged authenticity,' this subchapter related the cases of museum and conservation practices to the role of representation in content-making in heritage. Therefore, authenticity was explained to be an important criterion of the context, challenging the interpretation of heritage environments in digital forms while aiming to promote creative ways of interpretations. With this in mind, the first sub-question of this research is formulated as:

\section{sQ1: How can architectural heritage be translated into creative digital forms?}

The following subchapters are dedicated to the formulation of other two sub-questions, with sections (sub-subchapters) informing the background, theme, case and criterion in the same order as this subchapter (Table 6).

Table 6. The progress of formulation of research sub-questions.

\begin{tabular}{lll}
\hline Chapter & Sub-Questions & Progress (Formulated?) \\
\hline Chapter 3.2.4 & $\begin{array}{l}\text { sQ1: How can architectural heritage be } \\
\text { translated into creative digital forms? }\end{array}$ & Yes \\
\hline Chapter 3.3.4 & $\begin{array}{l}\text { sQ2: How can an immersive digital } \\
\text { heritage application convey virtuality to } \\
\text { the public via creative engagement? }\end{array}$ & Not yet \\
\hline Chapter 3.4.4 & $\begin{array}{l}\text { sQ3: What alternative knowledge of } \\
\text { virtuality lies in the participatory content- } \\
\text { making? }\end{array}$ & Not yet \\
\hline
\end{tabular}




\section{Subchapter Outline}

Purpose: The subchapter aims to appropriate the matters of the medium in digital heritage to the problematic of multimodality.

Design/Approach: The background knowledge of 'unmediated heritage' gives an overview of how the contemporary media are exploited by digital heritage projects. The theme of 'alternate realities' highlights the importance of multimodal engagement for the criterion of creative engagement. The Sub-question 2 (sQ2) is built on the convergence of 'unmediated heritage,' 'alternate realities' and 'creative engagement.'

Findings: It is reviewed that discussion on 'virtuality' unfolds critically on matters related to medium. Embodiment theories help envision alternate realities for a wired digital museum experience.

Limitations/Implications: Only immersive technology from a single research lab (ALiVE) is considered. In other disciplines, such as new media and interactive media, ALiVE projects that date back to 2013 may be outdated. However, these projects clearly represent the current situation in digital heritage as the research outcome of one of two prominent researchers, Sarah Kenderdine and Jeffrey Shaw, are highly cited and renowned. The subchapter analytically approaches this limitation while giving insight about 'virtuality' for the critique of digital heritage projects.

Originality/Value: Virtuality is one of the most frequently discussed topics in research about cyberspace, which forms the basis of virtual environments developed in digital heritage research. Research typically focuses on the technicality of the development of virtual environments. This subchapter reviews these aspects in relation to the concept of virtuality.

Keywords. Unmediated heritage; envisioning cyberspace; ALiVE; embodiment; alternate realities; creative engagement; virtuality.

In the previous subchapter the 'Matters of Context,' it is depicted that the criterion of finding the 'immateriality of tangible heritage' can be very extensive as it is subject to decoding via the digital medium. 'Matters of Medium' is pertinent to processes related to documenting heritage resources and disseminating as heritage outputs. This subchapter provides a novel understanding of 'unmediated heritage,' which is 'understood as heritage curated by individuals [...] not attached to authorised heritage institutions' (RICHES Taxonomy, 2014).

In the core of 'unmediated heritage' lies a correlation between democratisation of museums and new media technologies. The infamous quote of Derrida $(1976,158)$ that "there is nothing outside the text" (or translated as "there is no outside-text") refers to that which appears and contingently disappears like a contradictory "trace" (Spivak 1976: xviii). By playing between these moments of the 'found' and 'lost,' Derrida means that there is nothing unmediated and everything requires us to interpret and reinterpret endlessly. 
The proponents of 'unmediated heritage' in GLAMs (Galleries, Libraries, Archives and Museums) co-exist with the contemporary advocates of social inclusion and multiculturalism in heritage (Hall 1999, Message 2009a, 2009b, Waterton 2010). It is explained in this chapter that the concept of unmediated heritage is rather converse to the idealist guise of heritage that has legitimised and institutionalised selection and display processes.

The acceptance of new type of standards, content, medium and visitors has often been disputed as they were assumed to be 'errors' that needed correction. "The repression of error has served as the fulcrum of aesthetic autonomy whenever autonomy has been the standard of aesthetic perfection, and for good reason" (Singer 1998). Matters related to medium evolve on a concept of 'inclusiveness' in museums. This is to review not only the ways for objects and people but also alternate new media environments that cross over multiple installations.

\subsubsection{Background: Unmediated Heritage}

'Why Don't More Americans Go to Museums?' An arts blogazine, Hyperallergic, asked this to followers on Twitter, noting that "in the past we may have turned to pollsters or psychics, while today we turn to Twitter to look at the hive mind and discover why..." (Vartanian, 2015). This stance indicated a change in the way that museums look at communicating with the public. Although this example sounds hypothetical, it is undoubtedly an explicit representation of the current challenge faced in museums.

Heritage standards and their usage have always been attributed to authorised institutions such as galleries, museums and tourism boards (Bennett 1994; Smith 2006; Waterton and Watson 2013). But the rise of concern with public engagement has created a demand for change in the de facto acknowledged position of AHIs (Borowiecki, Forbes and Fresa 2016). Today, the prevalence of the internet and social media place pressure on museum curators to seek innovative ways to meet contemporary social demands.

In accord with Foucault's 'disciplinary societies,' museums served a specific audience in a specific way. They used to form exclusive and divisive platforms for the exposition of their collections (Ross 2004). From the 1980s onward, the New Museology movement constructed the first body of collective thinking focused on the democratisation of museums (Hall 1999; Message 2009; Waterton 2010). The introduction of new media permeated museums all over the world. Opponents of new technologies were concerned with "the death of the object and a reduction of knowledge to information" (Witcomb 2007, 35). In digital 
heritage, it has been understood as a transformation from museums as guardians of knowledge to museums as facilitators to share knowledge (Howell and Chilcott 2013).

One of the outcomes of this change is the RICHES project, which is the acronym for Renewal, Innovation and Change: Heritage and European Society. RICHES (2015) is an endeavour for "bringing cultural heritage and people together in a changing Europe and finding new ways of engaging with heritage in a digital world." RICHES is funded within the European Union's Seventh Framework Programme for research, technological development and demonstration. It promotes multi- and interdisciplinary research that closes the gap between science and humanities. The project encourages networks and collaboration for technological innovation via creative industries. As for digital heritage, the RICHES project provides a new taxonomy to help researchers converse with their multidisciplinary community (RICHES Taxonomy 2014).

Another multidisciplinary initiative is undertaken in the Europeana Space Project, which is also funded by an EU programme. The project focuses on digital cultural heritage with an objective to generate "new opportunities for employment and economic growth within the creative industries sector" (E-Space 2017). In this multidisciplinary environment in Europe, science and humanities are brought together to go beyond the epistemological boundaries between different fields of interest. The Pop-Up Museum is a know-how partnership of Noterik BV, NTUA, Sound and Vision (NISV) and KU Leuven. The modular installation of the Pop-Up Museum hybridises digital and physical exhibitions (MuPop 2017b).

The museum takes a process-based approach rather than outcome-oriented standard collection methods. In accord with the RICHES taxonomy, a Pop-Up Museum serves for "unmediated heritage" (RICHES Taxonomy 2014) as a user-centred curation independent of Authorised Heritage Institutions (AHIs). Open to anybody on the web and lowering participation boundaries, the Pop-Up Museum offers exhibition curators a platform for 'unmediated heritage.' It is a facilitator and helps exhibition designers facilitate a participatory content-making process, related to the past. Visitors can interact based on simple smart-phone gestures such as selecting and swiping content shown on their screen. The entire setup requires a large screen with an HDMI-connected laptop that displays a webbrowser in kiosk mode.

The Pop-Up Museum is designed for communal areas such as waiting rooms, entrance halls and even classrooms (MuPop 2017b). The Pop-Up Museum defines its own ecosystem of alternate realities through the merged lenses of the virtual and the real (E-Space 2017) (Fig. 36). “A Pop-Up Museum transforms a small portion of space into an island dedicated 
to art and culture, where visitors or passersby may dwell awhile, using their mobile phones or tablets to bring the screen or screens to life" (Mupop 2017a). Once set up, a Pop-Up Museum instantly becomes speculative to the function of modern museums. It contributes to the democratisation of museums by implicitly questioning their dominant role in defining ethical values within the context of authenticity and aesthetics.

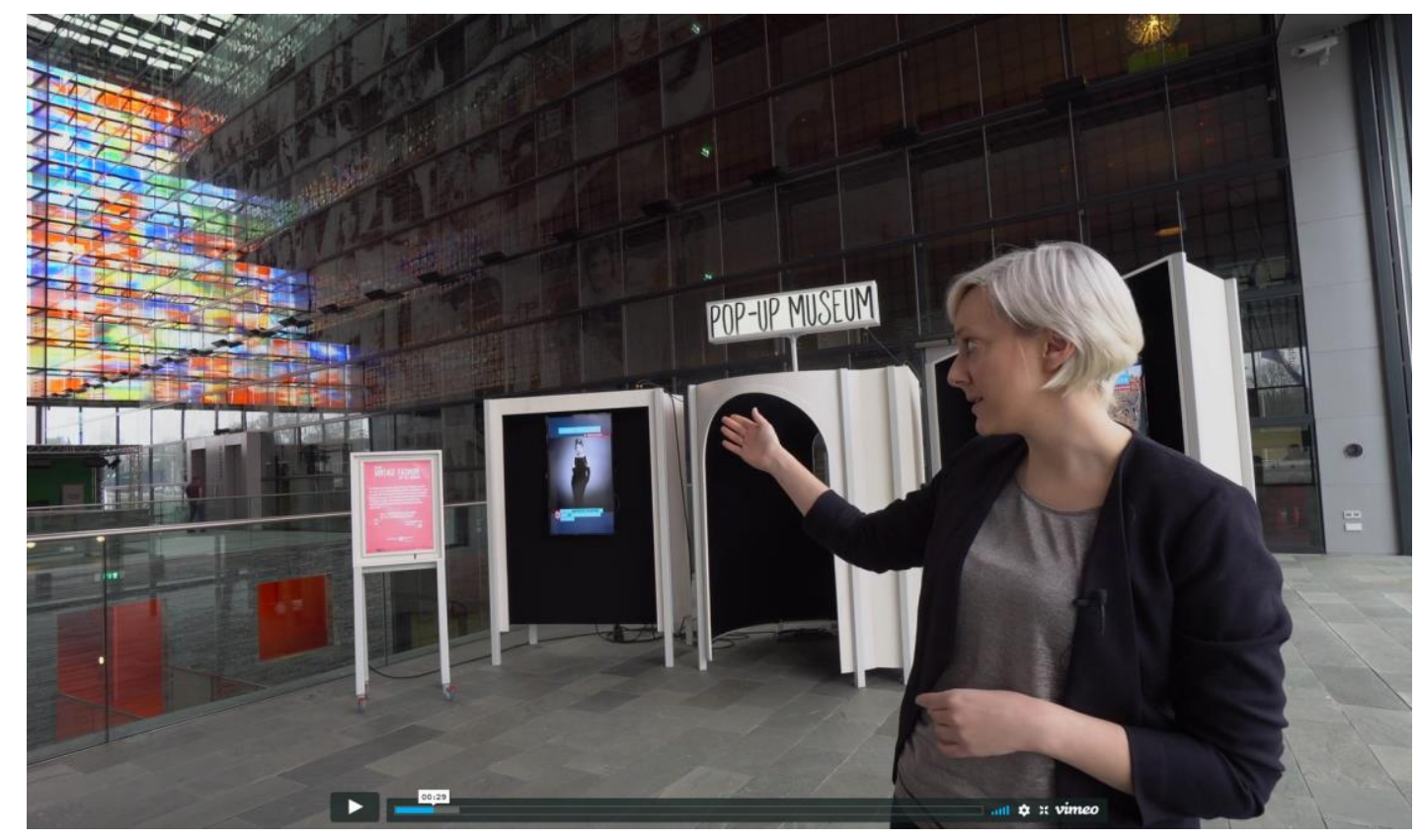

Fig. 36. Screenshot of a Pop-Up Museum set up at NISV in Hilversum, The Netherlands (January 12-24, 2017). Visitors login through a browser (without installing an app) to interact with three monitors. Adapted from MuPop (2017a).

What makes impractical sharing the role of content-making with the public is that there is no precise mechanism for controlling the process of an all-inclusive content generation (Adair, Filene and Koloski 2011). This will be elaborated in Chapter 3.3 .3 based on the visualisation projects of ALiVE (Applied Laboratory of Interactive Visualisation and Embodiment) at the City University of Hong Kong.

\subsubsection{Theme: Envisioning Alternate Realities}

The first interactive and photo-realistic 'virtual travel' project was the Aspen Moviemap developed in a 1980s collaboration at MIT involving the Architecture Machine Group and Negroponte's Media Lab. The Aspen Moviemap is an early experiment to experience that aimed to explore the streets of a city as a virtual traveller seated in an armchair that controls speed and direction (Naimark 2006). Similarly, Jeffrey Shaw's (1988) Legible City, which 
was exhibited in Nagoya, Japan in 1989, seeks a poststructuralist interpretation of textuality. The viewer tours in a virtual city made of text blocks by means of a stationary bike in front of a large screen. In her semiotic enquiry, Marie-Laure Ryan $(2015,2)$ conceptualises "textuality, mimesis, narrativity, literary theory, and the cognitive processing of texts" in the context of VR.

Envisioning a digital future in Placing Words: Symbols, Space and the City, Mitchell (2005) transfers Louis Kahn's metaphoric conversation about a brick. He asks, "what does a pixel want to be?" (87) to underline the "meta-materiality' in computer-based virtual technologies. Researchers have been working very hard to shed some light on these technologies, by means of 'genealogically' connected works of Understanding New Media (McLuhan 1964), The Language of New Media (Manovich 2001), and Optical Media (Kittler 2010).

The "Reality-Virtuality (RV) continuum" as developed by Milgram et al. (1994) is a highly cited work reference in the scholarship of VR. It is grounded in the 'Matters of Medium' with a linear relationship between reality and virtuality (Fig. 37). As described by its authors, it is "limited strictly to visual displays" (282). Given that, the RV Continuum reduces virtuality to reality (see other theoretical accounts in Chapter 2.4 and 2.5).

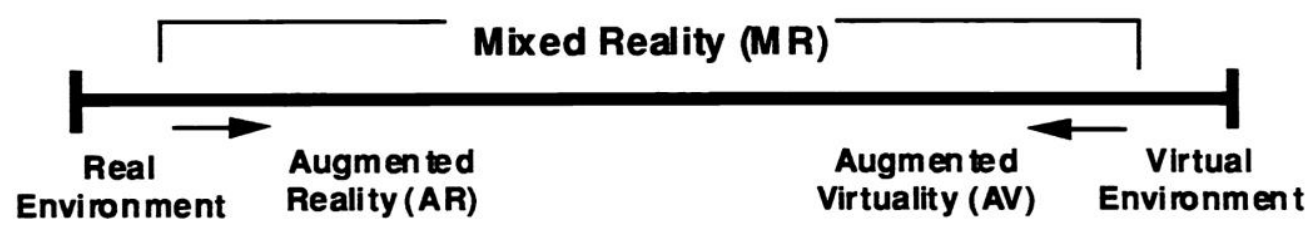

\section{Reality-Vir tuality (RV) Continuum}

Fig. 37. Diagram of the Reality-Virtuality (RV) continuum. Adapted from Milgram et al. (1994).

The diagram of the RV Continuum consists of a line between opposite ends. Anything in-between, unless entirely real or virtual, is named as Mixed Reality (MR). MR includes Augmented Reality (AR) and Augmented Virtuality (AV). Richens (2014) shows the incapability of RV Continuum to describe the complexity of the 'Matters of Medium.' His Virtuality Matrix (VM) is based not only on technology but in a tri-fold relation to visitorsite-content aspects (Table 7). 
Table 7. Virtuality Matrix (VM) (see further developments of it on p. 97 and 183). Adapted from Richens (2014).

\begin{tabular}{llll}
\hline Visitor & Site & Content & Richens' definition \\
\hline Real & Real & Real & Reality \\
\hline Real & Real & Virtual & Augmented Reality \\
\hline Real & Virtual & Real & Mixed Reality \\
\hline Real & Virtual & Virtual & - \\
\hline Virtual & Real & Real & Telepresence \\
\hline Virtual & Real & Virtual & - \\
\hline Virtual & Virtual & Real & Virtual Museum or Set \\
\hline Virtual & Virtual & Virtual & Virtual Reality \\
\hline
\end{tabular}

Increased use of digital applications in humanities has mitigated the entrenched dualism between 'real' and 'virtual' in recent years (Kenderdine 2016). Another critic of this persistent quarrel of the field is Andrea Witcomb (2007) whose critique of digital heritage calls for developing a materialist approach to understanding multimedia in museums.

In the 1990s, theoreticians focused on hypertext as a 'myth' of multimedia. They envisioned new modes of non-linear storytelling in cyberspace in the works of Brenda Laurel (1991), Marie-Laure Ryan (1991; 1999) George Landow (1992) Espen Aarseth (1997), Janet Murray (1998) and Katherine Hayles (1999). In literature, William Gibson's science fiction Neuromancer (1984) provided a powerful vision about technologically enhanced modern life. In cinema, The Matrix (1999) accounted for the interpretation of McLuhan's entirely wired 'global village.'

In Hamlet on the Holodeck, Janet Murray (1998) reframes matters pertaining to medium with what she calls the "three key pleasures of cyberspace." They are immersion, agency and transformation. Amongst these three, Milgram's RV continuum and Richens's VM only touch on the first one, immersion. Cyberspace starts with the second, agency (meaningful experience) and continues with the third, transformation (fully-fledged freedom granted in digital realities).

Ian Bogost (2006; 2007) and Alexander R. Galloway (2006) extend on games as new media instruments. The former's account is based on how games operate as mechanisms and as part of computer systems. The latter reflects on the impact of games in society by studying these mechanisms. At the instant of the participation of a video gamer, there emerges a mutual order statement between her/him and the game's algorithmically unfolding process (92). To play the game one should execute the code of the system and to 
win the game is to know the system (94). In contrast to traditional reader-text hierarchy, games reduce it on a horizontal plane, with the gamer in the act of gameplay (105). Galloway (106) refers cinema to Foucault's definition of 'disciplinary societies' and video games to Deleuze's 'informatic control.' The latter uses up the 'flexibility' of information superhighways. It uses the gamer in the act of "learning, internalizing, and becoming intimate with a massive, multipart algorithm" (90). It is a vertical mechanism, hiding the message in depth; whereas cinema privileges a horizontal mode of the discipline (88). On information superhighways, a gamer is an "experimental explorer" into our future of digital realities (Baudrillard 1993). Deleuze summarises superhighways as a new type of control:

A control is not (no longer) a discipline. In making freeways, for example, you don't enclose people but instead multiply the means of control. I am not saying that this is the freeway's exclusive purpose, but that people can drive infinitely and 'freely' without being at all confined yet while still being perfectly controlled. This is our future. (Deleuze 1998, 18)

This may be understood that there is no chance for 'unmediated heritage' in new media. But it no longer makes any sense to discuss a good-or-bad duality about the impact of new media on ethical issues such as authenticity. A contemporary focus that goes beyond this dichotomy is the issue of designing alternate realities. The issue is far beyond the scope of the RV continuum. It is not a mere issue of using highly realistic imagery based on "problematic" user questionnaires (Champion 2003, 253).

The underlying problem is that there is an empirical crystallisation bounded into the isolation of individual representations. Ryan and Thon (2014) introduce digital narratives that "span multiple instalments and transmedial storyworlds that are deployed simultaneously across multiple media platforms, resulting in a media landscape in which creators and fans alike constantly expand, revise, and even parody them" (Matz and Herman 2014, 1).

In Bogost's $(2006,36)$ review of media theorists, Kittler's interpretation of McLuhan is an ultimate imagination of "fully connected media systems" rather than 'segregated,' 'partial' and 'disembodied' media machines. Bogost appoints The Matrix to the former ontological, and William Gibson's Neuromancer to the latter's phenomenological account of McLuhan (35-36).

In this quarrel that Kittler positions computers as a "radical" win over culture, Manovich remains calmer and resolves computers as "influential" (40). In the next section, projects of the ALiVE lab, which is a significant contributor in the digital heritage field, are discussed parallel to the notion of alternate realities that have unfolded in this section. 


\subsubsection{Case: ALiVE Projects by Shaw and Kenderdine ( 1995-2015)}

Case studies of the 'Matters of Medium' are from the Applied Laboratory of Interactive Visualisation and Embodiment (ALiVE). Directed by Jeffrey Shaw and Sarah Kenderdine, the lab is described as "an incubator and innovations showcase for new forms of creativity and at the cutting edge of digital media in society." The summary of their core research themes is that "ALiVE promotes an integrated scientific and artistic research strategy" via embodied multimedia models to "enable the interpretation of tangible and intangible cultural knowledge" for museums (ALiVE 2017).

Set up in the Hong Kong Science Centre premises of the City University of Hong Kong, visualisation systems of ALiVE include 360-degree stereoscopic interactive visualization environments (AVIE and PLACE) and hemispherical projection systems (iDome and MediaDome) (Fig. 38). ALiVE's display systems have made a significant contribution to research and practice in digital heritage in accord with development in the New Museology movement (see Schettino and Kenderdine 2010; Kenderdine and Shaw 2015; Kenderdine 2015).
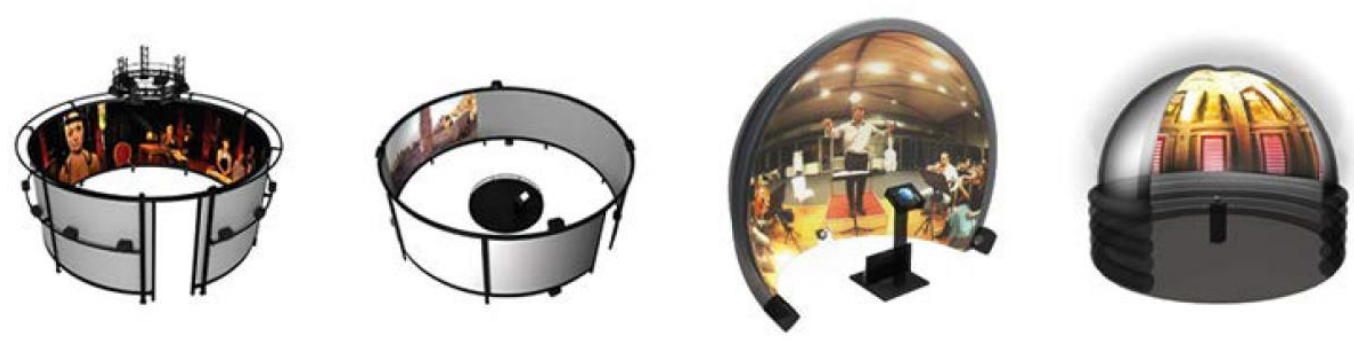

Fig. 38. 360-degree stereoscopic interactive visualization environments (AVIE and PLACE) and hemispherical projection systems (iDome and MediaDome). Adapted from ALiVE (2017).

ALiVE installations are designed by Jeffrey Shaw and Sarah Kenderdine. The former is a pioneering artist in interactive media, and the latter is a prominent researcher in digital heritage. Supported by substantial funding opportunities, ALiVE produce interdisciplinary research, converging creative industries with scientific research. Therefore, it is an excellent case study to situate the current level of new media usage in digital heritage. The overview in this part is based on the visualisation systems that are examined during the LuxLab workshop at the ALiVE studio in Hong Kong in September 2013 (Table 8). 
Table 8. Display systems examined at LuxLab.

\begin{tabular}{llllll}
\hline & AVIE & PLACE & iDome & MediaDome & Re-Actor \\
\hline Project & Pure Land & $\begin{array}{l}\text { PLACE- } \\
\text { Hampi }\end{array}$ & iTürkiye & LookUp & UNMAKEABLOVE \\
\hline Year & 2012 & 2012 & 2012 & 2012 & 2008 \\
\hline Interaction & joystick & $\begin{array}{l}\text { navigation } \\
\text { interface }+ \\
\text { rotating } \\
\text { platform }\end{array}$ & $\begin{array}{l}\text { navigation } \\
\text { via trackball }\end{array}$ & - & torchlight \\
\hline $\begin{array}{l}\text { Favourite } \\
\text { viewer } \\
\text { position }\end{array}$ & $\begin{array}{l}\text { standing in } \\
\text { the middle, } \\
\text { looking } \\
\text { outward }\end{array}$ & $\begin{array}{l}\text { standing in } \\
\text { the middle }\end{array}$ & $\begin{array}{l}\text { standing in } \\
\text { the middle }\end{array}$ & $\begin{array}{l}\text { lying on the } \\
\text { ground }\end{array}$ & $\begin{array}{l}\text { standing outside, } \\
\text { looking inward }\end{array}$ \\
\hline
\end{tabular}

The advanced digital imaging workshops of LuxLab have been organised twice so far in Hong Kong (2013) and in Sydney (2014) by Kenderdine (Fig. 39). The workshop is a one-day training programme on digital preservation and conservation and museum exhibition design. In LuxLab, the display technologies of the ALiVE lab are elemental to discussions and instrumental in hands-on training. Each display system in the lab has a narrative in which the viewer is invited to examine the aspect of immersiveness. Instructions for each media platform are text-based information sheets, provided along with additional apparatus such as polarised 3D glasses (Fig. 40).
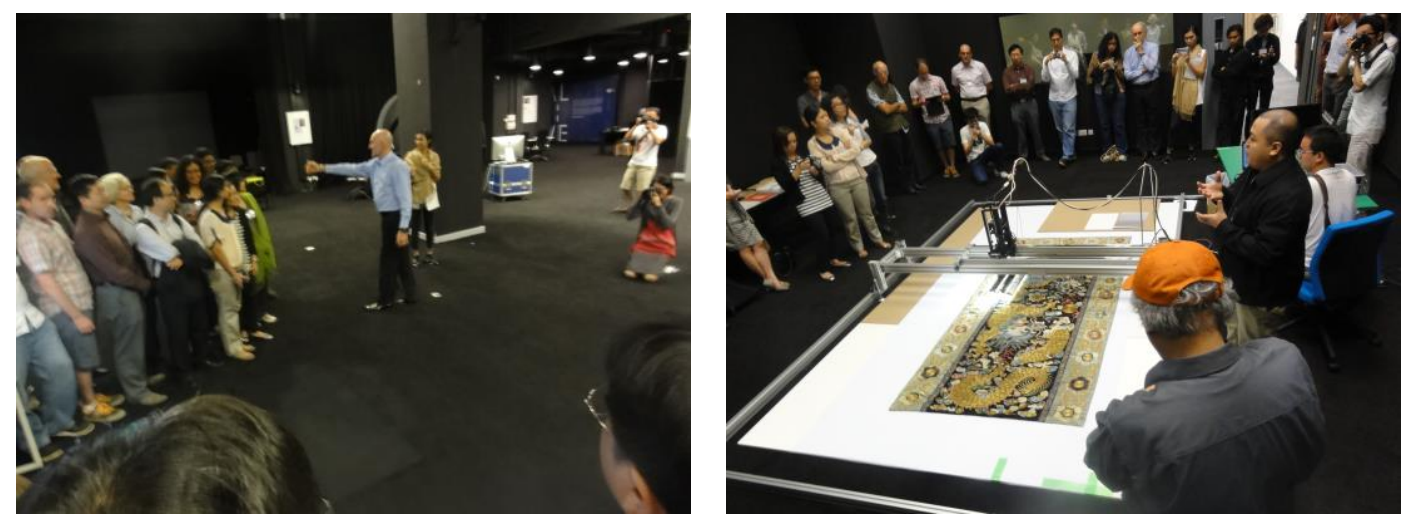

Fig. 39. Left: Jeffrey Shaw and Sarah Kenderdine while organising Luxlab workshop participants for a group photo. Right: Introduction to advanced high-definition scanning of heritage artefacts (up to 1,600 DPI). Photos by Author, September 2013, Hong Kong. 

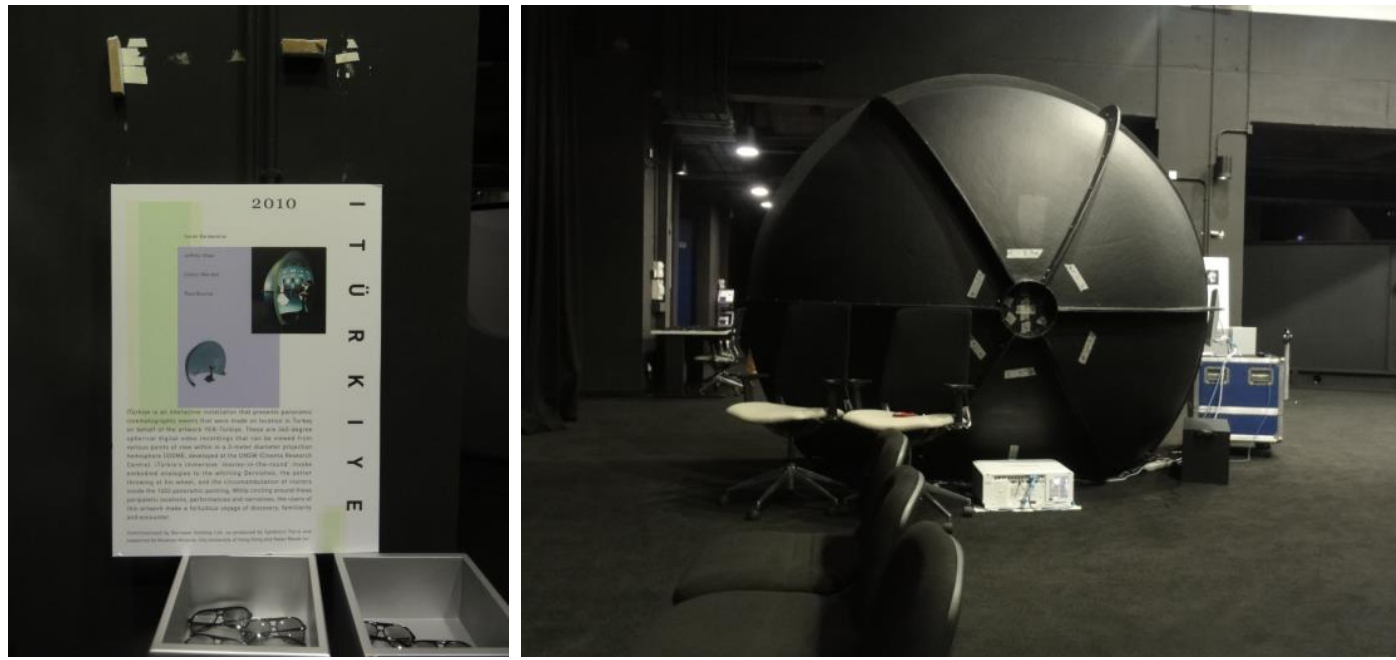

Fig. 40. Left: The information sheet of the iTürkiye installation using the iDome display system. Right: iDome's steel hemisphere from behind. Photos by Author, September 2013, Hong Kong.

Table 9 shows how the visualisation systems presented at LuxLab are conceived for three types of viewing modality (panoramic, hemispheric and linear spatial), two kinds of visual depth (stereoscopic and monoscopic) and in a variety of capacities (10 to 100 participants). Spatially each installation is confined by encircling screens projecting virtual scenes inward, with the 'Re-Actor' display system being an exception. It enables viewers to interact with a hexagonal box of space situated in the middle. The Re-Actor display system was initially conceived as a museum product named VROOM, for the Virtual Room (Kenderdine and Hart 2003). The primary purpose of VROOM was to engage the public with scientific visual data including cultural heritage sites.

With the involvement of Jeffrey Shaw, the display system was reoriented for an artistic installation. In this regard, it was a successful transformation connecting creative arts and science. In UNMAKEABLOVE (2008) using Re-Actor, the viewer using torchlight interacts with virtual content animated on each side of the hexagonal box (Kenderdine and Shaw 2012) (Fig. 41). Visitors interact with a virtual representation of a dystopian scene of animated human bodies. 
Table 9. ALiVE's display systems. Adapted and modified from Kenderdine and Shaw $(2015,199)$.

\begin{tabular}{lllllc}
\hline Display system & View mode & Depth & Capacity (in person) & Size & \\
\hline $\begin{array}{l}\text { AVIE } \\
\text { Pure Land }\end{array}$ & panoramic & stereo & 1 primary + 25-30 & H: 4 meters & $\bullet$ \\
\hline $\begin{array}{l}\text { PLACE } \\
\text { PLACE-Hampi } 10 \text { meters }\end{array}$ & & & & D: 10 meters & $\bullet$ \\
\hline $\begin{array}{l}\text { iDome } \\
\text { iTürkiye }\end{array}$ & panoramic & stereo & 1 primary + 25 & & \\
\hline $\begin{array}{l}\text { MediaDome } \\
\text { LookUp }\end{array}$ & hemispheric & mono & 1 primary + & D: 3 meters & $\bullet$ \\
\hline $\begin{array}{l}\text { Re-Actor } \\
\text { UNMAKEABLOVE }\end{array}$ & linear spatial & stereo & 6 primary + 20-100 & H: 3.5 meters & $\circ$ \\
\hline
\end{tabular}

\section{- Inward-screening $\quad$ Outward-screening}

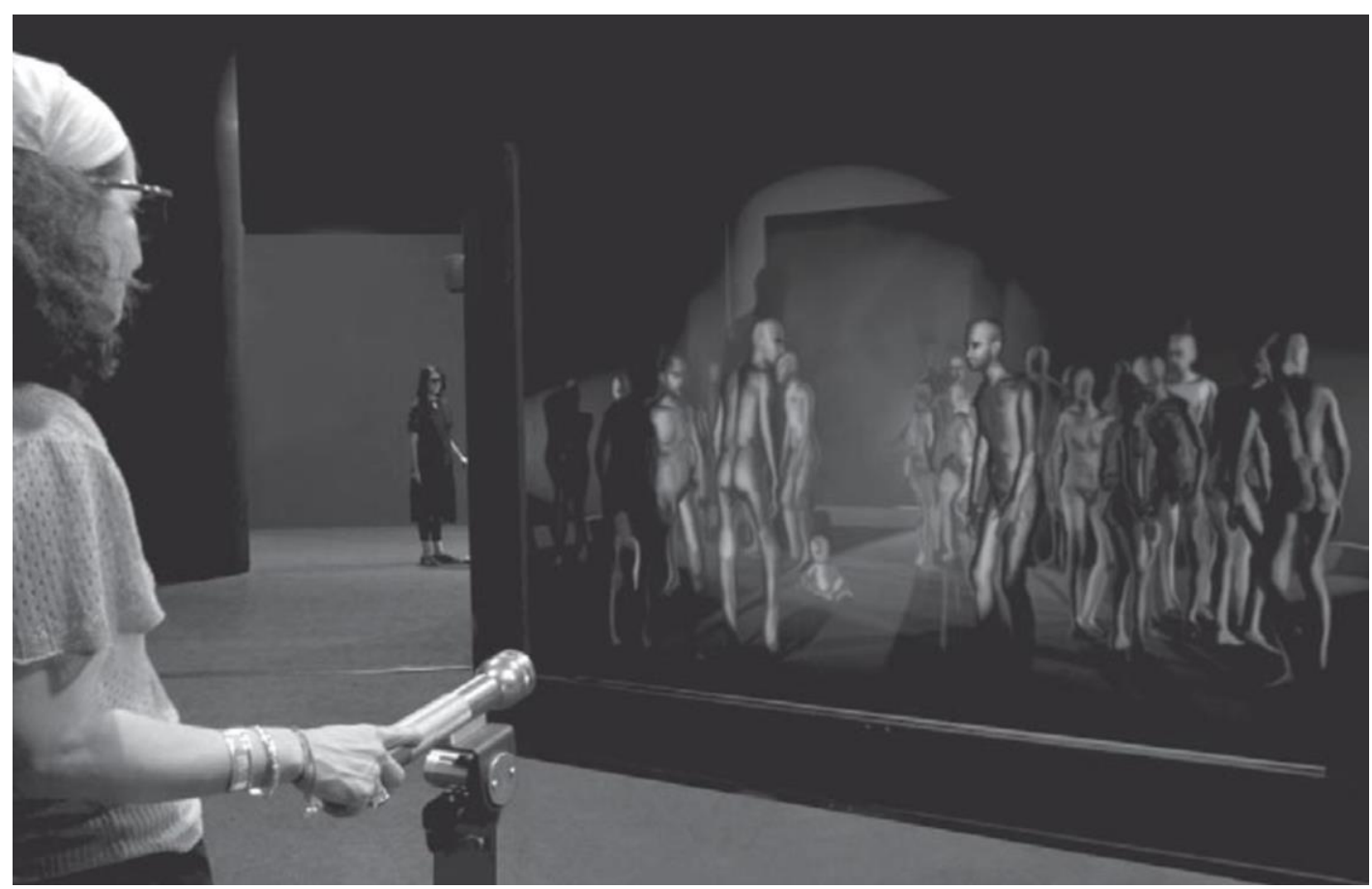

Fig. 41. UNMAKEABLOVE (2008). Adapted from Kenderdine and Shaw (2012)

In Re-Actor, the spectator view is not embedded into an encompassing screen like 360degree panoramas. It is therefore exemplary of the non-linearity of the notion of 'virtuality.' Re-Actor's hexagonal box is more like a fireplace in a cottage room that brings people together while leaving space behind for other activities. Six primary viewers around the hexagonal space can simultaneously interact with the virtual content via torches while others passively watch and wait for their turn. Other display systems are designed to engulf the viewers' visionary boundaries into a room-like space circular or spherical in form. With these features, Re-Actor is placed on Richens's Virtuality Matrix (VM) (Table 10). 
Table 10. Positioning the display system of 'Re-Actor' by ALiVE on Richens's VM. Adapted and modified from Richens (2014) (see the original p. 91 and further developed p. 183).

\begin{tabular}{llllr}
\hline Visitor & Site & Content & Narrative paradigm & $\bullet$ \\
\hline Real & Real & Real & Reality & $\bullet$ \\
\hline Real & Real & Virtual & Augmented Reality & $\bullet$ \\
\hline Real & Virtual & Real & Mixed Reality & $\bullet \bullet$ \\
\hline Real & Virtual & Virtual & Re-Actor system by ALiVE & $\bullet$ \\
\hline Virtual & Real & Real & Telepresence & $\bullet$ \\
\hline Virtual & Real & Virtual & - & $\bullet$ \\
\hline Virtual & Virtual & Real & Virtual Museum or Set & $\bullet$ \\
\hline Virtual & Virtual & Virtual & Virtual Reality & $\bullet$ Richens's definition \\
\hline
\end{tabular}

\subsubsection{Criteria: Creative Engagement}

Each display system of ALiVE is a unique means of virtuality, putting viewers into a variety of alternate realities operating differently. Kenderdine (2015) introduces these systems bounded to embodiment theory, which explains the corporeal attachment of the viewer's mind to the 'machine' through multisensory entanglement (visual, auditory, tactile, etc.). However, these systems are subject to an enquiry about the sustainability of communication between the mind and the machine. They are designed mostly in isolation from each other. They are alternate realities to themselves with no connection in between. Yet, such an integration is achieved at low-level between Pure Land: AVIE and Pure Land: Augmented Reality Edition. It is noticed that ALiVE projects are dominated by astonishment for achieving the integration of cutting-edge technologies. They, instead, identify with creative engagement secondarily, which is a prevailing attitude in the museum domain (see Chapter 2). But the outcome of medium-oriented practice is found to hardly sustain other aspects of engagement, such as the role of meaningful participation for immersiveness and the contribution of user-generated content to the generation of meaning.

During the LuxLab workshop 2013 in Hong Kong, the MediaDome display system, which virtualises the domes in Istanbul, is examined. Reflecting the dome of Sultan Ahmed Mosque in Istanbul on the inner surface of an inflated planetarium, MediaDome leaves visitors with an impression of virtually being there (Fig. 42). The reproduced version of the actual dome in the same spherical form makes it easy to identify with the actual space. However, in theory, this becomes a matter of a philosophical discussion about virtuality. In digital heritage, these discussions are related to a range of theories such as Baudrillard's 
'hyperreality' (Tiffin and Terashima 2001), Benjamin's 'aura' (Cameron 2010), Foucault's 'power and ethics' and Latour's 'Actor-Network Theory' (ANT) (Colley 2015).

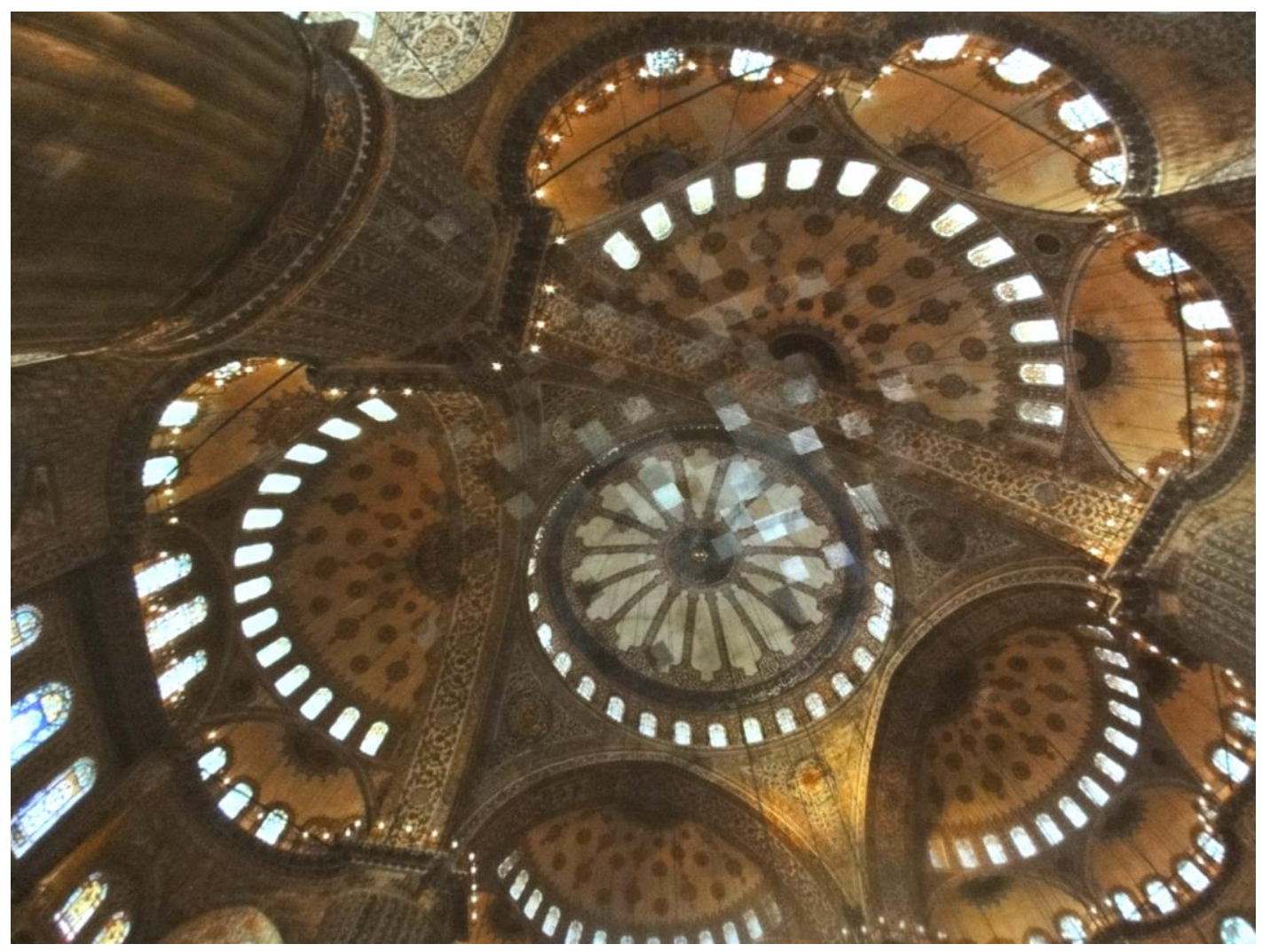

Fig. 42. The dome of Sultan Ahmet Mosque, Istanbul projected in MediaDome, LUXLAB Workshop. Photo by Author, September 2013, Hong Kong.

From an account of Baudrillard, 'hyperreality' deconstructs the objects that deconstruct us in turn. In an interview, titled "Forget Artaud," with Sylvere Lotringer, Jean Baudrillard says that the work of the French playwright and poet Antonin Artaud "belongs to a secret place, a reserved domain and talking about that, exposing that to the light would amount to making one's secret visible." Explaining the way that he followed in delivering his interpretation of Artaud's work, Baudrillard (2005) stresses the futility in attempting to place Artaud where he does not belong to. In this context, ALiVE's MediaDome makes a good sense of hyperreality that places the actual dome where it does not belong. It subjugates the audience while decontextualizing the dome of Sultan Ahmet Mosque in Istanbul. Attempting to interfere with the domain of the actual dome, its replica's concern with hyperreality tends to move the viewer further away from the objective of enquiry. It creates an event of a seductive advertisement hanging on a large board. This leads to alienation and estrangement with 'virtuality' instead of 'embodiment.' An enquiry of virtuality enforces the emancipation of the viewer instead of confinement. 
The alienation of the viewer within a 'staged authenticity' is somewhat eradicated in the Pop-Up Museum (MuPop) exhibitions (p. 89). Being a cost-effective interactive installation, MuPop offers a sustainable development for integration between different systems through which narratives are accumulated by the human agency. Murray's (1998) "three key pleasures of cyberspace" touch on the same point. Immersiveness, agency and transformation are all connected parameters of experience in cyberspace. Baudrillard incontestably represents the same critique. ALiVE installations with a focus on highly technological advancement are subject to review whether they are "moving ever closer to high definition, [...] to the useless perfection of images" (Baudrillard 2005).

The death of the virtual museum (see Chapter 2.5) is caused by the focus on the realist perfection of images rather than the creative power of illusion and virtuality. In this review chapter the 'Matters of Medium' are studied to find out that reinvigorating cultural assets involve considering a variety of latent factors. It depicts that an enquiry of virtuality requires emancipating the viewer instead of confining it in a function of the display system. Reviewing the medium-related matters in digital heritage, this subchapter formulates the second sub-question around the theme of envisioning alternate realities. Given that, designing alternate realities is so that users can intuitively find themselves in creative engagement, rather than the passive consumption of digital heritage, is drawn as the criterion of this subchapter. Based on this, a second sub-question in this research is defined as:

sQ2: How can an immersive digital heritage application convey virtuality to the public via creative engagement?

Having formulated the two sub-questions in the previous two subchapters, the thesis is conceived to describe the third sub-question in the following subchapter (Table 11).

Table 11. The progress of formulation of research sub-questions (further development of Table 6).

\begin{tabular}{lll}
\hline Chapter & Sub-Questions & Progress (Formulated?) \\
\hline Chapter 3.2.4 & $\begin{array}{l}\text { sQ1: How can architectural heritage be } \\
\text { translated into creative digital forms? }\end{array}$ & Yes \\
\hline Chapter 3.3.4 & $\begin{array}{l}\text { sQ2: How can an immersive digital } \\
\text { heritage application convey virtuality to } \\
\text { the public via creative engagement? }\end{array}$ & Yes \\
\hline Chapter 3.4.4 & $\begin{array}{l}\text { sQ3: What alternative knowledge of } \\
\text { virtuality lies in the participatory content- } \\
\text { making? }\end{array}$ & Not yet \\
\hline
\end{tabular}




\subsection{Matters of Audience}

Subchapter Outline

Purpose: User-generated content is one of several focused matters pertaining to the position of the audience. The purpose of this section is to unfold a contemporary understanding of user-generated content.

Design/Approach: The background knowledge of communication with audience through new media introduces a theme of aesthetics seen in user-generated content, called 'glitch aesthetics.' The projects of Lev Manovich and his team provide insight about how to generate alternative knowledge from user-generated content in digital platforms. The third sub-question derives from the criterion of generating alternative knowledge from user engagement contemporary media.

Findings: This section discovers a criterion of indexicality from content generated by audience in a digital platform.

Limitations/Implications: This section delivers the 'Matters of Audience' as part of the problematic of communication. In this context, the role of 'error' in the evaluation of user-generated content is emphasised and theorised. The subchapter is not confined to the boundaries of only digital heritage research and practice. It uses the cases of Lev Manovich to reach insight about the general aspects of digital engagement.

Originality/Value: Current digital heritage practice is studied under three major groups: documentation, visualisation and dissemination. This section replaces the third one with communication to the myth of the 'Matters of Audience,' which is reframed by a contemporary model of new media rather than the obsolete broadcasting model that drives 'dissemination' projects in digital heritage.

Keywords. Visual communication; communication; dissemination; visual storytelling; manga; user-generated content; abstraction; glitch.

Dissemination of digital heritage is an amalgam of a myriad of processes including interaction, visualisation, representation, presentation and communication. Today, many fields engage with playful solutions to receive the attention of The Emancipated Spectator (Rancière 2009). As an example from architecture, in Yes is More, an unconventional way of communication, based on manga style, is employed by Bjarke Ingels Group (BIG 2010). Schnabel's manga research underlines the importance of personalisation in visual communication in architecture. In bi-tonal architectural narratives, his research achieves 'embodiment' through a level of "abstraction" that becomes appealing to laypersons (Schnabel 2013). The embodiment research of Sarah Kenderdine, which is examined in the previous section, is correlated to affect studies that investigate the sensual, corporeal and kinaesthetic aspects. They pertain to user-oriented studies that are committed to the 'Matters of Audience' in the participatory content-making. From a museum management point of view, The Responsive Museum edited by Lang, Reeve and Woollard (2006) provides a list of theoretical and conceptual discussions on understanding and developing audiences. As 
was reviewed throughout the previous chapter, the notion of participation is continuously developing in parallel with the democratisation of museums. In digital heritage, understanding participation benefits from the use of communication models and tools. Addison (2000) defines three levels of study in digital heritage: documentation, representation and dissemination. Dissemination is akin to one-to-many broadcasting models of communication. Outlined in Chapter 2.5, the digital museum in information space requires us to update the model of communication to the offerings and standards of the current new media. Din and Hecht (2007) bring together a list of "guidance" articles to build The Digital Museum with new communication tools. In the same vein, Tallon and Walker (2008) extend the level of guidance to a more specific list of articles discussing Digital Technologies and the Museum Experience under the concepts of "Mobile, Digital and Personal" (xiii). These attempts represent the involvement of the New Museology scholarship in the emerging field of digital heritage in the 2000s.

With the New Museology thinking, the public role of museums started to be a concern from the 1980s onwards. New participatory exhibition models were tried. Participatory Action Research Methodologies (PARM) are used in heritage management and museum studies. All parties who are related to the problem on hand are invited to discuss what/how to represent. PARM enables a cyclical communication model, starting with a reflection on action. The participation in this model is meant for socially activating the dynamics of public 'discussion,' i.e. democratisation. It is meant to be an equaliser between identities and cultures for more inclusiveness.

\subsubsection{Background: Communication with Emancipated Spectators}

Ben M'Sik Community Museum (BMCM) in Casablanca is an example of participatory curatorship. The inhabitants of the largest shantytown in Casablanca are mostly ignored by the state that does not issue identity cards for some of them. To make their own museum, inhabitants donate their private possessions for display at the BMCM. Pieprzak (2014) pairs a Moroccan novel, Mother Comes of Age (Chraïbi 1984), with BMCM in Casablanca. Pieprzak studies how the novel and the museum create active participation through "the relinquishing of objects to a protective space within a greater public sphere." On the one hand, the character of the novel buries personal objects from her past (e.g. old combs) in a "beautiful tomb" to protect her self-identity from the totalitarian "ridicule" of modernisation. On the other hand, the museum collection represents the identity of the local. 
102

Both practices showcase activated individuals partaking in the protection of identity (Pieprzak, 2014).

Pieprzak's analysis represents an analogy to Ruskin's romanticising of the past through a notion of diversity in museums as "contact zones" (Clifford 1997) and "conversable civic spaces" (Bennett 1998, 370-371). One can read phenomenological underpinnings in Pieprzak's critique borrowing a museum definition as a "form of internment - a tomb with a view" from Barbara Kirshenblatt-Gimblett $(1998,57)$. In a poststructuralist reading by Lebbady $(2009,146)$, the novel, Mother Comes of Age, is examined based on the "norms of literacy" critiquing the burial of objects, i.e. endowment of identity, that can be read as equivalent to the relinquishing of objects to BMCM. Likewise, it can be thought that the Ben M'Sik Community surrenders the modern institutionalisation enforced by the standards of the state who tend to ignore them. Nordin (2012) examines the future of the world, as is illustrated in China, by analysing a digital media application that interacts with visitors of the Siemens pavilion at Expo 2010. Nordin argues that there is ambiguity in the freedom given by technology. The visitors who enter the pavilion are tracked on their faces and then turned into avatars on a big screen. Eventually, everybody is displayed as agents of a unilateral future, singing a song together. In the form of a pre-programmed design, every visitor has a chance to be a star provided that she/he agrees to the condition of being an avatar, "forfeiting identity." In conclusion, to allow plurality, there should be space for “contestation,” Nordin indicates (2012).

Participation can be studied as part of communication. The absence of communication between the represented subject (signified) and the object (signifier) jeopardises Pamuk's (2010) successful museum-novel, The Museum of Innocence. The moment Pamuk displays objects of his own imagination in the physical museums, the participation of the book reader in the collective imagination of objects conceived in the novel is somehow ill-treated. Readers might be encouraged to bring objects of their own imagination for permanency in communication. Yet, it also makes a paradox faced by the Moroccan examples because the collections formed by readers would mean the relinquishing of their imagination, i.e. identity. In a poststructuralist sense, participation is the multiplication of meaning, "the death of the author" (Barthes 1988), "the emancipation of the spectator" (Rancière 2007), and "deterritorialisation of subjectivity" (Deleuze and Guattari 1987), while, at the same time, it paradoxically becomes subject to hyperreality (Baudrillard 1994).

The 'Matters of Audience' have infrequently been an aggregable area in the poststructuralist school of thought. Within this theoretical ambiguity, Plato's 'logic' is disfavoured in humanities to a Spinoza-Whitehead-Bergson-Deleuze lineage of 'affect' 
(Wetherell 2013). In heritage, it stays as an "absence of theoretical noise" with a novel attempt to enliven the discourse (Waterton and Watson 2013). Original approaches, such as "more-than-representational" heritage (Waterton 2014), seek an emphasis on the sustainability of embodiment theories and affect studies. Following Marc Augé's distinguishing between anthropological places and 'non-places,' McKay (2007) diagrammatically conceives a meta-model of "affective communication" towards the personalisation of museum exhibitions. Today personalisation is commonplace on social media, which heritage and museum scholars refer to as an area of interest (Borowiecki, Forbes and Fresa 2016; Giaccardi, 2012). In this regard, Combi (2016) outlines the current communication preferences of digital natives for the many-to-many communication model in new media. These developments of participation, communication and personalisation are an attribution of 'affect' and 'embodiment.' Intriguingly, McKay (2007) brings personalisation from product development (outcome-based configurations) into museum exhibitions (process-oriented). The former is a problem-solving activity that may include optimisation and supervised learning algorithms. The latter is in nature a problem-finding that needs modelling negative and positive feedback cycles that are the subject-matter in cybernetics. Fun Palace (1964) is a cybernetic design by the British architect Cedric Price (1934-2003) (Mathews 2005, 74). Price's design represents an early 'affective communication' model that uses an embodying mechanism of corporeal, sensual and kinaesthetic relations (Fig. 43). The sketches below show Price's diagrammatic explanation of user-oriented feedback channels that give communication between architectural space and its occupants.

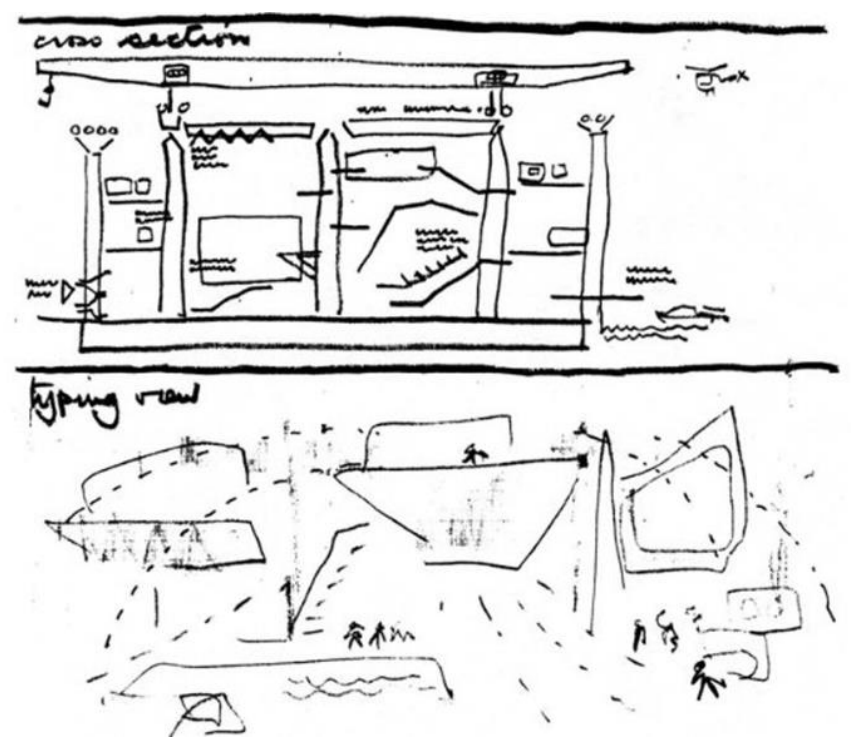

Fig. 43. Sketches by Price for Fun Palace (circa. 1964). Adapted from Mathews (2005, 74). 
As reviewed in the previous chapter, communication with the audience is an everevolving matter in museums and galleries. The idealist guise of the old museology used to be attributed to privileged strata and is still legitimised by institutionalised selection and display processes. In this regard, visitors are undesirable 'noise' emitters rather than partners. Weibel's (1999) essay “The Noise of the Observer" extends this to the context of art exhibitions. Philosophical accounts make deeper enquiries in the same vein. In Singer's (1998) words, "the repression of error has served as the fulcrum of aesthetic autonomy whenever autonomy has been the standard of aesthetic perfection, and for good reason." Built on this review of background knowledge, the next section puts forward a theme around glitch aesthetics with examples in which Author is involved.

\subsubsection{Theme: Glitch Aesthetics and User-Generated Content}

The ambiguous definition of the term 'artefact,' as a human-made object displayed in a museum, exposes a duality. The moment that an object is de-contextualised, it is an 'error,' but the displaced object creates its own narrative to guide understanding of its value beyond its materiality. Moving from object-centred to user-oriented functionality, the communication between the museum and the user results in a meaning-making process. This emancipates both the object and the spectator if she or he is involved in its creation. It goes beyond materiality and sits between the representational and the performative-subjectivist approaches (Smith 2006). The goal of this processual approach is "to explore the nature of the engagement itself; the ragged line between what is represented and what is understood or otherwise experienced" (Watson and Waterton 2010). The New Museology thinking entertains this emerging possibility of presenting transcending and conspicuous, however unexplored, aspects of participation.

The winners of the London Internet Museum architecture competition designed a platform that enables people with process-oriented personalisation. As a museum proposal of future, 404: NOT FOUND (the name of the winner project) questions how to build a museum space with the enactment of user "desires." The winners favour incorporation of people to authorial architecture with a crowdsourced process of "the glitch, the mismatch and the transgression" (McCallum and Belitskaja 2016). Fig. 44 shows, from left to right, the conceived construction-communication process/procedure, starting with users deciding building elements and completed with robotic assemblage. 


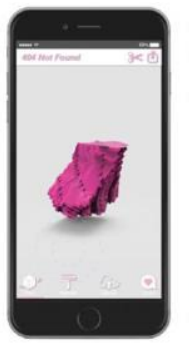

Form

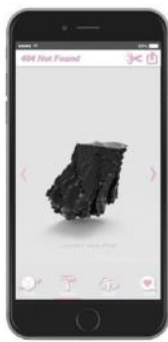

Assign

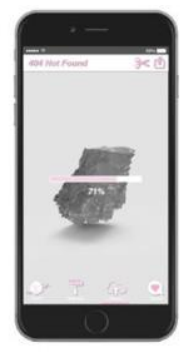

Upload

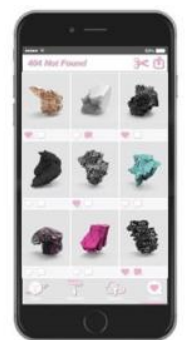

Share

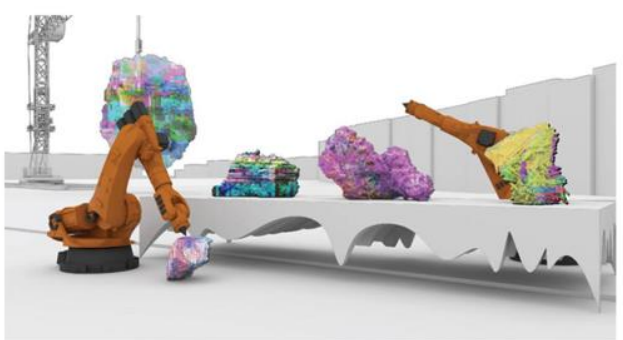

Unique Assembly

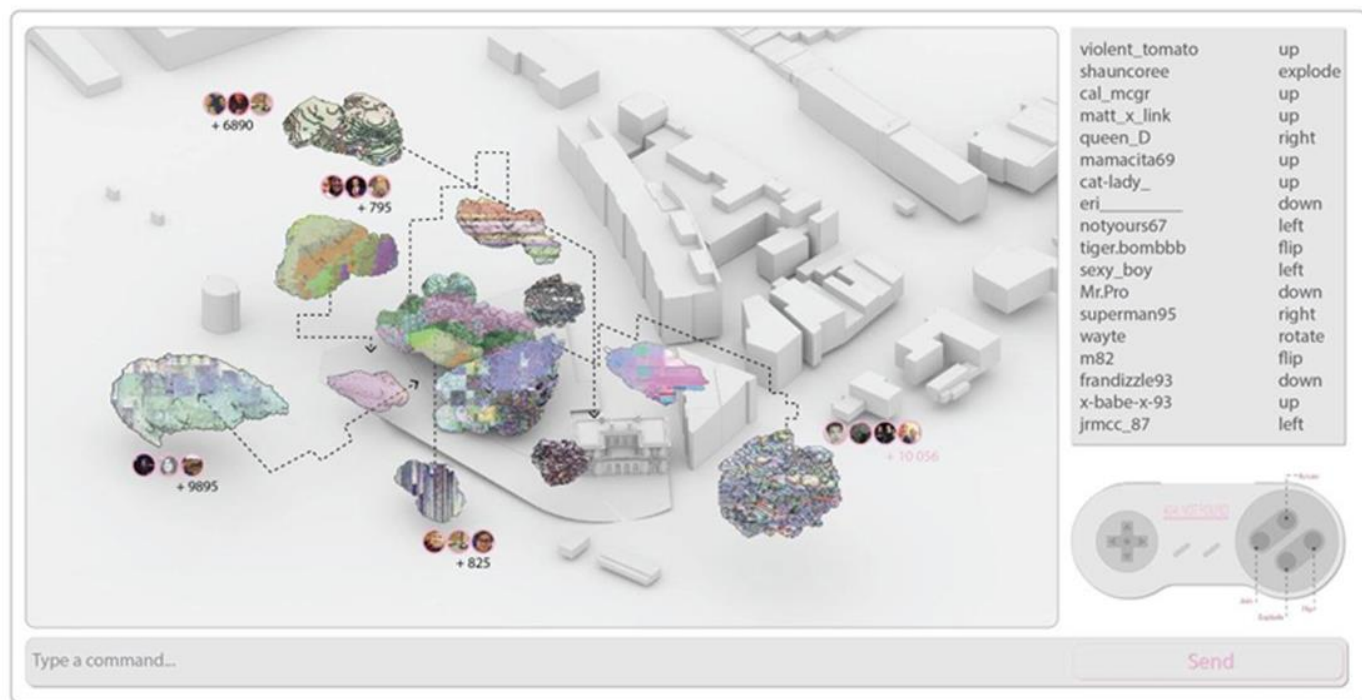

404: Not Found - User assembly

Fig. 44. Screenshots from the posters of the First Prize Winners of the London Internet Museum architecture competition. The winning entry design products ranging from a smartphone app to web interface. It shows how extensive the current mode of thinking is in architecture. Adapted from McCallum and Belitskaja (2016).

Multimodal user-engagement with production via new media ecologies results in an appreciation of glitch in 404: NOT FOUND (Haslop, Schnabel and Aydin 2017a). This reminds us of Shannon's choice, which describes an increase in entropy as an increase in information, giving a determinant role to 'error' in communication (Hayles 1990, 55; see also Nunes 2011, 12).

The studies that examine 'glitch' vary from visual art to sound recording technologies. They include works in music (Kelly 2009), in architecture (Weisskopf and Smoljo 2015), in furniture design (Laviani 2013), and in digital urban design (Retsin et al. 2015; Haslop, Schnabel and Aydin 2017a; 2017b; Haslop 2017) (Fig. 45 and Fig. 46). These works often remain controversial, raising questions as to what extent the aesthetics of 'glitch' should be related to randomness. 


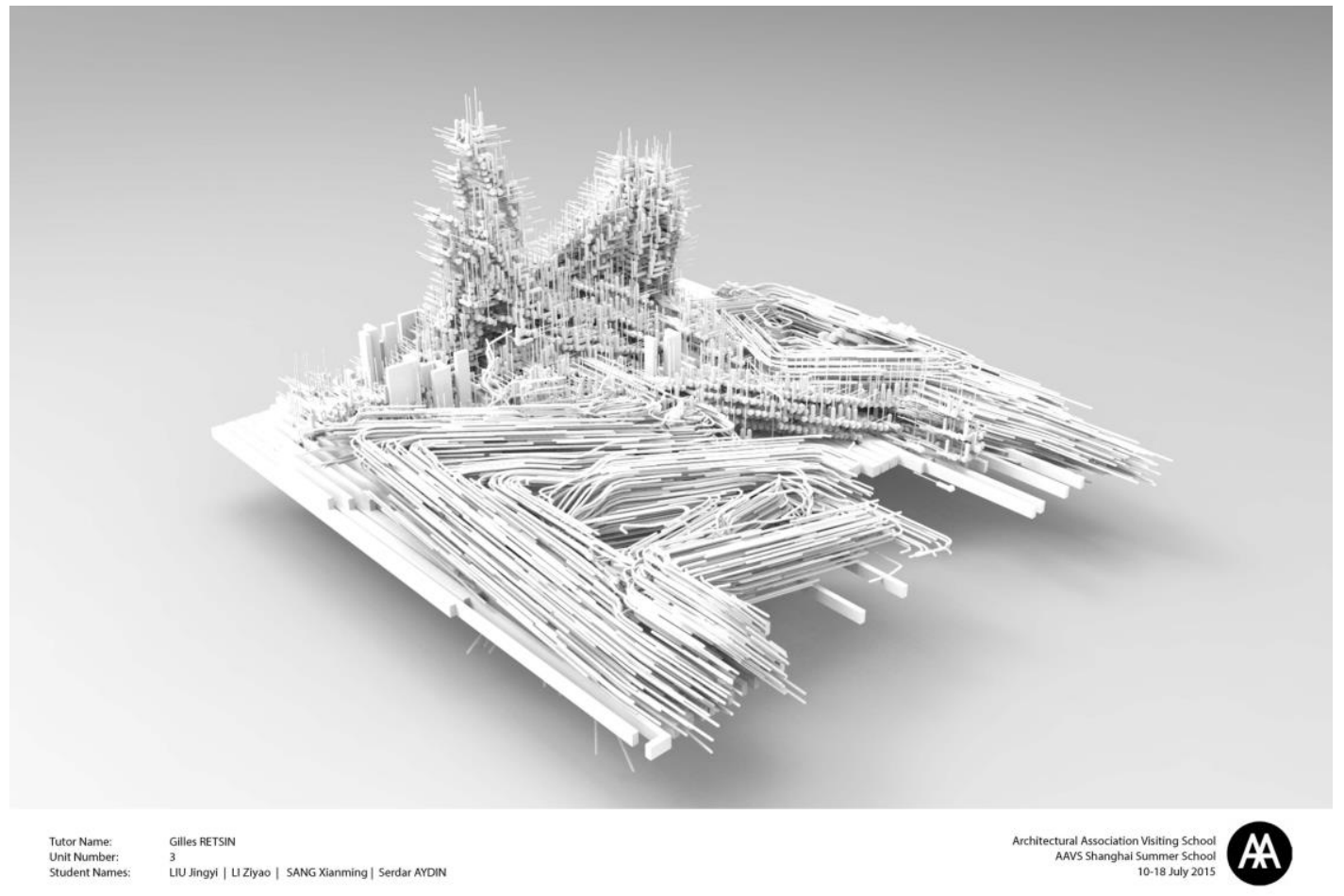

Fig. 45. Digital Spontaneity, an algorithmic design-research work of glitch in urban design, produced at AAVS Shanghai 2015, with Author involved (Retsin et al. 2015).

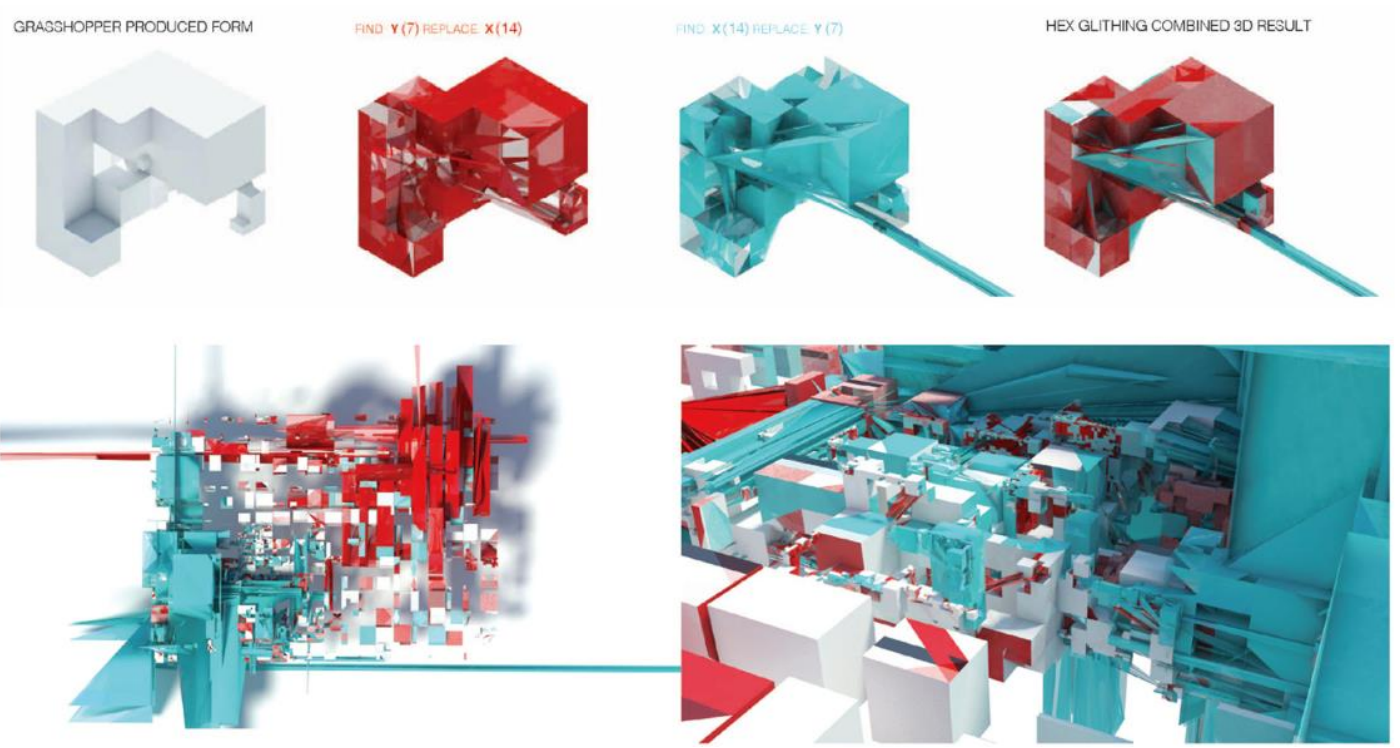

Fig. 46. 3D glitched anaglyph digital form. Illustration by Blaire Haslop, 2017 (Haslop, Schnabel and Aydin 2017b).

There is a correlation between the digital museum in information space and these works that study the idea of glitch, or that appear in other uses of interchangeable terms such as mathematical 'error,' cybernetic 'disjunction' and 'noise,' literary 'innocence' and artistic 'objets trouvés.' Research on the digital object has extended to theoretical repercussions of 
glitch in new media (Nunes 2011; Krapp 2011). The scholarship of glitch hints at new standards within the digital museum.

To study the role of glitch in the digital museum demands an examination of cybernetic design. One of the reasons is because of the roots of cybernetics in science and humanities, which digital heritage research aims to combine in principle. The recognition of 'error' in cybernetic design is key to mapping communication. "Try again. Fail again. Fail better" is the title of a famous essay of Ranulph Glanville (1946-2014) who shows that "cybernetics and design imply the same ethical qualities" (Glanville 2007). Considering the recent embodiment research breaking down the dualism between reality and virtuality, the cybernetic design is qualified to incubate new media of the museum in information space, in which communication is determinant to the evaluation of social systems based on the content generated by individuals of virtual communities.

\subsubsection{Case: The Language of New Media, Works of Manovich ( 2000-2010)}

Case studies of the 'Matters of Audience' are from Lev Manovich who is one of the most influential critics of new media. His research investigates virtual communities and their visual communication. Findings of Manovich are no less artistic and speculative than logical and scientific. So, he often shows research outcomes in exhibitions. This section briefly examines five of his project/exhibitions including Manga Style Space (2010), The Exceptional and the Everyday: 144 Hours in Kyiv (2014), Taipei Phototime (2014), Selfiecity (2014) and OnBroadway (2015). What these projects have in common is that they map online activities onto physical ones. These projects distinctively communicate individual, transient and temporal patterns anchored on permanent and encompassing urban space. They give hints about the indexicality concealed in the hybridisation of online activities and behaviours in cities. Table 12 shows the projects of Manovich, with information about collaborators, data and methods used and findings. Thumbnail images give a sense of how Manovich's works have evolved through the development of his own 'language of new media.'

Manovich, Douglass and Huber (2010) analyse visual features of over a million pages of manga. From about 900 manga series, they visually depict stylistic correlations (Fig. 47, left). During the 2014 revolution in the Ukrainian capital of Kyiv, there was a large amount of image-data generated by people (Fig. 47, middle). Manovich, Chow et al. (2014) computerises an analysis of 13,208 Instagram images of 6,165 people were tagged in the Kyiv location between February 17 and 22, 2014. Despite that international media depicted 
108

the city of Kyiv in an excessively desperate situation, people experienced a social upheaval very differently. The conclusion is that the power of medium (as enjoyed by international media) can be comparable to an index of visual aspects (generated by social media) (Manovich, Tifentale et al. 2014). Likewise, Chow and Manovich (2014) use Instagram images spotted in Taipei and New York to compare temporal visual patterns in two distant places (Fig. 47, right). Coloured squares of pixels stand for the average hue value of images, making it intelligible for exhibition visitors. By searching the indexicality of a big image database, the Taipei Phototime project discovers differences in style from online activities of our 'global village.'

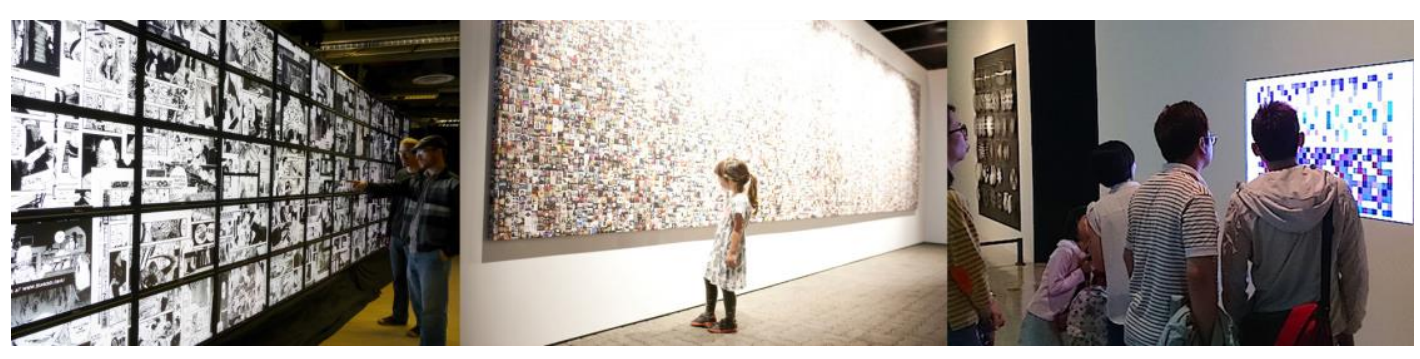

Fig. 47. Left. Manga Style Space, interactive exploration via 287-megapixel HIPerSpace. Adapted from Softwarestudies (2010). Middle. The Exceptional and the Everyday: 144 Hours in Kyiv, a child visitor facing 13,208 Instagram photos of a revolution in Kyiv in 2014. Adapted from TÊTE-

À-TÊTE (2016). Right. Taipei Phototime, visualisation of big data at The National Taiwan Museum of Fine Arts. Adapted from Softwarestudies (2014).

In the Selfiecity project, Manovich, Stefaner et al.'s (2014) customised software processes image data of about 3,200 Instagram selfie photos uploaded from five different cities in the world, including Bangkok, Berlin, Moscow, New York and Sao Paulo. In this project, the visualisation of big data with online interactive charts and diagrams emphasises similarities rather than the distinction between the styles of virtual communities in Instagram. The evolution of the projects led by Manovich shows a path from seeking distinctive visual features of distinct locations and genres to searching similarities between them.

In OnBroadway, Goddemeyer et al. (2015) segment the thirteen miles of Broadway in Manhattan into visual indices of image-based online activities. It intriguingly engages the viewer with the indexicality searched in dynamic datasets that range from over 650 thousand images from Instagram to 8 million Foursquare check-ins and to 22 million taxi pickups. The OnBroadway exhibition enables people to see themselves within a "transmodal engagement" (Ricardo 2013, 38), i.e. participation through different channels. 
Table 12. Selected projects of Lev Manovich between 2010 and 2015. Images and data adapted from Manovich (2018).

\begin{tabular}{|c|c|c|c|c|c|}
\hline Project & Authors & Data & Method & Information (Finding) & Image \\
\hline $\begin{array}{l}\text { OnBroadway } \\
(2015)\end{array}$ & $\begin{array}{l}\text { D. Goddemeyer } \\
\text { M. Stefaner } \\
\text { D. Baur } \\
\text { L. Manovich }\end{array}$ & $\begin{array}{l}\text { 660,000 Instagram photos, Twitter posts } \\
\text { with images, Google Street View images, } \\
\text { over } 8 \text { million Foursquare check-ins } \\
\text { (2009-2014), } 22 \text { million taxi pickups and } \\
\text { drop-offs (2013), income averages from } \\
\text { US Census Bureau (2013) } \\
\text { along } 13 \text { miles of Broadway throughout } \\
\text { Manhattan in New York }\end{array}$ & $\begin{array}{l}\text { interactive installation } \\
\text { of a multi-touch screen } \\
\text { web application } \\
\text { no maps }\end{array}$ & $\begin{array}{l}\text { a new visual metaphor for thinking } \\
\text { about the city } \\
\text { a vertical stack of image and data layers }\end{array}$ & \\
\hline Selfiecity (2014) & $\begin{array}{l}\text { L. Manovich } \\
\text { M. Stefaner } \\
\text { M. Yazdani } \\
\text { D. Baur } \\
\text { D. Goddemeyer } \\
\text { A. Tifentale } \\
\text { N. Hochman } \\
\text { J. Chow }\end{array}$ & $\begin{array}{l}\text { 3,200 Instagram selfie photos } \\
5 \text { global cities (Bangkok, Berlin, } \\
\text { Moscow, New York and Sao Paulo) }\end{array}$ & $\begin{array}{l}\text { visualizations of photos } \\
\text { with ad-hoc software }\end{array}$ & $\begin{array}{l}\text { Instagrammed selfie is part of history of } \\
\text { photography as a sub-genre of self- } \\
\text { portraiture } \\
\text { overarching processes similar across } \\
\text { social media platforms } \\
\text { selfie is a transnational genre as place- } \\
\text { making and styling of facial exposures }\end{array}$ & \\
\hline $\begin{array}{l}\text { Taipei } \\
\text { Phototime } \\
(2014)\end{array}$ & $\begin{array}{l}\text { J. Chow } \\
\text { L. Manovich }\end{array}$ & $\begin{array}{l}\text { Instagram images shared in Taipei and } \\
\text { New York }\end{array}$ & $\begin{array}{l}\text { real time capture and } \\
\text { display } \\
\text { comparison of two } \\
\text { streams of images } \\
\text { (Taipei and New York) }\end{array}$ & $\begin{array}{l}\text { compare temporal visual patterns of } \\
\text { Taiwan and New York } \\
\text { show differences in colour, contrast, } \\
\text { brightness, etc. }\end{array}$ & \\
\hline $\begin{array}{l}\text { The Exceptional } \\
\text { and the } \\
\text { Everyday: } 144 \\
\text { Hours in Kyiv } \\
\text { (2014) }\end{array}$ & $\begin{array}{l}\text { L. Manovich } \\
\text { J. Chow } \\
\text { A. Tifentale } \\
\text { M. Yazdani }\end{array}$ & $\begin{array}{l}\text { 13,208 Instagram images of } 6,165 \text { people } \\
\text { in Kyiv during } 2014 \text { Ukrainian revolution } \\
\text { (February } 17-22,2014 \text { ) }\end{array}$ & $\begin{array}{l}\text { computational and data } \\
\text { visualization techniques }\end{array}$ & $\begin{array}{l}\text { Instagram images tell a different story } \\
\text { about a place compared to what global } \\
\text { media and press depict during a social } \\
\text { upheaval }\end{array}$ & $=$ \\
\hline $\begin{array}{l}\text { Manga Style } \\
\text { Space (2010) }\end{array}$ & $\begin{array}{l}\text { L. Manovich } \\
\text { J. Douglass } \\
\text { W. Huber }\end{array}$ & $\begin{array}{l}883 \text { Manga series } \\
1,074,790 \text { unique pages }\end{array}$ & analyse visual features & reveal distinct stylistic styles & \\
\hline
\end{tabular}




\subsubsection{Criteria: Alternative Knowledge}

In a substantial phenomenological perspective, Tilley $(2008,46)$ stresses "the autonomy of the imagery" that does not need translation but studying its effects is important. Likewise, Kenderdine's $(2015,207)$ research, which is reviewed in the previous section $(3.4 .2)$, is grounded in a kinaesthetic enquiry that Tilley puts forward. Conversely, Manovich's research goes beneath the image to produce alternate knowledge about virtual communities (Fig. 48) without studying kinaesthetic and phenomenological aspects.

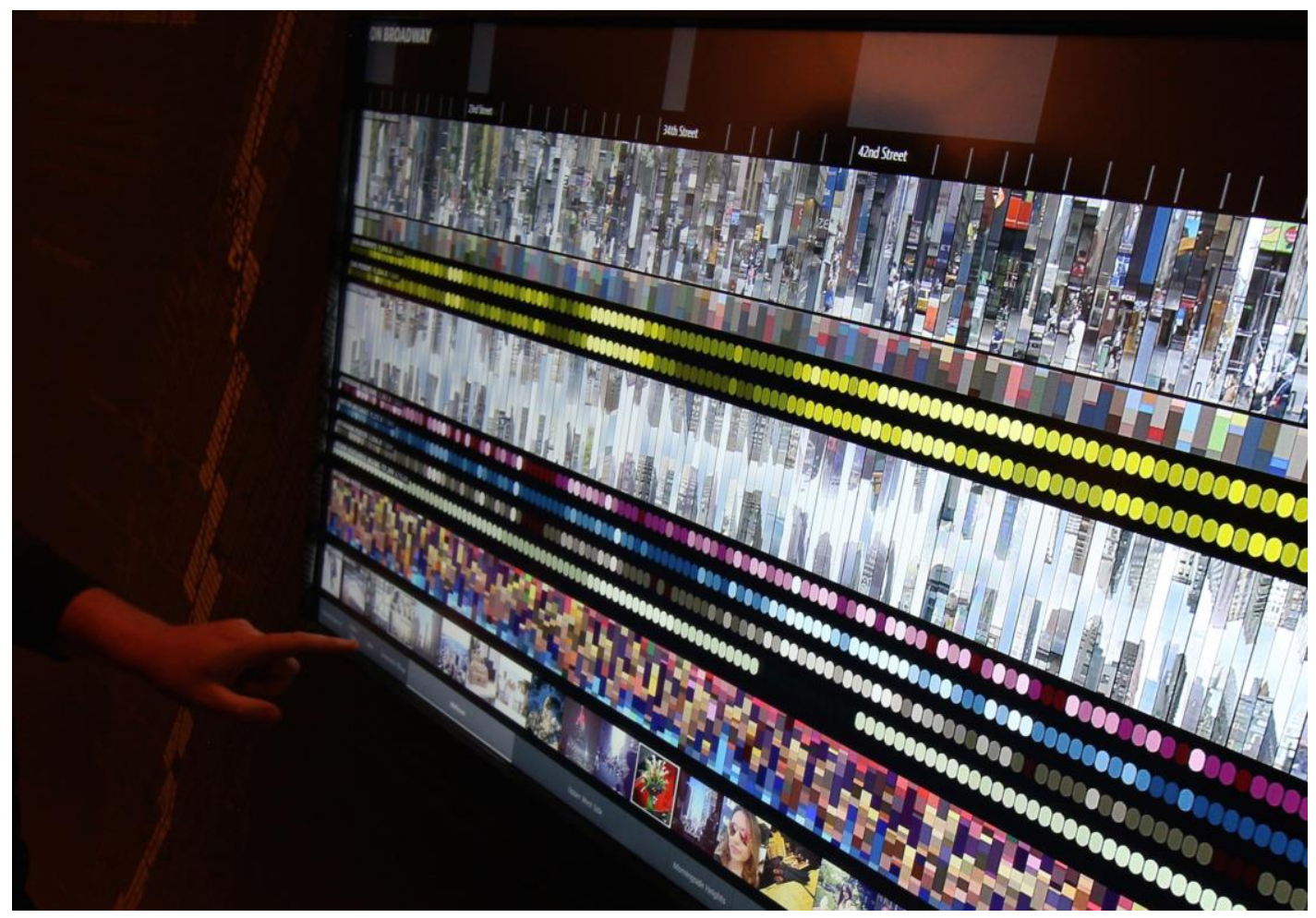

Fig. 48. The interactive installation, OnBroadway, 'representing the modern city' with non-textbased information extracted from Instagram. From top to bottom, the spectrum of rows of information is street names, street-view facades, façade colours, taxi drop-offs, taxi pick-ups, streetview top, Foursquare check-ins, twitter messages, Instagram photos, median household income, Instagram photo colours and Instagram photos. Adapted from Goddemeyer et al. (2015)

Manovich's team of new media artists achieves an assemblage of alternative realities through an indexical integration of the transitory online content with the permanency of actual place. To do so, his method searches 'indexicality' in ordinary content generated by online virtual communities. The iconographic approach in the projects of Manovich paves the way for systematic integration of alternate realities based on universalisation through what Bogost (2006) calls "unit operations." It is arguable that Kenderdine's disintegrated islands of installations do not fully make use of this connectivity of "transmodal engagement" (Ricardo 2013, 29). But evaluation should be independent of comparing these 
two distinguished artistic bodies of new media research. Manovich and Kenderdine contribute to the multidimensionality of new media usage in a social context, with the latter focusing on heritage. Their works bring together multi-, inter- and cross-disciplinary approaches.

The difference is that they do so in separate directions. Manovich extends research on the indexicality of user-generated content via new media, while Kenderdine ties its usage to embodiment theory that is interested in the effects of bodily engagement. In this regard, Huvila's (2006) $\mathrm{PhD}$ dissertation bridges the phenomenological discussion of heritage to information domain. He reviews the use of VR in archaeology. In his thesis, "a virtual reality needs to give an infrastructural framework to the information work by managing the constituent factors of engagement, interaction and dimensionality" (294).

This research is interested in testing both approaches (Chapter 4.4, 4.5 and 5). The review in this section reveals that audience produces perplexing amounts of data. The research translates this data into information. The result is not only statistical interpretation, which helps predict future behaviours, but also associations for discovering patterns between users. This potentially correlates activities in alternate realities when corresponding parameters fill a matrix of relations.

The 'Matters of Audience' reviewed a new research direction, such as those cases of Manovich. The value of the outcome in which audiences are involved is significant for revealing alternative knowledge about events. To figure it out, information retrieved from the audience is thematised as glitch aesthetics. Given that a glitch has creative features, it becomes important to know what alternative knowledge is implicit in them. From this, the research arrives at a third sub-question (Table 13):

sQ3: What alternative knowledge about virtuality lies in the participatory content-making?

Table 13. The progress of formulation of research sub-questions (further development of Table 11).

\begin{tabular}{lll}
\hline Chapter & Sub-Questions & Progress (Formulated?) \\
\hline Chapter 3.2.4 & $\begin{array}{l}\text { sQ1: How can architectural heritage be } \\
\text { translated into creative digital forms? }\end{array}$ & Yes \\
\hline Chapter 3.3.4 & $\begin{array}{l}\text { sQ2: How can an immersive digital } \\
\text { heritage application convey virtuality to } \\
\text { the public via creative engagement? }\end{array}$ & Yes \\
\hline Chapter 3.4.4 & $\begin{array}{l}\text { sQ3: What alternative knowledge of } \\
\text { virtuality lies in the participatory content- } \\
\text { making? }\end{array}$ & Yes \\
\hline
\end{tabular}




\subsection{Matters of Research}

\section{Subchapter Outline}

Purpose: This subchapter synthesises the previous matters pertaining to context, medium and audience in digital heritage. It aims at a critical and theoretical understanding of digital heritage, providing specific explanations for reducing the matters involved in research into a set of principles to be followed.

Design/Approach: The structure of this subchapter diverts slightly from the previous ones. Aside from the background and the theme sections, it undertakes a flow of analysis of the research matters divided into three sections.

Findings: Three matters are found central to research in digital heritage: overconcern with accuracy, epistemological crystallisation and information overload. They give rise to three themes to explore: error, multimethodology and abductive inference.

Limitations/Implications: Research in digital heritage can follow a scholarly approach driven by Critical Realism (CR). CR usually addresses theoretical social research while criticising positivist research approaches for quickly making general assumptions about reality. This subchapter outlines the evolution of CR from theoretical research into applied research.

Originality/Value: Having synthesised the qualitative findings of the case studies in the previous subchapters, 'Matters of Research' connects this chapter to the incoming chapter.

Keywords. Critical Realism; accuracy; information overload; epistemological crystallisation; multimethodology; error; abductive inference.

Having discussed the matters of context, medium and audience, this subchapter derives from the matters concerning the design of a digital heritage methodology for this research. Three sets of criteria that derive from these matters are studied here on the basis of the last set of criteria for mapping a mixed methodological approach.

One of the under-recognised issues in heritage is progress in 'theory-making' based on critical discourse. The methodological conventions draw the field into a recursive loop without solid theoretical underpinnings. The issue is identified as a part of the descriptive nature of the discourse in the field by Waterton (2010) in parallel with what Smith (2006) refers to the 'authorised heritage discourse (AHD).' As reviewed in the previous chapter, the field is driven by the 'descriptive' texts of charters, principles and guides. In this context, Waterton $(2010,19)$ urges us to 'remember that discourse also does things' (emphasis in original). In this scholarly atmosphere, digital heritage is recognised as an opportunity in terms of doing things, with novel methods (Cameron 2007, 49). Despite its early age, there are calls for theorisation in digital cultural heritage (Cameron and Kenderdine, 2007). However, Waterton and Watson's framing of theories shows that methodological experiments' inability to mix different approaches causes the "absence of theoretical noise 
surrounding the field" (Waterton and Watson 2013). In this section, an ad-hoc mapping of a digital heritage methodology is presented.

\subsubsection{Background: Accuracy versus Information Overload}

Each heritage charter and conservation principle, e.g. SPAB Manifesto ([1877] 2018), Venice Charter (1964) and The Nara Document on Authenticity (1994), have only reinforced permanency in the development of heritage discourse as being overconcerned with single universal integrity of values and practices. Likewise, their equivalents in digital heritage, e.g. the London Charter ([2006] 2009) and the Seville Principles (2011), have contributed less to the importance of unique cases than universality. Today's digital heritage discourse positions 'accuracy' in a central role in achieving the 'best' results for information retrieval. In the first chapter, it is shown that the roots of this advocacy stemmed from the virtual heritage boom when projects used to lack transparency over production processes, which has ever since been the main criticism about the loose use of the medium; yet the influence of information and management science methods on heritage should also be extended to their criticism of the positivism dominant both in digital heritage theorisation and practice. In the following sections, the underpinnings of this background section will unfold on Minger's (2014) analysis of multimethodological approaches in systems thinking. Minger's research is dedicated to using the Critical Realism (CR) of the British philosopher Bhaskar, which the heritage theory critic Waterton addresses, to criticise the dominance of the authorised heritage discourse (2010).

\section{- Digital Heritage Forensics}

The trend in digital heritage research is to celebrate the accuracy and the amount of detail documented. This thesis chooses to call this documentation approach "digital forensics" in reference to the digital architectural heritage research of Webb and Brown (2011a; 2011b; 2016; see also Webb and Buchanan 2017). With this approach, digital heritage forensics aim for "fuller examination of the original material" based on "incomplete illustrations" (Brown and Webb 2010). As a new branch of digital forensics attempting to complete missing information, Heritage Building Information Modelling (HBIM) focuses on the level of accuracy and detail of 3D geometries entangled with 2D material information for better preservation, documentation and maintenance (Arayici et al. 2017).

In the broader definition of this field, the recent state of computer forensics research indicates decreased impact factors for future research due to lack of methodological 
standardisation for data representation and forensic processing (Garfinkel 2010). Similarly lacking standardisation, digital heritage practice presents a unique case in every instance from the retrieval of data to generating meaningful information from it. Yet heritage forensics based on digital documentation and restitution techniques is a developing area since the historical context provides many challenges to be overcome. Documentation techniques vary from generating 3D point-cloud data to high-fidelity sound recordings. Applications from digital heritage forensics include a wide variety of topics ranging from underwater cultural heritage risk assessment in the vicinities of Cadiz Bay to the characterization of phototrophic biofilms deteriorating Indian stone monuments (RogerioCandelera 2014).

Since the beginning of museum computing in the 1960s, the evolution of acquiring accurate and detailed results in the field, now called digital heritage, has come a long way. Having passed nearly half a century since Alvarez et al. (1970) searched the hidden chimneys of pyramids in Egypt, the Scan Pyramids (HIP.Institute 2017) project of Cairo University documents their inner structures by using the same method called 'muon imaging' that requires highly sensitive detectors (Procureur 2018).

As accuracy in 3D documentation is a criterion for success and scientific reliability from data acquisition to visualisation, robust and expensive technologies are being developed. It may be another reason that most funding opportunities are related to "science and technology" (Münster and Ioannides 2015; Münster 2017), which has driven the field to focus on the research committed to state-of-the-art means of documentation. It can be deduced that accuracy as a de facto prerequisite in the field is a new form of the once obsessive trend for standardisation and automation in the early museum computing as a structuralist influence pointed out in the seminal study of Parry (2007).

\section{- Paradata}

Despite the growing role of accuracy and its emerging implications for 3D analysis and documentation in digital heritage forensics, there are issues around the standards of quality, the testability of output and validity of results. Overlooked within the boundaries of "scholarly make-believe" (Beacham 2012) constructed by digital heritage forensics, the issues of documenting the documentation process itself lead to an ethical conflict between transparency and intellectual properties. The London Charter for the Computer-based Visualisation of Cultural Heritage issued in 2009 and its offspring the Seville Principles for virtual archaeology in 2011 pointed out the necessity of intellectual transparency. While the notion of transparency was one of the most widely discussed topics in other fields (e.g. in 
media, culture and communication Galloway 2011), it has remained immature in digital heritage. The concepts of transparency rise in different labels such as provenance and "paradata" coined by Bentkowska-Kafel et al. (2012). Instead of "a more explicit articulation of theoretical premises" (Huvila 2012), these are bound to the implicit and technical underpinnings of the issue of transparency.

Under the influence of archaeology, the current state of the paradata research in the field contains arguments invariably bound to the technical, linear and standardised evidence of the applied methods of digital heritage forensics. Brusaporci (2017) states that thirdparties should be able to test simulated outcomes in the 'digital visualisation of architectural heritage.' In summary, digital means have shaken the conventional structure of heritage documentation fields such as archaeology. But later new definitions, e.g. paradata, have contributed to the traditional modes of thinking to assure the verification of accuracy in documentation. Therefore, it has created a vicious cycle as the field of digital heritage is driven mainly by archaeological research and technology companies who profit from the demands for more sensitive recording devices (Münster and Ioannides 2015). In other words, digital heritage can be said to have remained rather "computer-assisted" than “computer-led" [emphasis in original] (Parry 2007, 21).

\section{- Funding}

Based on over three hundred publications, the bibliometric investigation of Münster and Ioannides (2015) presents funding opportunities in digital heritage within a few decades of its relatively short history. According to the survey, the focus of the funding resources in digital cultural heritage is on development in 'science and technology' with around a hundred institutions, which are far greater than about only ten 'culture' institutions. Within this funding structure, the digital heritage researchers work to present competency with 'science and technology' by employing state-of-the-art instruments, whereas low-cost options are regarded as less successful and incomplete. However, no research has been committed to tracking the longevity of the achievements made by costly research schemes except a few reports.

This is noticed by Bentkowska-Kafel $(2009,150)$ who gives the example of the computer-assisted Domesday Project of the BBC in 1986. Despite the grand cost of a $£ 2.5$ million funding, the project turned obsolete in less than two decades, with rapid technological advancements making the digital heritage itself "endangered" (150; McKie and Thorpe 2002). This might be an accidental case, or it may still be counted as more of an indirect contribution to development in 'science and technology' than 'culture.' But 
unfortunately, there is no study analysing the 'futility' of digital heritage projects realised at enormous cost. This might lead to methodological novelties in the field.

The survey of Münster and Ioannides (2015) points out another relation between funding resources and nationality. Most funding opportunities in digital heritage are resourced and regulated by national institutions, whereas more transnational programmes remain less supportive, such as EU with less than a hundred bodies compared to over two hundred of their national counterparts. As is reviewed in the previous chapter, since the Nara Document on Authenticity, international charters have been inclined to appoint national agents for more contextual outcomes; yet it seems to generate a paradox that these funding resources are used to showcase national digital prowess. Bentkowska-Kafel (2009) touches on this "information overload" with an early 3D modelling example from the UK. Exhibited at the British Museum, the fly-through animation of the computer simulation of the AngloSaxon cathedral at Winchester is recognised as one of the "British achievements" at present (150). It seems that despite all technological changes in the last two centuries, the general objective of heritage applications is still bound to national representation.

As new technologies and platforms that can be used and populated by anyone at a reasonable cost, challenges to conventional ways of thinking in heritage are widely acknowledged and discussed (Smith 2006; Cameron 2007; Waterton 2010; Borowiecki, Forbes and Fresa 2016; Colley 2015). Despite these emerging voices, little progress has been made in the digital heritage discourse that can provocatively emphasise multimethodologies. Instead, the contentment with empiricist and positivist paradigm moves the epistemology of digital heritage onto linear and expensive chains of methods concerned with 'accuracy.'

The figure above shows the failure-prone structure of the field. Tim Winter makes a comparison of focus between two high-impact journals in the field, namely International Journal of Heritage Studies (IJHS) and Journal of Cultural Heritage (JOCH). Stressing them as "two cultures" of the sciences and humanities, his analysis tracks the same divide in these journals despite their claims to be "multi-disciplinary in approach" (Winter 2013, 540). The increasingly technical methodologies applied in digital heritage also draw on "scientific materialism of heritage" [emphasis in original] (537). The scientific and positivist methodologies of digital heritage are therefore prioritised through a symbiotic relationship with a problematic discourse that is "apolitical, objective and value neutral" (539). The rest of the section builds on this critical engagement based on a 'critical realist' view to mapping a multimethodological approach. 


\section{- Critical Realism (CR)}

Based on a critical realist perspective, the section gives insight about the divide between the science and humanities approaches in heritage that stems from its crystallised epistemology. Knowledge generation in heritage evolves through interaction with surrounding fields of different concepts such as 'tourist gaze' and 'over-commodification' in tourism. Its impact is most evident in theory-building activities. One of them is Latour's actor-network theory in sociology. It has influenced the contemporary scholarship of heritage, replacing the notion of 'affect' with 'logic' as subject-matter (Bagnall 2003; Holloway 2006; Smith 2006; Smith 2011; Byrne et al. 2011; Waterton and Watson 2013; Crouch, 2015).

Essentially, the resonance between actor-network theory and heritage emerges from reactions to interpretivist examinations of meaning in 'display.' Since triggered by Lowenthal (1985) questioning the material-based thinking of authenticity, representation and identity, interpretivist attempts have been covered in detail by Hooper-Greenhill (1995; 2000), Hall (1999), Kirshenblatt-Gimblett (1998) and Harrison (2013). Their questions pave the way for the notion of affect as a driving force in meaning-making where actor-network theory emerges as a philosophical 'utility' to satisfy some positive value in discourse. One of the strongest claims in this stream of thought is that heritage is "more-thanrepresentational" and even "non-representational" as coined in Watson and Waterton (2010). Their methodological argument advances 'phenomenological' analysis of heritage. These affect research in heritage as "guided by poststructuralist thinking and coupled with a foregrounding of present moments" [emphasis added] (Waterton and Watson 2013, 556). The focus on the human agency and the present time builds on a long traditional relationship with tourism that deals with tourists and temporality.

However, this overconcern may contrast with the primary aim of actor-network theory, which prioritises a 'complex network of agency' including objects, social processes and individuals. There are various types and qualities of agency in this immensely relational social mechanism. Limited by its traditional allies such as tourism, the heritage field prioritises either only the human actor or the technology. Therefore, these critical accounts are bound to an empirically-grounded and crystallised epistemology of the heritage field (Winter 2013). The "methodologically and conceptually" complicated task of actor-network theory and affect studies hinders further elaboration (Waterton and Watson 2013, 552). Their implementation requires the unfolding of overlapping practices and processes, i.e. not only our corporeal engagement with the content. John Law $(2009,141)$, a key proponent of actor-network theory, thinks that it will be "a disappointment for those seeking strong accounts" because "actor-network approach is not a theory." But this is celebrated in 
heritage as a strong theoretical basis that is about "what to study rather than how to understand" (Waterton and Watson 2013, 553).

Framing theories with regards to heritage studies, Waterton and Watson (2013) choose to stress the less subjective line of 'affect' rather than actor-network theory. However, in an earlier work, Waterton constructs a critical foundation for heritage discourse analysis based on the philosophy of Roy Bhaskar whose thoughts promoted a movement called Critical Realism (CR), which brought in more subjectivity in social science (for a general introduction, see Bhaskar 1998).

As mentioned in the previous chapter, amongst heritage management scholars Tilden (1957) started the defence of the interpretivist philosophies emerging in social sciences against the dominance of positivism, i.e. subjectivity against objectivity. In Bhaskar's (1978) CR, subjectivity is viewed as "judgemental relativity" in response to "epistemic relativity" (Mingers 2014, 341). Obliged to epistemic relativism, heritage scholars investigate with a crystallised empiricist view for knowing of objects only under a particular epistemology (Winter 2013).

Bhaskar $(1978,16)$ calls it "epistemic fallacy" in which "statements about being can always be transposed into statements about our knowledge of being." The foundation of this ontological questioning was long before laid by the nineteenth-century Gaussian mathematics that was later matured in philosophy by the semiotician Charles S. Peirce (1933) in his essay “Fallibilism, Continuity and Evolution.” Bhaskar's view of CR proposes judgemental relativity that resembles the Peircean notion of abduction.

The Peircean mechanism of abductive reasoning may be neglected for essentially being presumptive, defeasible and plausibilistic in explaining potential generative hypothetical mechanisms (Walton 2014, 1); yet abduction reappears as a fundamental component in CR that utilises a judgemental approach within a multitude of challenges in sociological research (Bhaskar 2014b, 306). Abduction, or in Bhaskar's words Retroduction, augments the position of multimethodology in CR (Mingers 2014, 348).

Therefore, it is incompatible with the Humean account of science that is epistemologically consolidated into deductive-statistical and inductive-probabilistic explanations (Bhaskar 2014b, 299). Bhaskar conceives four stages for a critical realist approach, called the RRRE model of explanation (305; see also Bhaskar 1978, 125):

(i) Resolution of a phenomenon into components (causal analysis)

(ii) Redescription of component causes (theoretically)

(iii) Retrodiction (or abduction) to possible causes of components

(iv) Elimination of possible alternative causes of components. 
Bhaskar's classical model of RRRE is then reformulated as DREIC (Description, Retroduction, Elimination, Identification and Correction), which is mostly the same form of understanding of generative causality. In summary, the use of DREIC is limited to theoretical research based on an asynchronous, i.e. a-historical, approach. To cover the applied research such as climate change that analyses event causality (Hartwig 2009, 238), i.e. diachronic where events have history with a beginning, duration and an end, DREIC is transformed into RRREI (Resolution, Redescription, Retrodiction or Retroduction, Elimination and Identification) (Mingers 2006, 23) and RRREIC (Bhaskar 2010, 4; Mingers 2014, 190; Mingers and Standing 2017, 176) (Table 14). In the Dictionary of Critical Realism, the $\operatorname{RREI}(C)$ schema is defined as "a form that is essential when conditions are fundamentally open" (Hartwig 2007, 196).

Table 14. Transformation of Critical Realism (CR) into applied research from the DREIC model to the RRREI schema (Mingers 2006, 23; 2017; Isaksen 2016, 249).

\begin{tabular}{|c|c|c|}
\hline $\begin{array}{r}\text { DREIC } \\
\text { (theoretical) }\end{array}$ & & $\begin{array}{l}\text { RRREI } \\
\text { (applied) }\end{array}$ \\
\hline--- & $\begin{array}{c}\text { Break an abstract phenomenon into causal } \\
\text { components (causal analysis) }\end{array}$ & Resolution \\
\hline Description & $\begin{array}{l}\text { Theoretical classification of components for } \\
\text { specific, meaningful explanations }\end{array}$ & Redescription \\
\hline Retroduction & $\begin{array}{l}\text { Abducting which mechanisms must be } \\
\text { hypothetically generative to components }\end{array}$ & $\begin{array}{l}\text { Retrodiction and } \\
\text { / or Retroduction }\end{array}$ \\
\hline Elimination & $\begin{array}{l}\text { Inference to the best explanation } \\
\text { amongst alternative hypotheses }\end{array}$ & Elimination \\
\hline Identification & $\begin{array}{l}\text { Find a coherent mechanism(s) to } \\
\text { generate alternative knowledge }\end{array}$ & Identification \\
\hline
\end{tabular}

A critical realist approach examines the underlying mechanisms of a social phenomenon such as cultural identity or heritage value. It hypothesises the causal components of these phenomena which may be the presence/absence of conceptual, social or material mechanisms. To do so, a critical realist approach employs a type of abductive inference that operates through transcendental arguments rather than arguments that are idealist and absolutist (Danermak et al. 2002, 80; Bhaskar 2014b, 91; Bhaskar 2014a, vii; Isaksen 2016, 247). With the addition of abduction, the logic of a researcher is based on plausible explanations, i.e. abduction or retroduction or 'inference to the best explanation' (Lipton, 2004) as well as inductive and deductive reasoning (Mingers and Standing 2017, 175). The role of abduction in CR is elaborated further in the sections below based on the matters identified in this chapter.

Chapter 3 has already covered the Resolution principle with a focus on its subjectmatter, namely 'transcoding' in digital heritage. As transcoding is a term in new media that sits between the "cultural layer" and the "computer layer" (Manovich 2001, 46), digital 
heritage research explains an "open-systemic event" in Bhaskar's (1978) terms. Firstly, the complexity of transcoding is compartmentalised into the 'Matters of Context, Medium and Audience,' with matters pertaining to research displaying the overlap between Resolution and Redescription. Secondly, these components of transcoding are theoretically reviewed with 'documentation' in context, 'representation' in medium and 'dissemination' to the audience (see Chapter 4.2, 4.3 and 4.4). In Retroduction, the hypothetical mechanisms are assessed qualitatively based on two events (a workshop and a demo show). The best alternative mechanism is adopted in the Elimination stage with an enquiry by 'design' (see Chapter 4.5). In Identification, an experiment is conducted (see Chapter 5). With a critical realist approach, this research is to:

(i) Resolve the heritage phenomena into matters of abstract components that are context, medium and audience

(ii) Redescribe them in a meaningful explanatory way that theoretically matches them with documentation, representation and dissemination that are more specific to the museum context

(iii) Retrodict and Retroduct hypothetically potential generative and explanatory mechanisms of participatory content-making in digital heritage

(iv) Eliminate (via qualified findings of user observation) the best alternative mechanism for combining the themes of error, multimethodology and information overload that arose from matters of context, medium and audience

(v) Identify causally efficacious mechanisms to generate alternative knowledge through experiments.

The following table demonstrates the RRREI schema in line with corresponding chapters.

Table 15. Matrix of the RRREI model and the implementation schema in this research.

\begin{tabular}{|c|c|c|c|c|}
\hline Principles & Enquiry of & Components & Chapter & $\begin{array}{l}\text { Minger's (2014) } \\
\text { classification }\end{array}$ \\
\hline \multirow[t]{3}{*}{ Resolution } & \multirow[t]{3}{*}{ Matters } & Context & 3.2 & \multirow{3}{*}{$\begin{array}{l}\text { Appreciation and } \\
\text { Analysis }\end{array}$} \\
\hline & & Medium & 3.3 & \\
\hline & & Audience & 3.4 & \\
\hline Redescription & Analysis & Research & 3.5 & Analysis \\
\hline \multirow{3}{*}{$\begin{array}{l}\text { Retroduction } \\
\text { and } \\
\text { Retrodiction }\end{array}$} & \multirow{3}{*}{$\begin{array}{l}\text { Computational } \\
\text { methods }\end{array}$} & Documentation & 4.2 & \multirow{3}{*}{$\begin{array}{l}\text { Analysis and } \\
\text { Assessment }\end{array}$} \\
\hline & & Representation & 4.3 & \\
\hline & & Dissemination & 4.4 & \\
\hline Elimination & Qualitative findings & Design & 4.5 & Assessment \\
\hline Identification & Experiment & Decoding & 5 & Actions \\
\hline
\end{tabular}


For CR, a natural system may exist as a combination of objects, structures and causal interactions between them (Law 2004, 158). To reveal these causal relations in the applied research, the RRREI model uses a multimethodological approach that provides "greater empirical generality" (Isaksen 2016, 250). To gain discursive power, CR promotes the conceptual exploitation of cybernetic principles to assess the Elimination of alternative explanations (Mingers 2003, 564-568). With the Retroduction principle, CR affiliates the researcher(s) to manage this dynamic communication for recognition of the "beauty" in error in the words of Singer (1998). By following these principles of applied CR, the goal of research is "not to discover new mechanisms, but rather to understand which mechanisms are functioning in an open-system to create some event" (Isaksen 2016, 249). While trying to understand this point, it is also critically analysed in the context of digital heritage. The following part explains the central themes to do so.

\subsubsection{Theme: Mapping a Digital Heritage Research}

Having discussed the underlying factors of the followed methodological approach, this section summarises the necessity of mixed methods in digital heritage. Covering at least two different phenomena, namely the 'digital' and 'heritage,' the current research needs to be integrated with the more profound ontological breadth and greater epistemological plurality. There are various ways of combining methods based on a range of theoretical background (e.g. Grounded Theory). CR offers digital heritage three advantages. They are "maximal ontological inclusiveness, greater epistemological generality and great methodological and heuristic fertility" (Bhaskar 2013, 20). To implement them, critical realists offer the RRREIC model. In summary, the model can be discussed at three levels, which are proposed by another critical realist Mingers (2003):

(i) Ontological

(ii) Epistemological

(iii) Axiological

Although they sound quite philosophical, it is possible to interrelate them to the language of applied research in digital heritage. The background section above implemented three matters of digital heritage research. They are accuracy, crystallised epistemology and information overload. The 'maximal ontological inclusiveness' offered in CR is directly related to the matter of accuracy in digital heritage. It points to design research for 'greater epistemological generality' since the current research methods are sterile studies causing "the absence of theoretical noise surrounding the field" in the words of Waterton and Watson 
(2013). Following accuracy and crystallised epistemology, understanding the relation of the last matter, i.e. information overload, provides an explanation to the axiological question of why, in the first place, CR is needed by digital heritage research (Table 16).

Table 16. Alignment of matters and themes for a multimethodological approach.

\begin{tabular}{llll}
\hline $\begin{array}{l}\text { Philosophical } \\
\text { matters }\end{array}$ & $\begin{array}{l}\text { Matters of } \\
\text { digital heritage research }\end{array}$ & Arising themes & $\begin{array}{l}\text { General } \\
\text { Enquiry of }\end{array}$ \\
\hline Ontological & Accuracy & Error & Being \\
\hline Epistemological & Crystallised epistemology & Multimethodology & Knowledge \\
\hline Axiological & Information overload & Abduction & Value \\
\hline
\end{tabular}

\subsubsection{Ontological Overview of 'Error'}

The first theme, error, refers to an understanding that is the opposite of what the positivist view of science once regarded as "something that must be corrected" (Nunes 2011, 10). In contrast, $\mathrm{CR}$ sympathises with error accounting for the empirical difference. Critical realists prefer probabilistic, revisable and 'judgemental relativity' based on transcendental realism instead of empirical realism.

In science, the probabilistic vision can be traced back to the semiologist Charles $\mathrm{S}$. Peirce (1839-1914) and his foreshadows in cybernetics developed by Norbert Wiener (1894-1964) in close proximity to the information theory of Claude Shannon (1916-2001) (Seising 2010). The convergence of these three fields, i.e. semiology, information theory and cybernetics, have led to an understanding of realism about the concepts of the sign, noise and error in communication science (Kockelman 2013). They provided methods to recognise error as valid input from the environment, as opposed to error regarded as invalid diversions from ideal results in universal empirical generalisations. The articulation of design as a social problem and transcendental phenomenon coincides with the rise of realists who negotiated the position of 'error' against the positivist counterparts. In the 1960s, the design was realised as a "wicked problem" (Buchanan 1992, 15). Referring to the same period in the history of science, the cybernetician Thomas Fischer (2017b) explains the role of design failures that have led to the evolution of spacecrafts. Interestingly, in the hands of positivist scientists, these failures were slyly swept under the rug, articulating the results as "great" achievements that a critical realist would still contend they are (Fischer 2017b). The transcendental and imaginary position of $\mathrm{CR}$ is different from the regularity seeking errorprone presuppositions of empirical realism. It holds the ability to recognise errors as 
something that reveals the truth. In Bhaskar's word, it is "the exercise of tendencies which may not be realised" (Bhaskar 1978, 16). The transcendental realism of Bhaskar follows a neo-Kantian tradition in opposition to the science of Humean causal laws that are empirical, universal, discrete and sterile generalisations. To characterise their ontological distinction, Bhaskar stratifies three distinct domains for real, actual and empirical (Table 17).

Table 17. Ontologically representing the domains of CR. Adapted from Bhaskar $(1978,13)$

\begin{tabular}{lccc}
\hline & Domain of real & Domain of actual & Domain of empirical \\
\hline Mechanisms & $\mathrm{X}$ & & \\
\hline Events & $\mathrm{X}$ & $\mathrm{X}$ & $\mathrm{X}$ \\
\hline Experiences & $\mathrm{X}$ & $\mathrm{X}$ & \\
\hline
\end{tabular}

There are mechanisms that exist independent of events and our experiences in the domain of real. Regardless of whether these mechanisms are activated, they exist in an intransitive dimension. Once activated, these mechanisms produce events in the domain of actual. But the empirical knowledge that can be measured or perceived is only based on those events that are experienced, hence held in a transitive dimension (Bhaskar 1978, 17; Wynn and Williams 2012, 790). This maximum ontological inclusivity of the domain of real is explained with "revisable theories and hypothesis" (Law 2004, 158). Similarly, Wiener's cybernetic theory equalises the balance between communicating entities by constantly operating in-between feedback loops. Appreciating mathematical probabilities rather than individual measurements, Wiener develops on the Peircean reading of error based on the use of "mean square approximation" (Nunes 2011, 11). In the mathematical background of this realist, contextual and yet transcendental idealism, given measurements of ideal equations only show deviant results of the observed events in the domain of actual (10). It is possible to see their influence on early museum computing efforts, e.g. the failure capacity of SELGEM designed at the Smithsonian Institute (see Chapter 2.2.4).

\subsubsection{Epistemological Overview of 'Multimethodology'}

The second theme of multimethodology arises from the matter of crystallised epistemology in heritage. As noted by Winter (2013) who surveyed articles in $J O C H$ and IJHS, there is "extremely little overlap" and "cross-fertilisation of ideas" because of methodological divisions. CR offers the possibility to dovetail methodologies in favour of "greater epistemological generality.” Despite being a strong advocate of CR, Mingers condemns 
124

Bhaskar's (2014, 65) theory for maintaining a political discourse rather than an epistemological critique.

Although Bhaskar's concept is transcendental, the elements of mechanisms, events and experiences become manifest only at discrete levels (Wynn and Williams 2012, 791-792); however, this does not sound genuinely transcendental, bound to an interrelation between mechanisms, events and experiences. With more abstraction, one event can be the mechanism of another event. Or an experience manifest in the domain of empirical can involve unobservable events akin to the domain of actual that may or may not activate another experience. In this style, CR would resemble actor-network theory. Now, this ontological distinction can be revised in the context of the digital heritage domain.

Epistemologically, digital heritage research seeks to acquire some transformative potential to reproduce heritage information rather than 'transcoding' power to cause a change in it. Digital representations of heritage environments transcode between two states in which an immense volume of information is ready for exchange. In their own ecology of communication, the medium of transcoding is prone to generate errors and noises. At its best, the system can recognise the error of communication. In the domain of digital, these errors have the transformative power to be the events of new experiences. The empty lots of Bhaskar's ontological stratification (Table 17) can be represented as an inversed dimension $\left(1^{x}\right)$ to indicate a truly transcendental sense of relativism (Table 18). The problem of the field is its epistemology is self-conditionality assured by charters and guides, e.g. requirements regarding paradata and transparency of 'methods' and processes instead of the applied ‘methodology' (see London Charter [2006] 2009).

Table 18. Revised ontological structure of Critical Realism (CR) in the context of digital heritage.

\begin{tabular}{lccc}
\hline & $\begin{array}{l}\text { Real } \\
\text { Domain of real }\end{array}$ & $\begin{array}{l}\text { Actual } \\
\text { Domain of heritage }\end{array}$ & $\begin{array}{l}\text { Empirical (subjective) } \\
\text { Domain of digital }\end{array}$ \\
\hline Mechanisms & $\mathrm{X}_{1}$ & $1^{\mathrm{x}}$ & $1^{\mathrm{x}}$ \\
\hline Events & $\mathrm{X}_{1}$ & $\mathrm{X}_{1}$ & $1^{\mathrm{x}}$ \\
\hline Experiences & $\mathrm{X}_{1}$ & $\mathrm{X}_{1}$ & $\mathrm{X}_{1}$ \\
\hline
\end{tabular}

From the revised ontological revision of $\mathrm{CR}$ arises an explanatory power to transform the epistemological underpinnings of digital heritage. We can make an analogy between the current position of digital heritage and a line between two points on a Cartesian graph (it can be any line for simplicity) (Fig. 49, left). The first point of the line represents the domain of heritage and the second one the current understanding of digital heritage. Although there 
is no physical or even chemical relation between the two value systems, contemporary research focuses on the domain of digital with the idiosyncrasies of heritage (Fig. 49, right); however, the domain of the digital is empirically freed from this line that should be referential ('more abstraction' - see the first paragraph of this section). And within the domain of digital, a representation is no longer on the same line and rather is a free point in space (Fig. 50, left). Mathematically, a Cartesian point has no value of inclination. However, there is an opportunity to see judgemental relativity between the reference line that is still in the domain of heritage and a line that can be redrawn between the free-dot and a secondary referential dot. By that, the free-dot can gain meaning and differential value in association with the original event (Fig. 50, right). This is a truly transcendental argument of CR for a 'greater epistemological generality.' It seeks flexibility and fallibility instead of accuracy.
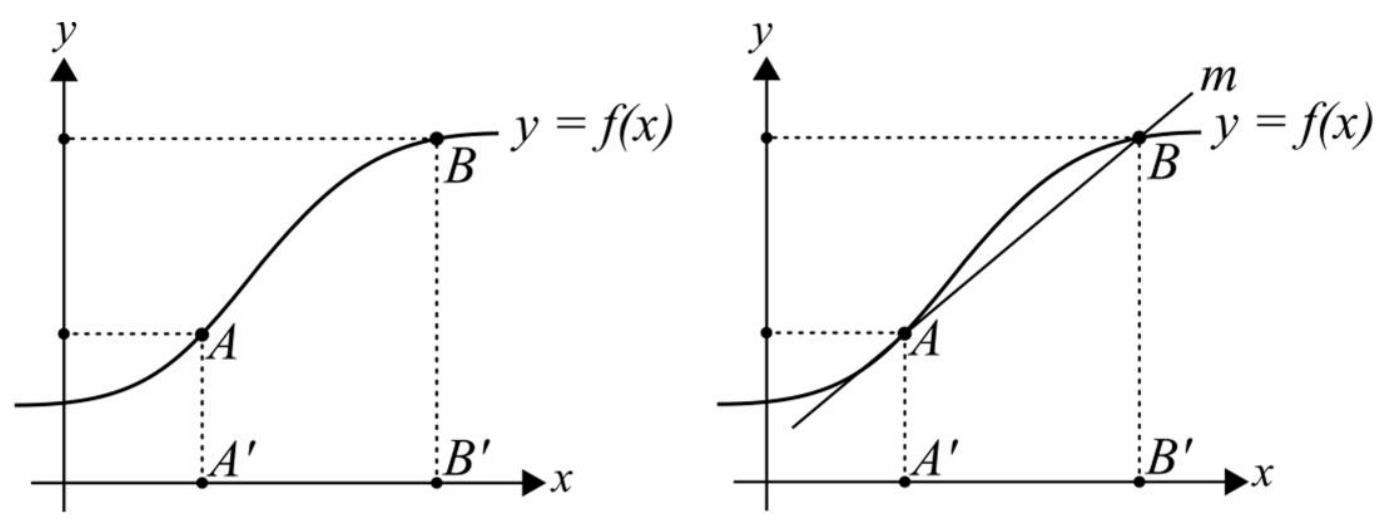

Fig. 49. Analogical Cartesian graphs represent the epistemological understanding of contemporary digital heritage research. Illustration by Author.
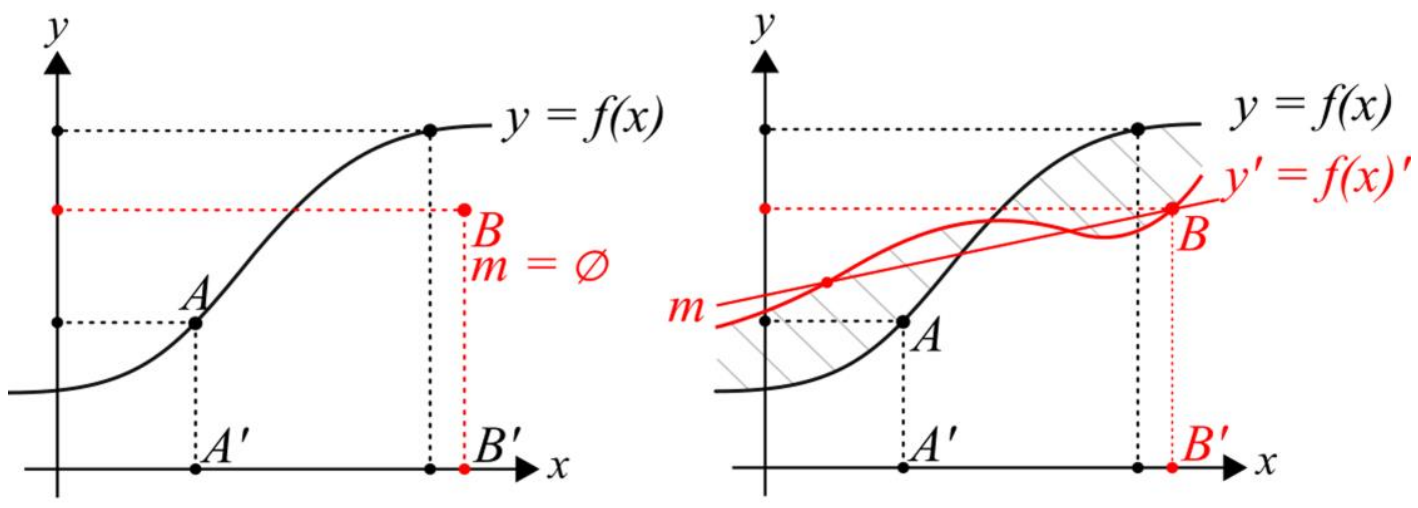

Fig. 50. Graphs represent the matters pertaining to digital heritage research that are independent empirically. Illustration by Author.

Although many novel methods are mixed in contemporary research in digital heritage, they are not meant to transform and advance heritage but rather to reproduce its scientific function, which has been proven impractical by heritage critics (Waterton and Watson 2013; 
Smith 2006; Bentkowska-Kafel 2009; Winter 2013; Smith 2011; Byrne et al. 2011; HooperGreenhill 1995; Hall 1997; Kirshenblatt-Gimblett 1998; Harrison 2012). In general, digital heritage forensics can be associated with the principles of the Humean notion of causality as they answer, "what caused Z to happen" but not "why did Z happen and not something else?" (Mingers and Standing 2017, 180). Research on this epistemological fault line incoherently scrutinises the domain of real in heritage that can be examined further at the axiological level to answer 'why?' The next section substantiates the necessity of abduction within the proposed greater epistemological generality via an axiological position of CR. In other words, it touches on the ethical side of the value of Bhaskar's normative argument.

\subsubsection{Axiological Overview of 'Abduction'}

The third theme arises as information overload in relation to Bhaskar's normative implications and asks, 'why CR in the first place?' Understanding the problem of information overload aids to grasp what value CR offers. Major factors of information overload are discussed above. On the one hand, the over-concern with accuracy in digital heritage forensics is determinant of information overload at present (Bentkowska-Kafel 2009, 150). On the other hand, crowdsourcing is subject to the interpretation of redundant information for future heritage platforms. This section will elaborate on the theme of abduction that is obliged as an ethically powerful instrument by CR. In the dialectic of CR and positivism, current research of 'digital heritage forensics' resembles the latter. The position of CR differs from that of the positivist position that offers empirical regularity. The use of abductive inference answers what the world 'may be like' - which is unlike the empirically crystallised field of heritage that is interpretivist and interactionist at its best. An equivalent notion is coined by Charles S. Peirce. Highlighting the role of the 'interpretant,' Peirce attempts to formulate abduction as a different reasoning method than deduction and induction. Peirce represents the interactionist perspective in social sciences. Augmenting the advocates of realism, his abductive reasoning is based on the examination of signs instead of a phenomenological focus on pure interactionism (Plummer 1996, 228). According to Peirce, the best explanation derived through abductive reasoning could fail empirically, but its conclusion would be sufficiently "plausible to warrant taking action" (Walton 2014, 7). This realist underpinning provides a philosophical basis of detachment from the conditions of empirical imperatives in heritage.

Sayer $(2000,177)$ writes that the refusal of axiological confrontation is an empirical denial of theory in favour of observation. The absence (or denial) of "theoretical noise" 
(Waterton and Watson, 2013) in heritage has already been reviewed above. Studying information overload in the context of new media is more appealing to the message of this section, which is axiologically related to ethics of abduction to reason from the superabundance of digital information. The thinkers and philosophers of the twentieth century are also concerned with information overload, or mass-production in sociological terms, (e.g. Baudrillard 2006; Rancière 2007; Galloway 2011). Chris Anderson (2008), the former editor-in-chief of Wired magazine (2001-2012), presumes an inevitable "end" for theory-making simply because of the challenges posed by the "deluge" of big-data. Nevertheless, CR prescribes a retroductive (or transcendental) argument. It considers the human activity in the "demi-reality," which is a critical connotation of the critique of the Hegelian 'demi-actual' that is the closed loop of the rational with the actual. Bhaskar's critique extends to the premises of error in which "falsity prevails over truth, evil over good, heteronomy over autonomy, war and repression over love and peace" that cannot be 'rationally transfigured' with a Hegelian account of philosophy (Hartwig 2007, 113). Within $\mathrm{CR}$, the ethical credit of error occurs via the transcoding of abduction from Peirce's semiology into retroductive arguments that infer the best explanation of a phenomenon. The axiological role of CR lies in the characterisation of abduction into retroduction that seeks a "hypothetical mechanism" to cast undisputed understanding on the unexplained phenomenon (Mingers 2006, 23).

The three themes that arose from the 'Matters of Context, Medium and Audience' display an appreciation of the intransitive and transitive components of the resolved phenomenon of virtuality in digital heritage. With the 'Matter of Research,' these components are redescribed to make meaningful explanations, based on a theoretical classification that is grounded in critical analysis of the field. Table 19 gives a summary of the stages followed in this and the subsequent chapters. 
Table 19. Summary of the course of the research from Resolution and Redescription to Retroduction and Retrodiction.

\begin{tabular}{|c|c|c|c|c|c|c|}
\hline \multicolumn{5}{|c|}{ Resolution and Redescription } & \multicolumn{2}{|c|}{ Retroduction and Retrodiction } \\
\hline $\begin{array}{l}\text { Matters of } \\
\text { (theoretical) }\end{array}$ & $\begin{array}{l}\text { Philosophy } \\
\text { (theoretical) }\end{array}$ & $\begin{array}{l}\text { Pragmatic issue } \\
\text { (applied) }\end{array}$ & $\begin{array}{l}\text { Consequent themes } \\
\text { (applied) }\end{array}$ & Questions & $\begin{array}{l}\text { Applied } \\
\text { methods }\end{array}$ & Task \\
\hline Context & Ontology & Accuracy & Error & $\begin{array}{l}\text { SQ1: How can architectural } \\
\text { heritage be translated into } \\
\text { creative digital forms? }\end{array}$ & Documentation & $\begin{array}{l}\text { Finding the 'humility' of } \\
\text { heritage }\end{array}$ \\
\hline Medium & Epistemology & $\begin{array}{l}\text { Crystallised } \\
\text { epistemology }\end{array}$ & Multimethodology & $\begin{array}{l}\text { sQ2: How can an immersive } \\
\text { digital heritage application } \\
\text { convey virtuality to the public via } \\
\text { creative engagement? }\end{array}$ & Representation & Enhancing 'virtuality' \\
\hline Audience & Axiology & $\begin{array}{l}\text { Information } \\
\text { overload }\end{array}$ & Abduction & $\begin{array}{l}\text { sQ3: What alternative knowledge } \\
\text { of virtuality lies in participatory } \\
\text { content-making? }\end{array}$ & Dissemination & $\begin{array}{l}\text { Generating alternative } \\
\text { knowledge }\end{array}$ \\
\hline Research & $\begin{array}{l}\text { Critical } \\
\text { Realism }\end{array}$ & & & $\begin{array}{l}Q: \text { How does a digital design } \\
\text { approach steer and diversify } \\
\text { creative content-making in digital } \\
\text { heritage }\end{array}$ & Design & Decoding 'Kashgar' \\
\hline
\end{tabular}





\title{
Chapter 4. Digitising Kashgar's Heritage: An Interdisciplinary Methodological Approach for Documentation, Representation and Dissemination
}

\author{
Any sufficiently advanced technology is indistinguishable from magic. \\ - Arthur C. Clarke, “Clarke's Third Law,” Profiles of the Future $(1973,39)$
}

\subsection{Introduction}

Having outlined the 'Matters of Context, Medium, Audience and Research' in the previous chapter, the following sections introduce methods that are employed in this research. Three research questions arise from each investigation based on the criteria derived by critically engaging with different matters. Therefore, it provides the Resolution (Chapter 3.2, 3.3 and 3.4) and Redescription (Chapter 3.5) of sophisticated digital heritage research. This chapter is pertinent to Retroduction and Elimination stages of the RRREI model.

Digital cultural heritage studies can be conducted through generally two headings: 'documenting' heritage resources and 'disseminating' heritage outputs (Cimadomo, 2013). In addition, Corsane $(2005,5)$ introduces heritage processes linking heritage resources and heritage outputs. Addison (2000) and Rahaman $(2012,5)$ discuss the study of digital heritage (or digital cultural heritage) under three headings: documentation, representation and dissemination.

Documentation is the transformation of data from heritage resources into digital formats. Representation is the process of virtual reconstruction and visualisation. Dissemination is when the visualised heritage output meets the audience (Addison 2000). Documentation, representation and dissemination are overlapping processes. In this chapter, 
methods used can best be studied under these three phases that simultaneously correspond with the themes of the previous chapter. Within an interrelated hierarchy, Resolution and Redescription of the research is articulated as a means to associate the 'methods' used (Chapter 4) with the three 'Matters' examined (Chapter 3).

Digital heritage studies include the use of advanced technologies, such as photogrammetry, laser scanning, virtual and augmented reality and high-fidelity sound recording. These methods interchangeably provide content for documentation, representation and dissemination. As discussed in the 'Matters of Research' (Chapter 3.5), one of the criteria for being scholarly acceptable is transparency and provenance of the processes and methods followed in digital heritage research. In this research, partly alternative and partly integrated methods are employed that will be introduced; however, it is highly important to note that this chapter aims not to boast a range of skillsets but to introduce what is achieved and failed through the course of the research. Apart from methods, each subchapter includes a section called 'challenges.' The findings from respective methods are discussed to pave the way for the hypothesis that is unveiled at the end of the chapter.

Firstly (Chapter 4.2), documentation methods are employed for capturing the modesty of alleyways of the Kashgar old-town. Fieldwork activities are introduced, varying from stereo street-sound recordings to aerial photography.

Secondly (Chapter 4.3), for representation, photogrammetric mesh modelling is the method used to lay a foundation for designing an environment of 'virtuality.' Photographs are stitched together with photogrammetry by using Autodesk ReCap and ReMake (formerly Memento Project; now ReCap Photo).

Thirdly (Chapter 4.4), with regards to dissemination, participatory events that are organised to gather data are introduced: a workshop in Melbourne during CAADRIA 2016 Conference and a demo-show at the Museum of New Zealand Te Papa Tongarewa as part of Te Papa Talks 2017. Methods that are utilised to design interactivity are introduced.

The subchapter (4.5) called 'research methodology' provides a synthesis of the methods used for documentation, representation and dissemination. Their combination is dedicated to searching alternative knowledge in participatory digital heritage making. This is followed by reflecting on the hypothesis of this research.

The novelty of the methodology for this research lies within the exercise of seeking alternative knowledge (Chapter 5) through alternate realities (Chapter 4). The method is designed to offer a contemporary way of translating 'things' into 'objects' in architectural 
heritage studies that can be built into their own digital museums where anybody can participate.

The purpose of digitising historical knowledge lies beneath an encompassing paradox of two distinct views about digital heritage forensics, which are "a mode of inquiry" and "form of communication" (Bentkowska-Kafel 2013). This can be further related to the “creativity versus preservation" paradigm (Delve and Anderson 2015), and even to the nineteenth-century division of architectural heritage between Ruskin's romanticism and Viollet-le-Duc's restoration (Howard 2003, 34).

There are conflicting de facto statements in the mind of a researcher to produce an acceptable theoretical discussion for novel approaches in digital heritage. BentkowskaKafel (2013) reframes the issue on an ethical basis where "scholarly visualisation of heritage may only be recognised as valid and ethical ... when accompanied by paradata, i.e. the documentation of the evaluative, analytical, deductive, interpretative, and creative decisions made in the course of research." This view on paradata is virtually identical to that of the London Charter version 2.1 ([2006] 2009, article 4.6), which notes that it "should be disseminated in such a way that the relationship between research sources, implicit knowledge, explicit reasoning, and visualisation-based outcomes can be understood." Yet the subjectivity of the researcher produces decisions to correspond with a gap in knowledge.

Based on this view, this research builds on a tailored approach that is directly related to the circumstances of the context. The rest of this chapter explicates the 'evaluative, analytical, deductive, interpretative, and creative' decisions and their derivative paradata information generated while processing heterogeneous sets of methods during documentation, representations and dissemination.

There is a wide variety of methods that can be undertaken for recording an architectural heritage site. In most cases, the decision to use which type of documentation technology depends on the predicted value of results for contributing humanity, the corresponding level of accuracy and the amount of detail for its achievement. Based on a review of different methods used in 3D digital documentation, Pavlidis et al. (2006) construct a table of nine criteria for choosing an appropriate system (Table 20). 
Table 20. Nine criteria for choosing an appropriate documentation method. Adapted from Pavlidis et al. (2006).

\begin{tabular}{ll}
\hline & Criterion \\
\hline 1 & Cost \\
\hline 2 & Material \\
\hline 3 & Size \\
\hline 4 & Portability of equipment \\
\hline 5 & Accuracy of the system \\
\hline 6 & Texture acquisition \\
\hline 7 & Productivity \\
\hline 8 & Required skills \\
\hline 9 & Compliance of outcome with standards \\
\hline
\end{tabular}

Laser scanning technologies can produce accurate models, measuring with a very high level of accuracy and detail. This research is built on a unique and original path to meet the standards of accuracy and the required level of detail via novel methods. Practically, the challenges for the level of accuracy and detail include, but are not limited to, dealing with the security measures taken in the research context (Kashgar), spending the research budget efficiently and the time required to finalise data collection. These practical considerations lead to a designerly way of decision-making that will be extrapolated in the following subchapters $(4.2,4.3,4.4$ and 4.5$)$. 


\subsection{Documentation}

Subchapter Outline

Purpose: In the 'Matters of Context' (Chapter 3.2), alleyways are found to construct the elemental profile of Kashgar's heritage. The subchapter extends on contextual matters by introducing what methods are used to document the old neighbourhood architecture of Kashgar.

Design/Approach: The activities in three fieldworks in Kashgar are demonstrated. The context is narrowed down into a neighbourhood division called Köziciyerbişi (Gaotai). From street-sound recordings to aerial photography, methods used are introduced hierarchically ranging from text-based information to non-textbased visual information.

Findings: In relation to the circumstances of Kashgar, they are identified as opportunities unique to the context.

Limitations/Implications: Documentation methods used in Kashgar are subject to challenges of accuracy, completeness and timeliness.

Originality/Value: Earlier research documented Köziciyerbişi (Gaotai) by using similar methods such as photography and measuring. But they are used to generate still 3D models without texture, focusing mainly on formal aspects of architecture. This chapter shows what kinds of data are collected to model a virtual Kashgar, and this data may be one of the last analyses of Köziciyerbişi (Gaotai) as the houses are being demolished one by one because of neglect, despite it being the only section of the old-town that survived a large-scale rebuilding project.

Keywords. Documentation; Köziciyerbişi; street-sound recordings; photography; aerial photography.

Previously, researchers from the Humanities, such as in modern and classical languages, anthropology, archaeology, history, comparative religion, culture studies, political science and architecture, have documented the cultural heritage in Kashgar (Beller-Hann 2008; Steenberg 2014; Pawan and Niyazi 2016); yet digital documentation activities have been limited to only a few cases. As mentioned in Chapter 3.2.2, The Kite Runner (2008) movie may be the first of its kind using aerial photography, digital modelling and post-production to document Kashgar's old-town. Scenes of Kashgar are refined with digital techniques, such as photogrammetry and 360-degree matte painting (Prince 2011, 168). But these efforts were to reconstruct an 'authentic' place that sufficiently resembles the city of Kabul in Afghanistan, where the movie was based on a story.

Scholarly works of documentation about the Kashgar old-town include a 3D model of the entire Köziciyerbişi (Gaotai) area embedded with survey information regarding each house (Wang et al. 2014), a study of cultural heritage sites in Kashgar with GIS and spatial modelling techniques (Gilad 2009), a morphological and typological analysis of houses in Kashgar old-town using digital techniques such as photogrammetry and web-museum 
136

(Florenzano, Courel and De Domenico 2010) and a study of dynamic monitoring of changes in the urban pattern between 2002 and 2009 by remote sensing (Tang and Zhou 2013).

\subsubsection{Fieldworks in Kashgar}

Kashgar was visited for documentation and data collection with three separate fieldworks between April 18 and May 18, 2015; September 17 and 27, 2015; and October 16 and 26, 2015. The focus area for data collection in Kashgar is Köziciyerbişi [Ch. 高台; pinyin. Gaotai; U. كوزنجيار ببشى], which is a neighbourhood section partially isolated from the rest of the old-town because of a motorway called Tuman Road (Fig. 51 and Fig. 52).

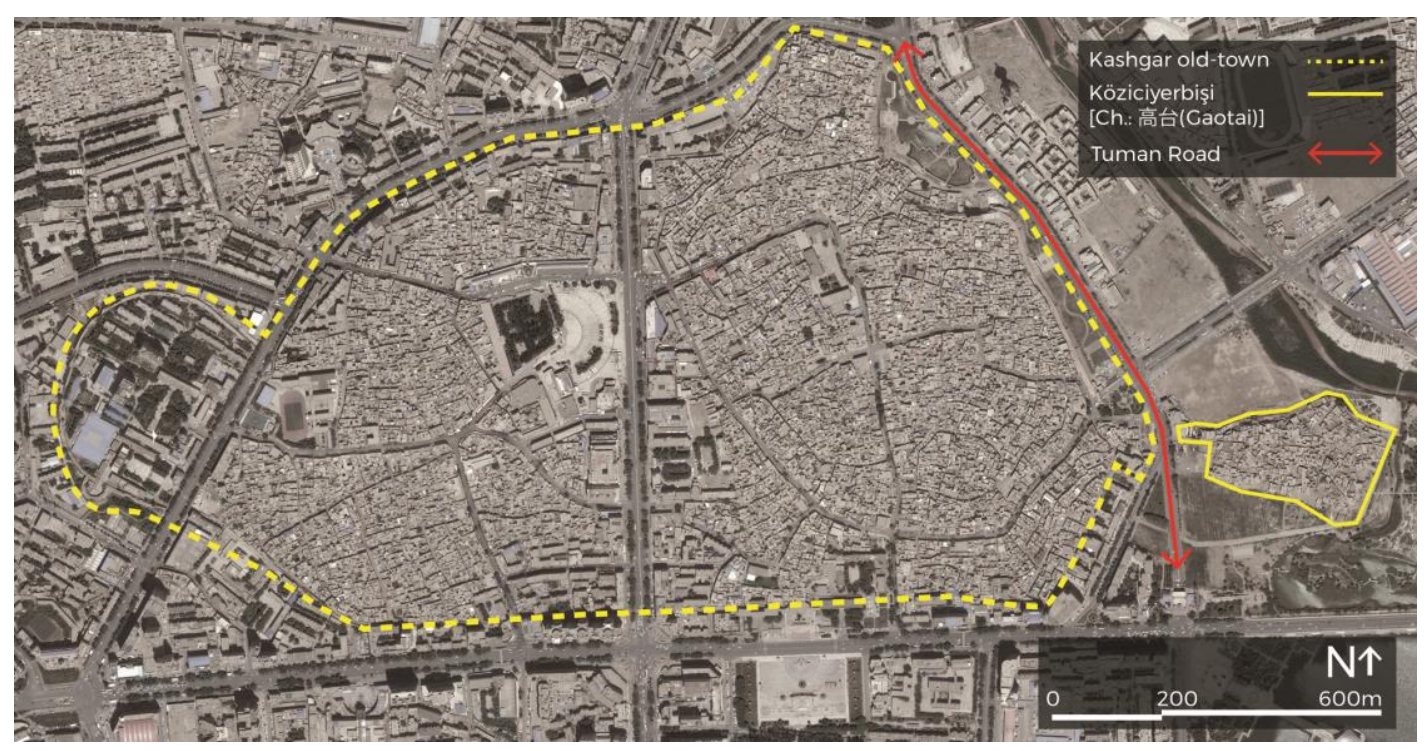

Fig. 51. Location of Köziciyerbişi (Gaotai) in the Kashgar old-town. Satellite image from Google.
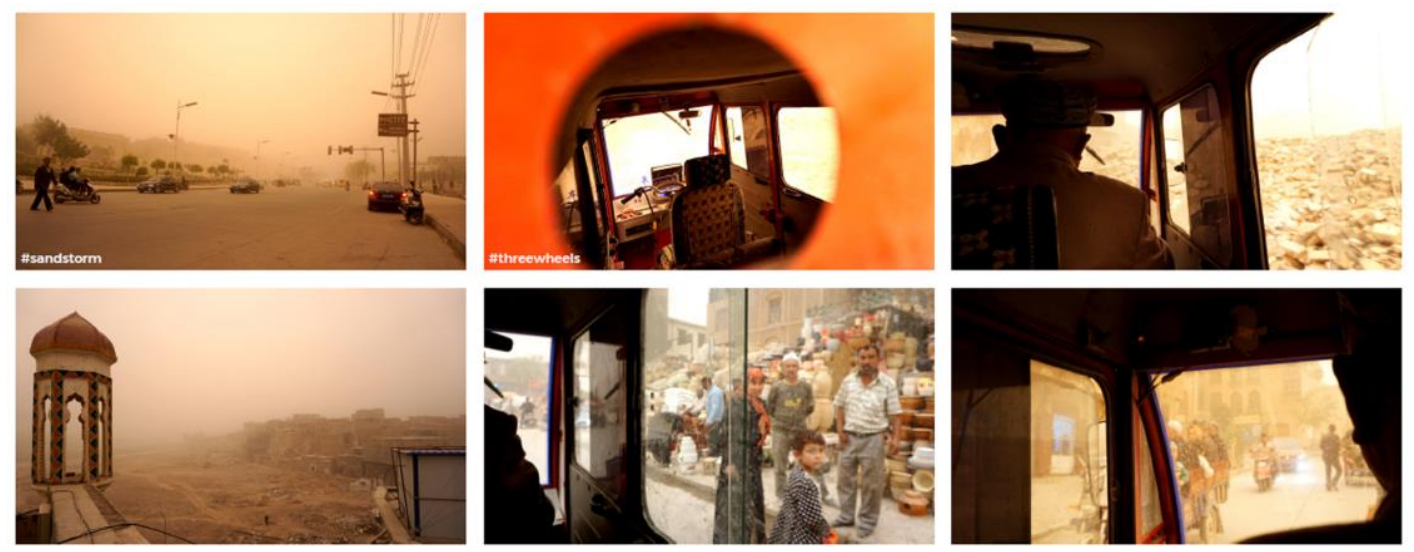

Fig. 52. Travelling in a modified three-wheel vehicle across Tuman Road (top-left) from Köziciyerbişi (Gaotai) to other parts of the old-town. Photos by Author from Aydin and Schnabel $(2017,16)$. 
The outdoor space in Köziciyerbişi (Gaotai) is composed of narrow alleyways that, if unfolded, measure more than a kilometre in length and two to three meters in width. They are defined by single to three-storey high house-walls that are occasionally linked with house units overpassing the alley to create tunnel-like passages underneath (Fig. 53).

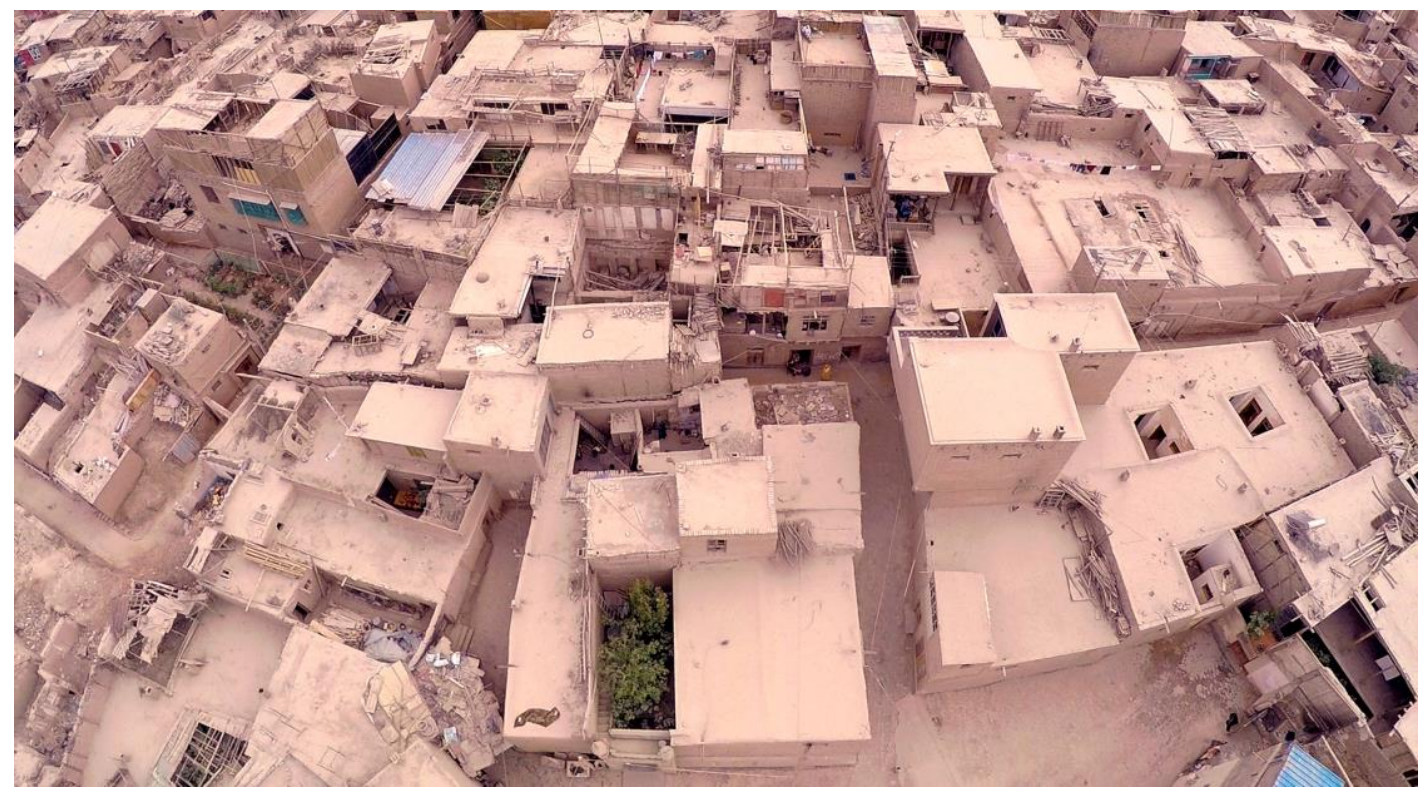

Fig. 53. Aerial view of houses with narrow alleys running through from the north of Köziciyerbişi (Gaotai). Photo by Author from Aydin and Schnabel $(2017,117)$.

Of three fieldworks, the first one was proposed as an overall examination of the life and culture in the context. On-ground and aerial photographic data collection for imagebased 3D modelling was instrumental to achieving successful fieldwork. Aerial photography was divided into three types of recording in Köziciyerbişi (Gaotai): (1) drawing on the perimeter of the neighbourhood, (2) following random pattern over the settlements and (3) ortho-photographic recording along three main routes of alleys. Other documentation activities included collecting CAD plan drawings (in .dwg format) of the old-town, finding resources about Kashgar architecture and improving communication in the local language for unstructured interviews. The methods involved in Fieldwork I between April 18 and May 18, 2015 varied from text notes to subjective observation to aerial photography. Fieldwork II (September 17-27, 2015) and Fieldwork III (October 16-26) were proposed to be largely complementary to the first one. The data collection activities during these two visits in Kashgar were conceived as a whole. Unstructured interviews were focused to sympathise with the local understanding and experience of the old architecture. Video and sound recordings were focused in these ten-day field studies (Table 21). 
Table 21. Data collection scheme for each fieldwork in 2015.

\begin{tabular}{|c|c|c|}
\hline Fieldwork / Date & Main purpose & Main methods \\
\hline $\begin{array}{l}\text { Fieldwork I } \\
\text { April 18-May } 18\end{array}$ & $\begin{array}{l}\text { Overall examination of life, culture and } \\
\text { architecture in the Kashgar old-town } \\
\text { Photographic collection (1/3) } \\
\text { Aerial photography: } \\
\text { 1. Draw a perimeter (free camera angle) } \\
\text { 2. Random pattern (free camera angle) } \\
\text { 3. Follow three different routes of } \\
\text { alleys (orthophotography) } \\
\text { Collect CAD drawings } \\
\text { Find books about the history of Kashgar } \\
\text { old-town } \\
\text { Learn the local language for } \\
\text { unstructured interviews in Fieldwork II } \\
\text { and Fieldwork III }\end{array}$ & $\begin{array}{l}\text { Text notes } \\
\text { Hand drawings } \\
\text { Photographs } \\
\text { Observation } \\
\text { Aerial photography }\end{array}$ \\
\hline $\begin{array}{l}\text { Fieldwork II } \\
\text { September 17-27 }\end{array}$ & $\begin{array}{l}\text { Photographic collection }(2 / 3) \\
\text { Learn history of architecture of the } \\
\text { Kashgar old-town from the locals }\end{array}$ & $\begin{array}{l}\text { Photographs } \\
\text { Unstructured interviews } \\
\text { Video recording } \\
\text { Sound recording }\end{array}$ \\
\hline $\begin{array}{l}\text { Fieldwork III } \\
\text { October 16-26 }\end{array}$ & $\begin{array}{l}\text { Photographic collection }(3 / 3) \\
\text { Aerial photography } \\
\text { Understand the perception of the } \\
\text { Chinese tourists about Kashgar } \\
\text { architecture }\end{array}$ & $\begin{array}{l}\text { Photographs } \\
\text { Aerial photography } \\
\text { Unstructured interviews } \\
\text { Video recording } \\
\text { Sound recording }\end{array}$ \\
\hline
\end{tabular}

Physically, to conduct these fieldworks was a challenging task because of the distance and the political circumstances of Kashgar. It is generously hard to conduct fieldwork in the western parts of China due to high-level security measures that would not help a foreign researcher move around as freely as planned beforehand. Documentation activities were limited to fast, easy and heterogenous recording techniques. For Fieldwork I, a digital camera was used for photography and sound and video recordings and a UAV device was borrowed for aerial photography.

A Sony $\alpha 6000$ mirrorless camera with stereo audio capture capability was used for photography, video and audio recording. Choosing a Sony $\alpha 6000$ was based on factors such as cost, size and quality for fast focus and shutter speed, which are instrumental to swiftly gathering image-based data of thousands of photographs for 3D photogrammetric modelling. 
It was important to intervene in the life of local people at a minimum. Fast focus and advanced shutter speed features of Sony $\alpha 6000$ aided the rapid performance of the photographic survey. This prevented suspicious gazes from the surrounding during the timeconsuming activity of photographing the walls that are equal to more than a kilometre.

\subsubsection{Statistical Analysis}

The documentation activities in Köziciyerbişi (Gaotai) section of the Kashgar old-town cover nearly $1 / 2 \mathrm{~km}^{2}$. Köziciyerbişi (Gaotai) contains four mosques and around 400 individual houses. The entire Kashgar old-town covers an area of more than eight squarekilometres in which more than 65,000 households accommodate more than 200,000 residents (Pawan and Niyazi 2016, 124) (Fig. 54).

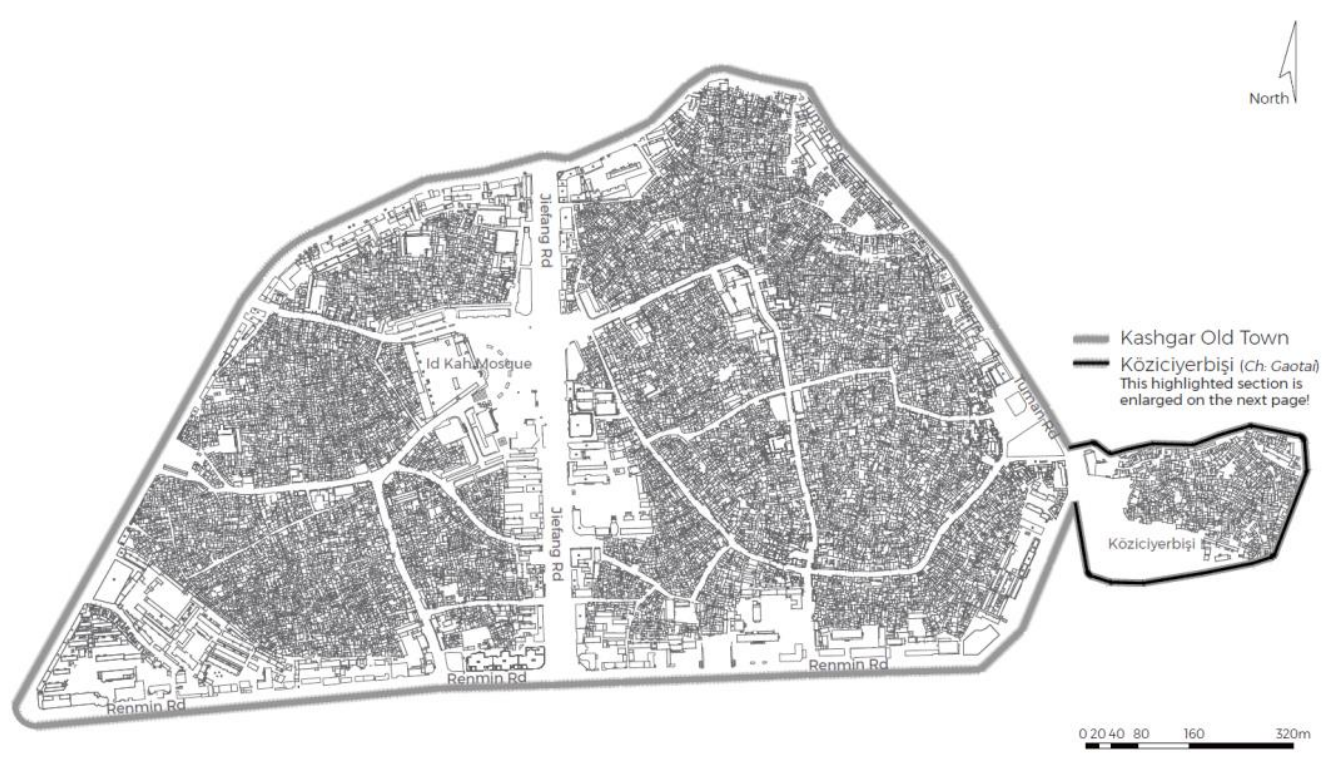

Fig. 54. Map of Köziciyerbişi (Gaotai) (at right bottom corner) divided from the rest of the old-town by Tuman Road. Illustration of a retrieved plan drawing by Author.

The old-town has been under transformation as a consequence of the renewal projects in the last two decades. Tang and Zhou's (2012) research analyses changes to the urban pattern of the Kashgar old-town. Comparing satellite images as a means of remote sensing monitoring, they report almost $10 \%$ decrease in the historic building area and more than $300 \%$ increase in open space area between 2002 and 2009. Their managerial view of research concludes that "open spaces may become modern architecture in the future" (Tang and Zhou, 2012). The renewal project that started in 2009 did not prove them wrong and open spaces in the old-town were largely turned into ‘modern' structures. But Köziciyerbişi 
(Gaotai) was rather exempted from the demolition process with an idea to make it an 'open museum' that can be translated as "staged authenticity" as coined by MacCannell (1992, 298). Employing remote sensing monitoring method based on satellite images, Table 22 comparatively depicts the transformation of Köziciyerbişi (Gaotai) between 2002 and 2017.

Table 22. Transformation of Köziciyerbişi (Gaotai) between 2002 and 2017. There was noticeable change in the density of houses after 2009 when the renewal project was initiated. Satellite images from Google Earth Pro.

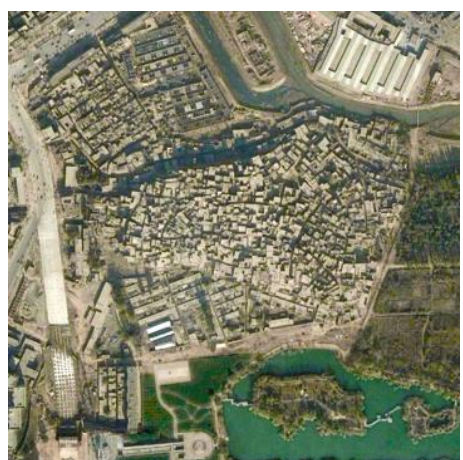

November 2002

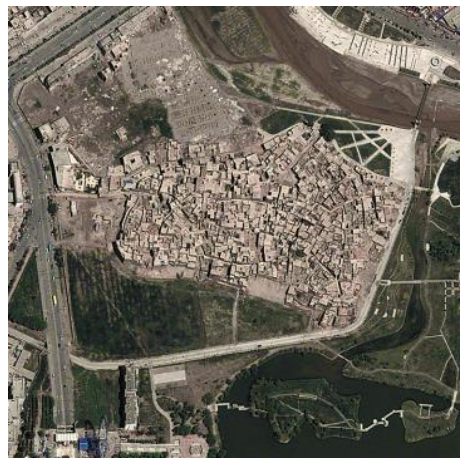

May 2012

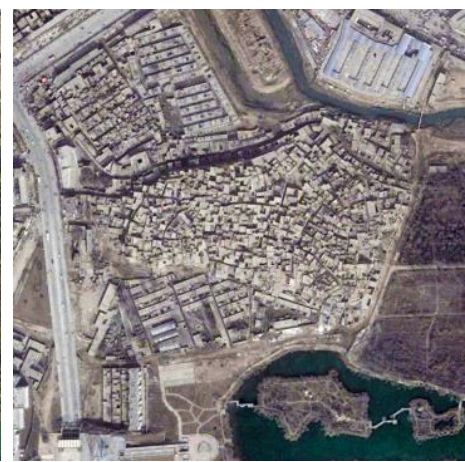

March 2005

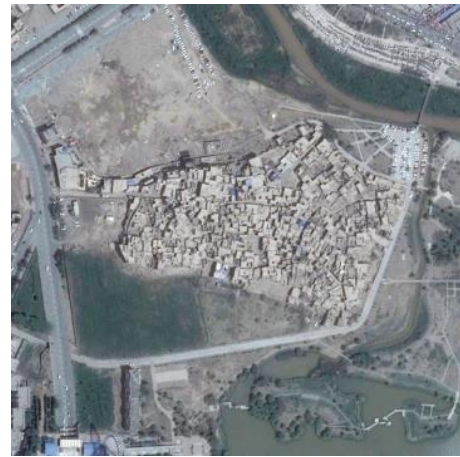

July 2015

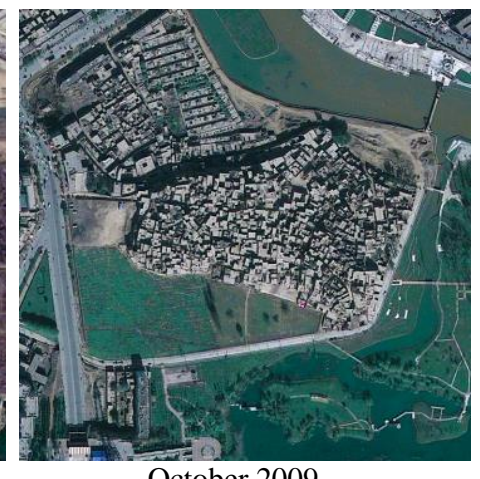

October 2009

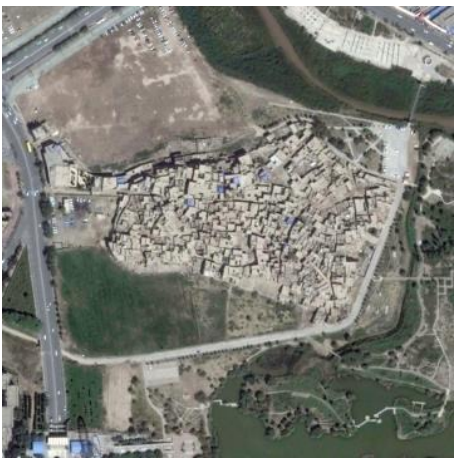

August 2017

Despite the importance of the socio-spatial characteristics in the labyrinthine maze-like structure made of alleyways, the "embraced communal identity" of the locals that has stood the test of time is challenged by the transformation of the urban pattern imposed by the renewal and relocation activities (Pawan and Niyazi 2016, 125). As a part of the documentation process, the important aspect of the spatial relations between courtyards and narrow alleys is recorded numerically below. Table 23 shows rough area calculations of the courtyard, floor, construction and staging based on the measurements obtained from Wang et al. (2014). Although many houses have been demolished since the survey that Wang's research team conducted in 2010 , it is a highly detailed information resource about the architectural features of the built environment in Köziciyerbişi (Gaotai). The team physically 
and digitally 3D modelled the entire area consisting of three-hundred-and-seventy-eight individual houses.

Table 23. Area measures in Köziciyerbişi (Gaotai) - calculations were made based on measurements in Wang et al. (2014).

\begin{tabular}{lccccc}
\hline & $\begin{array}{c}\text { Courtyard } \\
\text { area }(\boldsymbol{C} \boldsymbol{Y}) \mathbf{~ m}^{\mathbf{2}}\end{array}$ & $\begin{array}{c}\text { Plot area }(\boldsymbol{P}) \\
\mathbf{m}^{\mathbf{2}}\end{array}$ & $\begin{array}{c}\text { Floor area } \\
(\boldsymbol{F}) \mathbf{~ m}^{\mathbf{2}}\end{array}$ & $\begin{array}{c}\text { Gross floor area } \\
(\boldsymbol{G F A}) \mathbf{~ m}^{\mathbf{2}}\end{array}$ & $\begin{array}{c}\text { Floor area } \\
\text { ratio }(\boldsymbol{F A R})\end{array}$ \\
\hline Total & $>6,082.06$ & $>32,513.67$ & $>26,431.61$ & $>40,792.12$ & - \\
\hline Min & 0.00 & 8.06 & 8.06 & 8.37 & $19.20 \%$ \\
\hline Max & 180.73 & 412.98 & 322.83 & 622.07 & $100.00 \%$ \\
\hline Average & $\sim 16.16$ & $\sim 86.24$ & $\sim 70.11$ & $\sim 108.20$ & $83.74 \%$ \\
\hline
\end{tabular}

According to Wang (2014), there are more than 1500 residents (4 per house on average) living in Köziciyerbişi (Gaotai). Its boundary space totals more than $40,000 \mathrm{~m}^{2}$, whereas alleys, which offer space for a mixture of social and semi-private activities, amount to more than a space of $6,000 \mathrm{~m}^{2}$. Narrow roads with an average width of three meters are rather multifunctional spaces on which different daily activities are held. In her phenomenological PhD investigation, Niyazi (2016) divides outdoor spaces and alleyways into three levels: community level, street level and semi-private level (see also Pawan and Niyazi 2016, 124). As an extension of these three types of spaces, one enters into the private level with a courtyard where all levels act as a system contributing to the thermal comfort of the inhabitants. Also, the courtyard typology in Kashgar completes the hierarchical relations outside. The average courtyard area for an individual house is about $16.16 \mathrm{~m}^{2}$, while in total they account for approximately fifteen to twenty percent of the entire space in Köziciyerbişi (Gaotai). Courtyards and narrow roads together facilitate the vertical and horizontal circulation of movement.

The highest house has four floors, whereas the gross floor area is nearly equivalent to the entire land area. It can be deduced that the most critical factor in this balance is the intricately economic spatial configurations of courtyards within houses. Functionally, courtyards extend the cooling-down effect of alleys, reducing the thermal energy of walls from inside with plants and vines covering the space from above. To protect the corridorlike spaces from direct sunlight, house walls are extended to the boundary, causing minimal road space between houses (Fig. 55). 


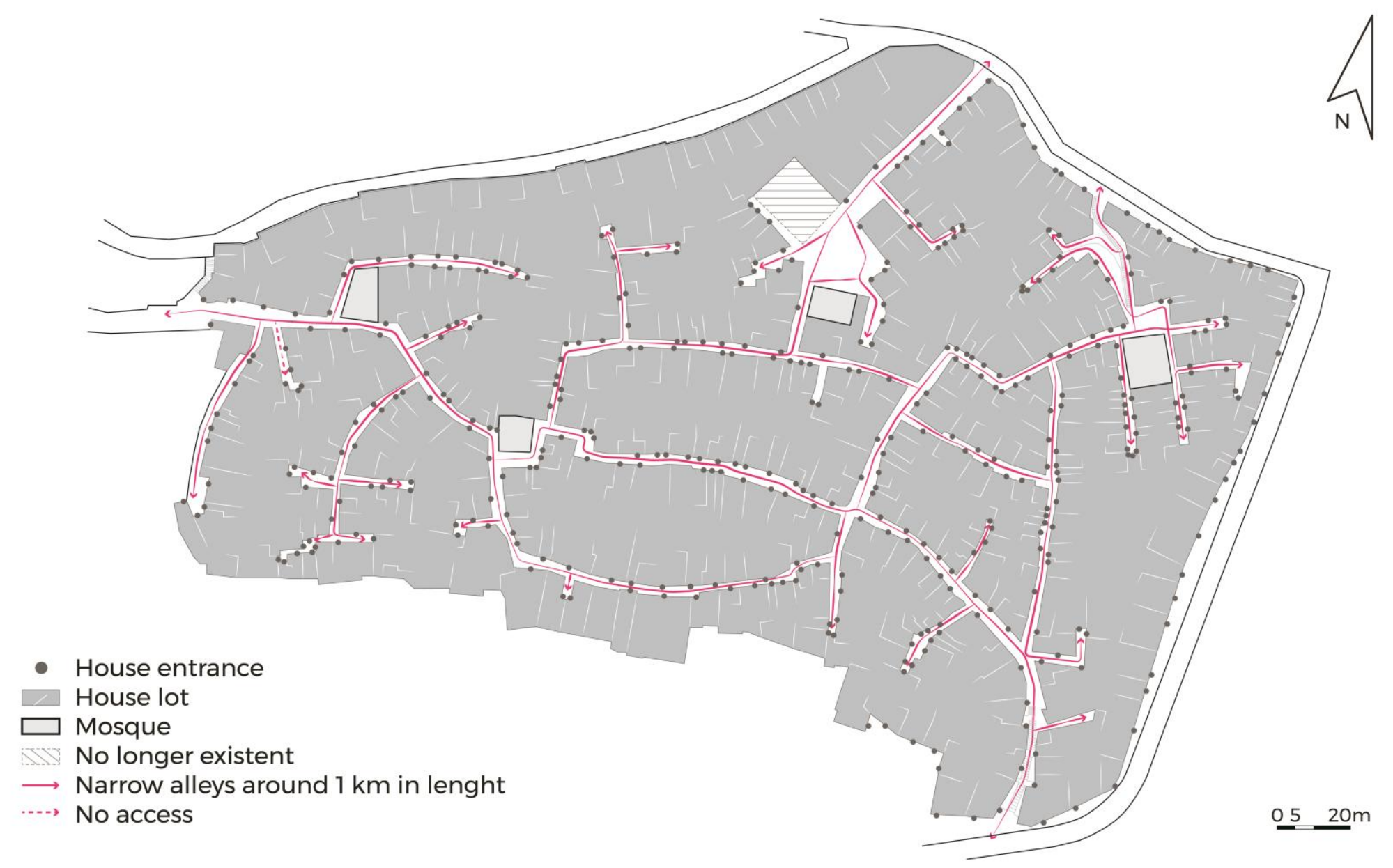

Fig. 55. Köziciyerbişi (Gaotai) in Kashgar. Illustration by Author. 
Research has shown a conceptual relationship between courtyards and lanes (Florenzano, Courel and De Domenico 2010; Pawan and Niyazi 2016; Steenberg 2014; Niyazi 2016). Yet, in the specific context of Köziciyerbişi (Gaotai), it remains unknown what superstructure may exist between different areas thanks to this relationship. Fig. 56 demonstrates the frequency distribution of courtyard areal data from Köziciyerbişi (Gaotai). The data is interpreted from an early report of Wang et al. (2014). For that, a Boolean number of courtyard-factor $(C Y b)$ is calculated. Four statistical figures $(C Y p, C Y f, C Y g$ and $C Y)$ generate the $C Y b$ Boolean factor for each house reported in Köziciyerbişi (Gaotai) (Table 24). These calculations are made with other figures given in Table 24 above. They are $\mathrm{CYa}$ (average courtyard area), $P$ (plot area), $\mathrm{Pa}$ (average plot area), $F$ (floor area), $F a$ (average floor area), GFA (gross floor area), GFAa (average gross floor area). From these calculations, $C Y b$ is distributed to each house recorded by Wang et al. (2014) in Köziciyerbişi (Gaotai). As mentioned, $C Y b$ is a Boolean factor for simplicity, i.e. either black ('zero') or white ('one'). By generating $C Y b$, it is aimed at mapping a superstructure (zoning) that may exist between different areas to understand the relationship between houses across their boundaries through the alleys of Köziciyerbişi (Gaotai). Three types of courtyard integration level are found. In the data chart, each is appointed a different colour, namely red (Level I), blue (Level II) and green (Level III). In the chart, they are identified through a visual analysis of a Boolean factor of $C Y b$ that is indicated in two ways: negative and positive (Fig. 56).

Table 24. Components of courtyard-factor $(C Y b)$ calculation.

\begin{tabular}{lll}
\hline Abb. & Factor & ${\text { Result in } \mathbf{~}^{2}}^{2}$ \\
\hline$C Y p$ & Courtyard-plot area factor & Plot-area-based prediction of $C Y$ size \\
\hline$C Y f$ & Courtyard-floor area factor & Floor-area-based prediction of $C Y$ size \\
\hline$C Y g$ & Courtyard-GFA factor & GFA-based prediction of $C Y$ size \\
\hline$C Y$ & Courtyard actual area & $C Y$ size \\
\hline
\end{tabular}

$\mathrm{CYP}=\mathrm{P}{ }^{*} \mathrm{CYa}\left(16.16 \mathrm{~m}^{2}\right) / \mathrm{Pa}(86.24)$

$\mathrm{CY} f=\mathrm{F}^{\star} \mathrm{CYa}\left(16.16 \mathrm{~m}^{2}\right) / \mathrm{Fa}(70.11)$ 
CYb Courtyard Boolean factor

Courtyard-floor area factor

Courtyard-CFA factor

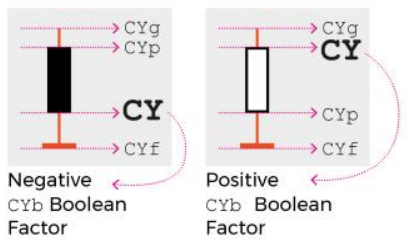

$\begin{array}{ll}\text { CYa } & \text { Average courtyard area } \\ \mathrm{P} & \text { Plot area } \\ \mathrm{Pa} & \text { Average plot area } \\ \mathrm{F} & \text { Floor area } \\ \mathrm{Fa} & \text { Average floor area } \\ \mathrm{GFA} & \text { Gross floor area } \\ \mathrm{GFAa} & \text { Average gross floor area } \\ \mathrm{CYP}=\mathrm{P} * \mathrm{CY}(16.16 \mathrm{~m} 2) / \mathrm{Pa}(86.24) & (1) \\ \mathrm{CYA}=\mathrm{F} * \mathrm{CY}(16.16 \mathrm{~m} 2) / \mathrm{Fa}(70.11) & (2) \\ \mathrm{CYg}=\mathrm{GFA} \times \mathrm{CYa}(16.16 \mathrm{~m} 2) / \mathrm{GFAa}(108.20) & (3)\end{array}$
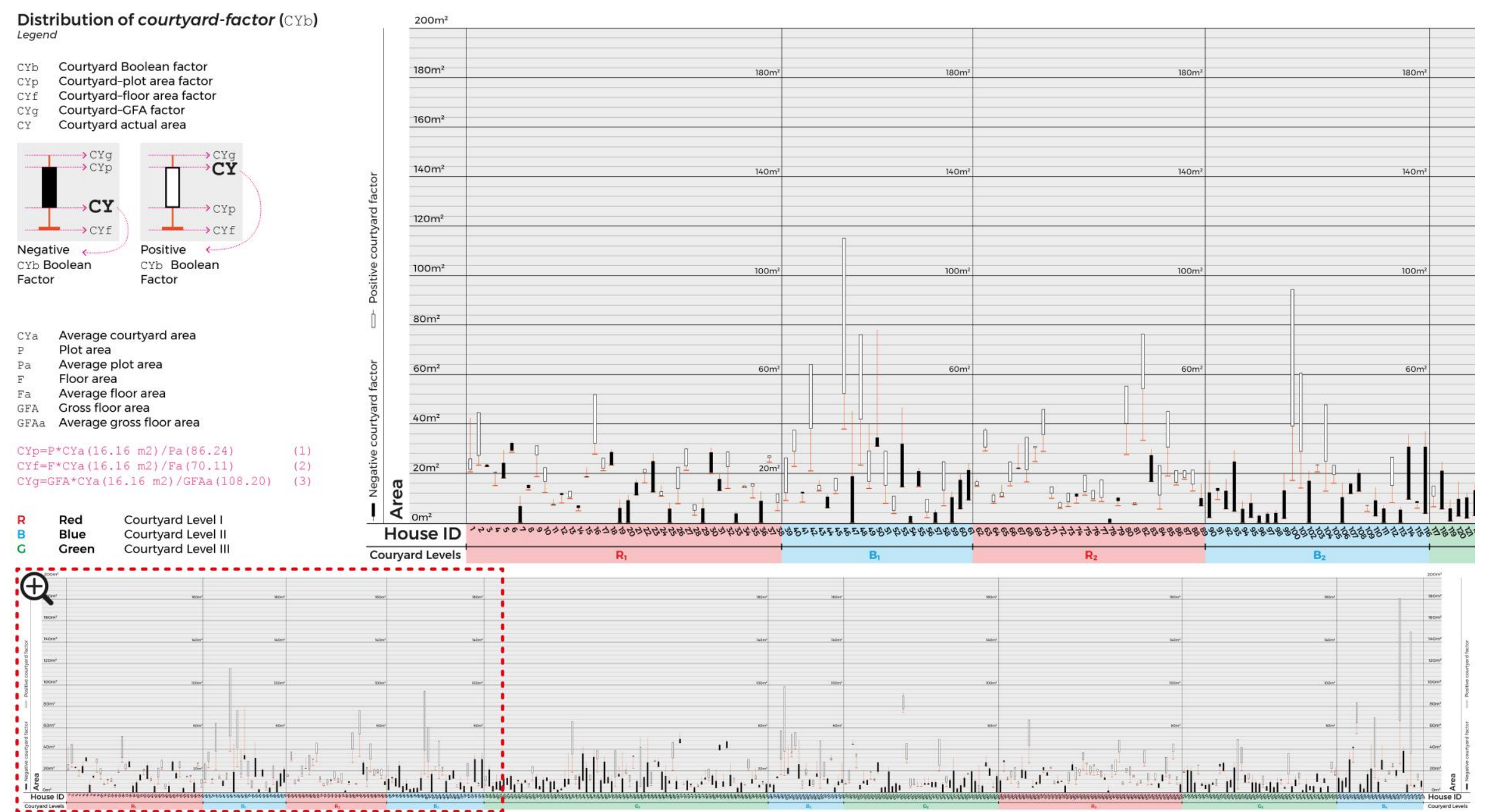

Fig. 56. Distribution of the courtyard factor $-C Y b$. The chart shows the courtyard area comparisons in Köziciyerbişi (Gaotai). The full chart is available in Appendix B. 
In Level I (red), houses present a rich combination of courtyard integration in comparison to each other. Houses in Level II (blue) represent outstandingly courtyardoriented integration with the neighbourhood. To really simplify, it can be deduced that courtyard area of these houses is considerably higher than average. Condensed in the southeast of Köziciyerbişi (Gaotai), the courtyard typology in Level II is understood to be relatively less significant. In Level III (green), houses are denser and courtyard areas are more limited. In the chart below, Level I is seen as mostly white, which means courtyard size is relatively above average, whereas Level II is the area of the chart where black indicators are dominant (see above Fig. 56). As mentioned, the calculation of the average size is made based on statistical courtyard size data taken from Wang et al. (2014). Equations (1), (2) and (3) are used to balance a relatively more sensitive approach. The finding of this chart is overlaid onto a map of the courtyard superstructure (Fig. 57). There appear three interest points emerging from the convergence of three levels of courtyard types. It means these areas are potentially more precious than any other part of Köziciyerbişi (Gaotai). They can be further studied through the design process of virtual environments that will be explained in the following sections of this chapter.

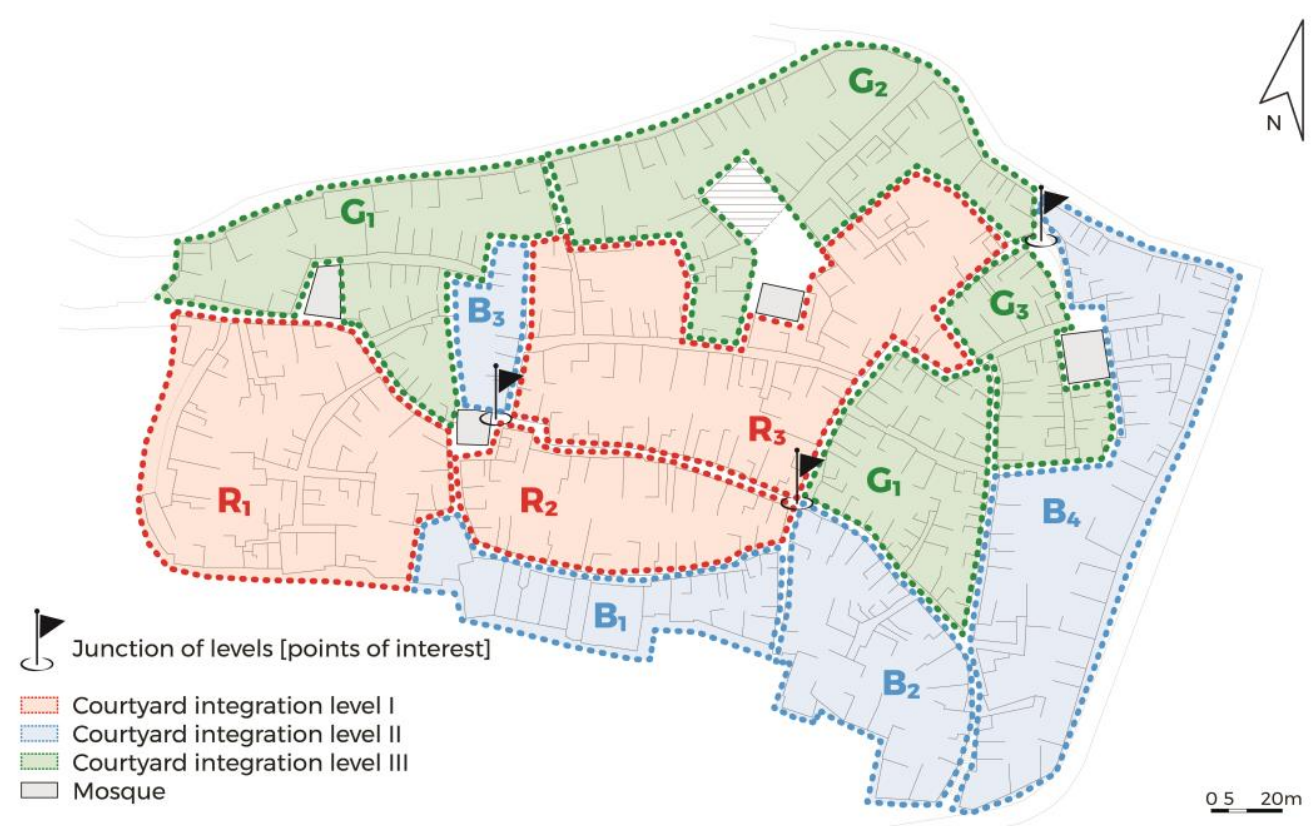

Fig. 57. Map colourised according to the courtyard analysis shows interrelations between courtyards spanning across lanes.

The convergence of all three courtyard levels is marked as points of interest that are presented with orthoptohographic images of aerial recording in Table 25. At the West Point, there is a mosque at which is likely to coincide different courtyard typologies. The central 
junction is occupied by houses of courtyards with most planting. The northeastern entrance of the neighbourhood has a unique identity that also appeared in the chart of the courtyard factor $(\mathrm{CYb})$ above (Fig. 56). The specialty of the northeast junction of courtyard levels is that it shows how the horizontal movements on alleyways are integrated with the vertical unfolding of courtyards. Ramps, stairs and bridges enhance the integration of courtyards and narrow roads, distinct from any other areas of the old-town.

Table 25. Points of interest at the convergence of three courtyard integration levels.

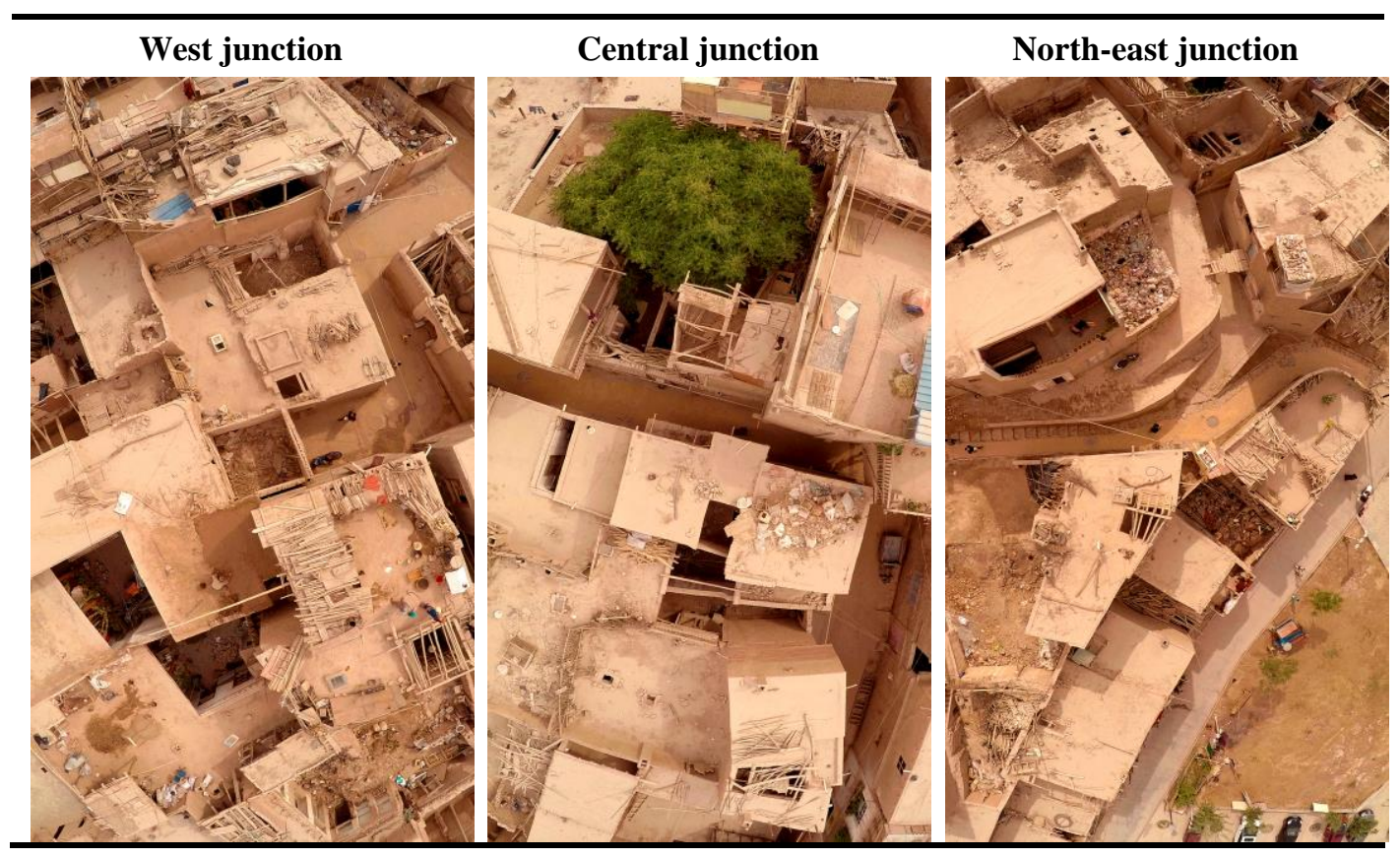

\subsubsection{Street-Sound Recording}

The digitisation of audio content is a field of intangible heritage documentation. Despite the long history of sound-recording technologies in our lives, there are many challenges and issues with regards to copyright and ethics (Prentice 2016). Nevertheless, high-fidelity sound recording technologies are increasingly more affordable, advanced and mobile for capturing the ambience of heritage environments. Recent research has focused on formulating sonic heritage as ecosystems of soundscapes (Abbazia et al. 2017). Doornbusch and Kenderdine (2004) studied the relationship betweeen sound and presence in VR based on their Angkor project. To document the sonic ambience of the Kashgar old-town, a series of field recordings were reproduced. In the presence of external noise, the ambience of the life on streets in the Kashgar old-town is captured via a digital camera of two-channel stereo audio recording (Table 26). 
Table 26. Audio specification.

\begin{tabular}{lll}
\hline Device: Sony $\mathbf{\alpha 6 0 0 0}$ camera & \\
\hline Video Input & Digital camera type & Mirrorless system \\
& Cont. shooting speed & $11 / 2.5 / 6$ frames per second \\
& Light sensitivity & ISO auto (100-25600) \\
& Digital Video Format & AVCHD, H.264 \\
& Frame rate & 25 \\
& Data rate & $16270 \mathrm{kpbs}$ \\
\hline Audio & Surround sound & Dolby Digital AC-3 (2 channel) recording \\
Specifications & Bitrate & $256 \mathrm{kpbs}$ \\
& Channels & $2($ stereo $)$ \\
& Audio sample rate & $48 \mathrm{kHz}$ \\
\hline
\end{tabular}

Recordings include various sounds including Uyghur music, radio news reports, conversations, adhan (calling for prayer), children playing in alleys and merchants' ambience. They are recorded as videos and converted into sounds that are uploaded to an online archive (Aydin, 2015). Inspired by the live-mixed webcast of field recordings in Kashgar by Shirley and Spinoza Internet Radio (2006), a playlist is generated to inform the audience about the track time, title, location, performer, instrument, and other relative information (Table 27) (Fig. 58).

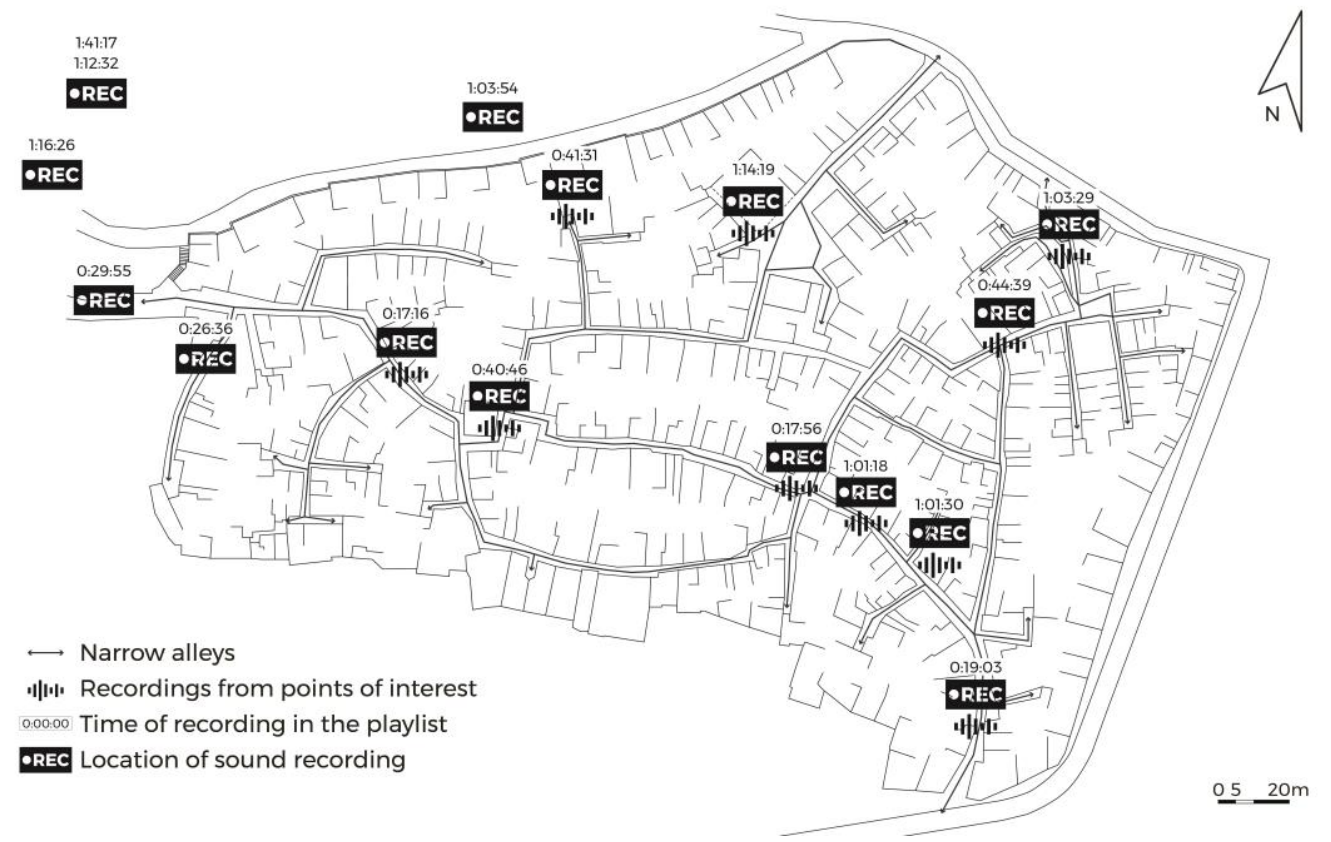

Fig. 58. Map of the tracks in the playlist of field recordings. 
148

Table 27. Playlist of street-sound recordings from Köziciyerbişi (Gaotai): (ø) Tracks, ( ): Playlists (available for listening via Aydin, 2015). A full list of sound recordings available in Appendix A.

\begin{tabular}{|c|c|c|c|c|}
\hline Time & Title & Location & Performer/Instrument & Information \\
\hline - $0: 17: 16$ & Narrow road recording & $\begin{array}{l}\text { In front of a little } \\
\text { shop in } \\
\text { Köziciyerbişi }\end{array}$ & $\begin{array}{l}\text { A bird singing in a cage and } \\
\text { two motorbikes passing } \\
\text { with one of them playing a } \\
\text { pop music }\end{array}$ & Narrow roads \\
\hline $0: 17: 56$ & $\begin{array}{l}\text { Noise of brick wall } \\
\text { masonry }\end{array}$ & Köziciyerbişi & & $\begin{array}{l}\text { Workers rebuilding a brick wall of } \\
\text { storage space, with a radio playing } \\
\text { Uyghur songs }\end{array}$ \\
\hline 0:19:03 & \multicolumn{3}{|c|}{$\begin{array}{ll}\text { Recording conversation in } & \text { A pottery } \\
\text { Uyghur } & \text { workshop in } \\
& \text { Köziciyerbişi } \\
\end{array}$} & $\begin{array}{l}\text { Introducing each other to children } \\
\text { of a pottery maker }\end{array}$ \\
\hline \multicolumn{5}{|l|}{$\cdots$} \\
\hline - $0: 26: 36$ & $\begin{array}{l}\text { Ambience of alleys in the } \\
\text { morning }\end{array}$ & Köziciyerbişi & & $\begin{array}{l}\text { Exploring the first encountered } \\
\text { roads in the neighbourhood }\end{array}$ \\
\hline $0: 29: 55$ & $\begin{array}{l}\text { Sound of children playing } \\
\text { on ground }\end{array}$ & Köziciyerbişi & $\begin{array}{l}\text { Three children playing with } \\
\text { little sand dunes }\end{array}$ & $\begin{array}{l}\text { Children turning narrow roads into } \\
\text { multifunctional space to generate } \\
\text { their own narrative }\end{array}$ \\
\hline \multicolumn{5}{|r|}{ 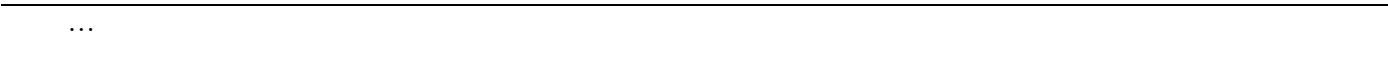 } \\
\hline - 0:40:46 & $\begin{array}{l}\text { Narrow road recording } \\
\text { after a two-day sand- } \\
\text { storm }\end{array}$ & $\begin{array}{l}\text { Mosque I in } \\
\text { Köziciyerbişi }\end{array}$ & & $\begin{array}{l}\text { A worker fixes the fallen thickness } \\
\text { of the mud-brick wall of a mosque } \\
\text { by hand while listening to Uyghur } \\
\text { music on a mobile radio }\end{array}$ \\
\hline $0: 41: 31$ & $\begin{array}{l}\text { Sound of children playing } \\
\text { football }\end{array}$ & $\begin{array}{l}\text { North of } \\
\text { Köziciyerbişi }\end{array}$ & & $\begin{array}{l}\text { Looking from an alley towards the } \\
\text { Grand Bazaar of Kashgar along the } \\
\text { rim of Tuman River }\end{array}$ \\
\hline 0:44:39 & $\begin{array}{l}\text { Recording conversations } \\
\text { in Uyghur }\end{array}$ & $\begin{array}{l}\text { North of } \\
\text { Köziciyerbişi }\end{array}$ & $\begin{array}{l}\text { A female, two males and a } \\
\text { child }\end{array}$ & \\
\hline \multicolumn{5}{|l|}{$\cdots$} \\
\hline - 1:01:18 & Street recording & Köziciyerbişi & & \\
\hline $1: 01: 30$ & Unknown Uyghur song & Köziciyerbişi & Abdurehim Heyit & $\begin{array}{l}\text { Recorded from a radio played } \\
\text { inside a house }\end{array}$ \\
\hline $1: 03: 29$ & Street recording & Köziciyerbişi & & $\begin{array}{l}\text { Recorded by following a cat in } \\
\text { alleys }\end{array}$ \\
\hline 1:03:54 & Unknown Uyghur song & Köziciyerbişi & $\begin{array}{l}\text { Unknown singer playing a } \\
\text { rawap }\end{array}$ & $\begin{array}{l}\text { Recorded from radio playing } \\
\text { outside a house }\end{array}$ \\
\hline \multicolumn{5}{|l|}{$\cdots$} \\
\hline - $1: 12: 32$ & $\begin{array}{l}\text { Recording Uyghur } \\
\text { traditional music calling } \\
\text { for an occasion }\end{array}$ & $\begin{array}{l}\text { Köziciyerbişi - } \\
\text { Tuman street }\end{array}$ & & Street recording of traffic \\
\hline \multicolumn{5}{|l|}{$\cdots$} \\
\hline - $1: 14: 19$ & \multicolumn{2}{|c|}{$\begin{array}{l}\text { Recording conversation in Köziciyerbişi } \\
\text { Uyghur }\end{array}$} & & \\
\hline$\cdots$ & & & & \\
\hline - $1: 26: 26$ & Rawab street recording & $\begin{array}{l}\text { Köziciyerbişi - } \\
\text { Tuman street }\end{array}$ & $\begin{array}{l}\text { Unknown musician playing } \\
\text { Rawab }\end{array}$ & Street shop owner playing rawab \\
\hline - $1: 41: 17$ & Unknown Uyghur song & $\begin{array}{l}\text { Köziciyerbişi - } \\
\text { Tuman street }\end{array}$ & $\begin{array}{l}\text { Unknown local musicians } \\
\text { playing Uyghur instruments }\end{array}$ & $\begin{array}{l}\text { Song rehearsal for Xinjiang } \\
\text { anniversary }\end{array}$ \\
\hline
\end{tabular}

\subsubsection{Photography}

Photography activities are related to photogrammetry processes in this research. Chapter 4.3 , with which this subchapter is integrated, is about the low-cost photogrammetry methods that explain the photographic survey and documentation. Apart from the photographs taken 
for photogrammetric modelling, many images are captured from and around the Kashgar old-town. Aydin and Schnabel (2017) is a book that depicts selected photographs with a narrative built around thirteen stories (Fig. 59). The specifics of the digital camera that is used are given in Table 28. The explanation of the rest of the photography documentation will be covered along with 3D photogrammetry covered in Chapter 4.3.

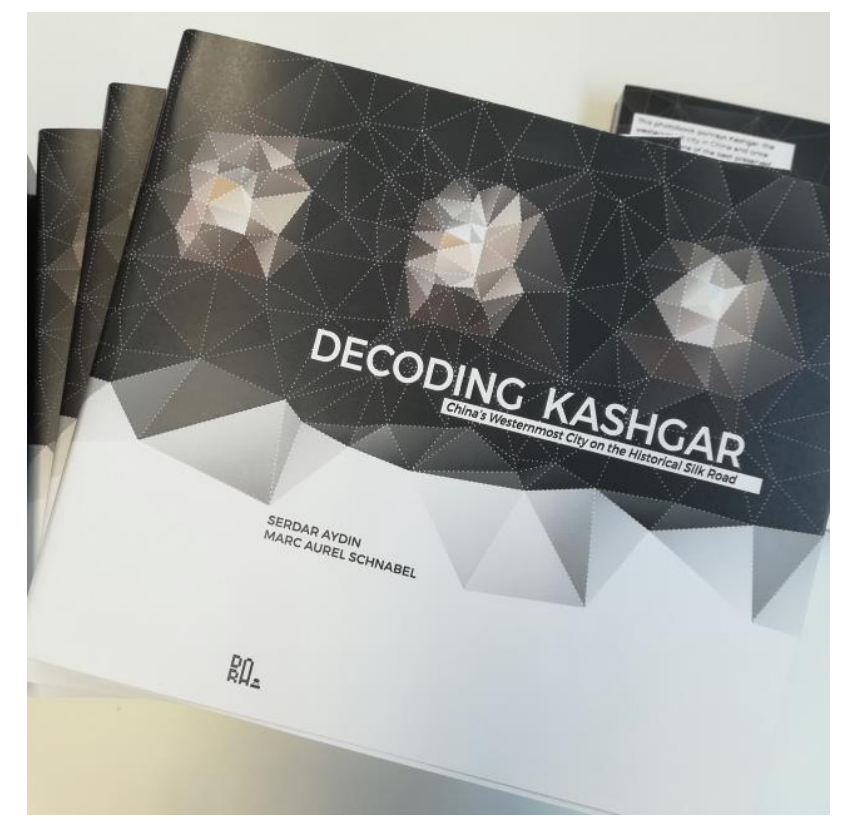

Fig. 59. Select photographs are documented in a photobook titled "Decoding Kashgar: China's westernmost city on the historical Silk Road" (Aydin and Schnabel 2017).

Table 28. Specification of the device and photographs.

\begin{tabular}{lll}
\hline Model: Sony $\mathbf{\alpha 6 0 0 0}$ / ILCE-6000 & \\
\hline Camera & Sensor resolution & 24.3 megapixel \\
Specifications & Optical sensor size & 15.6 x 23.5 mm \\
& Light sensitivity & ISO auto $(100-25600)$ \\
& Shutter speed & 30 secs $-1 / 4000 \mathrm{sec}$ \\
\hline Image & Width & 6000 pixels \\
Specifications & Hight & 3376 pixels \\
& Resolution & 350 dpi \\
& Bit depth & 24 \\
& Colour representation & Colour representation: sRGB \\
& Flash mode & No flash \\
\hline
\end{tabular}

\subsubsection{Aerial Imaging}

The intricate relations between houses in Kashgar are so detailed they are best viewed from above. Like most ancient towns, it is easy to get lost in this maze of alleys. When viewed 
from on high, the maze reveals itself as a combination of similar components that differ on closer inspection. Interlocked combinations of units reflect a historical fact that the houses are all produced with the same materials and construction techniques. This general homogeneity is broken up by local adaptations to suit the domestic functions of houses to their context.

Aerial imaging is used to view the complex structure of the old-town from above. This documentation is applicable to 3D photogrammetric modelling. A DJI Phantom 2 (mounted with a GoPro Hero4 camera) and a DJI Phantom 3 Professional (with an original built-in camera) are used in the recording of aerial views in Kashgar. Table 29 compares the features of the unmanned aerial vehicle (UAV) and of the cameras used.

Table 29. Unmanned aerial vehicles used to document the Kashgar old-town from above (specifications available from DJI's website: https://www.dji.com/).

\begin{tabular}{|c|c|c|}
\hline Model: & DJI Phantom 2 & DJI Phantom 3 Professional \\
\hline \multicolumn{3}{|l|}{ Aircraft Specifications: } \\
\hline Weight & 1000 gram & 1280 gram \\
\hline \multirow[t]{2}{*}{ Hover Accuracy } & Vertical: $0.8 \mathrm{~m}$ & Vertical: $\pm 0.5 \mathrm{~m}$ \\
\hline & Horizontal: $2.5 \mathrm{~m}$ & Horizontal: $\pm 1.5 \mathrm{~m}$ \\
\hline Max Angular Speed & $200 \% \mathrm{~s}$ & $150 \% \mathrm{~s}$ \\
\hline Max Tilt Angle & $35^{\circ}$ & $35^{\circ}$ \\
\hline Max Ascent/Descent Speed & Ascent: $6 \mathrm{~m} / \mathrm{s} ;$ Descent: $2 \mathrm{~m} / \mathrm{s}$ & Ascent: $5 \mathrm{~m} / \mathrm{s}$; Descent: $3 \mathrm{~m} / \mathrm{s}$ \\
\hline Max Flight Speed & $15 \mathrm{~m} / \mathrm{s}$ & $16 \mathrm{~m} / \mathrm{s}$ \\
\hline Flight Time & 25 mins & 23 mins \\
\hline Camera Specifications: & [GoPro Hero4] & [Built-in DJI camera] \\
\hline Sensor type & CMOS & CMOS \\
\hline Sensor resolution & 12.0 megapixel & 12.4 megapixel \\
\hline Light sensitivity & ISO auto (100-6400) & ISO auto (100-3200) \\
\hline Image size & $4000 \times 3000$ & $4000 \times 3000$ \\
\hline Image format & JPEG & JPEG, DNG (RAW) \\
\hline Video recording & $4 \mathrm{~K}$ & $4 \mathrm{~K}$ \\
\hline Video format & H.264 & H.264 \\
\hline Remote Controller: & Tablets and smartphones & Tablets and smartphones \\
\hline Battery: & [DJI Smart Battery] & [Intelligent Flight Battery] \\
\hline Type & 3S LiPo & 4S LiPo \\
\hline Capacity & $5200 \mathrm{mAh}, 11.1 \mathrm{~V}$ & $4480 \mathrm{mAh}, 15.2 \mathrm{~V}$ \\
\hline
\end{tabular}

Aerial images are produced by flying these two very similar aircraft (DJI Phantom 2 and DJI Phantom 3 Professional) (Fig. 60). The latter is a more compact and reliable version of the former that can be operated using more ad-hoc styles. A Phantom 2 is used in Fieldtrip $I$ and a Phantom 3 is brought to Kashgar for Fieldtrip II and III. As personal UAV devices 
become increasingly common for layperson's use, authorities seek ways to ensure safer experiences for everybody. Therefore they enact regulations for controlling GPS signals. It is even stricter in Kashgar as a consequence of the political conflict in the region. New regulations that were enacted before Fieldtrip II resulted in the new DJI Phantom 3 no longer being able to fly over Köziciyerbişi (Gaotai). Due to time restrictions, it was not possible to obtain official permission for the device ID to be recognised in the system for GPS tracking. Another UAV that had a registered ID for safely flying within China was rented on site.

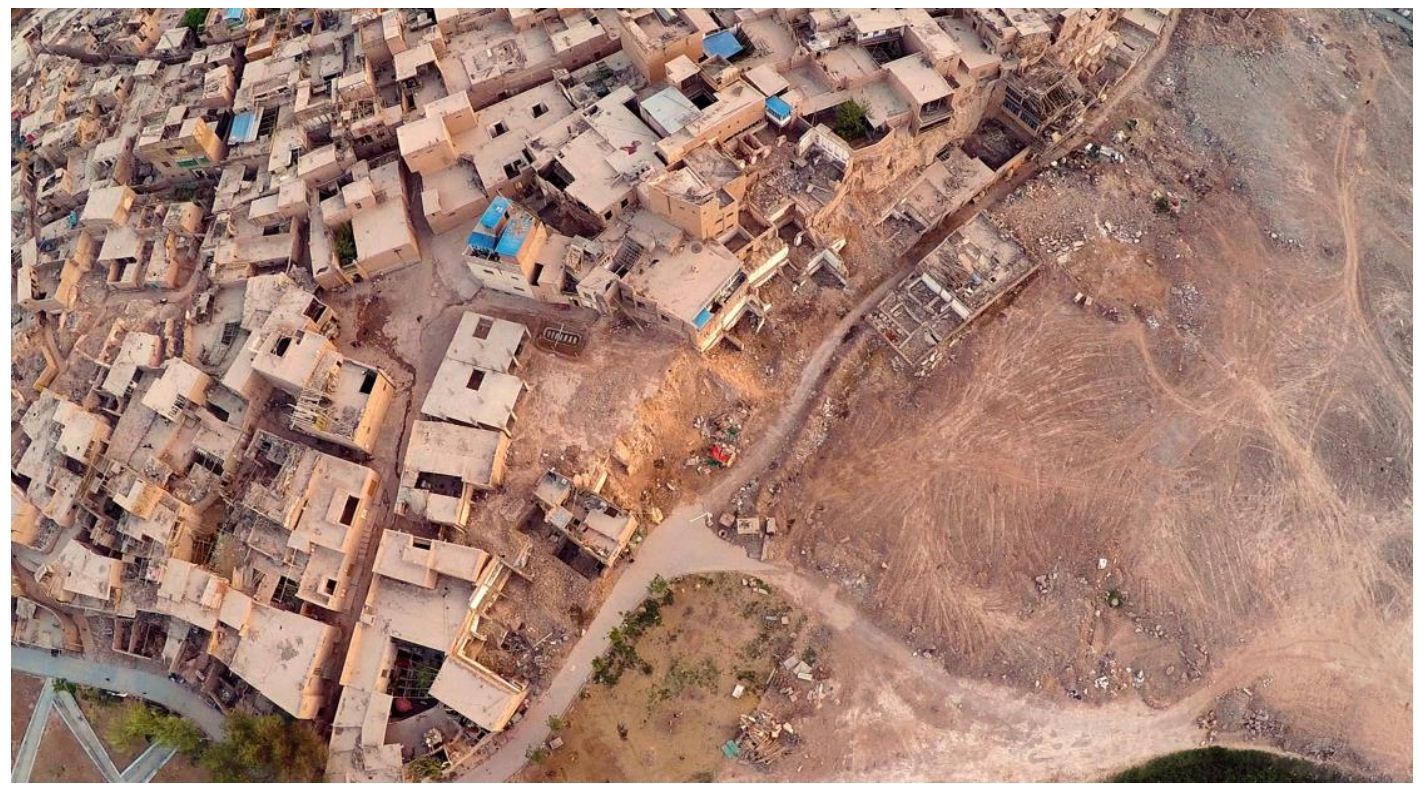

Fig. 60. Köziciyerbişi (Gaotai) from above (captured with DJI Phantom 2).

In Köziciyerbişi (Gaotai), the aerial documentation is divided based on three types of the followed flight path:

- Along the perimeter of the neighbourhood (A)

- Random pattern of the neighbourhood (B)

- On narrow roads $(\mathrm{C})$

Most recent UAV technologies are enabled with advanced intelligent features to organise the flight track before flying so that a user can define what type of pattern to follow. However, neither of the vehicles used in this research has this ability. With DJI Phantom 2 and 3 , it is important for the researcher to know how to manage a flying device on air under different forces, such as wind and loss of signal. Prior to flights, paths on air are designed to produce results as efficiently and accurately as possible. Table 30 summarises the three types of maps showing the respective path. Fig. 61 shows a result of the photogrammetric modelling by following one of the trails of the third flight path $\left(\mathrm{C}_{1}\right)$. 
Table 30. Aerial imaging types and flight paths.

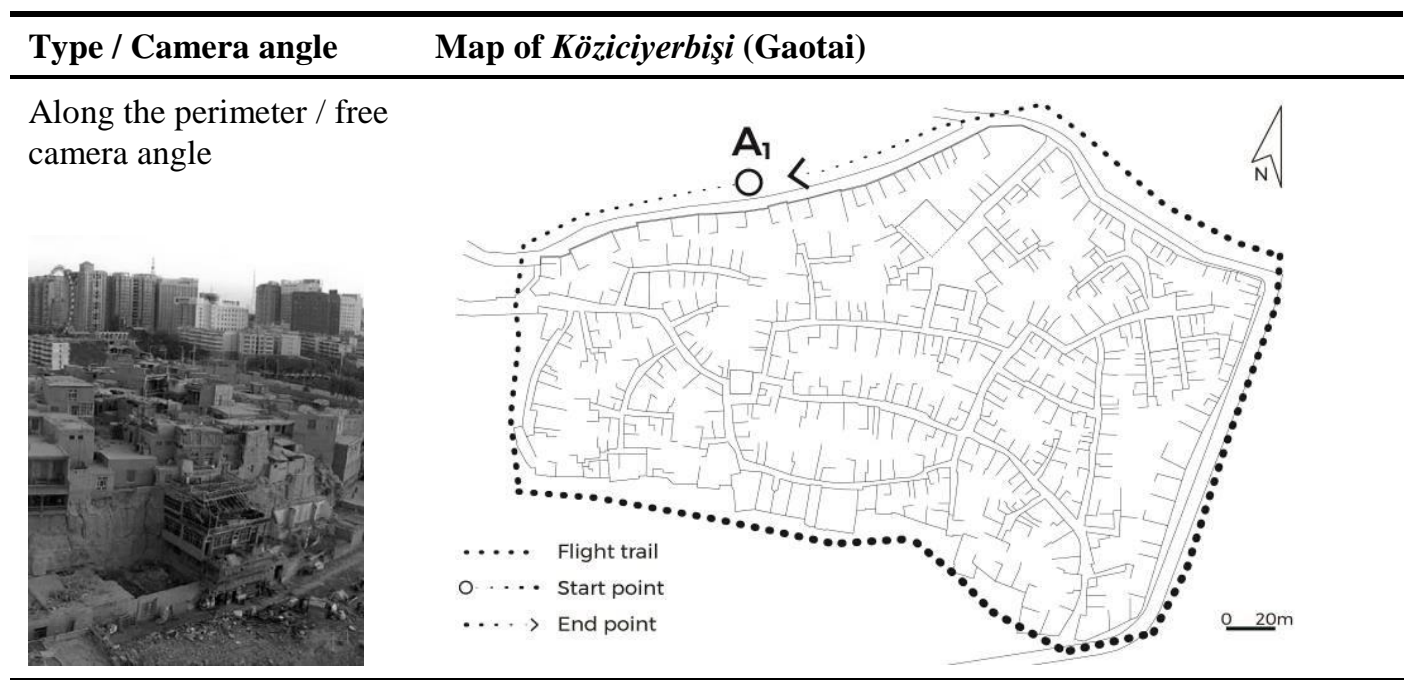

Random pattern / free camera angle
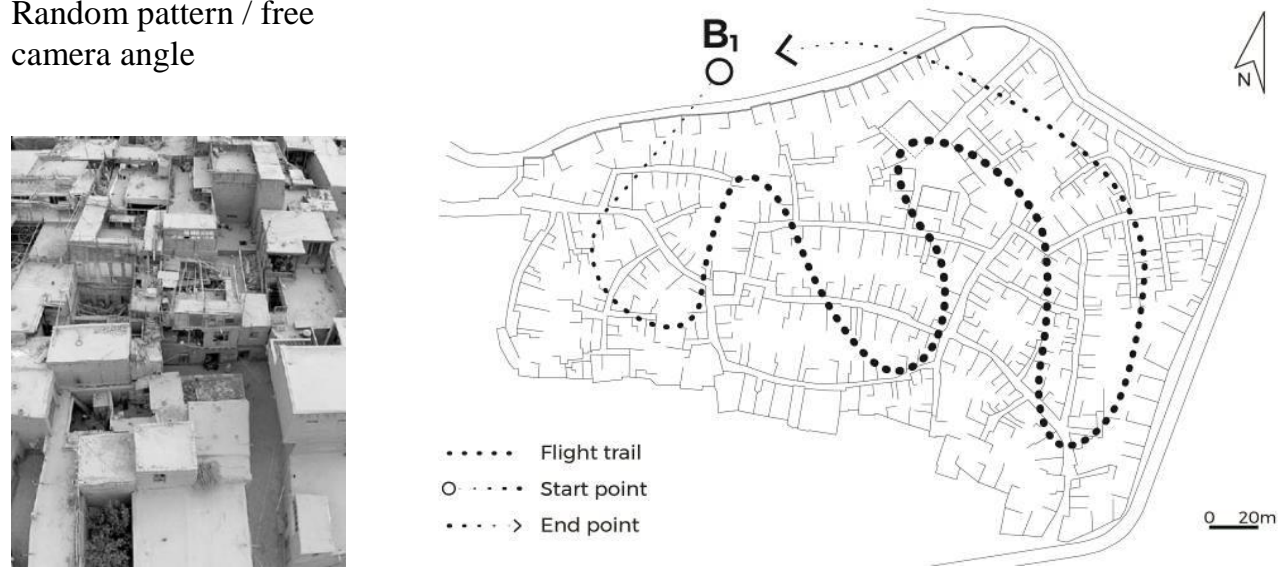

Narrow roads / orthophotography (90 degrees)
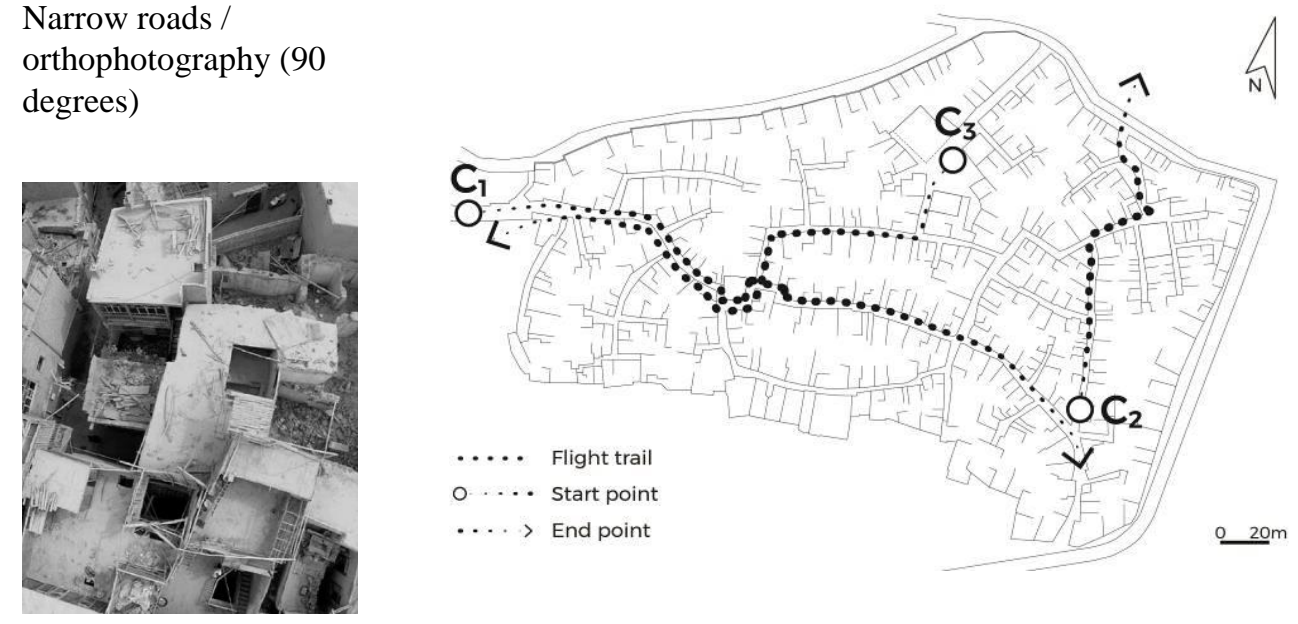


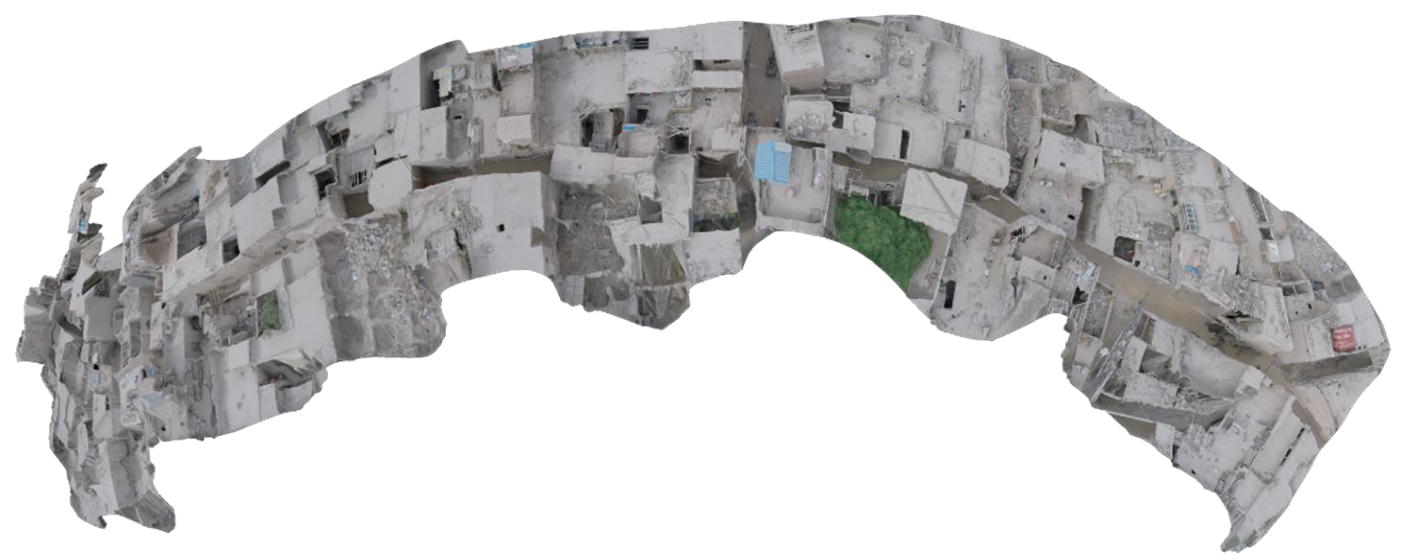

Fig. 61. The photogrammetric modelling result of the aerial imaging $\mathrm{C}_{1}$ in Köziciyerbişi (Gaotai).

Above image is a sample of the photogrammetric modelling method that will be elaborated in the following Chapter 4.3. High-resolution $4 \mathrm{~K}$ videos from aerial documentation were converted into still images, with their size reduced to remain manageable and reasonably accurate. In the following section, challenges of documentation activities are discussed based on the topics of accuracy, completeness and timeliness.

\subsubsection{Challenge}

Each method used for documentation in the fieldwork has its own limitations and challenges that led to concerns with a flexible methodology design. Table 31 shows the uses of each method in documenting the Köziciyerbişi (Gaotai) neighbourhood in Kashgar. One of the most challenging parts is interviewing the locals in Kashgar. The urban renewal project had been so controversial that residents were de facto forced to remain silent about expressing an opinion. In the current political atmosphere, structured interviews with people are difficult and subject to erratic comments. Therefore, interviews are kept unstructured at a minimum and only with regards to how the houses function at present and in the past without asking about the demolition process. In this regard, critical observation of daily works has been more helpful in the context of this research. Sound recordings are used as one of the intangible heritage documentation techniques. Field recordings are recorded in presentable formats based on a simple analysis method of waveform comparison. Visually comparing the waves of different street-sound pieces helps the design process in later stages to achieve a hierarchical order in association with the virtual visual experience. 
Table 31. Methods, data sources and their planned use.

\begin{tabular}{|c|c|c|c|}
\hline Method & Data source & Use & Location \\
\hline $\begin{array}{l}\text { Unstructured } \\
\text { interviews and } \\
\text { observation }\end{array}$ & Local people & N/A & $\begin{array}{l}\text { Throughout the entire } \\
\text { old-town }\end{array}$ \\
\hline CAD drawings & $\begin{array}{l}\text { Published and } \\
\text { unpublished materials } \\
\text { collected from local } \\
\text { administrative offices }\end{array}$ & 3D modelling & $\begin{array}{l}\text { Entire old-town, focus } \\
\text { on Köziciyerbişi } \\
\text { (Gaotai) }\end{array}$ \\
\hline Sound recording & $\begin{array}{l}\text { Field recordings from } \\
\text { shops, streets, houses } \\
\text { and public areas }\end{array}$ & $\begin{array}{l}\text { Interaction design, } \\
\text { modelling virtual } \\
\text { environment with } \\
\text { sound }\end{array}$ & $\begin{array}{l}\text { Throughout the entire } \\
\text { old-town }\end{array}$ \\
\hline Photography & $\begin{array}{l}\text { Houses, streets, and } \\
\text { public areas }\end{array}$ & $\begin{array}{l}\text { 3D modelling, } \\
\text { interaction design }\end{array}$ & $\begin{array}{l}\text { Throughout the entire } \\
\text { old-town, mostly in } \\
\text { Köziciyerbişi (Gaotai) }\end{array}$ \\
\hline Aerial video recording & $\begin{array}{l}\text { Neighbourhood from } \\
\text { above with }>30 \mathrm{~m} \\
\text { distance in } \\
\text { accordance with the } \\
\text { ethics approval }\end{array}$ & $\begin{array}{l}\text { 3D modelling, } \\
\text { interaction design }\end{array}$ & $\begin{array}{l}\text { Only Köziciyerbişi } \\
\text { (Gaotai) section of the } \\
\text { old-town }\end{array}$ \\
\hline
\end{tabular}

Considering all these challenges, the rest of the 3D documentation and representation methods are based on the concept of visual accuracy rather than on technical connotations imposed by the digital heritage forensics. 
Subchapter Outline

Purpose: The methods introduced in this section coincide with the 'Matters of Medium' (Chapter 3.3) of the 3D foundational aspects of virtuality. The primary goal is to show how the concept of representation can be understood from complementary geometrical and computational points of view.

Design/Approach: Low-cost photogrammetry is presented along with the introduction of software and methods that are used to process computational geometries. To extrapolate polygon mesh computing, this subchapter delineates the modelling process with tables, images and diagrams.

Findings: There are several aspects of digital geometry processing that are combined with the theoretical review of virtuality presented in Chapter 3.3. The concept of 'error' mentioned in Chapters 2.3.4 and 3.4.2 reappear here as polygon mesh processing causes glitches on $3 \mathrm{D}$ geometries.

Limitations/Implications: The findings of this section are connected to discussions on error, noise, entropy, innocence and indexicality.

Originality/Value: Representation of heritage environments in virtual reality is contingent on the suspension of disbelief about virtuality. Glitch concept remains misapprehended and undisclosed in the current digital heritage research. The myth of glitches on 3D representations are unveiled in this subchapter as to how it may enable the user's suspension of disbelief in the virtual museum.

Keywords. Representation; low-cost photogrammetry; glitch; Autodesk ReCap; computational geometry; polygon mesh.

To stimulate virtuality in the virtual museum, processing digital geometries is the first layer of the foundation, i.e. the main content, in the reconstruction of heritage environments. Digital geometry is "the geometry in the digital space" (Mukhopadhyay et al. 2013). In digital heritage, different types of geometries are computed via various means of representation. Methods of representation include a range of means such as those of 'digital forensics' (e.g. Webb and Brown 2016), high-definition scanning technologies (e.g. Kenderdine 2013), 3D digital reconstruction (e.g. Datta and Beynon 2014) and photogrammetry (e.g. Dorffner and Forkert 1998). Advanced digital technologies used for architectural and archaeological heritage generate a variety of computational geometrical representations that are addressed in research (Ippolito 2017; Zhou, Geng, and Wu 2012; Lu and Pan 2010; Ioannides and Quak 2014 and Münster et al. 2016). They are presented in biannual/annual conferences, seminars, workshops and meetings, such as "Digital Heritage Congress," "International Conference on Virtual Systems and Multimedia (VSMM)," "Eurographics Symposium on Grpahics and Cultural Heritage," "International Meeting on Archaeology, Graphic Informatics, Heritage \& Innovation (ARQUEOLOGICA 2.0)," 
“Computers Applications and Quantitative Methods in Archaeology (CAA)," “CIPA Heritage Documentation" and "World Cultural Heritage Conference (EuroMed)."

In this section, fundamentals of digital representation are explained as to how computational geometries are produced in a photogrammetric modelling process. This section is about the photogrammetric 3D reconstruction of the alleys in the neighbourhood of Köziciyerbişi (Gaotai) in Kashgar. This presents how processes of detailed capturing of wall surfaces that form the narrow lanes of Köziciyerbişi (Gaotai). Kashgar was once a farmstate owing to strong household structure based on courtyard typologies united along alleyways. The representation of this maze-like environment is instrumental to revealing the importance of the historic community-living in Kashgar.

3D digital reproductions help unfold the complexity of age-old structures. For this, custom methods are employed as built-in functions of commercial software in the current research. It is important to comprehend computational processes. In this section, polygon mesh modelling is explained based on photogrammetric data capture processes involved in this research.

\subsubsection{Low-Cost Photogrammetry}

Many media applications, from movie and game design industries to cultural heritage and architecture, prefer image-based modelling techniques to reconstruct real environments in virtual reality. Traditionally, museums have not been so interested in highly computational processes. It is explained in Chapter 2 that museums have been hindered from following state-of-the-art computing applications since the interest of computer scientists shifted away from museums. But museums were one of the first institutions that incorporated computer technologies into their workflow in the 1960s. Today, computing technologies become increasingly affordable and museums are interested in hiring professionals who can manage low-cost options. It can be predicted that new affordable technologies will play an essential role in bringing together professionals from the museum, heritage, architecture, computer science and many more fields. Photogrammetry is one of the buzzwords of this decade as anyone can capture 3D scenes from real environments by using low-cost or free software with user-friendly interface.

Photogrammetry is an image-based modelling method. It is often applied to situations when time and budget are limited to model sufficiently accurate 3D digital geometries. In photogrammetry, models can be produced as point-clouds that are produced by marking and aligning 2D photographs. Different methods of image-based modelling and laser scanning 
(e.g. Lidar surveys) are used in photogrammetry (Webb and Brown 2011b). A more scholarly term of Structure-from-Motion (SfM) is used in the field as well (Fukuda et al. 2017). Basically, photogrammetry can be understood as a science of measuring from photographs. It used to be a geological survey tool. With time and progression in technology, it has become a robust method for 3D modelling especially with sensitive camera lenses, advanced computers and low-cost software options. A low-cost photogrammetric modelling process demands a combination of understanding orientation, light and camera specs. The detail of the photographed content is vital for the quality of the model produced (Lievendag 2017). As presented in the previous section, the photogrammetric surveys in Kashgar are based on aerial and close-range techniques, which are two different techniques used in photogrammetry (Table 32). These two techniques require different comprehension of photographic resources. In aerial photogrammetry, geometries can be optimised by built-in functions of the software, such as the Nadir orientation if the camera angle is zero, i.e. perpendicular to the surface of the earth.

Table 32. Two main photogrammetry techniques.

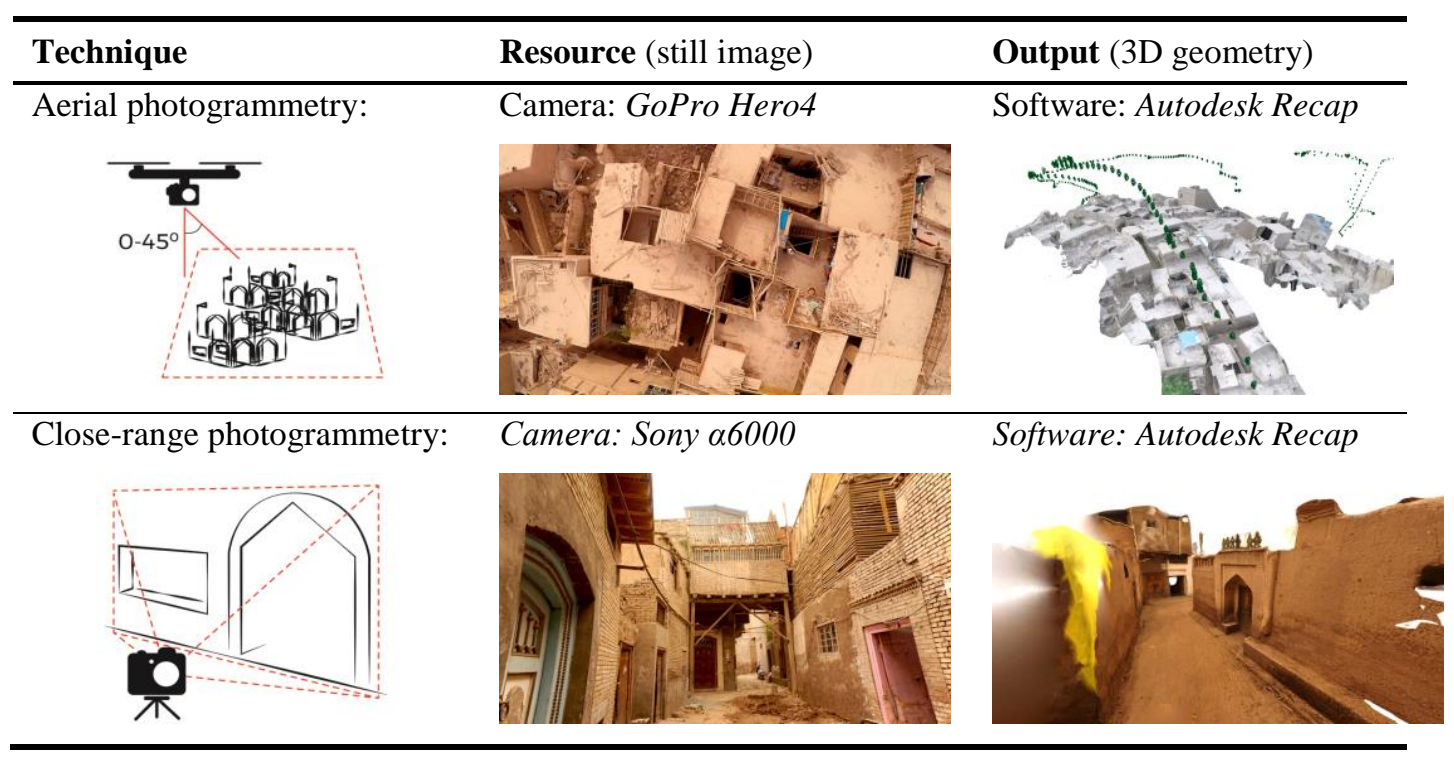

Software solutions come with monetary costs and choosing one for a specific task is an important step (Nikolov and Madsen 2016). Based on the three criteria they defined, an overall decision was made to use Autodesk products in the chain to have consistency between operations where Autodesk's FBX format through all steps could aid higherfidelity data exchange. 
158

\subsubsection{Instruction and Software}

There is a wide range of digital imaging techniques used for documentation in cultural heritage. Software packages including OpenMesh, CGAL and MeshLab provide mature algorithmic solutions for the end-user implementation of polygon mesh processing. But operations in these open-source tools may be laborious. To meet the commercial demands for studying heavy mesh models, powerful and specialised software, such as 3DF Zephyr, Agisoft and RealityCapture, offer options for low-cost photogrammetry. In this research, Autodesk ReCap 360, ReMake (formerly Memento Project; now ReCap Photo), Maya, 3ds Max and Mudbox are used. Alongside, McNeel's powerful NURBS modelling tool, Rhino is complementary. ReCap is the software deployed to do photogrammetric modelling by easily uploading photos (up to 250 images) on a cloud platform (Fig. 62).

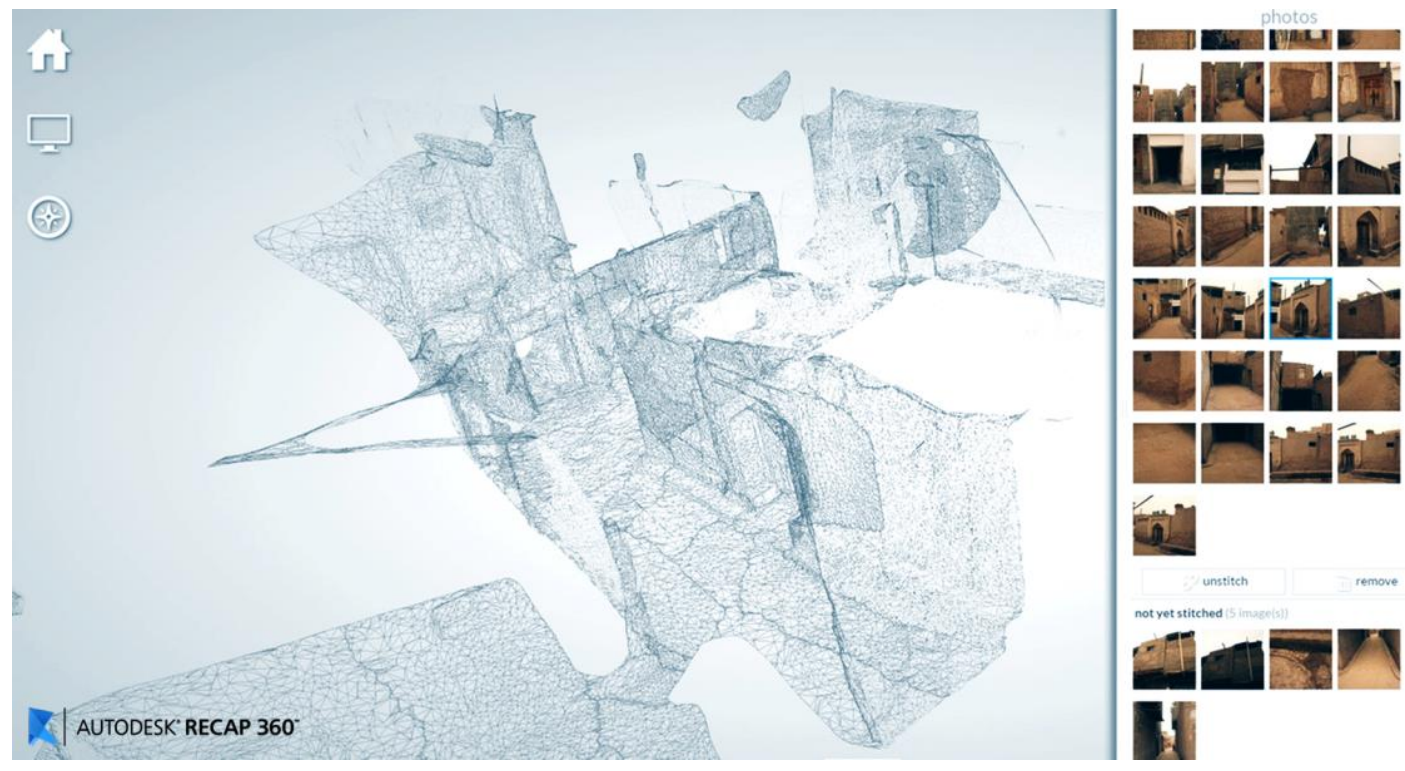

Fig. 62. Screenshot of a piece of raw Kashgar model in Autodesk Recap.

For the rest of the polygon mesh processing of SfM modelling, Autodesk ReMake is effective. It quickly reads files from Autodesk ReCap that operate the same Autodesk algorithm as ReCap. Polygon mesh processing is preferred because of two main reasons:

- In usage, it achieves a right balance between versatility and efficiency, with a user-friendly interface.

- In cost, it provides a free student license and is run on a cloud server, so there is no need to own high-end computer technology for the computationally intensive part of polygon mesh processing during image-based 3D modelling.

The following Table 33 summarises each software package used in this research for the 3D digital representations of Köziciyerbişi (Gaotai). 
Table 33. Software packages used in this research for $3 \mathrm{D}$ digital representation.

\begin{tabular}{|c|c|c|c|}
\hline Software & Usage & Formats & Information \\
\hline \multirow[t]{2}{*}{$\begin{array}{l}\text { Autodesk } \\
\text { ReCap } 360\end{array}$} & $\begin{array}{l}\text { Polygon-mesh photogrammetric } \\
\text { modelling (SfM) }\end{array}$ & \multirow[t]{2}{*}{$\begin{array}{l}\text { FBX, OBJ, } \\
\text { RCS, RCM }\end{array}$} & $\begin{array}{l}\text { Free education } \\
\text { license }\end{array}$ \\
\hline & Archiving models & & $\begin{array}{l}\text { Up to } 250 \text { photos } \\
\text { allowed to upload }\end{array}$ \\
\hline \multirow{7}{*}{$\begin{array}{l}\text { Autodesk } \\
\text { ReMake } \\
\text { (formerly } \\
\text { Memento } \\
\text { Project; } \\
\text { now ReCap } \\
\text { Photo) }\end{array}$} & $\begin{array}{l}\text { Polygon-mesh photogrammetric } \\
\text { modelling (SfM) }\end{array}$ & \multirow[t]{7}{*}{$\begin{array}{l}\text { FBX, RCM, } \\
\text { OBJ }\end{array}$} & \multirow[t]{7}{*}{$\begin{array}{l}\text { Free education } \\
\text { license }\end{array}$} \\
\hline & Joining separate mesh geometries & & \\
\hline & Remeshing & & \\
\hline & Smoothing & & \\
\hline & Simplification & & \\
\hline & Model repair & & \\
\hline & $\begin{array}{l}\text { Restoring models from Autodesk's } \\
\text { cloud service. }\end{array}$ & & \\
\hline \multirow[t]{2}{*}{ 3DF Zephyr } & \multirow{2}{*}{$\begin{array}{l}\text { Point-cloud photogrammetric } \\
\text { modelling (SfM) }\end{array}$} & \multirow[t]{2}{*}{ OBJ, ZEP } & Free limited license \\
\hline & & & Up to 50 photos \\
\hline \multirow{4}{*}{$\begin{array}{l}\text { Autodesk } \\
\text { Mudbox }\end{array}$} & Post-production & \multirow{4}{*}{$\begin{array}{l}\text { FBX, MUD, } \\
\text { OBJ }\end{array}$} & \multirow{4}{*}{$\begin{array}{l}\text { Free unlimited } \\
\text { education license }\end{array}$} \\
\hline & Retopologise mesh & & \\
\hline & Reduce mesh & & \\
\hline & Patch mesh & & \\
\hline \multirow{3}{*}{$\begin{array}{l}\text { Autodesk } \\
\text { Maya }\end{array}$} & Solid modelling & \multirow{3}{*}{$\begin{array}{l}\text { FBX, MB, } \\
\text { OBJ }\end{array}$} & \multirow{3}{*}{$\begin{array}{l}\text { Free unlimited } \\
\text { education license }\end{array}$} \\
\hline & Texture mapping & & \\
\hline & Photogrammetry post-production & & \\
\hline \multirow{4}{*}{$\begin{array}{l}\text { Autodesk } \\
\text { 3ds Max }\end{array}$} & Photogrammetry post-productions & \multirow{4}{*}{$\begin{array}{l}\text { FBX, MAX, } \\
\text { OBJ }\end{array}$} & \multirow{4}{*}{$\begin{array}{l}\text { Free unlimited } \\
\text { education license }\end{array}$} \\
\hline & $\begin{array}{l}\text { Combining large photogrammetric } \\
\text { mesh geometries }\end{array}$ & & \\
\hline & Mesh decimation & & \\
\hline & Texture mapping & & \\
\hline \multirow{3}{*}{$\begin{array}{l}\text { McNeel } \\
\text { Rhinoceros }\end{array}$} & Photogrammetry post-productions & \multirow{3}{*}{$\begin{array}{l}\text { FBX, 3DM, } \\
\text { OBJ }\end{array}$} & \multirow{2}{*}{$\begin{array}{l}\text { Paid education } \\
\text { license (university- } \\
\text { wide) }\end{array}$} \\
\hline & $\begin{array}{l}\text { Combining large photogrammetric } \\
\text { mesh geometries }\end{array}$ & & \\
\hline & $\begin{array}{l}\text { V-Ray rendering of solid models } \\
\text { imported from Autodesk Maya }\end{array}$ & & $\begin{array}{l}\text { Compatible with } \\
\text { Autodesk products }\end{array}$ \\
\hline
\end{tabular}

\subsubsection{Computational Geometry: Polygon Mesh Processing}

Computational geometry here delineates the production pipeline of polygon meshes as surface geometries that represent the walls of narrow laneways in Köziciyerbişi (Gaotai). Introduced above, digital geometry computation refers to polygon mesh processing here. Regarding the simplicity, flexibility and efficiency of polygon mesh representations, Botsch et al. (2010) examine digital processing techniques in several headings: surface 
representation, mesh data structure, differential geometry, smoothing, parameterisation, remeshing, simplification and approximation, model repair and deformation.

Surface representation, data structure, differential geometry and parameterisation are relatively abstract concepts; however, they are necessary to understand geometrical operations of polygon mesh processing. These abstract concepts are explained first. Smoothing, remeshing, simplification, repairing and deformation are less of an abstraction than operational definitions. Therefore they build on the explanation of abstract techniques. The following two sections (4.3.4 and 4.3.5) are aligned with these two concepts of digital geometry processing at the abstract and the operational levels. This section holds a place to combine the two separate approaches to scholarship in digital heritage. As reviewed in previous chapters, there is a lack of connection between humanities-based approaches (led by heritage discourse) and more scientific approaches (led by computer science experiments).

However, the concept of virtuality in a digital museum is mostly about the comprehension of both sides. Having presented theoretical views on it in Chapter 3.3, the following sections build a practical understanding of the $3 \mathrm{D}$ foundation of virtuality. Based on computational geometry processing, production pipeline of this research is extensively covered. Findings lead to the apprehension of glitch in polygon mesh processing, which will be discussed in the last section (4.3.6). Also, the delineation of the process in a transparent way with the following tables and figures is important for the paradata and prevalence criteria of the field. It is mostly based on polygon mesh processing in photogrammetric modelling, otherwise referred to as 'Structure from Motion' (SfM).

\subsubsection{Abstract Concepts of Digital Geometry Processing: Surface Representation, Data Structure, Differential Geometry and Parameterisation}

At the foundation of digital geometries lies the type of 'surface representation.' There are five main types of geometry representations used in digital heritage: polygon/triangle mesh, point cloud, solid surface models, volume graphics and procedural models (Zhou, Geng and $\mathrm{Wu} 2012$, 13). All representation types are examined in this research except volume graphics, which is used for analysis of 3D solid geometries that are common in medical imaging and scientific visualisation (Zhou, Geng and Wu 2012, 13). It is one of the major topics in computer graphics and rendering. But in this research, volume graphics is not examined directly. The rest of the surface representation types are documented in the following Table 34 . The content of images belongs to computational geometry activities in 
this research. Accordingly, all the software used to produce these images are informed as well.

Table 34. Five types of geometry representations used in this research.

\begin{tabular}{l}
\hline Representation \\
\hline Polygon/triangle mesh \\
Content: Section of 3D \\
photogrammetric Köziciyerbişi \\
(Gaotai) \\
Software: ReCap + ReMake+ \\
Mudbox
\end{tabular}

\section{Point cloud}

Content: Section of 3D photogrammetric Köziciyerbişi (Gaotai)

Software: 3DF Zephyr

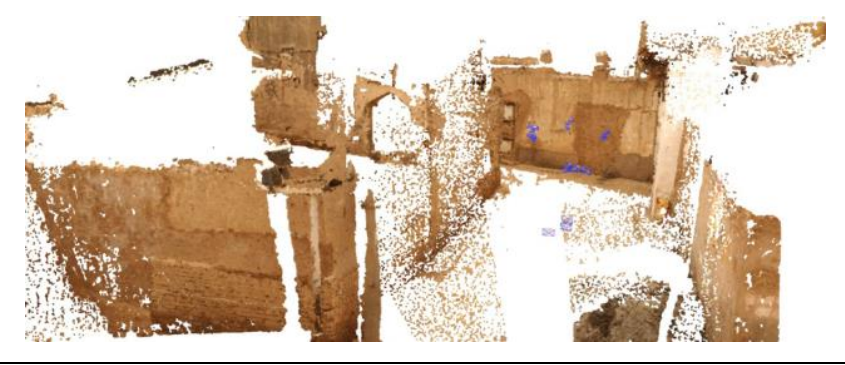

\section{Solid surface models}

Content: Low-poly 3D

Köziciyerbişi (Gaotai)

Software: Maya + Rhino

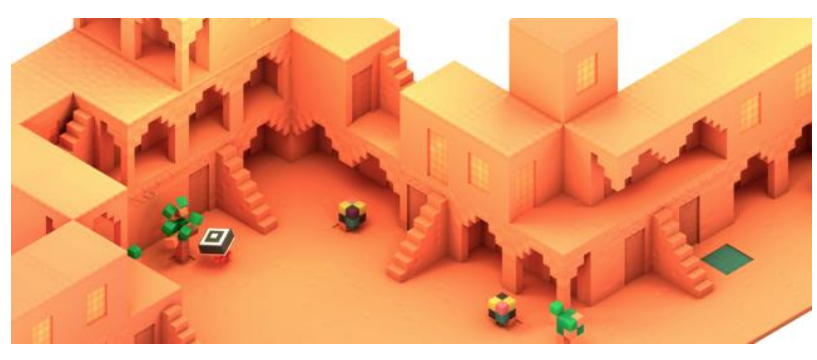

\section{Procedural models}

Content: Low-poly interactive version of 3D Köziciyerbişi (Gaotai) (Read from right to left)

Software: Maya + Rhino + Unity $3 D$

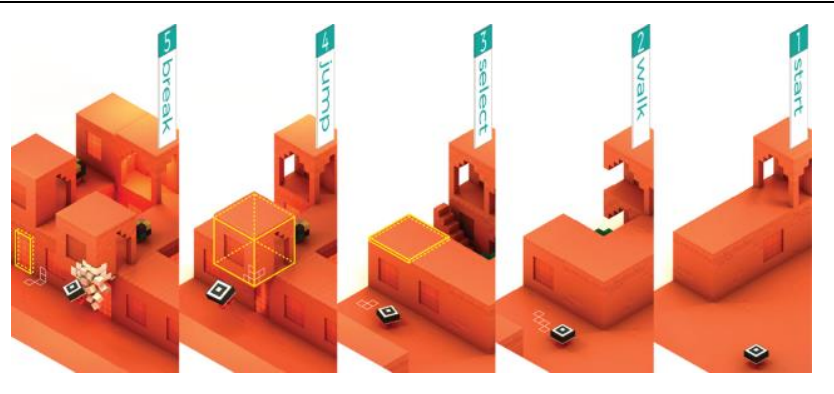

"The polygon mesh data structure is the most common and oldest modelling method for computer graphics" (SIGGRAPH Education 1999). The advantage of a polygon mesh model is fast and easy processing to obtain sufficiently good results. Despite low-level topological information attributed to each polygon mesh, the simplicity for Boolean operations is another property favoured as a data model in image-based modelling (Zhou, Geng and Wu 2012, 14). 
Input data of image-based scanning from SfM is represented as 3D free-form geometry in Euclidean space. This means that a multi-dimensional (i.e. volumetric) set of coordinate spaces is integral to the transformation of 2D images into 3D space. The background grid of these coordinates is a foundation to define and build the topological properties of a digital geometry. After revealing the findings about mesh data structure, the concept of a background grid is explained with differential geometry and parameterisation in polygon mesh processing. The process is examined in this research via different tools but mainly Autodesk ReMake (formerly Memento Project; now ReCap Photo). Table 35 summarises an assessment of polygon mesh representation based on three aspects realised during the photogrammetric modelling processes of Köziciyerbişi (Gaotai): fast modelling, large datasets and weak topological information.

Table 35. Assessment of polygon mesh representation.

\begin{tabular}{|c|c|c|}
\hline Aspects & Explanation & Observation \\
\hline Fast modelling & $\begin{array}{l}\text { Representation is based on linear } \\
\text { parameterisation without complex } \\
\text { mathematics, i.e. a piecewise } \\
\text { (referring to each triangle) linear } \\
\text { mapping of 3D space into 2D } \\
\text { parameter space (details in section } \\
\text { 'Parameterisation'). }\end{array}$ & $\begin{array}{l}\text { Almost all photogrammetric modelling } \\
\text { (SfM) software prefers polygon mesh } \\
\text { representation. Generating point-cloud } \\
\text { slows down the process and is subject } \\
\text { to the necessity of level of detail and } \\
\text { accuracy it provides. }\end{array}$ \\
\hline Large data & $\begin{array}{l}\text { Data structures of polygon mesh } \\
\text { are computationally designed to } \\
\text { maintain connectivity information } \\
\text { between all mesh polygons in } \\
\text { order to process faster the } \\
\text { manipulation methods. It comes at } \\
\text { the cost of massive datasets that } \\
\text { consume memory (details in the } \\
\text { section 'Mesh Data Structure'). }\end{array}$ & $\begin{array}{l}\text { It is usually required to decimate the } \\
\text { number of polygons after the } \\
\text { photographs are converted to 3D } \\
\text { geometrical mesh structures. In } \\
\text { Autodesk ReCap, models are often } \\
\text { good enough with around ninety } \\
\text { percent decimation. }\end{array}$ \\
\hline $\begin{array}{l}\text { Weak topological } \\
\text { information }\end{array}$ & $\begin{array}{l}\text { Piecewise linear mapping causes } \\
\text { the loss of control on entire mesh } \\
\text { at the global level (details in the } \\
\text { section 'Mesh Data Structure'). }\end{array}$ & $\begin{array}{l}\text { Manipulation of polygon mesh is time- } \\
\text { consuming. This is because of the lack } \\
\text { of control of overall geometry. } \\
\text { Although this is made by the structure } \\
\text { of data, it meanwhile conforms to the } \\
\text { criteria of fast modelling. }\end{array}$ \\
\hline
\end{tabular}

Representation types perform differently for the above-mentioned aspects. Upon preliminary tests, polygon mesh is proven as a more versatile SfM method in the context of this research that produces photogrammetric models. Accordingly, Autodesk ReCap and ReMake are selected as tools to continue reconstructing Kashgar's Köziciyerbişi (Gaotai) (Fig. 63). 


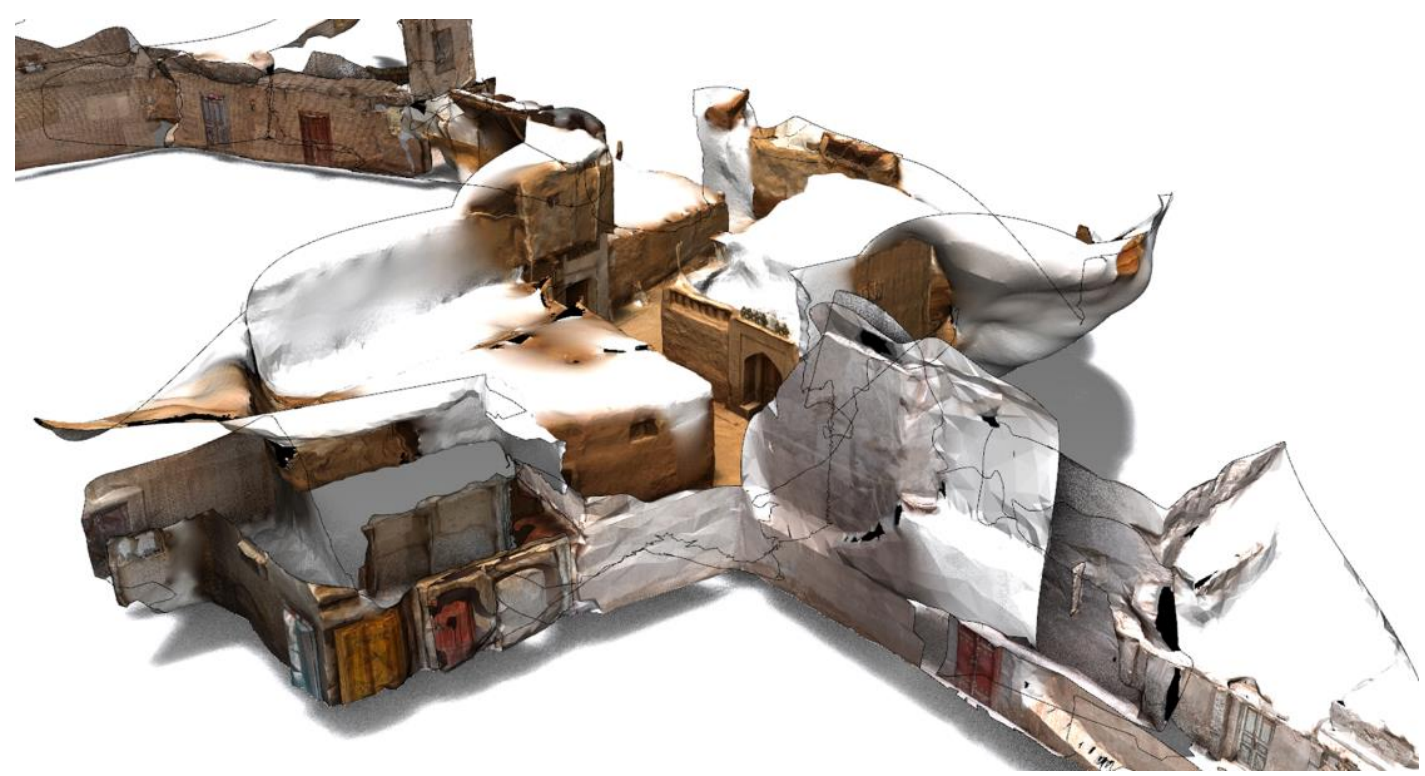

Fig. 63. Polygon mesh photogrammetric model of narrow roads in Köziciyerbişi (Gaotai) (image screenshot from Rhino; software in the production line: ReCap for photogrammetry, ReMake for joining digital geometries, Mudbox for combining files, Rhino for reducing the file size).

For efficient implementation, the type of the underlying 'mesh data structure' plays a role as well. The most generic type of polygon data structure is that of 'halfedge' method (Zhou, Geng and Wu 2012 13). In this data structure, the information embedded into each polygon triangle is stored by a relationship of three primitives that are vertices, faces and halfedges. The reason it is called halfedge is that each edge is attributed to two faces. By modelling the Kashgar old-town, it is figured out that ReMake uses halfedge data structure. In ReMake "the decimation algorithm [...] is following that of incremental decimation algorithm through half edge [sic] collapse, with several in-house optimization[s] to support large mesh data" (Aydin 2017b). Table 36 introduces the attributes of the halfedge-based data structure used in ReMake.

Table 36. Understanding the data structure of polygon mesh processing during photogrammetric modelling in SfM software used in reconstructing Köziciyerbişi (Gaotai).

\begin{aligned} & \hline Software $\begin{array}{l}\text { Autodesk ReCap } \\ \text { Autodesk ReMake }\end{array} \\ &$\hline Mesh data structure Halfedge-based data structure \\ & \hline Component Information restored in components \\ & Vertex Position $(p) \\ &$ Halfedge $(h) \\ &$ Face Face $(f) \\ &$ Halfedge Vertex $(v) \\ &$ Face $(f) \\ &$ Next halfedge $\left(h_{n}\right) \\ &$ Previous halfedge $\left(h_{p}\right) \\ &$ Opposite (inverse) halfedge $\left(h_{i}\right) \\ &$\hline\end{aligned}


Halfedge data structure helps search the neighbourhood relationships between the primitive components (vertices, faces and halfedges) (Botsch et al. 2010 21). Simple rules build connectivity information between 2-manifold mesh polygons. According to the Euler formula, the number of faces in 2-manifold geometries is about double the number of vertices and two-thirds of the number of edges. By splitting each edge into two, halfedge data structure actually turns an "unoriented" edge into two "oriented" edges (Botsch et al. 2010, 25). This, in turn, increases the cost of operation in comparison to other edge-based data structures. But it supplies simple topological information (connectivity) on the mesh. Fig. 64 illustrates the procedure of the halfedge data structure for forming mesh triangles.

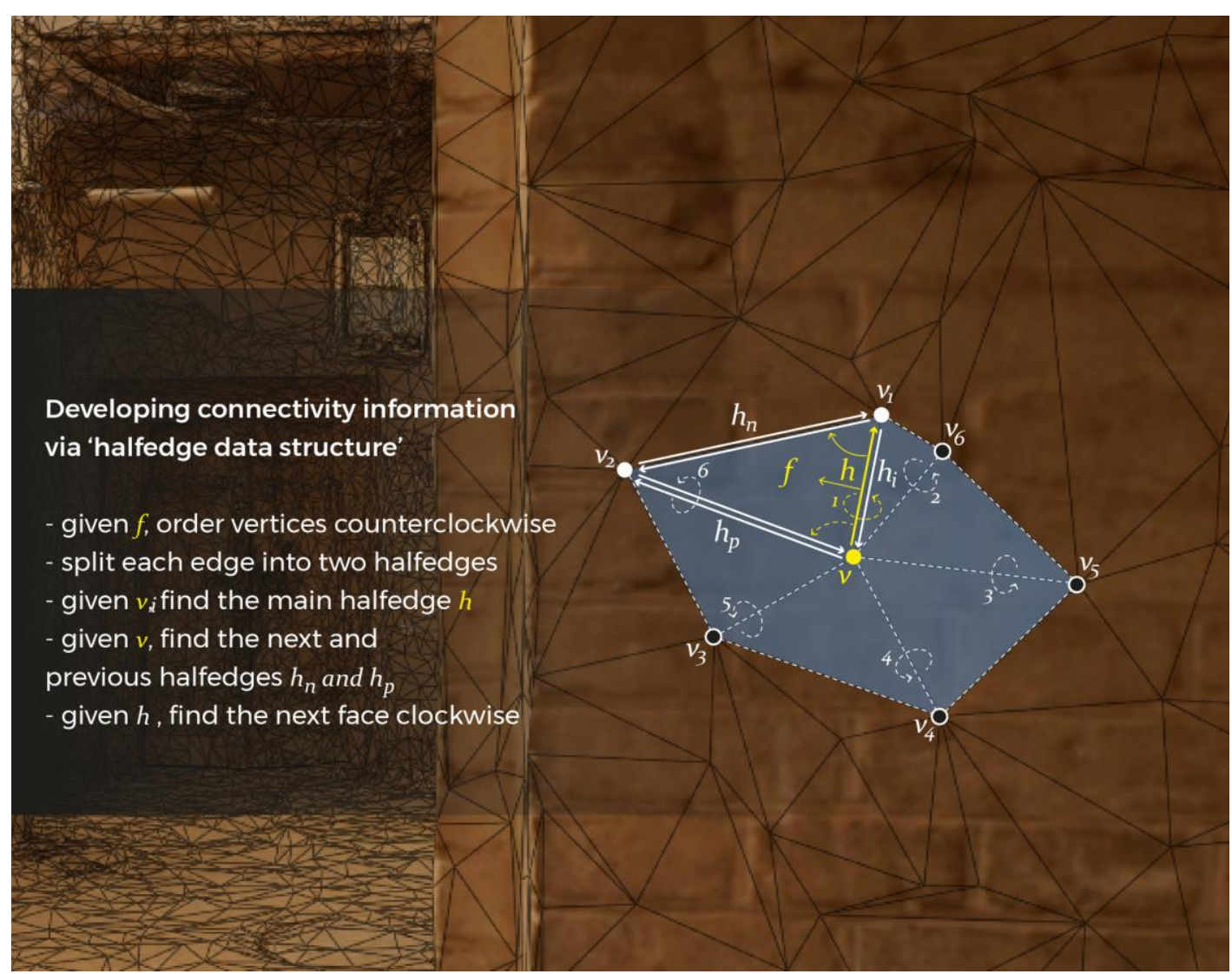

Fig. 64. A sketch of the halfedge data structure used in ReCap and ReMake for the implementation of connectivity information between polygons. Content: Section of 3D photogrammetric Köziciyerbişi (Gaotai). Illustration by Author.

Therefore, neighbour triangles recognise each other via information embedded locally; yet there is other information necessary to build a 3D digital geometry in SfM modelling. The location of vertices is needed to operate formulas during the transformation of $2 \mathrm{D}$ image-based information into polygons in three-dimension. These formulas are handled by 'differential geometry' and 'parameterisation' that are complementary concepts to each other. Mesh polygons are built on the scalar (e.g. length, curvature and area) and discrete 
attributes (e.g. vector and tensor) (Cohen-Steiner and Morvan 2006, 157; Botsch et al. 2010, 48). Smooth SfM surfaces of photogrammetric models stem from the derivation of these discrete elements (i.e. vectors) from $2 \mathrm{D}$ photographs. They are then calculated as 'differential' properties in 3D. These discrete elements allow mathematical operations. But this section is limited to explaining the role of 'vectors' with a simple diagram below to explain the underlying concept of 'differential geometry.' The image illustrates how polygon mesh processing is supported by the concept of differential geometry in order to generate 3D geometries from photographs (Fig. 65).

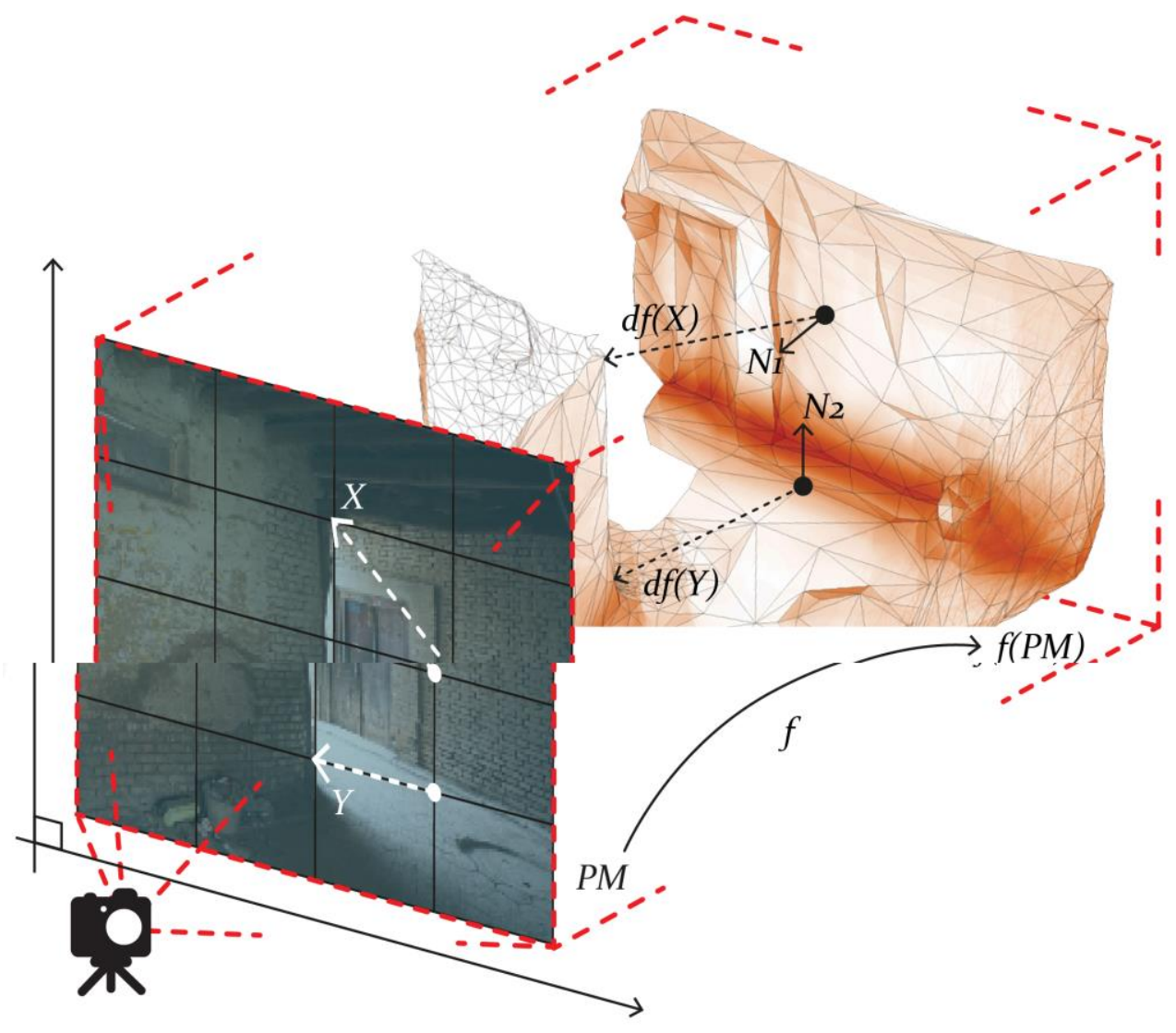

Fig. 65. The differential $d f$ of a photographic map $(P M)$ executes corresponding vectors $d f(X)$ and $d f(Y)$ on $f(P M)$. Vectors $X$ and $Y$ are stretched and transformed from one space to another. Content: $3 \mathrm{D}$ reconstruction of Köziciyerbişi (Gaotai). Illustration by Author.

Discrete properties are communicated by a differential $d f$ that operates a transformation matrix. By that, discrete information of the normal and other vectors is generated. This supports the calculation and implementation of smooth surfaces. This has many implications that make differential geometry a crucial concept in all sorts of polygon mesh processing. To give an example, differential geometry enables discrete parameterisation of surfaces that 
are conceived to construct dilapidated roof systems by maintaining constant thickness (Liu et al. 2006; Crane and Wardetzky 2017). Two main implications of differential geometry are commented on below.

The first one relates to the application processes of photogrammetry. All digital smoothing functions including remeshing, hole filling and mesh deformation are based on the concept of differential geometry (Botsch et al. 2010, 49). This means that glitches that may appear on photogrammetric models are caused by the lack of information to define discrete properties for differential geometry. In terms of virtuality, this will be defined as a challenge and opportunity in Chapter 4.3.6.

The second implication is related to the acquisition, i.e. image resources (photography and laser scanning data), that are processed during SfM modelling. Knowing what differential properties are relevant in relation to virtuality and the message that is aimed to be given helps take workable photographs. In general, it is not suggested to include photographs in poor lighting conditions. But in this research glitches are found to add value to virtuality, which is discussed in Chapter 4.3.6. Technically, the attribution of differential properties onto a 'geometric coordinate system' lays the first foundation of a parametric system (Botsch et al. 2010, 63). Table 37 positions the role of 'parameterisation' amongst the aforementioned abstract concepts of digital geometry.

Table 37. Purpose and implications of the abstract concepts of SfM digital geometry processing as happens in the 3D reconstruction of Köziciyerbişi (Gaotai).

\begin{tabular}{lll}
\hline Concept & Answer to & Implications \\
\hline Polygon mesh representation & $\begin{array}{l}\text { What type of } \\
\text { representation is used? }\end{array}$ & $\begin{array}{l}\text { Decision on software, data size, } \\
\text { modelling speed }\end{array}$ \\
\hline Halfedge-based data structure & $\begin{array}{l}\text { How are triangle faces } \\
\text { constructed? }\end{array}$ & $\begin{array}{l}\text { Simple topological information } \\
\text { (connectivity) }\end{array}$ \\
\hline Differential geometry & $\begin{array}{l}\text { How are surfaces defined } \\
\text { discretely (e.g. vectors and } \\
\text { normal) on polygons? }\end{array}$ & $\begin{array}{l}\text { Connectivity of surfaces onto } \\
\text { curvature dependencies and } \\
\text { smooth functions }\end{array}$ \\
\hline Parameterisation & $\begin{array}{l}\text { How can 3D digital } \\
\text { geometry be built from 2D } \\
\text { maps? }\end{array}$ & $\begin{array}{l}\text { Texture mapping, UV maps and } \\
\text { mesh operations (remeshing, } \\
\text { approximation, smoothing, etc.) }\end{array}$ \\
\hline
\end{tabular}

'Parameterisation' helps answer how to map texture on the mesh. Technically, it can be understood as a move before differential geometry in the hierarchy of processing polygon mesh. But it is closely related to differential geometry, which is the conceptualisation of discrete attributes on a parametric system. Simply put, differential geometry is the 
imagination of a system, e.g. Euclidean space that is already parameterised (Botsch et al. 2010, 63). Parameterisation explains how to construct the system.

There are mainly two groups of factors that influence the representation of geometry for efficiency. The first group is dependent on the process of acquisition and the second group is on the process of application. They are usually contradictory to each other. It means that a geometric representation that is most optimal for acquisition is likely to produce poor results for the application. Parameterisation of the geometry generates a mechanism between these two processes of acquisition and application.

A parametric representation of polygon mesh allows flexibility in operation between the processes of acquisition (resource) and application (outcome) (Botsch et al. 2010, 63). To do so, a 3D object is first aligned with a coordinate system and to facilitate conversion from one representation to another during different processes (Botsch et al. 2010, 64). In other words, it is a play of geometrical abstraction that enables the generation of different instances. Therefore, parameterisation is operational as well as conceptual.

There are two direct applications of parameterisation of geometry, which are texture mapping and basic operations, such as remeshing and decimation. The latter are presented in the next section (4.3.5). The former is used to generate UV maps to hold texture information on the polygon mesh. Texture mapping is the primary application of 'parameterisation' on which the visual glitches may appear if the algorithm is not able to correctly map the texture on the coordinates of the 3D geometry.

The photographs of Kashgar were taken at different times and in different environmental conditions such as cloudy days with evenly distributed light or sunny days that brighten white surfaces excessively, causing parts of the geometry to be unrecognised by the algorithm and thus creating random black holes. These are errors that are revealed in textures and other misalignments caused in the photogrammetric process. But it results in its own aesthetics, which is a result of an error in SfM modelling that misinterprets the pixels of a texture file (Fig. 66 and Fig. 67). It is a common parameterisation failure to introduce 'seams' to separate the polygon mesh into regions (Fig. 68). In this case, the algorithm of Autodesk fails to cull triangle data. Instead, it dispatches each pixel on texture onto polygon data based on the most extended list of triangle information. In these polygon mesh experiments, glitches appear at an abstract level of parameterisation. 


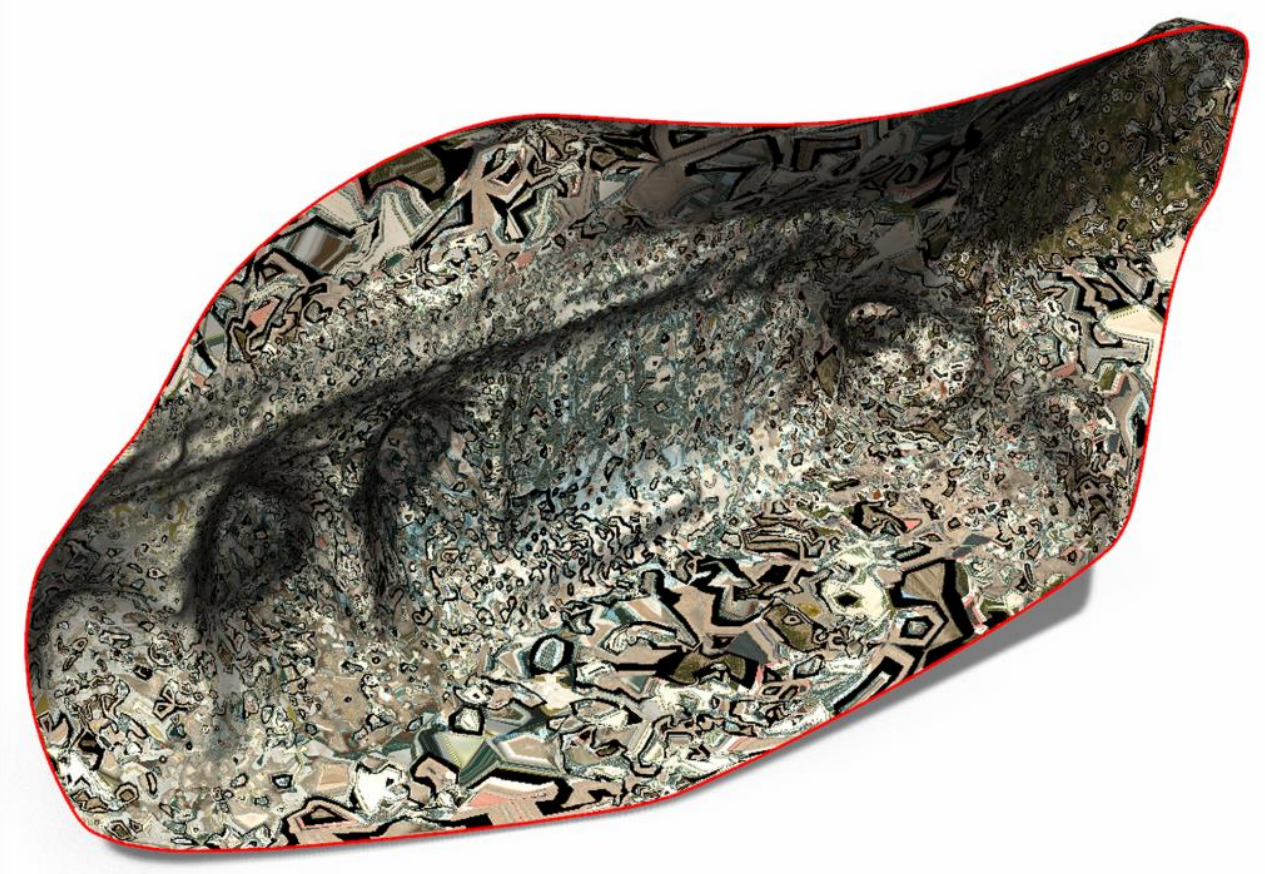

Fig. 66. Glitches during the modelling of the tomb of Mahmud Al-Kashgari (1005-1102) in Opal, Kashgar. Software: Autodesk ReCap. Illustration by Author.

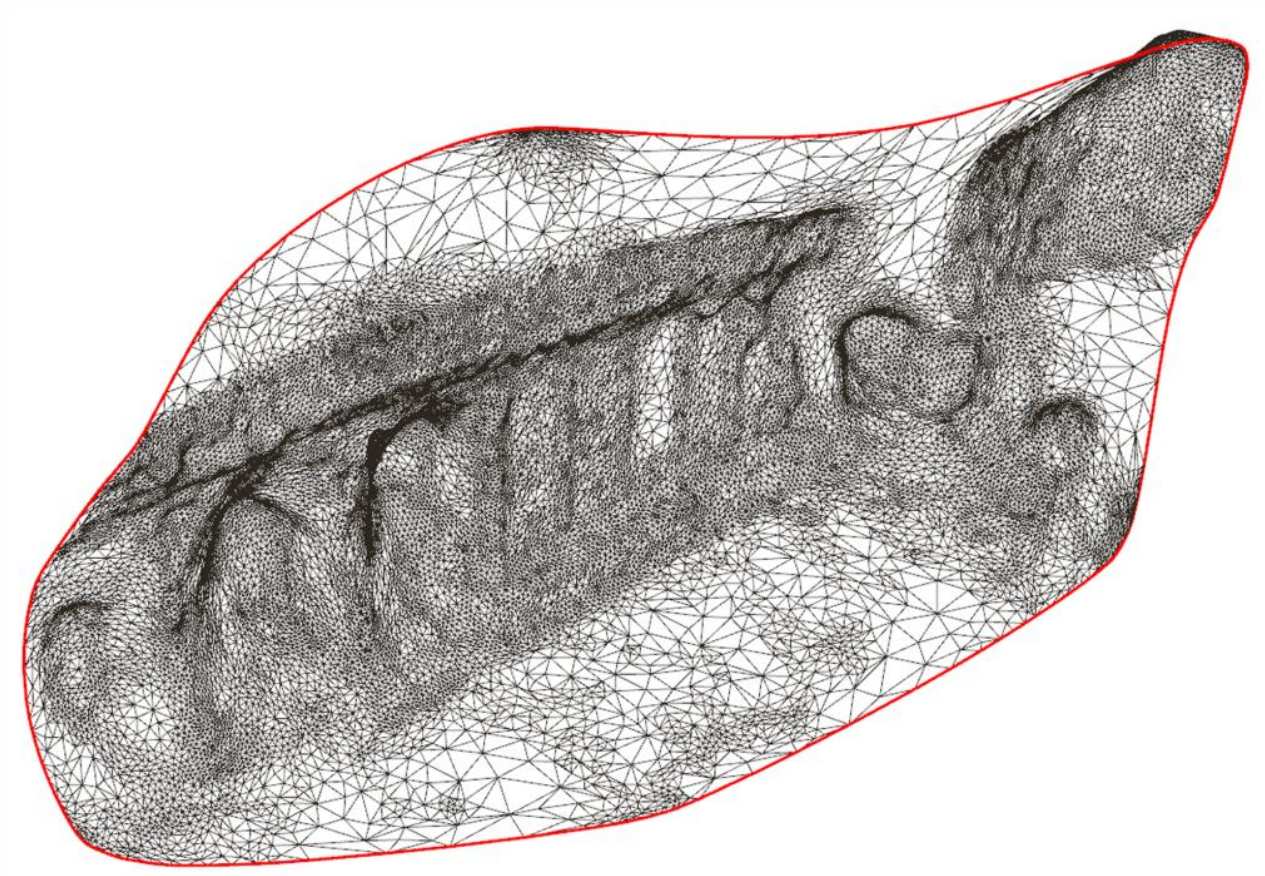

Fig. 67. Wireframe view of polygon mesh of the tomb of Mahmud Al-Kashgari (1005-1102) in Opal, Kashgar. Software: Autodesk ReCap. Illustration by Author. 


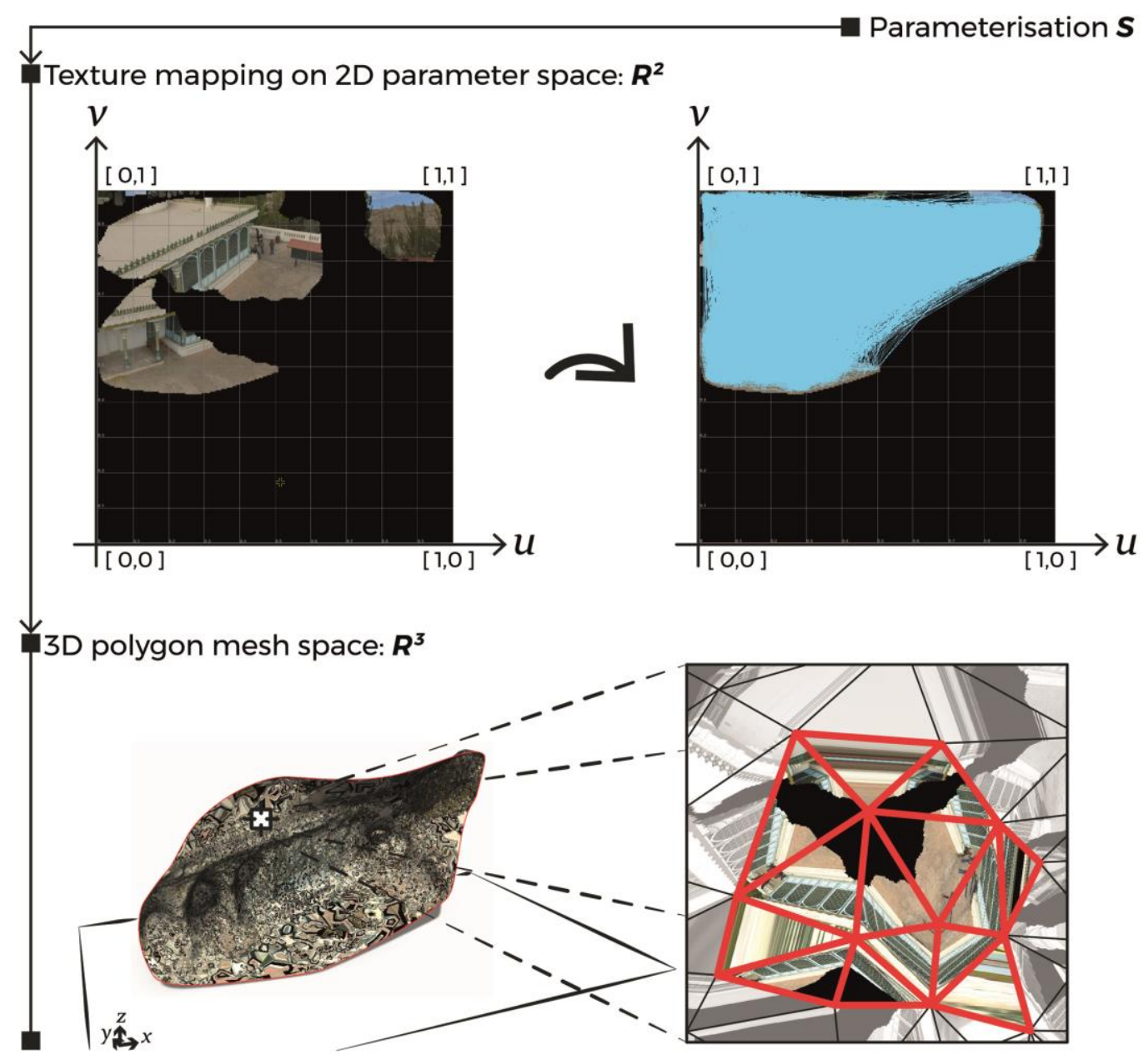

Fig. 68. Delineation of how glitches occur during parameterisation $(S)$ between $2 \mathrm{D}$ parameter domain $\left(R^{2}\right)$ and 3D mesh domain $\left(R^{3}\right)$. Software: Autodesk ReCap. Illustration by Author.

There may also be glitches in the operational domains of digital geometry processing. The next section will follow on this by presenting further glitch experiments during postprocessing in ReMake of SfM models from ReCap. These two software employ a virtually identical algorithm to process polygon mesh data.

\subsubsection{Operational Concepts of Digital Geometry Processing: Smoothing,}

Remeshing, Simplification and Approximation, Model Repair and

\section{Deformation}

This section explains operational techniques used in Autodesk ReMake (ReCap Photo as of December 2017). These operational concepts are encountered before and during the postprocessing of photogrammetric models. Original models that come from SfM (Structure from Motion) software (in this case, Autodesk ReCap) carry elaborate sets of information 
that are generated by an inbuilt algorithmic mechanism to handle the abstract concepts of the previous section. The output is usually incomplete and with flaws and defects. Operations like model repair, simplification and approximation are embedded as default functions in software to correct and complete the modelling process manually. While digitally reconstructing Köziciyerbişi (Gaotai), these operations are conducted to complete 3D digital models of narrow roads.

The first operation to decrease the computing power is 'simplification and approximation.' The decimation tool in ReMake decreases the number of polygons by percentage or number. It is observed that the model maintains a certain degree of similarity even if around 95 percent decimation is applied on the mesh. After decimation, texture images can hold more details compactly. Conversely, it reduces the degree of accuracy on polygon mesh representation. But digital models generated on ReCap and simplified on ReMake remain intelligible unless examined too closely (Fig. 69).

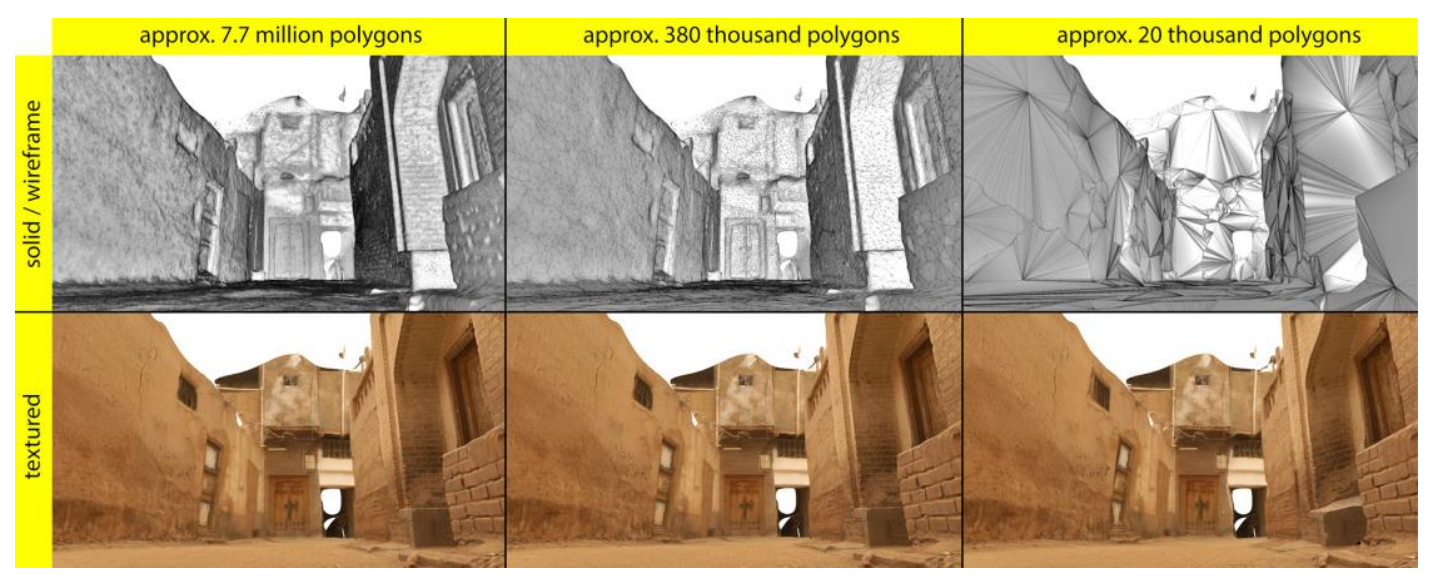

Fig. 69. The successive decimation of polygons by $95 \%$ from the left column to the right. Content: 3D reconstruction of Köziciyerbişi (Gaotai). Illustration by Author.

Having examined the abstract concepts of computational geometry in the previous section, this part is focused instead on the interface level instead of the mathematical background of each operational concept. Attene et al. (2013) provide a synopsis of flaws that can appear in most polygon mesh processing exercises. There are two main types of defects. In this regard, a glitch is either topological, which is generated by the application (in this case ReCap), or geometrical, which the software (in this case ReMake) offer users to fix methods. The former is examined in the previous section. In this part, the analysis is bounded to the taxonomy of the software used. It is understood in ReMake that there are three types of flaws and defects. Dangling 'particles,' 'holes' on mesh and 'self- 
intersections' of polygons are diagnosed and offered to be fixed by the software automatically (Table 38).

Table 38. Flaws and defects on polygon mesh diagnosed automatically in Autodesk ReMake during the 3D reconstruction of Köziciyerbişi (Gaotai).

\begin{tabular}{llll}
\hline & Particles & Holes & Self-intersections \\
\hline $\begin{array}{l}\text { Auto-fix } \\
\text { method }\end{array}$ & Remove & Fill (smooth or flat) & $\begin{array}{l}\text { Remove to create new } \\
\text { holes }\end{array}$ \\
\hline $\begin{array}{l}\text { Manual } \\
\text { tools }\end{array}$ & Smooth (surface or & Hole fill (smooth-flat) & Sculpt (push-pull) \\
& boundary) & Smooth (boundary) & Smooth (surface) \\
& Sculpt (push-pull) & Bridge gaps (smooth- & Re-triangulate \\
& Re-triangulate & flat) & Subdivide \\
& Subdivide & & Decimate \\
& Delete & & Delete \\
\hline
\end{tabular}

\begin{tabular}{l}
$\begin{array}{l}\text { Sample } \\
\text { (solid view } \\
\text { with } \\
\text { wireframe) }\end{array}$ \\
\hline $\begin{array}{l}\text { Sample } \\
\text { textured } \\
\text { view with } \\
\text { wireframe) }\end{array}$
\end{tabular}

Auto-fix methods are also offered by the software. Yet, it is often the case that these auto-fix methods result in more mesh errors, such as more holes generated after automatically erasing intersecting mesh triangles. However, polygon mesh processing is more complicated than isolating operational errors under three categories. In the production line, Autodesk Mudbox highlights a higher degree of categorisation with a taxonomy that defines twelve mesh errors for troubleshooting (Mudbox 2017). In this respect, Autodesk Maya, 3ds Max and Softimage are technically suggested for the most sophisticated clean-up purposes. As mentioned at the beginning of this section (Chapter 4.3.1), the concept of lowcost photogrammetry involves a user-friendly interface that is intelligible to standard 
172

laypersons. Although mesh tools of Mudbox (Fig. 70), Maya and 3ds Max are utilised in this research, the research in this section is interested in presenting results from ReMake (now ReCap Photo) as it is dedicated to the digital heritage field (Dzambazova 2015).

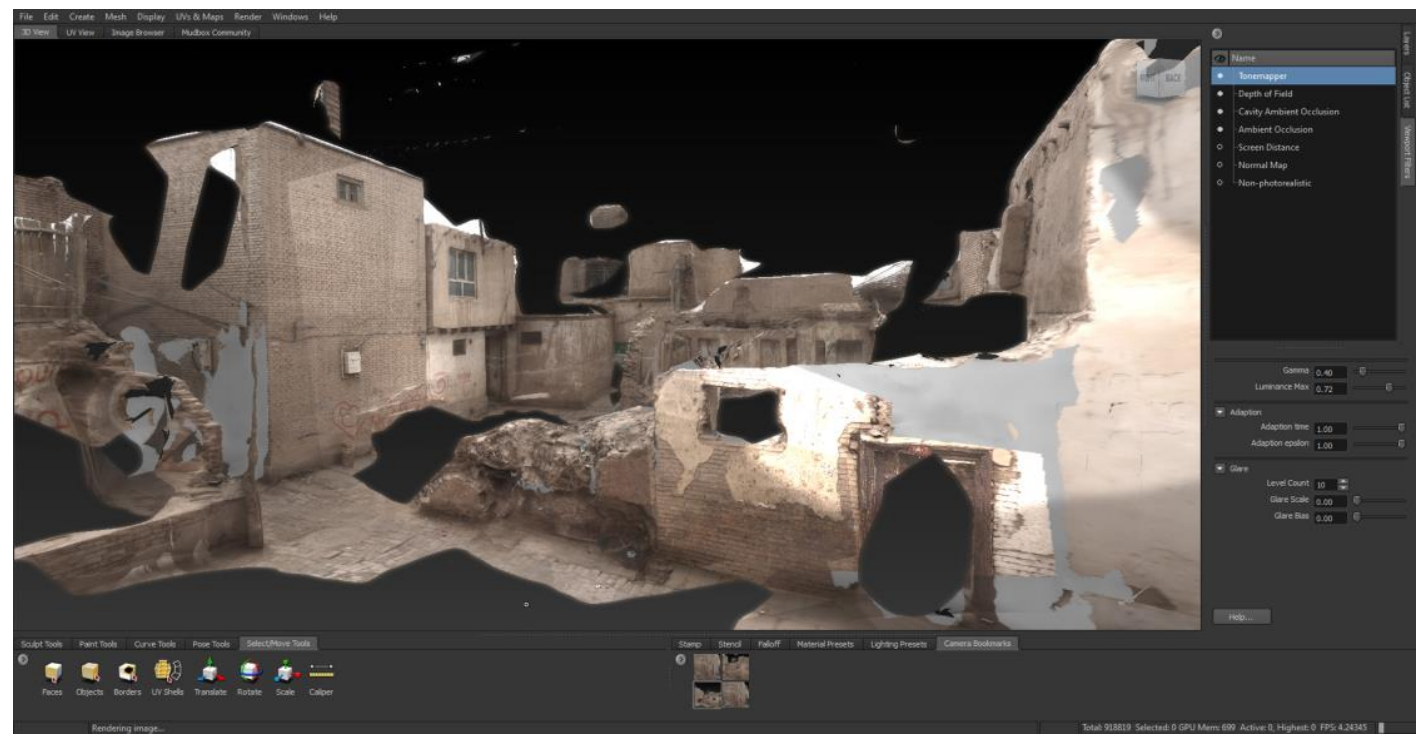

Fig. 70. Screenshot of the texture-mesh post-processing in Mudbox that offers fast solutions for inexpensive models. More complicated mesh models are resolved in Maya and 3ds Max.

\subsubsection{Challenge}

Overall, there is one purpose of 'correcting' polygon mesh, which is to generate a 2manifold geometry so that parameterisation does not fail (Chapter 4.3.4). Mesh connectivity that is held by the data structure, in this case the halfedge method (Chapter 4.3.4), is often compelled to fail by the complexity of actual heritage environments. In Kashgar, capturing reality involves tiny details, such as telephone cables and power lines wiring space between houses through alleyways. These trivial details may be important elements of authenticity. Their 'humility' may have the power to complete the last piece of a puzzle imagined in the 'virtuality' domain. Ironically, most software options in digital heritage are oriented towards 'fixing' these elements with their 'disappearance.' As such, glitch forces computational geometry to go beyond algorithmic definitions. But software engineering creates tools that are aimed for mass-production that is concerned with price. New tools are to be adapted to contemporary demand for mass-customisation that has to deal with variety. As stated in 'Shannon's Choice,' increase in entropy, which can be translated as maximum variety, noise, error, disorder or chaos, is equal to increase in information and meaning. Therefore, either topological or geometrical, failures of polygon mesh processing that are encountered 
in this research (see the glitch example on p. 169) are valuable, original and qualified findings. In this regard, developing cohesive apprehension of glitch aesthetics requires disclosing challenges in digital heritage that is sealed to an 'epistemological crystallisation' that risks the discourse into concealment (see Chapter 3.5).

Low-cost computation has become increasingly more prevalent for constructing content. Contemporary museums are entitled to integrate their managerial workflow with new strategies for benefiting user-generated content. These findings potentially generate new fields of interest in other subfields. Virtual object restoration is a topical subject in the field of archaeology (Papaioannou et al., 2017). In the object-centred field of archaeology, it is assumed negative if a generic object has missing parts that require more interpretation and lead to "greater ambiguity" (2). In contrast to the views of knowledge as a social construct, the criteria in these approaches are to seek information with no room for ambiguity; yet this view might be challenged in the context of the emerging notion of museums that is to promote the transformation of information into knowledge based more on social and participatory processes and relations than the objects.

Having demonstrated how polygons of the 3D mesh are represented and processed, this section builds a discussion on 'glitch aesthetics.' This triggers a discussion about authenticity, identity and human-machine communication that will be touched upon in the next section (Chapter 4.4). Thought-provoking exercises of glitch aesthetics enable the domain of 'virtuality' to be expressed. To recall from Chapter 2.4.2, the word 'virtual' comes from virtus (Latin: strength, manliness, virtue), which conceptualises "force or power" (Ryan 2015, 18). The qualitative findings from 3D representation exercises suggest that virtuality can be strengthened by escaping from, in the words of Heidegger (1977, 21), Ge-stell, or Enframing.

Glitches encountered during the entire computational geometry processing have reached an unmanageable amount of complexity and volume that was difficult to keep track entirely. So, a decision was therefore made to continue experimenting with a scenario that is sufficiently intelligible to draw conclusions. Glitched photogrammetric models were selected based on the criteria that the result is not too chaotic to fit in the volume of this research. The quality of the virtual environments made of the photogrammetric models is shown in Appendix C (Page 315). 


\subsection{Dissemination}

\section{Subchapter Outline}

Purpose: In Chapter 3.4, the 'Matters of Audience' were thematised with 'glitch aesthetics,' which were examined in the previous subchapter (4.3). Given that there occur glitches from processing photogrammetric geometry, they are also imputable to user-generated content on which this part is built. The goal of this section is to highlight the indexicality as a means to unfold the 'glitch aesthetics' ascribed to the active participation of users for heritage content-making in museums.

Design/Approach: It is presented how different methods are combined to generate alternate realities to tell a story of Kashgar's old-town. In a review of these methods, user-generated content is positioned in a systematic understanding of the key pleasures in cyberspace. Based on Janet Murray's classification of immersiveness, agency and transformation, this subchapter is designed to discuss the challenges of active participation in the technologies of the New Museology.

Findings: Based on experimental methods and demos, the research detects a focus area to evaluate the indexicality of user-generated content.

Limitations/Implications: Experimenting with participatory and interactive means of content generation in 'alternate realities,' the foundation of a specific research scenario is developed for further examination in the following subchapters.

Originality/Value: This subchapter connects the previous discussion of 'glitch aesthetics' to observations made on user-generated content through specific cases of dissemination methods used in this research.

Keywords. User-generated content; alternate realities; immersiveness; Hyve-3D; Unity3D; online sharing.

Having presented methods used for documentation and representation, this subchapter is focused on the dissemination of digital heritage based on two experiments. Immersiveness is tested based on content generated by participants. Additionally, unstructured interviews and observations are used to make conclusions about the user experience. The SfM models of the previous subsection are used as the original content in the experiments. The outcome of each experiment is analysed qualitatively.

The experiments were carried out as part of two public events. They are called Event I and Event II, whereas the former is occasionally described as the 'CAADRIA workshop' and the latter is the 'public demo at Te Papa Museum.' Table 39 describes these events with information about the events, organisers and hosting organisation and info including dates and venues. By that, the table acknowledges the contribution of organiser partners but the research activities entirely belong to Author, and this subchapter is focused on a presentation of the qualitative findings from Events I and II. These experiments precede a third one called Event III (it is demonstrated in Chapter 5) that was also conducted as part of Event I, which included different activities for workshop participants. 
Table 39. Event details to experiment with immerisiveness.

\begin{tabular}{|c|c|c|c|c|}
\hline Event & Organisation & Host occasion and info & Description & Poster \\
\hline $\begin{array}{l}\text { Workshop } \\
\text { Title: } \\
\text { User engagement } \\
\text { in Digital Heritage } \\
\text { via Hyve-3D }\end{array}$ & $\begin{array}{l}\text { Champions (Contribution): } \\
\text { Serdar Aydin (VUW) }(25 \%) \\
\text { Prof Marc Aurel Schnabel (VUW) }(25 \%) \\
\text { Prof Tomás Dorta (UdeM) (25\%) } \\
\text { Davide Pierini (UdeM) }(25 \%) \\
\\
\text { Institutional mentor: } \\
\text { Dr Dominik Holzer (UoM) } \\
\text { Dr Stanislav Roudavski (UoM) }\end{array}$ & $\begin{array}{l}\text { CAADRIA } 2016 \\
\text { Conference Workshops } \\
\text { Date: } \\
\text { March 29-30, } 2016 \\
\text { Venue: } \\
\text { Melbourne School of Design } \\
\text { University of Melbourne } \\
\text { Melbourne, Australia }\end{array}$ & $\begin{array}{l}\text { Organised to test digital } \\
\text { heritage content of this } \\
\text { research in an immersive } \\
\text { VR application. } \\
\text { The number of participants: } \\
8 \text { (on-site) }+2 \text { (remote) }\end{array}$ & 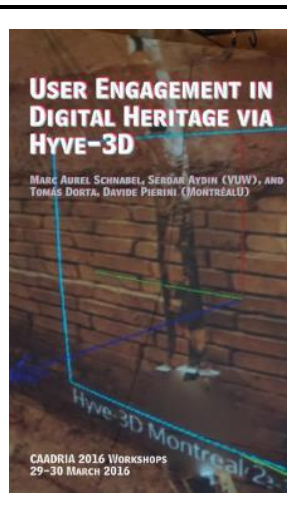 \\
\hline $\begin{array}{l}\text { Public demo show } \\
\text { Title: } \\
\text { Hyve-3D and the } \\
\text { Kashgar } \\
\text { Photogrammetry } \\
\text { Project }\end{array}$ & $\begin{array}{l}\text { Champions (Contribution): } \\
\text { Serdar Aydin (VUW) (60\%) } \\
\text { Prof Marc Aurel Schnabel (VUW) (15\%) } \\
\text { Tane Moleta (VUW) (15\%) } \\
\text { Assoc. Prof Leon Gurevitch (VUW) (10\%) } \\
\text { Institutional mentor: } \\
\text { Dr Hazel Bradshaw (Te Papa) }\end{array}$ & $\begin{array}{l}\text { Te Papa Talks } 2017 \\
\text { Virtual Realities } \\
\text { Date: } \\
\text { March 17-20, } 2017 \\
\text { Venue: } \\
\text { Te Papa Museum } \\
\text { [The Museum of New } \\
\text { Zealand] } \\
\text { Wellington, New Zealand }\end{array}$ & $\begin{array}{l}\text { Demonstrated to experiment } \\
\text { public engagement with } \\
\text { polygon mesh SfM models } \\
\text { of Kashgar screened through } \\
\text { a highly immersive VR tool } \\
\text { called Hyve-3D. } \\
\text { The number of participants } \\
\text { (and visitors): } \\
35 \text { (150) }\end{array}$ & $\begin{array}{l}\text { Te Papa } \\
\text { Talks }\end{array}$ \\
\hline
\end{tabular}




\subsubsection{A Hybrid Immersive Environment: Hyve-3D}

Hyve-3D is identified as "a new representational ecosystem," which means that it hybridises alternate realities of a myriad of media experiences (Dorta, Kinayoglu and Boudhraa 2016). Hyve-3D is used as part of an international collaboration that primarily served this research. The researcher partners from the School of Architecture at Victoria University of Wellington (VUW) and the School of Design at the University of Montreal (UdeM) worked together to visualise digital heritage content in Hyve-3D. It is used in this research as a means to allow collaborative and real-time sketching in 3D. Hyve-3D uses a user-friendly interface that is designed for anybody who can draw. The visualization system of Hyve-3D is an anamorphic trompe-l'oeil projection on a spherical concave screen that covers a five-to-five meter physical space. Of about twenty viewers within this space, one of them controls the navigation on screen. The size of virtual communities depends on the number of tablets connected to the system. It has a laptop station that stands in the middle of the space made by the concave screen (Fig. 71).

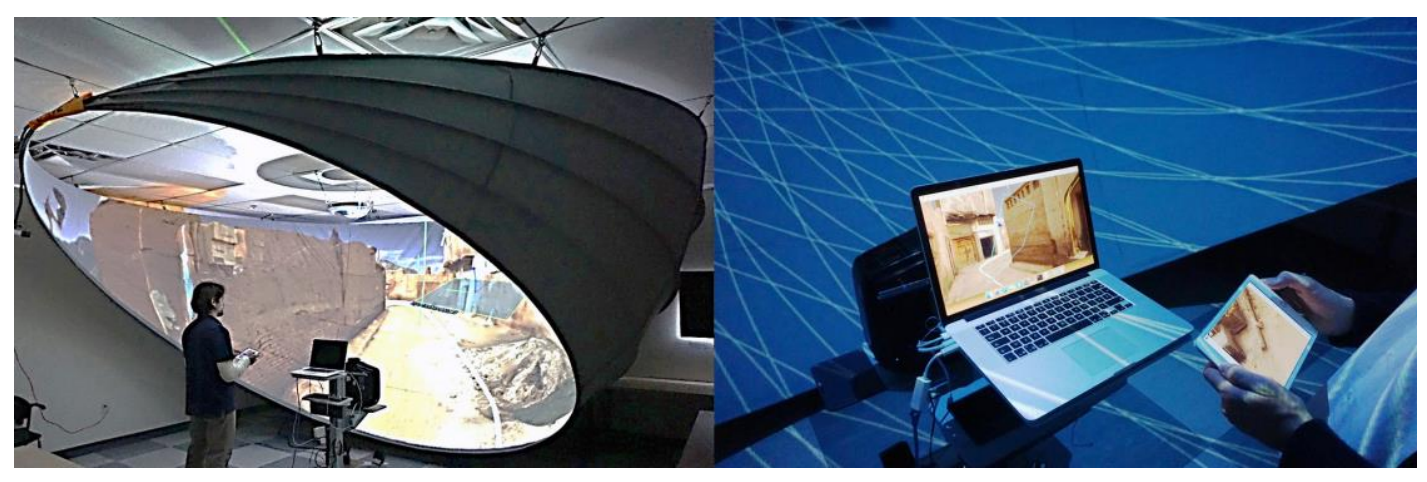

Fig. 71. Hyve-3D's concave screen and the laptop station. Photos by Tomás Dorta. Adapted from Schnabel et al. (2016).

Hyve-3D is an innovation of a virtual reality research CAAD (Dorta 2004). It has been extensively described in the literature and continuously evolved with updates. The research on the use of Hyve-3D is focused on several topical areas of user-oriented studies that are obtained from the evaluation of different aspects, such as cognitive learning (Calvo, Dorta and Robert 2014), collaborative design (Schnabel et al. 2016; Dorta, Kinayoglu and Hoffmann 2016), design ideation (Dorta and Perez 2006; Dorta 2008) and human-computer interaction in museums (Beaudry Marchand, Han and Dorta 2017). This section refers to previous research for further details on Hyve-3D. Below Table 40 introduces the features of Hyve-3D supported by brief explanation boxes and Fig. 72 diagrammatically shows the components of the hardware system constituting Hyve-3D. 
Table 40. Summary of Hyve-3D features.

\begin{tabular}{|c|c|c|}
\hline Features & Items & Explanation \\
\hline Immersive setup & $\begin{array}{l}\text { 1. Immersive } \\
\text { - Laptop station } \\
\text { - Half-dome mirror } \\
\text { - Concave curtain } \\
\text { - Tablets } \\
\text { 2. Non-immersive } \\
\text { - Desktop or laptop computer }\end{array}$ & $\begin{array}{l}\text { Immersive and non-immersive } \\
\text { setups are designed to help } \\
\text { produce content through } \\
\text { different interaction means. } \\
\text { They can be combined with a } \\
\text { local network. }\end{array}$ \\
\hline Navigation & $\begin{array}{l}\text { 1. Global navigation } \\
\text { - Controls the view screened on the concave } \\
\text { curtain } \\
\text { - Allows walkthrough in virtual space } \\
\text { 2. Local navigation } \\
\text { - Controls a rectangular reference plane on the } \\
\text { screened view } \\
\text { - Represents a viewer interacting with a tablet } \\
\text { - Translates sketches drawn on tablets to the } \\
\text { virtual reference plane }\end{array}$ & $\begin{array}{l}\text { The two navigation types } \\
\text { enable multi-dimensional } \\
\text { engagement. They play an } \\
\text { important role to generate a } \\
\text { special aura of a } \\
\text { 'representational ecosystem' } \\
\text { that blends hybrid ways of } \\
\text { interaction within virtual } \\
\text { space. }\end{array}$ \\
\hline $\begin{array}{l}\text { Content } \\
\text { making }\end{array}$ & $\begin{array}{l}\text { 1. 3D models (import) } \\
\text { 2. 3D sketches } \\
\text { 3. Point-cloud (import) }\end{array}$ & $\begin{array}{l}\text { The system does not support } \\
\text { 3D model-making. But the } \\
\text { visualisation of 3D geometries } \\
\text { and point-cloud data has a role } \\
\text { of control. }\end{array}$ \\
\hline File formats & $\begin{array}{ll}\text { 1. } & \text {.hyve (for Hyve-3D files) } \\
2 . & \text {.obj (for generic 3D data) } \\
3 . & \text {.ply (for point-cloud data) } \\
4 . & \text {.dxf (for exporting sketches) }\end{array}$ & $\begin{array}{l}\text { Only two file formats are } \\
\text { supported. '.hyve' is read by } \\
\text { default. }\end{array}$ \\
\hline Sketching & $\begin{array}{l}\text { 1. Physically drawn on tablets } \\
\text { 2. Virtually hold on reference planes that are } \\
\text { correspondent to tablets }\end{array}$ & $\begin{array}{l}\text { Many sketches drawn together } \\
\text { from different orientations of } \\
\text { angle and position can generate } \\
\text { visually 3D entities. }\end{array}$ \\
\hline Editing & $\begin{array}{l}\text { Basic transformations: } \\
\text { - Move } \\
\text { - Copy } \\
\text { - Delete } \\
\text { - Paste } \\
\text { - Scale }\end{array}$ & $\begin{array}{l}\text { These editing operations can } \\
\text { be applied on both imported } \\
\text { 3D digital geometries and user } \\
\text { sketches. }\end{array}$ \\
\hline Collaboration & $\begin{array}{l}\text { 1. Local network } \\
\text { 2. Remote network }\end{array}$ & $\begin{array}{l}\text { Distant collaboration is } \\
\text { supported via a remote } \\
\text { network connection. It can } \\
\text { connect all immersive and non- } \\
\text { immersive setups located in } \\
\text { different places in the world. }\end{array}$ \\
\hline Physical space & $\begin{array}{l}\text { Width: } 5 \text { meters } \\
\text { Length: } 5 \text { meters } \\
\text { Height: } 3 \text { meters }\end{array}$ & $\begin{array}{l}\text { The physical space is } \\
\text { sufficiently large to trigger } \\
\text { socialisation amongst viewers } \\
\text { who have to communicate in } \\
\text { order to navigate through } \\
\text { space. }\end{array}$ \\
\hline
\end{tabular}




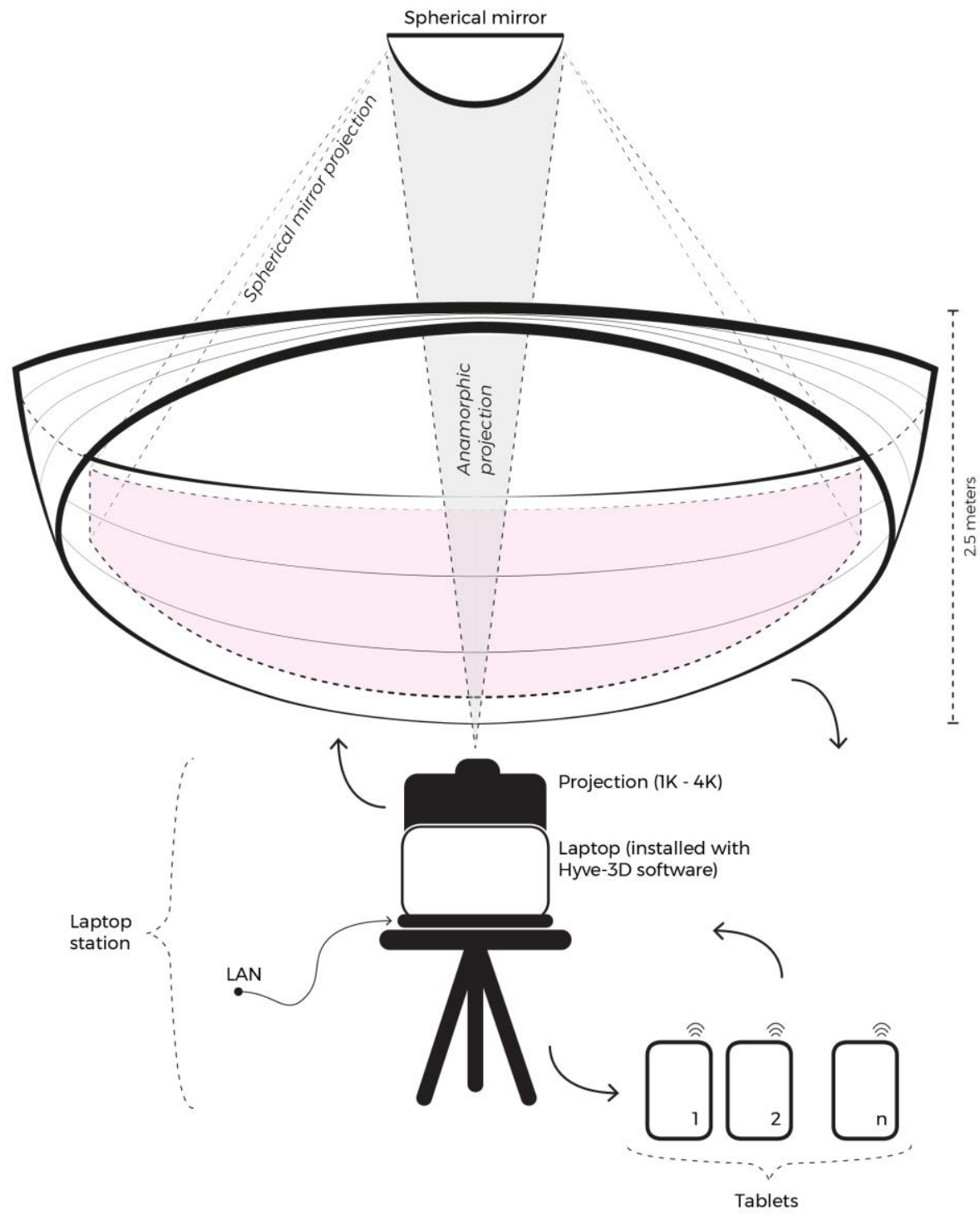

Immersive setup

Non-immersive setup

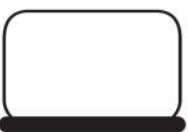
Laptop or desktop (installed with Hyve-3D software)<smiles>C1CCCC1</smiles>

$\longrightarrow$
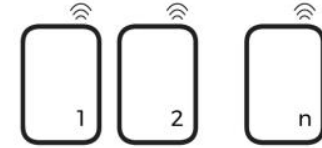

Tablets

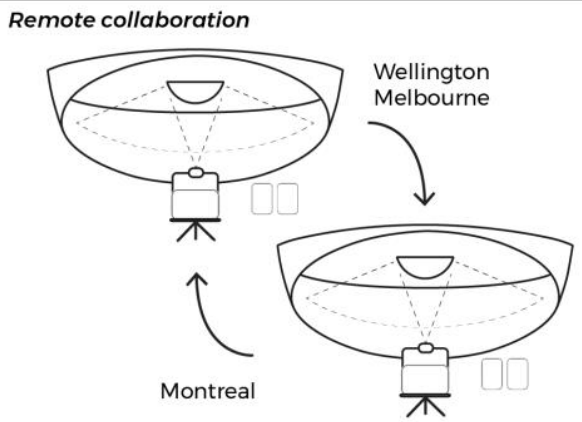

Fig. 72. Immersive and non-immersive Hyve-3D installations. In this research, remote collaboration has been tested between Melbourne-Montreal (Event I) and Wellington-Montreal (Event II). Illustration by Author. 


\subsubsection{Event I: Workshop at CAADRIA 2016}

The first event is a workshop organised as part of the CAADRIA 2016 Conference in Melbourne. The data collected from the workshop include participant observation and unstructured interviews. At the beginning of the workshop, the assembly line of this research that is explained in this Chapter 4 (i.e. documentation, representation and dissemination) is introduced to eight participants. Two more participants join from Montreal on the second day when Hyve-3D's immersive setup is used for remote collaboration. The workshop participants are from architecture-related fields. Participants were required to have no previous software skills to engage in the workshop.

The workshop activities include photography and aerial imaging, SfM modelling, polygon mesh processing, 3D sketching in immersive Hyve-3D and interaction design (Fig. 73). The below table shows the changes in the number of participants working on different activities. The timeline of the deployment of five different methods is divided into two days, each of which is four parts (two in the morning, Part A-D; two in the afternoon, Part E-H). Between Part D and G, SfM models of Köziciyerbişi (Gaotai) in Kashgar are visualised in both the immersive and non-immersive editions of Hyve-3D. Table 41 depicts the stages involved in each of Part D, E, and F in which Hyve-3D is embedded with 3D models of Kashgar. Participants were organised as individuals and groups at different stages. The remote connection between participants in Montreal and Melbourne is established in Part E.

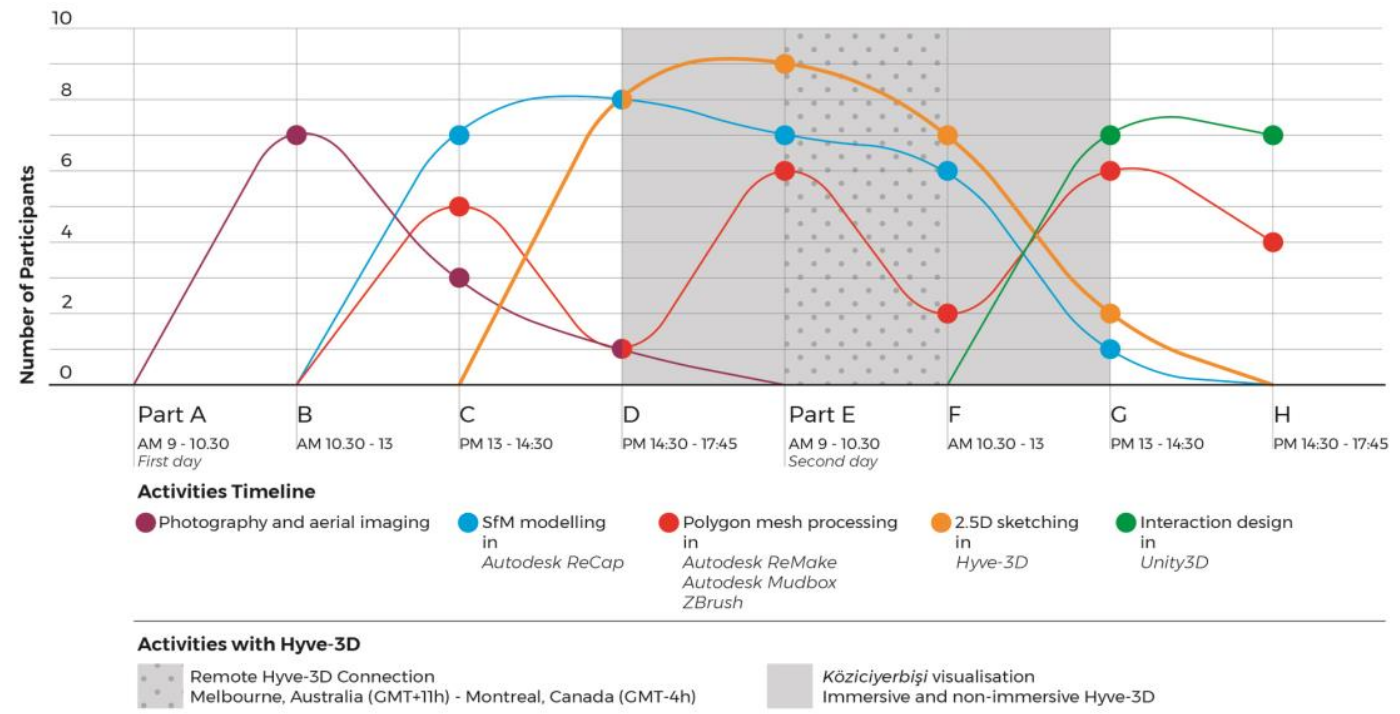

Fig. 73. The number of participants involved in different workshop activities during the two-day workshop in Melbourne. It depicts the integration of the immersive platform of Hyve-3D into the production line for digital heritage content-making. 
Table 41. Stages of the use of the Hyve-3D system with virtual Kashgar models.

\begin{tabular}{|c|c|c|c|c|c|}
\hline \multicolumn{6}{|l|}{ Part D } \\
\hline Stages & $\begin{array}{l}\text { 1. Introducing the system } \\
\text { and interface }\end{array}$ & $\begin{array}{l}\text { 2. Content-making as } \\
\text { individuals in immersive } \\
\text { Hyve }\end{array}$ & $\begin{array}{l}\text { 3. Group work in non- } \\
\text { immersive Hyve }\end{array}$ & $\begin{array}{l}\text { 4. Group work in } \\
\text { immersive Hyve }\end{array}$ & $\begin{array}{l}\text { 5. Comparing individual } \\
\text { works of sketches }\end{array}$ \\
\hline \multicolumn{6}{|l|}{ Part E } \\
\hline Stages & $\begin{array}{l}\text { 6. Setting up the content } \\
\text { and remote connection }\end{array}$ & $\begin{array}{l}\text { 7. Sketching in remote } \\
\text { collaboration }\end{array}$ & $\begin{array}{l}\text { 8. Sketching individually } \\
\text { with a remote partner }\end{array}$ & $\begin{array}{l}\text { 9. Sketching in groups with } \\
\text { remote partners }\end{array}$ & $\begin{array}{l}\text { 10. Sketching non- } \\
\text { immersive with remote } \\
\text { partners }\end{array}$ \\
\hline \multicolumn{6}{|l|}{ Part F } \\
\hline Stages & $\begin{array}{l}\text { 12. On-screen expert } \\
\text { analysis of different stages }\end{array}$ & $\begin{array}{l}\text { 11. On-screen discussion } \\
\text { of the content generated } \\
\text { immersively and remotely }\end{array}$ & $\begin{array}{l}\text { 13. On-screen expert } \\
\text { discussion on } \\
\text { immersiveness }\end{array}$ & $\begin{array}{l}\text { 14. General discussion on } \\
\text { user engagement in digital } \\
\text { heritage via immersiveness }\end{array}$ & \\
\hline
\end{tabular}


Four lectures were delivered to give the participants knowledge about Kashgar and digital heritage. The lectures were oriented towards 'creative participation' in the making of digital heritage content. The participants did not know about Kashgar prior to the workshop. But they (all with an architectural background) had personal opinions about heritage and conservation. It was asked of them to reflect the gained knowledge by drawing 3D sketches. The task was to complete the missing geometrical information on the virtual Kashgar. Ten participants (eight onsites and two remotes) engaged interactively with the pre-modelled replicas of alleys. Navigating through narrow spaces, participants superimposed digital sketches on the model to communicate their heritage interpretation (Fig. 74). The collaborative activity of $3 \mathrm{D}$ sketching is then analysed based on observations and unstructured interviews by an expert panel of the four workshop champions.

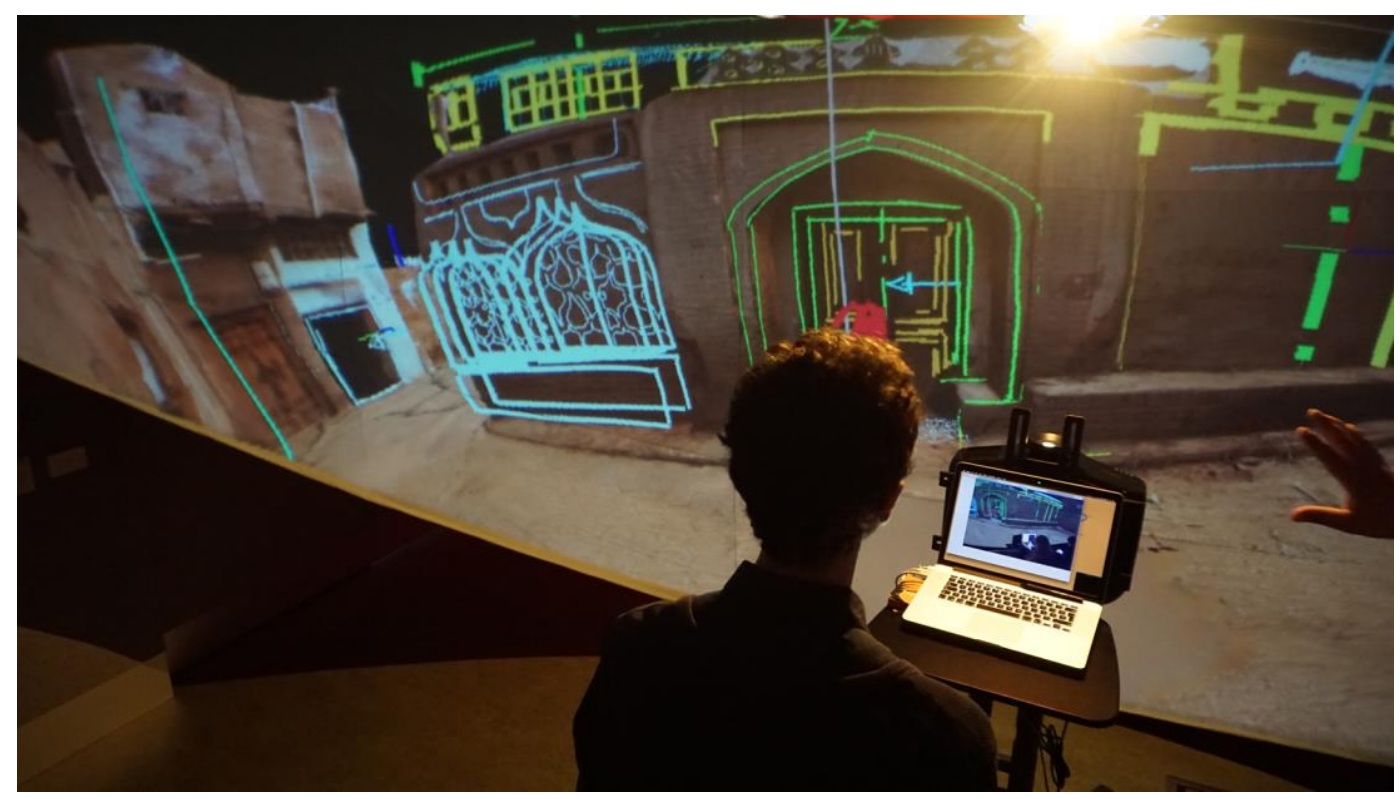

Fig. 74. 3D sketches at the end of remote collaboration between Melbourne and Montreal. Photo by Marc Aurel Schnabel.

\subsubsection{Workshop Outcome}

During lectures, it was taught that Kashgar is an Islamic city and its architecture has specific features, such as hexagonal cobblestones. Participants transferred this knowledge directly into the virtual world. One of them drew hexagonal patterns. Another participant added Islamic arches, influencing others to draw mashrabiya, timber latticework for window protection. Some participants also expressed their personality with graffiti. In summary, the insight gained from the workshop is that personalised reinterpretations of heritage in an 
immersive system produce the same desires as travellers in actual heritage sites. As studied by Urry (1990), MacCannell (1992), Leed (1991), Rojek (1997) and Staiff (2014), these tourist desires include nostalgia, intellectual fantasy, imaginary power, aesthetic expression and sensual and spiritual expositions. Evident in their 3D sketches, the essence of these desires is distilled by participants engrossed in interpretation (Fig. 75 and Fig. 76).

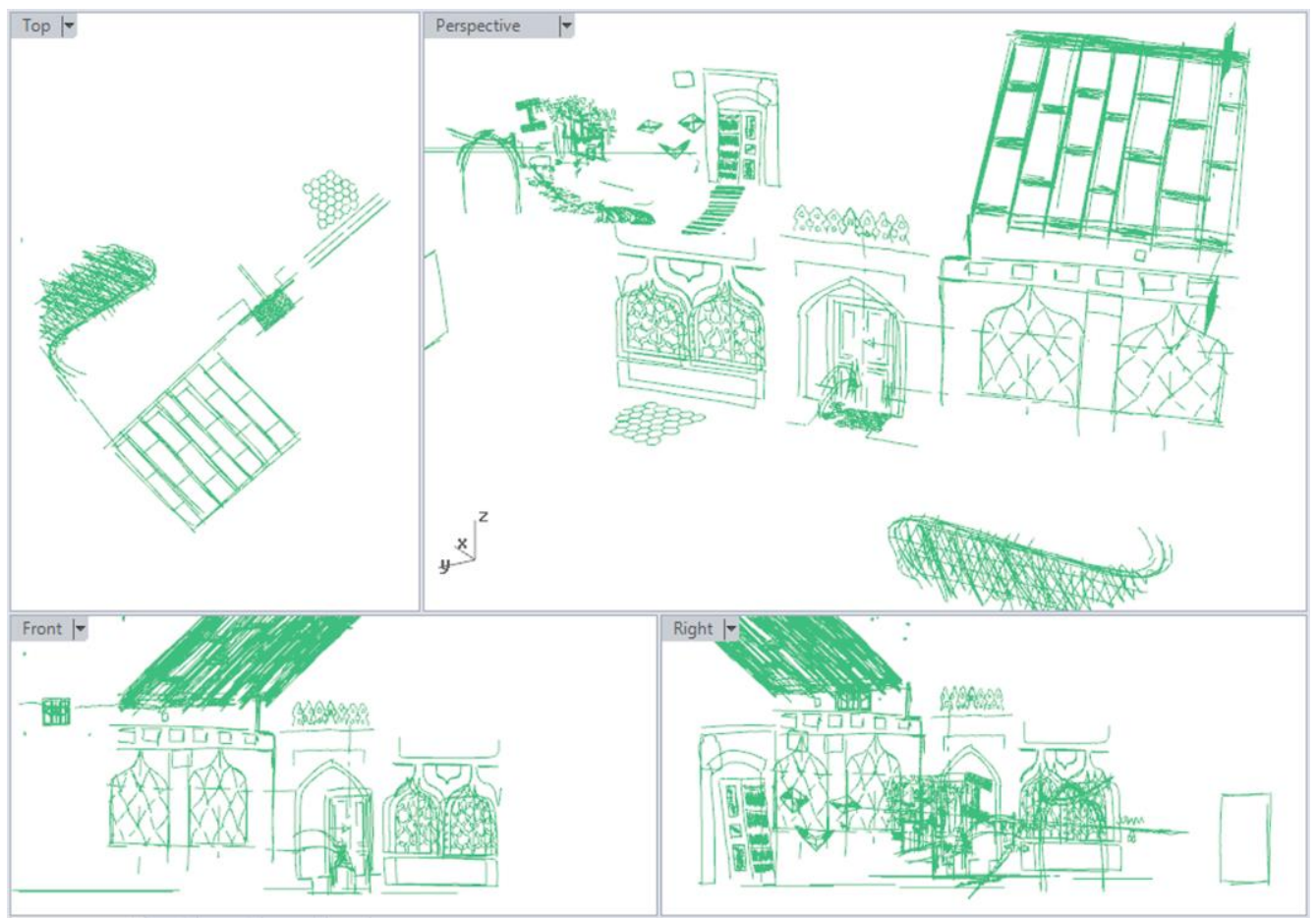

Fig. 75. 3D sketches (green) at the end of a remote collaboration session. The first activity of the user is nearly always the imitation of contour lines and edges of mesh surfaces.

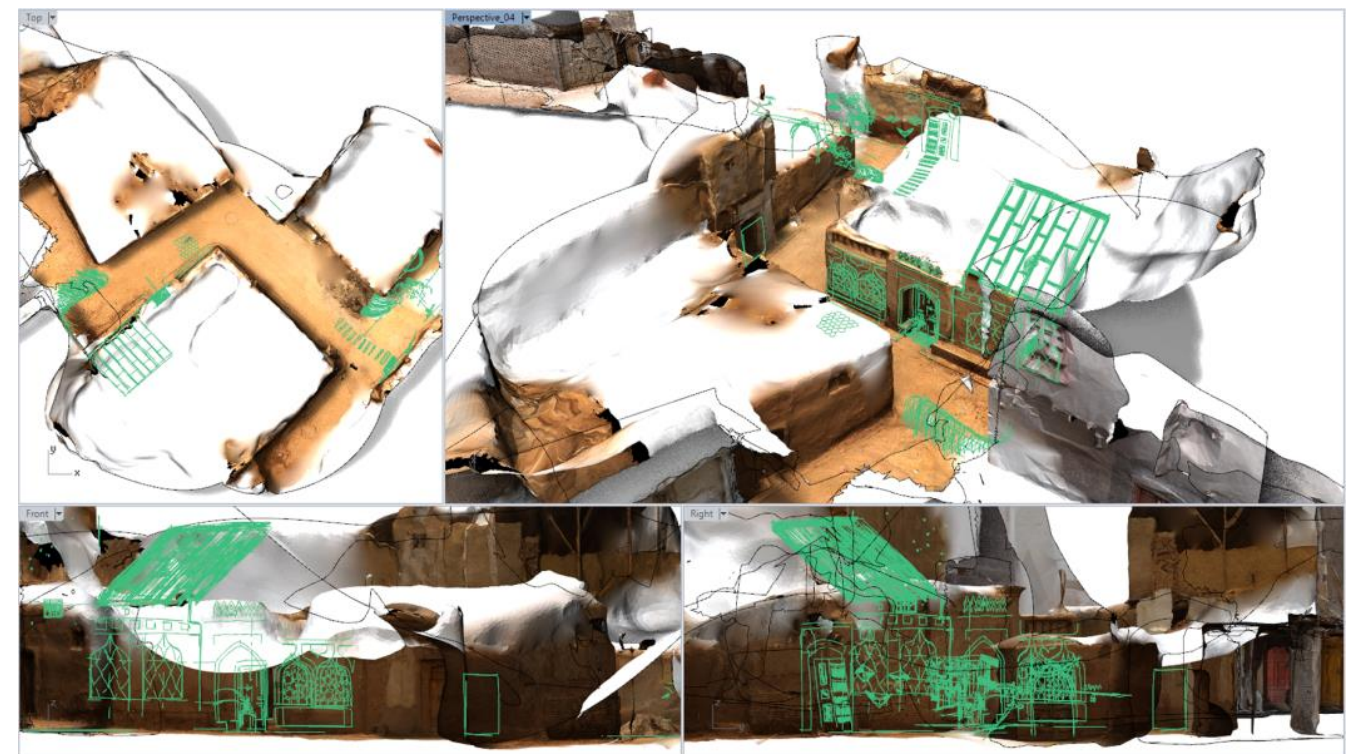

Fig. 76. 3D user sketches (green) together with the 3D model of Köziciyerbişi (Gaotai). 
In connection with the 'Matters of Research' (see Chapter 3.5), workshop findings are classified into three classes (Table 42). It is evident by observation that creative engagement should allow personalisation that is not predefined. This point is further explored in the next event. It is evidenced that digital design methods can be transferred to non-experts in a short time. The implication of this may be used by museums looking for 'citizen science' or online crowdsourcing projects (see Chapter 2.5.3) that ask the public to contribute to their workflow. The last finding is that participatory content-making can play a role in diversifying views on ethical issues such as authenticity.

Table 42. Workshop outcome and findings classified according to the 'Matters of Research.'

\begin{tabular}{lll}
\hline Matters of Research & Workshop outcome & Qualitative findings \\
\hline Error & 3D sketches & $\begin{array}{l}\text { Immersive 3D sketching resulted in 'noisy' } \\
\text { interpretations that are unique and grow } \\
\text { beyond the medium's technological } \\
\text { infrastructure. }\end{array}$ \\
\hline Multimethodology & Digital workflow & $\begin{array}{l}\text { It is relatively low-cost to train digital } \\
\text { heritage experts by using user-friendly and } \\
\text { inexpensive software that create a } \\
\text { compatible workflow between each other. }\end{array}$ \\
\hline Abduction & Ethics of digital heritage & $\begin{array}{l}\text { New viewpoints emerge on ethical topics, } \\
\text { such as authenticity. These issues are } \\
\text { topical in the digital age and require no } \\
\text { teaching as our lives are engrossed in } \\
\text { pondering them. }\end{array}$ \\
&
\end{tabular}

Along with these findings, Richen's Virtuality Matrix (VM) is further developed (see Chapter 3.3) (Table 43). Hyve-3D's immersive application with remote collaboration is positioned on the matrix. The remote connection with Montreal enabled virtual visitors to control the view of actual visitors on the other side. The communication over the network line allowed real and virtual visitors to sketch on the virtual models of the alleys in Kashgar.

Table 43. Further development of Richen's (2014) VM (see the earlier development p. 97 and the original p. 91) based on remote collaboration in a hybrid virtual reality environment.

\begin{tabular}{llllr}
\hline Visitor & Site & Content & Definition & $\bullet$ \\
\hline Real & Real & Real & Reality & $\bullet$ \\
\hline Real & Real & Virtual & Augmented Reality & $\bullet$ \\
\hline Real & Virtual & Real & Mixed Reality & $\bullet \bullet$ \\
\hline Real & Virtual & Virtual & Re-Actor (see Chapter 3.3.3) & $\bullet$ \\
\hline Virtual & Real & Real & Telepresence & $\bullet \bullet$ \\
\hline Virtual & Real & Virtual & Remote collaboration in Hyve-3D & $\bullet$ \\
\hline Virtual & Virtual & Real & Virtual Museum or Set & $\bullet$ \\
\hline Virtual & Virtual & Virtual & Virtual Reality & $\bullet$ Updated areas in this research \\
\hline & & $\bullet$ Richen's definition & $\bullet \bullet$ &
\end{tabular}


184

\subsubsection{Event II: Public Demo at Te Papa Talks 2017}

The second event is a public demo show organised as part of the Te Papa Talks 2017 hosted by The Museum of New Zealand Te Papa Tongarewa (Te Papa Museum). It builds on the previous event of the workshop in Melbourne. Participant observation and unstructured interviews of the previous event were extended with an event of 'public' engagement. In three days, the immersive installation was visited by around 150 people including children who were not under three years old (Table 44).

Table 44. Stages of the public event at Te Papa Museum with virtual Kashgar models.

\begin{tabular}{|c|c|c|c|c|}
\hline Day & Participants & Activities & Outcome & Image (Photos by Author) \\
\hline I & $\begin{array}{l}\text { Only the } \\
\text { attendants of } \\
\text { Te Papa } \\
\text { Talks } 2017\end{array}$ & $\begin{array}{l}\text { Immersive } \\
\text { navigation and } \\
\text { sketch } \\
\text { drawing on } \\
\text { virtual } \\
\text { Kashgar roads } \\
\text { inside Hyve- } \\
\text { 3D. }\end{array}$ & $\begin{array}{l}\text { Public interest in } \\
\text { immersive virtual } \\
\text { reality (VR) } \\
\text { Public awareness } \\
\text { about Kashgar's } \\
\text { heritage }\end{array}$ & 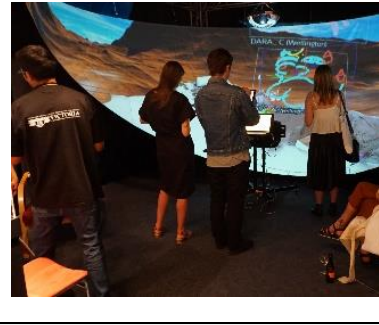 \\
\hline II & $\begin{array}{l}\text { Public: } \\
\text { Museum } \\
\text { visitors and } \\
\text { five remote } \\
\text { participants } \\
\text { from } \\
\text { Montreal }\end{array}$ & $\begin{array}{l}\text { Remote } \\
\text { collaboration } \\
\text { for creative } \\
\text { engagement } \\
\text { with virtual } \\
\text { heritage } \\
\text { model of } \\
\text { Köziciyerbişi } \\
\text { (Gaotai) in } \\
\text { Kashgar }\end{array}$ & $\begin{array}{l}\text { Record of } \\
\text { sketches in .dxf } \\
\text { format } \\
\text { Public } \\
\text { engagement and } \\
\text { interest in digital } \\
\text { heritage research }\end{array}$ & \\
\hline III & $\begin{array}{l}\text { Public: } \\
\text { Sunday- } \\
\text { visitors of } \\
\text { Te Papa } \\
\text { Museum }\end{array}$ & $\begin{array}{l}\text { Sketching in } \\
\text { mash-up } \\
\text { models of } \\
\text { Kashgar at } \\
\text { different } \\
\text { scales } \\
\text { Sketching on a } \\
\text { realistic scale }\end{array}$ & $\begin{array}{l}\text { Realistic scenes } \\
\text { more immersive } \\
\text { for children } \\
\text { Artistic mesh-up } \\
\text { scenes are more } \\
\text { engaging for } \\
\text { adults }\end{array}$ & \\
\hline
\end{tabular}

The audience was divided into groups for introducing the system, interface and content. Then they were granted access to draw sketches on virtual Kashgar models (Fig. 77). The day I and III were used to gather public opinion about the concept of the digital museum through unstructured interviews. Visitor engagement with heritage content in an immersive application was observed to make a general comparison with the previous workshop. It was directly distinguishable that the visitor motivation and drawing style in 'public' engagement were more diverse and 'creative' that is discussed subsequently (Chapter 4.4.4) (Fig. 78). 


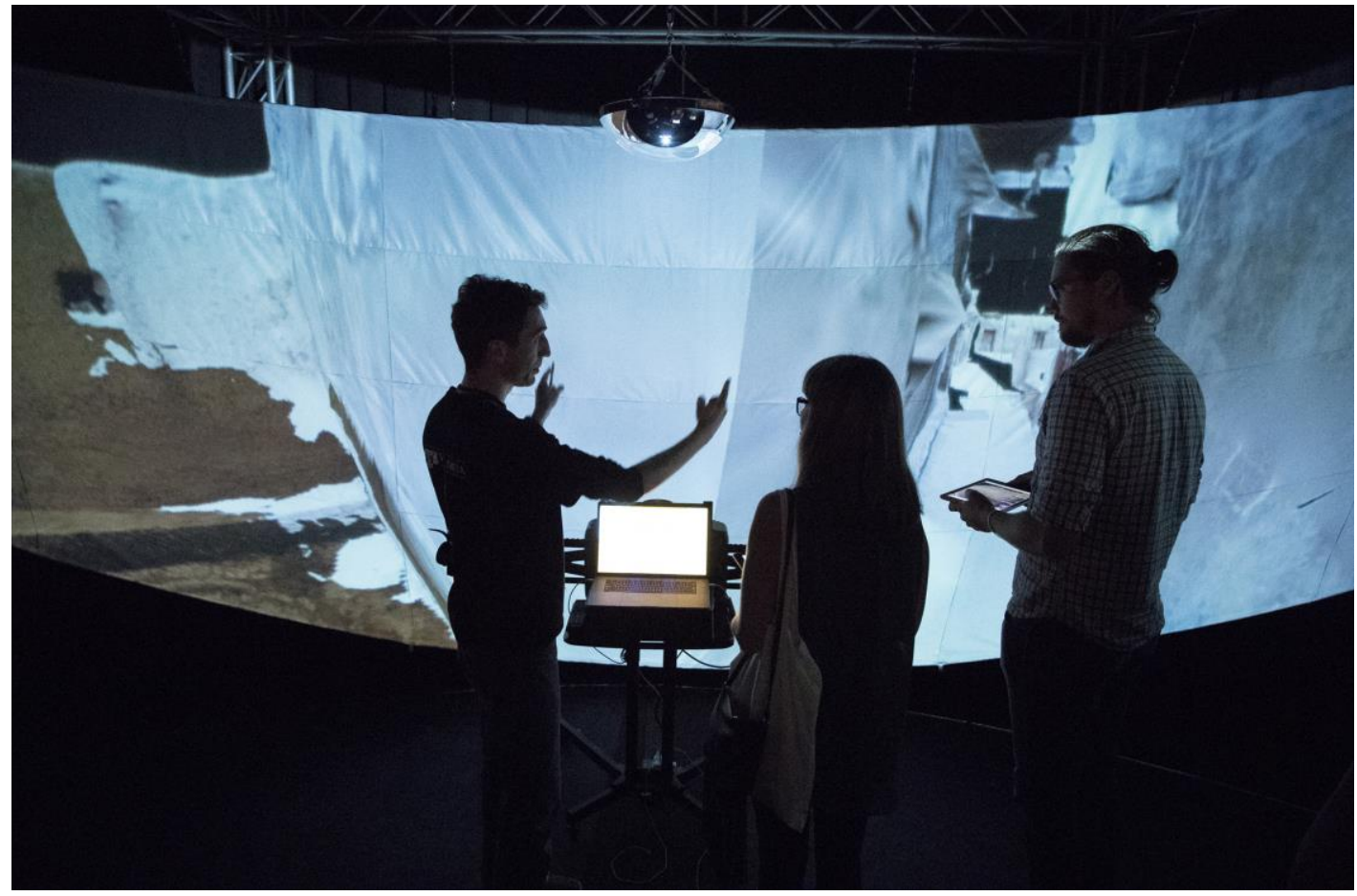

Fig. 77. Introducing the research to visitors at Te Papa Museum inside Hyve-3D. Photo by Sasja Mazurkiewicz.
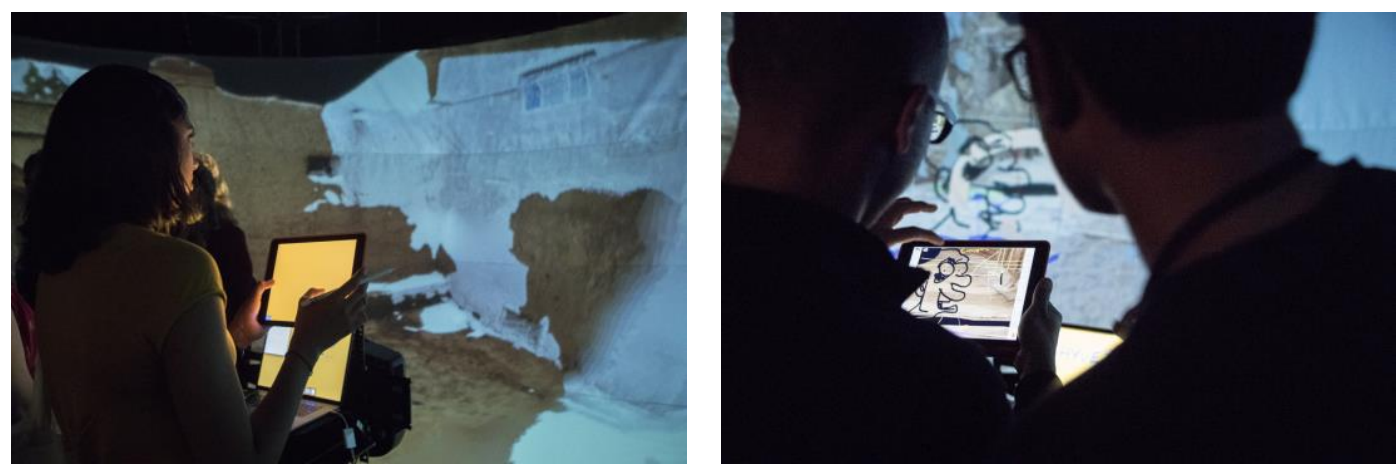

Fig. 78. Left. A visitor virtually travels through alleys by using the 'global navigation' which is calibrated by tablet orientation in 3D space. Right. A visitor is sketching while controlling his position through the local navigation of HYve-3D. Visitor motivation in 'public' engagement is more diverse than scholarly engagement at the previous workshop. Photos by Sasja Mazurkiewicz.

This section is focused on remote collaboration and meaningful role-playing. On Day II, two remote collaboration sessions with Montreal were conducted for three hours in total (Fig. 79). The overall aim of drawing 3D sketches in both sessions was to populate the empty alleys of the virtual Kashgar. Each session resulted in overwhelming visual complexity. Visitors entered in groups of three to ten people. As for meaningful role-playing, there were two types: low-level and high-level. The former is given top-down as a pre-defined task to follow, whereas the latter bottom-up is delineated by the participant while drawing. 
186
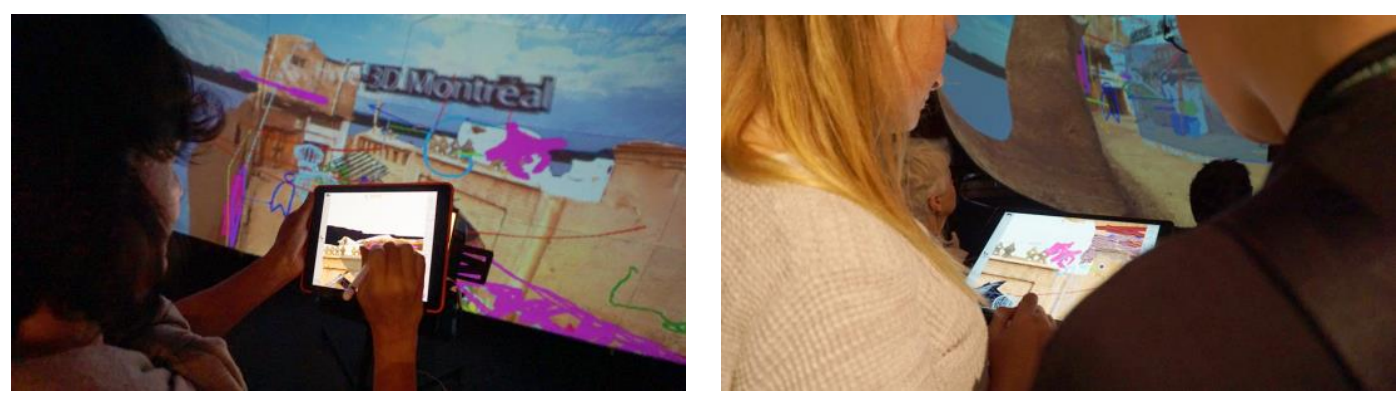

Fig. 79. Visitors engage with each other by navigating and sketching on virtual Kashgar models while being remotely connected to partners in Montreal. Photos by Author.

The low-level role was introduced to visitors as drawing sketches to complete the missing parts of 3D virtual mesh models. Remote participants from Montreal were already familiar with the virtual Kashgar model as they collaborated in the previous event. Therefore, visitors in Wellington were initially guided by remote participants from Montreal. The role of guidance became a crucial point of analysing communication between local and remote partners. Having learned how to draw, some Wellington visitors took over the role of guidance that inversed the communication of creativity. This crucial moment is the increase in the level of engagement in cyberspace from immersiveness to agency (Table 45). Earlier (Chapter 2.5.1); a similar 'digital crossover' was brought up with the Ripple Wall where workers taught parametric joint details to the architects.

Table 45. The shift from a role definition in the immersive experience to the full-fledged agency.

\begin{tabular}{llll}
\hline Immersive role & Hierarchy & Explanation & Agency \\
\hline Low-level role-playing & Top-down & Guided by experts or others & System-defined \\
\hline High-level role-playing & Bottom-up & Guide the experts or others & Self-defined \\
\hline Creative emancipation & No hierarchy & Beyond communication & Authentic, no agency \\
\hline
\end{tabular}

\subsubsection{Qualitative Findings}

"Index of representations" are the essential elements that make an everyday environment familiar to travellers of heritage sites, with "the process of indexing referring to the set of visual, textual and symbolic representations (Rojek 1997, 53). One of the implications of the dynamic exchange of guidance between Montreal and Wellington was the production of shared indices of representations. In this regard, the indexicality of 3D sketches was analysed visually. Indexicality reveals that both mimicking and preceding the guidance has creative aspects as to how pieces of lines are combined constructively. To give an example, sketches of architectonic interpretation indicate imitation of the remote guidance, whereas 
the horse cart drawings made by local participants in Wellington display novel interpretation (Fig. 80). The assemblage of these novelties and authentic contributions to the collaborative session inversed the role of guidance from Montreal to Wellington. The repositioning and exchange of the guidance role produced the hybridisation of tourist desires being guided and power of guidance as if the participant is a local in Kashgar, being familiar with the environment. Both types communicated creatively for bringing together their imagination with the remote guidance. Fig. 81 and Fig. 82 show the outcome of Session A and Fig. 83 and Fig. 84 belong to Session B. Each session is colour-coded for comparison.
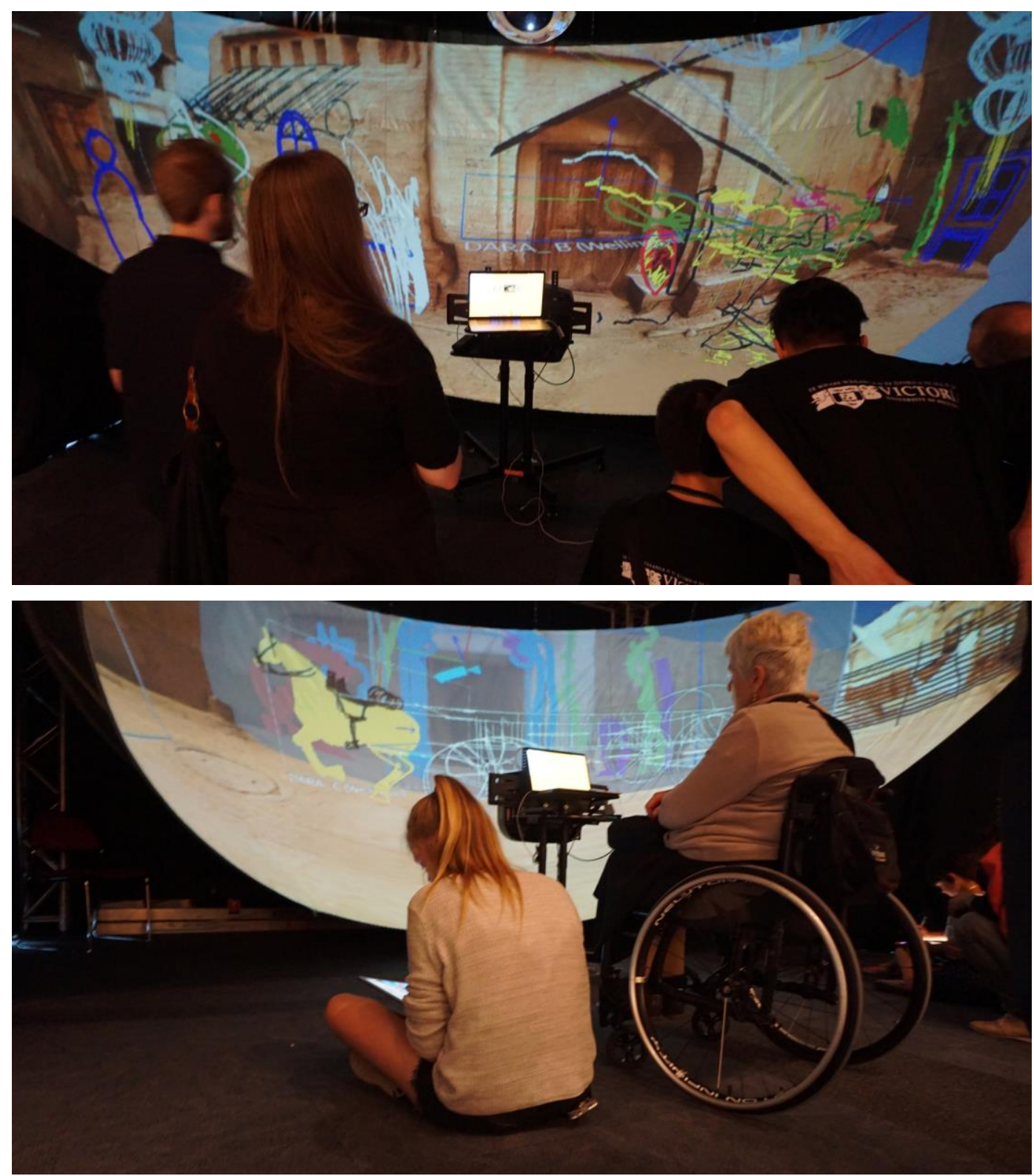

Fig. 80. Most visitors are guided to imitate architectonics (top) and a visitor taking the role of guidance from Montreal to influence more horse cart sketches (bottom). Photos by Author. 


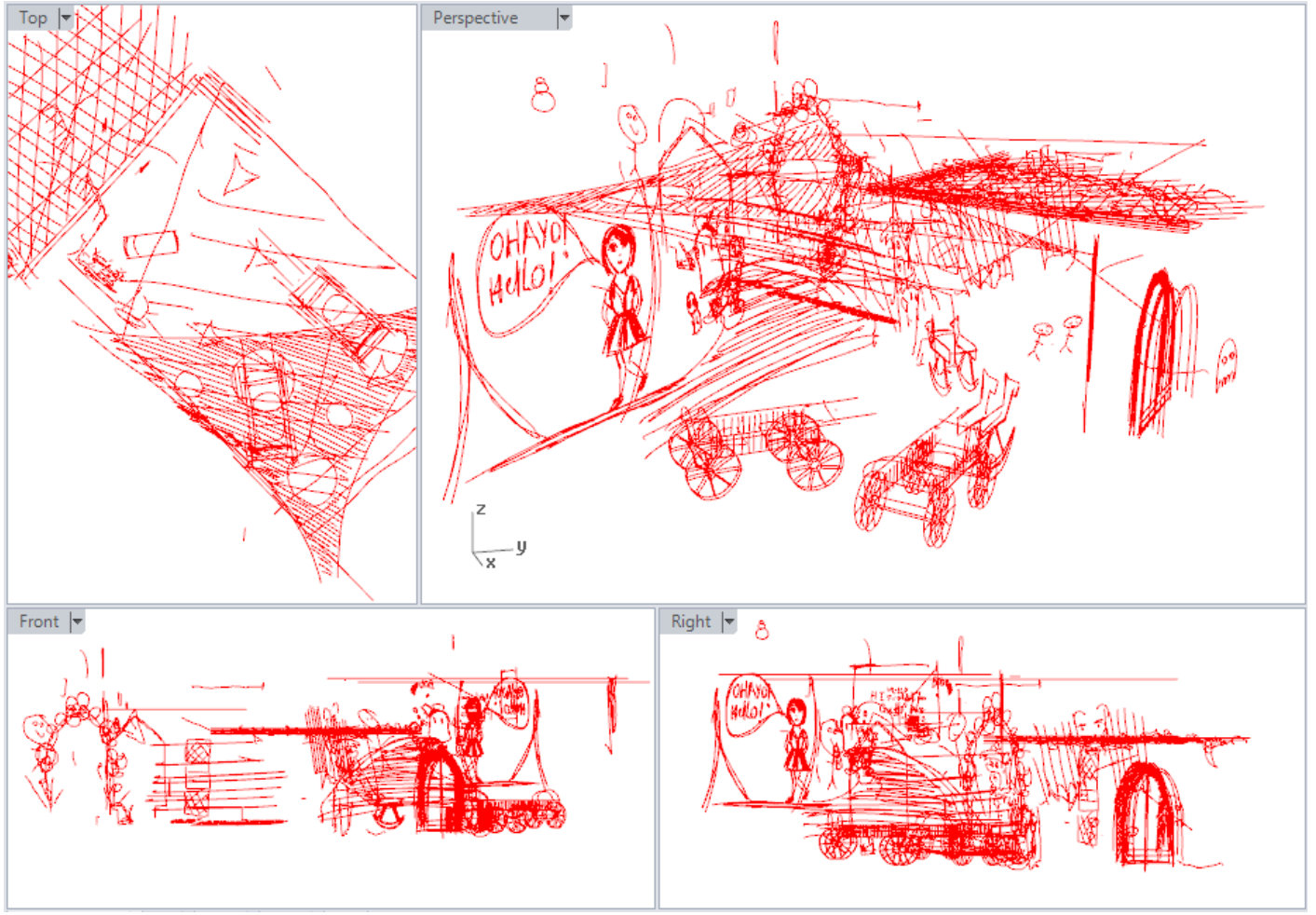

Fig. 81. 3D sketches (red) at the end of Session A.

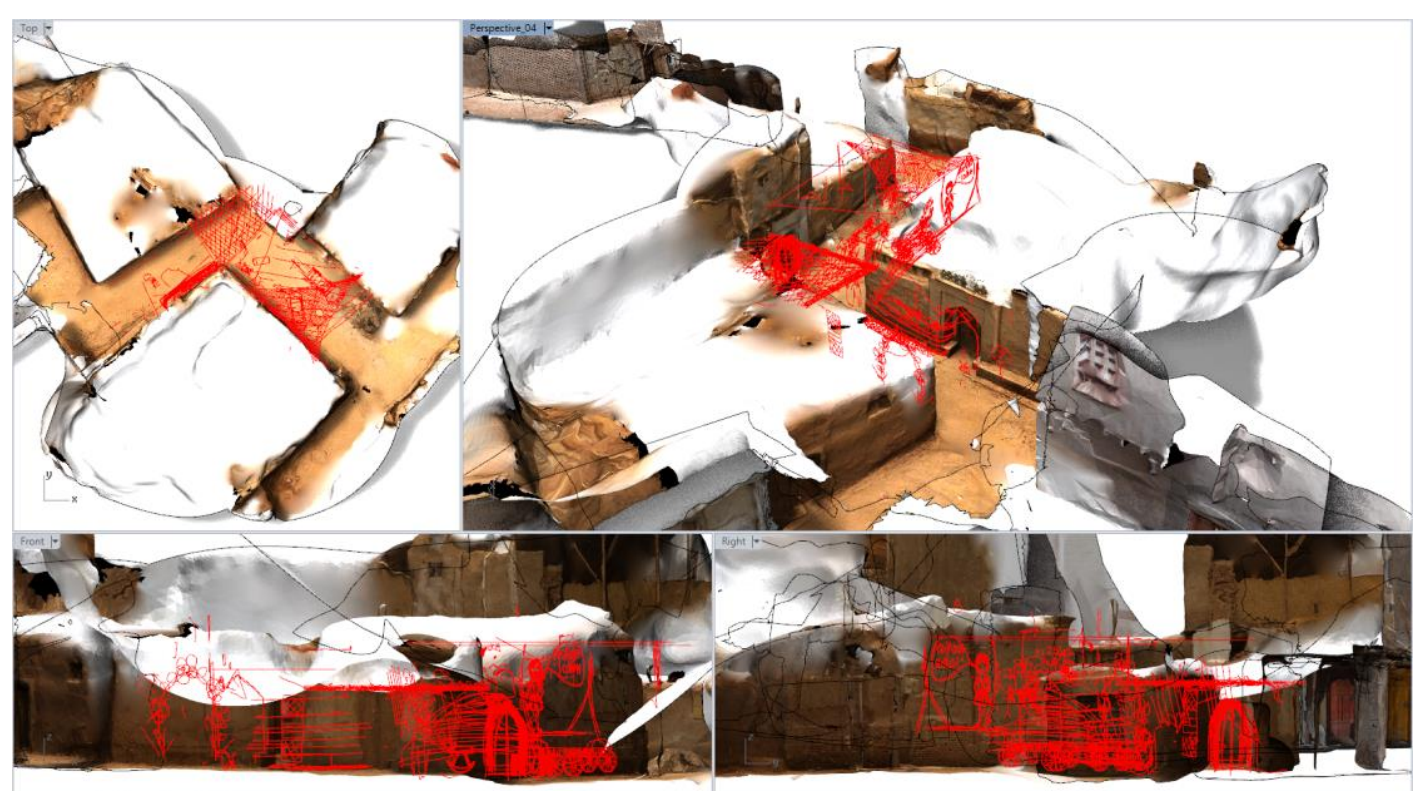

Fig. 82. 3D sketches (red) from Session A displayed on the model of Köziciyerbişi (Gaotai). 


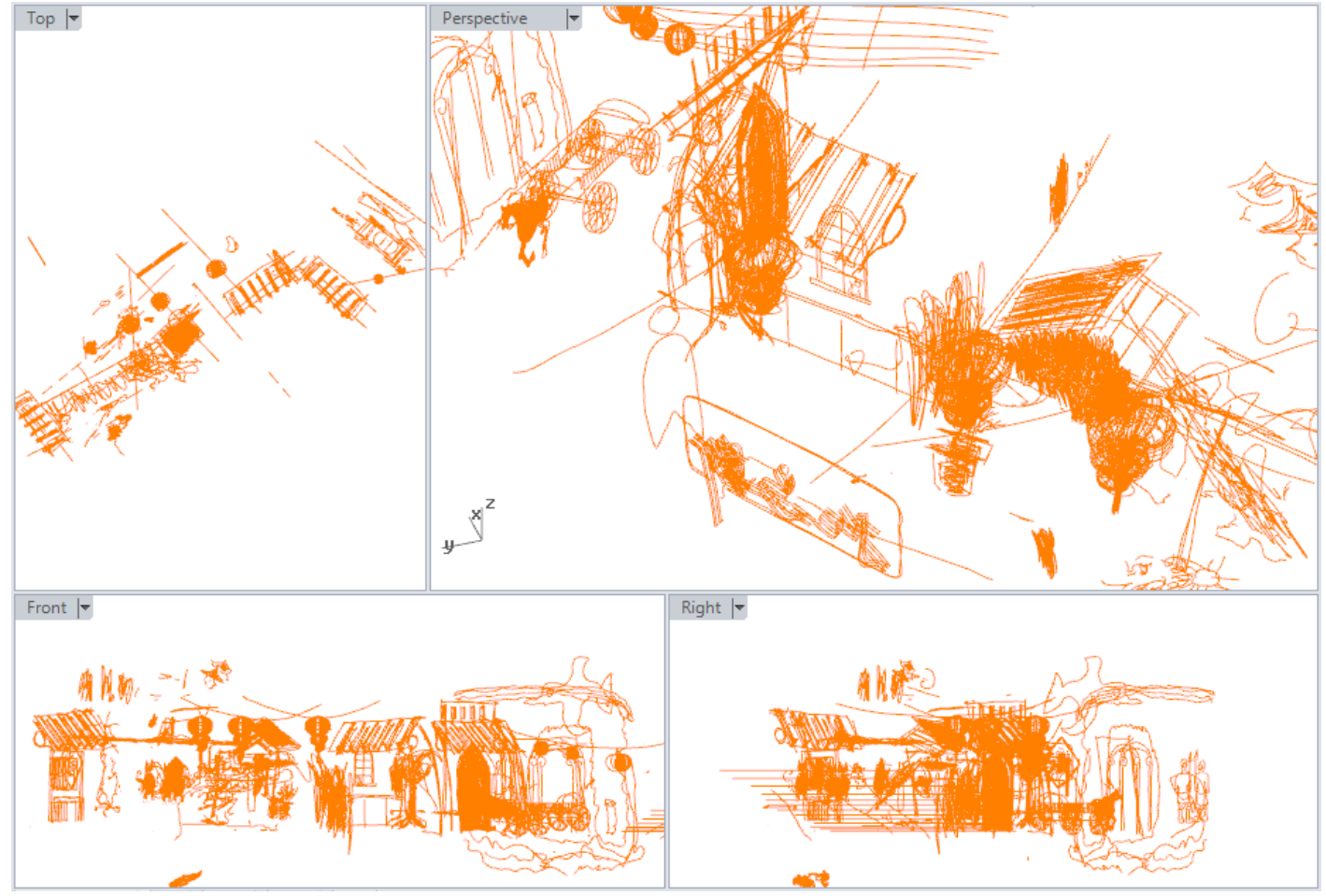

Fig. 83. 3D sketches (orange) at the end of Session B.

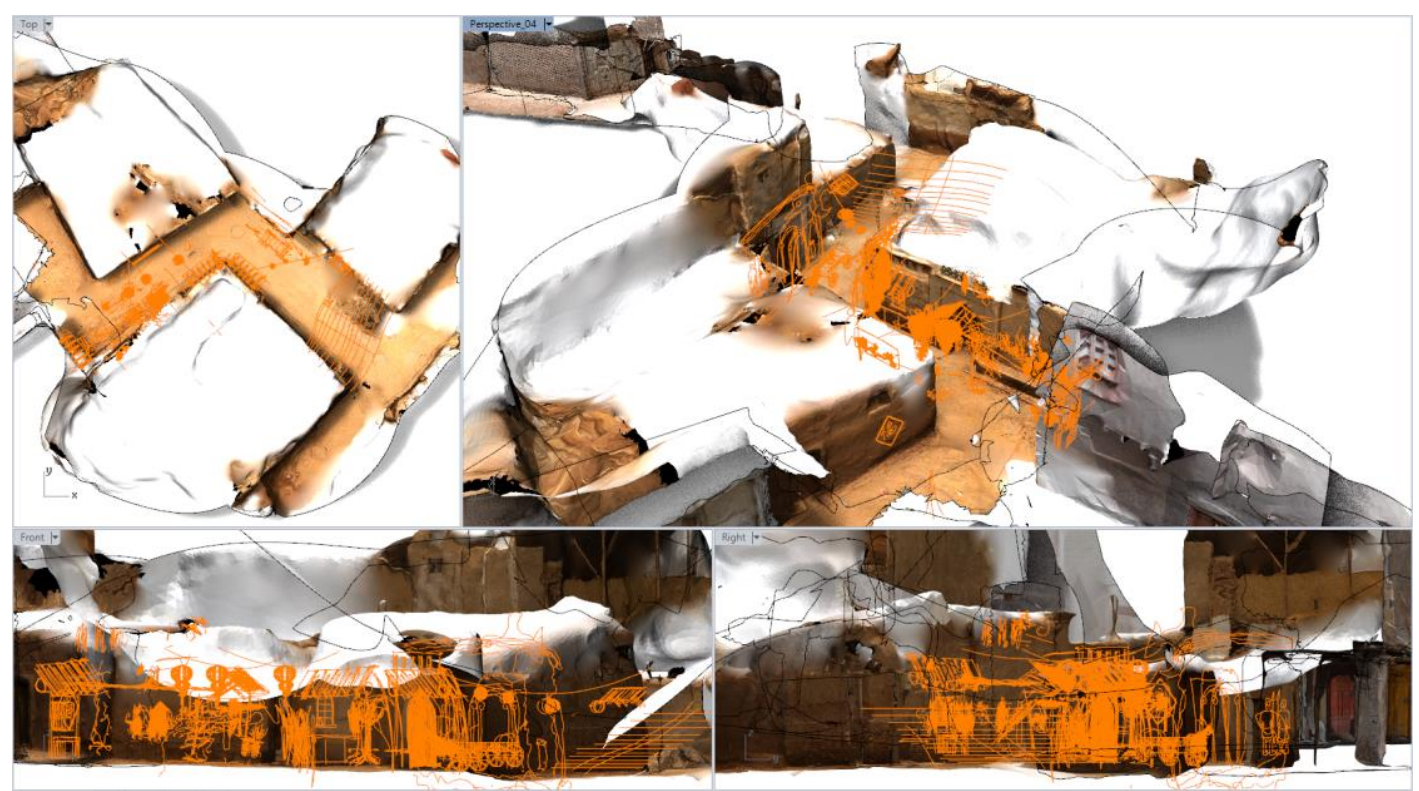

Fig. 84. 3D sketches (orange) from Session B displayed with the model of Köziciyerbişi (Gaotai). 
Overall, it is noticed that the communication was initially based on 3D sketch line expressions rather than verbal expressions. Visitors were not used to immediate contact with strangers, especially with Montreal. But the visual communication that occurred was a success of immersiveness as 3D sketching allowed intuitive communication. In the course of both sessions, this was enforced by the global navigation of the view on the concave curtain that only one person could control at a time. The person who wanted to navigate through space had to confirm with others who were already immersed through a personal connection to the content generated. This was the point of convergence between two aspects of cyberspace; immersiveness and agency. Ability to do the $3 \mathrm{D}$ intuitive sketching shifted the immersive experience of visitors into meaningful and personalised areas of role-playing.

As for Kashgar's heritage, the immersive application became a place of comparison between seemingly irrelevant lines. It can be attributed to Malraux's defence of museums as places of 'style' where different objects can be compared for a test of aesthetics. In this way, a Renaissance perspective painting could stay along with and be compared to a Chinese traditional landscape painting (Allan 2009, 246). Without the concept of a museum, these two different styles would have no chance to transfer and reterritorialize their authentic and aesthetic values. Similarly, the immersive hybrid environment created a place for comparing the styles of Kashgar and visitors' origin of place (Fig. 85).
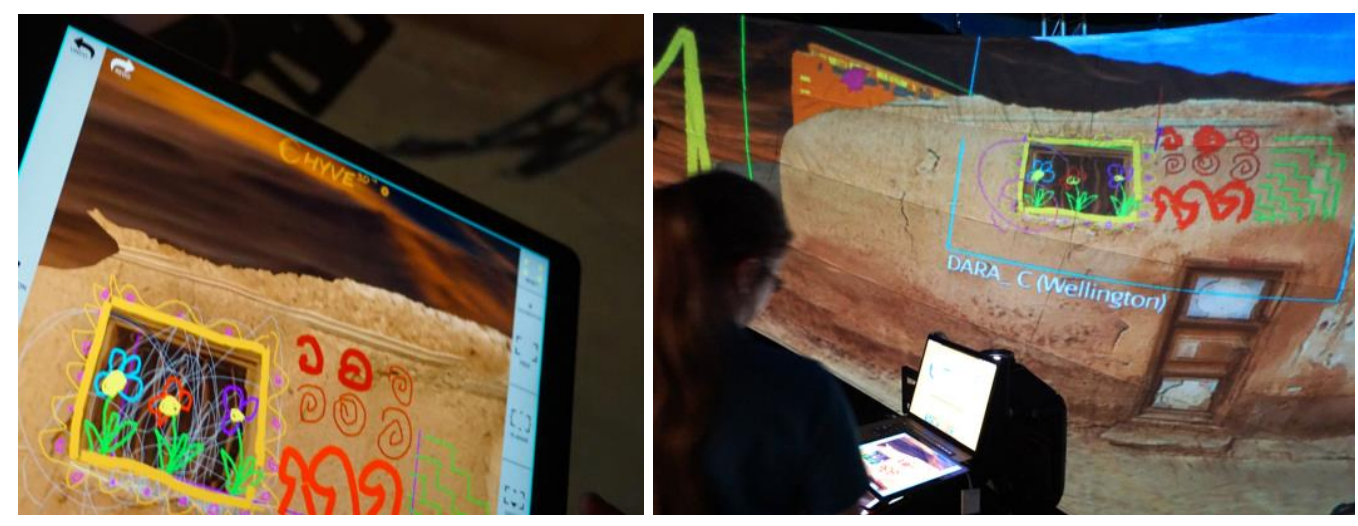

Fig. 85. Overlapping Māori patterns are drawn by a New Zealander on the Kashgar model.

The recognised patterns depict the freedom of choice in virtual reality. It is demonstrated that New Zealand's Māori patterns can be drawn on the walls of a house that represents the westernmost city in China. To understand this comparison between the styles, one way is to study the indexicality and decode meaning through indices. For an indexical generalisation of the user-generated complexity, visitors' sketches are conferred with Gero and Kannengiesser (2012)'s classification of representational affordances. In their classification system, reflexive, reactive and reflective representations are analogous to 
'routine,' 'innovative' and 'creative' designing, respectively. Likewise, in the immersive VR environment, 3D sketches of users represented (1) mimicry of the existing virtual content and guidance, (2) inverting the guidance with the same attributes in new interpretations and (3) emancipation from the conversation of guidance (Table 46).

Table 46. Three types of sketches observed qualitatively.

\begin{tabular}{llll}
\hline Type & Explanation & Immersive role & Agency \\
\hline Reflexive & $\begin{array}{l}\text { 'Routine' imitation of edges and outlines } \\
\text { of the pre-existing content }\end{array}$ & $\begin{array}{l}\text { Mimicry of the } \\
\text { guidance }\end{array}$ & System-defined \\
\hline Reactive & $\begin{array}{l}\text { 'Innovative' use of the pre-existing } \\
\text { digital content by adding new elements } \\
\text { that are still connected to edges and } \\
\text { outlines }\end{array}$ & $\begin{array}{l}\text { Inversing the } \\
\text { guidance }\end{array}$ & Self-defined \\
\hline Reflective & $\begin{array}{l}\text { 'Creative' 3D sketching without } \\
\text { guidance and guiding at the most } \\
\text { authentic level }\end{array}$ & $\begin{array}{l}\text { Emancipating from } \\
\text { the conversation of } \\
\text { the guidance }\end{array}$ & $\begin{array}{l}\text { Authentic, no } \\
\text { agency }\end{array}$ \\
\hline
\end{tabular}

The qualitative findings of the public demo event are collected in Table 47. In summary, the insight gained from the qualitative analysis of the outcome is that indexicality can be instrumental in gaining alternative knowledge generated by users. Visitors were entangled with a hybrid identity of having a tourist gaze and a local gaze that help the person identify with the place while moving from immersive engagement to the pleasure of agency. Chapter 5 will take this hypothetical statement further with a different type of engagement model.

Table 47. Summary of findings from the public demo show at Te Papa Museum.

\begin{tabular}{ll}
\hline Findings & Definition \\
\hline Tourist gaze & $\begin{array}{l}\text { Following from the previous workshop event in Melbourne, it is } \\
\text { found that immersive engagement stimulates desires similar to } \\
\text { tourists in actual heritage sites, such as the desire for nostalgia. }\end{array}$ \\
\hline $\begin{array}{l}\text { Dynamic role of guidance } \\
\text { (shift from immersiveness } \\
\text { to agency) }\end{array}$ & $\begin{array}{l}\text { It is inherently evident in 3D sketching that there is a shift from } \\
\text { immersive engagement to the pleasure of agency via } \\
\text { personalisation of drawings, which generates a sense of belonging } \\
\text { and authority to guide others. }\end{array}$ \\
\hline Comparison of 'style' & $\begin{array}{l}\text { Similar to Malraux's definition of the role of museums in response } \\
\text { to Merleau-Ponty, creative public engagement is found to animate } \\
\text { comparison between diverse drawing styles as well as the content. }\end{array}$ \\
\hline Indexicality & $\begin{array}{l}\text { The complexity of 3D sketches can be studied through an analysis } \\
\text { of the 'index of representations' (Rojek 1997, 53). A hypothetical } \\
\text { statement is made that indexicality analysis can help measure the } \\
\text { quality of above findings. }\end{array}$ \\
\hline
\end{tabular}


Index analysis of 3D sketches is made from the samples that are gained from Sessions A (red lines) and B (orange lines) (Fig. 86 and Fig. 87) (Table 48). The former is found to include nine recognisable patterns of drawings, whereas twelve patterns are recognised in the latter. Particularly medieval styles of gates and knights reflect the background of the visitors grounded in Western history. It is interesting to find horse carts drawn in both sessions, suggesting knowledge transfer between participants. People with an understanding of Chinese architecture made lanterns. It is an interesting coincidence that both Chinese officials and remote virtual visitors from New Zealand deem lanterns proper representations for Kashgar that does not have lanterns inherently in its history.
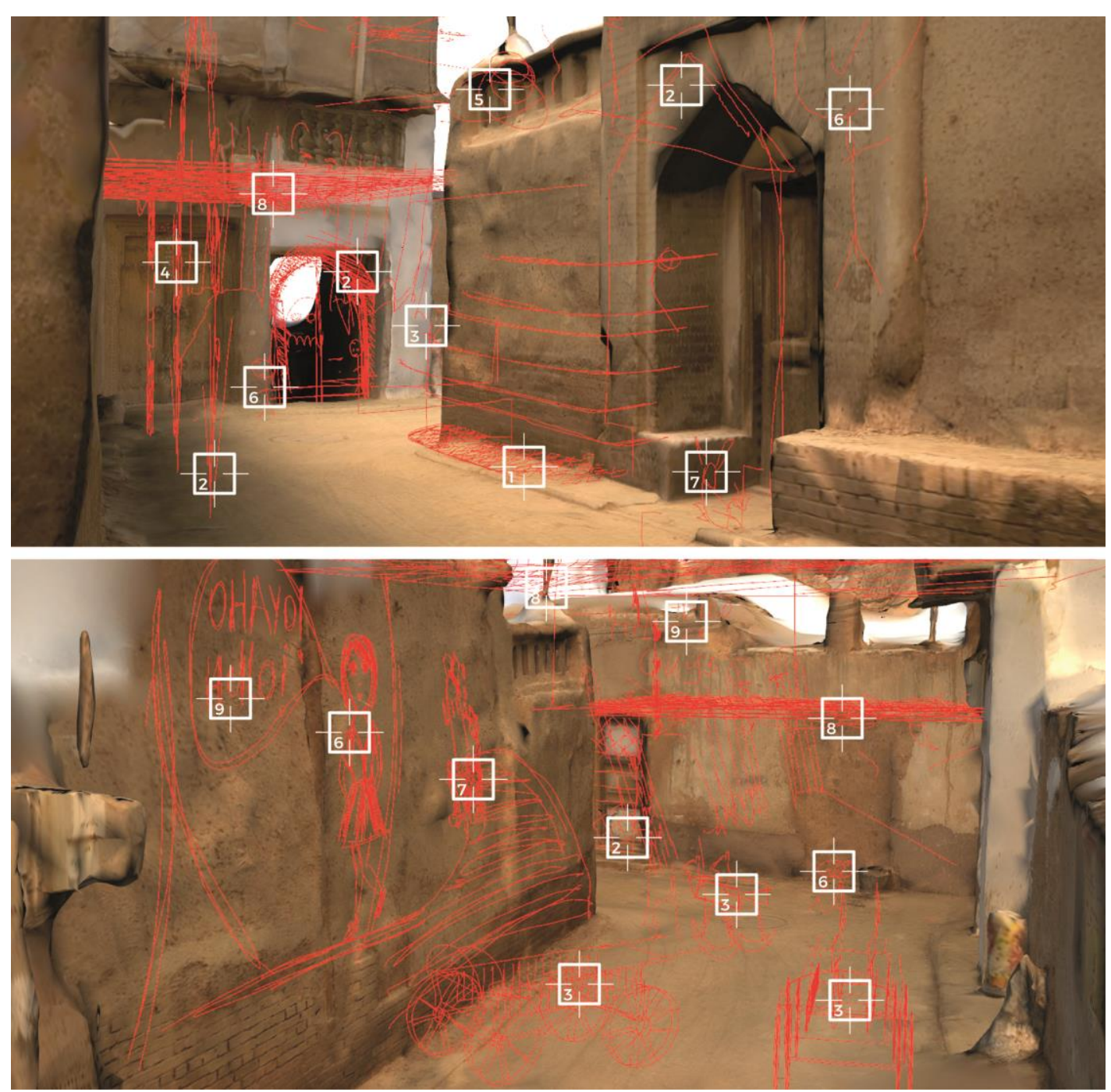

Fig. 86. Superimposing drawings (Session A) on polygon mesh model of the west junction in Köziciyerbişi (Gaotai). 1. Cobblestone, 2. Gate, 3. Horse cart, 4. Flowers, 5. Lantern, 6. Human, 7. Cat, 8. Shelter, 9. Text message. Illustration by Author. 

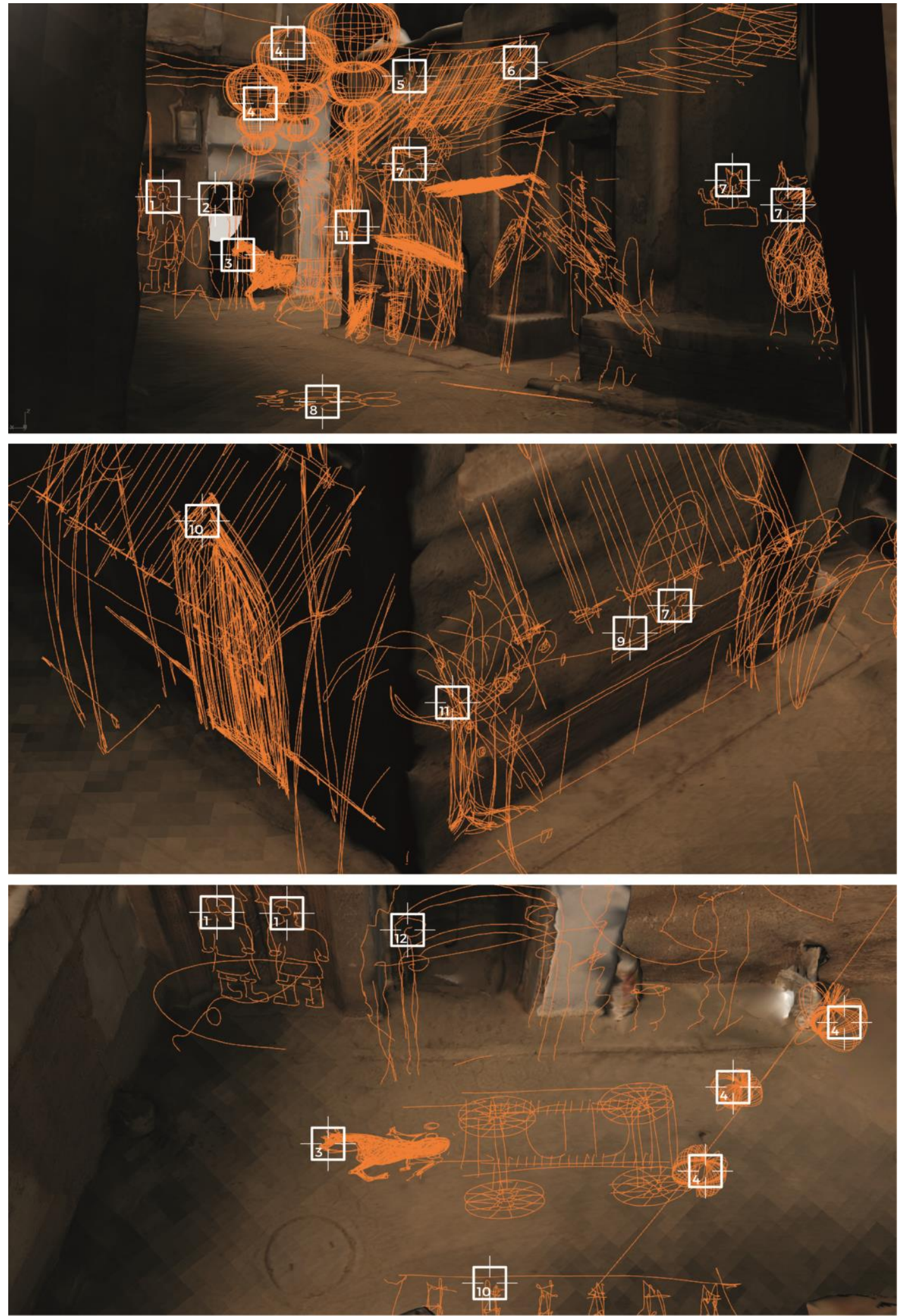

Fig. 87. Superimposing drawings (Session B) on a 3D model of the west junction in Köziciyerbişi (Gaotai). 1. Knights, 2. Human, 3. Horse cart, 4. Lanterns, 5. Māori pattern, 6. Shelter, 7. Cat, 8. Flower, 9. Window with an eave, 10. Door with an eave, 11. Tree, 12. Gate. Illustration by Author. 
Table 48. 'Index of representations' in Session A (red lines) and B (orange lines).

\begin{tabular}{|c|c|c|c|c|c|}
\hline ID & Session A & Index (A) & Index (B) & Session B & ID \\
\hline 1 & Cobblestone & & & Knights & 1 \\
\hline 2 & Gate & & & Human & 2 \\
\hline 3 & Horse cart & & & Horse cart & 3 \\
\hline 4 & Flowers & & & Lanterns & 4 \\
\hline 5 & Lantern & & & Māori pattern & 5 \\
\hline 6 & Human & & & Shelter & 6 \\
\hline 7 & Cat & & & Cat & 7 \\
\hline 8 & Shelter & & & Flower & 8 \\
\hline 9 & Text message & & & Window with eave & 9 \\
\hline & & & & Door with eave & 10 \\
\hline & & & & Tree & 11 \\
\hline & & & & Gate & 12 \\
\hline
\end{tabular}




\subsubsection{Challenge}

Digitising heritage information is a reflection of a subjective view on how best to map reality in virtual dimensions. Although this mapping seems to be protected, it remains possible for information to be lost, degraded, and even erased entirely. The chances increase when this information is disseminated to the audience. In this sense, digital objects representing their heritage counterparts in reality are not eternal, but vulnerable to ephemeral conditions of the digital space. This fragility is a stimulus for the transformation of both the content and the users (or decoders). These dynamic transformations in content produce an independent abstraction in the form of unusual conditions in the digital realm, which become metadata generated from user-interaction and interpretation. This relational knowledge can be searched in relations between user-generated content to validate alternative meanings that are unknown until searched (Fig. 88).

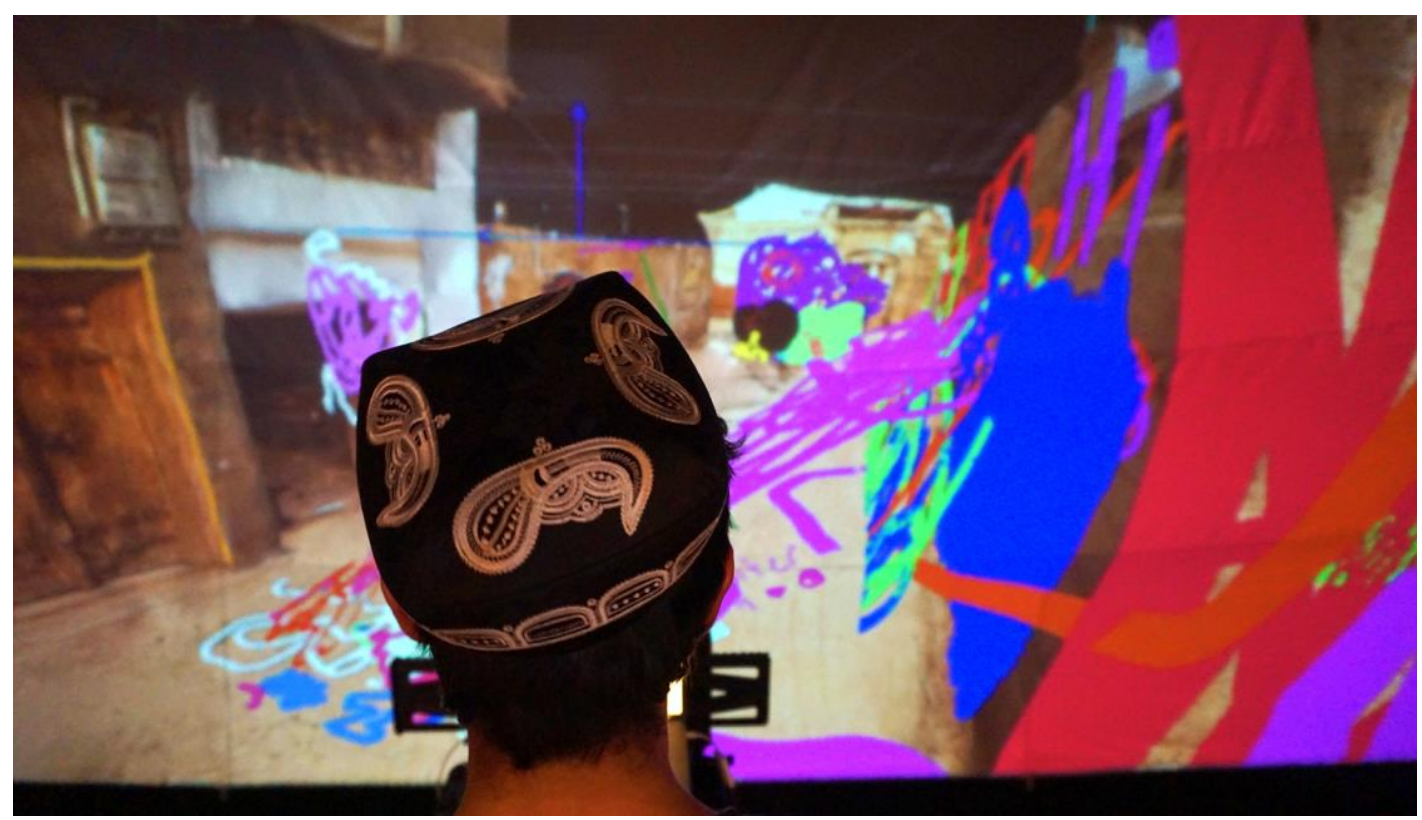

Fig. 88. A visitor wearing a duppa hat, a tradition of Kashgar, captured in a scene after children engaged with the virtual models of the historic town. The visitor looking confused confronting the complexity of user-generated content.

Event I (the workshop) and II (the public demo) produced overwhelming details about immersive engagement (Fig. 89). Museum visitors are granted direct and free access to the content at the cost of complexity. This can be aligned with the concepts of noise, chaos and disorder in information theory (see Chapter 2.2.5). Having realised the challenge of retrieving alternative knowledge from creative public participation, the indexicality of content is further studied with an innovative application of a data mining method in digital architectural heritage research (see Chapter 5). 


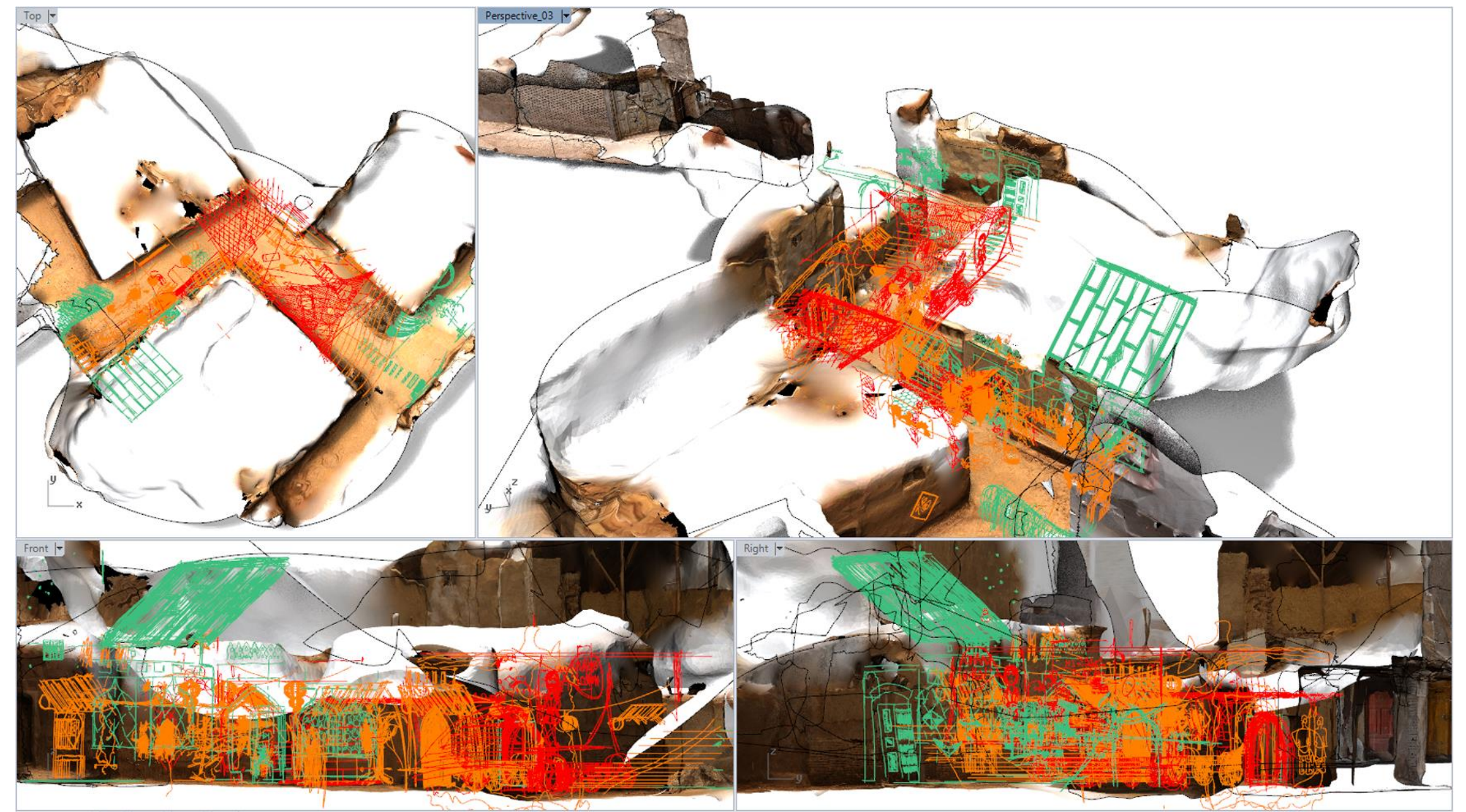

Fig. 89. 3D user sketches together with the 3D model of Köziciyerbişi (Gaotai). Green lines represent the CAADRIA workshop event in Melbourne in March 2016. Red lines represent Session A and orange Session B in the public demo show at Te Papa Museum in March 2017. 
In the two events committed to examining immersiveness in digital heritage, it was discovered that an offspring of immersiveness is the agency that starts when the user takes meaningful action. The role of agency reflects a mechanism of intelligence interpreting 'authenticity' in digital heritage. Making a correlation analysis of 3D sketches as usergenerated content may reveal insight into this novel mechanism of authenticity. Fig. 90 below shows a preliminary correlations analysis between three sessions (one from the workshop, two from the public demo). Associations of indices are found to occur in a single session, two or all three sessions, with a different style of line attributed to each case. In this sense, these correlations explain 'shared indexicality' between alternate realities based on select correlations including horse carts, doors with eave, lanterns, mashrabiya windows, flowers, gates and cats.

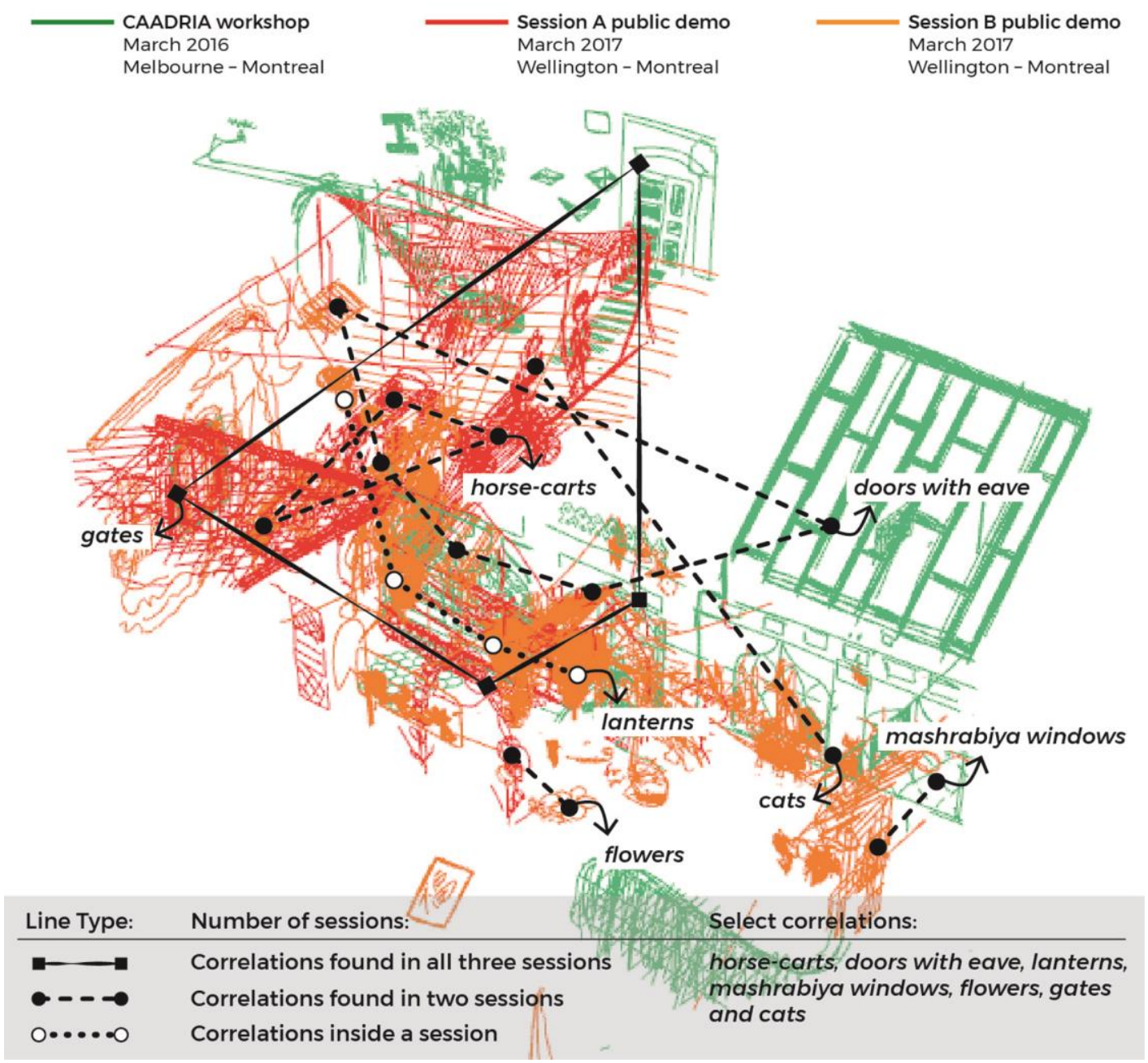

Fig. 90. Assemblage of 3D user sketches in two events. Green lines represent the CAADRIA workshop event in Melbourne in March 2016. Red lines represent Session A and orange Session B in the public demo show at Te Papa Museum in March 2017. 
198

Despite a year gap between the two events, user-generated 3D sketches were still found to correlate. Horse cart drawings are correlated in the two sessions of the public demo, whereas a specific style of gate arches presents a correlation between all three sessions. On the theoretical basis of this practice, it is mentioned earlier (Chapter 2.3.3 and 3.2.4) that, being plural in meaning, "each generation views authenticity in a new guise, reflecting its new needs for truth, new standards of evidence, and new faiths in the uses of heritage" (Lowenthal 1999). A correlation analysis exposes 'new standards of evidence' to define authenticity based on participation and conversation instead of solo-authorship. The lack of local opinion may result in inauthenticity; therefore, it should be considered as a limitation or an opportunity. Touching on this challenge, Chapter 5 is about resolving associations in user-generated content. 
Purpose: 'Design' is appended to the procedures (documentation, representation and dissemination) of digital heritage research. The use of the term 'design' does not refer to designing a product but to the completion of the methodology defined in Chapter 3.5. It reframes qualitative findings from the two events of the previous subchapter.

Design/Approach: This section reframes previous findings of creative engagement examined in the previous section. Diagrams and tables help position a relational and process-oriented design procedure within the context of this research to move on the subsequent chapter.

Findings: A diagram called MAC (Medium, Audience and Content) is designated to a system of correlations between immersiveness, agency and transformation.

Limitations/Implications: This section provides a systematic conceptual framework to study the participatory content-making in digital heritage.

Originality/Value: The designerly way of knowing is often neglected in digital heritage. Conventional methodologies follow certain procedures to synthesise creative engagement with digital heritage content in museums. This section relates to a previous publication of Author (Aydin, Schnabel and Sayah 2017; Aydin and Schnabel 2017) with substantial modification to fit into this thesis.

Keywords. Design; immersiveness; agency; transformation; cybernetics.

In Chapter 3.5, it is pointed out there is theoretical inactivity, based on the compartmentalisation of the heritage field into science and humanities as two separate schools of thought. According to Winter (2013), this causes "epistemological crystallisation," which in turn generates inertia in heritage discourse (Waterton and Watson 2013). Moving on the subsequent chapter, it is stated that 'digital heritage can best be studied in three processes: documentation, representation and dissemination.' This research claims to append 'design' to these three procedures. The current research highlighted the importance of design in resolving the matters embedded in digital heritage (Parry, Page and Moseley, forthcoming; Vavoula and Mason 2017; Mason 2016; Popple and Mutibwa 2016; Gottlieb 2011; 2015; Pujol-Tost and Economou 2009; Macleod 2005); however, the field of digital heritage is deficient in positioning design as a robust instrument. The focus of this scholarship is on the role of design within the managerial boundaries of museum exhibitions.

In this subchapter, the design is mainly pertinent to the schematisation of the 'immaterial' aspects of 'creative engagement.' A cybernetic design scheme is conceived to reframe the immaterial aspects of creative engagement, which will lead to the introduction of the hypothesis in Chapter 4.5.3. These immaterial aspects revisit the analysis of the gap 
in knowledge explained in the 'Matters of Research' (Chapter 3.5). In this way, the design objective is grounded on the Elimination stage of the RRREI model (see Table 15, p. 120). In this context, the attributed design approach reconceptualises creative public engagement for a design scenario to be developed in the next chapter. Therefore, it makes vertical associations between Chapters 3 and 5, while making parallel connections between the previous subchapters of Chapter 4 (Table 49).

Table 49. The amalgamation of Chapter 4.5 and Chapter 3.5 in the RRREI schema; the vertical connection between chapters and the horizontal combination of subchapters.

\begin{tabular}{|c|c|c|}
\hline RRREI & Chapters & \\
\hline Resolution & 3.2. Matters of Context & 3.4. Matters of Audience \\
\hline
\end{tabular}

Redescription 3.5. Matters of Research

Retroduction 4.2. Documentation 4.3. Representation 4.3. Dissemination

\begin{tabular}{ll} 
Elimination & 4.5. Design \\
\hline Identification & 5.3. Experiment
\end{tabular}

\subsection{Synthesis}

In the previous section, i.e. in the Retroduction stage of the model, the hypothetical components of this research are defined through explanatory mechanisms (Redescription) of documentation, representation and dissemination. These processual mechanisms provided qualitative assessment outcomes from the workshop and the demo show, based on 3D user sketches, observations and unstructured interviews. Now, in the Elimination stage, this research looks at drawing a path to the best explanation for measuring 'creative public engagement.' The next chapter will then use it to demonstrate an assessment mechanism that is to discover alternative knowledge from participatory content-making.

In Chapter 3.5 at the Resolution stage of the RRREI model, the review of the matters pertaining to digital heritage research demonstrated a lack of critical engagement in research. Based on the abstract components as 'Matters of Context, Medium and Audience,' the 'Matters of Research' are grouped in three levels, with each descending onto a theme (see Table 16, page 122). Each of these three themes, namely 'error,' 'multimethodology' and 'abduction,' is committed to 'Documentation' (Chapter 4.2), 'Representation' (Chapter 4.3) and 'Dissemination' (Chapter 4.4), respectively. As a result, three design objectives are defined (Table 50). 
Table 50. Revisiting the matters of digital heritage research.

\begin{tabular}{llll}
\hline $\begin{array}{l}\text { Matters of } \\
\text { Research }\end{array}$ & Arising themes & Methods in & Design objective \\
\hline $\begin{array}{l}\text { Accuracy } \\
\text { (context-related) } \\
\text { (Chapter 3.2) }\end{array}$ & Error & $\begin{array}{l}\text { Documentation } \\
\text { (Chapter 4.2) }\end{array}$ & $\begin{array}{l}\text { Establishment of order through } \\
\text { disorder for creative engagement }\end{array}$ \\
\hline $\begin{array}{l}\text { Crystallised } \\
\text { epistemology } \\
\text { (medium- } \\
\begin{array}{l}\text { related) } \\
\text { (Chapter 3.3) }\end{array}\end{array}$ & Multimethodology & $\begin{array}{l}\text { Representation } \\
\text { (Chapter 4.3) }\end{array}$ & $\begin{array}{l}\text { Escape from conventional } \\
\text { methodological structure of the } \\
\text { field to extend the capacity of } \\
\text { understanding virtuality }\end{array}$ \\
\hline $\begin{array}{l}\text { Information } \\
\text { overload } \\
\text { (audience- } \\
\text { related) } \\
\text { (Chapter 3.4) }\end{array}$ & Abduction & $\begin{array}{l}\text { Dissemination } \\
\text { (Chapter 4.4) }\end{array}$ & $\begin{array}{l}\text { Discovering alternative knowledge } \\
\text { in creative participatory content }\end{array}$ \\
\hline
\end{tabular}

Based on these objectives, a particular role is attributed to design (Table 51). It is not meant to be a product design but a process-oriented description of the Elimination step of the RRREI model. But it is more than assisting the methodological model being followed in this research. There is a hypothetical motivation underlining the involvement of design thinking in this process. This is elaborated upon in Chapter 4.5.2 that the hypothetical motivation of the 'design' procedure is to revisit the concept of virtuality in the cyber museum. Therefore it foregrounds the third sub-question (sQ3) that asks: What alternative knowledge of virtuality lies in the participatory content-making? So Chapter 5 is conceived to address $s \boldsymbol{Q 3}$ while assessing the hypothesis (see Chapter 4.5.2) outlined to find a coherent mechanism for alternative knowledge of virtuality.

The other two sub-questions ( $\boldsymbol{Q} \mathbf{1}$ and $s Q 2)$ have been addressed previously. Based on qualitative findings, they are analysed and assessed as part of the computational processes of documentation, representation and dissemination. In this subchapter of 'design,' a hypothetical foundation is provided to discuss the 'connection' of the assessment of $s \boldsymbol{Q} 3$ to the previous assessments. Therefore this research aims to discuss the main question $(\boldsymbol{Q})$ in Chapter 6: How does a digital design approach steer and diversify creative engagement in digital heritage? 
Table 51. Positioning design as a procedure of digital heritage studies in the context of this research. The classification involves overlaps between procedures. But the demonstration of these overlaps is excluded from the table for simplicity.

\begin{tabular}{|c|c|c|}
\hline & Explanation & As a digital heritage procedure \\
\hline $\begin{array}{l}\text { Documentation } \\
\text { Chapter } 4.2\end{array}$ & $\begin{array}{l}\text { Revisits the arising theme (error) of the } \\
\text { 'Matters of Context' to find 'the } \\
\text { immateriality of tangible heritage' }\end{array}$ & $\begin{array}{l}\text { Connects research to heritage, } \\
\text { museum, archaeology, } \\
\text { architecture, geography, etc. }\end{array}$ \\
\hline $\begin{array}{l}\text { Representation } \\
\text { Chapter } 4.3\end{array}$ & $\begin{array}{l}\text { Revisits the arising theme } \\
\text { (multimethodology) of the 'Matters of } \\
\text { Medium' by demonstrating the } \\
\text { challenges of translating tangible } \\
\text { architectural heritage into creative } \\
\text { digital forms (see } \mathbf{Q} \mathbf{1} \text { ) }\end{array}$ & $\begin{array}{l}\text { Connects research to computer } \\
\text { science, digital forensics }\end{array}$ \\
\hline $\begin{array}{l}\text { Dissemination } \\
\text { Chapter } 4.4\end{array}$ & $\begin{array}{l}\text { Revisits the arising theme (abduction) of } \\
\text { the 'Matters of Audience' based on } \\
\text { qualitative findings from an immersive } \\
\text { digital heritage application conveying } \\
\text { virtuality to public for creative } \\
\text { engagement (see sQ2) }\end{array}$ & $\begin{array}{l}\text { Connects research to HCI, New } \\
\text { Media, creative industries such } \\
\text { as art, games, interaction through } \\
\text { 'product-oriented' design } \\
\text { involvement }\end{array}$ \\
\hline $\begin{array}{l}\text { Design } \\
\text { Chapter } 4.5\end{array}$ & $\begin{array}{l}\text { Revisits the 'Matters of Research' } \\
\text { hindering the sustainability of digital } \\
\text { heritage applications which is } \\
\text { instrumental in assessing alternative } \\
\text { knowledge generation of virtuality in the } \\
\text { participatory content-making (see } \mathbf{Q 3} \text { ) }\end{array}$ & $\begin{array}{l}\text { Connects research to system } \\
\text { thinking, cybernetics, machine } \\
\text { learning, etc. through 'process- } \\
\text { oriented design' }\end{array}$ \\
\hline
\end{tabular}

In the previous section, creative participation is examined in a hybrid immersive VR tool. In the workshop and the public demo, immersiveness is taken for granted by employing the Hyve-3D system (see Chapter 4.4); however, this is limited to a medium-oriented view on immersiveness as a critical component of the virtuality concept in VR. To study further, this section revises phenomenological theories of the 1990s that are discussed in Chapter 3.3.2 to build a systematic perspective on designing virtuality.

Amongst theoreticians, Murray (1998) classifies digital narratives in cyberspace using three aspects, namely immersiveness, agency and transformation. Kenderdine (2015) defines the issue of immersion as an event evoked by a concomitance of embodiment (medium oriented) and embeddedness (user-oriented). The implication of this mutuality between medium-oriented and user-oriented is a lack of focus on content, which is the foundation of virtuality because its transformation is a measure of interaction. In a football game, there are agents (spectators, players and referees) and a medium (grass in the middle of the stadium). But without considering the transformation of the ball itself between the goals, the virtuality of football cannot be qualified. In the same vein, without a way to measure the transformation of content, interactivity is poorly defined. In this research, the 
correlation between medium, audience and content is mapped on Murray's vision of narrative in cyberspace, i.e. immersiveness, agency and transformation, respectively (Fig. 91).

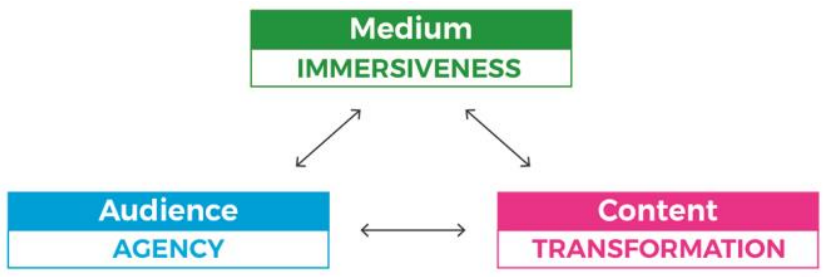

Fig. 91. Murray's " 3 key pleasures in cyberspace" are shown in relation to the medium-, the audience- and the content-centric factors.

\subsubsection{Systematisation of Design as a Novel Procedure in Digital Heritage}

In the previous section, the synthesis of creative engagement is shown to be pertaining to the "Matters of Research" (see Chapter 3.5) in digital heritage. It is concerned with the configuration of these matters with a digital design approach. "Design research is a systematic search and acquisition of knowledge related to design and design activity" (Bayazit 2004). This research embodies the configuration of immersiveness, agency and transformation through a "designerly way of thinking" (Cross 2006). Immersiveness is about the quality of feeling in a digital environment as if it is real. Agency means meaningful role-playing and the digital identity of the immersed subject. Transformation is an aggregation of the changes in content. These three concepts are not crystallised by absolute boundaries. They overlap into each other's domain to construct an entire experience. For example, the transformation includes not only the changes in content but also in users' cognitive abilities; immersiveness is not merely a medium-oriented event but also concomitant to the role of agency.

In this connection, the three components are conceived to be linked via feedback mechanisms. The MAC diagram below is influenced by a cybernetics lecture of Pangaro (2017) as part of an interaction design programme at the Institute of Design and Communication, FH Joanneum. Starting from the inner feedback loop, the transformation of content provides information in order to maintain creative public engagement (Fig. 92). To give an example from the previous section, 3D user sketches are qualitatively classified as to how 'routine,' 'innovative' or 'creative' they appeared (see Chapter 4.4.4). This is the inner feedback loop of the system. Meanwhile, it is pertaining to the matters of the audience by generating assumptions about how to improve participatory content-making. To continue 
with the same example, the research did not develop on giving feedback related to the medium. Since this thesis is not about developing or improving an immersive tool itself, such as Hyve-3D, the findings are narrowed down by referring to research conducted elsewhere (Fig. 92).

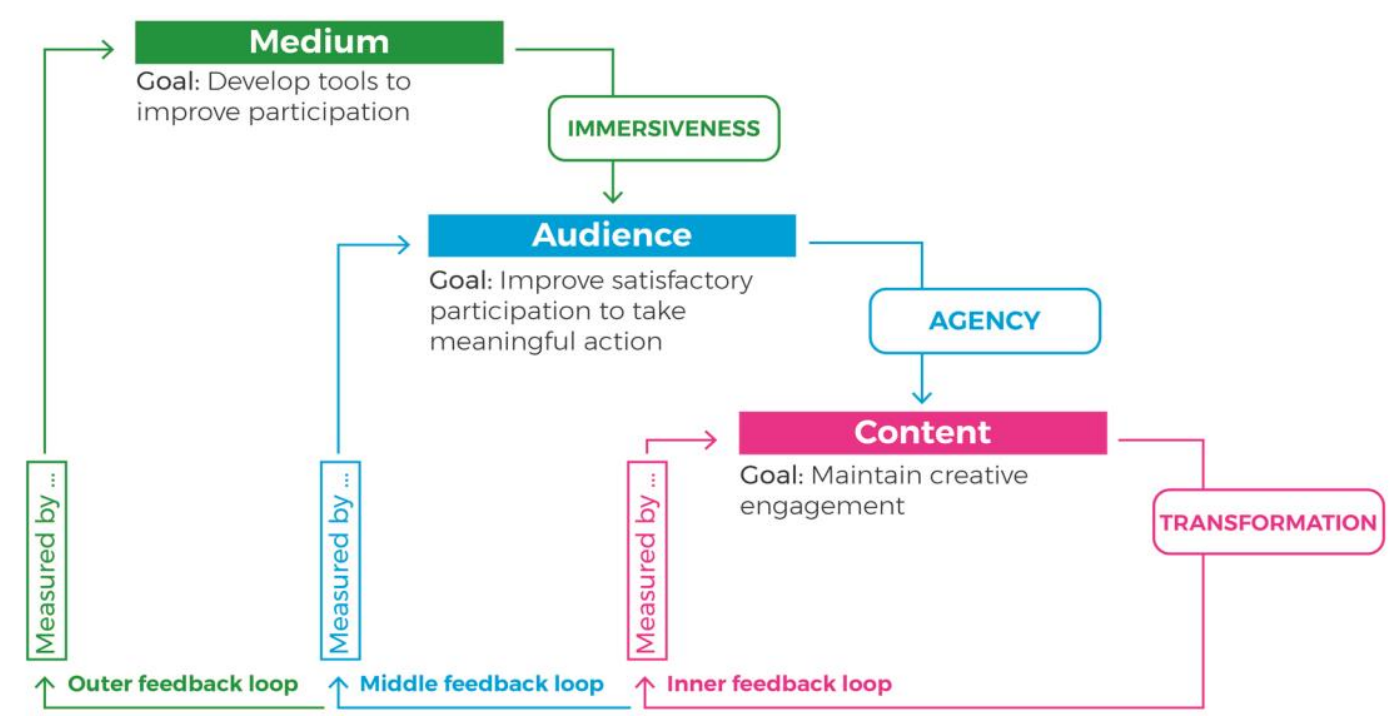

Fig. 92. Systematisation of a process-oriented design procedure in digital heritage. It is called the MAC diagram in this research. Illustration by Author.

Turning back to the inner feedback loop, which will be the focus in Chapter 5, the transformation of content creates a new layer of ambience. Zimmerman and Salen (2004, $305)$ analyse the transformative power of games with a ball bouncing against a wall:

[Transformative play] is a special case of play that occurs when the free movement of play alters the more rigid structure in which it takes shape. The play doesn't just occupy and oppose the interstices of the system, but actually transforms the space as a whole [...] bouncing a ball against a wall is at odds with more utilitarian uses of the architecture. At the same time, the action conforms to certain rules afforded by the formal structure of the building, leading to a particular type of architecture.

It is demonstrated in Chapter 4.4 that the transformation of architectural virtual space into a place of creative engagement alters the rigid conditions of computational geometries, which are delineated in Chapter 4.3.

This section relates to Aydin, Schnabel and Sayah (2017), Aydin and Schnabel (2017a) and Aydin (2017a) that give further explanations on the "goal" of the model schematised above and the "description" of its components and processes. For this thesis, it is summarised here with an overview of the above design schema. It conceptualises a processual examination of alternative knowledge generation from user-generated content. 
In Chapter 3.4, it is found that Manovich's research team uses 'indexicality' as a way of understanding visual content generated by social media users. In their research, data mining and data visualisation techniques are employed to translate the transformation of online visual content into the alternate knowledge of virtuality. In this regard, the above schematisation of medium, audience and content situates 'indexicality' instrumental in measuring the transformation of content. The below version of the MAC diagram shows which part of the subsequent Chapter 5 is focused as a means to generate alternate knowledge of virtuality (Fig. 93).

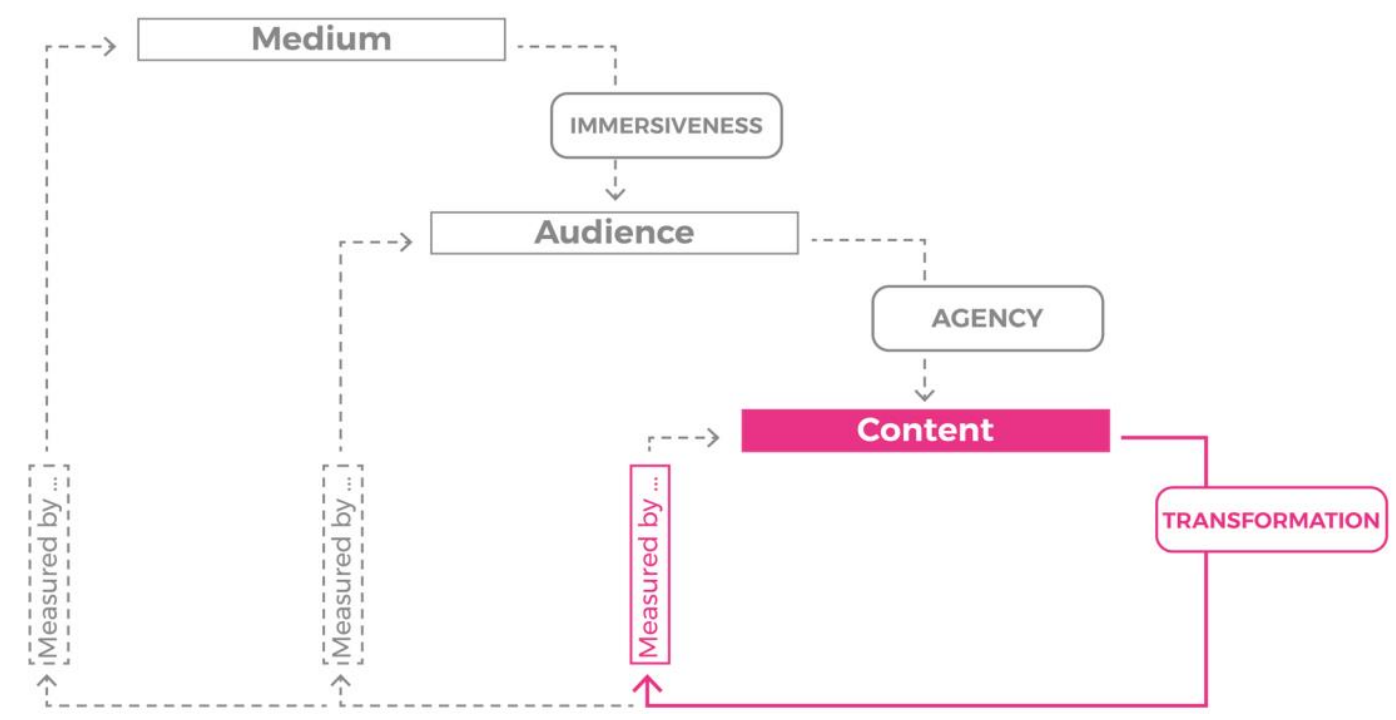

Fig. 93. The focused part of the MAC diagram in this thesis within Chapter 5 that assesses alternate knowledge generation from participatory content-making. Other parts of MAC are greyed out. Illustration by Author.

In the previous publications (Aydin, Schnabel and Sayah 2017; Aydin and Schnabel 2017a; Aydin 2017a), two interactive environments are used to explain the integration of feedback cycles conceived as part of the MAC diagram. This thesis only employs one of these two platforms for the assessment of the hypothesis given below. It is used as an experimental platform in this thesis that is explained in Chapter 5. This experimental platform is designated as a hypothesis to be assessed. The other platform is excluded from this thesis for clarity, whereas its correlation to this research is included in the discussion (Chapter 6). 


\subsubsection{Hypothesis}

The MAC diagram conceptualises a relational structure for examining virtuality in the computational museum. Digital design methods are examined in the previous subchapters for forming an understanding of a computational mechanism whereby the contemporary museum can be altered. Meanwhile, the two sub-questions ( $\boldsymbol{Q} \mathbf{1}$ and $s Q 2)$ are addressed. Last but not least, the third sub-question $(s Q 3)$ is about finding what alternative knowledge of virtuality lies in the participatory content-making (Table 52).

Table 52. Research questions

\begin{tabular}{l}
\hline Research Questions \\
\hline $\begin{array}{l}\text { Q: How does a digital design approach steer and diversify creative engagement in digital } \\
\text { heritage? }\end{array}$ \\
\hline sQ1: How can architectural heritage be translated into creative digital forms? \\
$\begin{array}{l}\text { sQ2: How can an immersive digital heritage application convey virtuality to the public via } \\
\text { creative engagement? }\end{array}$
\end{tabular}

sQ3: What alternative knowledge of virtuality lies in the participatory content-making?

It is found pertaining to Murray's (1998) classification of cyberspace narrative that immersiveness, agency and transformation can be directly corresponding to the 'Matters of Medium, Audience and Content.' Three hypotheses arise from the fact that the source of alternative knowledge can be either the medium, audience or content in creative public engagement. Having outlined the systematisation of the MAC diagram, it is inferred that 'transformation of content' is instrumental to measuring alternative knowledge of virtuality in participatory content-making. Therefore, the best explanation of these three hypotheses as to whether medium, audience or content is the basis of alternative knowledge of virtuality can be translated as:

Hypothesis: Alternative knowledge of virtuality in digital heritage is entangled with the transformation of content in creative engagement. 


\section{Chapter 5. Decoding Kashgar via User-Generated Content}

We turn our own lives into information archive by storing all our emails, SMS, digital photos, and other digital traces of our existence.

- Lev Manovich, The Shape of Information (2005)

\subsection{Introduction}

In this chapter, the development of an interactive experiment platform in a game engine is presented. Game design elements are composed to construct the experiment platform. The section about the development of game mechanics and gameplay includes the description of concept elements, game assets, codes and a database (Fig. 94).

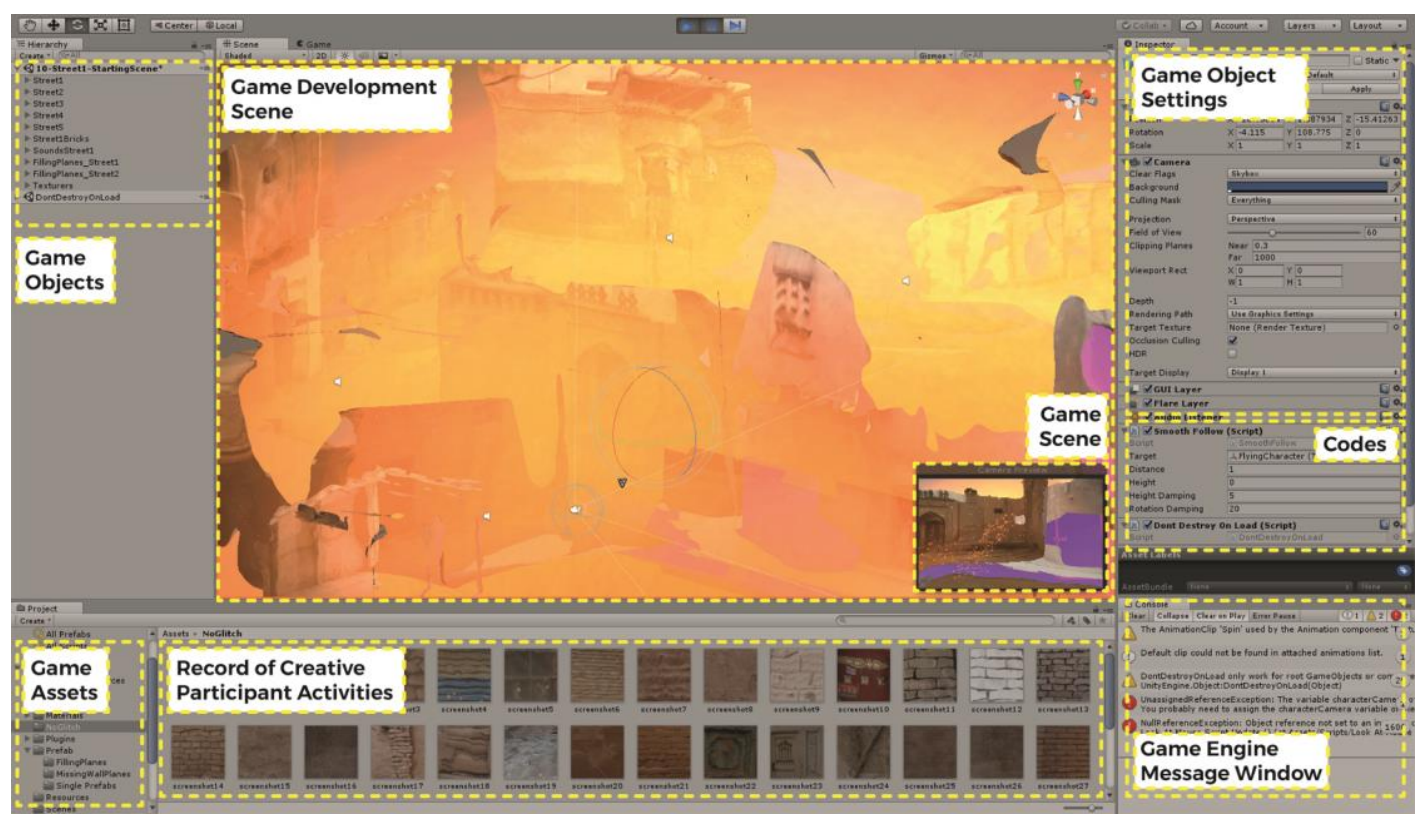

Fig. 94. The outlook for the game engine software where an experiment platform is constructed. 
During the design process of the experiment platform, concept elements driven by the context are represented as game objects. Interactivity between game objects is described by codes. A database folder is built to automatically record the creative outcome of the participant in the experiment. Association Rule Finding is introduced as a novel method to disclose correlations between lists of participant outcome.

An experiment is contrived to draw conclusions from an analysis of alterations in content. Other details about participants, variables, analysis, findings and limitations are described in due order.

Subsequently, a synthesis is derived from a qualitative assessment of the quantitative experiment results. The synthesis part utilises the findings from the experiment to respond to the three sub-questions of this research. Therefore it elaborates how the findings from the experiment confirm the hypothesis that alternative knowledge of virtuality in digital heritage is entangled with the transformation of content in creative engagement.

By introducing the use of a game data mining technique, namely Association Rule Finding, for the first time in the digital heritage field, this chapter demonstrates what alternative knowledge of virtuality lies in participatory creative engagement. The content of this chapter relates to Aydin, Schnabel and Sayah 2017. To mark this chapter in connection with previous documentation methods, Fig. 95 depicts a superimposition of the SfM photogrammetric models on the map of Köziciyerbişi (Gaotai). These models are used as the prime resource in the construction of the experiment platform.

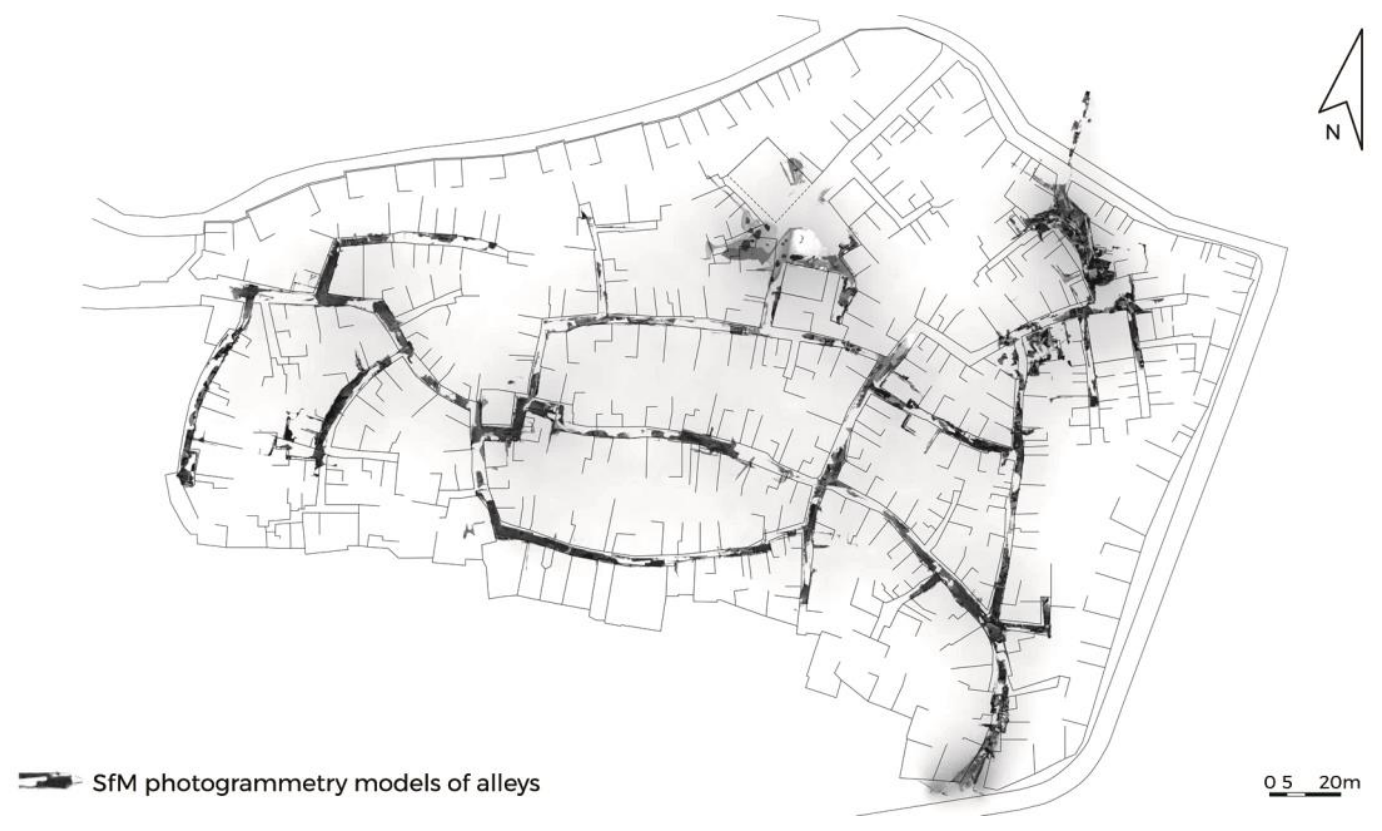

Fig. 95. SfM photogrammetry models of narrow roads in Köziciyerbişi (Gaotai) are used to construct the experiment platform. 


\subsection{Experiment Platform}

\section{Subchapter Outline}

Purpose: This section details the design of the experimental platform along with a data mining method introduced. It highlights the role of design in bringing together the concepts of architectural heritage, games and data mining.

Design/Approach: It borrows terminology from game design, e.g. the use of 'player' refers to the previous 'participant' and 'user.' It is conceived as a brief game design document that informs design concepts, mechanics of the gameplay and database development for collecting data to be assessed.

Findings: Low-cost game development platforms offer excellent value for conveniently transforming SfM models into game objects for interactivity.

Limitations/Implications: Low-cost design methods are used as a criterion of 'productivity' and 'required skills.'

Originality/Value: The first interactive virtual platform of the Kashgar oldtown is produced. Mechanics from other games are decomposed and uniquely contextualised according to the objectives of this thesis. This section relates to Aydin, Schnabel and Sayah 2017.

Keywords. Interaction; MDA; game mechanics; Unity; explorable game objects; game data; frequent pattern finding; Association Rule Finding.

In the previous chapter, a diagram with the abbreviated title MAC is constructed to delineate a hierarchy of connectivity and association between 'Medium, Audience and Content' (see Fig. 92, p. 204). MAC matches with Murray's (1998) classification of engagement in cyberspace with immersiveness, agency and transformation, which are essential to computer games, whereas the "gamer is a wizard in virtuality" (Coulter 2007) and plays the role of adventurer into the future of humanity in cyberspace (Baudrillard 1993).

Research in digital architectural heritage interprets game elements as primarily 'spatial' simulation technologies (Champion 2011). These game-based spatial studies deal with the level of immersiveness while the role of agency is examined as complementary. Agency and immersiveness in those works are focused on architectural education as to how game-like engagement improves the learning of spatial aspects (Richens and Nitsche 2005; Varinlioglu et al. 2017; Şahbaz and Özköse 2017). The strategic use of games in non-game contexts is called serious games (and gamification), which are investigated in digital heritage (Champion 2015; Ioannides, Magnenat-Thalmann, and Papagiannakis 2017).

In this research, spatial immersiveness plays an essential role, but the focus is on the transformation of content. The role of agency is excluded from the study since the objective is not to build a serious game environment to test immersiveness, which is examined in Chapter 4.4 through a VR application. 
In game design and research literature, the Mechanics, Dynamics and Aesthetics (MDA) framework of Hunicke, LeBlanc, and Zubek (2004) is a highly cited methodological approach. It formalises games with three essential design elements pertaining to rules, challenges and emotions, respectively (Fig. 96). Mechanics are concentrated in this section to demonstrate the design of an interactive scenario. In this way, Dynamics and Aesthetics are integrated into the scenario and discussed on a broader scope in Chapter 6.

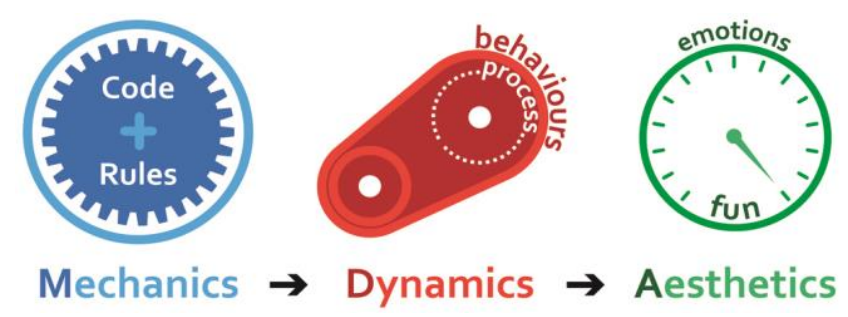

Fig. 96. MDA framework (Hunicke, LeBlanc, and Zubek 2004). Illustration by Author.

\subsubsection{Mechanics and Gameplay Development}

In this section, the development of the mechanics and gameplay is studied under four categories including concept elements, game objects, codes (scripts) and finally naming (Table 53).

Table 53. Mechanics and gameplay development of the experimental platform.

\begin{tabular}{lll}
\hline & Categories & Explanation \\
\hline 1 & Concept elements & Driven from the context, including repairing, protecting, etc. \\
\hline 2 & Game assets & Digital content that populates the experimental platform \\
\hline 3 & Codes & JavaScript and C\# files that describe how the game objects interact \\
\hline 4 & Database & The file where the player interaction is recorded in \\
\hline
\end{tabular}

\section{- Concept Elements}

A conceptual framework is developed based on the findings from the 'Matters in Context' (Chapter 3.2). They are the sources for growing a meaningful design scenario. Conceptual actions, such as to protect, to repair, to give away, to place love, to mimic and to return home, are driven from the context in Kashgar. Interaction design decisions are made based on these concepts. In due course of the entire construction of the experiment platform, they support the idea of 'digitally preserving' the architectural heritage of narrow roads in Kashgar's Köziciyerbişi [Ch. 高台; pinyin. Gaotai; U. كوزجيار بنشى]. Unity (formerly 
Unity3D) game development platform is used to translate the design concept into game mechanics. The development of the experimental platform also borrows concepts and terms from the game design field. The terms 'participant' and 'user' that are used in previous chapters are mostly omitted in this section because the term 'player' is adapted, instead.

\section{- Game Assets}

Mechanics of other games, e.g. Flower ${ }^{\circledR}$ (2018), are decomposed and customised in an interactive environment to shape an experiment platform. The development of this virtual environment in Unity was assisted by Scott Meekings for writing the 'codes' of interaction between game objects as designed by the Author. In this virtual environment, nine game objects in two groups (explorable and non-explorable) are composed to construct an acceptable level of interactivity at an inexpensive cost of computational power (Table 54).

Table 54. Game objects used in the experiment.

\begin{tabular}{ll}
\hline Explorable Objects & Non-explorable Objects \\
\hline 3D photogrammetric SfM models & Skydome \\
\hline Flying first-person controller (FP) & Surrounding environment image (2D texture) \\
\hline Brick checkpoints (3D cubes and 2D textures) & Speedway (3D invisible tunnels) \\
\hline Filling planes (2D purple highlights) & Speed transfer object (invisible) \\
\hline Sound files & \\
\hline
\end{tabular}

Firstly, separate 3D models are combined based on the maps presented in Chapter 4.2. The SfM photogrammetry models of Köziciyerbişi (Gaotai) are imported as new game assets in five groups in a Unity scene (Fig. 97). The glitched and incomplete SfM models are combined with the idea that imperfection induces the player to participate in the making of digital heritage content (Fig. 98). This confirms with the critique of the over-concern with materiality in heritage studies in general and with the level of accuracy as a predominant criterion of success in the contemporary digital heritage research. The player must project her/his own intuitive understanding in order to complete the holes and gaps in models. This activity corroborates participatory content-making in digital heritage, which is presented earlier as one of the main gaps in knowledge in Chapters 2.3 and 3.4 (see also Chapter 3.3.1). The participant is planned to advance beyond the provided factual heritage information by using her/his creative power of imagination. 


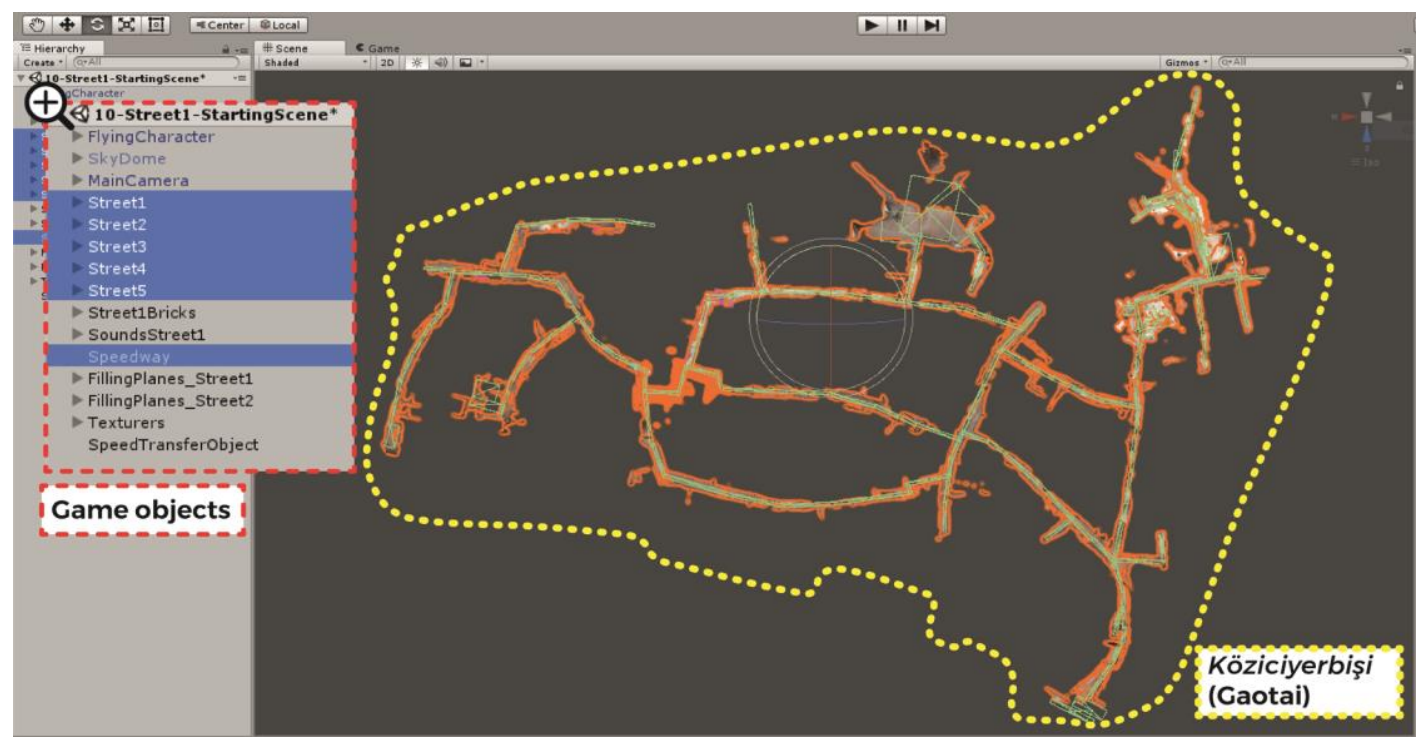

Fig. 97. The top view of alleys in Köziciyerbişi (Gaotai) transformed into Unity as game objects (compare its alignment with the actual map of the neighbourhood on p. 142).
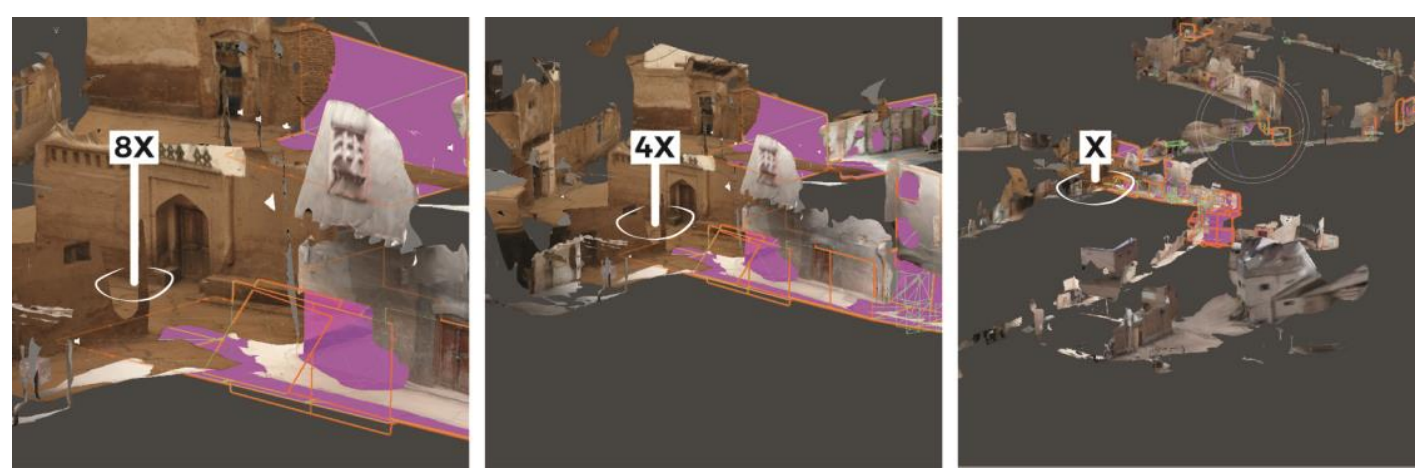

Fig. 98. Imperfect SfM models (with zoom scales $8 X, 4 X$ and $X$ ) with glitches and holes to stimulate the player to project her/his understanding to complete them.

In connection to the idea of the player repairing the SfM models, an analogy with 'brick,' the basic construction element in Kashgar, is made, enriching the gameplay. The brick collection is used as the main activity. Brick collection checkpoints are placed alongside the alleys of virtual Köziciyerbişi (Gaotai) model. There are two types of brick checkpoints (Fig. 99). The first is made of cubes and placed as visible objects on the road. The second type of collectable bricks is hidden behind semantic elements of the SfM models such as doors and windows. This is conceived as an indirect message to the player to intuitively add 'smart' information about where a door or a window is on digital mesh geometries that are, by default, devoid of the definition of these substances. The collection of the first type of bricks prompts visual and textual feedback (Fig. 100). Approaching the second type of bricks generates glitches on display and textual feedback is provided as the 'Brick Count' shows increase (Fig. 101). 


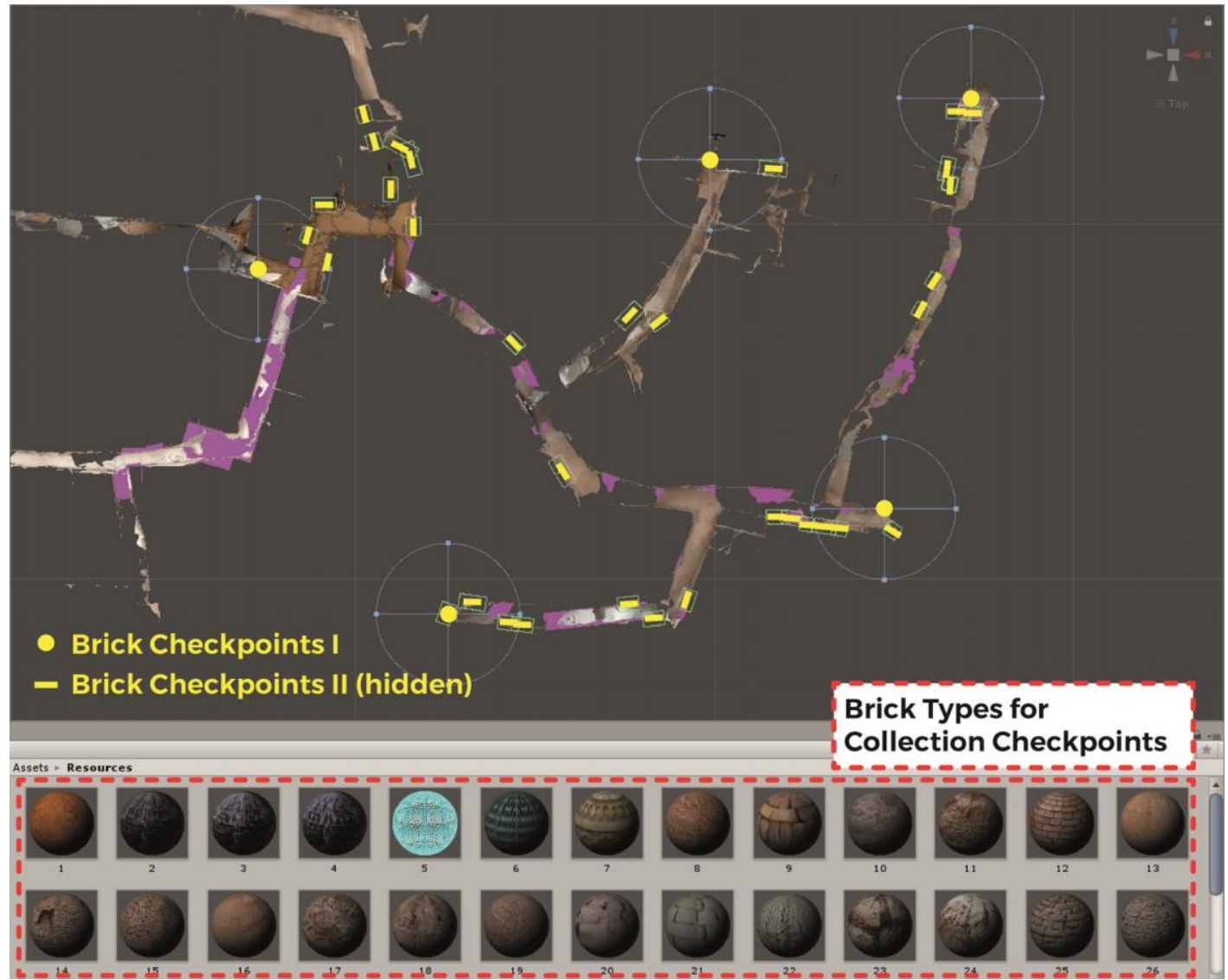

Fig. 99. Top view of brick collection checkpoints placed on the alleys.
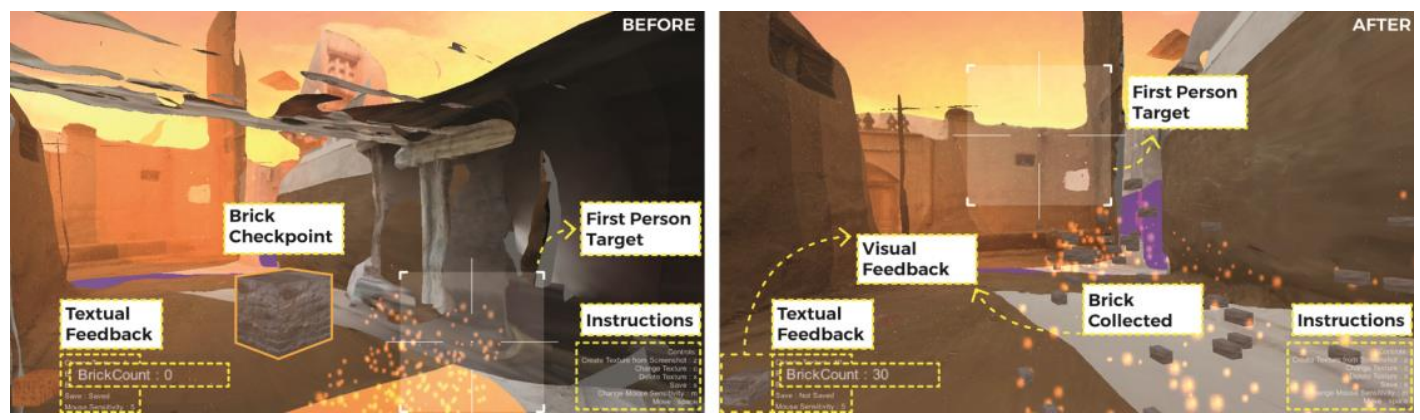

Fig. 100. Brick collection (Type I - Visible): A brick collection checkpoint (cube) before collection (left). It is shrunk into pieces after collection (right). 'Brick Count' gives textual feedback.
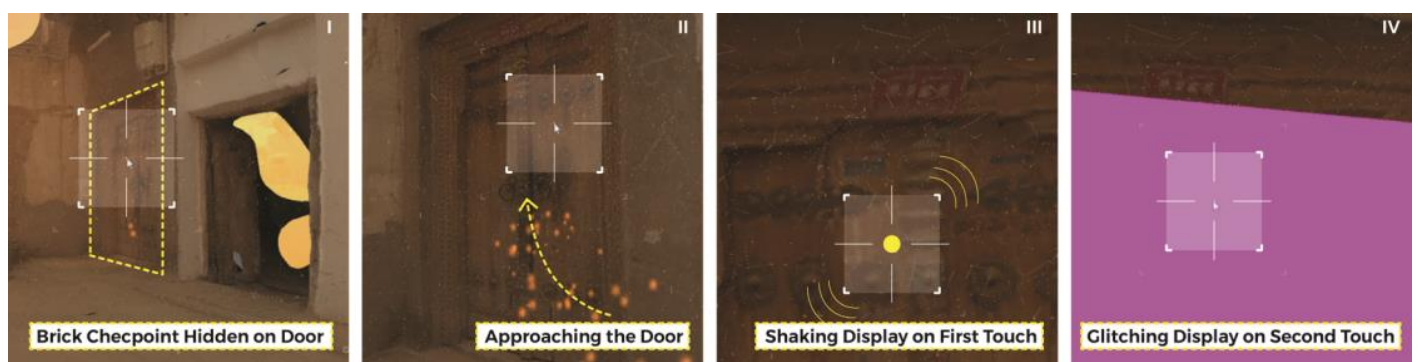

Fig. 101. Brick collection (Type II - Hidden): From left to right, a brick collection checkpoint (a door) is guessed, approached, touched and glitched as visual feedback. 
The missing parts of the SfM models are visually highlighted with a fluorescent purple colour to indicate that the player can fill them with items collected (Fig. 102). But their function is explained in the subsequent section. Moving on to the flying first-person character (FP), speedways and audio files, this part further elaborates on how the collision detection function in Unity is used to control its speed and the volume of the embedded audio objects.

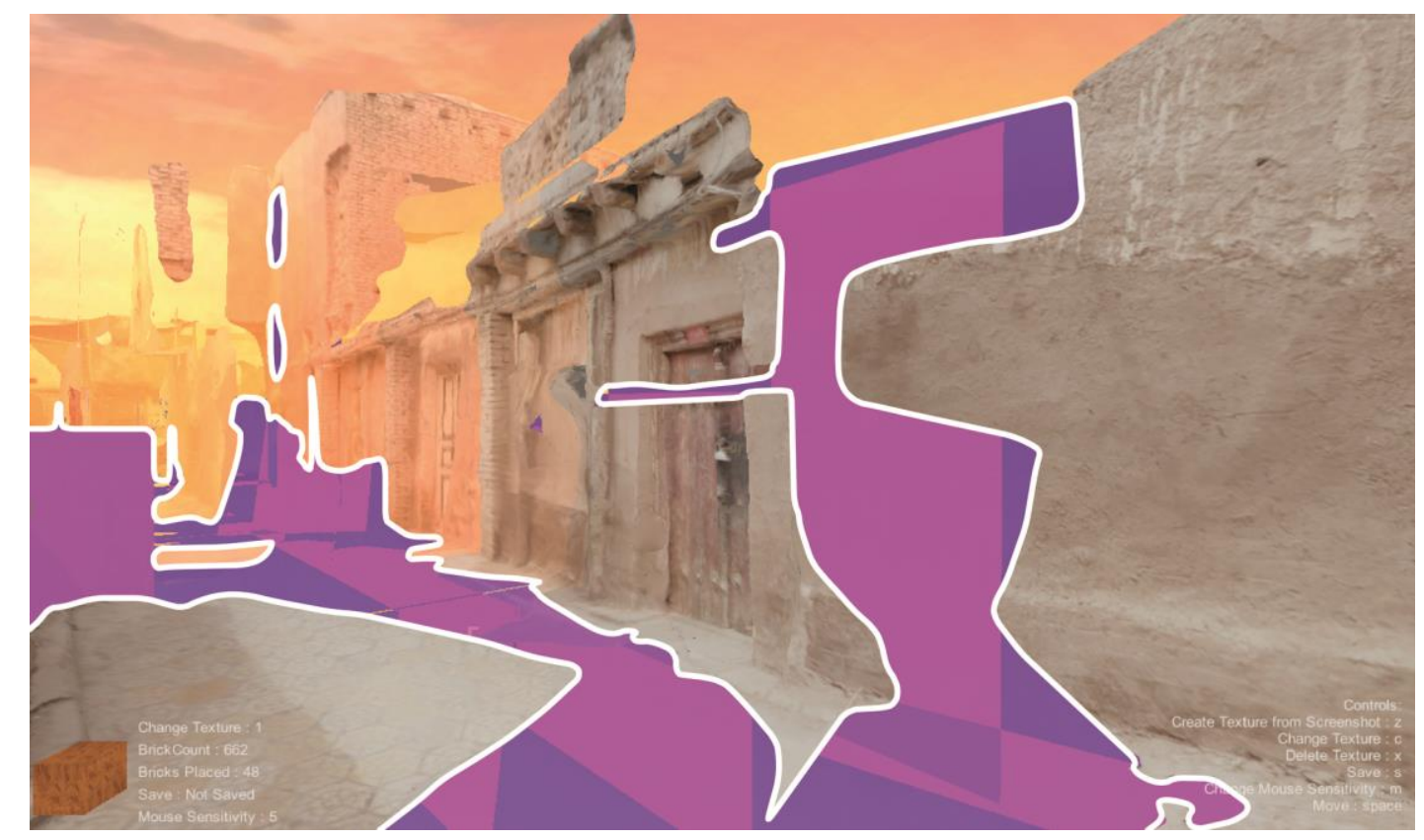

Fig. 102. Filling planes of the virtual Köziciyerbişi (Gaotai) model, highlighted with a fluorescent tone of purple to attract the player's attention to interaction.

Controlling a flying first-person character (FP) associates a sense of freedom to the confined spaces of alleyways. This is increased by adding particle objects from the standard game assets in Unity. These particles trace the movement of the FP character of the player. However, the course of the flying FP is restricted by speedway tunnels that are placed inside alleys and invisible in the play mode. The last explorable game objects in the platform are audio files (.ogg). To mimic the soundscape of Köziciyerbişi (Gaotai), a list of street sound recordings (see Chapter 4.2.3) was planted in the environment, with their volumes turned up and down by player proximity (Fig. 103). Both audio files and speedway tunnels are instigated by the player's movement. The position of the flying FP attracts these invisible game objects by means of collision detection that triggers the assigned function once the collision boundary of the game object is affected. 


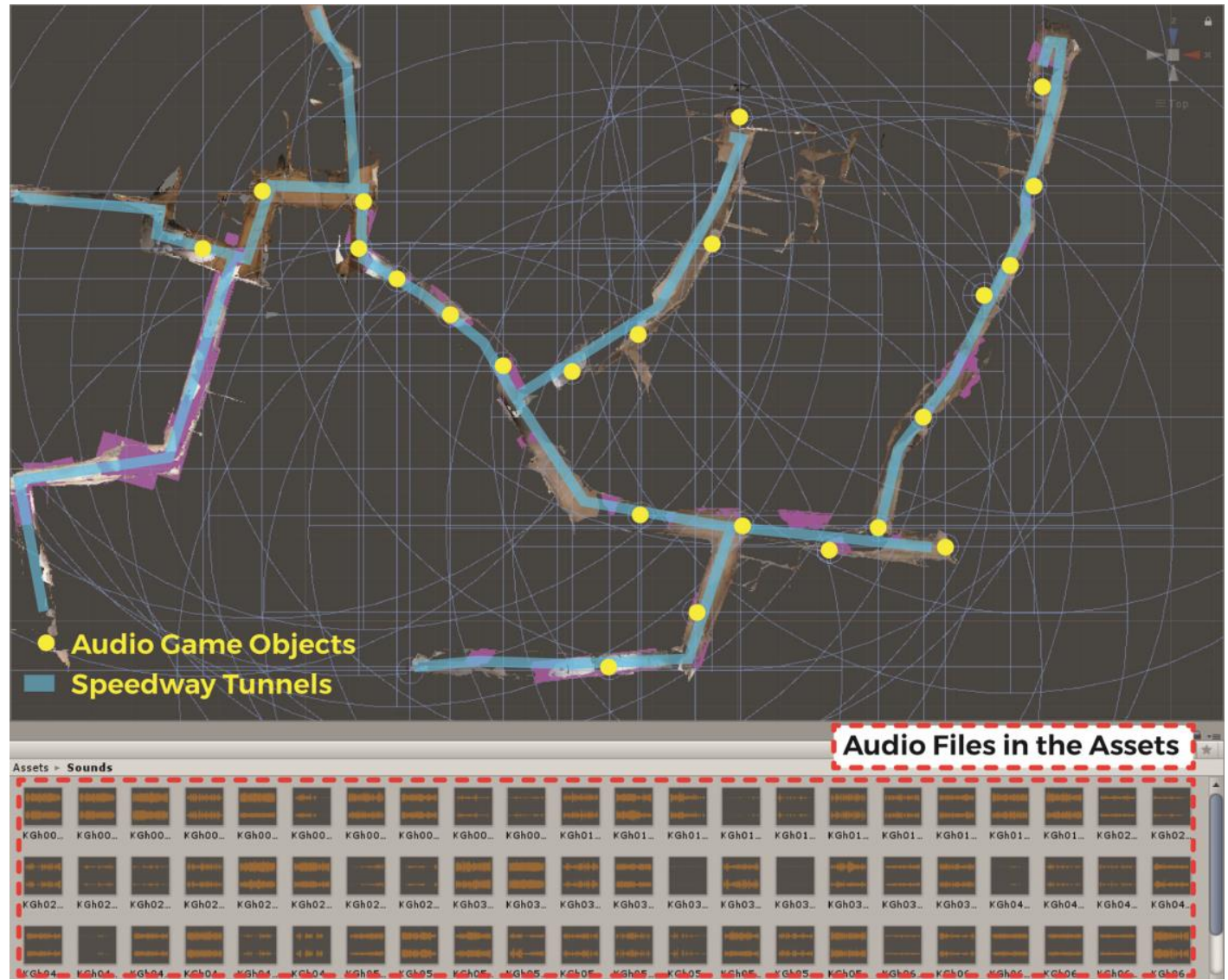

Fig. 103. Top view of the positions of audio game objects used for generating the ambience of streets in Kashgar's Köziciyerbişi (Gaotai) (see street sound recording in Chapter 4.2.3).

\section{- Codes}

Collecting bricks from the roads of virtual Köziciyerbişi (Gaotai) represents the essential activity of museums as collectors. In the experimental platform, the 'collection activity' is integrated into the gameplay via interaction between game objects (see the previous section). More intuitive interaction is achieved with the design of a 'selection activity.' A selection role is conceived so that the player can identify with the role of a museum curator whose appetite is determinant to deciding content for exhibitions. Therefore the 'selection activity' within the gameplay is designed for more artistic and creative expression than the 'collection activity' (Table 55). In this way, a transition of the role is defined for the player. A similar role change was observed in the experiments of immersiveness via Hyve-3D (see Chapter 4.4). Intuitively drawn 3D sketches of museum visitors enabled creative engagement during the transition of the experience from being purely immersive to agency, i.e. meaningful roleplaying (see Chapter 4.4.4 and 4.4.5). These connections between the experiments of Chapter 4 and this Chapter 5 will be made in the discussion Chapter 6 . 
Table 55. Principal gameplay roles in the experimental platform.

\begin{tabular}{ll}
\hline Selection Activity & Collection Activity \\
\hline Bottom-up & Top-down \\
\hline High-level role playing & Low-level role-playing \\
\hline Content generative & Content consumptive \\
\hline Catharsis & Mimesis \\
\hline Creative & Communicative \\
\hline
\end{tabular}

Having designed interaction around the collection activity by mainly means of the collision detection function in Unity, the gameplay of the experimental platform is enhanced with the design of a 'selection activity' by scripting a higher layer of engagement. Codes are written in JavaScript (.js) and C\# (.cs) supported by Unity (by August 2018). The selection role ensures creativity in the sense that the activity is based on intuitive decisionmaking. To allow this, previously mentioned 'filling planes' are used (Fig. 104). Some of the holes of the SfM models are filled with these planes coloured differently. Navigating through the virtual narrow roads of Köziciyerbişi (Gaotai), it is designed that the player fills these planes with patterns at their disposal. By using the target of the flying FP, the player is tasked to collect texture patterns from the polygon mesh surfaces. A texture pattern can be saved by pressing on a keyboard button which is, in this case, ' $z$.'

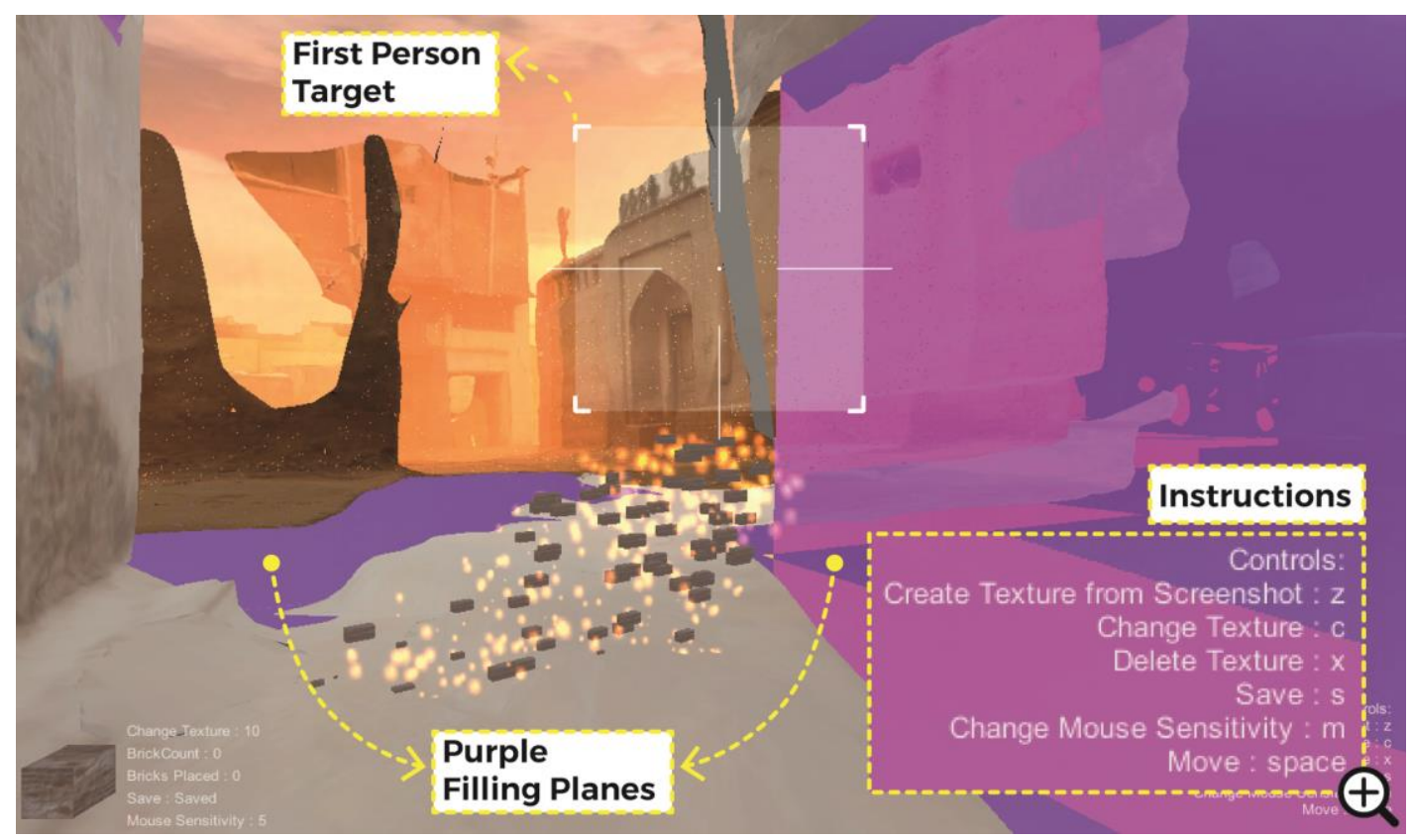

Fig. 104. The 'selection activity' in the experiment platform consists of saving a texture pattern by casting a screenshot within the boundaries of the FP target frame and then placing one of the hitherto saved patterns onto filling planes. 


\section{- Database}

Together with concept elements, game objects and codes, the database folder is the last element for elaboration in the mechanics and gameplay development. The gameplay development is terminated with the design of a 'selection activity' and constructed in Unity. Flying through the alleys, the player searches patterns to save in the library. Having created a pattern by casting a screenshot bound to the target square of the FP, the player is ready to patch holes (Fig. 105). Repairing the missing parts of the model, the player is allowed to change or delete patterns that are patched on filling planes coloured in purple (Fig. 106). All these activities reflect the player's aesthetical interpretation of the virtual Köziciyerbişi (Gaotai). The library of patterns being selected, shot and saved by the player generates a visual narrative in the form of thumbnail images. The library is recorded in a separate data collection folder. By that, the mechanics and gameplay development of the experiment platform is wrapped up.
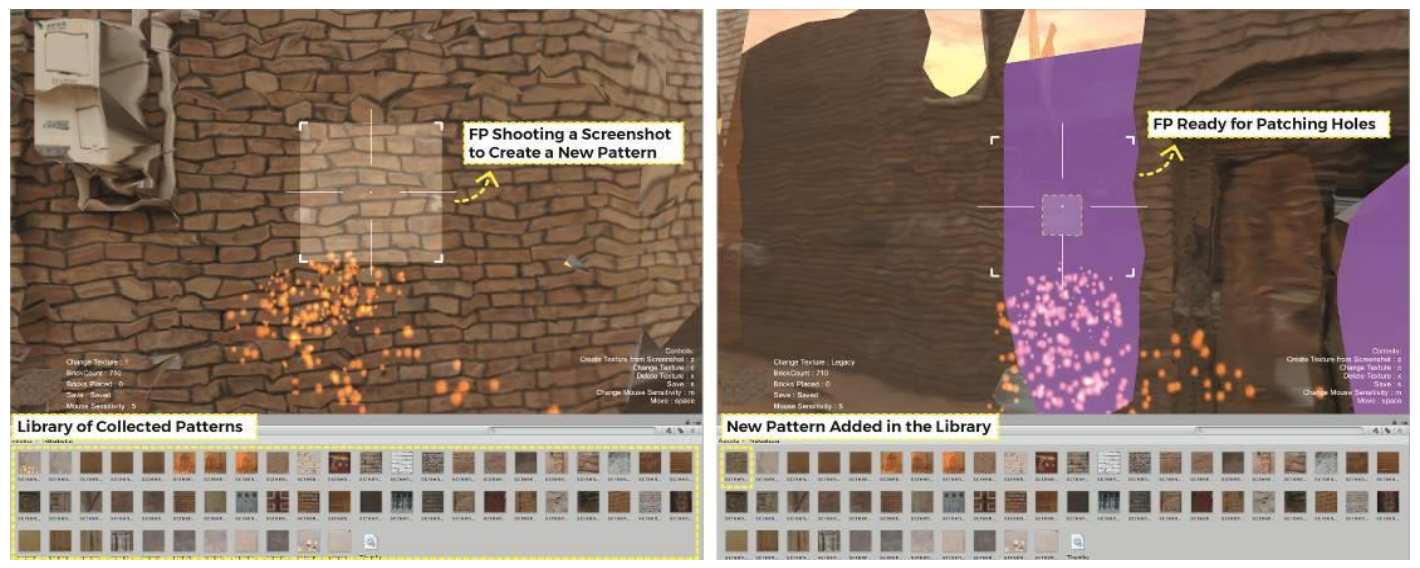

Fig. 105. The flying FP character moves freely within the alleys, looking for patterns to patch on holes indicated with purple filling planes. A brick wall pattern is selected (left) and added in the library (right).
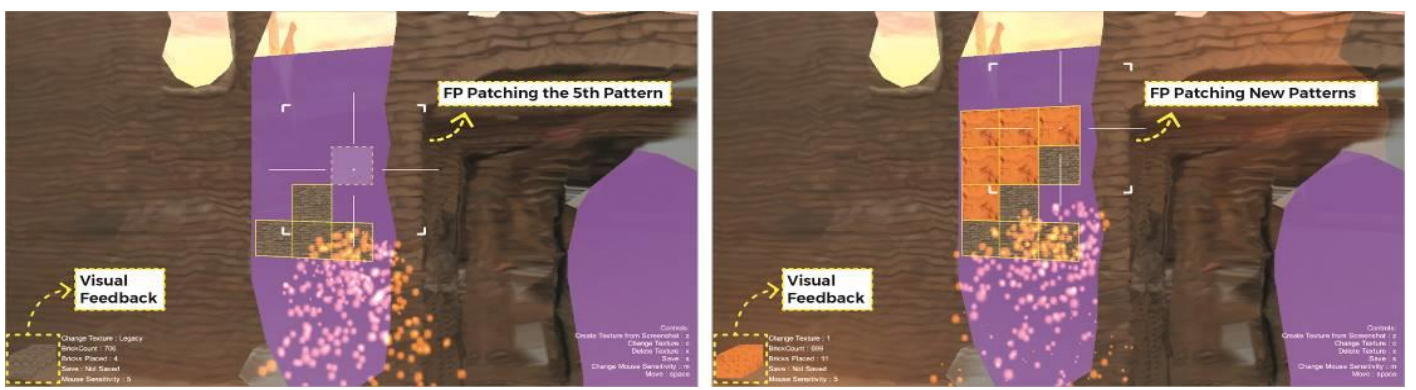

Fig. 106. The flying FP patches the units of a hole with a brick pattern (left) with the visual feedback illustrating the selected pattern from the library. Having called a new pattern, the FP continues the virtual masonry of patching holes with wall patterns (right). 
To summarise the gameplay in five steps, the player engages with heritage by: (1) navigating as a flying FP in the alleys of Köziciyerbişi (Gaotai), (2) exploring the style, (3) collecting existing bricks, intuitively generating new ones and storing them, (4) tracing glitches, (5) selecting patterns from the library and patching them on highlighted parts (Fig. 107).

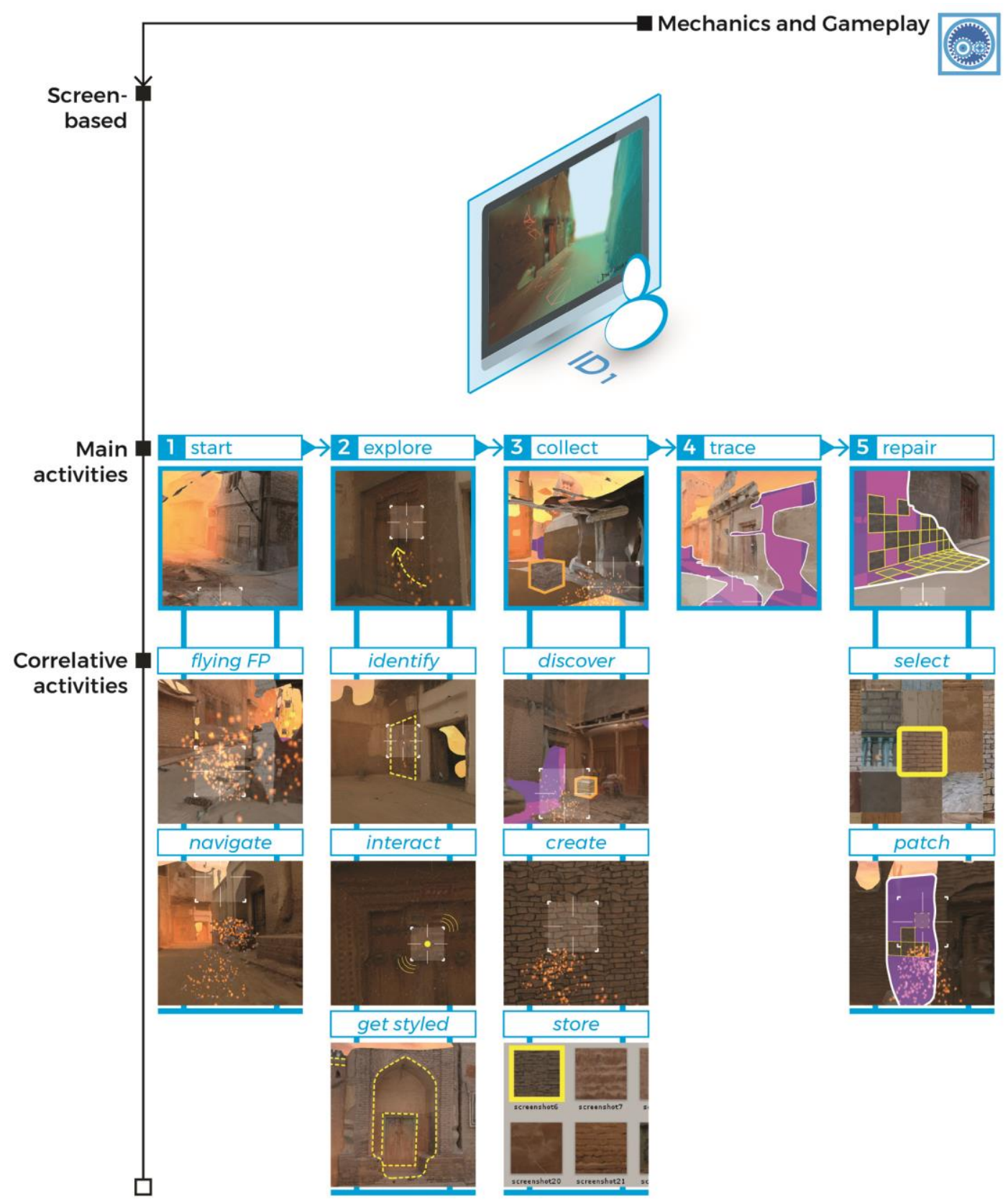

Fig. 107. Mechanics and gameplay summary are showing participant activities in the experiment. Illustration by Author. 


\subsubsection{Association Rule Finding (ARF) Method}

In this section, an essential data mining technique called Association Rule Finding (ARF) is introduced in relation to setting up a database folder to record the player activities. This database is a visual summary of the participant's creative engagement in the experiment platform. In Chapter 3.4, it is demonstrated based on Manovich's data mining projects that the visual stories of online social media users potentially generate alternative knowledge. At this level, potentially useful alternative knowledge is unknown until a correlative study is performed. Similarly, this experiment platform is designed to discover alternative knowledge from the transformation of content in digital heritage. Based on the pattern library of the player, a visual storyline provides a large dataset to analyse semantically, including a study of the basic visual units of these patterns.

Image-based historical research has been in use since the emergence of early museum computing activities in the 1960s. Henri van de Waal implemented a comprehensive system called "Decimal Index of the Art of the Low Countries; D.I.A.L" for photo-cataloguing the artworks in the Netherlands (Ellin 1968). Since then, deriving meaningful information from image-based data has been limited to "the capture of images, rather than analysis" (Dee et al. 2016). There are many features that can be extracted from user-generated image-based content to make an indirect observation without requiring demographic information about the audience. In the design scenario of this experimental platform, a large virtual community is assumed to be a potential user group about which it is nearly impossible to acquire demographic information. In this scenario, engagement is presumed to be online as part of a futuristic prospective of the museum in information space mentioned in Chapter 2.5.

Therefore indirect observation methods for data gathering are investigated from the field of game analytics. Transferring such methods and studies from game data mining to digital heritage and virtual reality research is earlier recommended by Champion, Dave, and Bishop (2003). Indirect observation in games, which is called gameplay metrics, is one of the approaches under development in the field of procedural games and game analytics (Drachen and Canossa 2009). These approaches are utilised in many ways and sometimes in hybrid forms in order to achieve 'player modelling' for gameplay improvement (Seif ElNasr, Drachen, and Canossa 2013). Game metrics may involve the duration of a player in a specific area in the virtual space of the game world or player itemsets that consist of a collection of game elements throughout a level of gameplay.

In game data mining, choosing one of the analytic methods relies on what information is needed to be extracted from player experiences. In this research, the information that 
needs to be extracted is based on the curiosity about what relations might exist between usergenerated content to examine the shift from 'agency' to 'transformation.' In general, there are two approaches, supervised and unsupervised, which are the terms that the game designers and researchers borrow from the field of machine learning (Drachen 2013; Betts 2014). The selection and collection activities described above are subject to examining the transformation of content in user engagement. As the gameplay is designed in order for the player to create a collection of texture patterns, the correlations between the item libraries of players can unveil information about the game world.

ARF, described as an unsupervised machine learning method, is a data mining method used in game design to understand correlations between player activities. ARF is an increasingly important subject in the game design field. Establishing helpful and unfamiliar data from player transactions, rules are discovered from associations to gain insight into user experience. Association rules between pieces of data that represent individuals' collection of objects are conditional statements such as 'if there is a door, there is a handle.' These rules help uncover relations between discrete pieces of data in a relational database.

Therefore association rules inform about relationships of player data, which are otherwise not noticeable. In summary, ARF performs a correlation (in this thesis the terms association and correlation are used interchangeably) analysis to find attributes that are possibly contingent on each other. In this research, ARF is used to measure and disclose these attributes from the content under transformation (Fig. 108).

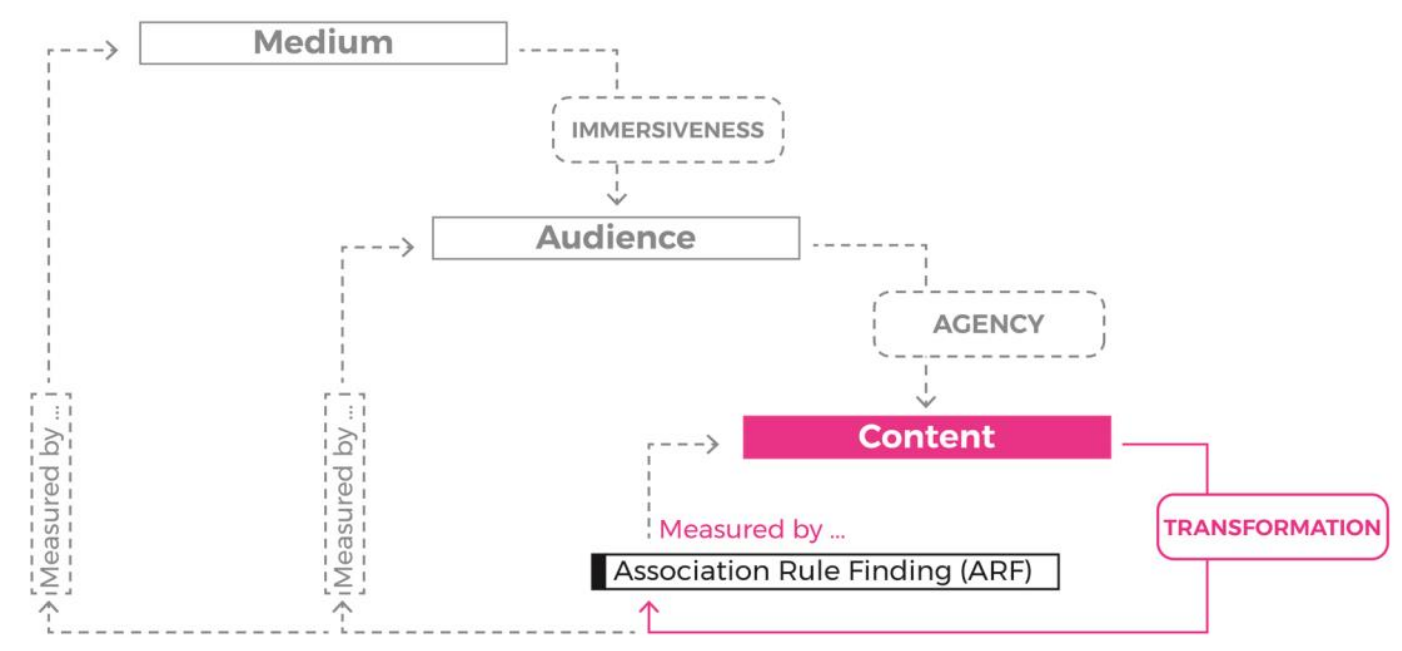

Fig. 108. Further development of Fig. 93 p. 205: Association Rule Finding positioned in the box of 'Measured by...' as part of the inner feedback loop within the MAC (Medium, Audience and Content) diagram. Illustration by Author. 
In ARF, attributes of quantitative data are analysed to disclose correlations between individual items generated by the player. For example, players collecting unique sets of items in Minecraft, inadvertently generate associations between their own itemsets and that of other players (Drachen 2012). These associations remain unknown until analysed. Likewise, every participant exhibits a unique interest in different parts and patterns in a digital heritage environment such as the experiment platform design for this research. In this scenario, a list of collected items tells the story of a unique virtual journey.

Calculating the frequent sets of instances is the first method to analyse user-generated content, which does not take attributes into account. It is followed by an FP-Growth algorithm in search of association rules between player itemsets. The frequency list is then reformulated by threshold factors. The measures of 'support,' 'confidence' and 'lift' condition some threshold values used to find stronger associations.

The 'support' measure indicates the frequency of an itemset in the entire database. It means that an itemset with $1 \%$ support is contained in $1 \%$ of all transactions in a database. In the context of this research, a transaction consists of the patterns collected by the participant for patching holes in the experiment platform. Therefore a transaction may contain only one item or as many items as available variables in the experiment (see variables in Chapter 5.3.2).

The 'confidence' measures the strength of an association rule. It is the frequency of two or more items as to how often they exist together in all transactions. If an association rule between a brick pattern $(\mathrm{X})$ and a cobblestone $(\mathrm{Y})$ has $8 \%$ confidence, it means that $8 \%$ of the transactions contain both items together.

The 'lift' measures the attractiveness of an item to another item. If an item is not very frequent in relation to all transactions but co-exists very often when it comes together with another item or items, it means the item is lifted. It may be the other way around. Despite being very frequent amongst in the whole transaction database, an item may be repulsed in relation to a specific item or items. In this case, a reverse association rule is discovered to suggest that no association exists. In this case, an item is disqualified from forming an interesting correlation with a specific itemset even if the item is statistically frequent.

The functions of 'support,' 'confidence' and 'lift' show that ARF technique allows non-linear and statistically independent data interpretation. These three functions of ARF are employed to generate association rules in the experiment (see findings in Chapter 5.3.4). Table 56 shows an example of four player itemsets. 
Table 56. Transactional data for 4 players as an example.

\begin{tabular}{cl}
\hline ID & List of items \\
\hline 1 & Thick Brick, Darwaza Gate, Pink Curtain, Red Door \\
\hline 2 & Thick Brick, Mud Wall, Red Door \\
\hline 3 & Thick Brick, Red Door, Darwaza Gate \\
\hline 4 & Darwaza Gate, Red Door, Mud Wall, Pink Curtain \\
\hline
\end{tabular}

In this case, a possible 2-itemset frequency would be: $\{$ Red Door, Darwaza Gate $\}$, as three players' transactions contain both Red Door and Darwaza Gate. In this example, we can show the association rule (with support 3 and confidence 1) as:

DarwazaGate $\rightarrow$ RedDoor [support $=75 \%$, confidence $=100 \%$ ]

However, Darwaza Gate $\rightarrow$ Red Door and Thick Brick is most likely not a useful association rule because although support condition is met, confidence stays $1 / 3$. A rule A $\rightarrow \mathrm{B}$ is the percentage of the transactions in the whole list that contains both $\mathrm{A}$ and $\mathrm{B}$, i.e. $\mathrm{P}(\mathrm{A} \cup \mathrm{B})$. The confidence of the same rule $\mathrm{A} \rightarrow \mathrm{B}$ is the percentage of transactions in the list that contains A provided that it also contains $\mathrm{B}$, i.e. $\mathrm{P}(\mathrm{B} \backslash \mathrm{A})$.

$\operatorname{support}(A \rightarrow B)=P(A \cup B)$
$\operatorname{confidence}(A \rightarrow B)=P(B \backslash A)$

As a frequent pattern finding technique, association rule mining is an unsupervised method accompanied by a measure of support and confidence. To generate strong association rules, a 'minimum support threshold' and a 'confidence factor' are required. This enables the elimination of trivial rules from results.

To summarise the entire process hitherto explained, ARF is used to complement the findings of the two previous events, i.e. the CAADRIA workshop (Chapter 4.4.3) and the public demo at Te Papa Museum (Chapter 4.4.5) where an overlap between immersiveness and agency was observed (Fig. 109). In this experiment, the qualitative findings are focused on the transformation of content measured by a qualitative assessment of association rules. These rules are discovered in relation to the 'interestingness' of a thumbnail image in an entire relational database made of one million transactions and the transaction of the participant (ID1). This experiment platform is designed to observe an overlap between agency and the transformation of content. In this section, the relationship diagram between immersiveness, agency and transformation, i.e. the MAC diagram, is used to explain the role of the experiment platform. 


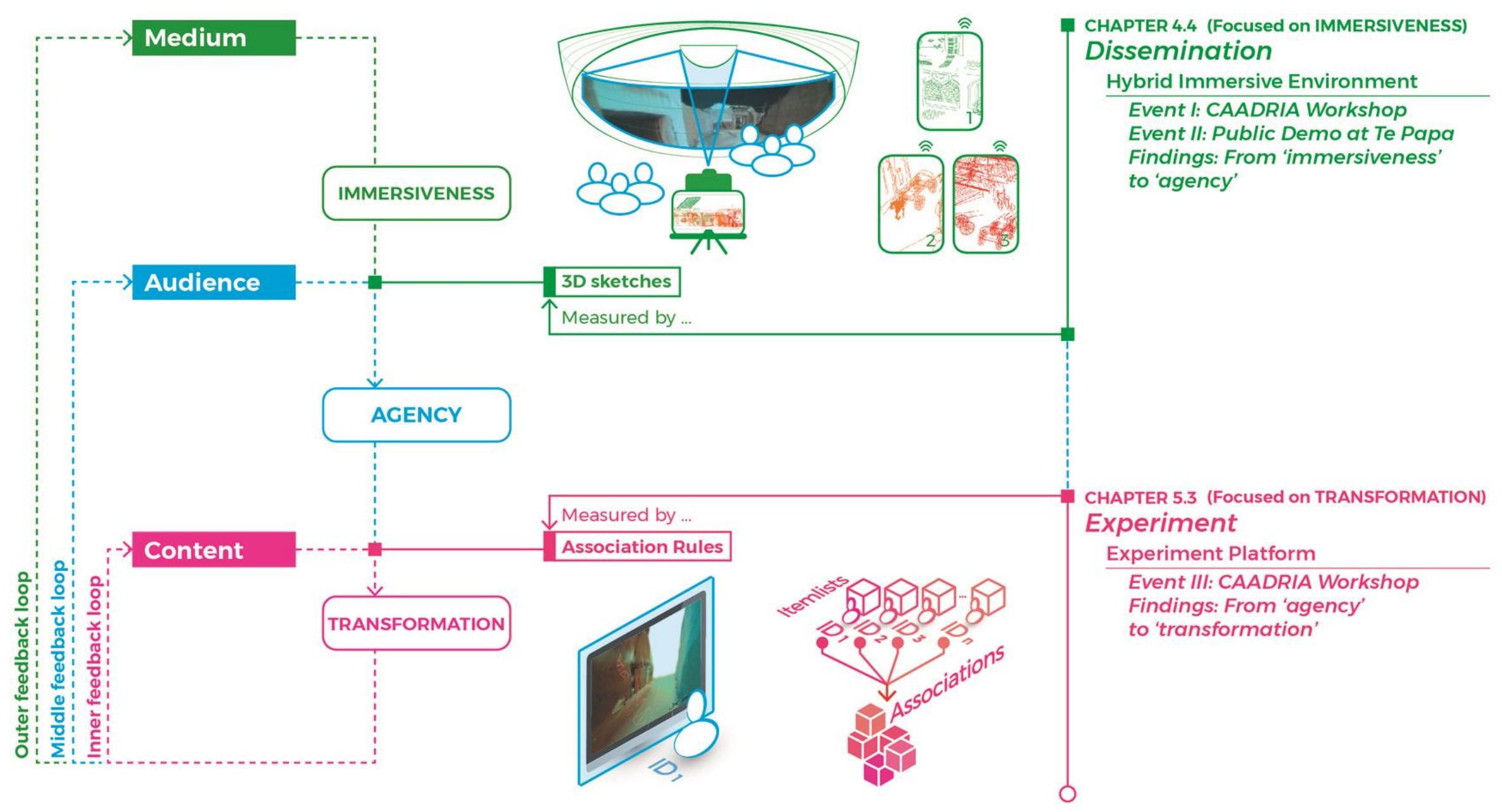

Fig. 109. Correlation of findings in Chapter 4 and 5 . 
Fig. 110 gives an overview of the development of the experiment as part of the design process from immersiveness to agency and to transformation. ARF is used to synthesise findings from these three events. The glitches of the SfM photogrammetric models are turned useful within this scenario by designing gameplay that allows the player to repair them. It is aimed to generate a sense of participation in digital heritage content-making.

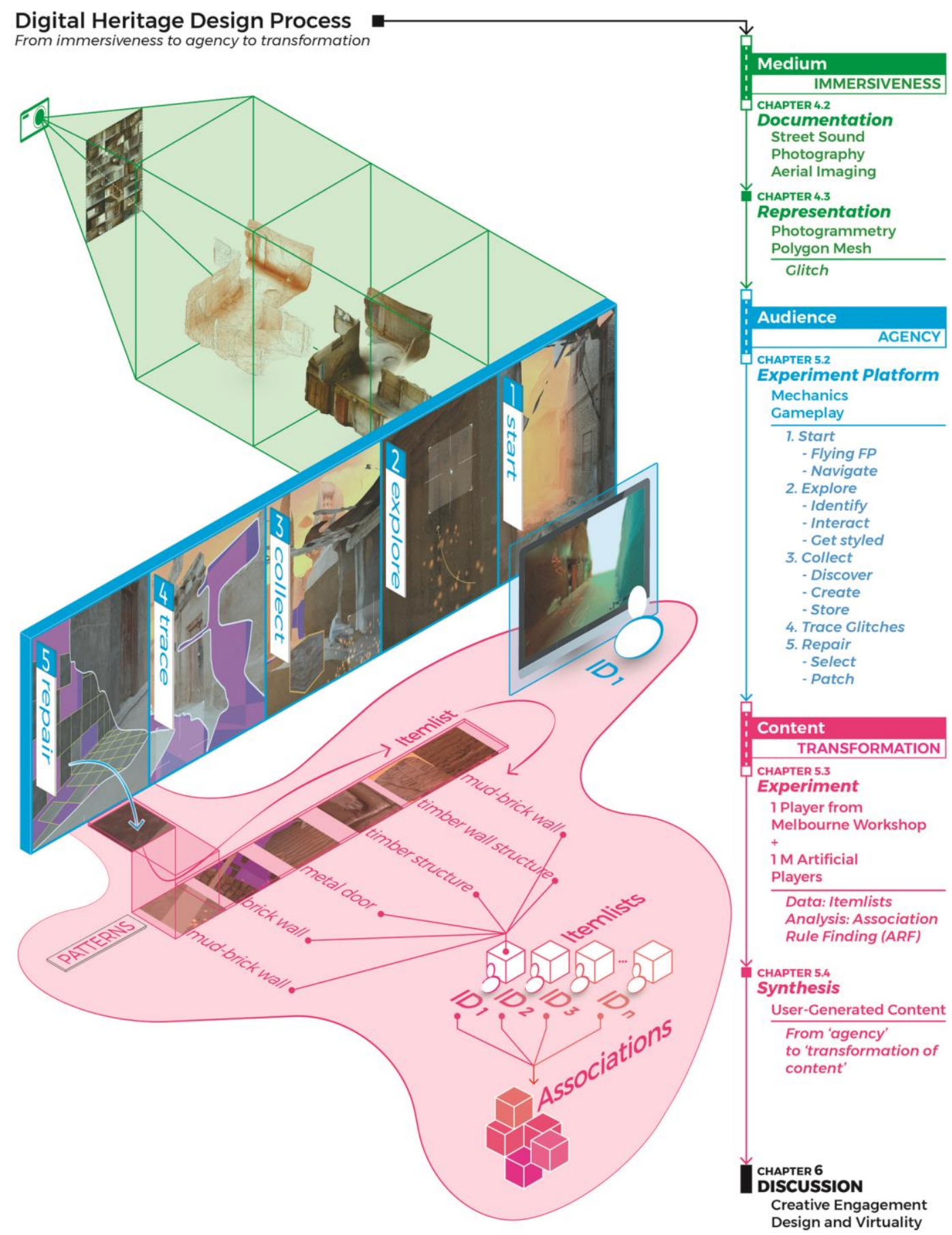

Fig. 110. The process of the experiment development in line with chapters and the MAC (Medium, Audience and Content) diagram. Illustration by Author. 


\subsection{Experiment}

Subchapter Outline

Purpose: This section is complementary to the findings of two previous experiments (see Chapter 4.4). It connects to earlier results of immersiveness, agency and transformation. This section examines the hypothesis given in Chapter 4.5 that alternative knowledge of virtuality in digital heritage is entangled with the transformation of content in creative engagement.

Design/Approach: This section is dedicated to describing the analysis of data generated in the experiment platform. It consists of six parts that are about: participants, variables, assessment criteria, analysis, findings and limitations. Qualitative findings are presented based on the ARF analysis.

Findings: It is shown possible that indirect observation without requiring demographic information about audience can provide useful feedback for discovering meaning from a digital heritage engagement. Association rules are found from user-generated content.

Limitations/Implications: As a large data mining technique, the use of ARF in this small-scale experiment is subject to further discussion. ARF is theoretically functional if the dataset is extensive. Therefore, the data that are gathered from a single-participant experiment are enlarged with the data of one million artificial participants. This synthetic data is generated by randomising the activities of the single-participant. Synthetic data is used to test the scenario of the experiment platform, which is designed for a large number of online participants.

Originality/Value: This experiment is the first to investigate the ARF analysis in relation to heritage interpretation. It supports the notion of creativity in digital heritage as a field of knowledge generation rather than highlighting facts. This section relates to Aydin, Schnabel and Sayah 2017.

Keywords. Association Rule Finding; FP-growth algorithm; transformation; user-generated data.

The previous subchapter described the construction of the experiment platform and discussed the data mining approach used to analyse the data made of user-generated content. Earlier, two events were presented and discussed in terms of how users move from immersiveness to agency during a creative engagement in digital heritage (see Chapter 4.4). This chapter describes the analysis of data gathered from the last experiment. It was conducted as part the CAADRIA workshop that was explained in Chapter 4.4.2. The immersive experiments in the workshop and the public demo at the Te Papa Museum were called 'Event I' and 'Event II.' The experiment in this chapter is coded as 'Event III,' which is conceived to support the qualitative findings of the previous events by quantifying results. The analysis is followed by a synthesis that relates to the last sub-question: What alternative knowledge of virtuality lies in the participatory content-making? (see Chapter 1, 3.5 and 4.5.2). Data are analysed to identify, describe and explore the role of creative engagement in the transformative power of user-generated content. This section is divided into five parts. 
The first part provides the definition of the participant (the player). The second one is dedicated to informing the data variables that define the scope. In the analysis part, the deployed ARF method is elaborated in detail. Then the findings of the ARF analysis are provided, with limitations explained in the last part.

\subsubsection{Participants}

Having tested the immersive VR application, the participants of the workshop were invited for a screen-based experience of the alleys of Köziciyerbişi (Gaotai). The data generated by one of the participants is taken as a sample. Based on this workshop participant's interactive session, a synthetic dataset is generated to expand the number of players to a million. There are two reasons for using synthetic data. The first one is that this thesis draws conclusions from qualitative findings. By generating a sizeable synthetic database, it aims to go beyond the quantitative findings. In support of this, the second reason is the ARF is an analysis technique that produces more reliable results when the data is extensive. In this scenario, the background information about the participant is not necessary as the study does not focus on a user-centred analysis but user-generated content. The participant's activity is used to contextualise the generation of a million synthetic itemsets, i.e. one million artificial players, since the focus is on the design process instead of the design product. The experiment is facilitated by a mouse-keyboard control in front of a large HDTV (Fig. 111).

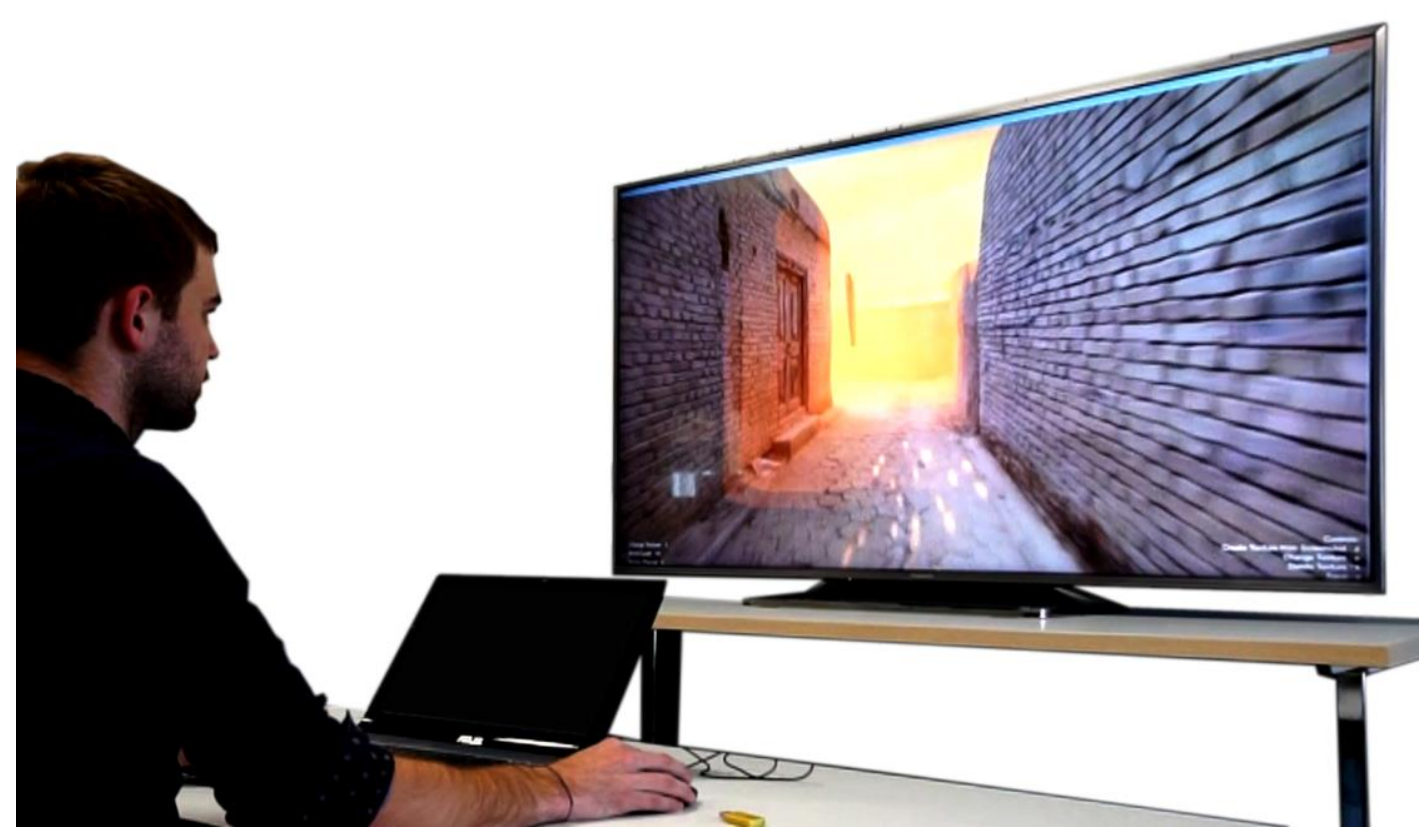

Fig. 111. The participant is navigating in the photogrammetric model of Kashgar's narrow alleyways. 


\subsubsection{Variables}

The participant is tasked with completing the incomplete model by facilitating a dynamic interpretation process stimulated by digital errors such as glitches. In this way, the variables are generated by the player targeting textures, which is similar to first-person shooter (FPS) games. Unlike the moving character targets of FPS games, the player here focuses on immobile items, i.e. the mesh model of Kashgar's narrow roads. The number of items that can be collected from the experiment platform is limited to 10. They are Mud-Brick Wall, Timber Wall Structure, Timber Structure, Metal Door, Rec Cobblestone, Hex Cobblestone, Red Door, Blue Door, Mosque Gate and Brick Wall (Table 57).

Table 57. Pattern items that can be collected from the experiment platform. The size of each sample thumbnail image is $250 \times 250$ pixels.

\begin{tabular}{|c|c|c|c|c|c|}
\hline Abb. & Item & $\begin{array}{l}\text { Sample } \\
\text { thumbnail }\end{array}$ & Abb. & Item & $\begin{array}{l}\text { Sample } \\
\text { thumbnail }\end{array}$ \\
\hline$M B V$ & Mud-Brick Wall & & $H C$ & Hex Cobblestone & \\
\hline$T W S$ & $\begin{array}{l}\text { Timber Wall } \\
\text { Structure }\end{array}$ & & $R D$ & Red Door & \\
\hline$T S$ & Timber Structure & & $B D$ & Blue Door & \\
\hline$M D$ & Metal Door & & $M G$ & Mosque Gate & \\
\hline$R C$ & Rec Cobblestone & & $B W$ & Brick Wall & \\
\hline
\end{tabular}


The workshop participant generated six patterns, shifting the centroid of the digital model by generating new walls and roads, developing correlations between items for analysis. These patterns are saved as $250 \times 250$ (.png) pixel frames in the library (Fig. 112). In the database, the convention for file names is \#IDParticipantNumber.PatternNumber. In this way, a pattern name is generated. The single participant of the experiment is named as 'ID1' and there are six collected patterns. In this case, patterns are \#ID1.001 to ID1.006.

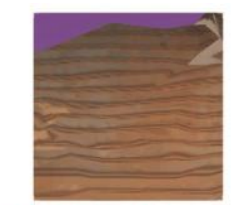

$\rightarrow$ \#ID1.001

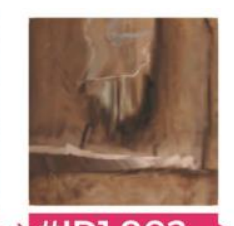

\#ID1.002

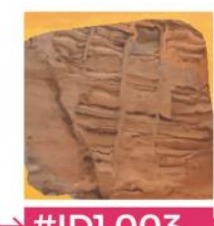

\#ID1.003

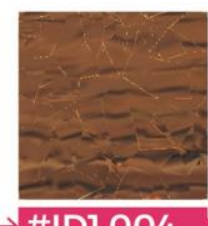

\#ID1.004

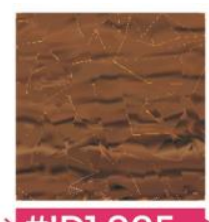

\#ID1.005

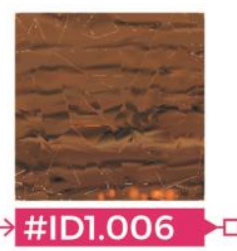

Fig. 112. Patterns captured by the participant $(A)$ with pattern IDs, \#ID1.001 to \#ID1.006.

The collection of the workshop participant (ID1), which consists of six patterns, is then manually purged, based on their equivalent semantics as described in Table 58. The similar patterns \#ID1.004, \#ID1.005 and \#ID1.006 are merged. The unrecognised pattern \#ID1.002 is disqualified from further analysis. Therefore, the optimal outcome of ID1 is a 3-itemset sample that includes only Metal Door, Timber Wall Structure and Brick Wall.

Table 58. A 3-itemset generated by the participant (ID1).

\begin{tabular}{llll}
\hline Pattern & Abb. & Item & Thumbnail \\
\hline \#ID1.001 & MD & Metal Door & \\
\#ID1.003 & TWS & Timber Wall Structure & \\
& & & \\
\#ID1.004 & BW & Brick Wall & \\
& & & \\
\hline
\end{tabular}




\subsubsection{Analysis}

The algorithm used in the ARF analysis follows a two-step process to calculate association rules in the database: firstly collecting frequent items and constructing a frequent pattern tree (FP tree) and then mining of the FP tree. This method is called FP-growth. It recursively grows frequent patterns by partitioning the database. In this experiment, the conditional FPtree of ID1's 3-itemset within the database of all transactions is built from its conditional pattern-base. The implementation of the mining method of the FP tree is as the below pseudocode provided by (Han and Kamber 2011, 550):

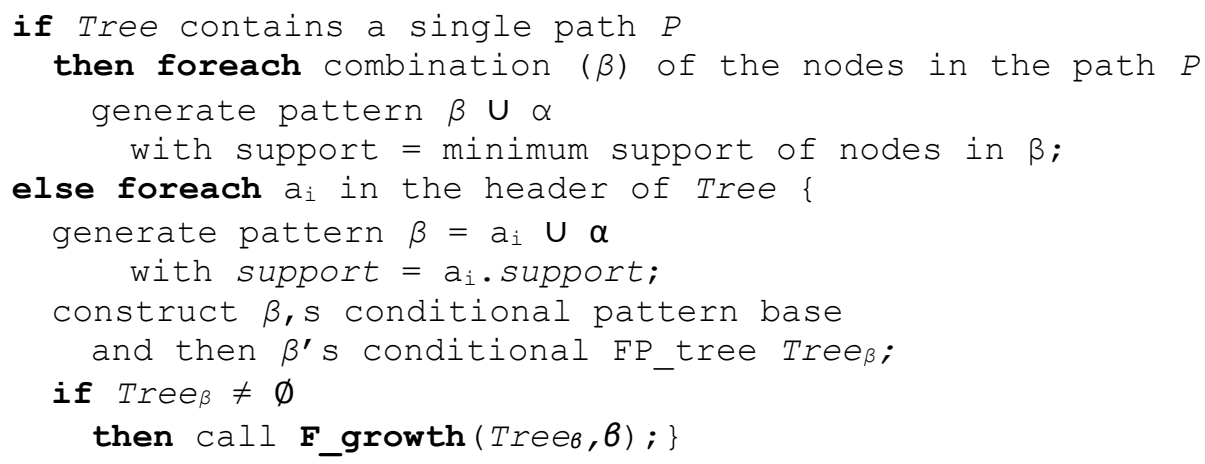

After the purpose of the algorithm is defined to search associations, the algorithm is written in MATLAB, which can handle a large database of one million itemsets (Aydin, Schnabel and Sayah 2017). In addition, a script was created to randomly sparse the available pattern list of the experiment platform into a synthetic database of itemsets of one million players, which are random in size ranging from 1-itemsets to 10 -itemsets.

\subsubsection{Findings}

Merged with one million synthetically generated itemsets, the transaction of ID1 \{Metal Door, Timber Wall Structure, Brick Wall\} is found to have associations with other 1itemsets in the following order $\{$ Rec Cobblestone $\},\{$ Timber Structure $\},\{$ Mud-Brick Wall $\}$, $\{$ Hex Cobblestone $\},\{$ Blue Door $\},\{$ Mosque Gate $\},\{$ Red Door $\}$ (Fig. 113). In this scenario, we can presume that the workshop participant (ID1) is more likely to collect a $\{$ Rec Cobblestone $\}$ pattern than a $\{$ Red Door $\}$ pattern. Therefore, an association rule is discovered: 


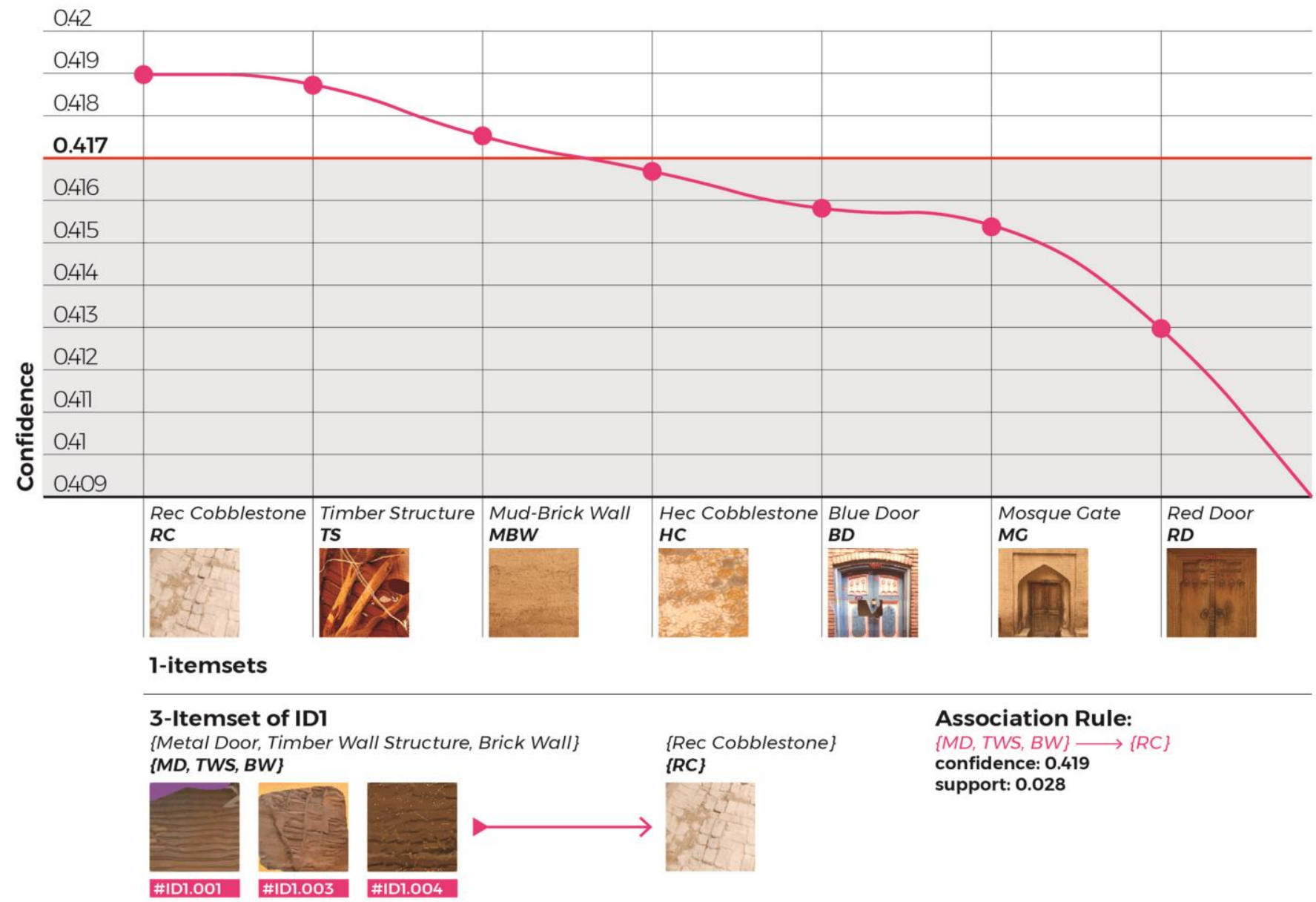

Fig. 113. Confidence graph amongst 1-itemsets associated with the 3 -itemset of ID1, i.e. \{Metal Door, Timber Wall Structure, Brick Wall\}. 
Depending on the set boundary of support and confidence, new association rules can be accepted in this situation. To give an example, a confidence rate is set as the lowest limit, such as [minimum confidence $=0.417$ ] (see the red line on the chart above). In this situation, two new association rules are added to the list of association rules:

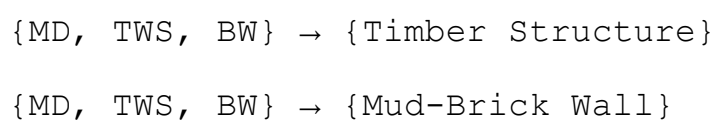

Furthermore, new correlations are sought with 2-itemsets and 3-itemsets in the database. New association rules found in relation to 2-itemsets based on the 3-itemset of ID 1 , with minimum confidence set at 0.165 and support 0.011 .

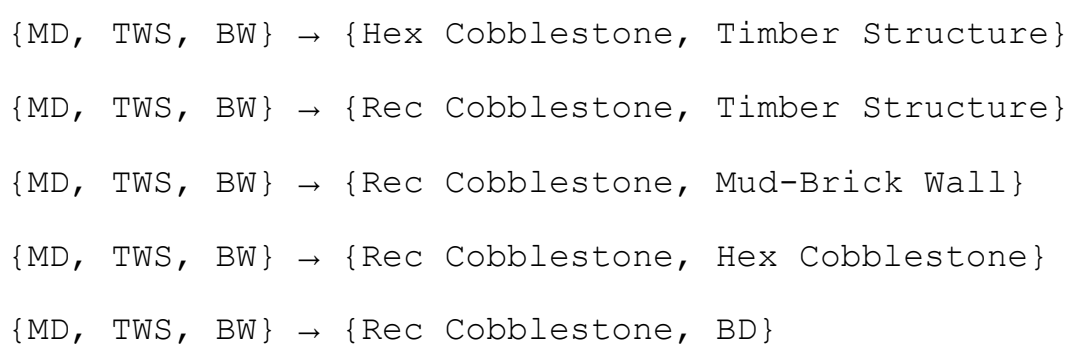

In comparison, it appears that there is a strong association between ID1's 3-itemset $\{$ Metal Door, Timber Wall Structure, Brick Wall $\}$ and the 2-itemset transaction of $\{\mathrm{Hex}$ Cobblestone, Timber Structure\}. From the infinite number of possibilities offered by the association rule mining method, higher confidence does not always provide the best finding. To discover more interesting correlations based on the interactions of ID1, 'lift' count is searched. If an itemset is not very frequent but is attracted in relation to another itemset, it may be an interesting result. In the chart below (Fig. 114), three 2-itemsets are found outstanding from others by lift count. One of these lifts is repulsive, i.e. the association is not as strong as the neighbour associations. The other two lifts demonstrate an increase of the chances of getting into association with the 3 -itemset of ID1. The repulsive lift belongs to one of the association rules extracted above (see formula 13). That is to say, the 2-itemset of $\{$ Rec Cobblestone, Hex Cobblestone $\}$ is less attractive to ID1's 3-itemset of \{Metal Door, Timber Wall Structure, Brick Wall\}. Therefore, this association rule is found unreliable and omitted. Further examination reveals that there are two 2-itemsets that are attracted by ID1's transaction with their lift count making a sudden leap upward. So these associations are added to the list, although they were disqualified by minimum confidence:

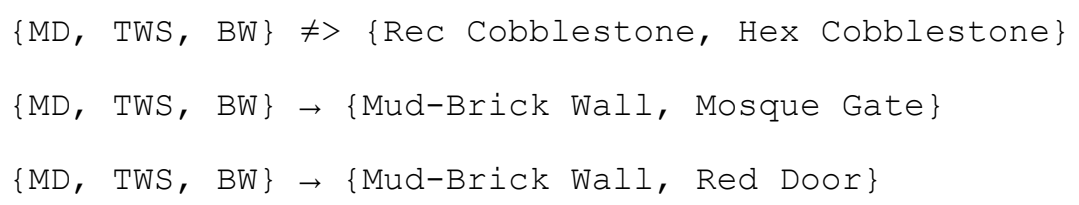




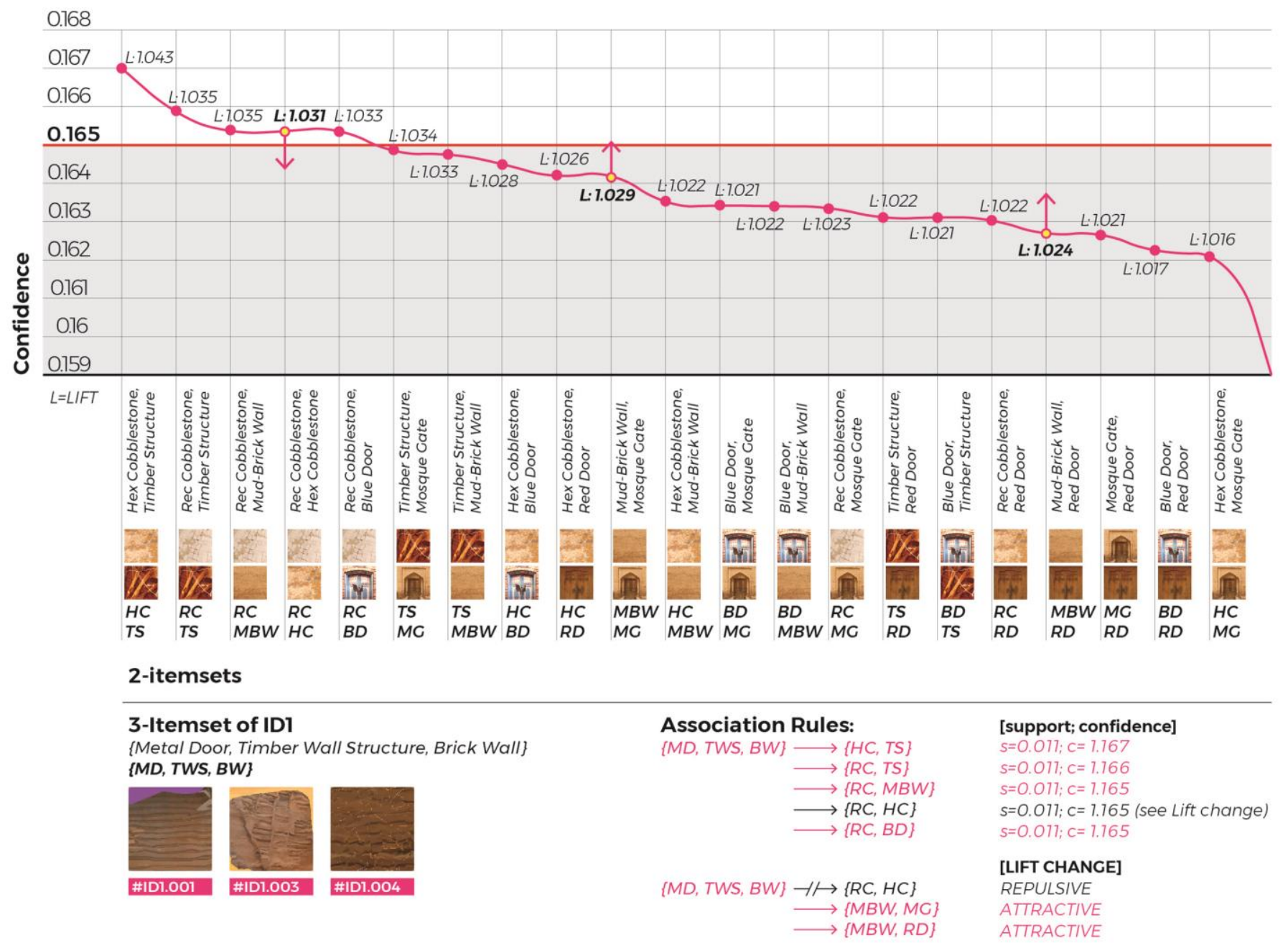

Fig. 114. Confidence graph amongst 2 -itemsets associated with the transaction of ID1, i.e. $\{$ Metal Door, Timber Wall Structure, Brick Wall $\}$. 
The concept of lift count helps distinguish ARF from other statistical analysis by allowing non-linear interpretation. This aspect of the ARF analysis strengthens the reliability of findings, providing a filter into initial assumptions about data. Having used two different approaches based on 'minimum confidence' and 'confidence-oriented lift count,' this study also discloses associations of player itemsets by using a third approach that is 'support-oriented lift count.' In this approach, the association rules of 3-itemsets with [support $=0.004$ ] and [minimum confidence $=0.06$ ] are searched first. Having put in order all 3-itemsets according to their association with ID1's transaction, minimum support is defined to seek itemsets with 'lift count' outstanding within the list. First, three association rules are generated based on the classic approach of minimum confidence (in this case 0.06 ):

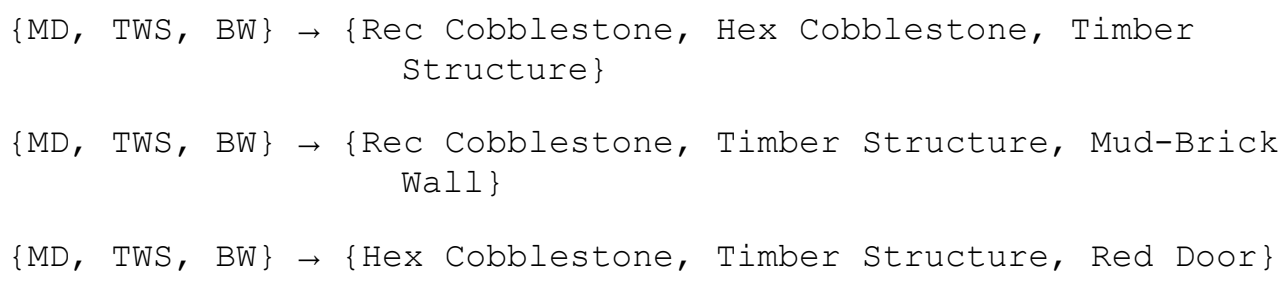

In addition to the above rules, one more association is discovered based on 'lift count' provided that its support is more than the minimum (defined as 0.004):

$\{\mathrm{MD}, \mathrm{TWS}, \mathrm{BW}\} \rightarrow$ TTimber Structure, Mud-Brick Wall, Mosque Gate

The new rule suggests that there may be a strong association between $\{$ Metal Door, Timber Wall Structure, Brick Wall\} and \{Timber Structure, Mud-Brick Wall, Mosque Gate\} as the second transaction is lifted far more than other neighbour transactions. Breaking the routine increase of 'lift count' with a stronger figure, it is added to the list of association rules generated from 3-itemsets. Other 3-itemsets are excluded as their support does not meet the minimum support criteria (in this case 0.004) (Fig. 115).

As explained above, three types of interpretation of an ARF analysis are made in this research. The first one was the use of 'minimum confidence,' the second was about generating new rules or disqualifying old ones based on 'lift count' and finally the third one offered criteria of 'support-oriented lift count.' The entire data pertaining to the transaction of the player (ID1) is provided in the appendices. The list of all association rules discovered in the experiment includes thirteen associations with the player ID1's transaction of a 3itemset. Therefore, potential association rules between itemsets generated by the participant (ID1) of the experiment and one million artificial players are studied. The findings serve to demonstrate a scenario of a shift from 'agency' to 'transformation of content' in creatively engaging with a digital heritage application. 


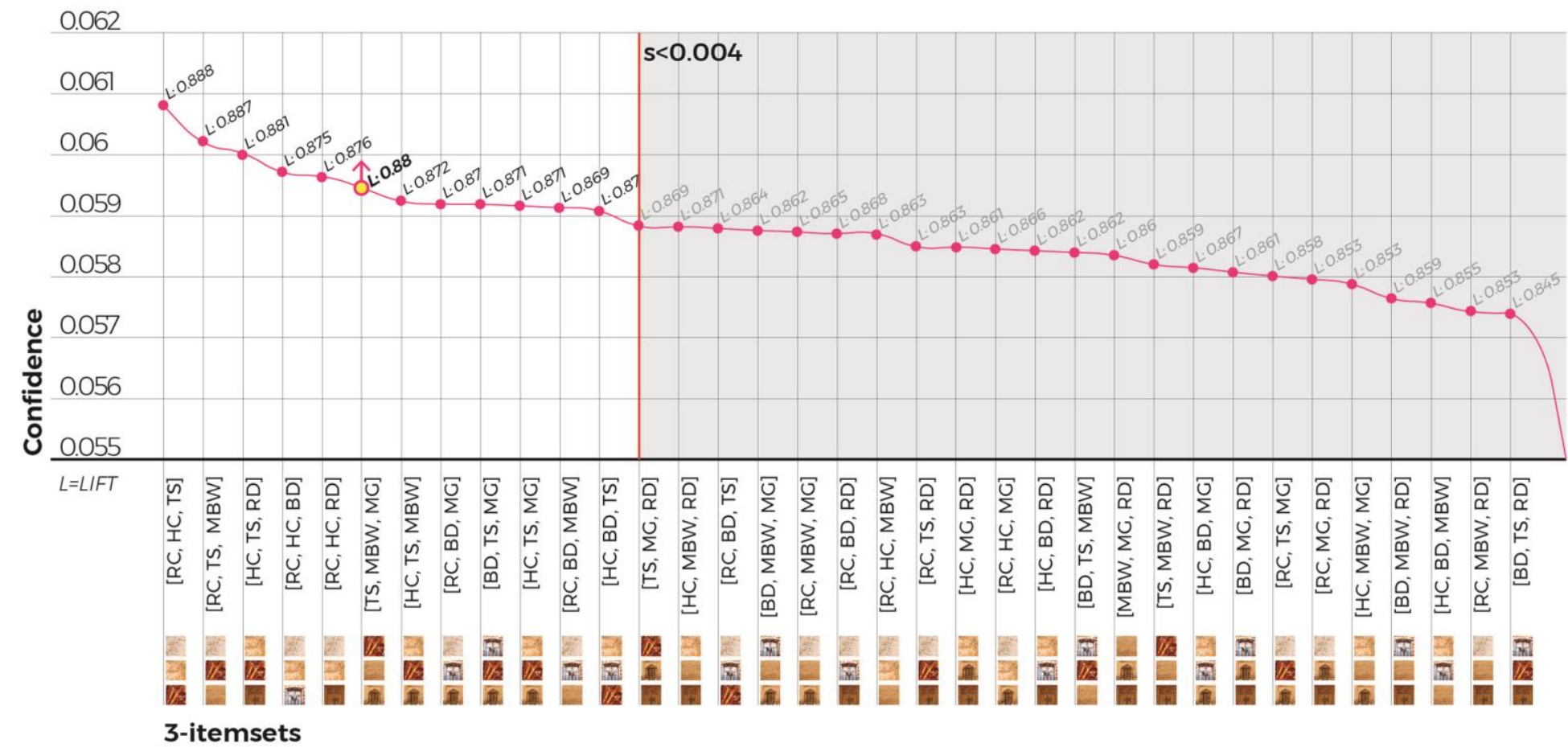

3-Itemset of IDI

\{Metal Door, Timber Wall Structure, Brick Wall\} $\{M D, T W S, B W\}$
Association Rules:
$\{M D, T W S, B W\} \longrightarrow\{R C, H C, T S\}$
[support; confidence] $\longrightarrow\{R C, T S, M B, T S, R D\}$
$s=0.004 ; c=0.06$
[LIFT CHANGE] / support <! 0.04
$s=0.004: c=0.059: L=0.88$

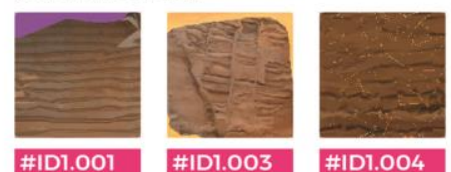

Fig. 115. Confidence graph amongst 3 -itemsets associated with the transaction of ID1, i.e. $\{$ Metal Door, Timber Wall Structure, Brick Wall $\}$. 


\subsubsection{Limitations}

This experiment has described a list of association rules found in a relational database. Thirteen association rules are identified, describing quantitative findings. This study opted for using synthetic data to draw conclusions from a qualitative assessment that go beyond quantitative findings. The data generated by one participant is used as a sample as the basis of the synthetic data. Based on the sample participant data, the number of records is extended to a million itemsets.

The use of synthetic data allowed the ARF analysis to produce more reliable information because data is large. Similar approaches are available in the Artificial Intelligence (AI) research where there is no precedent work from which to inhere data. Due to lack of precedent data, one million itemsets are procedurally generated based on the activities of the real participant. This enables this thesis to make conclusions qualitatively based on an assumption of how a similar case would exist in reality with an extensive database produced within the experiment platform.

The quantitative findings of the experiment are bound to single-dimensional associations. The rules only disclose intra-attribute relationships between user-generated content items. Since itemsets in the experiment are recorded as single-dimensional data, inter-attribute relations for more reliable association rules have not been explored. The itemsets in the experiment distinguish user-generated patterns by name, i.e. limited to semantic information. The collected patterns of the participant actually carry information about the precise location from which they were collected. But this information is excluded from the conventional representation of itemsets as $\left\{i_{1}, i_{2}, \ldots, i_{\mathrm{k}}\right\}$. Therefore it is not possible to make cross-object associations between itemsets integrated with more information such as $\left\{i_{1}\left(\Delta_{1}\right), i_{2}\left(\Delta_{2}\right), \ldots, i_{\mathrm{k}}\left(\Delta_{\mathrm{k}}\right)\right\}$. More details are given within the next subchapter.

The qualitative findings of this experiment suggest that measuring the transformation of content in creative engagement via ARF analysis is potentially consistent and generalizable. In this experiment, the user-generated content as components of itemsets is tagged with semantic information manually. In fact, this information needs to be generated by the player for robust correlation analysis. For simplicity, the experiment is limited to the researcher's subjective intervention for synthesising the quantitative findings qualitatively. More details are given in the next subchapter.

The last limitation to acknowledge is that the player is not provided with a real reward because participation in the experiment was entirely voluntary and the research is bound rather to content than user-experience analysis. 


\section{Subchapter Outline}

Purpose: The findings of the experiment are synthesised as a means to explain their implication in this research.

Design/Approach: The synthesis of findings is made of three states of the same thread corresponding with the three sub-questions of this research.

Findings: Association Rule Finding (ARF) is confirmed to be a universal method for gaining insight into the processes of documentation, representation, dissemination and design in digital heritage. It is presented how uniquely ARF can be applied to digital heritage applications.

Limitations/Implications: The analysis and findings of the experiment in the previous subchapter are extended. The synthesis of findings is merged to move into a discussion in Chapter 6 pertaining to answering the main question of this research. It combines the findings gained from the experiment with the findings of previous experiments explained in Chapter 4.4.

Originality/Value: The synthesis contributes to the discussion chapter of the thesis by merging qualitative and quantitative results of the analyses.

Keywords. Synthesis; creative digital heritage, virtuality of creative engagement; alternative knowledge.

This section provides a synthesis of previous findings in relation to association rules discovered in the experiment. Table 59 lists all association rules with the threshold measures of support, confidence and lift. The full list of associations is available in Appendix D.

Table 59. Association rules found in the database of the experiment, in relation to ID1's 3 -itemset transaction of $\{$ Metal Door, Timber Wall Structure, Brick Wall $\}$

\begin{tabular}{|c|c|c|c|c|}
\hline Size & Association with & Sup. & Conf. & Lift \\
\hline \multirow{3}{*}{ 1-itemsets } & $\{$ Rec Cobblestone $\}$ & 0.028 & 0.419 & 1.079 \\
\hline & $\{$ Timber Structure $\}$ & 0.028 & 0.419 & 1.08 \\
\hline & $\{$ Mud-Brick Wall $\}$ & 0.028 & 0.418 & 1.078 \\
\hline \multirow[t]{6}{*}{ 2-itemsets } & $\{$ Hex Cobblestone, Timber Structure $\}$ & 0.011 & 0.167 & 1.043 \\
\hline & $\{$ Rec Cobblestone, Timber Structure $\}$ & 0.011 & 0.166 & 1.035 \\
\hline & $\{$ Rec Cobblestone, Mud-Brick Wall $\}$ & 0.011 & 0.165 & 1.035 \\
\hline & $\{$ Rec Cobblestone, Blue Door $\}$ & 0.011 & 0.165 & 1.033 \\
\hline & \{Mud-Brick Wall, Mosque Gate $\}$ & 0.011 & 0.164 & 1.029 \\
\hline & $\{$ Mud-Brick Wall, Red Door $\}$ & 0.011 & 0.163 & 1.024 \\
\hline \multirow[t]{4}{*}{ 3-itemsets } & $\{$ Rec Cobblestone, Hex Cobblestone, Timber Structure $\}$ & 0.004 & 0.061 & 0.888 \\
\hline & $\{$ Rec Cobblestone, Timber Structure, Mud-Brick Wall $\}$ & 0.004 & 0.06 & 0.887 \\
\hline & $\{$ Hex Cobblestone, Timber Structure, Red Door $\}$ & 0.004 & 0.06 & 0.881 \\
\hline & \{Timber Structure, Mud-Brick Wall, Mosque Gate $\}$ & 0.004 & 0.059 & 0.88 \\
\hline
\end{tabular}




\subsubsection{Retrospective Synthesis of Sub-Questions and Theories}

Chapter 5.3 demonstrated the experiment results. The data were analysed to identify, describe and explore association rules based on the 3-itemset of the participant (ID1). The itemset consisted of three patterns including Metal Door (MD), Timber Wall Structure $(T W S)$ and Brick Wall $(B W)$. Based on a single 3-itemset, synthetic transaction data were generated. Then one million synthetic itemsets were edited as to how correlated they were with ID1. Eventually, thirteen association rules were distilled. In this section, the results are interpreted in connection to the three sub-questions of this research (Table 60). To synthesise them as part of the same thread, the sub-questions are reduced to three concepts. Three subquestions are analysed in a reverse order (i.e. $s Q 3-s Q 2-s Q 1)$. However, the analysis of three concepts is for increasing the readability of the synthesis. It means that each analysis overlaps with each other. This subchapter inaugurates the discussion of how generalizable the outcome of the research is.

Table 60. Synthesis of experiment findings with the three sub-questions of this research.

\begin{tabular}{ll}
\hline Sub-Questions & Addressed concept \\
\hline $\begin{array}{l}\text { sQ1: How can architectural heritage be translated into } \\
\text { creative digital forms? }\end{array}$ & ...creative digital heritage... \\
\hline $\begin{array}{l}\text { sQ2: How can an immersive digital heritage application } \\
\text { convey virtuality to the public via creative engagement? }\end{array}$ & $\begin{array}{l}\text {...virtuality of creative } \\
\text { engagement... }\end{array}$ \\
\hline $\begin{array}{l}\text { sQ3: What alternative knowledge of virtuality lies in the } \\
\text { participatory content-making? }\end{array}$ & ...alternative knowledge.... \\
\hline
\end{tabular}

\section{- Alternative Knowledge}

In relation to $s Q 3$, the alternative knowledge of virtuality consists of association rules between pieces of data. Thirteen rules found in the experiment help uncover relations between seemingly unrelated data in a relational database. These rules depict the relationship of the transaction of ID1 with the rest of the data, which are otherwise not obvious or hard to find. Before the experiment, there was no evidence about the relationship between the 3-itemset of $\{$ Metal Door, Timber Wall Structure, Brick Wall $\}$ and $\{$ Rec Cobblestone $\}$. The rule of $\{M D, T W S, B W\} \rightarrow\{R C\}$ [support=0.028, confidence=0.419] implies an assessment that it is the strongest association. Basically, a metal door, some part of timber wall structure, and a section of a brick wall are somehow associated with rectangular cobblestones on the ground. The generation of an association rule resources an alternative piece of knowledge about the tangible architectural heritage of Kashgar. 
However, when analysed with 2-itemsets, the association with hexagonal cobblestones and timber strcutures becomes more significant than that of rectangular cobblestones. It means that association rules are statistically independent and contextual while generating alternative meanings from an already alternative piece of knowledge.

The rationale behind this contextuality is that the alternative knowledge is not dependent upon static meanings but a rule of 'relativity' between user-generated content. Association rules take over the control to define a new understanding of authenticity that becomes, instead of an outcome, a process-oriented myth. Usually, the expectation from a digital heritage platform is to achieve a realist perfection of imagery qualities; however, they fail to facilitate the alternative knowledge of virtuality. With ephemeral, contextual and relative association rules, the research proves that digital engagement does not only conform with descriptive information, which generally conditions heritage knowledge to the past, but also is generative. The production of the alternative knowledge turns heritage information into the knowledge of the future.

\section{- Virtuality of Creative Engagement}

With regards to $\mathbf{s} \mathbf{2}$, there are lessons to be learnt in terms of the role of the creative engagement in digital heritage platforms. Spatial indices found in the association rules illustrate the virtuality of the alleyways in Kashgar. Thus the creative engagement of the participant helps heritage information transcend the boundaries of 'actuality.' The participant enriches the meaning of content by interpreting it in different ways. Each pattern recorded as a thumbnail image by the participant represents a unique spatial sign. The assemblage of these patterns propagates alternative knowledge generated via a signification process.

The knowledge of association rules may be indeterminate and transitory because of resulting from statistically independent analysis of the user-generated content; however, the creative engagement generates a causal mechanism between the material aspects and the meaningful expressions of heritage. Of ten patterns available for collection, the participant created three meaningful samples. The 3 -itemset of $\{M D, T W S, B W\}$ is extracted from a virtual world and inserted into the worlds of others' perceptions of virtuality. The design activities created relations amongst individual worlds of virtuality that were contingent on the materiality of heritage. An assemblage of user-generated content gives insight into the heterogeneous components of virtuality and heritage knowledge.

The gameplay of the experiment platform blends heterogeneous activities. In the context of Bloom's Taxonomy (Krathwohl 2002) these activities are represented in a 
hierarchy evolving from remembering and understanding to analysing, evaluating and creating (Fig. 116). Starting with a flying FP character, the participant navigated through the virtual narrow roads of Kashgar while trying to generate a mechanism of remembrance. The gameplay required the participant to interact with different parts of the virtual environment so that the participant could identify and learn an architectural style. By collecting bricks, the participant applied a sense of responsibility to the virtual heritage of Kashgar. Next, the participant had to analyse, question and distinguish different parts of the $3 \mathrm{D}$ representation of the narrow roads. After evaluating the selected patterns, the participant made a creative impact on the virtual Kashgar by intuitively judging, reimagining and proposing alternative visual expressions. Based on a process of creative engagement, the result is an assemblage of visual components that form a novel and authentic expression of virtuality in a digital heritage platform.

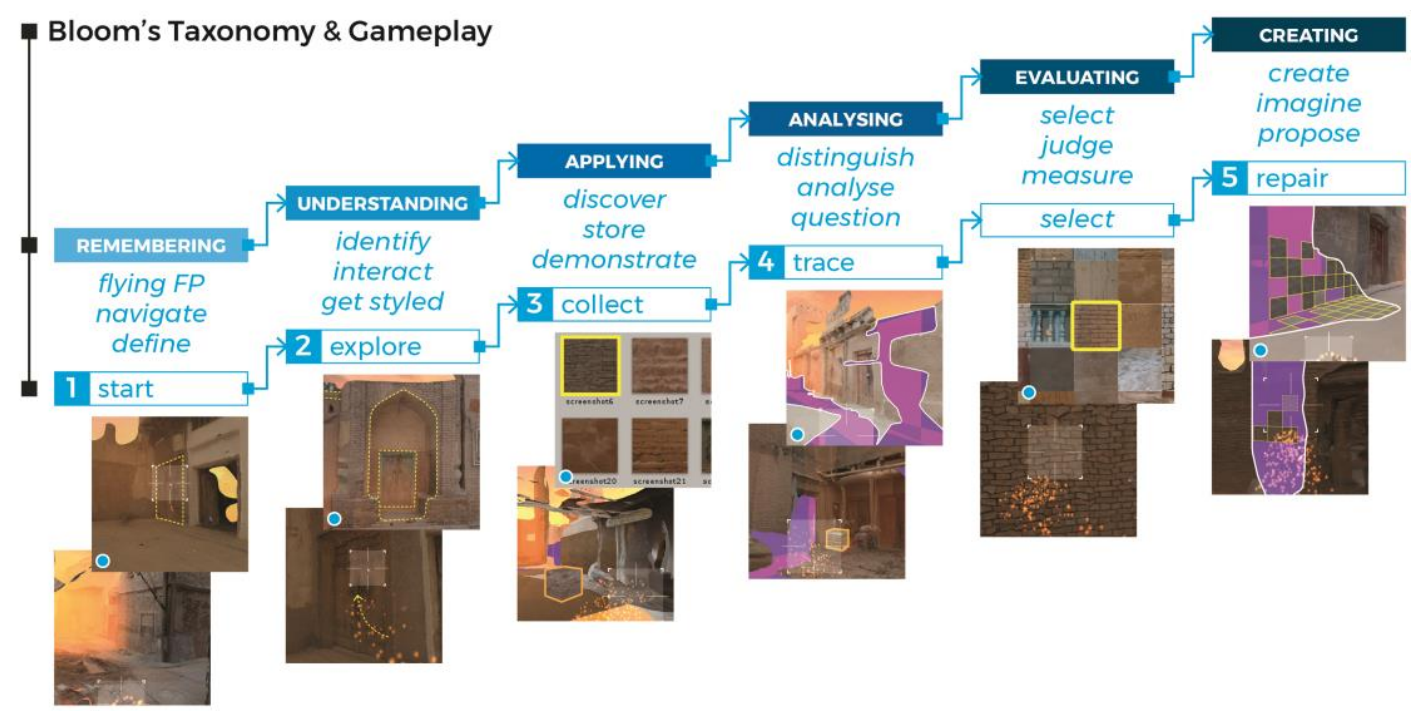

Fig. 116. Matching the gameplay activities in the experiment with Bloom's Taxonomy used in cognitive and pedagogical studies. In the revised version of the taxonomy, there are remembering (retrieval of relevant knowledge), understanding (determining the meaning of graphic communication), applying (carrying out a procedure), analysing (detecting how the components relate), evaluating (making criteria-based judgements) and creating (combining elements to form a novel and original product) (Krathwohl 2002). Illustration by Author.

The platform enabled a higher level of engagement based on the creative act of completing a virtual model. This operates as an endless signification process of combining patterns and glitches, the appropriate and the inappropriate, the good and the bad. This paves the way for generalising a language of visual conversation that can be sustained through a search mechanism, which is, in the case of the experiment during Event III, managed by the ARF analysis. 


\section{- Creative Digital Heritage}

In relation to $\boldsymbol{Q} \mathbf{1}$, the participant translates architectural heritage into creative digital forms. A dialog between appropriate patterns and inappropriate glitches is sustained by the participant creatively engaging with the original content. The participant is invited to this process through an evolution of activities starting from retrieving relevant knowledge by navigating through alleys and ending with combining patterns to make a novel interpretation of the original content. It is important to recognise that this process is stimulated mostly by gaps, i.e. holes and glitches, rather than high-resolution imagery that perfectly resemble the actual site. Thanks to being imbued with imperfection and glitches, each thumbnail image patched by participants is conceived to bestow further signification on the final meaning of the original content. Therefore, the participant is positioned to join the 'designerly way of knowing' used as a novel procedure for content-making in digital heritage. This design procedure contrasts with the prevailing studies of digital heritage that are overconcerned with 'accuracy,' which is discussed in Chapter 3.5 as part of the 'Matters in Research.'

The qualitative findings of the experiment are synthesised with the semiotician Peirce's elaboration on the sign. This inaugurates a discussion of the main question addressed in this thesis that presents a digital design approach to 'steer and diversify' creative engagement in digital heritage. Peirce states that (1) the final meaning is always the result of a series of successive interpretants, (2) whose never-ending combinations give rise to the language of a system of signs, and (3) the interpreter enriches each sign with further signification (Peirce and Moore 1998; Merrell 1997). Peirce considers three sign types, i.e. icon, index and symbol, whereas a sign can be one of them or all simultaneously. Peirce's logic suggests that a general rule may not apply in all cases since a sign is transient between different states. But the indexicality of this progressive signification can be used as a means to steer the generation of alternative knowledge and it is devoted to permanent modification and diversification.

To connect Peirce's logic with the experiment results, the 'lift' measure of ARF is instrumental in illustrating the processual quality of creative engagement maintained by users. Initially, $\{M D, T W S, B W\} \rightarrow\{R C\}$ is found to be the strongest association according to the confidence threshold. However, the lift function intensifies the importance of \{ Timber Structure in association with the transaction of ID1 (see above Table 59). This is to say, the significance of timber structure ( $\{$ Lift_TS $\}=1.080406)$ is lifted superior to a pattern of rectangular cobblestones ( $\{$ Lift_ $R C\}=1.078894)$. The knowledge about the strongest association is updated as part of the intuitive communication of the participant with the 
virtual Kashgar streets. It is the creative engagement of the participant that enables a sign to move between inter-related states of being an icon, an index or a symbol.

These indexical findings are translated as new insight for, in Parry's (2007) words, "recalibrating authenticity" in digital heritage. The primary axis of authenticity has always been related to the mode of representation. With the increasing influence of digital tools, this axis has now been broken into discrete and unexplored units (Galloway 2011). Heritage theories have evolved from material culture to its beyond with social context and even further to our bodily engagements with heritage objects (Waterton and Watson 2013; Watson and Waterton 2010). Analysing indices of heritage artefact no longer stipulates a physical interaction with the original. Although digitising the complexity of an authentic experience is virtually impossible, basic units of visual language are capable of carrying a degree of indexicality to re-examine the question of "what is authentic heritage?" in digital.

As Peirce notes, signification is not a result of a simple dual relationship between sign and object. Creative engagement produces authentic content. It is central to the signification process that generates a special, intuitive, natural and ordinary language for conversation and dialog between sign users (Peirce and Moore 1998). In an analogy to the Peircean understanding of sign, the experiment correlated the indexicality of the user-generated content with the alternative knowledge of virtuality. The interconnected aspects of designing a digital heritage application are demonstrated. Conceivably, experiment results in alternate realities can be united via the notion of 'index of representation,' about which an initial discussion was brought earlier in Chapter 4.4.5. Deepening the use of digital information is shown instrumental in conceiving a generalised language that can commence different engagement types.

Fig. 117 communicates an account of this generalisation via the feedback system of the MAC diagram. The association rules and 3D sketches (see Chapter 4.4.5) represent the indexicality of creative engagement in digital heritage. 3D sketches were used to examine the progression from immersiveness to agency. For example, horse carts, gates and lanterns drawn by participants give insight into the signification process in an immersive application. The thirteen association rules driven by the experiment present the progression from agency to transformation. Discovering correlations between different itemsets, ARF promotes the generalisation of the MAC diagram. 


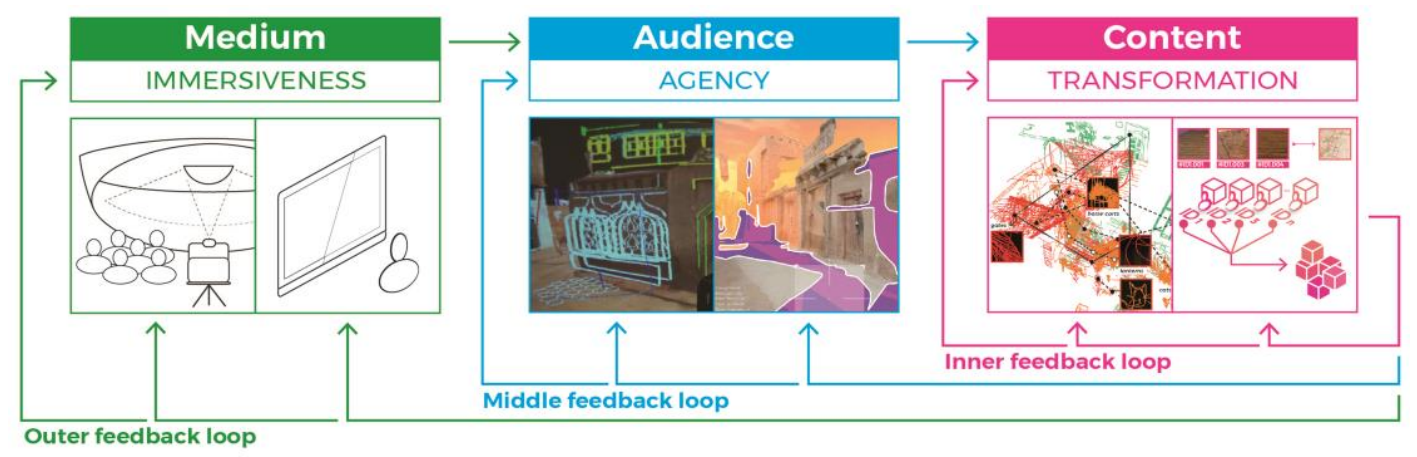

Fig. 117. Distribution of feedback loops from the inner to the middle and to the outer loop. Conceivably, ARF promotes the generalisation of the MAC diagram by allowing the communication of messages generated in different platforms at various times and remote locations. Illustration by Author.

\subsubsection{Prospective (Future) Synthesis}

In this section, results are synthesised with an overview of the future direction for improving the findings of the experiment. Analysing the hidden associations of user-generated content reveals the impact of transformation in the construction of virtuality. The level of detail that a transaction data holds can be extended by additional information. The findings are bound to single-dimensional associations that disclose intra-attribute relationships between usergenerated content items. Additionally, inter-attribute relations can be involved in searching for stronger associations. Conceivably, the itemsets may contain figurative information including destination, dimension, brightness and saturation. Such figurative information helps discover multi-dimensional associations in order to seek deeper and stronger insight. However, this requires some alteration in the representation of the itemsets. The classical definition of an itemset $\left\{i_{1}, i_{2}, \ldots, i_{\mathrm{k}}\right\}$ has to be modified to contain a secondary data. In this case, it is called cross-object association rules and represented as $\left\{i_{1}\left(\Delta_{1}\right), i_{2}\left(\Delta_{2}\right), \ldots, i_{\mathrm{k}}\left(\Delta_{\mathrm{k}}\right)\right\}$ (Feng and Tsang 1999). To discover such cross-object associations, itemsets can include location-based information, such as \{Timber Structure (Rd 1), Mud-brick Wall (Rd 5), Red Door (Rd 3), Timber Structure ( $R d$ 8)\}. These cross-object associations can increase the translatability of indices from the actual heritage environment.

Further research may give insight into the relationship between virtuality and heritage by using sound and destination information in the ARF analysis. The current experiment showed that the participant makes faster creative decisions when conversations are played in the background. The map in Fig. 118 depicts the approximate location of each collected pattern of ID1. There are four patterns extracted from where the background sound consists of street conversations of people in Uyghur, the local language in Kashgar. However, there 
is no pattern collected when the background sound is a piece of Uyghur music recorded in Kashgar. It may be too general to claim that music is less effective for creative activities than conversations, or that the participant accelerates the navigation of the FP character only because of music. It may be interesting to further study these relationships to deduce binary findings but it is more advisable to follow the concept of plurality in interpretation about the virtuality of a digital heritage platform. The future research can synthesise the destination of collected patterns with the corresponding sound information to reveal stronger association rules that generate knowledge about 'relativity' beyond binary conclusions.

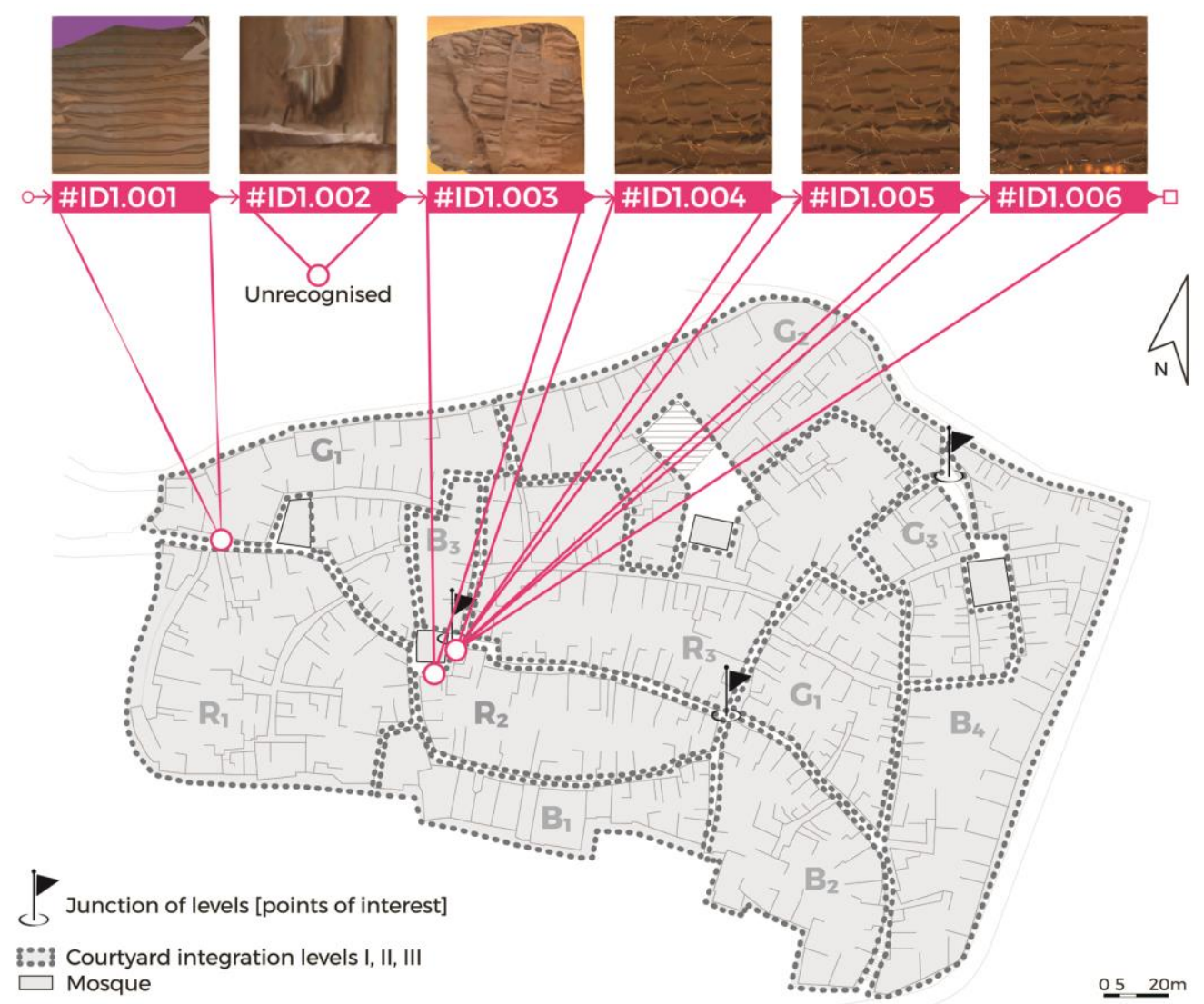

Fig. 118. Further development of Fig. 57. Location of user-collected patterns (the participant: ID1) drawn on a map of Köziciyerbişi (Gaotai) that depicts the points of interest and courtyard integration levels which are analysed in Chapter 4.2.

With the increased level of information used in the ARF analysis, the basic visual units of patterns collected by the participant can extend to the concept of 'index of representation' (see Chapter 4.4.5). Here, defining effective resolution, frequency and range values can be instrumental to executing inter-attribute relations between itemsets. Fig. 119 depicts a hypothetical analysis of the visual values of a pattern. This may help supplement transaction datasets with the digital idiosyncrasies of representing heritage. It is plausible that cross- 
referencing the values of resolution, frequency and range can help discover more interesting association rules. The parameterisation of thumbnail images can potentially enhance the outcome of the ARF analysis. In a broader context, this can contribute to research that seeks to demonstrate how to generate endless knowledge from relatively smaller sets of data instead of requesting big data; that is to say, the generation of knowledge should not be dependent upon big data. Digital data offers deep levels to extract more findings.

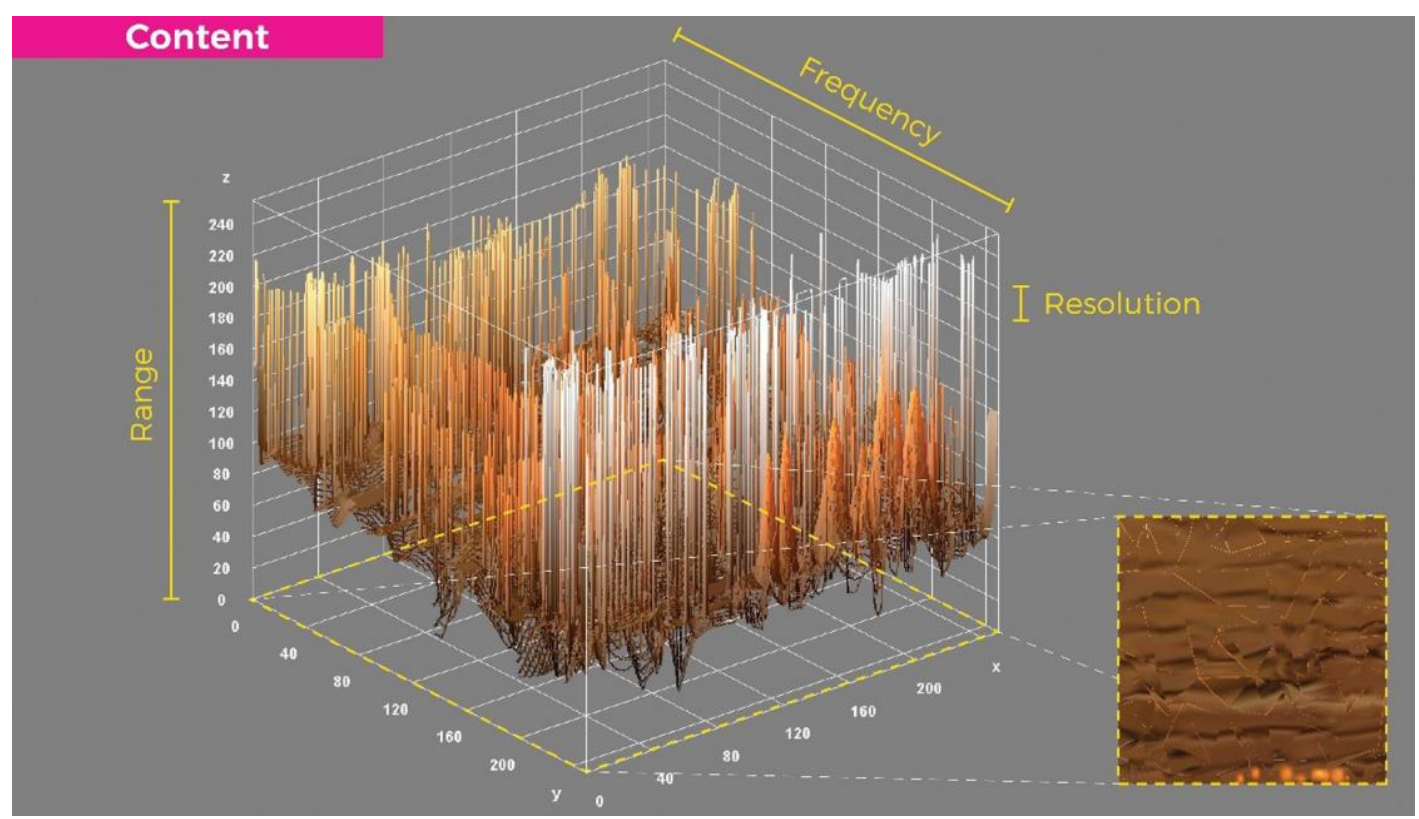

Fig. 119. Defining the resolution, the frequency and the range values of user-generated content based on the RGB information visualised three-dimensionally. The open-source software, ImageJ, is used to generate the three-dimensional view of a two-dimensional pattern.

These experiments dealing with associations between basic units of the visual content merit an understanding of the indexicality that the heritage object holds. The role of these correlated indices of digital engagement can be parameterised. The findings of the ARF method demonstrated patterns of images that are more likely to receive attention from the participant. Evidently, this data mining technique allowed non-linear interpretations of usergenerated data. The statistically independent method of ARF is proven efficacious for the first time in increasing the depth of knowledge in a digital heritage study. In essence, the synthesis of qualitative and quantitative findings from experiments depicted how creative engagement in digital heritage is entangled with the transformation of content. In this connection, the findings of the experiment corroborate this research's hypothesis that alternative knowledge of virtuality in digital heritage is entangled with the transformation of content in creative engagement. 



\section{Chapter 6. Discussion: Design as Virtuality in Digital Heritage}

This new kind of a cultural institute would not only not be a museum of 'art' statistically speaking, but not even a 'museum.' This new kind could be compared to a power station rather, to a generator of new forces.

- Alexander Dorner, as quoted in Claus (1999)

\subsection{Introduction}

The research conceived a new role for participatory content-making and evaluated the creative outcome of user engagement. The experiments implemented a 'design-based,' 'generative,' 'active' and 'creative' mode of engagement with heritage information for alternative knowledge production. By contextualising a design-research problem around the architectural heritage of Kashgar, the westernmost city in China, this study contributed to the field of digital heritage in three ways. The first contribution is briefly summarised in this introduction subchapter that is also a review of the thesis chapters. The second contribution is discussed in the previous chapters as outlined below. The third contribution is discussed subsequently in the next subchapter of the statement. The discussion part combines a discourse of the past, present and future in the field of digital heritage, contingent on the organisation of the previous chapters.

The first contribution lies in the overall arrangement of the chapters. The organisation of the thesis chapters has conceived a foundational research model that addresses the past (Chapter 2), present (Chapter 3 and 4) and future (Chapter 5) of the digital heritage field.

The literature review in Chapter 2 contributes to knowledge on how to make a history of digital heritage. To date, research has expounded on the history of digital heritage beginning with the early museum computing activities in the 1960s. Museum and heritage 
studies have evolved parallel discourses and the scholarship of authorised heritage discourse (AHD) does not explicitly distinguish between them for discussing research outcome. In this thesis, the overview of digital heritage reveals the depth of the field associated with a range of disciplines that exceed the narrow field of museum computing. The discussion touches on the development of museums (e.g. authorised heritage institutions (AHIs)) from being instrumental in controlling heritage information to losing significance because of contemporary people seeking new standards from museums. It is discussed here that digital heritage facilitates the change that museums are obliged to undergo in the twenty-first century.

Chapter 3 takes on the 'present' matters of context, medium and audience to increase the 'resolution' of the thesis' position in the field. It makes a contribution to the literature on Kashgar by revealing the significance of narrow laneways for the local identity. In reviewing the history of Kashgar beginning from the eighteenth century was found a strong relationship between the concepts of house, courtyard, narrow road, neighbourhood and farm-state. Apart from the context, the matters of the medium and the audience disclose a set of criteria to consider in the development of digital heritage research.

Chapter 4 focused on the transparency of all processes of digitisation tested in the context of Kashgar. The documentation activities and statistics contribute to architectural knowledge about Kashgar's historical architecture. With the representation part, the research supplies insight into the mystery of photogrammetric modelling processes. The details of how to generate polygon mesh models impart a new understanding of how to delve into the paradata as a standard requirement in digital heritage. In the dissemination subchapter, the findings from two experiments give a comprehensive analysis of the outcome of a digital design approach for steering and diversifying content-making.

In Chapter 5, the development of gameplay and this experiment platform shows how digital heritage research can integrate design processes with research objectives. The objective of the present study was to employ a digital design approach to steer and diversify creative content-making in digital heritage. The design of the experiment platform is an assemblage of thought processes for finding an abstract form of virtuality rather than actuality. The design process shows how to operate in a flux of appearances by trial and error. The research contributes to the field of digital heritage on how to deal with demands for constant modification in the application of design as a critical form of virtuality. In this regard, the investigations introduce a design research approach that acts as an intelligent filter of the flow of information distilled from the uncertainties of these processes. As a result, the datamining method, Association Rule Finding (ARF), used to distill a set of 
alternative knowledge, provided for the first time in the field, a statistically independent analysis of user-generated digital heritage content. The synthesis of findings in Chapter 5 brings about a prospective discussion on the implications of the research.

As for the second major contribution, earlier analyses and syntheses are significant contributions, including the discussions of:

- the matters involved in digital heritage research (Chapter 3.5),

- the metamorphosis of users' 'tourist gaze' in an 'immersive' virtual environment into a sense of belonging through 'agency' (meaningful role-taking) and intuitive involvement (Chapter 4.4.3),

- creative engagement containing 'shared indexicality' that was measured qualitatively (Chapter 4.4.5 and 4.4.6),

- 'design' as a novel procedure in the development of digital heritage platforms (Chapter 4.5),

- the virtuality, relativity, contextuality, and processeshood of creative contentmaking under continuous transformation (Chapter 5.4.1).

With regards to the third major contribution, this chapter makes a more extensive discussion of the generalisation of knowledge gained through the investigations, analyses and syntheses. In summary, this chapter discusses a statement of the research results; a set of limitations and implications to be aware; and finally, an agenda for future research.

\subsection{Statement of Results}

The primary motivation of the research was to define a new role for participation with heritage information through digital media. The thesis coined 'creative engagement' to describe the integration of the participant into content-making processes in digital heritage. The investigations concentrated on the assessment of creative engagement where the participant becomes operational in the production of new knowledge. The research developed a digital design approach to 'steer and diversify' creative content-making in digital heritage. The design activities operated on a macro and a micro level creative engagement. In the thesis, Chapter 4 focused on the former as a problem-finding, whereas Chapter 5 examined the latter as a problem-solving task. The combination of the design activities grows into a digital design approach that this chapter discusses.

In Subchapter 4.5, three design components were identified as interdependent parameters (see Fig. 92, Page 204). Coined by Murray (1998) as "three key pleasures in cyberspace," they were immersiveness, agency and transformation, which, in the context of 
this research, were aligned with medium, audience and content, respectively. The table below explains the type of quality that each design parameter addresses and the consecutive outcomes that are identified in the experiments. Of these three design components proposed for all digital heritage applications, transformation of content was the focus of the research. Testing the hypothesis that was given following the identification of the three design components, Chapter 5 showed that alternative knowledge of virtuality in digital heritage is entangled with the transformation of content in creative engagement, demonstrating how design decisions were involved in the process.

Table 61. Digital heritage design parameters and their roles in the thesis.

\begin{tabular}{lll}
\hline $\begin{array}{l}\text { Digital Heritage } \\
\text { Design Parameters }\end{array}$ & The quality of ... & $\begin{array}{l}\text { Consecutive outcomes identified in the } \\
\text { thesis }\end{array}$ \\
\hline Immersiveness & Being virtually 'there' & $\begin{array}{l}\text { N/A (this is a medium related domain, i.e. at } \\
\text { this stage, the thesis does not drive any } \\
\text { particular outcome related to the improvement } \\
\text { of the medium in technical means.) }\end{array}$ \\
\hline Agency & Meaningful role-taking & Role of creativity \\
\hline Transformation & $\begin{array}{l}\text { Changes made in content } \\
\text { and user perception }\end{array}$ & $\begin{array}{l}\text { Alternative knowledge recognised in the } \\
\text { changes via user-generated content. }\end{array}$ \\
\hline
\end{tabular}

On the macro level, the results of the design activities were qualitative findings from two experiments on an immersive VR platform. The experiments were conceived to 'find' the problems of 'participatory' content-making pursuing the processes of digital documentation, representation and dissemination. Based on qualitative findings, 'creative participation' was verified to be a catalyst that stipulates the transition from an immersive experience to an experience of agency (meaningful role-taking) in a digital heritage platform. The findings were translated into a holistic diagram that unites the issues of Medium, Audience and Content (MAC) (see Chapter 4.5). The proposal of the diagram contrives a macro-level design approach for use in digital heritage.

On the micro level, a third experiment provided quantitative results regarding the transformation of content made by a participant on a virtual heritage platform. The experiment platform was conceived to 'solve' a problem of 'creative' content-making. The development of gameplay presented how designerly ways of engagement can enhance the virtual expression of narrow roads in Kashgar as creative products of tangible architectural heritage. The experiment results uncovered the transition from agency to transformation. The intuitive design role of agency bestows the transformation of content for a sustainable experience in the virtual heritage environment of Kashgar. The analysis of the 
transformation of content unveils undisclosed associations between digitally represented elements of heritage information. Thirteen association rules gave quantitative descriptions to the meaning of user-generated heritage information that would otherwise remain unknown or difficult to recognise.

Table 62 summarises the macro-level and micro-level design activities in all three experiments conducted in this research. The outcome of the macro- and micro-level design activities is nonstandard and instrumental in designing and developing any digital heritage platform. The primary objective of digitisation is to increase the 'virtue' of heritage information. VR research is responsible for enhancing the 'virtuality' of heritage sites by achieving this objective. The thesis evidenced that the best way to reach the goal of enhanced virtuality is to allow the creative engagement of people. The research gap was that there had been no methodology to distill the 'virtue' of creative engagement in digital heritage. The synthesis of qualitative and quantitative findings from both macro- and micro-levels of design engagement gives insight into the effects of creative engagement on building 'virtuality' in digital heritage. The investigations showed that alternative knowledge of virtuality in digital heritage is implicit in the transformation of content and proved that intuitive engagement with the development of a sense of virtuality 'steers and diversifies' the process of participatory content-making. While addressing this point, the discussion will continue by detailing the contribution of the research in connection with the topics raised in the previous chapters.

Table 62. Macro-level and micro-level design activities observed in the research.

\begin{tabular}{|c|c|c|}
\hline & Macro-level & Micro-level \\
\hline Observation & From immersiveness to agency & From agency to transformation \\
\hline Data & 3D sketches & 2D thumbnail images \\
\hline Method & $\begin{array}{l}\text { Qualitative analysis through an 'Index } \\
\text { of Representation' method }\end{array}$ & $\begin{array}{l}\text { Quantitative analysis - Association Rule } \\
\text { Finding (ARF) method }\end{array}$ \\
\hline Results & $\begin{array}{l}\text { 'Shared indexicality,' i.e. correlations } \\
\text { between different sets of user-generated } \\
\text { content, such as horse carts, doors with } \\
\text { an eave, lanterns, mashrabiya windows, } \\
\text { flowers, gates and cats. }\end{array}$ & $\begin{array}{l}\text { Thirteen association rules, such as } \\
\{\text { Metal Door }, \text { Timber Wall Structure, } \\
\text { Brick Wall }\} \rightarrow\{\text { Rec Cobblestone }\}\end{array}$ \\
\hline Implication & $\begin{array}{l}\text { MAC diagram for the design and } \\
\text { development of participatory and } \\
\text { interactive platforms in digital heritage }\end{array}$ & $\begin{array}{l}\text { Association Rule Finding for the } \\
\text { assessment of user-generated content in } \\
\text { participatory and interactive digital } \\
\text { heritage platforms. }\end{array}$ \\
\hline
\end{tabular}


The choice of the contemporary people leans towards the new standards of the social context where participatory and continuous generation and interpretation of facts are more valuable than watching them broadcast. In reviewing the history of digital heritage, Chapter 2 presented the journey of museums to become social platforms for disseminating heritage knowledge. In the last two centuries of museums, the purpose of displaying heritage information evolved "from being about something to being for somebody" (Weil 1999). The review of the New Museology movement showed how a conversation started between museum professionals and new media technologists. The movement encouraged the use of display technologies for easier public access to museum collections.

Since the New Museology, display technologies used in museum and heritage studies have been required less to be creative than technological. The overwhelming interest in HD displays has captivated the standards of the use of technology in heritage visualisation. In fact, the interest in the realist perfection of images has been a mature form of 'actuality' and 'materiality.' However, the language of new media transforms itself into information space with the changing conditions and use of virtual technologies. In other words, the end of the era of obsession for the realist perfection of images indicates a novel understanding of the creative power of virtuality.

The findings respond to the challenges regarding the dissemination of heritage. Dissemination mostly requires a function of display that can be discussed regarding the power of imagery 'representation.' The discussion of representation pertains to revealing the 'creative power of virtuality.' To address the 'creative power of virtuality' in heritage, AHD patrols the routes for discussing representation. In AHD, one of the universal topics is authenticity, which museums are entitled to evaluate regarding heritage value.

Patrolling the discursive path of 'authenticity' is the chief function of museums as the guardians of heritage knowledge. The authoritative role of museums and heritage institutions has been a permanent thread of 'control' which is an elongated appearance of politics, literature, media, technology and science. In "Allegories of Control," Galloway (2006; 85-106) likens the Foucauldian term of the 'disciplinary societies' and the Deleuzian term of the 'societies of control' to the cinema and video games, respectively.

From the 'birth' of the modern museum to the 'death' of the virtual museum, the extensive review in Chapter 2 made an entry to discuss how AHIs control 'content' and 'audience' through 'medium' for defining the value of heritage. In the beginning, museums as 'cabinets of curiosities' served the 'society of sovereignty.' Museums used heritage information in partly scientific and partly ostentatious exhibitions that displayed the 
belongings of the elite, the noble, the royal and the rich for an audience superior to the general public.

In the post-industrial world of the Foucauldian 'disciplinary society,' computers penetrated AHIs for cataloguing, recording and retrieving information about collections. Automation in museum computing helped control the archiving and dissemination of heritage information. However, the early computing in AHIs was far from offering room for virtual communities.

After the 1960s, vast muséal spaces of enclosure started to enter the virtual world that the Deleuzian concept of the 'societies of control' could explain as 'information superhighways.' In all three phases, the power of control in AHD is comparable to the primary medium of the corresponding era. There appear three dualities: the museum and the theatre, the museum and the cinema and finally the museum and video games (Table 63).

Table 63. The connectivity of museum concepts to the concepts of control for ruling the progress of AHD in different eras.

\begin{tabular}{lll}
\hline Concept of control & Museum concepts ruling AHD & The medium of the era \\
\hline Society of sovereignty & Cabinets of curiosities & Theatre and architecture \\
\hline Disciplinary society & $\begin{array}{l}\text { Museum computing } \\
\text { New Museology }\end{array}$ & Cinema and TV \\
\hline Societies of control & $\begin{array}{l}\text { Virtual Museum } \\
\text { Digital heritage }\end{array}$ & Internet and video games \\
\hline
\end{tabular}

AHIs originate from the fall of empires and the rise of new institutions within the modern state. There were two main reasons behind their 'birth'-the first being about the past and the second about the present. The former reason was to claim ownership on the history. The latter was to acquire credibility from the public opinion for the new hierarchy within the modern state. In an earlier phase, the dissemination of content in the theatre of the 'society of sovereignty' was based on direct contact between the content and the audience, which was conceptualised as the 'cabinets of curiosities' [De. Wunderkammer or Kunstkammer] displaying content to an exclusive audience. One of the most 'authentic' objects in the 1599 museum of Ferrante Imperato in Naples was a taxidermy of a crocodile hanged from the ceiling (see Fig. 2, p. 17). But, as the medium of the era, the theatre ushered museums into a strictly hierarchical relationship between the audience and the author. The author had a full-fledged authority over the medium, audience as well as content. 
Chapter 2 gave the example of Altes Museum in Berlin, which has a remarkable position in the history of heritage discourse. The use of marble replica sculptures, 'whiter than their originals in Greece,' disclosed the concerns of the modern national state about embarking on a quest for credibility sought in the past and liability in the present. As instruments for seeking and feeding public opinion with heritage information, AHIs evolved from being exclusive to inclusive platforms. Predominantly, the 'cabinets of curiosities' used to praise the elite, the royal and the advocates of Enlightenment.

Later on, the museum became a 'conversable civic space' to facilitate plurality and participation in the interpretation of the past; however, the director of the cinematic text has had an authoritative role over the content during the 'disciplinary society.' The cinema and TV facilitated the modern museum with instruments to control the medium, the audience and the content. With the influence of the New Museology, the poststructuralist thinkers made a belated impact on the dissemination of heritage information. The plurality of heritage interpretation extended the materialistic views of museum exhibitions to imagery reproductions.

Afterward, the Internet and virtual communities promoted the concept of the virtual museum in the 'societies of control.' The virtual museum follows the instructions that video games ushered into the contemporary experience of the medium in more active forms of engagement through interactive applications. In this research, the outcome of intuitive engagement shows the importance of developing a sense of "virtuality' through participation and creativity. The role of the author shifts from being a 'guardian of knowledge' about authenticity to a 'facilitator of shared knowledge' that reveals new authentic correlations.

Table 64. The connectivity of museum concepts to the concepts of control for ruling the progress of AHD in different eras.

\begin{tabular}{llll}
\hline Theoretical structure & Medium & Audience-author & Content \\
\hline Binary codes of heritage & Theatre and architecture & One-to-one & Materialistic \\
\hline $\begin{array}{l}\text { Plurality in heritage } \\
\text { interpretation }\end{array}$ & Cinema and TV & $\begin{array}{l}\text { One-to-many } \\
\text { (passive) }\end{array}$ & Imagery \\
\hline $\begin{array}{l}\text { Interaction with heritage } \\
\text { information }\end{array}$ & $\begin{array}{l}\text { Internet and video } \\
\text { games }\end{array}$ & $\begin{array}{l}\text { One-to-many } \\
\text { (active) }\end{array}$ & Information \\
\hline
\end{tabular}

The investigations of the creative engagement shown in this research are a novel contribution to AHD. Since the emergence of AHIs, there has been no attempt to change the destiny of 'heritage' from being solely about the 'past.' In this research, a novel design approach presents how the 'past' can generate new knowledge which is otherwise unknown. 
Finding association rules between user-generated content contributes to the making of the post-virtual museum. It is a museum in the information space where the boundary between the author and audience is blurry. The research presents a more democratic way of engagement that does not only enable the present audience to take part in the definition of authenticity but also leaves space for future generations to contribute.

The 'death' of the virtual museum brings in a non-linear, non-actual, nonstandard understanding of the past, present and future of heritage information. The authentic information transcends the past into the future. As the present signs of the non-absent past, it sustains the heritage value in a close relationship with the contemporary standards of authenticity. The assessment method of ARF used in this research gives flexibility to the progression of knowledge generation with a statistically independent approach. With that, the outcome of creative engagement 'steers and diversifies' the development of virtuality through heritage information. By proving the hypothesis through experiments, quantitative and qualitative findings, the research shows how to alleviate the problem of sustainability of digital heritage.

The thesis contributes to the progression of AHD into the digital domain where a plurality of different voices facilitates the end of the laws of 'materiality.' The investigations support the permeation of AHIs into 'information space' where 'ephemeral' and 'elusive' objects go beyond the corporeal qualities of 'actuality.' AHIs initially entered information space with the arrival of computers in the 1960s primarily for recording and cataloguing purposes. In the beginning, the information space was not necessarily inclusive of heritage information because of the dominant computational legacy and lack of skills. Since then, the enthusiasm of computer scientists, who want to transfer their knowledge and skillsets, has been persistent in heritage and museum studies. Because of this influence, it is still not the information space where AHIs can go beyond 'materiality' and 'actuality' of the heritage object. Today, uncertain definitions, such as digital heritage and the virtual museum, represent a conflict of interests between different fields that want to generate 'knowledge' through heritage. On the one hand, there are purely scientific approaches and on the other hand, there are types of approaches akin to the humanities.

With an interdisciplinary methodology, the research brings together the two separate fields. The thesis outlines a series of principles that derive from a critical review of the field. The research conceives principals that attempt to generate 'discursive novelty' and 'effective innovation.' For the former, the multi-methodological model of RRREI (Resolution, Redescription, Retrodiction or Retroduction, Elimination and Identification) offers principals to follow in digital heritage research. For the latter, the diagram of MAC 
(Medium, Audience and Content) provides principles for digital heritage design with a goal of effectiveness that is to integrate immersiveness, agency and transformation in experiencing a digital heritage platform. No digital heritage study is concerned with a network of tightly entangled relationships between immersiveness, agency and transformation. Creative engagement provokes a metamorphosis of the virtual experience from immersiveness to agency and from agency to transformation.

Table 65. From museum computing to digital heritage, the growth of the field.

\begin{tabular}{lll}
\hline Society & Spatial concept & Axiom \\
\hline Museum computing & Control room & The exclusive power of automation \\
\hline New Museology & Non-place & The social power of inclusivity \\
\hline Virtual Museum & Virtual space & The representative power of imagery \\
\hline Digital heritage & Information space & The transformative power of creativity \\
\hline
\end{tabular}

Chapter 3.3 demonstrated that the medium-oriented research in digital heritage is overconcerned with high-definition display standards of immersiveness. As renowned research outcome, the display technologies of ALiVE by Sarah Kenderdine and Jeffrey Shaw are analysed. The case study of ALiVE showed that design of actuality is still highly esteemed in the field. The over-concern with actuality via immersiveness and embodiment theory is linearly linked to the level of accuracy as a predominantly sought element for validation in digital heritage research. In the 'Matters of Research' (Chapter 3.5), it is revealed that the over-concern with accuracy leads to the crystallisation of epistemology in the field as a result of the positivist postulation of conventional methodologies. The thesis looked into the problem with a hands-on examination of the conventional methodologies of digital heritage.

In Chapter 4, three conventional processes of knowledge generation are employed, namely documentation, representation and dissemination. Eventually, Chapter 4.5 confirmed the analysis of Chapter 3.5 that research overlooks the role of the researcher as 'subject-in-action.' Therefore design as a new process of knowledge generation is attributed to formerly defined processes of digital heritage; however, design as a new process is undertaken here with a focus on a cybernetic understanding of conversation between a system and an observer. It restores the position of the researcher with design as a subjectin-action that does things to contribute to heritage discourse, which was identified to be in silence in Chapter 3.5 (see Waterton and Watson 2013; Winter 2013; Smith 2006; Harrison 2013). It is the disassociation of the researcher from making 'intentional' reasoning that 
generates a circulation of problems raised in the 'Matters of Research' (Chapter 3.5). Conversely, it is argued here that design in practice uses intention to overcome the congestion of conventional processes.

Based on the empirical evidence in Chapter 4.4 that demonstrated two dissemination events, a crucial moment of creative engagement is identified while participants were shifting from immersiveness to agency. The main factor of this moment was observed as the transformation of content. Then the best explanation of the dynamics of creative engagement is delineated as a feedback mechanism between immersiveness, agency and transformation. The hypothesis suggested that alternative knowledge of virtuality in digital heritage is entangled with, first and foremost, the transformation of content in creative engagement.

In Chapter 5, an interactive experiment platform was designed as a means to generate alternative knowledge from user-generated content. The experiments illustrated that usergenerated content did not reveal alternative knowledge of virtuality until a statistically independent approach is used to discover associations between user activities. In cybernetics, research translates intuitive and subjective engagement of the researcher as a 'designerly way of knowing.' In second-order cybernetics, the role of the observer in research through design approach requires intuitional involvement.

The results of experiments generated two main conclusions that can be revolutionary contributions to digital heritage. The first one is about the role of the researcher, which is overlooked by the previous research. The second one is the role of content in generating alternative knowledge of virtuality. They are interrelated aspects of creative engagement in digital heritage. The former derives from a hands-on examination of the conventional means of digital heritage, i.e. documentation, representation and examination. By following the linearity of these processes, it is observed that creative engagement in digital heritage is independent of epistemological postulations of digital heritage, which are dominated by universal principles, guidelines and charters. According to the requirements of the field, such as paradata and prevalence, a digital heritage application may look successful and even outstanding; however, there is usually a problem of sustainability because of continually evolving technologies. Therefore, a role of a researcher as subject-in-action is taken in this thesis, which required the addition of design as an intuitive involvement in the process. Mainstream virtual reality research in digital heritage often employs embodiment theory to deal with the evaluation problem. Conclusions of this approach remain bounded to useful feedback on how to improve the technology for the realist perfection of images representing architectural heritage. In this thesis, it is argued that a novel understanding of the creative power of virtuality is hidden in the transformation of content. 


\subsection{Limitations and Implications}

The investigations relied on a Medium, Audience and Content diagram developed in this research, but only the last element of MAC is tested quantitatively. For each of these three items, there are limitations to consider. The research made a deeper engagement with the content-related aspects of the MAC diagram, e.g. Chapter 5 focused solely on the transformation of content, using synthetic user-data generated from a single participant.

First of all, the experiments were based on two media: an immersive VR cave and a screen-based virtual environment. There are limitations related to the media, but the purpose of the research was not to develop them as new instruments. Therefore, the limitations of the media have been omitted in the research. However, the results may be used to improve tools for virtual experiences for the dissemination of heritage information. Two experiments were implemented within a hybrid VR environment that projects content on a concave screen creating a cave-like physical space. It was discussed in Chapter 4.4 that the immersiveness of the VR environment depends on the role of agency. However, further investigation of the medial role of the VR environment remains to be established.

Secondly, the research starts to address the issues of participation in digital heritage; however, the number of participants is limited to minimal numbers. The generalisability of the syntheses between qualitative and quantitative findings of participation is subject to more specific user-profiles. The role of audience in the research was unspecific and mostly qualitatively assessed based on unstructured interviews and observations. User-centred research in digital heritage usually aims to improve the media utilised or contribute to the learning-based outcome. This research deliberately omits the medium- and audience-related limitations because of the primary objective to contribute to the creative side of digital heritage that pays more attention to the content-related limitations and the implications of designerly ways of engagement - the critique of which is dependent upon the transformation of the content being deployed and generated by users.

Thirdly, the digital heritage content consisted of polygon mesh data. The contentrelated limitations include a lack of audio-visual combination and inadequate types of 3D representations deployed in the development of experimental virtual environments. The role of design in the development of content is limited to standard procedures of heritage for digitally documenting and visualising the narrow roads of Kashgar. The digital content used in the research was semantically open in the beginning. The meaning-making process was integrated with the experiments of participatory content-making; however, the participants were not allowed to describe the visual content they generated. For example, the 3D sketches 
analysed in Chapter 4.4 are embedded with meaning by the researcher as part of the qualitative assessment. Therefore, it was a significant limitation that, on the contrary, enabled the research to eliminate some additional conditions that remain to be considered in future research.

Despite these limitations, the design activities have a range of implications that extend beyond the MAC diagram that is offered for the design and development of digital heritage platforms. The investigations foster the advancement of research and practice in digital heritage beyond the frontiers of current knowledge. The experiments become the beginning of a larger project that is explained in the next subchapter. In the context of the design approach of the thesis, the interconnectedness between three components, which are immersiveness, agency and transformation (see Fig. 92, page 204), suggest how the changes in content can be interpreted in terms of user experience. Further, the outcome of the experiments can be critically evaluated. The user-generated and computer-generated data can be compared by reconnecting the outcomes of the three experiments to the actual site conditions. The creation of such a repository of user-generated content that can be iteratively generated while reconnecting to the site in Kashgar remains a part of future studies. Therefore, one implication of the experiment results is the circular loops between three components of digital heritage design, which would bring this research closer to the domain of cybernetic design principles.

In a narrower sense, the workflow of the research has implications on the digital design methods used in architecture. At the School of Architecture, Victoria University of Wellington, the design-oriented approach of the research has been implemented in an elective course titled Digital Representation and Documentation. In this elective course, third- and fourth-year students are taught digital documentation, representation and dissemination methods with a primary objective to cast their designerly understanding. The results cannot be shown in this dissertation, but they are available for viewing upon request from the course coordinator, Tane J. Moleta, who has the authority to share student works (https://www.victoria.ac.nz/courses/sarc/363/2017/offering?crn=18285). About seventy students in two years in this course have been taught how to make photogrammetric models interactive by using the software solutions in the way used in this research.

Aside from this, the school has established a new design-research studio where the methodology of this research has been partly integrated into projects that address real problems. The studio is called Virtual and Augmented Studio Environment (VASE) and directed by Prof Marc Aurel Schnabel (Lo and Schnabel 2018). The research activities reinforce the validity of the generalisability of the methodology used in this research. The 
results have been presented as research papers at international conferences including eCAADe (Rogers and Schnabel 2018-forthcoming), CAADRIA (Rogers, Schnabel and Lo 2018), AMPS (Architecture, Media, Politics and Society) (Rushton et al. 2018).

In a broader sense, the design activities in the research imply the possibility of more intuitive and democratic ways of engagement with heritage information. The findings can be applied to VR research in general. There is a number of fields that can apply the results of this research, including cultural heritage, computer-aided architectural design, museology, new media, TV, education, MOOC, streaming, as well as culture and game studies. Regarding the role that users play, the research has implications on the progress of content-making platforms in heritage.

These implications are similar to the progressive impacts of the changing standards of broadcasting. The launch of YouTube changed the nature of television use. There has been a move from one-to-many broadcasting to many-to-many storytelling. Individuation has been increased with Facebook. Live video posting on social media, such as Facebook and Instagram, is a blend of YouTube and the television. In the post-war era, the public demanded new standards from AHIs for the dissemination of the information that only AHIs were authorised to select and release. The rise of information over knowledge in the 1960s caused AHIs to change their retrieval methods from analog to computational systems. The progress of computerisation in AHIs has been largely completed recently; however, the public demand for new standards from AHIs is an ever-increasing complexity, now leading to new concerns about personalisation.

Similar to the impacts of social media on the television, this research brings the use of creative input into the domain of AHIs. The investigations present new means of digital heritage reinterpretation in a new social context. The results address the fact that participatory and social context has become more significant than the archival and curatorial position of the AHIs in the contemporary society. The methods and findings of the research show how to distill meaning from the information generated within the contemporary social context.

\subsection{Future Research: Decoding $X$ in The Museum of Gamers}

The future research will include other historical towns from around the world with a specific interest in the architecture of Central Asia and its surrounding regions. A new initiative has 
been made in the context of Lahore. The research on Lahore's historical walled city focuses on the transformation of phenomenological aspects into VR environments (Abid, Aydin and Schnabel 2017). The virtual experiences of the niches alongside the narrow roads are under investigation based on photogrammetry and participatory content-making. The objective of the 'Decoding Lahore' project is to develop new storyworlds through participatory experiences in VR (Fig. 120).

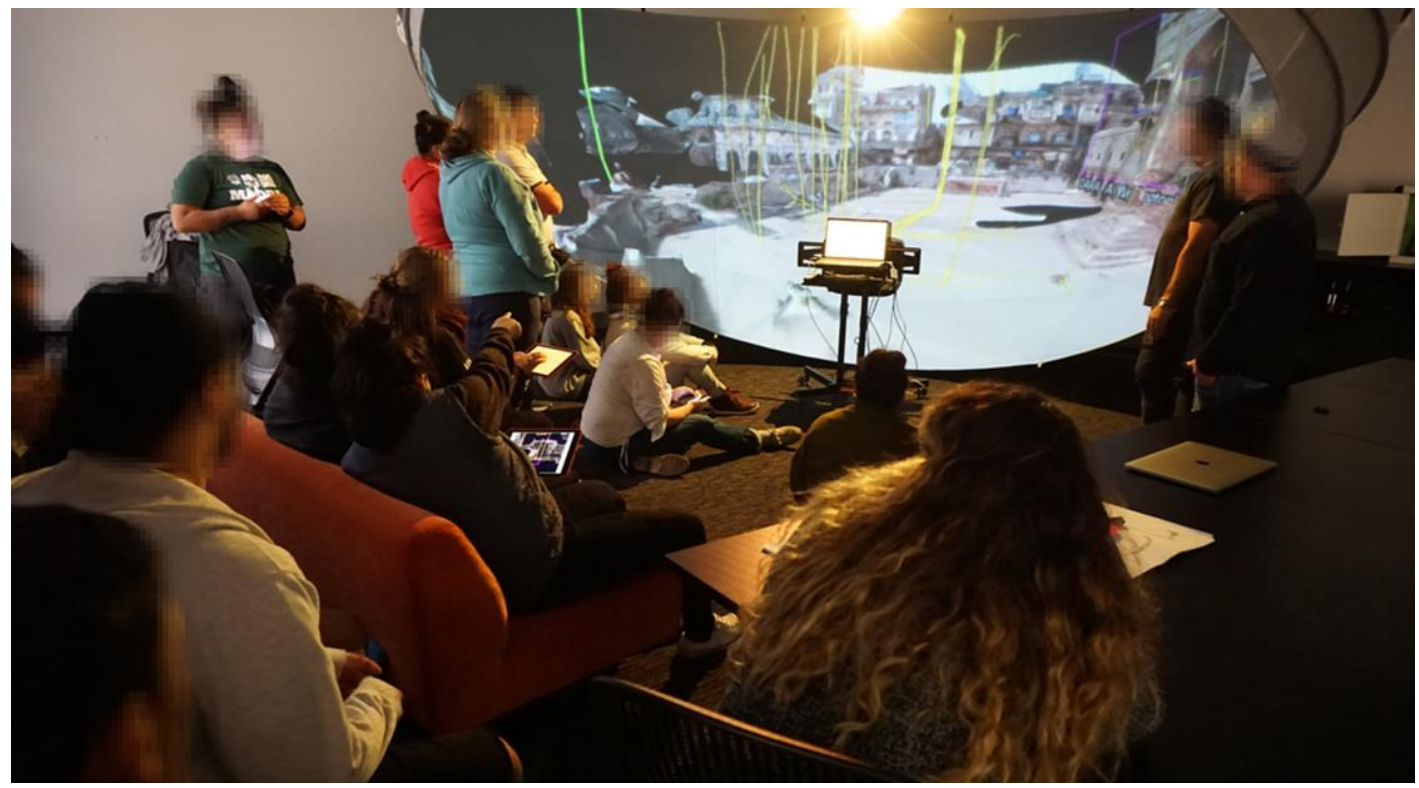

Fig. 120. Participants remotely (from Wellington, New Zealand) visiting the front outer courtyard of Masjid Wazir Khan, Lahore. Photo by Author.

To exhibit the present 'Decoding Kashgar' and future 'Decoding X' projects, a website has been created under the name of The Museum of Gamers (http://www.themuseumofgamers.org/) where Decoding Kashgar is the first. The website homepage opens with a TV-like video-content running continually on the cover page (Fig. 121). The website interprets the need for an organisational change in AHIs as a provocation to embark on a task for supporting collective intelligence in digital heritage. The Museum of Gamers aims for a novel museum experience instead of merely developing new media applications. Bridging design expertise to alternate realities in a digitally-enhanced online museum experience, the website draws on the role of transformation in participatory content generation. By combining cutting-edge applications, the website aims for an online ecosystem that is extended on the use of televisions and smart gadgets to augment the sense of reality in a digitally conceived museum experience. The Museum of Gamers chooses to salute the television and aims to integrate its facilities into the designed platforms through alternate realities that are run via the shared aspects of cyberspace. 


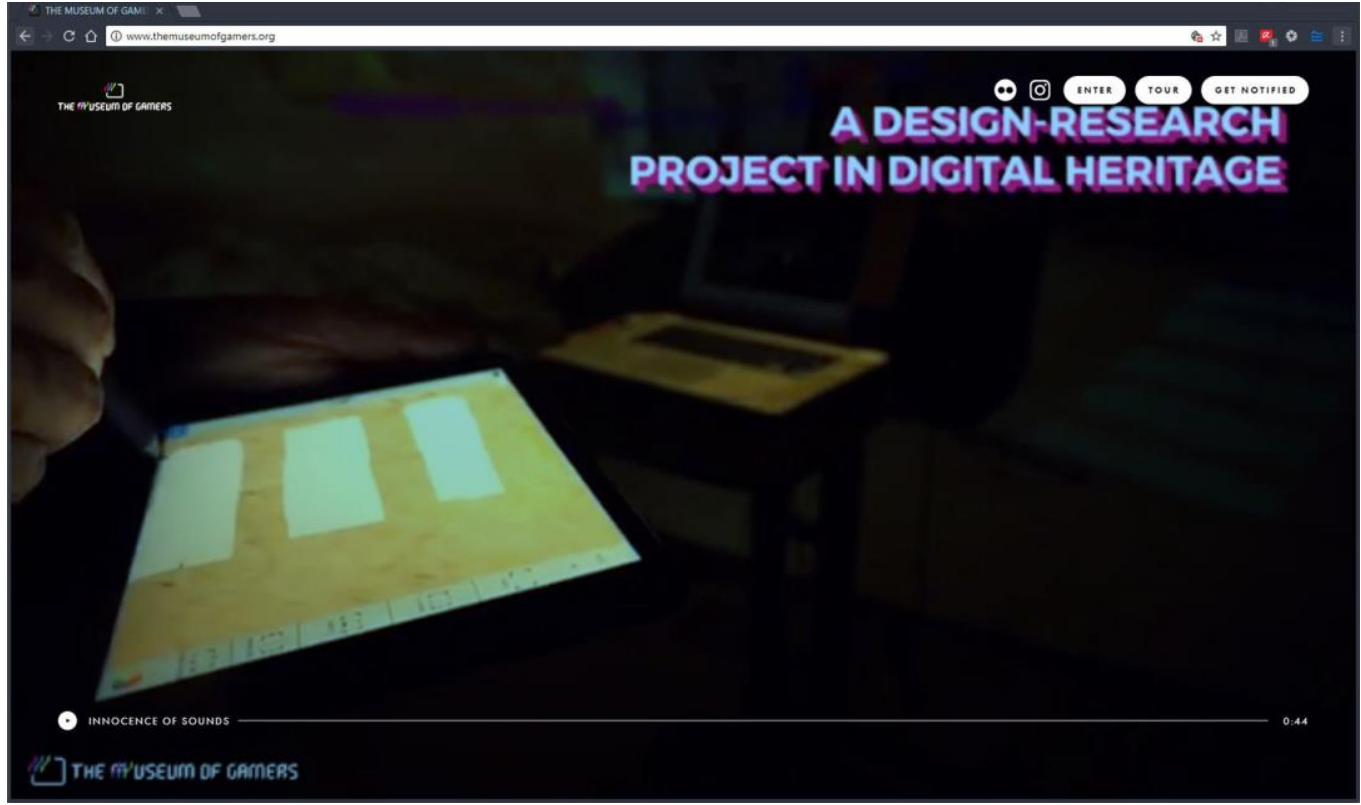

Fig. 121. The cover page of The Museum of Gamers. The website is designed to contain all 'Decoding $X$ ' projects to be conducted in the future. It is an online conceptual museum where usergenerated content defines the curation of exhibitions. The online platform is conceived to present the digital heritage of historical towns.

Currently, the website offers online game experiences for public engagement with Decoding Kashgar. Furthermore, The Museum of Gamers is interested in the deployment of beacons, smart watches, second screen companion apps, such as Game of Thrones for iTV, that move the world ever closer to perfection in connecting alternate realities.

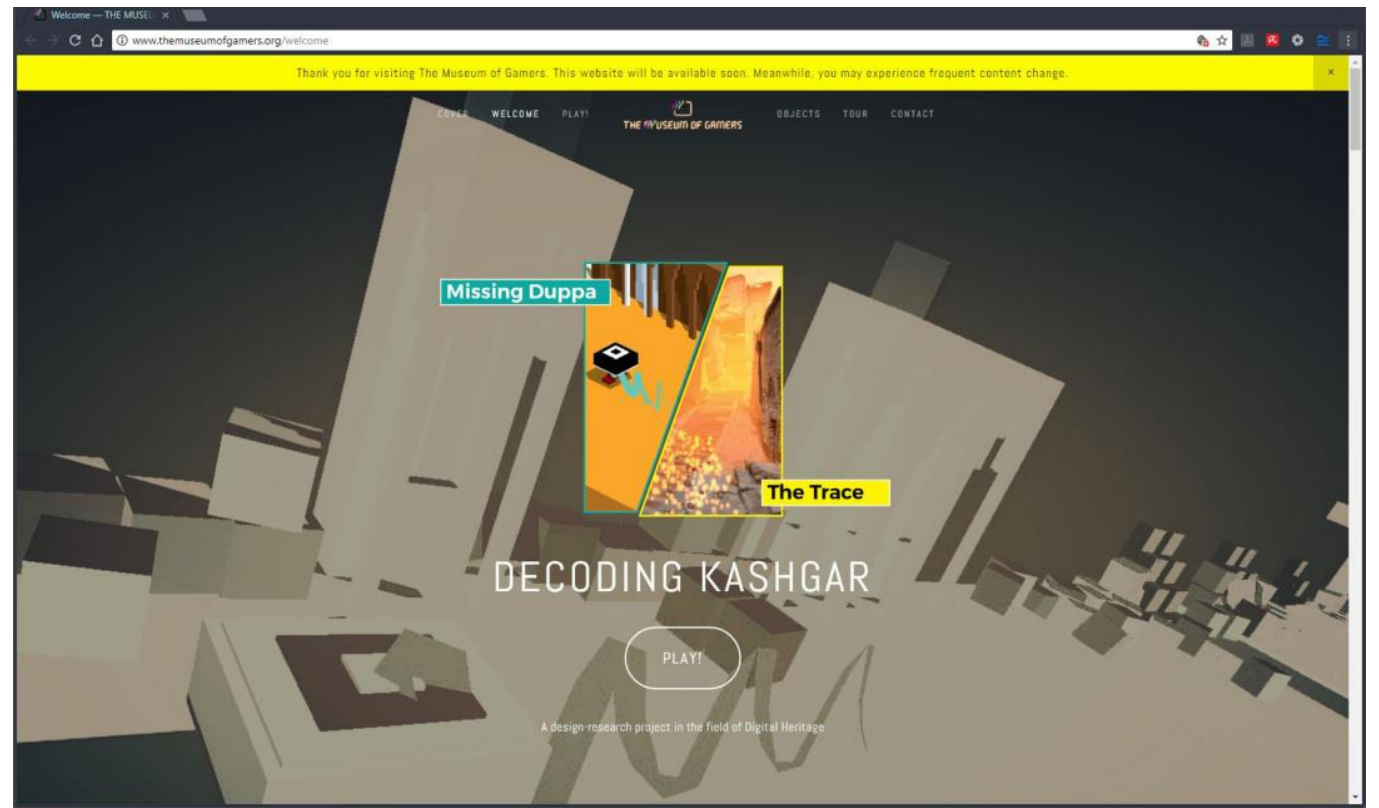

Fig. 122. Decoding Kashgar in The Museum of Gamers. 

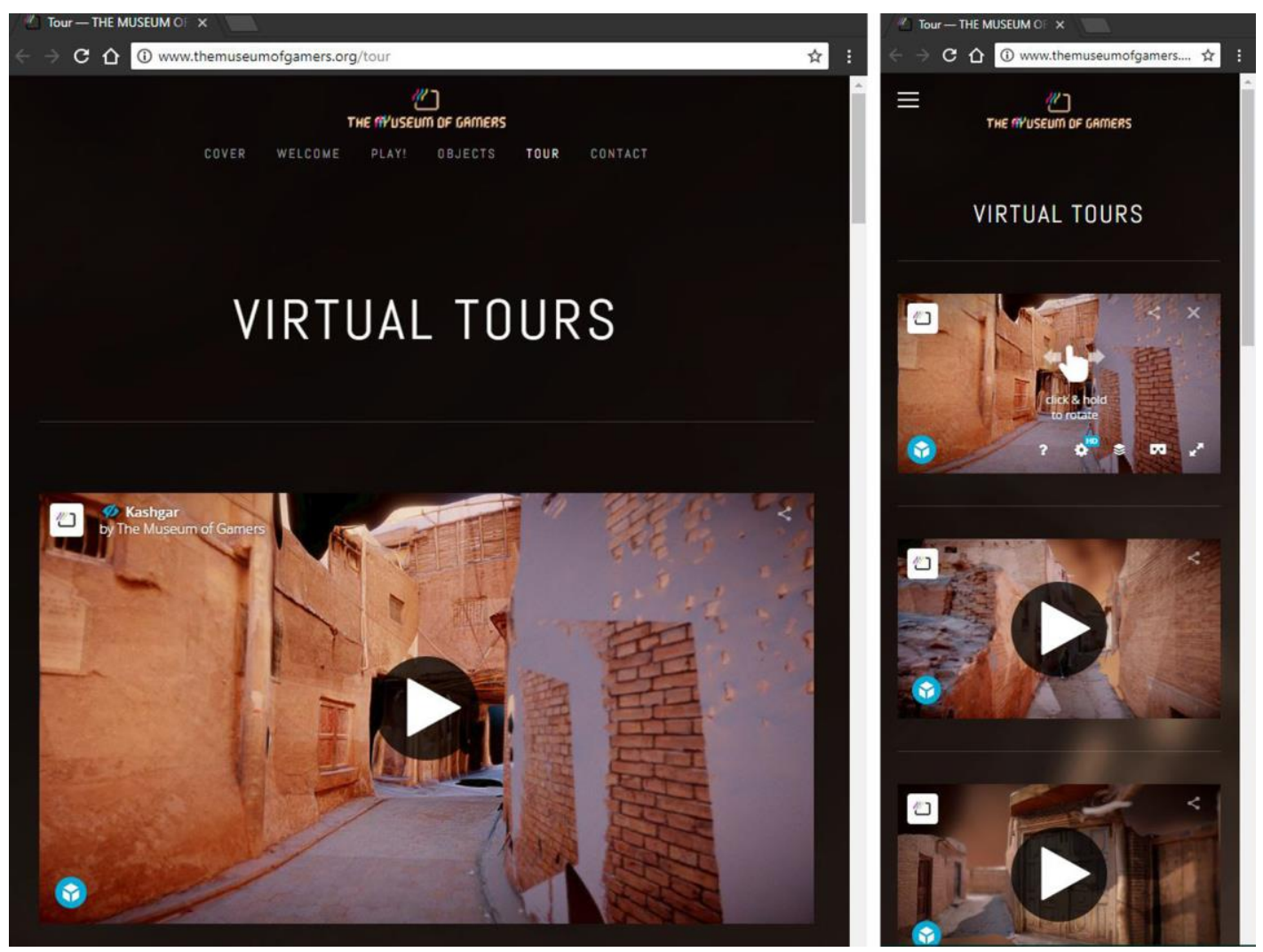

Fig. 123. The responsive website of The Museum of Gamers demonstrate the quality of virtual environments supported by Sketchfab (https://sketchfab.com/), which is an online platform to publish 3D content with different interaction options such as viewing with a VR headset or a cardboard.

Future research requires a contemporary technological integration between alternate realities based on an assessment scheme that focuses on the user-generated content in digital heritage. To do so, this research showed how to design feedback cycles for information management between different media. In an ecosystem of display technologies that are programmed to sense the low-proximity of mobile devices, it is possible to hand over the control to participants. Therefore, the goal of further studies aims to bring closer the domains of museology and television. Therefore, The Museum of Gamers is to be the first to combine the television industry and AHIs, such as museums as part of the GLAMs sector. 



\section{Chapter 7. Conclusions}

The main motivation behind the research was the lack of a holistic understanding of the potentials of digital engagement that offers new ways of expressing 'authenticity' and 'virtuality' in heritage. This research was undertaken to conceive a new role for participatory content-making and evaluate the creative outcome of user engagement. A 'design-based,' 'generative,' 'active' and 'creative' mode of engagement with heritage information is sought as a stimulus of alternative knowledge production. In this investigation, the unique case of Kashgar, which is an old city on the historic Silk Road in China, is used as a context to build a scenario for experimentation and research.

The findings from three experiments make several contributions to the current literature. The contributions categorically have significance for (1) bringing novelty to the heritage discourse, (2) providing innovation to available methodologies, (3) demonstrating a successful interdisciplinary approach to practice, (4) producing alternative knowledge in heritage and virtual reality, and (5) adding on the preservation of Kashgar's architectural heritage in digital formats.

First, the research brings novelty to heritage discourse by analysing the matters involved in research. This study resolves epistemological obsessions of the current research with 'accuracy' through a critical realist approach. With it, the research follows principles to combine methodologies, and thereby to synthesise quantitative and qualitative results of the experiments.

Second, the standard heritage methodologies, comprised of digital 'documentation,' 'representation' and 'dissemination,' are supplemented with the domain of 'design.' A digital design approach is conceived to document not only the facts of heritage but also the creative outcome of user engagement for future generations. Accordingly, a corrected workflow of the standard heritage activities is established, centred on a correlation between medium, audience and content for designing digital applications. 
Third, the present study confirms the position of Murray's (1998) three key pleasures (i.e. immersiveness, agency and transformation) in engaging with virtual reality and contributes additional evidence that suggests analysing user-generated content for exposing alternative knowledge through undisclosed patterns. Experiment results suggest that studying neither immersiveness nor agency (meaningful role-taking) provide sufficient information for knowledge production. Analysing user-generated content is critical to understand the impact of engagement with heritage and the success of a digital application. Creative activities enable a higher level of engagement in digital heritage, wherein the participant is stimulated to advance from having a 'tourist gaze' in a virtual heritage environment to generating a new 'local gaze' that deeply identifies with the place.

Fourth, the most obvious finding to emerge from this study is that alternative knowledge of virtuality in digital heritage lies in the transformation of content as a part of the creative engagement. Association Rule Finding (ARF) method borrowed from the data mining field revealed that the assemblage of alterations made by participants in a digital heritage platform retains information of undisclosed patterns. With ARF's non-linear and statistically independent data interpretation, correlations between user-generated content are revealed. The patterns identified by association propagate alternative and new knowledge generated via a signification process throughout the creative engagement.

This is the first time that ARF has been used to assess user-generated content for 'creativity' and 'virtuality' in digital heritage. The most important limitation lies in that synthetic data is used to support a qualitative assessment of quantitative findings from an ARF analysis. Although the current study is based on the synthesis of a sample participant and artificial user data, the findings suggest that creative engagement of the participant helps heritage information transcend from the boundaries of 'actuality' and that the participant enriches the meaning of content by interpreting it in different ways. The analysis of usergenerated content undertaken here has extended our knowledge of Kashgar heritage. Thirteen association rules are distilled, uncovering relationships between architectural features of narrow roads in Kashgar, which were otherwise unknown.

A natural progression of the present ARF analysis is to add more layers of information into data. More detailed information on user-generated content would help us establish a greater number of associations between architectural features of heritage. It is suggested that the association of additional factors such as the time and location of user-generated content in a virtual heritage environment is investigated in future studies. More broadly, research is also needed to determine the interdependency between medium, audience and content by 
testing and assessing changes in creative outcome through feedback cycles that are formulated quantitatively.

Fifth, the findings of the ARF analysis visualise the invisible mystery of narrow alleys in Kashgar, by documenting the outcome of creative user engagement with virtual Kashgar. The digital preservation of Kashgar's architectural heritage provides a resource and record for future generations. The investigations fill a gap in knowledge about the architectural heritage of Kashgar, once an important place along the historical Silk Road. Proceeding from few other studies, the research preserves the cultural heritage of Kashgar from an architectural and digital perspective. It is the first time that research 'analytically' displays the significance of narrow roads in Kashgar, which are fundamental to the local history and identity. Experiments organised as part of public events in this research are not bound to lab conditions and rather act as catalysts to build awareness about Kashgar amongst distant audiences.

Taken together, these findings suggest a new role for user-generated content for building 'virtuality' through creativity, which is discovered here as a higher level of engagement with heritage than merely testing the success of embodiment and immersiveness for educating users about historical places. Although more research in connection with the assessment of user experience is required to determine the efficacy of analysing the transformation of content, the methods used for this research for decoding Kashgar can be applied to other historical cities elsewhere in the world.

This research will serve as a base for future studies in digital heritage that is concerned with the transformative power of creative engagement. The investigations foster the advancement of research and practice in digital heritage beyond the frontiers of current knowledge. It may be the first time that cultural heritage presents unique research challenges in the domain of digital design. There is a number of fields that can apply the results of this research, including cultural heritage, computer-aided architectural design, museology, new media, TV, education, MOOC, streaming, as well as culture and game studies. 


\section{Reference List}

* Please refer to 'International Charters and Principles' and 'Artworks and Projects' at the end of the reference list.

Aarseth, Espen J. 1997. Cybertext: Perspectives on Ergodic Literature. Baltimore, Md.: Johns Hopkins University Press.

Abbazia, Danielle, Judith Opoku-Boateng, Agnieszka Slomska, and Aparna Tandon. 2017. Unlocking Sound and Image Heritage: Selected Readings from the 2015 SOIMA Conference. Rome; Brussels: ICCROM \& KIK-IRPA.

Abid, Mehwish, Serdar Aydin and Marc Aurel Schnabel. 2017. "Reclaiming Heritage by Retelling 'The Thing' in Virtual Reality: Decoding Walled City of Lahore." In Back to the Future: The Next 50 Years, Proceedings of the 51st International Conference of the Architectural Science Association (ANZAScA), Wellington, New Zealand, November 29-December 2, 2017. 15-24.

Adair, Bill, Benjamin Filene, and Laura Koloski, eds. 2011. Letting Go?: Sharing Historical Authority in a User-Generated World. Philadelphia, Pa.: Pew Center for Arts \& Heritage.

Addison, Alonzo C. 2000. "Emerging Trends in Virtual Heritage." IEEE MultiMedia 7 (2):22-25. doi: 10.1109/93.848421.

Affleck, Janice, and Thomas Kvan. 2005. "Reinterpreting Virtual Heritage." In A. Bhatt, ed. Proceedings of the 10th International Conference on Computer-Aided Architectural Design Research in Asia (CAADRIA 2005), Volume 1, New Delhi, India, April 28-30, 2005. 169178.

Affleck, Janice, and Thomas Kvan. 2008. "A Virtual Community as the Context for Discursive Interpretation: A Role in Cultural Heritage Engagement." International Journal of Heritage Studies 14 (3):268-280. doi: 10.1080/13527250801953751.

ALiVE (Applied Laboratory for Interactive Visualization and Embodiment). 2014. Accessed December 5, 2017, http://alive.scm.cityu.edu.hk/.

Allan, Derek. 2009. Art and the Human Adventure: André Malraux's Theory of Art. Amsterdam; New York: Rodopi.

Alvarez, Luis W., Jared A. Anderson, F. El Bedwei, James Burkhard, Ahmed Fakhry, Adib Girgis, Amr Goneid, Fikhry Hassan, Dennis Iverson, Gerald Lynch, Zenab Miligy, Ali Hilmy Moussa, Mohammed Sharkawi, and Lauren Yazolino. 1970. "Search for Hidden Chambers in the Pyramids." Science 167 (3919):832-839. doi: 10.1126/science.167.3919.832. 
272

Anders, Peter. 1999. Envisioning Cyberspace: Designing 3D Electronic Spaces. New York; London: McGraw-Hill.

Anderson, Chris. 2008. "The End of Theory: The Data Deluge Makes the Scientific Method Obsolete." Wired, issued June 23, 2008. Accessed January 14, 2018, https://www.wired.com/2008/06/pb-theory/.

Andreotti, Libero, and Nadir Lahiji. 2016. The Architecture of Phantasmagoria: Specters of the City. Florence: Taylor and Francis. Accessed December 10, 2017, ProQuest Ebook Central.

Andro, Mathieu, and Imad Saleh. 2017. "Digital Libraries and Crowdsourcing: A Review." In Collective Intelligence and Digital Archives: Towards Knowledge Ecosystems, edited by Samuel Szoniecky and Nasreddine Bouhaï, 135-161. Hoboken, N.J.; London: Wiley-ISTE.

Appel, Arthur, T. P. Dankowski, and R. L. Dougherty. 1968. "Interactive Graphics in Data Processing: Aspects of Display Technology." IBM Systems Journal 7 (3.4):176-187. doi: $10.1147 /$ sj.73.0176.

Aquilina, Janick Daniel. 2011. "The Babelian Tale of Museology and Museography: A History in Words." Museology, International Scientific Electronic Journal 6:1-20.

Aquilina, Janick Daniel. 2017. Email conversation with author, October 12, 2017

Arayici, Yusuf, John Counsell, Lamine Mahdjoubi, Gehan Nagy, Suhayr Zakī Hawwās, and Khaled Dweidar, eds. 2017. Heritage Building Information Modelling. Abingdon, Oxon; New York, N.Y.: Routledge.

Argyris, Chris, and Donald A. Schön. 1996. Organizational Learning II: Theory, Method, and Practice. New York: Addison-Wesley.

Ascott, Roy. 1999. "Gesamtdatenwerk: Connectivity, Transformation and Transcendence." In Ars Electronica: Facing the Future: A Survey of Two Decades, edited by Timothy Druckrey with Ars Electronica, 86-89. Cambridge, Mass.; London: The MIT Press.

Attene, Marco, Marcel Campen, and Leif Kobbelt. 2013. "Polygon Mesh Repairing: An Application Perspective." ACM Computing Surveys 45 (2):1-33. doi: 10.1145/2431211.2431214.

Augé, Marc. 2008. Non-Places: Introduction to an Anthropology of Supermodernity. London: Verso.

Aydin, Serdar. 2017a. "Decoding Kashgar: Participatory Digital Heritage Making via Digital Online Interaction and Gamification." Adjunct Publication of the 2017 ACM International Conference on Interactive Experiences for TV and Online Video, Hilversum, The Netherlands. 93-97.

Aydin, Serdar. 2017b. "Type of Decimation Algorithm Used in Remake." ReMake Forum, October 10, 2017. Accessed January 16, 2018, https://forums.autodesk.com/t5/remake-forum/typeof-decimation-algorithm-used-in-remake/m-p/7449193/highlight/true.

Aydin, Serdar, and Marc Aurel Schnabel. 2017a. "A Collective Intelligence Assessment Approach through Alternate Realities in 'The Museum of Gamers'." In A. Ulisses, M. Armstrong, B. Shirley, B. Malheiro, J. Foss and S. Kepplinger, eds., Proceedings of the In-Programme Personalisation for Broadcast (IPP4B) Workshop, ACM TVX2017, Hilversum, The Netherlands, June 14-16, 2017. 1-5. doi:10.6084/m9.figshare.c.3793435.v1.

Aydin, Serdar, and Marc Aurel Schnabel. 2017b. Decoding Kashgar: China's Westernmost City on the Historical Silk Road. Wellington: DARA. 
Aydin, Serdar, Marc Aurel Schnabel, and Tane Moleta. 2016. "Transcoding of Game Design into Museology: An Object-Oriented Perspective." In S. Uddin, M. Sahin, A.Ö. Torun and Ü.C. Bayazitoğlu, eds., Inclusiveness in Design: Proceedings of the 2016 European Design Communication Conference (DCA-E 2016), Istanbul, Turkey, May 11-14, 2016. 375-384.

Aydin, Serdar, Marc Aurel Schnabel, and Iman Sayah. 2017. "Association Rule Mining to Assess User-Generated Content in Digital Heritage." In Computer-Aided Architectural Design. Future Trajectories: 17th International Conference, CAAD Futures 2017, Istanbul, Turkey, July 12-14, 2017, Selected Papers, edited by Gülen Çağdaş, Mine Özkar, Leman Figen Gül and Ethem Gürer, 231-251. Singapore: Springer.

Aygen, Zeynep. 2013. International Heritage and Historic Building Conservation: Saving the World's Past. New York: Routledge.

Bærenholdt, Jørgen Ole, Michael Haldrup, Jonas Larsen, and John Urry. 2004. Performing Tourist Places. Aldershot: Ashgate.

Bagnall, Gaynor. 2003. "Performance and Performativity at Heritage Sites." Museum and Society 1 (2):87-103.

Ballantyne, Andrew. 2015. John Ruskin. London: Reaktion Books.

Barcel'o, Juan A., Maurizio Forte, and Donald H. Sanders, eds. 2000. Virtual Reality in Archaeology: Computer Applications and Quantitative Methods in Archaeology (CAA). Oxford: Archaeopress.

Barthes, Roland. 1988. Image, Music, Text. Translated by Stephen Heath. New York: Noonday Press.

Batkin, Norton. 1997. "Conceptualizing the History of the Contemporary Museum: On Foucault and Benjamin." Philosophical Topics 25 (1):1-10, http://www.jstor.org/stable/43154248.

Battro, Antoni M. 2010. "From Malraux's Imaginary Museum to the Virtual Museum." In Museums in a digital age, edited by Ross Parry, 136-147. London: Routledge.

Baudrillard, Jean. 1993. The Transparency of Evil: Essays on Extreme Phenomena. London; New York: Verso.

Baudrillard, Jean. 1994. Simulacra and Simulation. Translated by Sheila Faria Glaser. Ann Arbor: University of Michigan Press.

Baudrillard, Jean. 2005. The Conspiracy of Art: Manifestos, Interviews, Essays. Sylvère Lotringer. Translated by Ames Hodges. New York: Semiotext(e).

Bautista, Susana Smith. 2014. Museums in the Digital Age: Changing Meanings of Place, Community, and Culture. Lanham, Md.: AltaMira Press, a division of Rowman \& Littlefield.

Bayazit, Nigan. 2004. "Investigating Design: A Review of Forty Years of Design Research." Design Issues 20 (1):16-29. doi: 10.1162/074793604772933739.

Bearman, David. 2008. "Representing Museum Knowledge." In Museum Informatics: People, Information, and Technology in Museums, edited by Paul F. Marty and Katherine Burton Jones, 35-57. New York; Oxon: Routledge.

Beaudry Marchand, Emmanuel, Xueying Han, and Tomás Dorta. 2017. "Immersive Retrospection by Video-Photogrammetry: UX Assessment Tool of Interactions in Museums, a Case 
Study." In A. Fioravanti, S. Cursi, S. Elahmar, S. Gargaro, G. Loffreda, G. Novembri and A. Trento, eds., ShoCK! - Sharing Computational Knowledge!: Proceedings of the 35th Annual International Conference of eCAADe - Education and Research in Computer Aided Architectural Design in Europe (eCAADe 2017), Volume 2, Rome, Italy, September 20-22, 2017. 729-738.

Bell, Jeffrey A. 1998. The Problem of Difference: Phenomenology and Poststructuralism. Toronto: University of Toronto Press.

Bellér-Hann, Ildiko. 2013. "The Bulldozer State: Chinese Socialist Development in Xinjiang." In Ethnographies of the State in Central Asia: Performing Politics, edited by Madeleine Reeves, Johan Rasanayagam and Judith Beyer, 173-197. Bloomington: Indiana University Press.

Bellér-Hann, Ildikó. 2008. Community Matters in Xinjiang, 1880-1949: Towards a Historical Anthropology of the Uyghur. Leiden: Brill.

Bellér-Hann, Ildikó. 2016. "Uyghur Peasants Remember Collectivisation in Southern Xinjiang." In Inside Xinjiang: Space, Place and Power in China's Muslim far Northwest, edited by Anna Hayes and Michael E. Clarke, 15-31. London: Routledge.

Belogolovsky, Vladimir. 2016. "Interview with Asymptote Architecture: "We Are Spatial Engineers"." ArchDaily, issued February 19, 2016. Accessed January 10, 2018, https://www.archdaily.com/782384/interview-with-asymptote-architecture-we-are-spatialengineers.

Benedikt, Michael L., ed. 1991. Cyberspace: First Steps. Cambridge, Mass.: The MIT Press.

Benjamin, Andrew E., and Beatrice Hanssen. 2002. Walter Benjamin and Romanticism. London; New York: Continuum.

Bennett, Tony. 1995. The Birth of the Museum: History, Theory, Politics. London; New York: Routledge.

Bennett, Tony. 1998. "Pedagogic Objects, Clean Eyes, and Popular Instruction: On Sensory Regimes and Museum Didactics." Configurations 6 (3):345-371. doi: 10.1353/con.1998.0020.

Bentkowska-Kafel, Anna. 2009. "The Fix v. the Flux. Which Digital Heritage?" In Net Pioneers 1.0, edited by Günther Reisinger, 55-72. Sternberg Press in Association with the Ludwig Boltzmann Institute.

Bentkowska-Kafel, Anna, Trish Cashen, and Hazel Gardiner, eds. 2009. Digital Visual Culture: Theory and Practice. Bristol: Intellect books.

Bentkowska-Kafel, Anna, Hugh Denard, and Drew Baker. 2012. Paradata and Transparency in Virtual Heritage. Farnham, Surrey; Burlington, Vt.: Ashgate.

Betts, Tom. 2014. "Procedural Content Generation." In Handbook of Digital Games, edited by Marios C. Angelides and Harry Agius, 62-91. John Wiley \& Sons, Inc.

Bhaskar, Roy. 1978. A Realist Theory of Science. Sussex: Harvester Press. Original edition, Leeds Books, 1975.

Bhaskar, Roy. 1998. "General Introduction." In Crtical Realism: Essential Readings, edited by Margaret Archer, Roy Bhaskar, Andrew Collier, Tony Lawson and Alan Norrie, ix-xxiv. London; New York: Routledge. 
Bhaskar, Roy. 2013. "Prolegomenon." In Engaging with the world: agency, institutions, historical formations, edited by Margaret Scotford Archer, Roy Bhaskar and Andrea Maccarini, 1121. London: Routledge.

Bhaskar, Roy. 2014a. "Forward." In Studying Organizations Using Critical Realism: A Practical Guide, edited by P. K. Edwards, Joe O'Mahoney and Steve Vincent, v-xv. Oxford: Oxford University Press.

Bhaskar, Roy. 2014b. The Possibility of Naturalism: A Philosophical Critique of the Contemporary Human Sciences. Florence: Taylor and Francis.

BIG (Bjarke Ingels Group). 2010. Yes is More: An Archicomic on Architectural Evolution. Köln: Taschen.

Binford, Lewis R. 1962. "Archaeology as Anthropology." American Antiquity 28 (2):217-225. doi: $10.2307 / 278380$.

Binford, Lewis R., and Sally R. Binford. 1966. "A Preliminary Analysis of Functional Variability in the Mousterian of Levallois Facies." American Anthropologist 68 (2):238-295. doi: 10.1525/aa.1966.68.2.02a001030.

Bogost, Ian. 2006. Unit Operations: An Approach to Videogame Criticism. Cambridge, Mass.: MIT Press.

Bogost, Ian. 2007. Persuasive Games : The Expressive Power of Videogames. Cambridge, Mass.: MIT Press.

Borbein, Adolf H. 2000. "Zur Geschichte der Wertschätzung und Verwendung von Gipsabgüssen antiker Skulpturen (insbesondere in Deutschland und in Berlin) [On the History of the Appraisal and Use of Plaster Casts of Ancient Sculpture (especially in Germany and in Berlin)]." Last modified March 22, 2013. Accessed January 10, 2018, http://www.digitalsculpture.org/casts/borbein/.

Borgo, Rita, Paolo Cignoni, and Roberto Scopigno. 2001. "An Easy-to-Use Visualization System for Huge Cultural Heritage Meshes." In D. B. Arnold, A. Chalmers and D. Fellner, eds., Proceedings of the 2001 Conference on Virtual Reality, Archeology, and Cultural Heritage (VAST 2001), Glyfada, Greece, November 28-30, 2001. 121-130. doi:10.1145/584993.585013.

Borowiecki, Karol Jan, Neil Forbes, and Antonella Fresa, eds. 2016. Cultural Heritage in a Changing World.

Borowiecki, Karol Jan, and Juan Prieto-Rodriguez. 2015. "Video Games Playing: A Substitute for Cultural Consumptions?" Journal of Cultural Economics 39 (3):239-258. doi: 10.1007/s10824-014-9229-y.

Botsch, Mario, Leif Kobbelt, Mark Pauly, Pierre Alliez, and Bruno Lévy. 2010. Polygon Mesh Processing. Natick, Mass.: A K Peters.

Bridge, David. 2016a. "SELGEM: Designing IT applications in the 1970s." Smithsonian Institution Archives. Accessed October 29, 2017, http://si-siris.blogspot.co.nz/2016/08/selgemdesigning-it-applications-in.html.

Bridge, David. 2016b. "SELGEM: The Data Structure." Smithsonian Institution Archives. Accessed October 29, 2017, http://si-siris.blogspot.co.nz/2016/07/selgem-data-structure.html. 
Bridge, David. 2016c. "SELGEM: The Logical Structure." Smithsonian Institution Archives. Accessed October 29, 2017, http://si-siris.blogspot.co.nz/2016/07/selgem-logicalstructure.html.

Brown, Andre, and Nicholas Webb. 2010. "Examination of the Designs by Auguste Perret Using Digitally-Enabled Forensic Techniques." International Journal of Architectural Computing 8 (4):537-555. doi: 10.1260/1478-0771.8.4.537.

Brusaporci, Stefano. 2017. "The Importance of Being Honest: Issues of Transparency in Digital Visualization of Architectural Heritage." In Handbook of Research on Emerging Technologies for Architectural and Archaeological Heritage, edited by Alfonso Ippolito, 66-93. Hershey, Pa.: IGI Global.

Buchanan, Richard. 1992. "Wicked Problems in Design Thinking." Design Issues 8 (2):5-21. doi: 10.2307/1511637.

Burridge, Jane M., Brian M. Collins, B. Neil Galton, Alan R. Halbert, Thomas R. Heywood, William H. Latham, Robert W. Phippen, Peter Quarendon, Paul Reilly, M. William Ricketts, John Simmons, Stephen J. P. Todd, Andrew G. N. Walter, and John R. Woodwark. 1989. "The WINSOM Solid Modeller and its Application to Data Visualization." IBM Systems Journal 28 (4):548-568. doi: 10.1147/sj.284.0548.

Burton, Virginia. 1967. "Computers Confront the Curator." The Metropolitan Museum of Art Bulletin 26 (1):20-23, http://library.metmuseum.org:80/record=b1179343 S1.

Byrne, Sarah, Anne Clarke, Rodney Harrison, and Robin Torrence. 2011. "Networks, Agents and Objects: Frameworks for Unpacking Museum Collections." In Unpacking the Collection: Networks of Material and Social Agency in the Museum, edited by Sarah Byrne, Anne Clarke, Rodney Harrison and Robin Torrence, 3-26. New York, NY: Springer New York.

Calvo, Ignacio, Tomás Dorta, and Jean-Marc Robert. 2013. "An Empirical Study on the User's Context in Mobile Videoconferencing Devices." Proceedings of the 25th ACM Conference on l'Interaction Homme-Machine (IHM 2013), Talence, Bordeaux, France, November 12 15, 2013. 79-88. doi:10.1145/2534903.2534914.

Cameron, Fiona. 2010. "Beyond the Cult of the Replicant: Museums and Historical Digital ObjectsTraditional Concerns, New Discourses." In Theorizing Digital Cultural Heritage: A Critical Discourse, edited by Fiona Cameron and Sarah Kenderdine, 49-75. Cambridge, Mass.: The MIT Press.

Cameron, Fiona, and Sarah Kenderdine, eds. 2007. Theorizing Digital Cultural Heritage: A Critical Discourse. Cambridge, Mass.; London: The MIT Press.

Carbonell, Bettina Messias. 2004. Museum Studies: An Anthology of Contexts. Malden, MA: Blackwell Pub.

Castells, Manuel. 2000. The Rise of the Network Society. Oxford: Blackwell Publishers, Inc.

Champion, Erik. 2006. "Evaluating Cultural Learning in Virtual Environments." PhD diss., University of Melbourne.

Champion, Erik. 2011. Playing with the past. London; New York: Springer.

Champion, Erik. 2015. Critical Gaming: Interactive History and Virtual Heritage. Farnham, Surrey: Ashgate. 
Champion, Erik. 2016. "Cross-cultural Learning, Heritage, and Digital Games." In Re-orientation: Trans-cultural, Trans-lingual, Transmedia Studies in narrative, language, identity and knowledge, edited by John Hartley and Weiguo Qu, 218-233. Fudan: Fudan University Press.

Champion, Erik, Bharat Dave, and Ian Bishop. 2003. "Interaction, Agency and Artefacts." In M. L. Chiu, J. Y. Tsou, T. Kvan, M. Morozumi and S. S. Jeng, eds., Digital Design - Research and Practice: Proceedings of the 10th International Conference on Computer Aided Architectural Design Futures (CAAD Futures 2003), Tainan, Taiwan, October 13-15, 2003. 249-258.

Chandler, Daniel. 2002. Semiotics: The Basics. London; New York: Routledge.

Chandler, Daniel. 2017. "Semiotics for Beginners: Encoding/Decoding." Last modified May 7, 2017. Accessed January 16, 2018, http://visualmemory.co.uk/daniel/Documents/S4B/sem08c.html.

Checkland, Peter, and Jim Scholes. 1990. Soft Systems Methodology in Action. Chichester: Wiley.

Chen, Xiaolei, and Yehuda Kalay. 2008. "Making a Liveable 'Place': Content Design in Virtual Environments." International Journal of Heritage Studies 14 (3):229-246. doi: 10.1080/13527250801953710.

Chenhall, Robert G. 1978. Nomenclature for Museum Cataloging: A System for Classifying ManMade Objects. Nashville: American Association for State and Local History.

Chenhall, Robert G., and David Vance. 1988. Museum Collections and Today's Computers. New York: Greenwood Publishing Group Inc.

Chraïbi, Driss. 1984. Mother Comes of Age. Translated by Hugh Harter. Washington D.C.: Three Continents Press.

Cimadomo, Guido. 2013. "Documentation and Dissemination of Cultural Heritage: Current Solutions and Considerations about its Digital Implementation." Proceedings of the 2013 Digital Heritage International Congress (DigitalHeritage 2013), Volume 1, Marseille, France, October 28-November 1, 2013. 555-562. doi:10.1109/DigitalHeritage.2013.6743796.

Clarke, Arthur C. 1973. Profiles of the Future: An Inquiry into the Limits of the Possible. London: Pan Books. Original edition, London: Gollancz, 1962.

Claus, Jürgen. 1999. "Expansion of Media Art: What Will Remain of the Electronic Age?" In Ars Electronica: Facing the Future: A Survey of Two Decades, edited by Timothy Druckrey with Ars Electronica, 180-184. Cambridge, Mass.; London: The MIT Press.

Claypool, Lisa. 2005. "Zhang Jian and China's First Museum." The Journal of Asian Studies 64 (3):567-604, http://www.jstor.org/stable/25075826.

Clifford, James. 1997. Routes: Travel and Translation in the Late Twentieth Century. Cambridge, Mass.: Harvard University Press.

Cohen-Steiner, David, and Jean-Marie Morvan. 2006. "Differential Geometry on Discrete Surfaces." In Effective Computational Geometry for Curves and Surfaces, edited by Jean-Daniel Boissonnat and Monique Teillaud, 157-179. Berlin, Heidelberg: Springer Berlin Heidelberg.

Cole, Andrew. 2015. "Those Obscure Objects of Desire." Artforum, 318-323. 
Colley, Sarah. 2015. "Ethics and Digital Heritage." In The Ethics of Cultural Heritage, edited by Tracy Ireland and John Schofield, 13-32. New York, NY: Springer New York.

Combi, Mariella. 2016. "Cultures and Technology: An Analysis of Some of the Changes in Progress-Digital, Global and Local Culture." In Cultural Heritage in a Changing World, edited by Karol Jan Borowiecki, Neil Forbes and Antonella Fresa, 3-15. Cham: Springer International Publishing.

Cooper, Seth, Firas Khatib, Adrien Treuille, Janos Barbero, Jeehyung Lee, Michael Beenen, Andrew Leaver-Fay, David Baker, and Zoran Popovic. 2010. "Predicting Protein Structures with a Multiplayer Online Game." Nature 466:756-760. doi: 10.1038/nature09304.

Corsane, Gerard. 2005. Heritage, Museums and Galleries: An Introductory Reader. London; New York: Routledge.

Coulter, Gerry. 2007. "Jean Baudrillard and the Definitive Ambivalence of Gaming." Games and Culture 2 (4):358-365. doi: 10.1177/1555412007309530.

Coyne, Richard. 2011. Derrida for Architects. London; New York: Routledge.

Crane, Keenan, and Max Wardetzky. 2017. "A Glimpse into Discrete Differential Geometry." Notices of the American Mathematical Society 64 (10):1153-1159. doi: 10.1090/noti1578.

Cross, Nigel. 2006. Designerly Ways of Knowing. London: Springer-Verlag.

Crouch, David. 2015. "Affect, Heritage, Feeling." In The Palgrave Handbook of Contemporary Heritage Research, edited by Emma Waterton and Steve Watson, 177-190. London: Palgrave Macmillan UK.

Cruz-Neira, Carolina, Daniel J. Sandin, Thomas A. DeFanti, Robert V. Kenyon, and John C. Hart. 1992. "The CAVE: Audio Visual Experience Automatic Virtual Environment." Communications of the ACM 35 (6):64-72. doi: 10.1145/129888.129892.

DAM (Digital Art Museum). 2018. "Digital Art Museum: Artists." Accessed January 10, 2018, http://dam.org/artists.

Danermark, Berth, Mats Ekström, Liselotte Jakobsen, and Jan Ch. Karlsson. 2002. Explaining Society: Critical Realism in the Social Sciences. London; New York: Routledge.

Datta, Sambit, and David Beynon. 2014. Digital Archetypes: Adaptations of Early Temple Architecture in South and Southeast Asia. Farnham, Surrey; Burlington, Vt.: Ashgate Publishing Company.

Davis, Daniel. 2013. "Modelled on Software Engineering: Flexible Parametric Models in the Practice of Architecture." PhD diss., RMIT University.

De Landa, Manuel. 1997. A Thousand Years of Nonlinear History. Swerve ed. New York: Zone Books.

De Lusenet, Yola, and Vincent Wintermans, eds. 2007. Preserving the Digital Heritage: Principles and Policies, Selected Papers of the International Conference Organized by Netherlands National Commission for UNESCO, National Library of the Netherlands, The Hague, 4-5 November 2005. Amsterdam: Netherlands National Commission for UNESCO, European Commission on Preservation access, https://www.ica.org/sites/default/files/WG_2007_PAAG-preserving-the-digitalheritage_EN.pdf. 
Debevec, Paul E., Camillo J. Taylor, and Jitendra Malik. 1996. "Modeling and Rendering Architecture from Photographs: A Hybrid Geometry- and Image-Based Approach." Proceedings of the 23rd Annual Conference on Computer Graphics and Interactive Techniques (ACM SIGGRAPH 1996), New Orleans, L.A., USA, August 4-9, 1996. 11-20. doi:10.1145/237170.237191.

Dee, Hannah, Lorna Hughes, Gareth Lloyd Roderick, and Alexander David Brown. 2016. "Visual Digital Humanities: Using Image Data to Derive Approximate Metadata." In Managing Digital Cultural Objects, edited by Allen Foster and Pauline Rafferty, 89-110. London: Facet Publishing.

DeLeon, Victor, and Robert Berry. 2000. "Bringing VR to the Desktop: Are You Game?" IEEE MultiMedia 7 (2):68-72. doi: 10.1109/93.848433.

Deleuze, Gilles, and Felix Guattari. 1987. A Thousand Plateaus: Capitalism and Schizophrenia. Translated by Brian Massumi. Minneapolis; London: University of Minnesota Press. Original edition, Mille Plateaux, volume 2 of Capitalisme et Schizophrenic, 1980.

Deloche, Bernard. 2001. Le musée virtuel: Vers une éthique des nouvelles images. Paris: Presses universitaires de France.

Delve, Janet, and David Anderson, eds. 2014. Preserving Complex Digital Objects. London: Facet Publishing.

Denard, Hugh. 2012. "A New Introduction to the London Charter." In Paradata and Transparency in Virtual Heritage, edited by Anna Bentkowska-Kafel, Hugh Denard and Drew Baker, 5771. Farnham, Surrey; Burlington, Vt.: Ashgate.

Derrida, Jacques. 1976. Of Grammatology. Translated by Gayatri Chakravorty Spivak. Baltimore, Md.; London: Johns Hopkins University Press.

Desvallées, André, and François Mairesse. 2010. Key Concepts of Museology. Translated by Suzanne Nash. International Council of Museums: Armand Colin.

Deterding, Sebastian, Dan Dixon, Rilla Khaled, and Lennart Nacke. 2011. "From Game Design Elements to Gamefulness: Defining "Gamification"."Envisioning Future Media Environments: Proceedings of the 15th International Academic MindTrek Conference (MindTrek 2011), Tampere, Finland, September 28-30, 2011. 9-15. doi:10.1145/2181037.2181040.

Di Giuseppantonio Di Franco, Paola. 2014. "Talking about Things: A Cognitive Approach to Digital Heritage and Material Culture Studies in Archaeology." PhD diss., University of California, Merced.

Din, Herminia, Darrell L. Bailey, and Fang-Yin Lin. 2015. "Using New Media for Exhibit Interpretation: A Case Study, Yuan Ming Yuan Qing Emperors' Splendid Gardens." In Digital Heritage and Culture: Strategy and Implementation, edited by Herminia Din and Steven Wu, 131-148. Singapore: World Scientific Publishing.

Din, Herminia, and Phyllis Hecht, eds. 2007. The Digital Museum: A Think Guide. Washington, D.C.: American Association of Museums.

Dong, Wen, and Xiaolei Zhang. 2011. "Urumqi." Cities 28 (1):115-125. doi: 10.1016/j.cities.2010.10.002. 
Donovan, Andrea Elizabeth. 2008. William Morris and the Society for the Protection of Ancient Buildings. London: Routledge.

Doornbusch, Paul, and Sarah Kenderdine. 2004. "Presence and Sound; Identifying Sonic Means to "Be there"." In R. Ascott, ed. Qi and Complexity - Consciousness Reframed 2004: Proceedings of the 6th International Research Conference, 氣·複雜：意識的再現：國際學術論壇, Beijing, China, November 24-27, 2004. 67-70.

Dorffner, L., and Gerald Forkert. 1998. "Generation and Visualization of 3D Photo-Models Using Hybrid Block Adjustment with Assumptions on the Object Shape." ISPRS Journal of Photogrammetry and Remote Sensing 53 (6):369-378. doi: 10.1016/S0924-2716(98)000203.

Dorta, Tomás. 2001. "L'influence de la réalité virtuelle non-immersive comme outil de visualisation sur le processus de design." PhD diss., University of Montréal.

Dorta, Tomás. 2004. "Drafted Virtual Reality: A New Paradigm to Design with Computers." In H. S. Lee and J. W. Choi, eds., Proceedings of the 9th International Conference on Computer Aided Architectural Design Research in Asia (CAADRIA 2004), Seoul, South Korea, April 28-30, 2004. 829-844.

Dorta, Tomás. 2008. "Design Flow and Ideation." International Journal of Architectural Computing 6 (3):299-316. doi: 10.1260/1478-0771.6.3.299.

Dorta, Tomás, Gokce Kinayoglu, and Michael Hoffmann. 2016. "Hyve-3D and the 3D Cursor: Architectural Co-Design with Freedom in Virtual Reality." International Journal of Architectural Computing 14 (2):87-102. doi: 10.1177/1478077116638921.

Dorta, Tomás, and Edgar Perez. 2006. "Immersive Drafted Virtual Reality: A New Approach for Ideation within Virtual Reality." In G. A. Luhan, P. Anzalone, M. Cabrinha and C. Clarke, eds., Synthetic Landscapes: Proceedings of the 25th Annual Conference of the Association for Computer-Aided Design in Architecture (ACADIA 2006), Louisville, Ky., USA, October 12-15, 2006. 304-316.

Drachen, A. 2012. "Frequent Itemset and Association Rule Mining." Accessed February 15, http://www.gameanalytics.com/blog/frequent-itemset-and-association-rule-mining-orhow-to-know-if-shirts-follows-pants-or-the-other-way-around.html.

Drachen, Anders, and Alessandro Canossa. 2009. "Towards Gameplay Analysis via Gameplay Metrics."Everyday Life in the Ubiquitous Era: Proceedings of the 13th International MindTrek Conference, Tampere, Finland. 202-209. doi:10.1145/1621841.1621878.

Drachen, Anders, Christian Thurau, Julian Togelius, Georgios N. Yannakakis, and Christian Bauckhage. 2013. "Game Data Mining." In Game Analytics: Maximizing the Value of Player Data, edited by Magy Seif El-Nasr, Anders Drachen and Alessandro Canossa, 205-253. London: Springer London.

Dreier, Franz Adrian. 1985. "The Kunstkammer of the Hessian Landgraves in Kassel." In The Origins of Museums: The Cabinet of Curiocities in Sixteenth and Seventeenth Century Europe, edited by Oliver Impey and Arthur MacGregor, 102-109. Oxford: Clarendon Press.

Drioli, Alessandra. 2006. "Contemporary Aesthetic Forms and Scientific Museology." Journal of Science Communication 5 (1):1-10, https://jcom.sissa.it/archive/05/01/A050102. 
Druckrey with Ars Electronica, Timothy, ed. 1999. Ars Electronica: Facing the Future: A Survey of Two Decades, Electronic Culture: History, Theory, Practice. Cambridge, Mass.; London: The MIT Press.

Dzambazova, Tatjana. 2015. "Autodesk Memento." Keynote speech at Digital Heritage 2015 Congress, Granada, Spain, September 29, 2015.

E-Space (Europeana Space). 2014. "Europeana Space, Best Practice Network: Spaces of Possibility for the Creative Reuse of Digital Cultural Content." Accessed January 14, 2018, http://www.europeana-space.eu/.

Eco, Umberto. 1976. "Peirce's Notion of Interpretant." MLN 91 (6):1457-1472. doi: $10.2307 / 2907146$.

Ellin, Everett. 1968. "An International Survey of Museum Computer Activity." Computers and the Humanities 3 (2):65-86, http://www.jstor.org/stable/30199290.

Ellin, Everett. 1969. "Museums and the Computer: An Appraisal of New Potentials." Computers and $\begin{array}{lll}\text { the Humanities } & \text { Hers-30, }\end{array}$ https://www.jstor.org/stable/30199319?seq=1\#page_scan_tab_contents.

Ellin, Everett. 1971. "Considerations in the Formation of Museum Data Banks in the United States of America." Museum International 23 (1):18-21. doi: 10.1111/j.14680033.1971.tb01747.x.

Emerick, Keith, ed. 2014. Conserving and Managing Ancient Monuments: Heritage, Democracy, and Inclusion. Woodbridge, Suffolk, UK; Rochester, NY: The Boydell Press.

ESA (Entertainment Software Association). 2017. "Essential Facts about the Computer and Video Game Industry." [Annual research conducted by Ipsos Connect]. Accessed January 12, 2018, http://www.theesa.com/about-esa/industry-facts/.

Esposito, Joseph L. 2017. "Virtuality." In Digital Encyclopedia of Charles S. Peirce. Accessed December 11, 2017, http://www.digitalpeirce.fee.unicamp.br/home.htm.

Exeter, Open Research. 2009. "Section of the Rotunda, Leicester Square." [Bill Douglas collection BD085143]. Accessed January 20, 2018, http://hdl.handle.net/10472/2843.

Fairclough, Graham J., ed. 2008. The Heritage Reader. London; New York: Routledge.

FDL (La Fondation Daniel Langlois = Daniel Langlois Foundation). 2000. "Lynn Hershman: Room of One's Own." Accessed Janurary 10, 2018, http://www.fondationlanglois.org/html/e/page.php?NumPage=169.

Feng, Ling, and Eric Tsang. 1999. "Finding Cross-Object Relationships from Large Databases." Proceedings of the 1999 IEEE International Conference on Systems, Man, and Cybernetics (IEEE SMC '99), Volume 5, 1999. 876-881. doi:10.1109/ICSMC.1999.815669.

Findlen, Paula. 2000. "The Modern Muses: Renaissance Collecting and the Cull of Remembrance." In Museums and Memory, edited by Susan A. Crane, 161-178. Stanford, Calif.: Stanford University Press.

Fischer, Thomas. 2017a. "A Cybernetic Perspective on Determinability and Design Research." Kybernetes 46 (9):1588-1596. doi: doi:10.1108/K-10-2016-0269. 
Fischer, Thomas. 2017b. "From "Failure is Not an Option" to "Try Again. Fail Again. Fail Better"." Public lecture at Victoria University of Wellington, Wellington, New Zealand, May 17, 2017.

Florenzano, Michel, Marie-Françoise Courel, and Francesca De Domenico. 2010. "Digitally Conserving an Endangered Built Heritage in Kashgar, An Oasis City of the Taklimakan." In Water and Sustainability in Arid Regions: Bridging the Gap Between Physical and Social Sciences, edited by Graciela Schneier-Madanes and Marie-Francoise Courel, 165-179. Dordrecht: Springer Netherlands.

Flower ${ }^{\circledR}$. 2018. [Video game developed by "thatgamecompany"]. Sony Computer Entertainment. Accessed January 20, 2018, https://www.playstation.com/en-nz/games/flower-ps4/.

Foster, Allen, and Pauline Rafferty. 2016. Managing Digital Cultural Objects: Analysis, Discovery and Retrieval. London: Facet Publishing.

French, Howard W. 2006. "Where to Shoot an Epic About Afghanistan? China, Where Else?" The Newyork Times, issued December 31, 2006. Accessed January 14, 2018, http://www.nytimes.com/2006/12/31/movies/31 fren.html?pagewanted=all\&_r=0.

Friendly, Alfred. 1973. "Modern Science and Ancient Egypt." Expedition Magazine, 24-29.

Frischer, Bernard, Franco Niccolucci, Nick S. Ryan, and Juan Barceló. 2000. "From CVR to CVRO: The Past, Present, and Future of Cultural Virtual Reality." Virtual Archaeology between Scientific Research and Territorial Marketing. In F. Niccolucci, ed. Virtual Archaeology: Proceedings of the VAST EuroConference, Volume 1, Arezzo, Italy, November 24-25, 2000, https://kar.kent.ac.uk/21942/.

Fukuda, Tomohiro, Hideki Nada, Haruo Adachi, Shunta Shimizu, Chikako Takei, Yusuke Sato, Nobuyoshi Yabuki, and Ali Motamedi. 2017. "Integration of a Structure from Motion into Virtual and Augmented Reality for Architectural and Urban Simulation." In ComputerAided Architectural Design. Future Trajectories: 17th International Conference, CAAD Futures 2017, Istanbul, Turkey, July 12-14, 2017, Selected Papers, edited by Gülen Çağdaş, Mine Özkar, Leman Figen Gül and Ethem Gürer, 60-77. Singapore: Springer Singapore.

Galloway, Alexander. 2011. "Are Some Things Unrepresentable?" Theory, Culture \& Society 28 (78):85-102. doi: 10.1177/0263276411423038.

Galloway, Alexander R. 2006. Gaming: Essays on Algorithmic Culture. Minneapolis: University of Minnesota Press.

Garfinkel, Simson L. 2010. "Digital Forensics Research: The Next 10 years." Digital Investigation 7:S64-S73. doi: 10.1016/j.diin.2010.05.009.

Gero, JohnS, and Udo Kannengiesser. 2012. "Representational Affordances in Design, with Examples from Analogy Making and Optimization." Research in Engineering Design 23 (3):235-249. doi: 10.1007/s00163-012-0128-y.

Giaccardi, Elisa. 2012. Heritage and Social Media: Understanding Heritage in a Participatory Culture. London: Routledge.

Gibson, William. 1994. Neuromancer. London: HarperCollins. Original edition, Gollancz, 1984.

Gilad, Uri. 2009. "Kashgar: GIS and Spatial Modeling of Cultural Heritage Sites at the Heart of the Silk Road." PhD diss., Monash University. 
Gladney, Dru C. 1994. "Representing Nationality in China: Refiguring Majority/Minority Identities." The Journal of Asian Studies 53 (1):92-123. doi: 10.2307/2059528.

Google Cultural Institute. 2015. Google Arts \& Culture. Accessed January 10, 2018, https://www.google.com/culturalinstitute/beta/.

Gottlieb, Halina. 2011. "Designing Engagement for Art, Exploring Interfaces and Interpretative Content of Digital Heritage Artifacts in Museum Environments." PhD diss., University College Dublin.

Gottlieb, Halina. 2015. "Designing Digital Heritage Competence Centers: A Swedish Model." In Digital Heritage and Culture: Strategy and Implementation, edited by Herminia Din and Steven Wu, 21-39. Singapore: World Scientific Publishing.

Guinness. 2013. "DreamHack Winter 2013." [Largest LAN party, 22,810 people]. Guinness World Records. Accessed August 28, 2017, http://www.guinnessworldrecords.com/worldrecords/largest-lan-party.

Haber, Alicia. 2000. "MUVA: A Virtual Museum in Uruguay." Museum International 52 (1):26-31. doi: 10.1111/1468-0033.00241.

Hall, Colin Michael, and Simon McArthur. 1998. Integrated Heritage Management: Principles and Practice. London: Stationery Office.

Hall, Stuart. 1999. "Un-settling 'The Heritage', Re-imagining the Post-Nation: Whose Heritage?" Third Text 13 (49):3-13. doi: 10.1080/09528829908576818.

Han, Jiawei, and Micheline Kamber. 2001. Data Mining: Concepts and Techniques. San Francisco; London: Morgan Kaufmann Publishers.

Haraway, Donna. 1987. "A Manifesto for Cyborgs: Science, Technology, and Socialist Feminism in the 1980s." Australian Feminist Studies 2 (4):1-42. doi: 10.1080/08164649.1987.9961538.

Harrison, Rodney. 2013. Heritage: Critical Approaches. Milton Park, Abingdon; New York: Routledge.

Hartwig, Mervyn, ed. 2007. Dictionary of Critical Realism, Critical Realism--Interventions. London: Routledge.

Hartwig, Mervyn. 2009. "'Orthodox’ Critical Realism and the Critical Realist Embrace." Journal of Critical Realism 8 (2):233-257. doi: 10.1558/jocr.v8i2.233.

Haslop, Blaire. 2017. "Digital Decay: Glitch Architecture." MArch thesis, Victoria University of Wellington. Accessed January 12, 2018, https://issuu.com/blairehaslop/docs/blairehaslop_digitaldecaythesis_red.

Haslop, Blaire, Marc Aurel Schnabel, and Serdar Aydin. 2017a. "Digital Decay." International Journal of Parallel, Emergent and Distributed Systems:1-7. doi: 10.1080/17445760.2017.1390092.

Haslop, Blaire, Marc Aurel Schnabel, and Serdar Aydin. 2017b. "Glitch Space: Experiments on Digital Decay to Remap the Anatomy of Glitch in 3D." In P. Janssen, P. Loh, A. Raonic and M. A. Schnabel, eds., Protocols, Flows, and Glitches: Proceedings of the 22nd International Conference on Computer-Aided Architectural Design Research in Asia (CAADRIA 2017), Suzhou, China, April 5-8, 2017. 591-600. 
284

Hayes, Anna. 2015. "Space, Place and Ethnic Identity in the Xinjiang Regional Museum." In Inside Xinjiang: Space, Place and Power in China's Muslim Far Northwest, edited by Anna Hayes and Michael E. Clarke, 52-72. New York, N.Y.: Routledge.

Hayles, N. Katherine. 1984. The Cosmic Web: Scientific Field Models and Literary Strategies in the Twentieth Century. Ithaca, N.Y.; London: Cornell University Press.

Hayles, N. Katherine. 1990. Chaos Bound: Orderly Disorder in Contemporary Literature and Science. Ithaca, N.Y.: Cornell University Press.

Hayles, N. Katherine. 2012. How We Think: Digital Media and Contemporary Technogenesis. Chicago, Ill.: The University of Chicago Press.

Heidegger, Martin. 1967. What is a Thing? Translated by W. B. Barton Jr. and Vera Deutsch with an analysis by Eugene T. Gendlin. South Bend, Ind.: Regnery/Gateway.

Heidegger, Martin. 1977. The Question Concerning Technology, and Other Essays. Translated by William Lovitt. New York; London: Harper and Row.

Heilig, Morton L. 1960. Stereoscopic-Television Apparatus for Individual Use. edited by US Patent: Heilig, Morton L.

Heilig, Morton L. 1962. Sensorama Simulator. edited by US Patent.

Hildebrand, Michiel, Maarten Brinkerink, Riste Gligorov, Martijn Van Steenbergen, Johan Huijkman, and Johan Oomen. 2013. "Waisda?: Video Labeling Game." Proceedings of the 21st ACM international conference on Multimedia (ACM MM 2013), Barcelona, Spain, October 21-25, 2013. 823-826. doi:10.1145/2502081.2502221.

Holdstock, Nick. 2015. China's Forgotten People: Xinjiang, Terror and the Chinese State. London: I.B.Tauris. Accessed January 14, 2018, ProQuest Ebook Central.

Holloway, Julian. 2006. "Enchanted Spaces: The Séance, Affect, and Geographies of Religion." Annals of the Association of American Geographers 96 (1):182-187. doi: 10.1111/j.14678306.2006.00507.x.

Hooper-Greenhill, Eilean. 1992. Museums and the Shaping of Knowledge. London; New York: Routledge.

Hooper-Greenhill, Eilean. 1995. Museum, Media, Message. London; New York: Routledge.

Hooper-Greenhill, Eilean. 2000. "Changing Values in the Art Museum: Rethinking Communication and Learning." International Journal of Heritage Studies 6 (1):9-31. doi: $10.1080 / 135272500363715$.

Howard, Peter. 2003. Heritage: Management, Interpretation, Identity. London; New York: Continuum.

Howe, Jeff. 2006. "The Rise of Crowdsourcing." Wired, issued June 1, 2006. Accessed December 13, 2017, https://www.wired.com/2006/06/crowds/.

Howell, Ray, and Matt Chilcott. 2013. "A Sense of Place: Re-purposing and Impacting Heritage Resource Evidence through Digital Heritage and Interpretative Practice." International Journal of Intangible Heritage 8:165-177, http://www.ijih.org/volumeMgr.ijih?cmd=volumeView\&volNo=8\#none. 
Huber, William. 2010. "Kingdom Hearts Game Play Visualizations." [with Lev Manovich]. Accessed January 14, 2018, http://lab.softwarestudies.com/2012/02/kingdom-hearts-game-playvisualizations.html.

Hughes, Joe. 2009. Deleuze's Difference and Repetition: A Reader's Guide. London: Continuum.

Huhtamo, Erkki. 2002. "On the Origins of the Virtual Museum." Paper presented at Nobel Symposium (NS 120): Virtual Museums and Public Understanding of Science and Culture, Stockholm, Sweden, May 26-29, 2002. Accessed January 10, 2018, https://www.nobelprize.org/nobel_organizations/nobelfoundation/symposia/interdisciplina ry/ns120/about.html.

Hunicke, Robin, Marc LeBlanc, and Robert Zubek. 2004. "MDA: A Formal Approach to Game Design and Research." Proceedings of the AAAI Challenges in Game AI Workshop, http://www.aaai.org/Library/Workshops/2004/ws04-04-001.php.

Huvila, Isto. 2006. "The Ecology of Information Work: A Case Study of Bridging Archaeological Work and Virtual Reality Based Knowledge Organization." PhD diss., Åbo Akademi University.

ICC. 1991. "The Museum Inside The Telephone Network." InterCommunication Center. Accessed January 10, 2018, http://www.ntticc.or.jp/en/exhibitions/1991/intercommunication-91-themuseum-inside-the-telephone-network/.

Impey, Oliver, and Arthur MacGregor, eds. 1985. The Origins of Museums: The Cabinet of Curiocities in Sixteenth and Seventeenth Century Europe. Oxford: Clarendon Press.

Ingwersen, Peter. 1992. "Information and Information Science in Context." Libri - International Journal of Libraries and Information Studies 42 (2):99-135. doi: 10.1515/libr.1992.42.2.99.

Ioannides, Marinos. 1993. "3D Object Reconstruction Using 4D-Laser Digitalizing." In J. Rix and E. G. Schlechtendahl, eds., Proceedings of the IFIP TC5/WG5.10 Working Conference on Interfaces in Industrial Systems for Production Engineering, Darmstadt, Germany, March 15-17, 1993. 257-266.

Ioannides, Marinos, Nadia Magnenat-Thalmann, and George Papagiannakis. 2017. Mixed Reality and Gamification for Cultural Heritage. Cham: Springer.

Ioannides, Marinos, and Ewald Quak, eds. 2014. 3D Research Challenges in Cultural Heritage: A Roadmap in Digital Heritage Preservation, Lecture Notes in Computer Science 8355. Heidelberg; New York: Springer. doi: 10.1007/978-3-662-44630-0.

Ippolito, Alfonso, ed. 2017. Handbook of Research on Emerging Technologies for Architectural and Archaeological Heritage. Hershey, Pa.: IGI Global. doi: 10.4018/978-1-5225-0675-1.

Isaksen, Robert K. 2016. "Reclaiming Rational Theory Choice as Central: A Critique of Methodological Applications of Critical Realism." Journal of Critical Realism 15 (3):245262. doi: 10.1080/14767430.2016.1169369.

Johnston, John. 2008. The Allure of Machinic Life: Cybernetics, Artificial Life, and the New AI. Cambridge, Mass.: The MIT Press.

Jones-Garmil, Katherine. 1997. "Laying the Foundation: Three Decades of Computer Technology in the Museum." In The Wired Museum: Emerging Technology and Changing Paradigms, edited by Katherine Jones-Garmil, 35-62. Washington, D.C.: American Association of Museums. 
Jones, Katherine Burton. 2008. "The Transformation of the Digital Museum." In Museum Informatics: People, Information, and Technology in Museums, edited by Paul F. Marty and Katherine Burton Jones, 9-25. New York; Oxon: Routledge.

Kalay, Yehuda E., Thomas Kvan, and Janice Affleck. 2008. New Heritage: New Media and Cultural Heritage. London; New York: Routledge.

Kawrykow, Alexander, Gary Roumanis, Alfred Kam, Daniel Kwak, Clarence Leung, Chu Wu, Eleyine Zarour, Luis Sarmenta, Mathieu Blanchette, and Jérôme Waldispühl. 2012. "Phylo: A Citizen Science Approach for Improving Multiple Sequence Alignment." PLoS ONE 7 (3):1-9. doi: 10.1371/journal.pone.0031362.

Kelly, Caleb. 2009. Cracked Media: The Sound of Malfunction. Cambridge, Mass.: The MIT Press.

Kenderdine, Sarah. 1998. "Sailing on the Silicon Sea: The Design of a Virtual Maritime Museum." Archives and Museum Informatics 12 (1):17-38. doi: 10.1023/a:1009099116304.

Kenderdine, Sarah. 2007. "Speaking in Rama: Panoramic Vision in Cultural Heritage Visualization." In Theorizing Digital Cultural Heritage: A Critical Discourse, edited by Fiona Cameron and Sarah Kenderdine, 301-331. Cambridge, Mass.; London: The MIT Press.

Kenderdine, Sarah. 2013. "“Pure Land": Inhabiting the Mogao Caves at Dunhuang." The Museum Journal 56 (2):199-218. doi: 10.1111/cura.12020.

Kenderdine, Sarah. 2015. "Embodiment, Entanglement, and Immersion in Digital Cultural Heritage." In A New Companion to Digital Humanities, edited by Susan Schreibman, Ray Siemens and John Unsworth, 22-41. Chichester: John Wiley \& Sons, Ltd.

Kenderdine, Sarah, and Tim Hart. 2003. "This is Not a Peep Show!: The Virtual Room at the Melbourne Museum." the International Cultural Heritage Informatics Meeting (ICHIM 2003), Paris, France, September 8-12, 2003. Accessed January 14, 2018, http://www.archimuse.com/publishing/ichim03/003C.pdf.

Kenderdine, Sarah, and Jeffrey Shaw. 2012. "Making UNMAKEABLELOVE: The Relocation of Theatre." In Bastard or Playmate?: Adapting Theatre, Mutating Media and Contemporary Performing Arts, edited by Robrecht Vanderbeeken, Christel Stalpaert, David Depestel and Boris Debackere, 102-120. Amsterdam University Press.

Kenderdine, Sarah, and Jeffrey Shaw. 2015. "A Cultural Heritage Panorama: Trajectories in Embodied Museography." In Digital Heritage and Culture: Strategy and Implementation, edited by Herminia Din and Steven Wu, 197-218. Singapore: World Scientific Publishing.

King, Laura, James F. Stark, and Paul Cooke. 2016. "Experiencing the Digital World: The Cultural Value of Digital Engagement with Heritage." Heritage \& Society 9 (1):76-101. doi: $10.1080 / 2159032 X .2016 .1246156$.

Kirshenblatt-Gimblett, Barbara. 1998. Destination Culture: Tourism, Museums, and Heritage. Berkeley: University of California Press.

Kitchin, Rob. 1998. Cyberspace: The World in the Wires. Chichester: Wiley.

Kittler, Friedrich. 2010. Optical Media: Berlin lectures 1999. Translated by Anthony Enns. Cambridge: Polity. Original edition, in German as Optische Medien / Berliner Vorlesung 1999, Merve Verlag Berlin, 2002. 
Kockelman, Paul. 2013. "Information is the Enclosure of Meaning: Cybernetics, Semiotics, and Alternative Theories of Information." Language \& Communication 33 (2):115-127. doi: 10.1016/j.langcom.2013.01.002.

Krapp, Peter. 2011. Noise Channels: Glitch and Error in Digital Culture. Minneapolis: University of Minnesota Press.

Krathwohl, David R. 2002. "A Revision of Bloom's Taxonomy: An Overview." Theory Into Practice 41 (4):212-218. doi: 10.1207/s15430421tip4104_2.

Krueger, Myron W. 1977. "Responsive Environments." In R. R. Korfhage, ed. Proceedings of the 1977 National Computer Conference (AFIPS 1977), Dallas, Texas, USA, June 13-16, 1977. 423-433. doi:10.1145/1499402.1499476.

Krueger, Myron W. 1991. Artificial Reality II. Reading, Mass.; London: Addison-Wesley. Original edition, Artificial Reality, Reading: Addison-Wesley, 1983.

Kwastek, Katja. 2013. Aesthetics of Interaction in Digital Art. Cambridge, Mass.: The MIT Press.

Landow, George P. 1992. Hypertext: The Convergence of Contemporary Critical Theory and Technology. Baltimore: Johns Hopkins University Press.

Lang, Caroline, John Reeve, and Vicky Woollard, eds. 2006. The Responsive Museum: Working with Audiences in the Twenty First Century. Aldershot; Burlington, Vt.: Ashgate.

Latour, Bruno, and Peter Weibel. 2005. Making Things Public: Atmospheres of Democracy. Cambridge, Mass.; Karlsruhe: MIT Press; ZKM/Center for Art and Media in Karlsruhe.

Laurel, Brenda. 1991. Computers as Theatre. Reading, Mass.; Wokingham: Addison-Wesley.

Law, John. 2004. After Method: Mess in Social Science Research. London: Routledge.

Law, John. 2009. "Actor-Network Theory and Material Semiotics." In The New Blackwell Companion to Social Theory, edited by Bryan S. Turner, 141-158. Malden, Mass.: WileyBlackwell.

Leask, Anna, and Ian Yeoman. 1999. Heritage Visitor Attractions: An Operations Management Perspective. London: Cassell.

Lebaddy, Hasna. 2009. Feminist Traditions in Andalusi-Moroccan Oral Narratives. New York: Palgrave Macmillan.

Leed, Eric J. 1991. The Mind of the Traveler: From Gilgamesh to Global Tourism. New York, N.Y.: Basic Books.

Lévy, Pierre. 1997. Collective Intelligence: Mankind's Emerging World in Cyberspace. Translated by Robert Bononno. Cambridge, Mass.: Perseus Books.

Lévy, Pierre. 1998. Becoming Virtual: Reality in the Digital Age. Translated by Robert Bononno. New York: Plenum Trade.

Lévy, Pierre. 2010. "Building a Universal Digital Memory." In Museums in a Digital Age, edited by Ross Parry, 107-115. London: Routledge.

Li, Jia, Lei Yao, Ella Hendriks, and James Z. Wang. 2012. "Rhythmic Brushstrokes Distinguish Van Gogh from His Contemporaries: Findings via Automated Brushstroke Extraction." IEEE 
Transactions on Pattern Analysis and Machine Intelligence 34 (6):1159-1176. doi: 10.1109/TPAMI.2011.203.

Lievendag, Nick. 2017. "The Beginners Guide to 3D Scanning \& Photogrammetry on a Budget." Accessed January 14, 2018, https://3dscanexpert.com/beginners-guide-3d-scanningphotogrammetry.

Liu, Yang, Helmut Pottmann, Johannes Wallner, Yong-Liang Yang, and Wenping Wang. 2006. "Geometric Modeling with Conical Meshes and Developable Surfaces." Transactions on Graphics 25 (3):681-689. doi: 10.1145/1141911.1141941.

Lo, Tian Tian, and Marc Aurel Schnabel. 2018. "Virtual \& Augmented Studio Environment (VASE): Developing the Virtual Reality Eco-System for Design Studios."Learning, Adapting and Prototyping: Proceedings of the 23rd International Conference on Computer-Aided Architectural Design Research in Asia (CAADRIA 2018), Beijing, China, May 17-19, 2018. 443-452.

Loukaki, Argyro. 2008. Living Ruins, Value Conflicts. Aldershot: Ashgate.

Lourie, Janice. 1968. "An Example of Computer-Graphics Tools for Execution Aesthetic Decisions." Computers and Their Potential Applications in Museums: A Conference Sponsored by The Metropolitan Museum of Art Supported by a Grant from the IBM Corporation, New York, USA, April 15-17, 1968. 221-235.

Lowenthal, D. 1999. "Authenticity: Rock of Faith or Quicksand Quagmire?" Getty Newsletter 14 (3), http://www.getty.edu/conservation/publications_resources/newsletters/14_3/feature1_2.ht $\mathrm{ml}$.

Lu, Dongming, and Yunhe Pan. 2010. Digital Preservation for Heritages: Technologies and Applications. Hangzhou; Dordrecht; London: Zhejiang University Press; Springer.

Lu, Tracey Lie Dan. 2015. Museums in China: Power, Politics and Identities. Oxon; New York: Routledge.

MacCannell, Dean. 1992. Empty Meeting Grounds: The Tourist Papers. London; New York: Routledge.

Macleod, Suzanne. 2005. Reshaping Museum Space: Architecture, Design, Exhibitions. London; New York: Routledge.

Malraux, André. 1953. The Voices of Silence. Translated by Stuart Gilbert. New York: Doubleday \& Company.

Mania, Katerina, and Alan Chalmers. 2001. "The Effects of Levels of Immersion on Memory and Presence in Virtual Environments: A Reality Centered Approach." CyberPsychology \& Behavior 4 (2):247-264. doi: 10.1089/109493101300117938.

Manovich, Lev. 1999. "Database as Symbolic Form." Convergence 5 (2):80-99. doi: $10.1177 / 135485659900500206$.

Manovich, Lev. 2001. The Language of New Media. Cambridge, Mass.: MIT Press.

Manovich, Lev. 2005. "The Shape of Information." [Summary of the main themes of Info-Aesthetics, London: Bloomsbury, 2005]. Accessed January 20, 2018, http://manovich.net/index.php/projects/the-shape-of-information. 
Manovich, Lev. 2018. "Exhibitions." Accessed January 14, 2018, http://manovich.net/index.php/exhibitions.

Manovich, Lev, Alise Tifentale, Mehrdad Yazdani, and Jay Chow. 2014. "The Exceptional and the Everyday: 144 Hours in Kiev." In J. Lin, ed. Proceedings of the 2014 IEEE International Conference on Big Data (IEEE Big Data 2014), Washington, D.C., USA, October 27-30, 2014. 72-79. doi:10.1109/BigData.2014.7004456.

Marchand, Suzanne. 2000. "The Quarrel of the Ancients and the Moderns in the German Museums." In Museums and Memory, edited by Susan A. Crane, 179-199. Stanford, Calif.: Stanford University Press.

Marty, Paul F. 2008. "Information Professional in Museums." In Museum Informatics: People, Information, and Technology in Museums, edited by Paul F. Marty and Katherine Burton Jones, 269-274. New York; Oxon: Routledge.

Mason, Marco. 2016. "The MIT Museum Glassware Prototype: Visitor Experience Exploration for Designing Smart Glasses." Journal on Computing and Cultural Heritage (JOCCH) 9 (3):128. doi: $10.1145 / 2872278$.

Mathews, Stanley. 2006. "The Fun Palace as Virtual Architecture: Cedric Price and the Practices of Indeterminacy." Journal of Architectural Education (1984-) 59 (3):39-48. doi: $10.2307 / 40480644$.

Matz, Jesse E., and David Herman. 2014. "Introduction." In Storyworlds across Media, edited by Marie-Laure Ryan, Jan-No Thon, Jesse E. Matz and David Herman, 1-22. Lincoln, Nebraska; London: University of Nebraska Press.

McCall, Vikki, and Clive Gray. 2014. "Museums and the 'New Museology': Theory, Practice and Organisational Change." Museum Management and Curatorship 29 (1):19-35. doi: 10.1080/09647775.2013.869852.

McCallum, Shaun, and Aleksandra Belitskaja. 2016. "404: Not Found." [1st prize winners]. London Internet Museum: International Architecture Competition. Accessed January 12, 2018, https://londoninternetmuseum.beebreeders.com/.

McCarty, Willard. 2014. Humanities Computing. Basingstoke: Palgrave Macmillan.

McKay, Alison. 2007. "Affective Communication: Towards the Personalisation of a Museum Exhibition." CoDesign 3 (sup1):163-173. doi: 10.1080/15710880701333183.

McKie, Robin, and Vanessa Thorpe. 2002. "Digital Domesday Book Lasts 15 Years Not 1000." The Guardian, issued March 3, 2002. Accessed January 12, 2018, https://www.theguardian.com/uk/2002/mar/03/research.elearning.

McLuhan, Marshall. 1964. Understanding New Media: The Extensions of Man. New York: McGrawHill Book Company.

Menzhausen, Joachim. 1985. "Elector Augustus's Kunstkammer: An Analysis of the Inventory of 1587." In The Origins of Museums: The Cabinet of Curiocities in Sixteenth and Seventeenth Century Europe, edited by Oliver Impey and Arthur MacGregor, 69-75. Oxford: Clarendon Press.

Merrell, Floyd. 1997. Peirce, Signs, and Meaning. Toronto; Buffalo: University of Toronto Press. 
Message, Kylie. 2009a. "Museums in the Twenty-First Century: Still Looking for Signs of Difference." Konsthistorisk Tidskrift/Journal of Art History 78 (4):204-221. doi: 10.1080/00233600903461388.

Message, Kylie. 2009b. "New Directions for Civil Renewal in Britain: Social Capital and Culture for All?" International Journal of Cultural Studies 12 (3):257-278. doi: $10.1177 / 1367877908101571$.

Michel, Jean-Baptiste, Yuan Kui Shen, Aviva Presser Aiden, Adrian Veres, Matthew K. Gray, Joseph P. Pickett, Dale Hoiberg, Dan Clancy, Peter Norvig, Jon Orwant, Steven Pinker, Martin A. Nowak, and Erez Lieberman Aiden. 2011. "Quantitative Analysis of Culture Using Millions of Digitized Books." Science 331 (6014):176-182. doi: 10.1126/science.1199644.

Michell, George, Marika Vicziany, Tsui Yen Hu, and John Gollings. 2008. Kashgar: Oasis City on China's Old Silk Road. 1st Frances Lincoln ed. London: Frances Lincoln.

Miele, Chris. 2005. From William Morris: Building Conservation and the Arts and Crafts Cult of Authenticity, 1877-1939. New Haven; London: Yale University Press for the Paul Mellon Centre for Studies in British Art; the Yale Center for British Art.

Milgram, Paul, Haruo Takemura, Akira Utsumi, and Fumio Kishino. 1995. "Augmented Reality: A Class of Displays on the Reality-Virtuality Continuum." In H. Das, ed. Proceedings of the SPIE 2351, Telemanipulator and Telepresence Technologies, December 21, 1995. 282-292. doi:10.1117/12.197321.

Miller, Gavin. 1992. "The Virtual Museum: Interactive Exploration of a Virtual Museum." [A work of Apple Computer Inc.]. Presented at SIGGRAPH 92 Final Program. Accessed January 12, 2018, http://www.siggraph.org/ fujii/etech/1992_43.html.

Miller, Vincent. 2011. Understanding Digital Culture. London: SAGE.

Millward, James A. 1998. Beyond the Pass: Economy, Ethnicity, and Empire in Qing Central Asia, 1759-1864. Stanford, Calif.: Stanford University Press.

Millward, James A. 2007. Eurasian Crossroads: A History of Xinjiang. New York: Columbia University Press.

Mingers, John. 2003. "A Classification of the Philosophical Assumptions of Management Science Methods." Journal of the Operational Research Society 54 (6):559-570. doi: 10.1057/palgrave.jors.2601436.

Mingers, John. 2006. Realising Systems Thinking: Knowledge and Action in Management Science. New York: Springer.

Mingers, John. 2014. Systems Thinking, Critical Realism and Philosophy: A Confluence of Ideas. London: Routledge.

Mingers, John, and Craig Standing. 2017. "Why Things Happen - Developing the Critical Realist View of Causal Mechanisms." Information and Organization 27 (3):171-189. doi: https://doi.org/10.1016/j.infoandorg.2017.07.001.

Misunas, Marla, and Richard Urban. 2007. "A Brief History of the Museum Computer Network." In Encyclopedia of Library and Information Sciences. Accessed January 10, 2018, http://mcn.edu/about/history/. 
Mitchell, William J. 2005. Placing Words: Symbols, Space, and the City. Cambridge, Mass.: The MIT Press.

Morgan, Peter. 1988. "Ruskin's Queen of the Air." In Poetics of the Elements in the Human Condition: Part 2 The Airy Elements in Poetic Imagination: Breath, Breeze, Wind, Tempest, Thunder, Snow, Flame, Fire, Volcano ... edited by Anna-Teresa Tymieniecka, 301-307. Dordrecht: Springer Netherlands.

Morris, William. [1877] 2018. The SPAB Manifesto. edited by The Society for the Protection of Ancient Buildings (SPAB). London.

Moyano, Steven. 1990. "Quality vs. History: Schinkel's Altes Museum and Prussian Arts Policy." The Art Bulletin 72 (4):585-608. doi: 10.2307/3045763.

Mudbox. 2017. "Troubleshoot Mesh Errors." [Help documentation]. Autodesk. Accessed January 16, 2018 , http://docs.autodesk.com/MBXPRO/2017/ENU/\#!/url=./files/Troubleshoot_mesh_errors.ht $\mathrm{m}$.

Mujoo-Munshi, Usha, and Bidyut Baran Chaudhuri. 2014. Multimedia Information Extraction and Digital Heritage Preservation. Singapore: World Scientific Publishing. Accessed January 12, 2018, ProQuest Ebook Central.

Mukhopadhyay, Jayanta, Partha Pratim Das, Samiran Chattopadhyay, Partha Bhowmick, and Biswa Nath Chatterji. 2013. Digital Geometry in Image Processing. Boca Raton, Fl.: Chapman and Hall/CRC.

Münster, Sander. 2017. "A Survey on Topics, Researchers and Cultures in the Field of Digital Heritage." ISPRS Annals of Photogrammetry, Remote Sensing and Spatial Information Sciences IV-2/W2:157-162. doi: 10.5194/isprs-annals-IV-2-W2-157-2017.

Münster, Sander, and Marinos Ioannides. 2015. "A Scientific Community of Digital Heritage in Time and Space." Proceedings of the 2015 Digital Heritage International Congress (DigitalHeritage 2015), Volume 2, Granada, Spain, September 28-October 2, 2015. 267274. doi:10.1109/DigitalHeritage.2015.7419507.

Münster, Sander, Mieke Pfarr-Harfst, Piotr Kuroczyński, and Marinos Ioannides. 2016. 3D Research Challenges in Cultural Heritage II: How to Manage Data and Knowledge Related to Interpretative Digital 3D Reconstructions of Cultural Heritage. Cham: Springer.

MuPop (The Pop-Up Museum). 2017a. "Pop-Up Exhibition in Hilversum." [Interactive installation at The Netherlands Institute for Sound and Vision (NISV)]. Accessed January 14, 2018, https://www.mupop.net/index.php/portfolio/pop-up-exhibition-in-hilversum/.

MuPop (The Pop-Up Museum). 2017b. "Pop-Up Exhibitions: Digital Meets Physical." [interactive virtual exhibitions]. Accessed January 14, 2018, https://www.mupop.net/.

Murray, Janet Horowitz. 1998. Hamlet on the Holodeck: The Future of Narrative in Cyberspace. Cambridge, Mass.: The MIT Press.

Naimark, Michael. 2006. "Aspen the Verb: Musings on Heritage and Virtuality." Presence: Teleoperators and Virtual Environments 15 (3):330-335. doi: 10.1162/pres.15.3.330.

Navarrete, Trilce, and John Mackenzie Owen. 2016. "The Museum as Information Space: Metadata and Documentation." In Cultural Heritage in a Changing World, edited by Karol Jan 
Borowiecki, Neil Forbes and Antonella Fresa, 111-123. Cham: Springer International Publishing.

Negroponte, Nicholas. 1996. Being Digital. 1st ed. New York: Knopf.

Newby, Laura. 2005. The Empire and the Khanate: A Political History of Qing Relations with Khoqand c. 1760-1860. Leiden; Boston: Brill.

Nikolov, Ivan, and Claus Madsen. 2016. "Benchmarking Close-Range Structure from Motion 3D Reconstruction Software under Varying Capturing Conditions." In Digital Heritage. Progress in Cultural Heritage: Documentation, Preservation, and Protection: 6th International Conference, EuroMed 2016, Nicosia, Cyprus, October 31 - November 5, 2016, Proceedings, Part I, edited by Marinos Ioannides, Eleanor Fink, Antonia Moropoulou, Monika Hagedorn-Saupe, Antonella Fresa, Gunnar Liestøl, Vlatka Rajcic and Pierre Grussenmeyer, 15-26. Cham: Springer International Publishing.

Niyazi, Abiguli. 2016. "空间的韵味一新疆维吾尔自治区喀什老城 整体风貌研究 [Flavour of Space: Research on the Architectural Style of the Kashgar Old-Town in Xinjiang-Uyghur Autonomous Region]." (title translated by Author), PhD diss., China Central Academy of Fine Arts.

Nordin, Astrid H. M. 2012. "Taking Baudrillard to the Fair: Exhibiting China in the World at the Shanghai Expo." Alternatives: Global, Local, Political 37 (2):106-120. doi: $10.1177 / 0304375412444816$.

Nunes, Mark. 2011. Error: Glitch, Noise, and Jam in New Media Cultures. London: Continuum.

Olmi, Giuseppe. 1985. "Italian Cabinets of the Sixteenth and Seventeenth Centuries." In The Origins of Museums: The Cabinet of Curiocities in Sixteenth and Seventeenth Century Europe, edited by Oliver Impey and Arthur MacGregor, 5-16. Oxford: Clarendon Press.

Orna, Elizabeth, and Charles Pettitt. 2010. "What is Information in the Museum Context?" In Museums in a Digital Age, edited by Ross Parry, 28-38. London: Routledge.

Osborne, Robin. 2004. Studies in Ancient Greek and Roman Society. Cambridge, UK; New York, NY, USA: Cambridge University Press.

Otto, Peter. 2011. Multiplying Worlds: Romanticism, Modernity, and the Emergence of Virtual Reality. Oxford; New York: Oxford University Press.

Pamuk, Orhan. 2010. The Museum of Innocence. Translated by Maureen Freely. Main Edition ed. London: Faber \& Faber.

Pamuk, Orhan. 2015. "A Modest Manifesto for Museums." Accessed January 14, 2018, http://en.masumiyetmuzesi.org/page/a-modest-manifesto-for-museums.

Pangaro, Paul. 2017. "Designing Our World: Cybernetics as Conversation for Action." [Lecture at the Institute of Design and Communication, FH Joanneum]. Accessed January 20, 2018, http://www.pangaro.com/graz17/index.html.

Papaioannou, Georgios, Tobias Schreck, Anthousis Andreadis, Pavlos Mavridis, Robert Gregor, Ivan Sipiran, and Konstantinos Vardis. 2017. "From Reassembly to Object Completion: A Complete Systems Pipeline." Journal on Computing and Cultural Heritage 10 (2):1-22. doi: 10.1145/3009905. 
Parry, Ross. 2007. Recoding the Museum: Digital Heritage and the Technologies of Change. London: Routledge.

Parry, Ross, and Nadia Arbach. 2007. "Localised, Personalised, and Constructivist: A Space for Online Museum Learning." In Theorizing Digital Cultural Heritage: A Critical Discourse, edited by Fiona Cameron and Sarah Kenderdine, 281-298. Cambridge, Mass.; London: The MIT Press.

Parry, Ross, Ruth Page, and Alex Moseley, eds. forthcoming. Museum Thresholds: The Design and Media of Arrival, Routledge Research in Museum Studies: Routledge.

Pavlidis, George, Anestis Koutsoudis, Fotis Arnaoutoglou, Vassilios Tsioukas, and Christodoulos Chamzas. 2007. "Methods for 3D Digitization of Cultural Heritage." Journal of Cultural Heritage 8 (1):93-98. doi: 10.1016/j.culher.2006.10.007.

Pawan, Sawut, and Abiguli Niyazi. 2016. "From Mahalla to Xiaoqu: Transformations of the Urban Living Space in Kashgar" Inner Asia 18 (1):121-134. doi: 10.1163/22105018-12340056.

Peirce, Charles S. 1933. Collected Papers of Charles Sanders Peirce. Charles Hartshorne and Paul Weiss. Cambridge, Mass.: Harvard University Press.

Peirce, Charles S., and Edward C. Moore. 1998. Charles S. Peirce: The Essential Writings. Amherst, N.Y.: Prometheus Books.

Peters, John Durham. 2016. The Marvelous Clouds: Toward a Philosophy of Elemental Media. Chicago: The University of Chicago Press.

Pieprzak, Katarzyna. 2014. "A Beautiful Grave: Innocent Objects, Museums, and the Modern Self in Driss Chraïbi's La Civilisation, ma Mère!... and the Ben M'Sik Community Museum." Studies in 20th \& 21st Century Literature 38 (2):Article 7. doi: http://dx.doi.org/10.4148/2334-4415.1026.

Pietroni, Eva, and Andrea Adami. 2014. "Interacting with Virtual Reconstructions in Museums: The Etruscanning Project." Journal on Computing and Cultural Heritage 7 (2):1-29. doi: $10.1145 / 2611375$.

Pletinckx, Daniel, Dirk Callebaut, Ann E. Killebrew, and Neil A. Silberman. 2000. "Virtual-Reality Heritage Presentation at Ename." IEEE MultiMedia 7 (2):45-48. doi: 10.1109/93.848427.

Plummer, Ken. 1996. "Symbolic Interactionism in the Twentieth Century: The Rise of Empirical Social Theory." In The Blackwell Companion to Social Theory, edited by Bryan S. Turner, 223-251. Oxford: Blackwell.

Popple, Simon, and Daniel H. Mutibwa. 2016. "Tools You Can Trust? Co-design in Community Heritage Work." In Cultural Heritage in a Changing World, edited by Karol Jan Borowiecki, Neil Forbes and Antonella Fresa, 197-214. Cham: Springer International Publishing.

Prentice, Will. 2016. "Digital Preservation of Audio Content." In Managing Digital Cultural Objects: Analysis, Discovery and Retrieval, edited by Allen Foster and Pauline Rafferty, 129-140. London: Facet Publishing.

HIP.Institute (Heritage Innovation Preservation). 2017. "About ScanPyramids." Accessed January 14 , http://www.scanpyramids.org/assets/components/pyramids/pdfs/About_ScanPyramidsen.pdf. 
Prince, Stephen. 2012. Digital Visual Effects in Cinema: The Seduction of Reality. New Brunswick, N.J.: Rutgers University Press.

Procureur, Sébastien. 2018. "Muon Imaging: Principles, Technologies and Applications." Nuclear Instruments and Methods in Physics Research Section A: Accelerators, Spectrometers, Detectors and Associated Equipment 878:169-179. doi: 10.1016/j.nima.2017.08.004.

Pujol-Tost, Laia, and Maria Economou. 2009. "Worth a Thousand Words? The Usefulness of Immersive Virtual Reality for Learning in Cultural Heritage Settings." International Journal of Architectural Computing 7 (1):157-176. doi: 10.1260/147807709788549367.

Pujol, Laia, and Erik Champion. 2012. "Evaluating Presence in Cultural Heritage Projects." International Journal of Heritage Studies 18 (1):83-102. doi: 10.1080/13527258.2011.577796.

Quiccheberg, Samuel. (1565) 2013. The First Treatise on Museums: Samuel Quiccheberg's Inscriptiones 1565. Translated by Mark A. Meadow and Bruce Robertson. Los Angeles, Calif.: Getty Publications.

Ötkür, Abdurrahim. (1985) 2017. İz. Istanbul: Boyalıkuş Yayınları.

Raben, Joseph. 1977. Computer-Assisted Research in the Humanities: A Directory of Scholars Active. New York: Pergamon Press.

Radio, Shirley and Spinoza Internet. 2006. "Under a Dim Crescent Moon - Part I." [Live-mixed webcast of field recordings, edited by Fausto Caceres]. Accessed January 14, 2018, http://compound-eye.org/radio/playlists/ss2006-01-15.html.

Rahaman, Hafizur. 2012. "A Framework for Digital Heritage Interpretation." PhD diss., National University of Singapore.

Rancière, Jacques. 2009. The Emancipated Spectator. Translated by Gregory Elliott. London: Verso.

Rancière, Jacques. 2007. The Future of the Image. Translated by Gregory Elliot. London; New York: Verso.

Ranulph, Glanville. 2007. "Try Again. Fail Again. Fail Better: The Cybernetics in Design and the Design in Cybernetics." Kybernetes $36 \quad$ (9/10):1173-1206. doi: 10.1108/03684920710827238.

Rashid, Hani. 1999. "The Museum as a Digital Experience." Proceedings of the ICOMON meetings, Madrid, Spain. 30-32, www.icomon.org/file_download/160/Rashid+1999.pdf.

Rectanus, Mark W. 2002. Culture Incorporated: Museums, Artists, and Corporate Sponsorships. NED - New edition ed. Minneapolis, Minn.; London: University of Minnesota Press.

Refsland, Scot Thrane, Takeo Ojika, Tom Defanti, Andy Johnson, Jason Leigh, Carl Loeffler, and Xiaoyuan Tu. 1998. "Virtual Great Barrier Reef: A Theoretical Approach Towards an Evolving, Interactive VR Environment Using a Distributed DOME and CAVE System." In Virtual Worlds: First International Conference (VW'98), Paris, France, July 1-3, 1998, edited by Jean-Claude Heudin, 323-336. Berlin, Heidelberg: Springer Berlin Heidelberg.

Rekrei. 2015. "Project Mosul." [Online virtual museum]. Accessed January 12, 2018, https://projectmosul.org/about. 
Reynolds, Jack. 2015. "Time Out of Joint." In Performance and Temporalisation: Time Happens, edited by Stuart Grant, Jodie McNeilly and Maeva Veerapen, 101-113. London: Palgrave Macmillan UK.

RFA (Radio Free Asia). 2010. "Kashgar's Vanishing Memory." [Online multimedia platform]. Accessed January 14, 2018, http://www.rfa.org/english/multimedia/kashgardemolition03232010120925.html.

Rheingold, Howard. 2000. The Virtual Communities: Homesteading on the Electronic Frontier. Cambridge, Mass.; London: The MIT Press. Original edition, Addison Wesley, 1993. Reprint, Harper Collins, 1994.

Ricardo, Francisco, J. 2013. The Engagement Aesthetic: Experiencing New Media Art through Critique. London; New York: Bloomsbury.

Richens, Paul. 2014. "Virtual Reality and Cultural Landscapes." May 14-17, 2014.

Richens, Paul, and Michael Nitsche. 2005. "Mindstage: Towards a Functional Virtual Architecture." In Computer Aided Architectural Design Futures 2005: Proceedings of the 11th International CAAD Futures Conference held at the Vienna University of Technology, Vienna, Austria, on June 20-22, 2005, edited by Bob Martens and Andre Brown, 331-340. Springer Netherlands.

RICHES (Renewal, Innovation and Change: Heritage and European Society). 2015. "Recalibrating Relationships." [Project description]. Accessed November 1, 2017, http://www.richesproject.eu/project.html.

RICHES Taxonomy. 2014. RICHES. Accessed December 5, 2017, http://resources.richesproject.eu/research/taxonomy/terms-and-definitions/.

Ridge, Mia, ed. 2014a. Crowdsourcing Our Cultural Heritage, Digital Research in the Arts and Humanities. Farnham, Surrey: Ashgate.

Ridge, Mia. 2014b. "Introduction." In Crowdsourcing Our Cultural Heritage, 1-13. Farnham, Surrey: Ashgate.

Roberts, Andrew. 2001. "The Changing Role of Information Professionals in Museums." MDA Information 5

(3):15-17, http://network.icom.museum/fileadmin/user_upload/minisites/cidoc/ConferencePapers/199 9/4.pdf.

Robertshaw, Andrew. 2006. "Live Interpretation." In Heritage Interpretation, edited by Alison Hems and Marion R. Blockley, 41-54. New York: Routledge.

Robins, Claire. 2007. "How did the Reticent Object Become so Obliging?: Artists' Interventions as Interpretive Strategies in Galleries and Museum." Engage 20:23-28, https://www.engage.org/downloads/215024FE1_EJ_20_C.Robins.pdf.

Rogerio-Candelera, Miguel Ángel, ed. 2014. Science, Technology and Cultural Heritage. Boca Raton: CRC Press.

Rogers, Jessie, and Marc Aurel Schnabel. 2018 (forthcoming). "Digital Design Ecology: An Intricate Framework for the Analysis of Architectural Design."Computing for a Better Tomorrow: Proceedings of the 36th Annual International Conference of eCAADe - Education and Research in Computer Aided Architectural Design in Europe (eCAADe 2018), Lodz, Poland, September 17-21, 2018. pgs. 10. 
Rogers, Jessie, Marc Aurel Schnabel, and Tian Tian Lo. 2018. "Digital Culture: An Interconnective Design Methodology Ecosystem."Learning, Adapting and Prototyping: Proceedings of the 23rd International Conference on Computer-Aided Architectural Design Research in Asia (CAADRIA 2018), Beijing, China, May 17-19, 2018. 493-502.

Rojek, Chris. 1997. "Indexing, Dragging and the Social Construction of Tourist Sights." In Touring Cultures: Transformations of Travel and Theory, edited by Chris Rojek and John Urry, 214 : ill ; 24 cm. London: Routledge.

Ross, Max. 2004. "Interpreting the New Museology." Museum and Society 2 (2):84-103, https://journals.le.ac.uk/ojs1/index.php/mas/article/view/43.

Roued-Cunliffe, Henriette, and Andrea Copeland Japzon, eds. 2017. Participatory Heritage. London: Facet Publishing.

Roussou, Maria. 2004. "Learning by Doing and Learning through Play: An Exploration of Interactivity in Virtual Environments for Children." ACM Computers in Entertainment (CIE) 2 (1):1-23. doi: 10.1145/973801.973818.

Roussou, Maria, and Dimitris Efraimoglou. 1999. "High-End Interactive Media in the Museum." Proceedings of the 26th International Conference on Computer Graphics and Interactive Techniques (ACM SIGGRAPH 1999), Los Angeles, Calif., USA, August 08-13, 1999. 5962. doi:10.1145/311625.311682.

Rushton, Hannah, David Silcock, Marc Aurel Schnabel, Tane Moleta, and Serdar Aydin. 2018. "The Moving Image in Digital Heritage: Architectural Heritage in Virtual Reality." International Conference, Mediated City 4: Moving Images Static Spaces: Architectures, Media Film, Communication, Digital Art and Design, Istanbul, April 12-13, 2018.

Ruskin, John. [1880] 1989. Seven Lamps of Architecture. New York: Dover Publications. Reprint, Sunnyside, Orpington, Kent: G. Allen.

Ryan, Marie-Laure. 1991. Possible Worlds, Artificial Intelligence, and Narrative Theory. Bloomington: Indiana University Press.

Ryan, Marie-Laure. 1999. Cyberspace Textuality: Computer Technology and Literary Theory. Bloomington: Indiana University Press.

Ryan, Marie-Laure. 2015. Narrative as Virtual Reality 2: Revisiting Immersion and Interactivity in Literature and Electronic Media. Baltimore, Md.: Johns Hopkins University Press.

Ryan, Marie-Laure, and Jan-Noël Thon, eds. 2014. Storyworlds across Media: Toward a MediaConscious Narratology, Frontiers of Narrative. Lincoln, Nebraska; London: University of Nebraska Press.

Salen, Katie, and Eric Zimmerman. 2004. Rules of Play: Game Design Fundamentals. Cambridge, Mass.: MIT Press.

Sandberg, Mark B. 2014. "Location, "Location": On the Plausibility of Place Substitution." In Silent Cinema and the Politics of Space, 23-46. Indiana University Press.

Santana Quintero, Mario. 2003. "The Use of Three-Dimensional Techniques of Documentation and Dissemination in Studying Built Heritage." PhD diss., Katholieke Universiteit Leuven.

Sayer, R. Andrew. 2000. Realism and Social Science. London; Thousand Oaks, Calif.: Sage. 
Schettino, Patrizia, and Sarah Kenderdine. 2010. "Place-Hampi: Narratives of Inclusive Cultural Experience." International Journal of the Inclusive Museum 3 (3):141-156.

Schnabel, Marc Aurel. 2004. "Architectural Design in Virtual Environments: Exploring Cognition and Communication in Immersive Virtual Environments." PhD diss., University of Hong Kong.

Schnabel, Marc Aurel, Serdar Aydin, Tane Moleta, Davide Pierini, and Tomás Dorta. 2016. "Unmediated Cultural Heritage via Hyve-3D: Collecting Individual and Collective Narratives with 3D Sketching." In S. F. Chien, S. Choo, M. A. Schnabel, W. Nakapan, M. J. Kim and S. Roudavski, eds., Living Systems and Micro-Utopias: Towards Continuous Designing: Proceedings of the 21st International Conference on Computer-Aided Architectural Design Research in Asia (CAADRIA 2016), Melbourne, Australia, March 30April 2, 2016. 683-692.

Schnabel, Marc Aurel, Thomas Kvan, Steve K.S. Kuan, and Weidong Li. 2004. "3D Crossover: Exploring Objets Digitalisé." International Journal of Architectural Computing 2 (4):475490. doi: 10.1260/1478077042906212.

Schön, Donald A. 1983. The Reflective Practitioner: How Professionals Think in Action. New York: Basic Books.

Schön, Donald A. 1992. "Designing as Reflective Conversation with the Materials of a Design Situation." Research in Engineering Design 3 (3):131-147. doi: 10.1007/bf01580516.

Schumacher, Patrik S. 2011. The Autopoiesis of Architecture: A New Framework for Architecture, Volume I. Chichester, West Susex: John Wiley \& Sons Ltd.

Seif El-Nasr, Magy, Anders Drachen, and Alessandro Canossa, eds. 2013. Game Analytics Maximizing the Value of Player Data. London: Springer.

Seiler, Uwe Tobias, Volker Koch, and Petravon Both. 2015. "Immersive Virtual Simulation of Spaces." In B. Martens, G. Wurzer, T. Grasl, W. E. Lorenz and R. Schaffranek, eds., Real Time: Extending the Reach of Computation: Proceedings of the 33rd Annual International Conference of eCAADe - Education and Research in Computer Aided Architectural Design in Europe (eCAADe 2015), Volume 1, Vienna, Austria, September 16-18, 2015. 77-88.

Seising, Rudolf. 2010. "Cybernetics, System(s) Theory, Information Theory and Fuzzy Sets and Systems in the 1950s and 1960s." Information Sciences 180 (23):4459-4476. doi: 10.1016/j.ins.2010.08.001.

Shannon, Claude Elwood. 1948. "A Mathematical Theory of Communication." Bell System Technical Journal 27 (3):379-423. doi: 10.1002/j.1538-7305.1948.tb01338.x.

Shepherd, Robert J., and Larry Yu. 2013. "The Social Impact of Heritage." In Heritage Management, Tourism, and Governance in China: Managing the Past to Serve the Present, edited by Robert J. Shepherd and Larry Yu, 67-83. New York, N.Y.: Springer New York.

SIGGRAPH Education. 1999. "Polygon Mesh Data Structure for 3D Graphics." Accessed January 16 , https://www.siggraph.org/education/materials/HyperGraph/modeling/polymesh/polymesh. htm.

Simon, Nina. 2010. The Participatory Museum. Santa Cruz, Calif.: Museum 2.0. 
Singer, Alan. 1998. "Beautiful Errors: Aesthetics and the Art of Contextualization." boundary 225 (1):7-34. doi: 10.2307/303934.

Sito, Tom. 2013. Moving Innovation: A History of Computer Animation. Cambridge, Mass.; London: The MIT Press.

Smith, George S., Phyllis M. Messenger, and Hilary A. Soderland. 2010. Heritage Values in Contemporary Society. Walnut Creek, Calif.: Left Coast Press.

Smith, Laurajane. 2006. Uses of Heritage. London; New York: Routledge.

Softwarestudies. 2010. "Manga Style Space." [Interactive exhibition]. Accessed January 12, 2018, http://lab.softwarestudies.com/2010/11/one-million-manga-pages.html.

Softwarestudies. 2014. "Taipei Phototime." [Big data visualisation]. Accessed January 12, 2018, http://lab.softwarestudies.com/2014/05/our-new-instagram-visualization-project.html.

Šola, Tomislav. 1997. Essays on Museums and Their Theory: Towards the Cybernetic Museum. Helsinki: Suomen Museoliitto = Finnish Museums Association.

Spingarn-Koff, Jason. 2000. "Guggenheim Going Virtual." Wired, issued June 9, 2000. Accessed January 10, 2018, http://archive.wired.com/culture/lifestyle/news/2000/06/36741.

Spivak, Gayatri Chakravorty. 1976. "Translater's Preface." In Of grammatology. Jacques Derrida, ix-1xxxvii. Baltimore, Md.; London: Johns Hopkins University Press.

Staiff, Russell. 2014. Re-imagining Heritage Interpretation: Enchanting the Past-Future. Farnham, Surrey; Burlington, Vt.: Ashgate Publishing.

Starr, S. Frederick. 2013. Lost Enlightenment: Central Asia's Golden Age from the Arab Conquest to Tamerlane. Princeton, N.J.: Princeton University Press.

Steenberg, Rune. 2014. "Transforming Houses - The Changing Concept of the House in Kashgar." Internationales Asien Forum. International Quarterly for Asian Studies 45 (1):171-191, https://search.proquest.com/docview/1628647141?pq-origsite=gscholar.

Stevens, Terry. 1989. "The Visitor - Who Cares? Interpretation and Consumer Relations." In Heritage Interpretation: The Visitor Experience, edited by David L. Uzzell, 103-107. New York: Belhave Press.

Stuckey, Helen, Nick Richardson, Melanie Swalwell, and Denise De Vries. 2015. "What Retrogamers can Teach the Museum." MWA2015: Museums and the Web Asia 2015, Melbourne, $\begin{array}{llll}\text { Victoria. } & \text { Accessed } & \text { January } & \text { 2018, }\end{array}$ https://mwa2015.museumsandtheweb.com/paper/what-retrogamers-can-teach-themuseum/.

Stuckey, Helen, and Melanie Swalwell. 2014. "Retro-Computing Community Sites and the Museum." In Handbook of Digital Games, edited by Marios C. Angelides and Harry Agius, 523-547. John Wiley \& Sons, Inc.

Su, Donghai. 2008. "Chinese Museums' Tradition and Changes." Museologia e Patrimônio 1 (1):120-122, http://revistamuseologiaepatrimonio.mast.br/index.php/ppgpmus/article/viewFile/13/9.

Sutherland, Ivan E. 1964. "Sketchpad a Man-Machine Graphical Communication System." Simulation 2 (5):R-3-R-20. doi: 10.1177/003754976400200514. 
Swarbrooke, John. 1995. The Development and Management of Visitor Attractions. Oxford: Butterworth-Heinemann.

Szoniecky, Samuel, and Nasreddine Bouhaï. 2017. Collective Intelligence and Digital Archives: Towards Knowledge Ecosystems. Hoboken, N.J.; London: Wiley-ISTE.

Şahbaz, Eray, and Aysun Özköse. 2017. "Experiencing Historical Buildings through Digital Computer Games." International Journal of Architectural Computing:1478077117749960. doi: $10.1177 / 1478077117749960$.

Tallon, Loïc, and Kevin Walker, eds. 2008. Digital Technologies and the Museum Experience: Handheld Guides and Other Media. Lanham, Md.: AltaMira Press.

Tan, Beng-Kiang, and Hafizur Rahaman. 2009. "Virtual Heritage: Reality and Criticism." In T. Tidafi and T. Dorta, eds., Joining Languages, Cultures and Visions: Proceedings of the 13th International Conference on Computer Aided Architectural Design Futures (CAAD Futures 2009), Montréal, Canada, June 17-19, 2009. 143-156.

Tang, Jianbo, and Wensheng Zhou. 2013. "Research of National Historic City Dynamic Monitoring by Remote Sensing: A Case Study of Kashgar Historic Urban Area." In Proceedings of the 2012 International Conference on Information Technology and Software Engineering, edited by Wei Lu, Guoqiang Cai, Weibin Liu and Weiwei Xing, 329-337. Springer Berlin Heidelberg.

TÊTE-À-TÊTE. 2016. "The Exceptional and the Everyday: 144 Hours in Kyiv." [Big data visualisation]. Accessed January 12, 2018, http://tat.lv/en/photo/270916-opening-of-theexhibition-light-at-the-end-of-the-cable-latvian-art-in-the-digital-era-/.

Teyssot, Georges. 2013. A Topology of Everyday Constellations. Cambridge, Mass.: The MIT Press.

Thellefsen, Torkild, and Bent Sørensen, eds. 2014. Charles Sanders Peirce in His Own Words: 100 Years of Semiotics, Communication and Cognition. Boston: De Gruyter Mouton.

Tiffin, John, and Nobuyoshi Terashima. 2001. HyperReality: Paradigm for the Third Millennium. London; New York: Routledge.

Tilden, Freeman. 2007. Interpreting Our Heritage. R. Bruce Craig. Chapel Hill, N.C.: University of North Carolina Press.

Tilley, Christopher Y. 2008. Body and Image: Explorations in Landscape Phenomenology. Walnut Creek, Calif.: Left Coast Press Inc.

Urry, John. 1990. The Tourist Gaze: Leisure and Travel in Contemporary Societies. London: Sage Publications.

Uzzell, David L. 1989. "Introduction: The Visitor Experience." In Heritage Interpretation: The Visitor Experience, edited by David L. Uzzell. New York: Belhave Press.

Uzzell, David L. 1998. "Interpreting Our Heritage: A Theoretical Interpretation." In Contemporary Issues in Heritage and Environmental Interpretation: Problems and Prospects, edited by David L. Uzzell and Roy Ballantyne, 11-25. London: Stationery Office.

Van der Wetering, Ernst. 2005. "'Self-Portrait'." In A Corpus of Rembrandt Paintings: The SelfPortraits, edited by Ernst Van der Wetering, 486-497. Dordrecht: Springer Netherlands. 
Van Hoff, Arthur. 2017. "Virtual Reality and the Future of Immersive Entertainment." Proceedings of the 2017 ACM International Conference on Interactive Experiences for TV and Online Video (ACM TVx 2017), Hilversum, The Netherlands. 129-129. doi:10.1145/3077548.3080529.

Van Ouwerkerk, Marieke, and Jürgen Rosemann, eds. 2001. Research by Design: International Conference, Faculty of Architecture Delft University of Technology, in Co-operation with the EAAE/AEEA. Vol. Proceedings A. Delft: Delft University Press, https://repository.tudelft.nl/islandora/object/uuid:b9f3682a-e36b-41f0-960a$165587 \mathrm{fe} 0 \mathrm{c} 62$ ? collection=research

Varinlioglu, Guzden, Ali Aslankan, Gazihan Alankus, and Gokhan Mura. 2017. "Raising Awareness for Digital Heritage through Serious Game." In A. Fioravanti, S. Cursi, S. Elahmar, S. Gargaro, G. Loffreda, G. Novembri and A. Trento, eds., ShoCK! - Sharing Computational Knowledge!: Proceedings of the 35th Annual International Conference of eCAADe Education and Research in Computer Aided Architectural Design in Europe (eCAADe 2017), Volume 2, Rome, Italy. 647-654.

Vartanian, Hrag. 2015. "Why Don't More Americans Go to Museums?" Hyperallergic, issued September 4, 2015. Accessed January 14, 2018, http://hyperallergic.com/230554/why-dontmore-americans-go-to-museums/.

Vavoula, Giasemi, and Marco Mason. 2017. "Digital Exhibition Design: Boundary Crossing, Intermediary Design Deliverables and Processes of Consent." Museum Management and Curatorship 32 (3):251-271. doi: 10.1080/09647775.2017.1282323.

Vergo, Peter. 1989a. The New Museology. London: Reaktion Books.

Vergo, Peter. 1989b. "The Reticent Object." In The New Museology, edited by Peter Vergo, 41-59. London: Reaktion Books.

Wahba, Malak, Reem Bahgat, and Fathi Saleh. 2001. "Cairo's Architectural Heritage: The Downtown Area." the International Cultural Heritage Informatics Meeting (ICHIM 2001), Milan, Italy, September 3-7, 2001. 279-290. Accessed January 14, 2018, http://www.archimuse.com/publishing/ichim01_vol1/wahba.pdf.

Waldmeir, Patti. 2014. "Pingyao: The Great Wall Street of China." Financial Times Magazine, issued March 15, 2014. Accessed January 14, 2018, http://www.ft.com/cms/s/2/74be78cc-a98011e3-9b71-00144feab7de.html\#slide0.

Walsh, Peter. 2007. "Rise and Fall of the Post-Photographic Museum: Technology and the Transformation of Art." In Theorizing Digital Cultural Heritage: A Critical Discourse, edited by Fiona Cameron and Sarah Kenderdine, 19-34. Cambridge, Mass.; London: The MIT Press.

Walton, Douglas N. 2004. Abductive Reasoning. Tuscaloosa, Ala.: University of Alabama Press.

Wang, Shu-Yi. 2012. "From a Living City to a World Heritage City: Authorised Heritage Conservation and Development and Its Impact on the Local Community." International Development Planning Review 34 (1):1-17. doi: 10.3828/idpr.2012.2.

Wang, Xiaodong, Hui Song, Jing Liu, and Ni Yiding Zhu. 2014. 喀什高台民居[Kashi gao tai min ju = Terraced Household Kashgar]. Nanjing: Southeast University Press.

Waterton, Emma. 2010. Politics, Policy and the Discourses of Heritage in Britain. Basingstoke: Palgrave Macmillan. 
Waterton, Emma. 2014. "A More-Than-Representational Understanding of Heritage? The 'Past' and the Politics of Affect." Geography Compass 8 (11):823-833. doi: 10.1111/gec3.12182.

Waterton, Emma, and Steve Watson. 2013. "Framing Theory: Towards a Critical Imagination in Heritage Studies." International Journal of Heritage Studies 19 (6):546-561. doi: 10.1080/13527258.2013.779295.

Watson, Steve, and Emma Waterton. 2010. "Reading the Visual: Representation and Narrative in the Construction of Heritage." Material Culture Review 71:84-97, https://journals.lib.unb.ca/index.php/MCR/article/view/18377.

Webb, Nicholas, and Andre Brown. 2011a. "Augmenting Critique of Lost or Unbuilt Works of Architecture Using Digitally Mediated Techniques."Respecting Fragile Places: Proceedings of the 29th Annual International Conference of eCAADe - Education and Research in Computer Aided Architectural Design in Europe (eCAADe 2011), Ljubljana, Slovenia, September 21-24, 2011. 942-950.

Webb, Nicholas, and Andre Brown. 2011b. "Digital Forensics as a Tool for Augmenting Historical Architectural Analysis: Case Study: The Student Work of James Stirling." In H. Christiane, N. Gu, S. Roudavski and M. A. Schnabel, eds., Circuit Bending, Breaking and Mending: Proceedings of the 16th International Conference on Computer-Aided Architectural Design Research in Asia (CAADRIA 2011), Newcastle, Australia, April 27-29, 2011. 505-514.

Webb, Nicholas, and André Brown. 2016. "Digital Re-analysis of Lost Architecture and the Particular Case of Lutyens' Liverpool Metropolitan Cathedral." Frontiers of Architectural Research 5 (2):265-275. doi: 10.1016/j.foar.2016.01.004.

Webb, Nicholas, and Alexandrina Buchanan. 2017. "Tracing the Past: A Digital Analysis of Wells Cathedral Choir Aisle Vaults." Digital Applications in Archaeology and Cultural Heritage 4:19-27. doi: 10.1016/j.daach.2017.01.001.

Weibel, Peter. 1999. "The Noise of the Observer." In Ars Electronica: Facing the Future: A Survey of Two Decades, edited by Timothy Druckrey with Ars Electronica, 140-148. Cambridge, Mass.; London: The MIT Press.

Weil, Stephen E. 1999. "From Being About Something to Being For Somebody: The Ongoing Transformation of the American museum." Daedalus 128 (3):229-258, http://www.jstor.org/stable/20027573.

Weisskopf, Carmen, and Domagoj Smoljo. 2015. "H3333333K." [Glitch art on the facade of the House of Electronic Arts Basel]. !Mediengruppe Bitnik. Accessed January 12, 2018, https://wwwwwwwwwwwwwwwwwwwwww.bitnik.org/h3333333k/.

Wells, Jeremy C. 2007. "The Plurality of Truth in Culture, Context, and Heritage: A (Mostly) PostStructuralist Analysis of Urban Conservation Charters." City and Time 3 (2):1-13, https://docs.rwu.edu/saahp_fp/19/.

Westberg Gabriel, Lýsa, and Thessa Jensen. 2017. "Who is the Expert in Participatory Culture?" In Participatory heritage, edited by Henriette Roued-Cunliffe and Andrea Copeland Japzon, 87-95. London: Facet Publishing.

Wetherell, Margaret. 2013. "Affect and Discourse - What's the Problem? From Affect as Excess to Affective/Discursive Practice." Subjectivity 6 (4):349-368. doi: 10.1057/sub.2013.13.

White, Ed. 2012. How to Read Barthes' Image-Music-Text. London: Pluto. 
Wilkinson, William D. 1968. "Computerized Museum Networks: Introductory Remarks by Chairman of Session." Computers and Their Potential Applications in Museums: A Conference Sponsored by The Metropolitan Museum of Art Supported by a Grant from the IBM Corporation, New York, USA, April 15-17, 1968. 305-306.

Williams, David. 2010. "A Brief History of Museum Computerization." In Museums in a Digital Age, edited by Ross Parry, 15-21. London: Routledge.

Winfield Smith, Ray. 1967. "The Akhenaten Temple Project." Expedition Magazine 10.1, 24-32.

Winter, Tim. 2013. "Clarifying the Critical in Critical Heritage Studies." International Journal of Heritage Studies 19 (6):532-545. doi: 10.1080/13527258.2012.720997.

Witcomb, Andrea. 2003. Re-imagining the Museum: Beyond the Mausoleum. London; New York: Routledge.

Witcomb, Andrea. 2007. "The Materiality of Virtual Technologies: A New Approach to Thinking about the Impact of Multimedia in Musuems." In Theorizing Digital Cultural Heritage: A Critical Discourse, edited by Fiona Cameron and Sarah Kenderdine, 35-48. Cambridge, Mass.; London: The MIT Press.

Wynn, Donald, and Clay K. Williams. 2012. "Principles for Conducting Critical Realist Case Study Research in Information Systems." MIS Quarterly 36 (3):787-810.

Yazdani, Mehrdad, Jay Chow, and Lev Manovich. 2017. "Quantifying the Development of UserGenerated Art during 2001-2010." PLOS ONE 12 (8):e0175350. doi: 10.1371/journal.pone.0175350.

Yuan, Philip F., Mei Zhang, and Li Han. 2013. "Low-Tech Digital Fabrication: Traditional Brick as Material in Digital Practice." In Global Design and Local Materialization: 15th International Conference, CAAD Futures 2013, Shanghai, China, July 3-5, 2013, edited by Jianlong Zhang and Chengyu Sun, 139-148. Berlin, Heidelberg: Springer Berlin Heidelberg.

Zhang, Xiaolei, and Jun Lei. 2006. "Trend of Urban System Structure under the Restriction of Water and Land Resources in Xinjiang." Chinese Science Bulletin 51 (1):179-188. doi: 10.1007/s11434-006-8224-x.

Zhou, Mingquan, Guohua Geng, and Zhongke Wu. 2012. Digital Preservation Technology for Cultural Heritage. New York; Dordrecht; London; Beijing: Springer Publishing Company; Higher Education Press.

Zhu, Yujie. 2017. "Authenticity and Heritage Conservation in China: Translation, Interpretation, Practices." In Authenticity in Architectural Heritage Conservation: Discourses, Opinions, Experiences in Europe, South and East Asia, edited by Katharina Weiler and Niels Gutschow, 187-200. Cham: Springer International Publishing. 


\section{International Charters and Principles}

Athens Charter. 1931. The Athens Charter for the Restoration of Historic Monuments. Adopted at the First International Congress of Architects and Technicians of Historic Monuments, Athens, International Council on Monuments and Sites (ICOMOS). Accessed January 10, 2018, https://www.icomos.org/en/charters-and-texts/179-articles-enfrancais/ressources/charters-and-standards/167-the-athens-charter-for-the-restoration-ofhistoric-monuments.

Burra Charter. (1979) 2013. The Australia ICOMOS Charter for Places of Cultural Significance (The $\begin{array}{llll}\text { Burra Charter). } & \text { Accessed } & \text { January }\end{array}$ http://australia.icomos.org/publications/charters/.

London Charter. (2006) 2009. The London Charter for the Computer-Based Visualisation of Cultural Heritage. First drafted at an expert seminar on "Making 3D Visual Research Outcomes Transparent", King's College London, London, February 25, 2006. Accessed October 20, 2017, http://www.londoncharter.org/introduction.html.

Nara Document. 1994. The Nara Document on Authenticity. Nara Conference on Authenticity, UNESCO World Heritage Centre, Nara, Japan, November, 1-6 1994. Accessed January 12, 2018, https://www.icomos.org/charters/nara-e.pdf.

Seville Principles. 2011. International Principles of Virtual Archaeology (The Seville Principles). Accessed October 20, 2017, http://smartheritage.com/seville-principles/seville-principles.

SPAB Manifesto. (1877) 2018. The Principals of the Society for the Protection of Ancient Buildings as Set Forth upon its Foundation (The SPAB Manifesto). Written by William Morris and Philip Webb. Accessed January 12, 2018, https://www.spab.org.uk/about-us/spabmanifesto.

UNESCO. 2003. The Charter on the Preservation of Digital Heritage. General Conference, Paris, September 29-October 17, 2003. Accessed October 20, 2017, http://portal.unesco.org/en/ev.php-

URL_ID=17721\&URL_DO=DO_TOPIC\&URL_SECTION=201.html.

Venice Charter. 1964. International Charter for the Conservation and Restoration of Monuments and Sites (The Venice Charter). The Second International Congress of Architects and Technicians of Historic Monuments, Venice, May 25-31, 1964, International Council on Monuments and Sites (ICOMOS). Accessed January 10, 2018, https://www.icomos.org/venicecharter2004/. 


\section{Artworks and Projects}

Bernstein, Shelley. 2008. Click!: A Crowd-Curated Exhibition. [Participatory photography exhibition]. Brooklyn, New York: Brooklyn Museum, June 27-August 10, 2008. Accessed January 10, 2018, https://www.brooklynmuseum.org/exhibitions/click.

Chow, Jay, and Lev Manovich. 2014. Taipei Phototime. [Real time comparative visualization of $\begin{array}{lllll}\text { Instagram images]. } & \text { Accessed }\end{array}$ http://manovich.net/index.php/exhibitions/taipei-phototime.

Dion, Daniel. 1991. The Moment of the Truth. [Video installation, duration: 100 seconds]. With the collaboration of Su Schnee. Accessed January 12, 2018, http://www.virtualmuseum.ca/sgccms/expositions-exhibitions/arts_mediatiques-media_arts/artiste-artist/daniel_dioneng.php.

Dufour, Joseph. 1804. Les Sauvages de la Mer Pacifique. [20-panel panorama wallpaper painting]. $\begin{array}{lll}\text { Accessed January } & 18, & \end{array}$ https://www.artgallery.nsw.gov.au/collection/works/356.1989.a-d/.

Eliasson, Olafur. 2015. Demented Architecture. [Participatory art installation]. The Cubic Structural Evolution Project (2004). Wellington, New Zealand: City Gallery Wellington, June 27$\begin{array}{llllll}\text { November } & 8, & 2015 . & \text { Accessed } & \text { January }\end{array}$ http://citygallery.org.nz/exhibitions/demented-architecture.

Goddemeyer, Daniel, Moritz Stefaner, Dominikus Baur, and Lev Manovich. 2015. OnBroadway. [Interactive installation through a compilation of images and data]. New York: New York Public Library for its exhibition "The Public Eye". Accessed January 14, 2018, http://www.on-broadway.nyc/.

Hershman Leeson, Lynn. 1993. Room of One's Own. [Interactive art installation]. Lin, Austria: Ars Electronica 1993.

Hochman, Nadav, and Lev Manovich. 2014. A View from Above: Exploratory Visualizations of the Thomas Walther Collection. [An Online Project of The Museum of Modern Art]. New York: The Museum of Modern Art, January 14, 2018, http://www.moma.org/interactives/objectphoto/assets/essays/Manovich_Hochman.pdf.

Krueger, Myron W. 1969. Glowflow. [Computer art project]. Memorial Union Gallery, University of Wisconsin, April 1969. Accessed January 20, 2018, http://dada.compartbremen.de/item/artwork/1347.

Laviani, Pietro Ferruccio. 2013. Fratelli Boffi: Good Vibration: Studio Laviani. Accessed January 12, 2018, www.laviani.com/good-vibrations.

Manovich, Lev, Jay Chow, Alise Tifentale, and Mehrdad Yazdani. 2014. The Exceptional and the Everyday: 144 Hours in Kyiv. [First project to analyze the use of Instagram during a social 
upheaval]. Accessed January 14, 2018, http://manovich.net/index.php/projects/hours-inkiev-copy.

Manovich, Lev, Jeremy Douglass, and William Huber. 2010. Manga Style Space. [High resolution visualizations of 1 million manga pages]. Accessed January 14, 2018, http://manovich.net/index.php/exhibitions/manga-style-space.

Manovich, Lev, Moritz Stefaner, Mehrdad Yazdani, Dominikus Baur, Daniel Goddemeyer, Alise Tifentale, Nadav Hochman, and Jay Chow. 2014. Selfiecity. [Investigation of the style of self-portraits (selfies)]. Accessed January 14, 2018, http://selfiecity.net/.

Mohr, Mafred. 1969. Random Walk.

Molnar, Vera. 1968. No Title.

Noll, A. Michael. 1964. Computer Composition With Lines.

Reihana, Lisa. 2015. in Pursuit of Venus [infected]. [Panoramic screen-based interactive exhibition]. Accessed January 14, 2018, https://www.aucklandartgallery.com/whats-on/exhibition/inpursuit-of-venus-infected.

Retsin, Gilles, Jingyi Liu, Ziyao Li, Xianming Sang, and Serdar Aydin. 2015. Digital Spontaneity. [Algorithmic glitch design-research]: AAVS Shanghai 2015. Accessed January 12, 2018, http://shanghai.aaschool.ac.uk/gallery/aavs-shanghai-2015/.

Sanborn, John, and Dean Winkler. 1985. Luminare. [video art installation]. Vancouver, B.C., Canada: Expo 86. Accessed January 12, 2018, https://youtu.be/5ojlyR2ODe8.

Shaw, Jeffrey. 1988. Legible City. [Interactive art installation]. Maastricht, Netherlands: Bonnefantenmuseum. 


\section{Appendix A}

Playlist of street-sound recordings: (๘) Track, (•) Playlist.

\begin{tabular}{|c|c|c|c|c|}
\hline Time & Title & Location & Performer/Instrument & Information \\
\hline - 0:00:00 & $\begin{array}{l}\text { Uyghur Mukam } \\
\text { recordings }\end{array}$ & $\begin{array}{l}\text { A music shop in } \\
\text { the old-town }\end{array}$ & $\begin{array}{l}\text { Two instrument craftsmen / } \\
\text { ghijek and dutar }\end{array}$ & $\begin{array}{l}\text { Ghijek and dutar are major } \\
\text { instruments of Uyghur music. } \\
\text { Ghijek is a bowed string } \\
\text { instrument; Dutar is a two-string } \\
\text { instrument widely played by both } \\
\text { Uyghur men and women. Ghijek is } \\
\text { known as the Uyghur violin. }\end{array}$ \\
\hline 0:05:55 & Levant music & $\begin{array}{l}\text { A music shop in } \\
\text { the old-town }\end{array}$ & $\begin{array}{l}\text { A craftsman playing a } \\
\text { ghijek }\end{array}$ & \\
\hline 0:07:31 & $\begin{array}{l}\text { Ethno-nationalistic } \\
\text { Uyghur music recording }\end{array}$ & Rural Kashgar & Unknown solo / dutar & \\
\hline $0: 13: 13$ & $\begin{array}{l}\text { Pastoral Uyghur music } \\
\text { recording }\end{array}$ & Rural Kashgar & Unknown solo / dutar & \\
\hline 0:17:03 & $\begin{array}{l}\text { Gidiyorum Gündüz Gece } \\
\text { (Turkish) }\end{array}$ & $\begin{array}{l}\text { A night club in } \\
\text { Kashgar }\end{array}$ & Unknown / keyboard & $\begin{array}{l}\text { A folk song from Turkey is re- } \\
\text { contextualised for entertainment in } \\
\text { a night club in China. }\end{array}$ \\
\hline - $0: 17: 16$ & Narrow road recording & $\begin{array}{l}\text { In front of a little } \\
\text { shop in } \\
\text { Köziciyerbişi }\end{array}$ & $\begin{array}{l}\text { A bird singing in a cage and } \\
\text { two motorbikes passing } \\
\text { with one of them playing a } \\
\text { pop music }\end{array}$ & Narrow roads \\
\hline $0: 17: 56$ & $\begin{array}{l}\text { Noise of brick wall } \\
\text { masonry }\end{array}$ & Köziciyerbişi & & $\begin{array}{l}\text { Workers rebuilding a brick wall of } \\
\text { storage space, with a radio playing } \\
\text { Uyghur songs }\end{array}$ \\
\hline 0:19:03 & $\begin{array}{l}\text { Recording conversation in } \\
\text { Uyghur }\end{array}$ & $\begin{array}{l}\text { A pottery } \\
\text { workshop in } \\
\text { Köziciyerbişi }\end{array}$ & & $\begin{array}{l}\text { Introducing each other to children } \\
\text { of a pottery maker }\end{array}$ \\
\hline - 0:23:06 & Noise of construction & Old-town & & Workers composing concrete \\
\hline $0: 24: 54$ & $\begin{array}{l}\text { Recording conversation in } \\
\text { Uyghur with construction } \\
\text { workers }\end{array}$ & Old-town & & \\
\hline $0: 25: 22$ & Silent street & $\begin{array}{l}\text { Id Kah Bazaar in } \\
\text { the old-town }\end{array}$ & & Recorded after shops closed \\
\hline - $0: 26: 36$ & $\begin{array}{l}\text { Ambience of alleys in the } \\
\text { morning }\end{array}$ & Köziciyerbişi & & $\begin{array}{l}\text { Exploring the first encountered } \\
\text { roads in the neighbourhood }\end{array}$ \\
\hline $0: 29: 55$ & $\begin{array}{l}\text { Sound of children playing } \\
\text { on ground }\end{array}$ & Köziciyerbişi & $\begin{array}{l}\text { Three children playing with } \\
\text { little sand dunes }\end{array}$ & $\begin{array}{l}\text { Children are turning narrow roads } \\
\text { into multifunctional space to } \\
\text { generate their own narrative. }\end{array}$ \\
\hline - $0: 35: 18$ & $\begin{array}{l}\text { Adhan calling for Friday } \\
\text { prayer }\end{array}$ & $\begin{array}{l}\text { Id Kah Mosque } \\
\text { courtyard }\end{array}$ & & $\begin{array}{l}\text { Adhan calling is a stereotype of } \\
\text { Islamic cities in the world. In } \\
\text { Kashgar no electronic system is } \\
\text { used because of regulations. All } \\
\text { adhan callings are performed } \\
\text { naturally. }\end{array}$ \\
\hline 0:37:09 & Noise of the Friday crowd & $\begin{array}{l}\text { Id Kah Mosque } \\
\text { square and Id Kah } \\
\text { Bazaar }\end{array}$ & Thousands of people & $\begin{array}{l}\text { Walking out of Id Kah Mosque } \\
\text { after Friday prayer }\end{array}$ \\
\hline $0: 37: 54$ & Noise of dok preparation & Id Kah Bazaar & Street vendor & $\begin{array}{l}\text { Dok is a cold drink consumed } \\
\text { widely by Uyghurs on street in } \\
\text { summers }\end{array}$ \\
\hline - 0:39:01 & $\begin{array}{l}\text { Merchant ambience and } \\
\text { various ambiences }\end{array}$ & Id Kah Bazaar & & $\begin{array}{l}\text { Walking from Id Kah towards } \\
\text { Renmin Road }\end{array}$ \\
\hline
\end{tabular}


310

\begin{tabular}{|c|c|c|c|c|}
\hline Time & Title & Location & Performer/Instrument & Information \\
\hline - $0: 40: 34$ & Noise of tourists & Apak Khoja Tomb & & \\
\hline - 0:40:46 & $\begin{array}{l}\text { Narrow road recording } \\
\text { after a two-day sand- } \\
\text { storm }\end{array}$ & $\begin{array}{l}\text { Mosque I in } \\
\text { Köziciyerbişi }\end{array}$ & & $\begin{array}{l}\text { A worker fixes the fallen thickness } \\
\text { of the mud-brick wall of a mosque } \\
\text { by hand while listening to Uyghur } \\
\text { music on a mobile radio }\end{array}$ \\
\hline D $0: 41: 31$ & $\begin{array}{l}\text { Sound of children playing } \\
\text { football }\end{array}$ & $\begin{array}{l}\text { North of } \\
\text { Köziciyerbişi }\end{array}$ & & $\begin{array}{l}\text { Looking from an alley towards the } \\
\text { Grand Bazaar of Kashgar along the } \\
\text { rim of Tuman River }\end{array}$ \\
\hline 0:44:39 & $\begin{array}{l}\text { Recording conversations } \\
\text { in Uyghur }\end{array}$ & $\begin{array}{l}\text { North of } \\
\text { Köziciyerbişi }\end{array}$ & $\begin{array}{l}\text { A female, two males and a } \\
\text { child }\end{array}$ & \\
\hline - $0: 45: 55$ & $\begin{array}{l}\text { Recording wedding } \\
\text { rituals }\end{array}$ & $\begin{array}{l}\text { Payzawat / the } \\
\text { groom's house }\end{array}$ & & $\begin{array}{l}\text { Recorded during the wedding meal } \\
\text { where the groom is rationally } \\
\text { forced to eat a lot for fun }\end{array}$ \\
\hline 0:49:10 & Unknown Uyghur song & $\begin{array}{l}\text { Payzawat / the } \\
\text { bride's house }\end{array}$ & Unknown singer & Recorded during a wedding party \\
\hline $0: 50: 25$ & Unknown Uyghur song & Payzawat & Unknown singer & $\begin{array}{l}\text { Recorded during the bride's } \\
\text { farewell party }\end{array}$ \\
\hline 0:50:56 & Uyghur dance music & Payzawat & & Wedding dance on street \\
\hline 0:53:10 & Old Chinese song & Payzawat & Unknown singer & $\begin{array}{l}\text { Recorded during transportation } \\
\text { from Payzawat to Kashgar }\end{array}$ \\
\hline - $0: 56: 28$ & $\begin{array}{l}\text { Recording conversation in } \\
\text { Uyghur with children of } \\
\text { alleys }\end{array}$ & Kashgar old-town & & \\
\hline - 1:00:52 & Unknown Uyghur song & Id Kah Bazaar & Unknown singer & Recorded on street at night market \\
\hline - 1:01:18 & Street recording & Köziciyerbişi & & \\
\hline 1:01:30 & Unknown Uyghur song & Köziciyerbişi & Abdurehim Heyit & $\begin{array}{l}\text { Recorded from a radio played } \\
\text { inside a house }\end{array}$ \\
\hline 1:03:29 & Street recording & Köziciyerbişi & & $\begin{array}{l}\text { Recorded by following a cat in an } \\
\text { alleyway }\end{array}$ \\
\hline - $1: 03: 54$ & Unknown Uyghur song & Köziciyerbişi & $\begin{array}{l}\text { Unknown singer playing a } \\
\text { rawap }\end{array}$ & $\begin{array}{l}\text { Recorded from radio playing } \\
\text { outside a house }\end{array}$ \\
\hline - 1:05:13 & Street recordings & $\begin{array}{l}\text { In front of a } \\
\text { butcher on Arayer } \\
\text { Road, old-town }\end{array}$ & & $\begin{array}{l}\text { Pop music played by a scooter } \\
\text { driver }\end{array}$ \\
\hline - $1: 05: 22$ & Rhythm of labour & $\begin{array}{l}\text { Arayer Yoli, old- } \\
\text { town and }\end{array}$ & Hammers & Recordings from blacksmith shops \\
\hline - 1:08:28 & Recording inside shops & $\begin{array}{l}\text { South section of } \\
\text { the old-town }\end{array}$ & & Uyghur radio news about the USA \\
\hline 1:08:33 & $\begin{array}{l}\text { Noise of construction } \\
\text { tools }\end{array}$ & $\begin{array}{l}\text { South section of } \\
\text { the old-town }\end{array}$ & Hammer and wood revolver & Construction workers, carpenters \\
\hline 1:11:01 & $\begin{array}{l}\text { Recording the work and } \\
\text { conversation of } \\
\text { construction workers }\end{array}$ & $\begin{array}{l}\text { South section of } \\
\text { the old-town }\end{array}$ & & Road-work and chat with workers \\
\hline $1: 11: 18$ & $\begin{array}{l}\text { Recording adhan calling } \\
\text { for prayer from a small } \\
\text { neighbourhood mosque }\end{array}$ & $\begin{array}{l}\text { South section of } \\
\text { the old-town }\end{array}$ & & \\
\hline - $1: 12: 12$ & Noise of carpenters & Old-town & Revolver & \\
\hline $1: 12: 32$ & $\begin{array}{l}\text { Recording Uyghur } \\
\text { traditional music calling } \\
\text { for an occasion }\end{array}$ & $\begin{array}{l}\text { Köziciyerbişi - } \\
\text { Tuman street }\end{array}$ & & Street recording of traffic \\
\hline - $1: 12: 57$ & $\begin{array}{l}\text { Recording vendors' voice } \\
\text { calling for customers }\end{array}$ & Old-town central & & Samsa bun seller \\
\hline - 1:14:19 & $\begin{array}{l}\text { Recording conversation in } \\
\text { Uyghur }\end{array}$ & Köziciyerbişi & & \\
\hline $1: 14: 43$ & $\begin{array}{l}\text { Recording the noise of } \\
\text { turmoil }\end{array}$ & $\begin{array}{l}\text { The entrance of } \\
\text { the Grand Bazaar }\end{array}$ & & $\begin{array}{l}\text { Recorded while a big sand storm } \\
\text { approaching }\end{array}$ \\
\hline 1:14:52 & $\begin{array}{l}\text { Recording the sound of } \\
\text { people in a turmoil }\end{array}$ & $\begin{array}{l}\text { The exit of the } \\
\text { Grand Bazaar }\end{array}$ & & $\begin{array}{l}\text { Greeting store-owners before they } \\
\text { close early because of a sand storm }\end{array}$ \\
\hline 1:15:04 & Noise of transportation & Köziciyerbişi & Electric motor & $\begin{array}{l}\text { Inside motor-car to run away from } \\
\text { the sand storm }\end{array}$ \\
\hline - $1: 15: 53$ & $\begin{array}{l}\text { Noise of construction and } \\
\text { vendors }\end{array}$ & Old-town & A concrete mixer & Recorded in alleyways \\
\hline - $1: 19: 10$ & $\begin{array}{l}\text { Recording noise inside a } \\
\text { school }\end{array}$ & Old-town & & Primary school students \\
\hline
\end{tabular}




\begin{tabular}{|c|c|c|c|c|}
\hline Time & Title & Location & Performer/Instrument & Information \\
\hline - 1:19:51 & & Old-town & & $\begin{array}{l}\text { Children playing with a doll } \\
\text { donkey }\end{array}$ \\
\hline - 1:20:05 & & Old-town & & $\begin{array}{l}\text { Construction activities, lifting sand } \\
\text { from ground to rooftop }\end{array}$ \\
\hline - $1: 20: 18$ & $\begin{array}{l}\text { Uyghur traditional } \\
\text { wedding song performed } \\
\text { on street }\end{array}$ & Id Kah Bazaar & $\begin{array}{l}\text { Three people behind a car, } \\
\text { with one of them blowing a } \\
\text { sunayi, and the two others } \\
\text { drumming a dap. }\end{array}$ & $\begin{array}{l}\text { Sunayi is a wind instrument, } \\
\text { around fifty centimetres. Dap is a } \\
\text { membranous percussion } \\
\text { instrument. }\end{array}$ \\
\hline 1:21:01 & $\begin{array}{l}\text { Recording coppersmith } \\
\text { shops }\end{array}$ & $\begin{array}{l}\text { Old-town, Arayer } \\
\text { Yoli, Grand } \\
\text { Bazaar }\end{array}$ & & $\begin{array}{l}\text { Coppersmith hammering heard } \\
\text { from inside a mosque courtyard }\end{array}$ \\
\hline - $1: 26: 26$ & Rawab street recording & $\begin{array}{l}\text { Köziciyerbişi - } \\
\text { Tuman street }\end{array}$ & $\begin{array}{l}\text { Unknown musician playing } \\
\text { Rawab }\end{array}$ & Street shop owner playing rawab \\
\hline - $1: 41: 17$ & Unknown Uyghur song & $\begin{array}{l}\text { Köziciyerbişi - } \\
\text { Tuman street }\end{array}$ & $\begin{array}{l}\text { Unknown local musicians } \\
\text { playing Uyghur instruments }\end{array}$ & $\begin{array}{l}\text { Song rehearsal for Xinjiang } \\
\text { anniversary }\end{array}$ \\
\hline - $1: 44: 34$ & $\begin{array}{l}\text { Recording the noise of } \\
\text { entertainment in daytime }\end{array}$ & Kashgar pond & & Water skiing \\
\hline - 1:47:02 & $\begin{array}{l}\text { Recording the sound of } \\
\text { Sama dance performed in } \\
\text { large numbers to } \\
\text { celebrate an Islamic } \\
\text { festival }\end{array}$ & Id Kah Mosque & $\begin{array}{l}\text { Three people on top of the } \\
\text { darwaza (the main gate) of } \\
\text { Id Kah Mosque, with one of } \\
\text { them blowing a sunayi, and } \\
\text { the two others drumming a } \\
\text { dap. }\end{array}$ & $\begin{array}{l}\text { Sama dance after a long time ban, } \\
\text { reluctant and surprised locals. In } \\
\text { fact, it was allowed because of a } \\
\text { casting of a Chinese documentary. } \\
\text { It was a big surprise for people so } \\
\text { they initially hesitated to join. }\end{array}$ \\
\hline
\end{tabular}




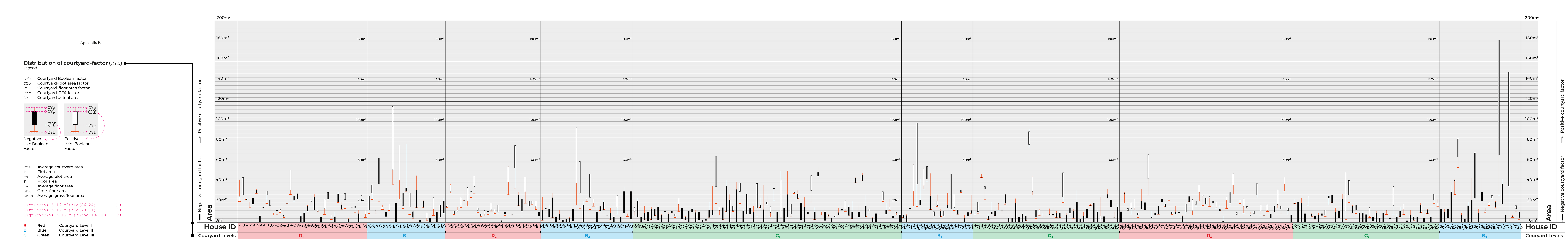




\section{Appendix C}

The below images show the quality of virtual environments generated with photogrammetry. These 3D models are made in Autodesk ReCap and uploaded in Sketchfab.

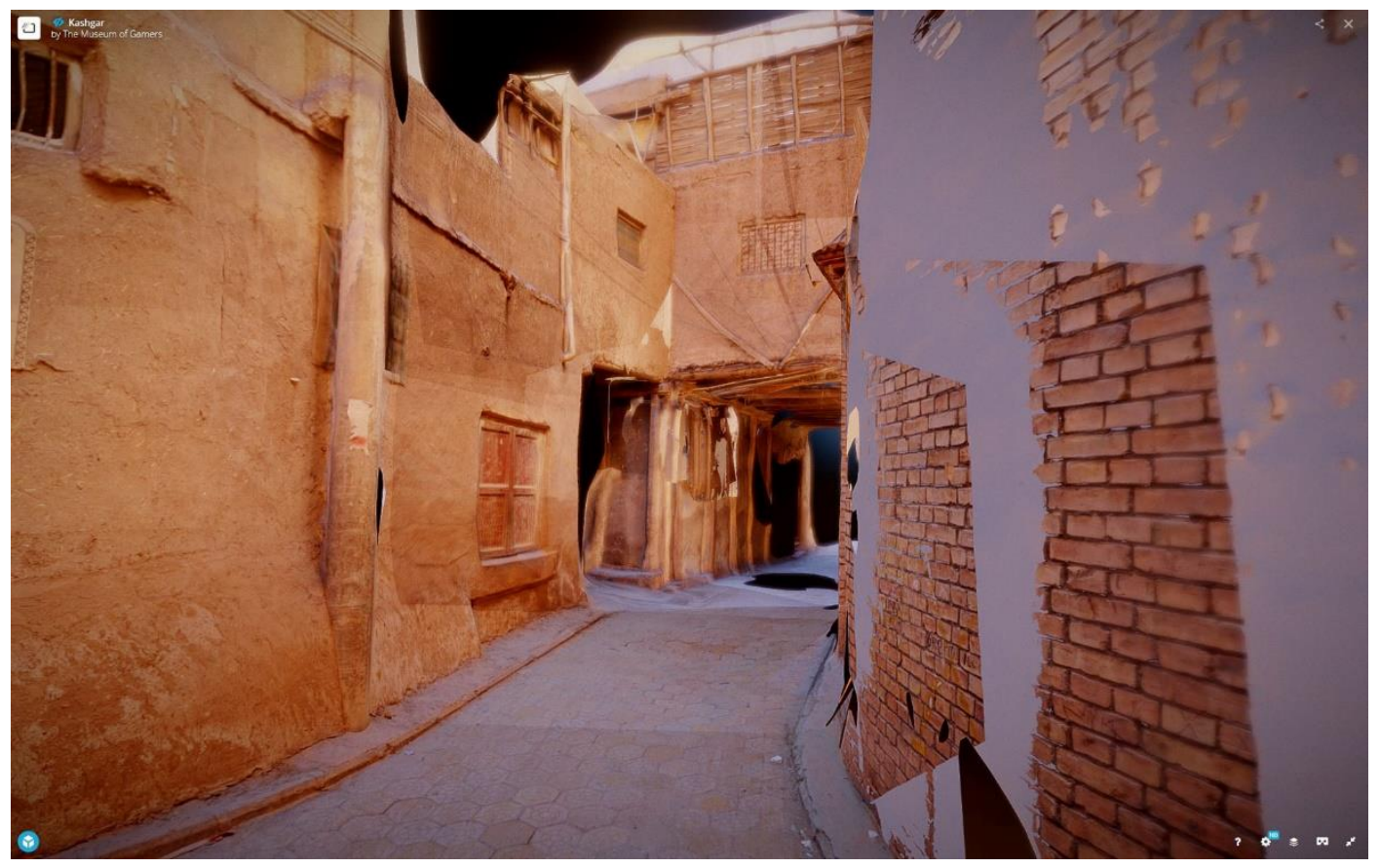

Fig. 124. A view of the virtual model of the narrow lanes in Köziciyerbişi (Gaotai). 
316

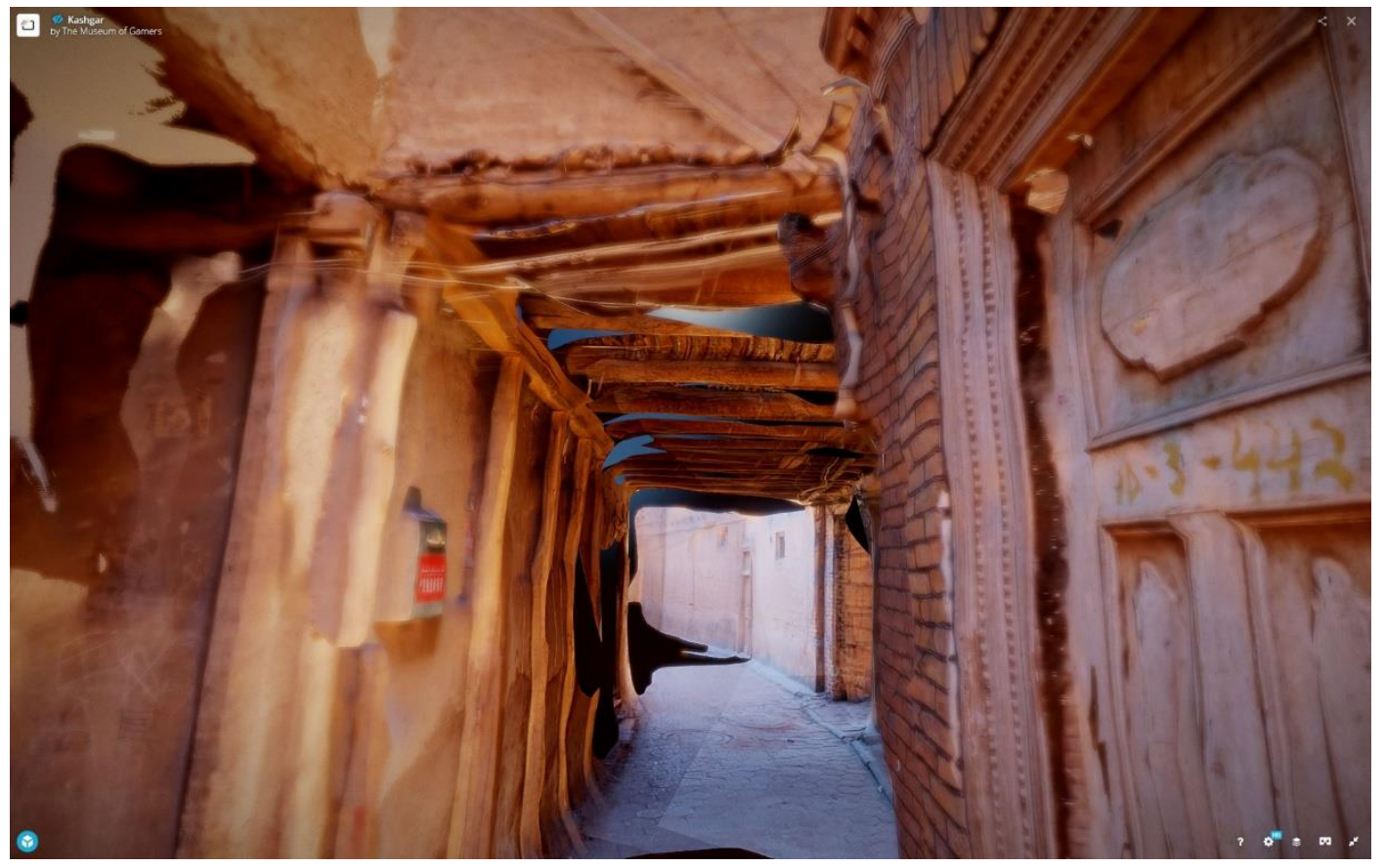

Fig. 125. A view of the virtual model of a passage lane in Köziciyerbişi (Gaotai).

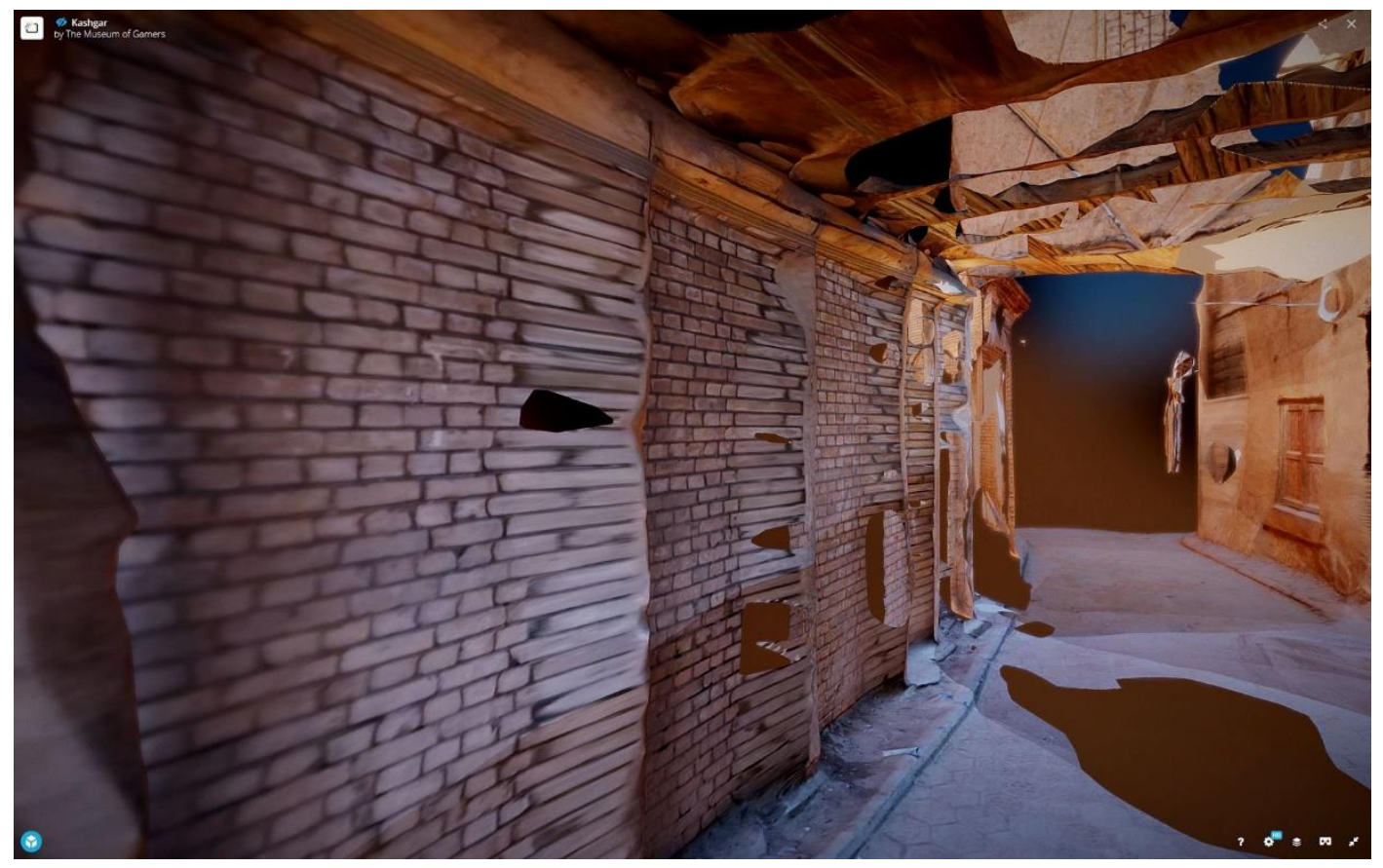

Fig. 126. A view of glitches seen on the virtual model of a passage lane in Köziciyerbişi (Gaotai). 


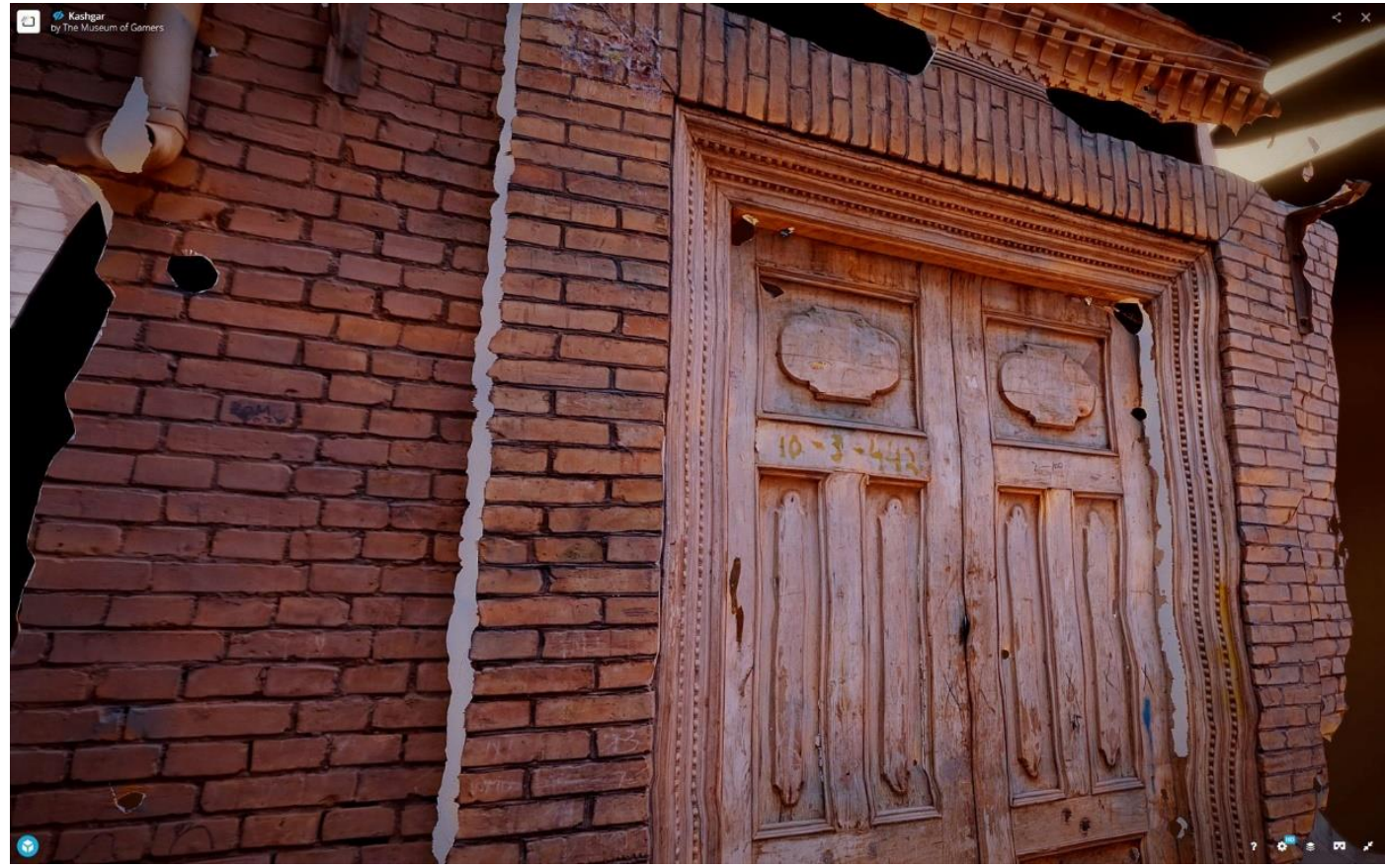

Fig. 127. A view of the virtual model of a timber door in Köziciyerbişi (Gaotai).

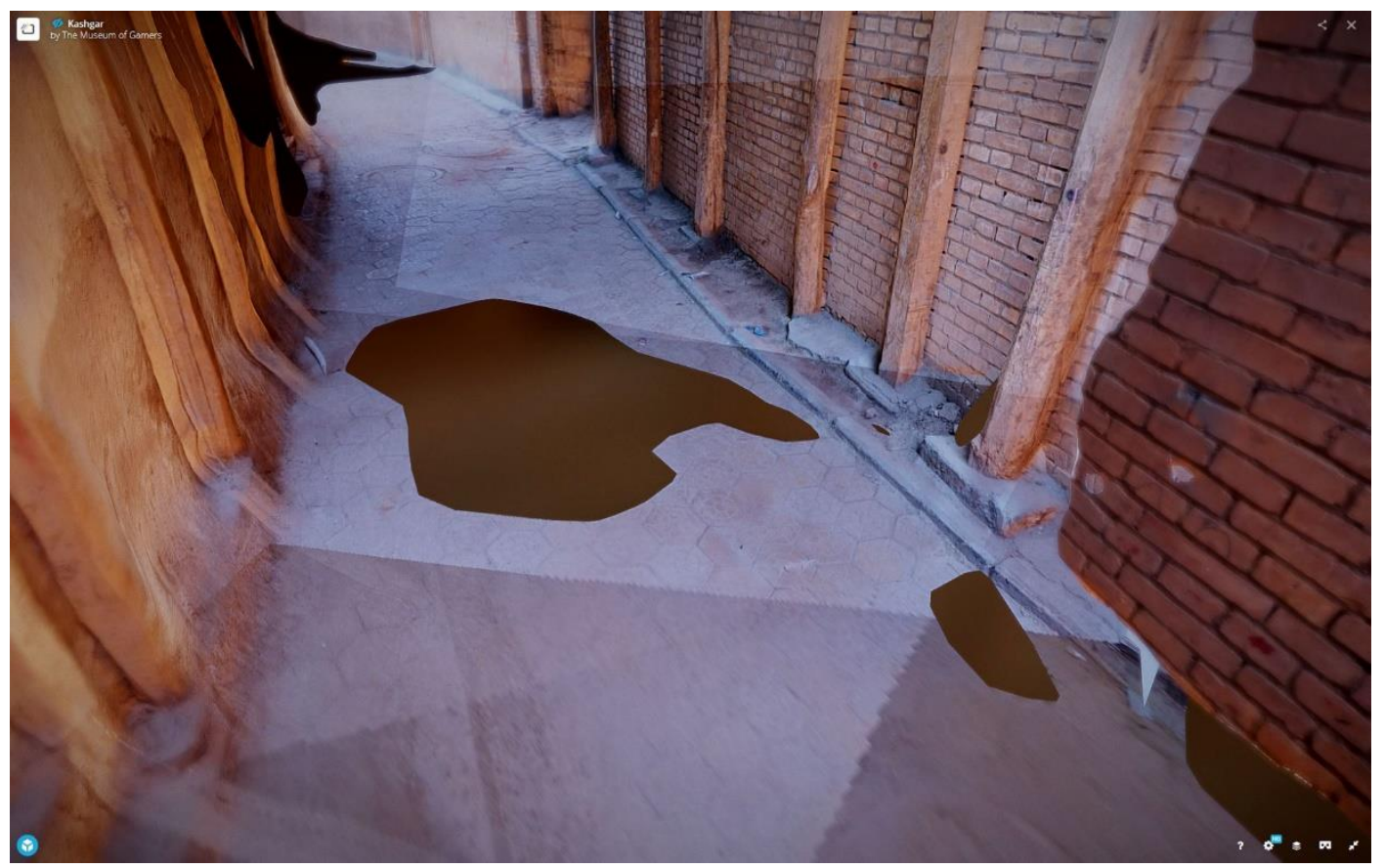

Fig. 128. A detail of the virtual model of a pavement section in Köziciyerbişi (Gaotai). Holes appear in the middle of a passage lane where light was limited during taking the photos. 
318

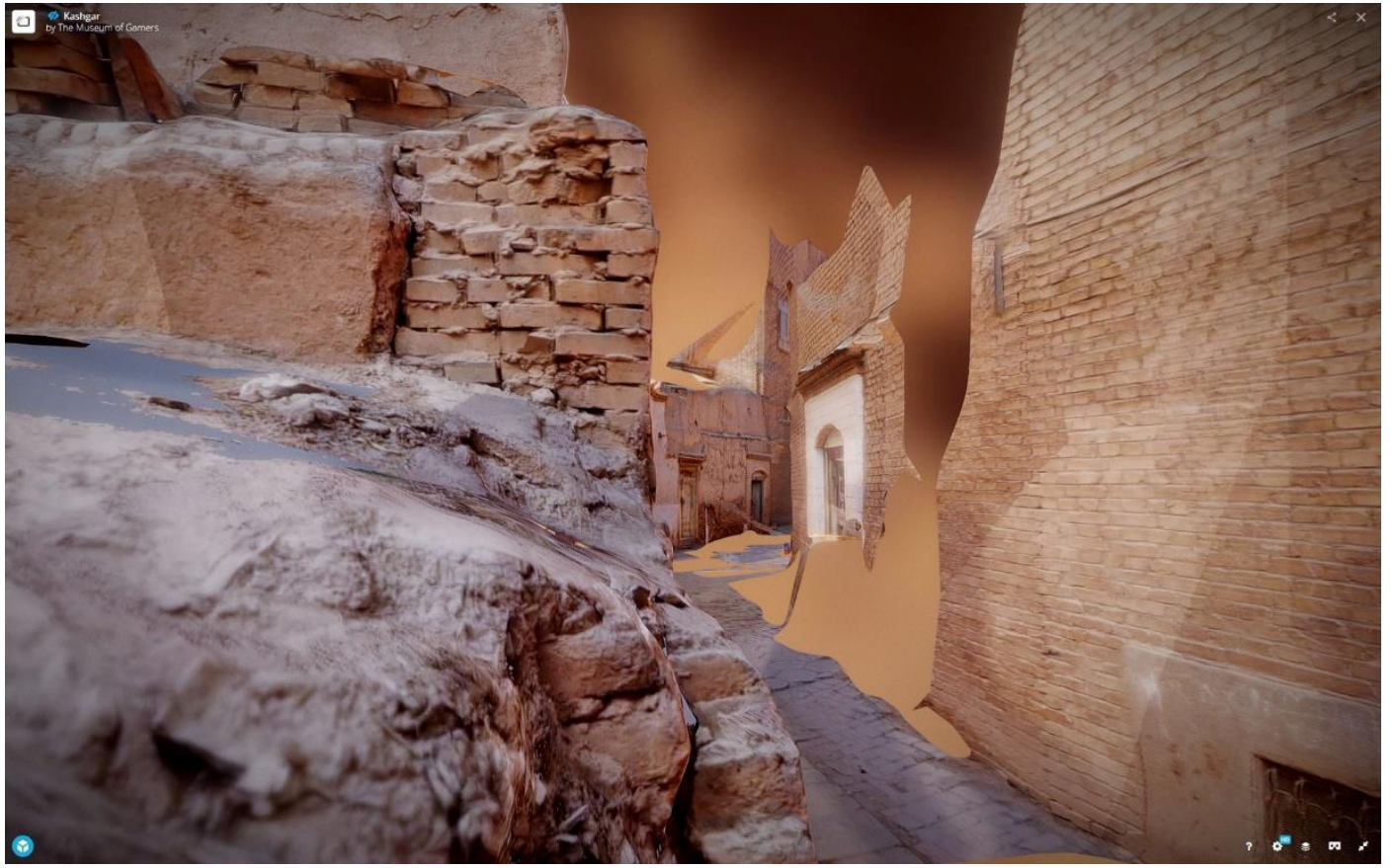

Fig. 129. A detail of the virtual model of a brick wall section being demolished in Köziciyerbişi (Gaotai).

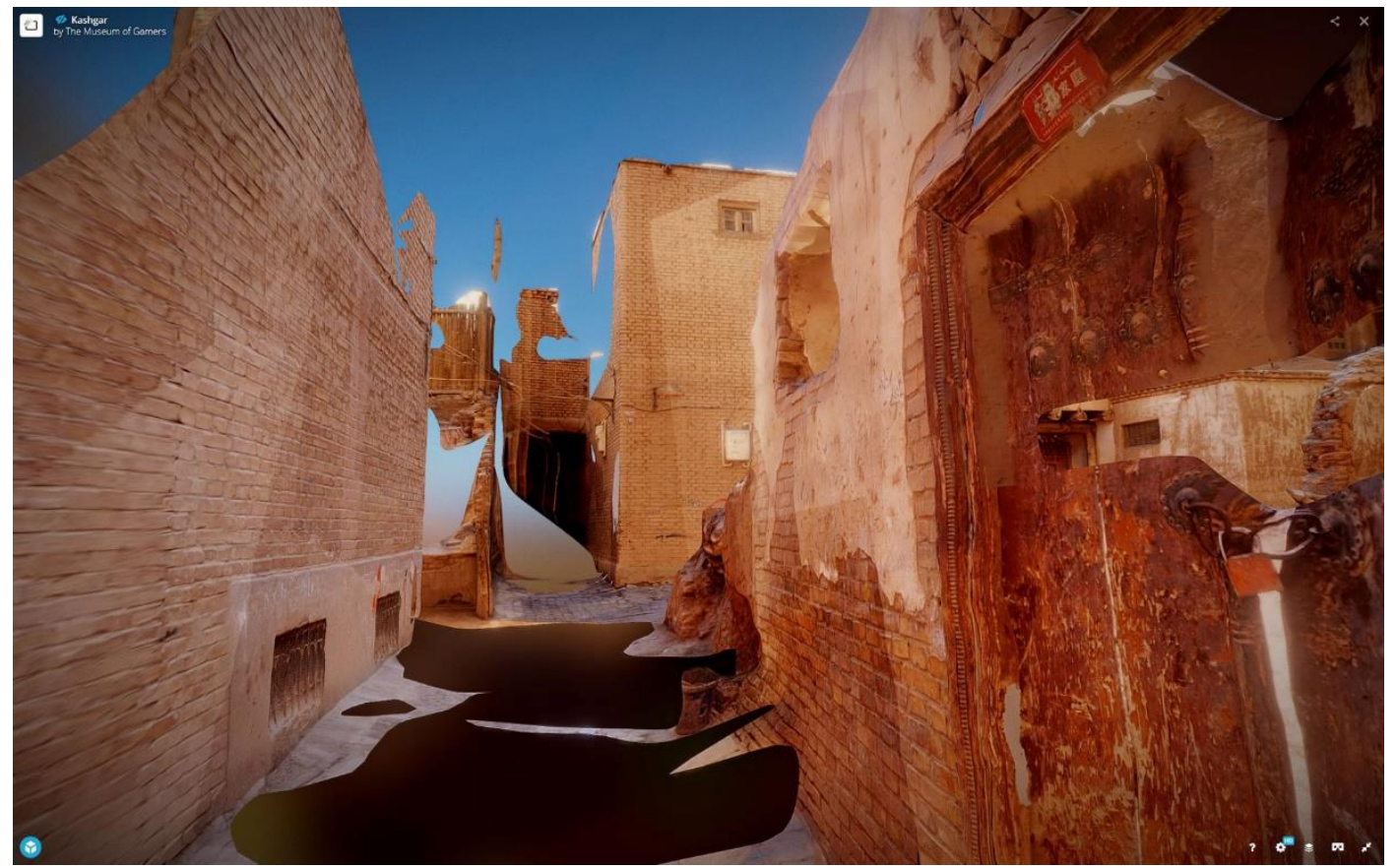

Fig. 130. A view of the virtual model of a narrow lane in Köziciyerbişi (Gaotai), with holes that appeared on the ground because of the shade of the surrounding walls. 


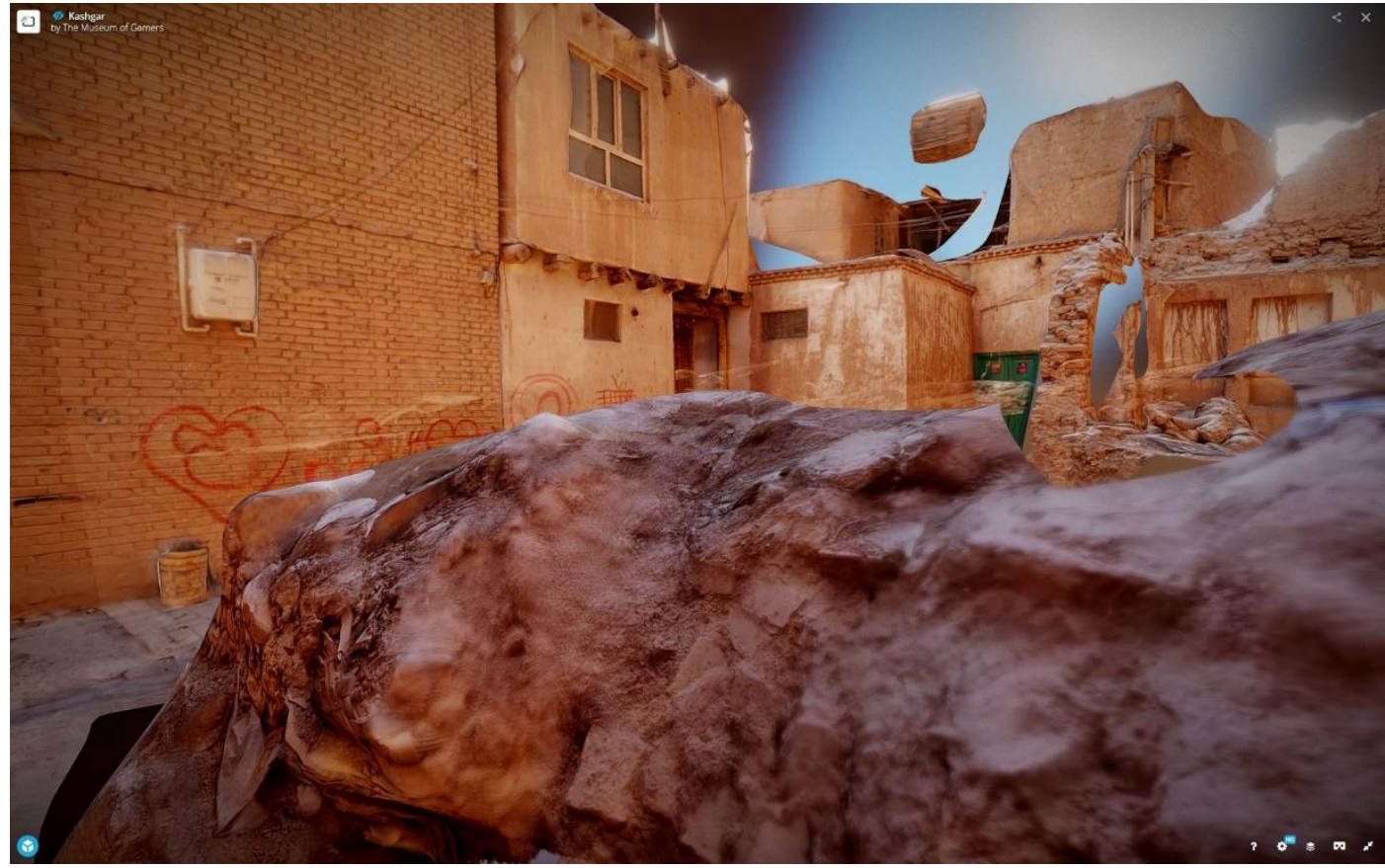

Fig. 131. A detail of the virtual model of a brick wall section being demolished in Köziciyerbişi (Gaotai).

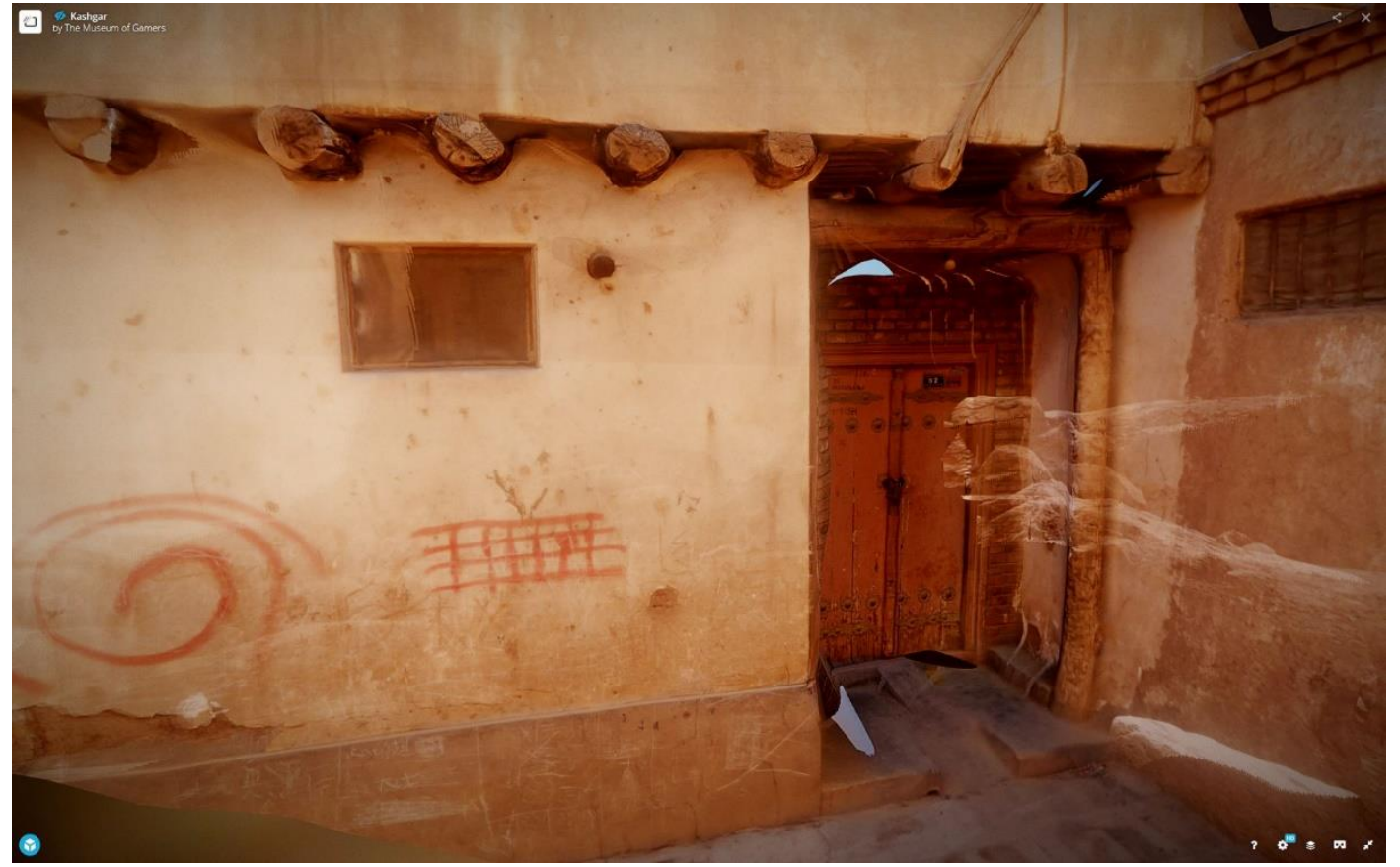

Fig. 132. A view of the virtual model of a house entrance with timber structure extending from the wall in Köziciyerbişi (Gaotai). 


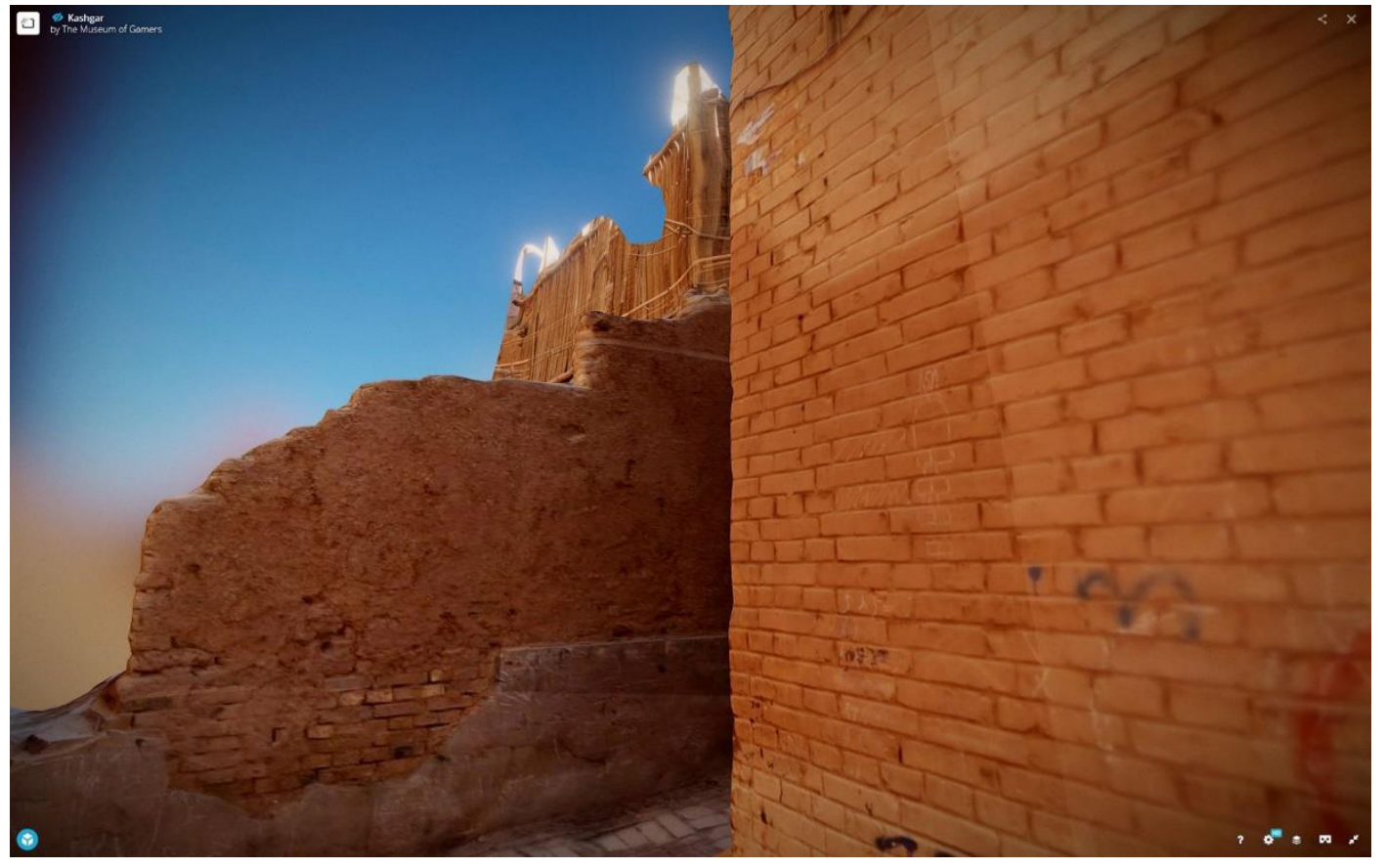

Fig. 133. A view of the virtual model of a junction in Köziciyerbişi (Gaotai).

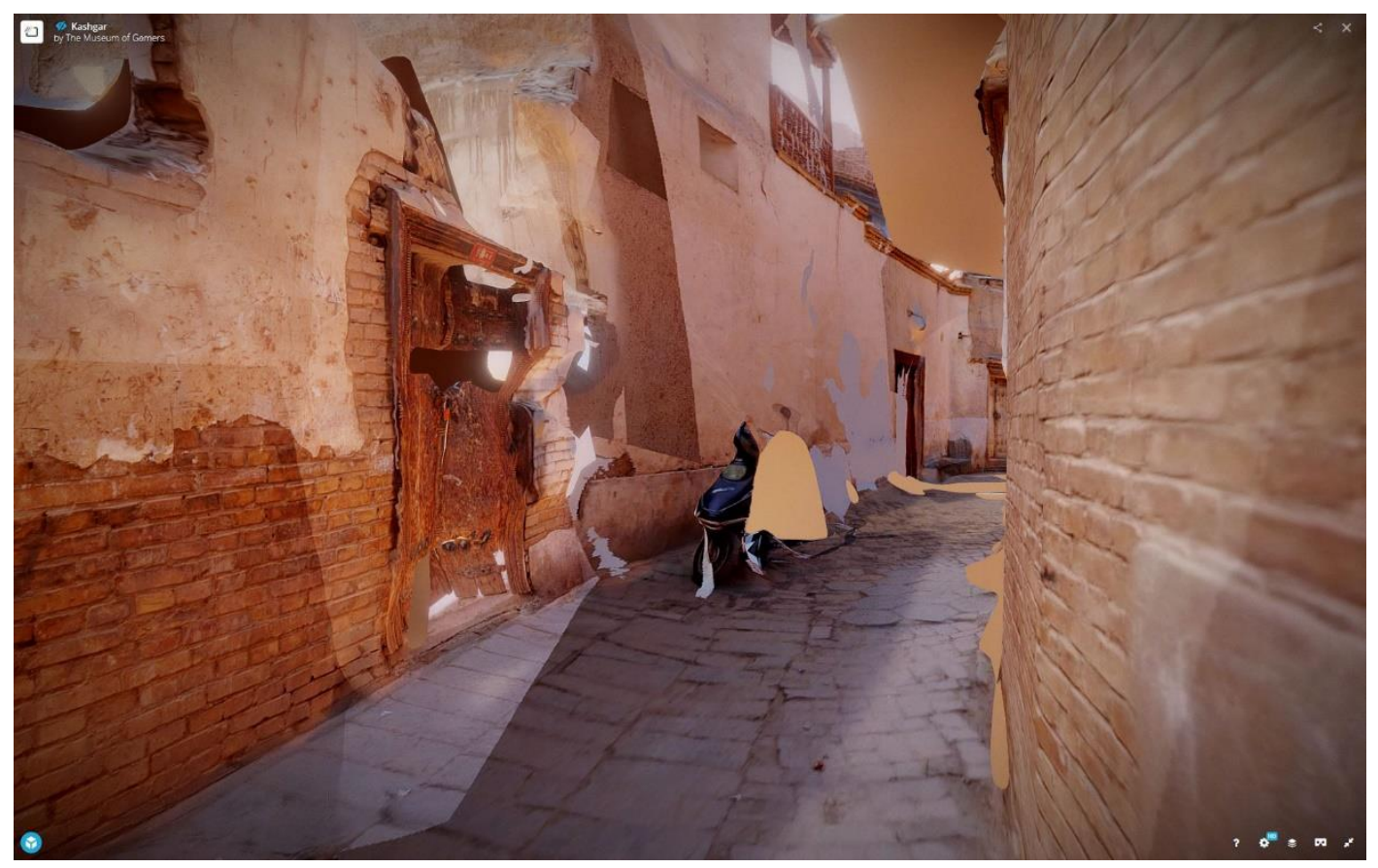

Fig. 134. A view of the virtual model of a narrow lane in Köziciyerbişi (Gaotai), with glitches that appeared on the texture of the geometry. 


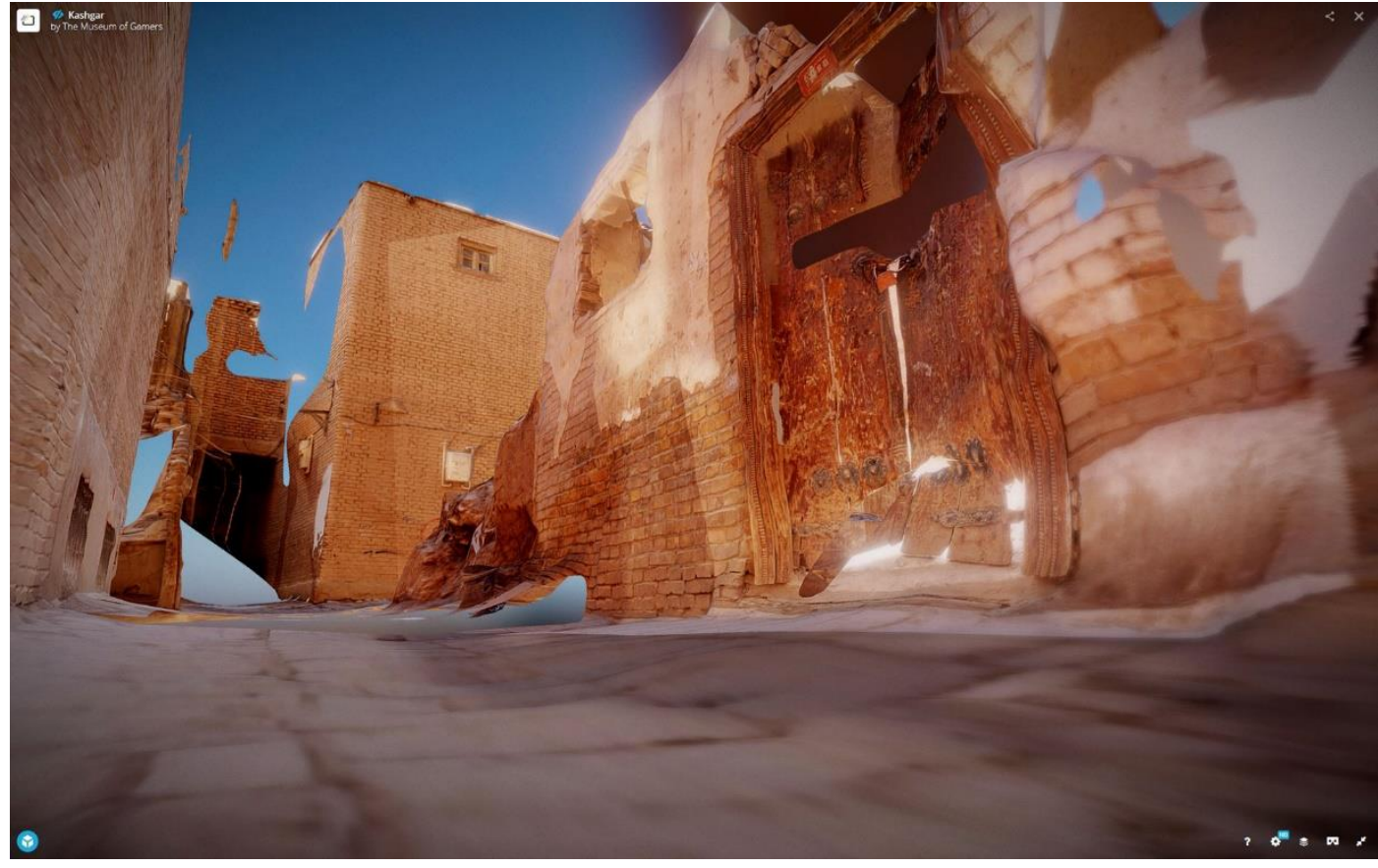

Fig. 135. A view of the virtual model of a narrow lane in Köziciyerbişi (Gaotai).

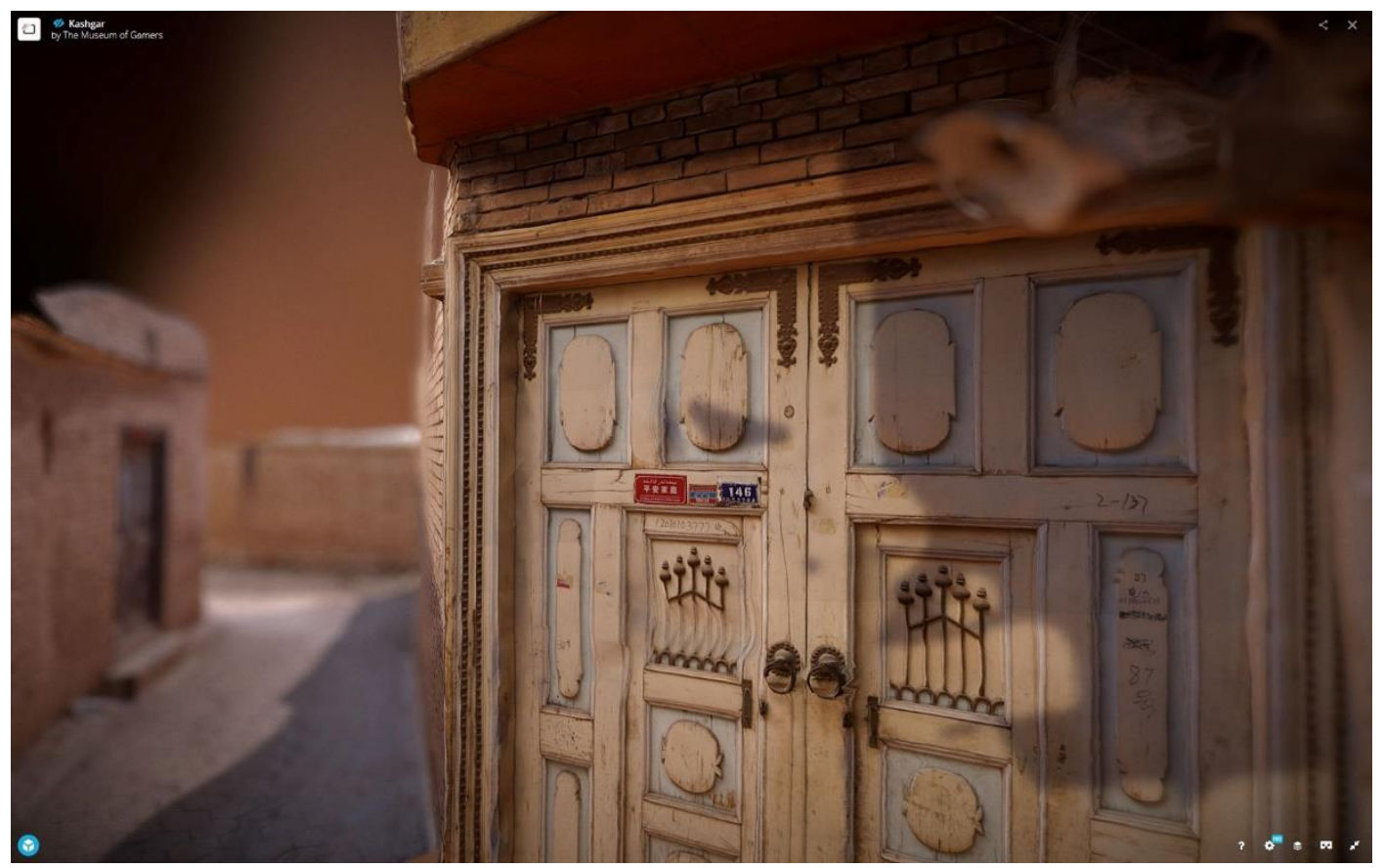

Fig. 136. A view of the virtual model of a timber door in Köziciyerbişi (Gaotai). 
322

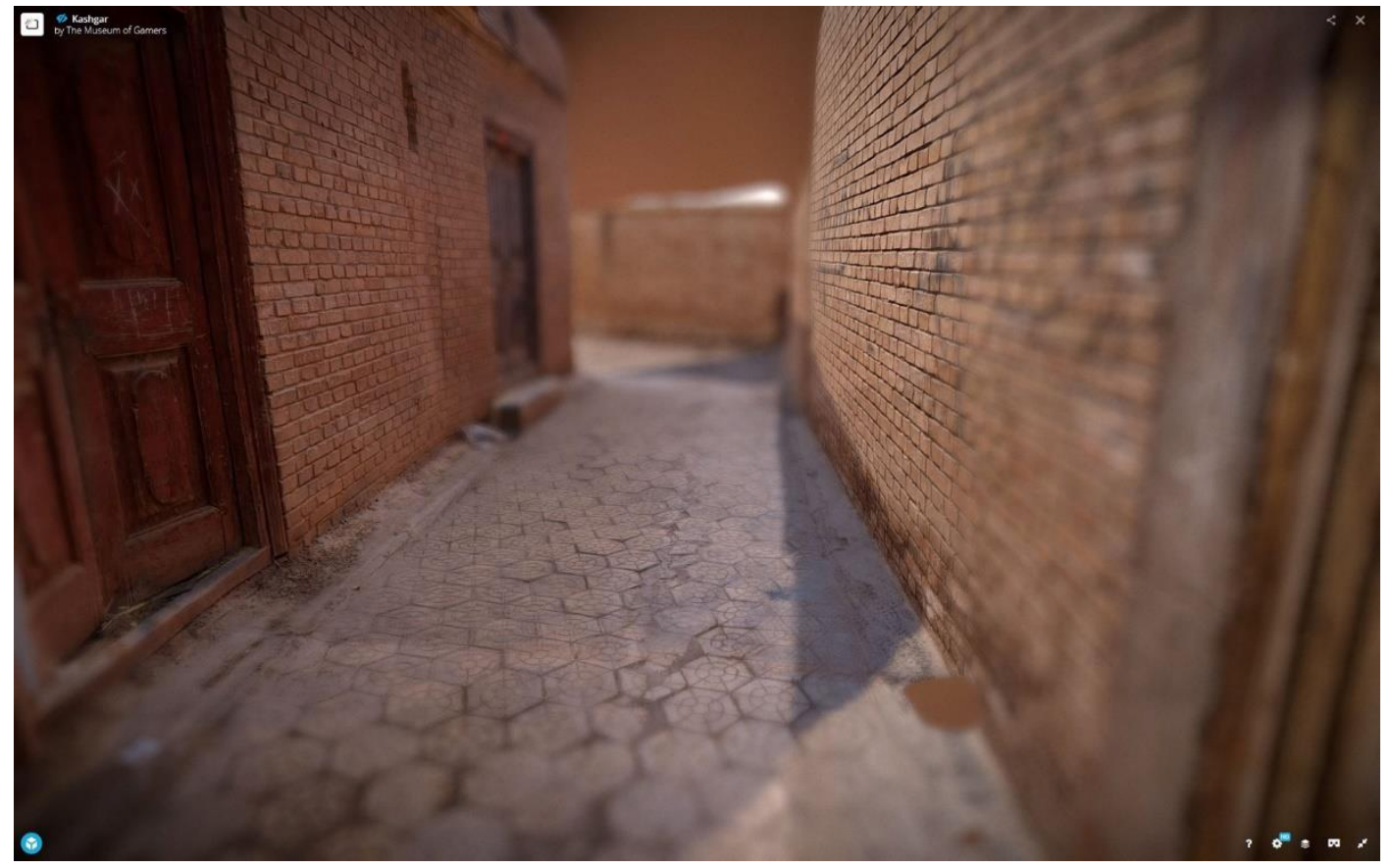

Fig. 137. A view of the virtual model of a narrow lane in Köziciyerbişi (Gaotai).

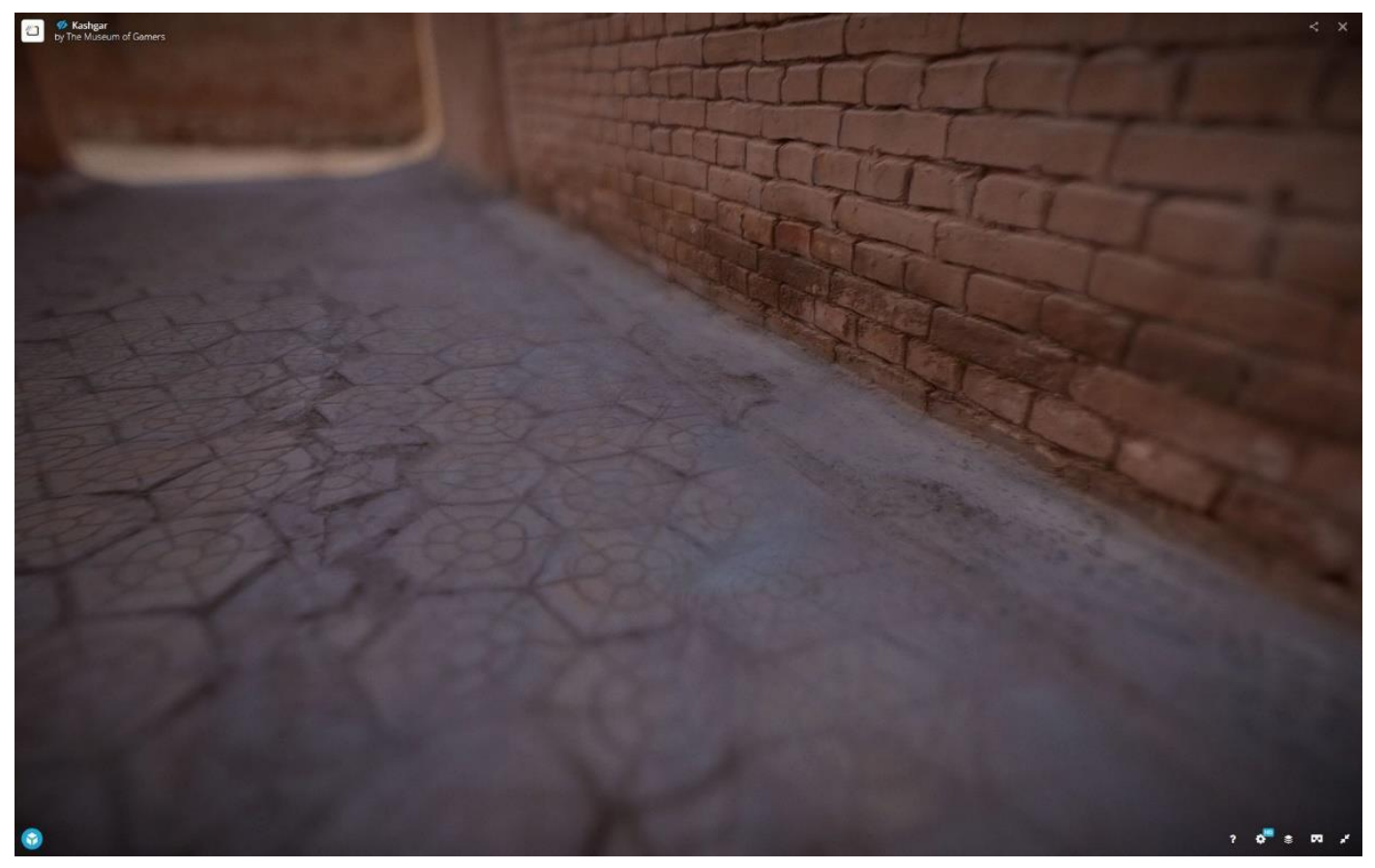

Fig. 138. A detail of the virtual model of a ground section in Köziciyerbişi (Gaotai), with a texture of the hexagonal pattern. 


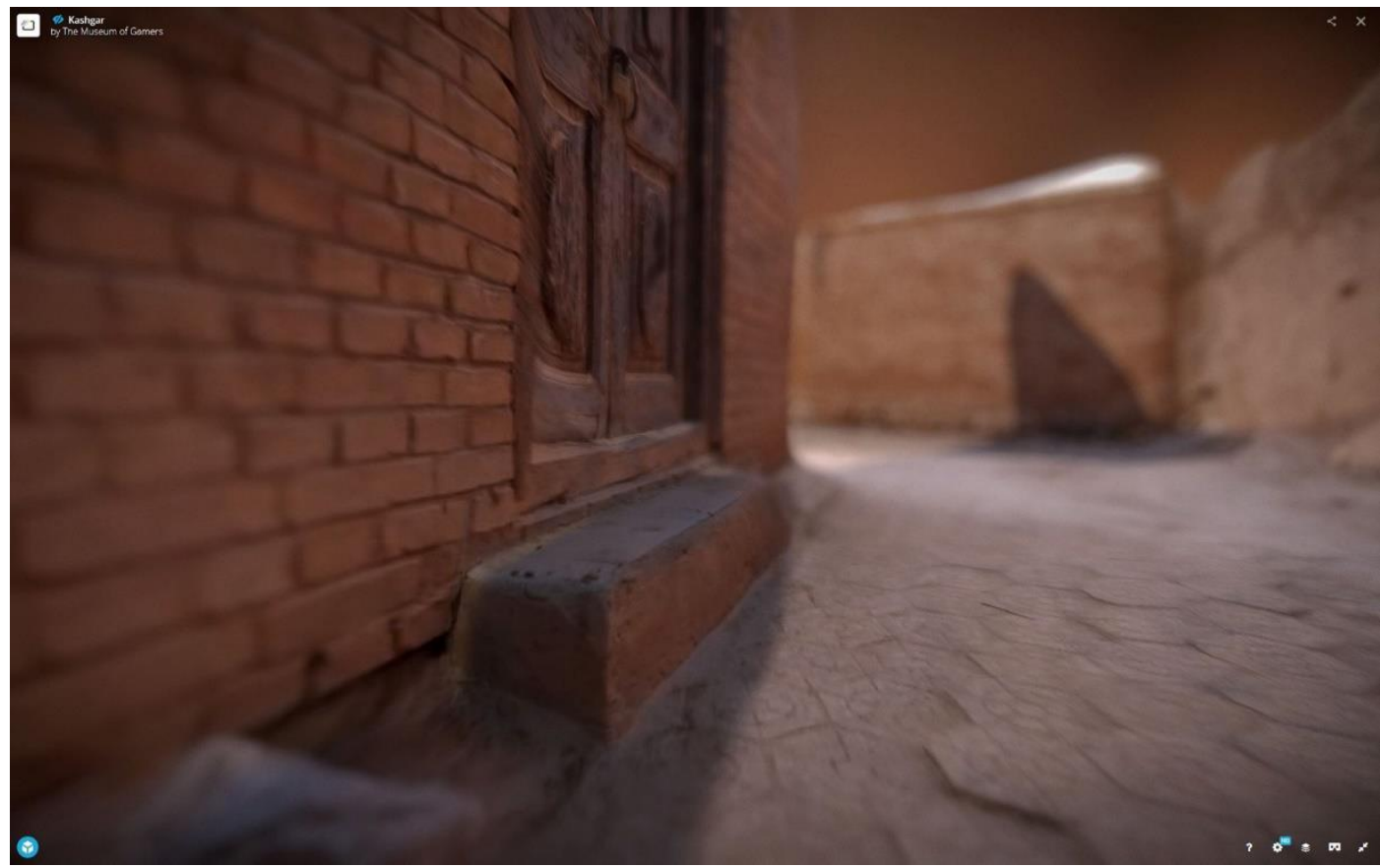

Fig. 139. A detail of the virtual model of a house entrance in Köziciyerbişi (Gaotai).

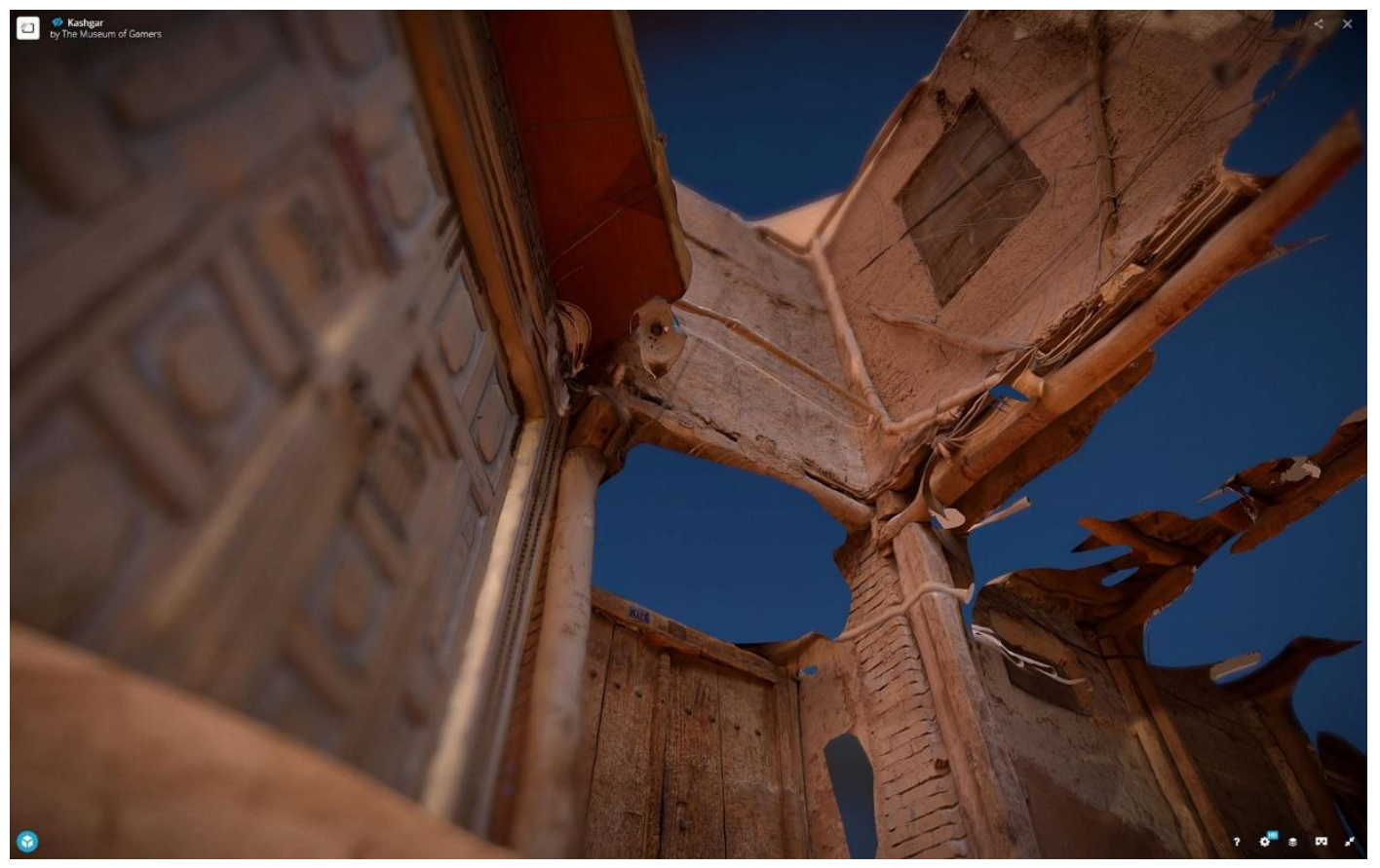

Fig. 140. An upward view of the virtual model of mud-brick walls and timber doors in Köziciyerbişi (Gaotai). 
324

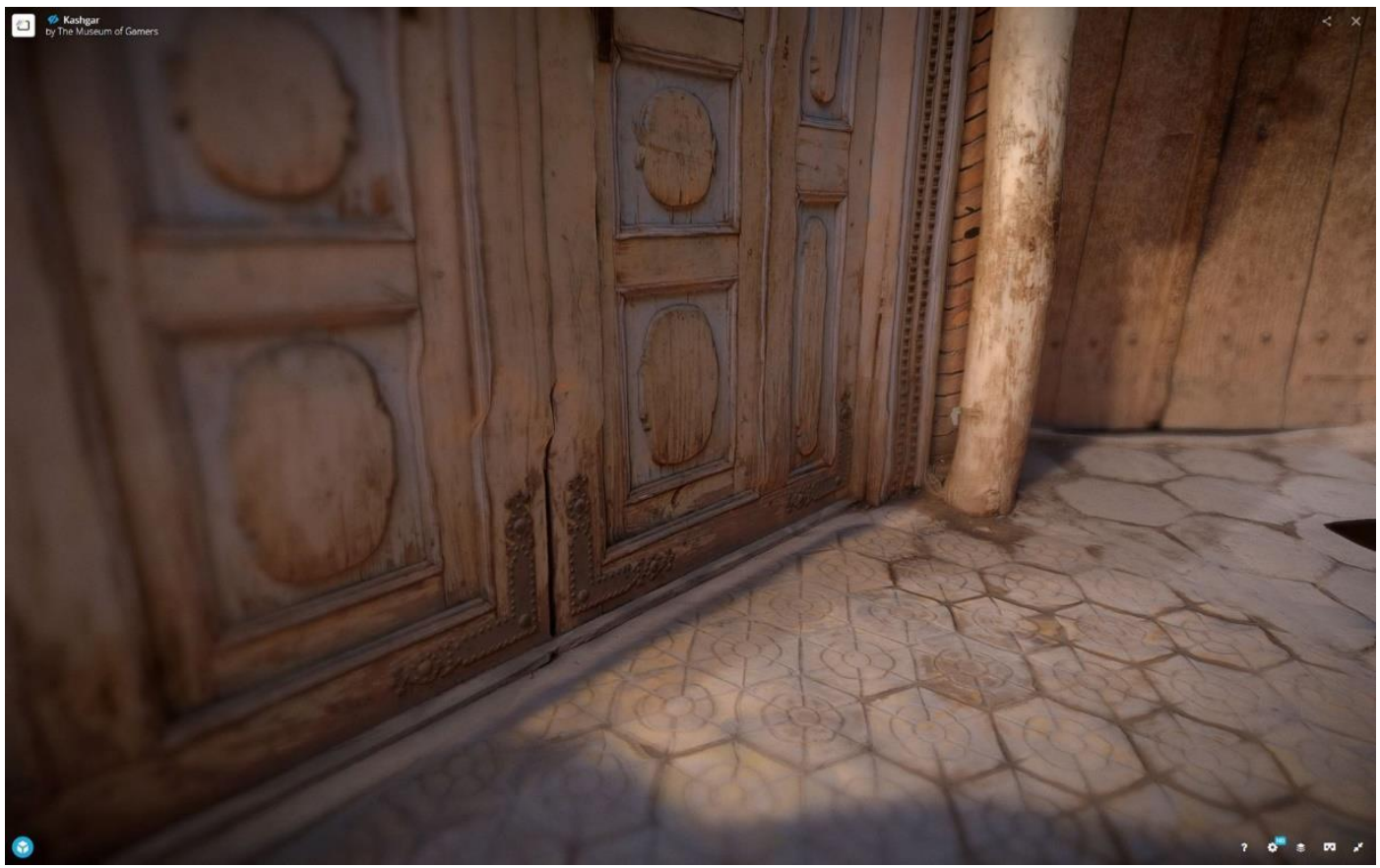

Fig. 141. A view of the virtual model of a timber door with ground pattern detail in Köziciyerbişi (Gaotai).

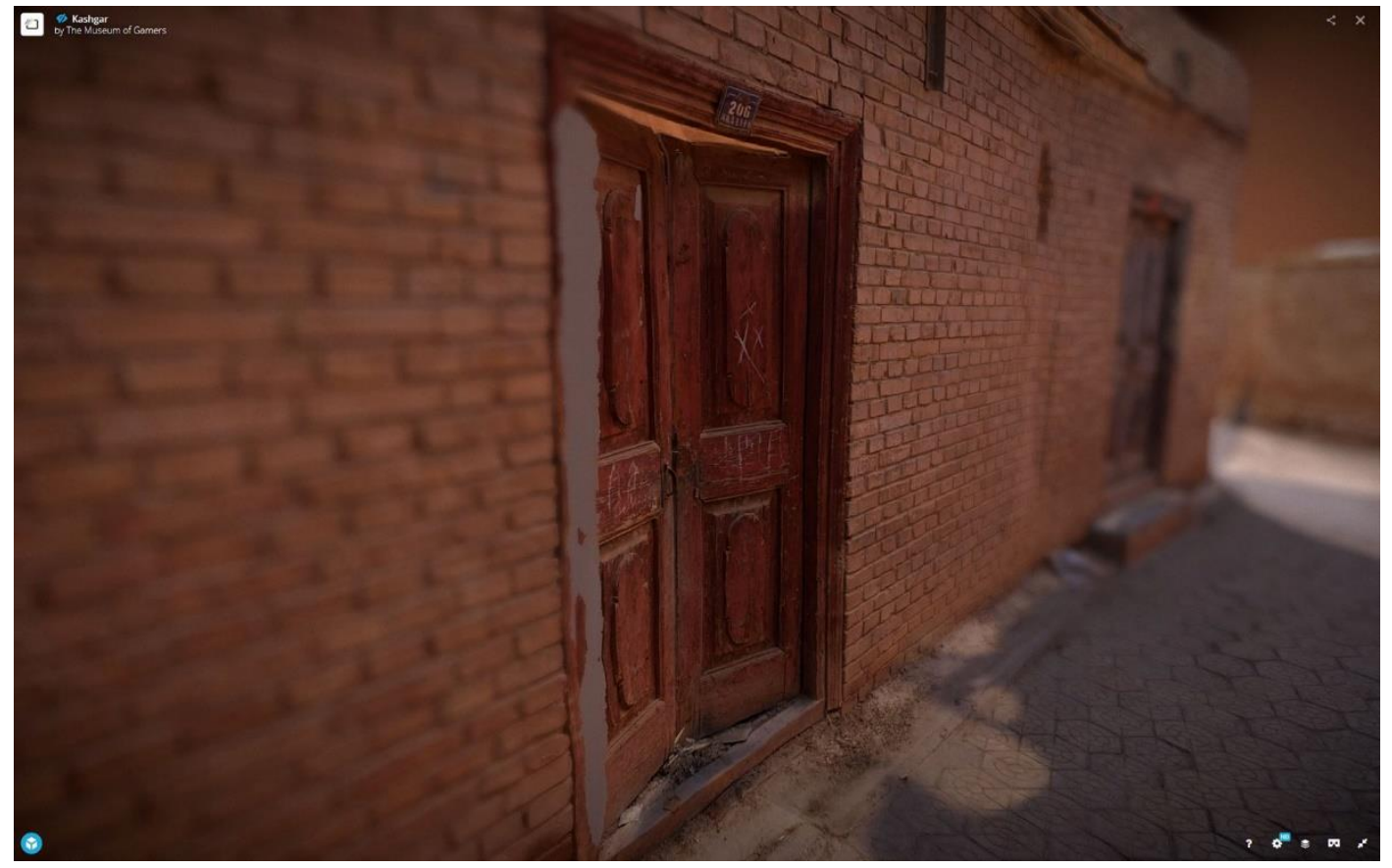

Fig. 142. A view of the virtual model of a timber door on a brick wall in a narrow lane in Köziciyerbişi (Gaotai). 


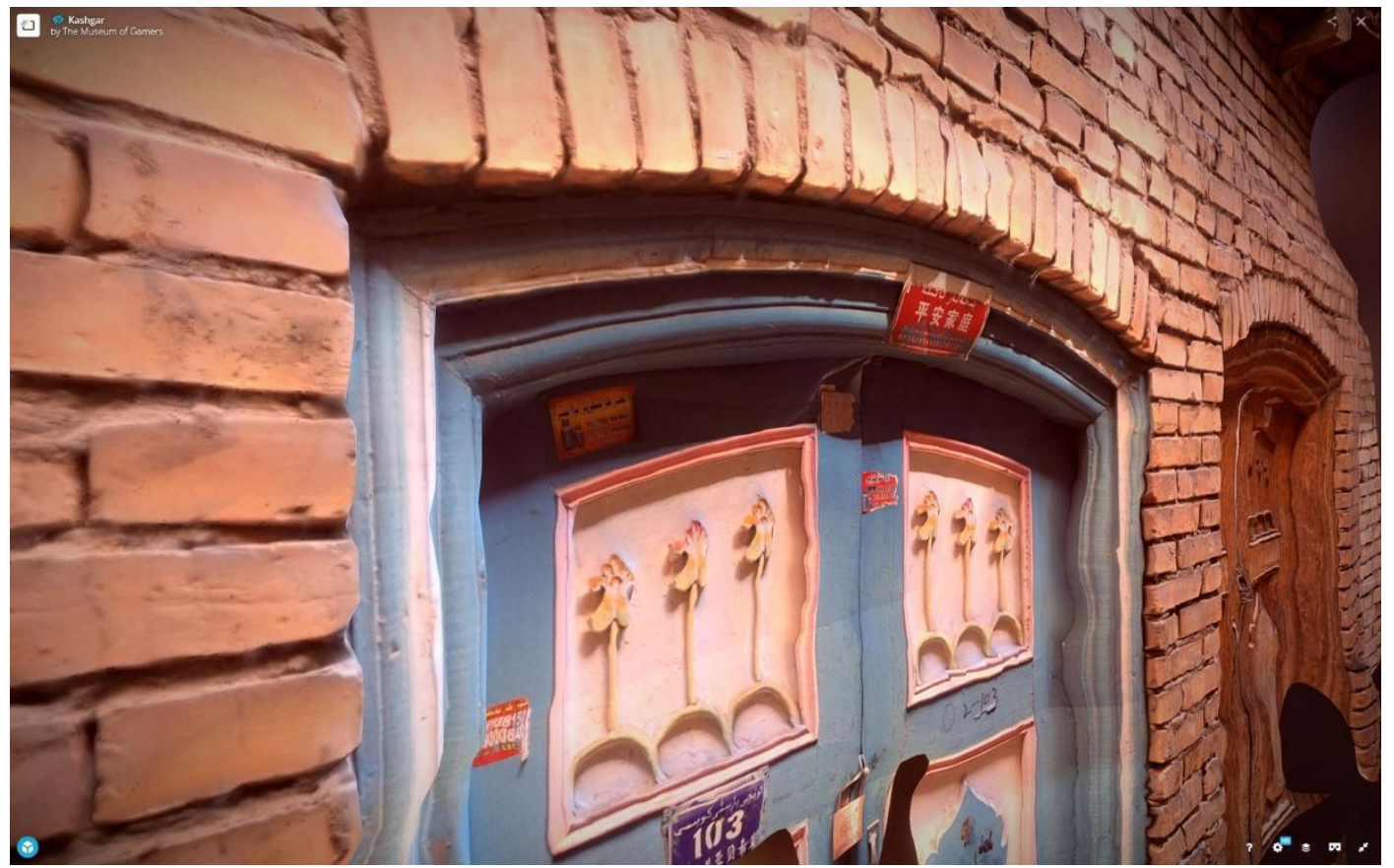

Fig. 143. A detail of the virtual model of a brick arch over a timber wall in Köziciyerbişi (Gaotai).

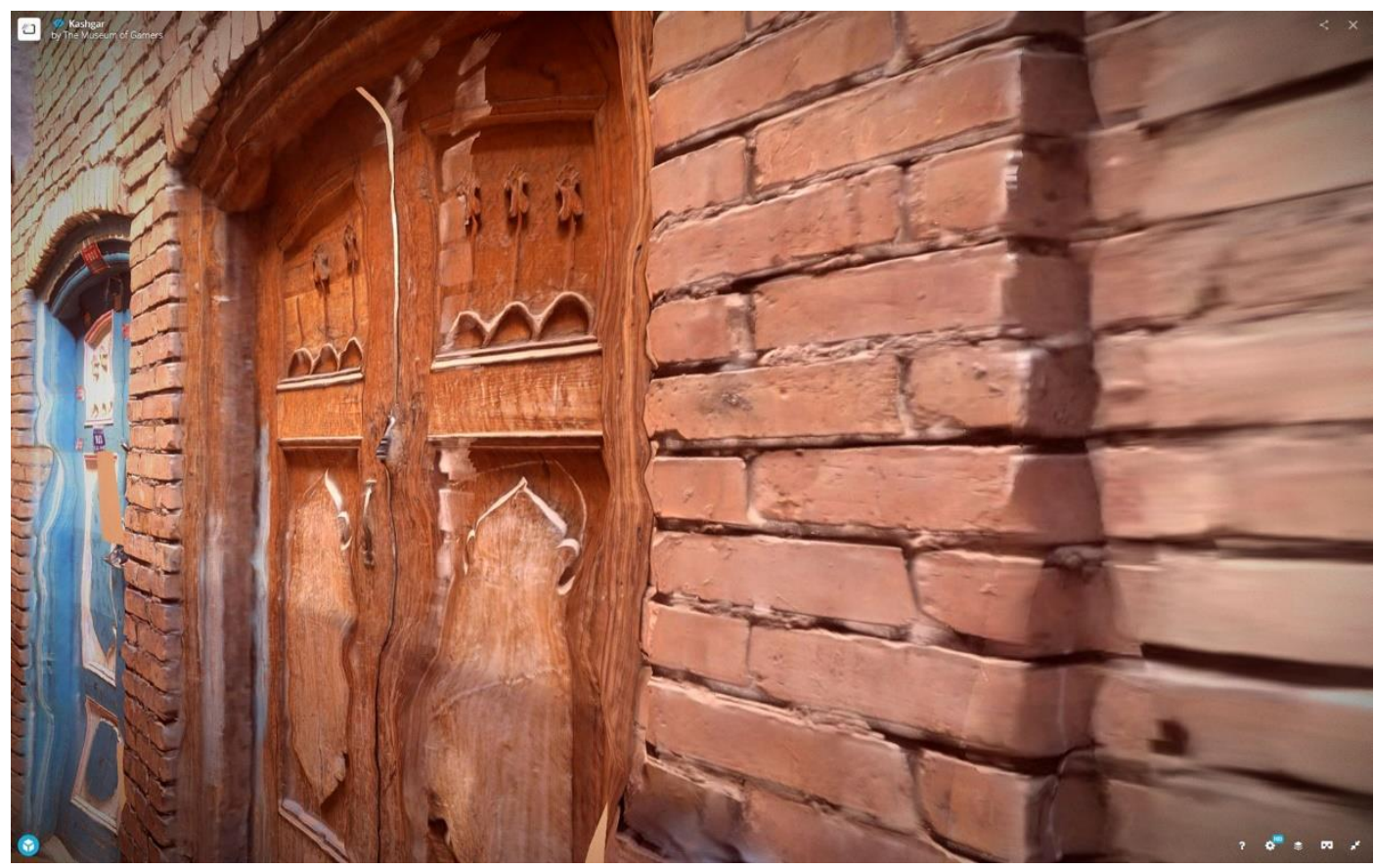

Fig. 144. A view of the virtual model of a timber door in Köziciyerbişi (Gaotai). 
326

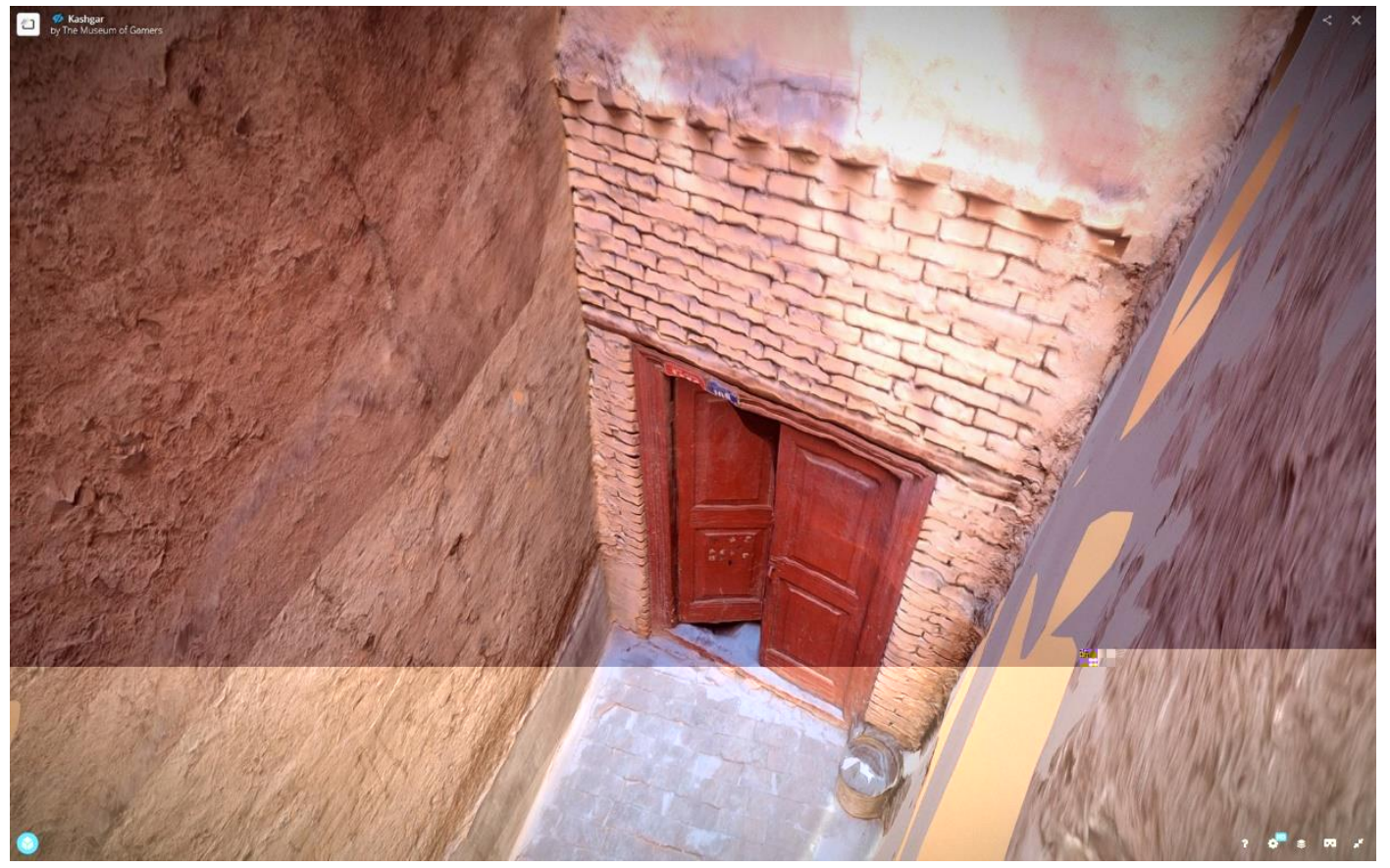

Fig. 145. A downward view of the virtual model of a timber door at the end of a cull-de-sac in Köziciyerbişi (Gaotai).

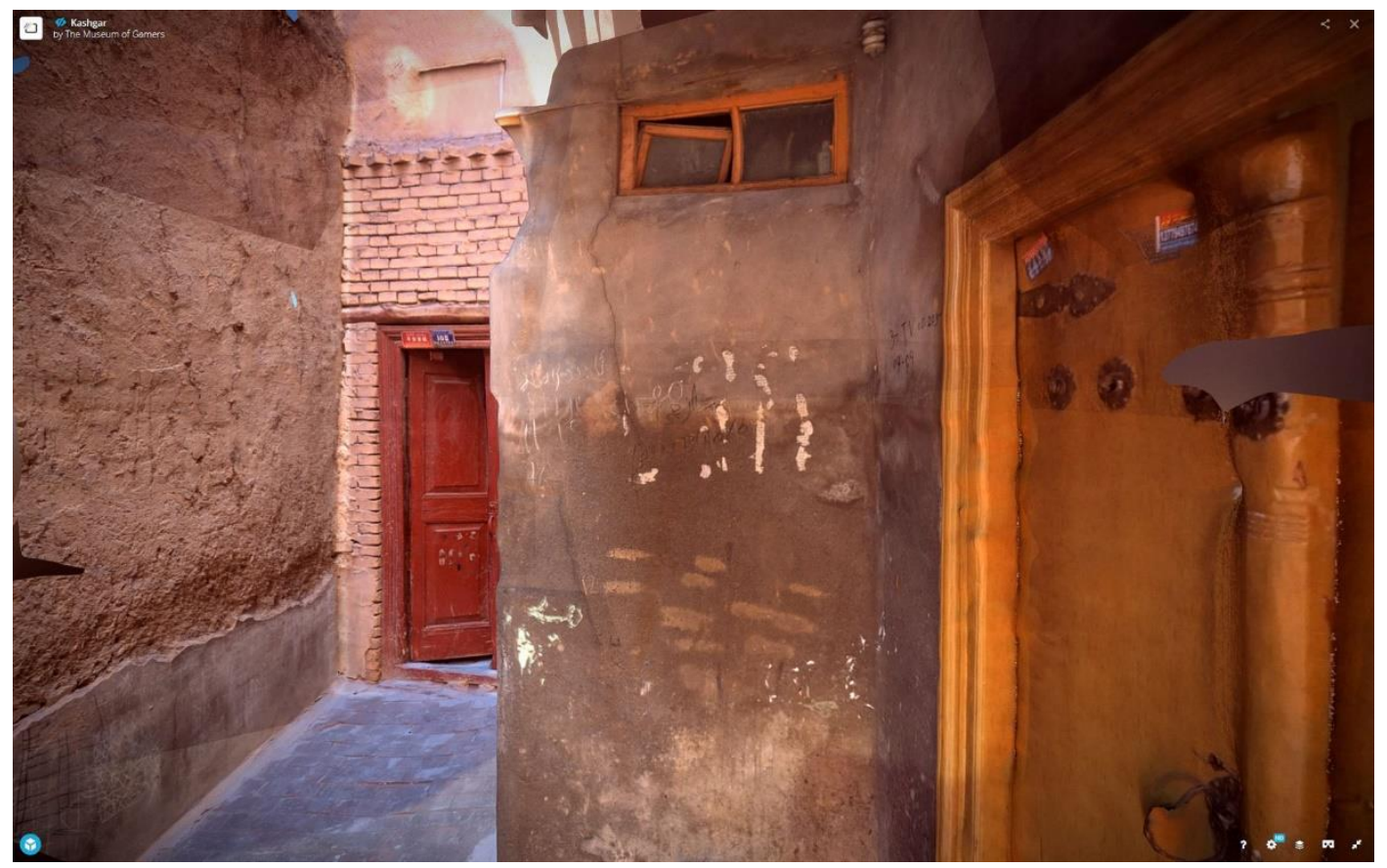

Fig. 146. A view of the virtual model of a cull-de-sac in Köziciyerbişi (Gaotai). 


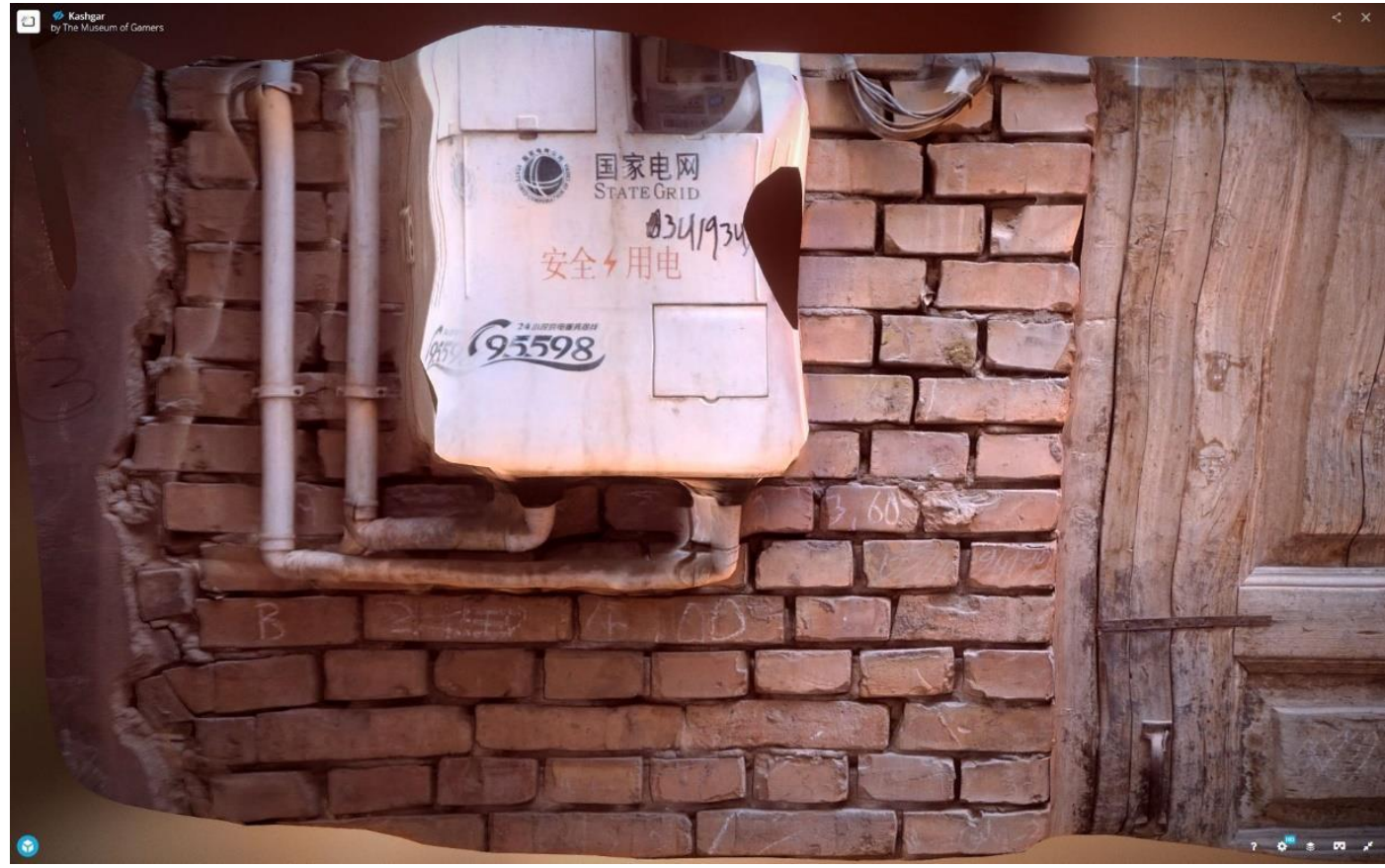

Fig. 147. A view of the virtual model of a gas meter hung on a brick wall in Köziciyerbişi (Gaotai).

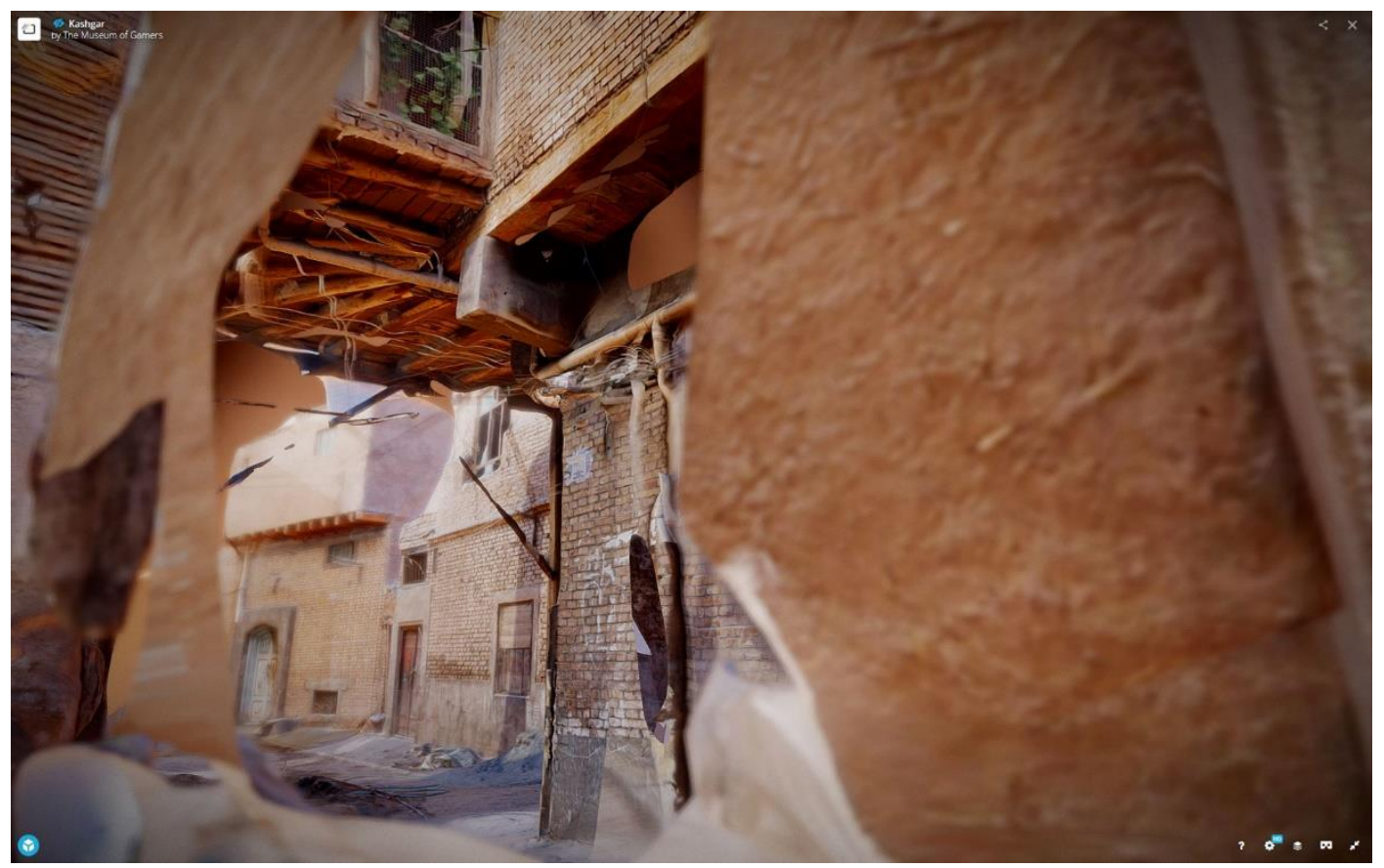

Fig. 148. An upward view of the virtual model of a junction of narrow lanes in Köziciyerbişi (Gaotai). 
328

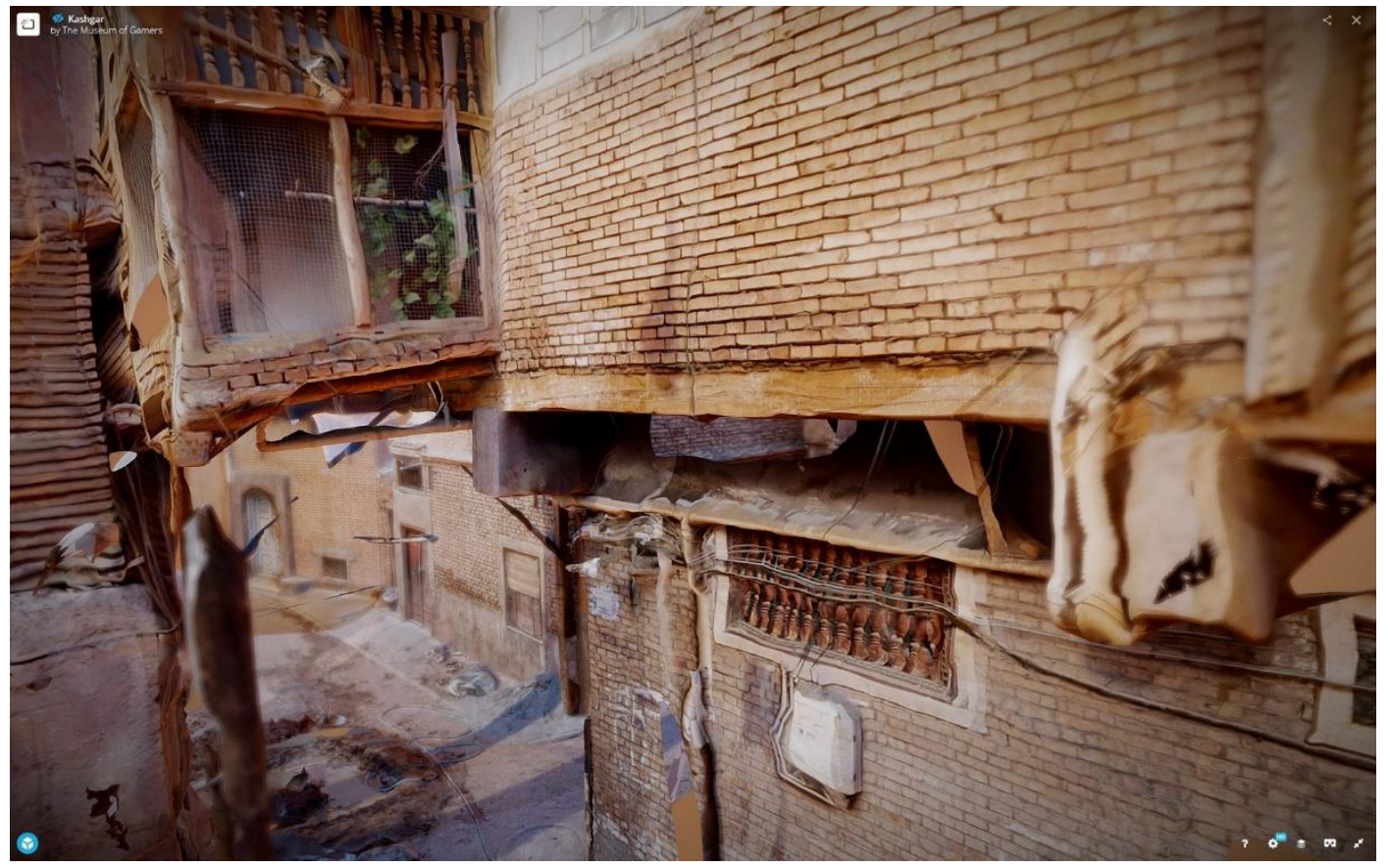

Fig. 149. A view of the virtual model of a timber extension overpassing a junction of narrow lanes in Köziciyerbişi (Gaotai).

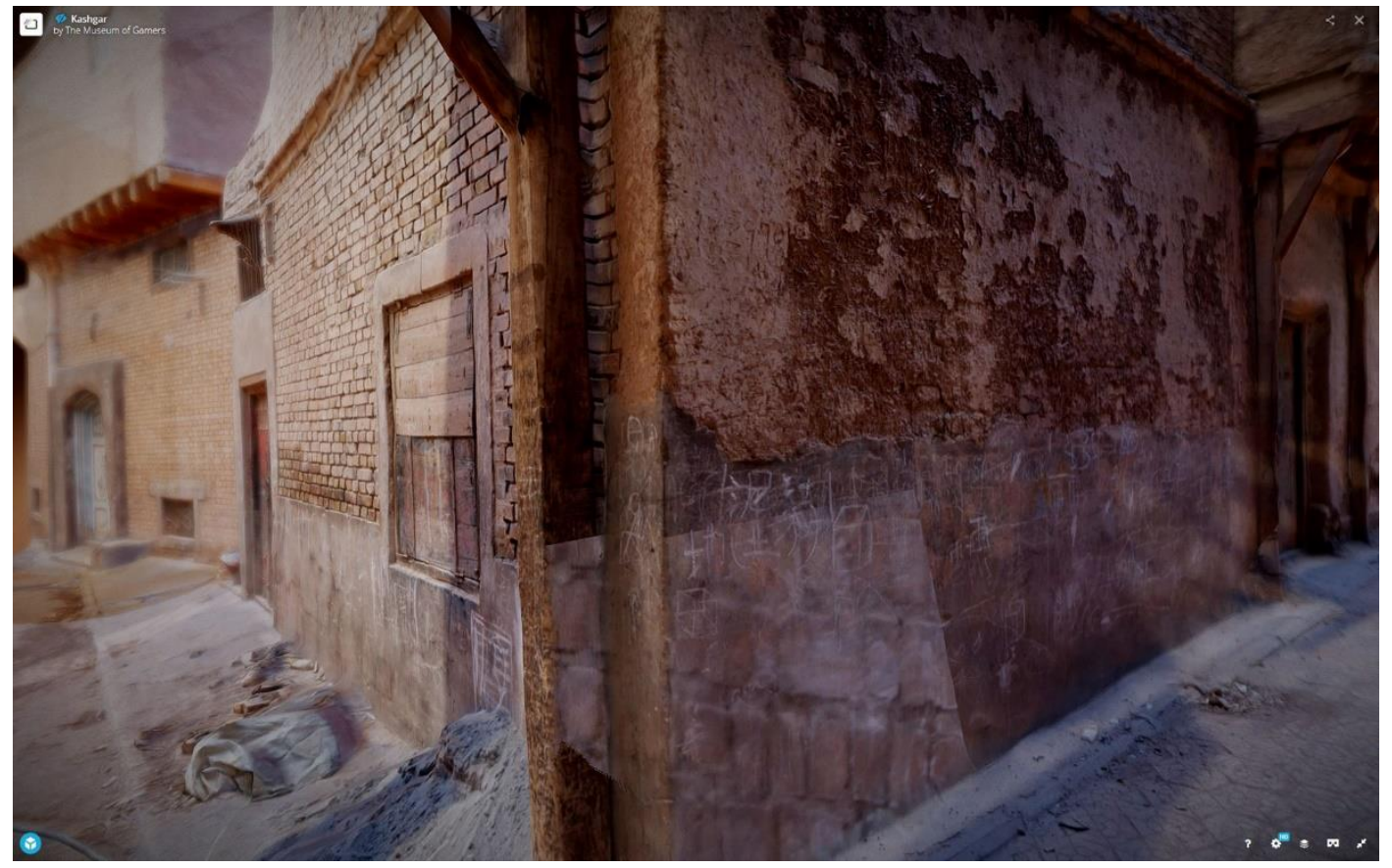

Fig. 150. A detail of the virtual model of a timber structure that carries an overpassing extension of a house in Köziciyerbişi (Gaotai). 


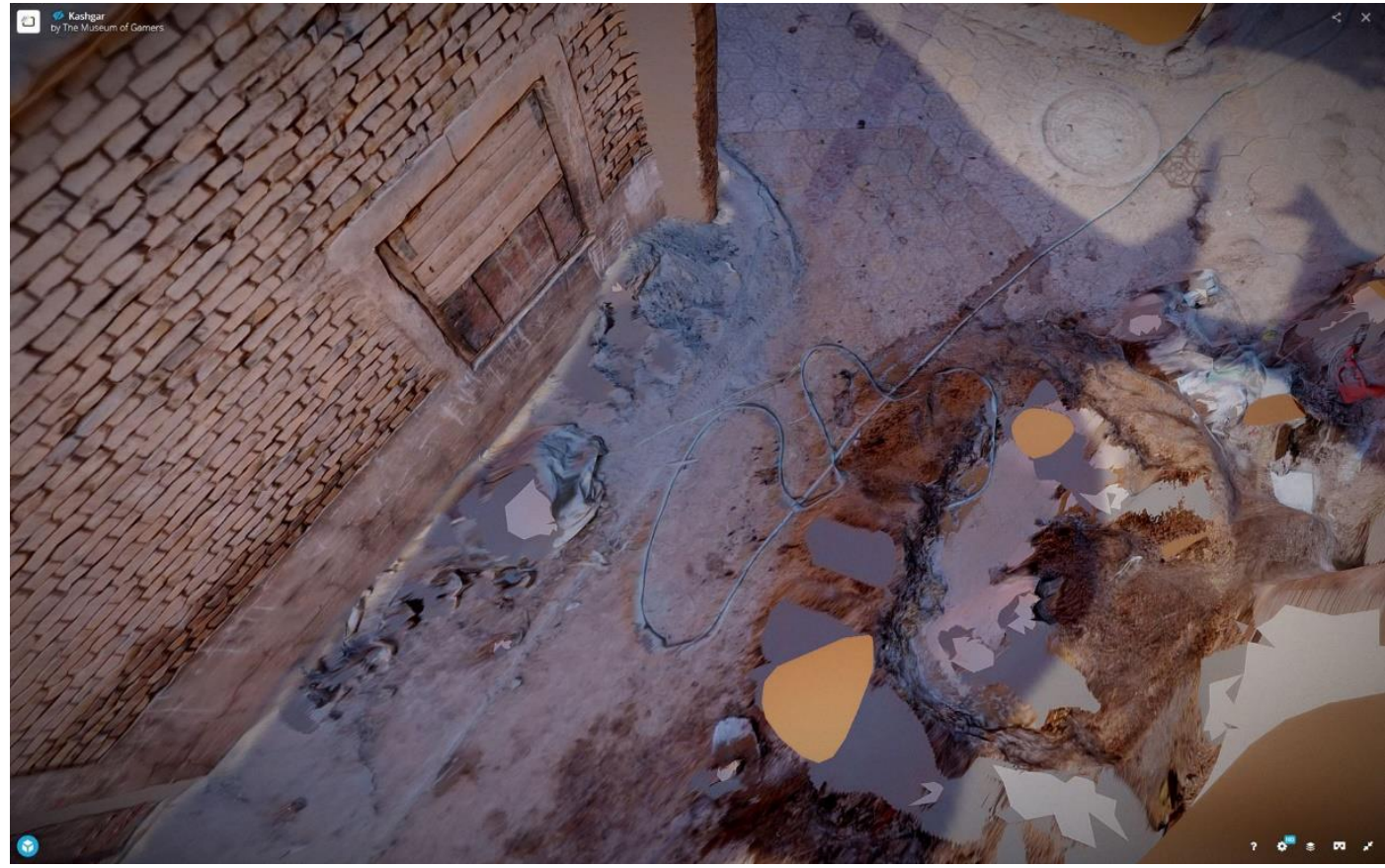

Fig. 151. A top view of the virtual model of a narrow lane in Köziciyerbişi (Gaotai).

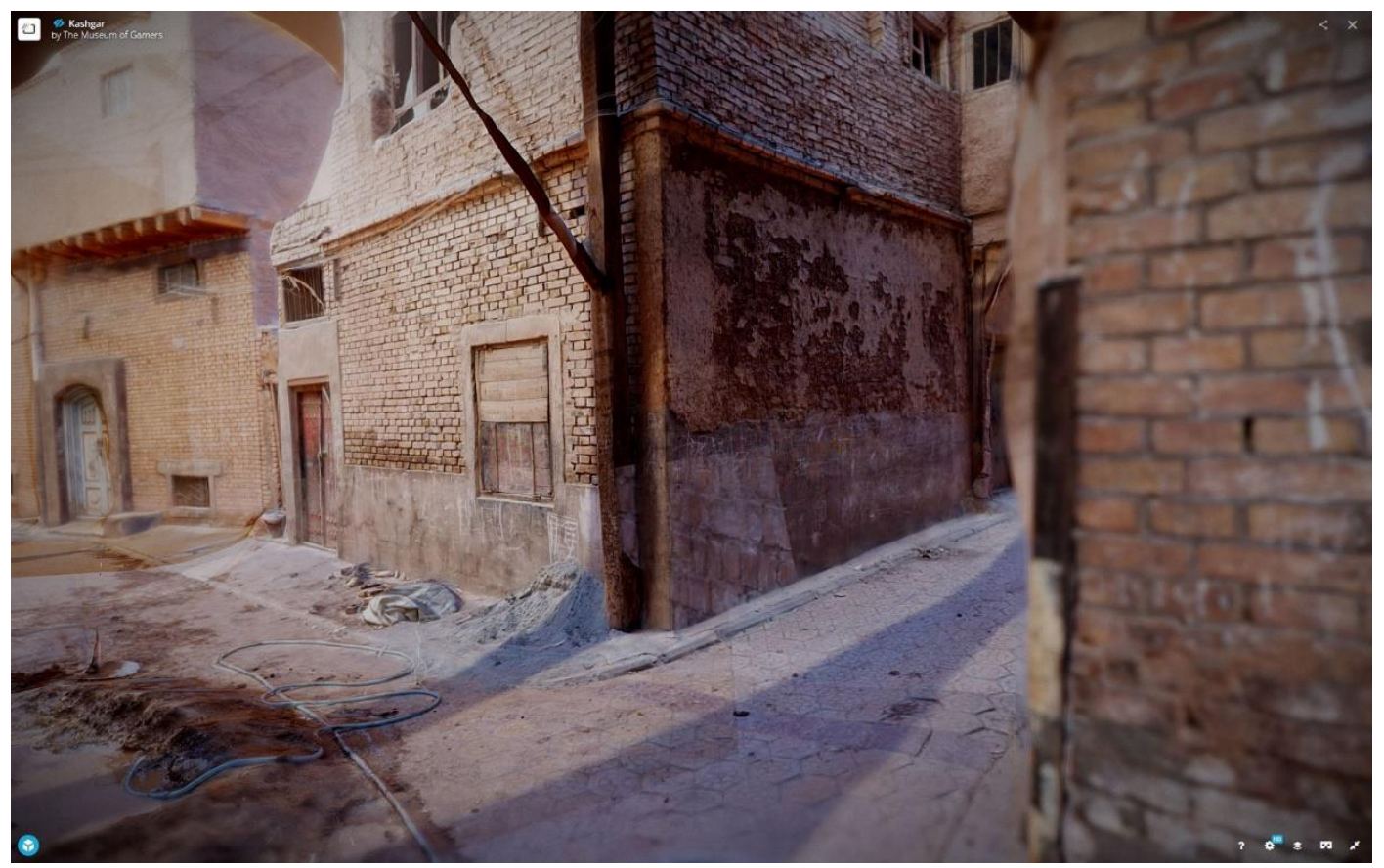

Fig. 152. A view of the virtual model of a junction of narrow lanes in Köziciyerbişi (Gaotai). 
330

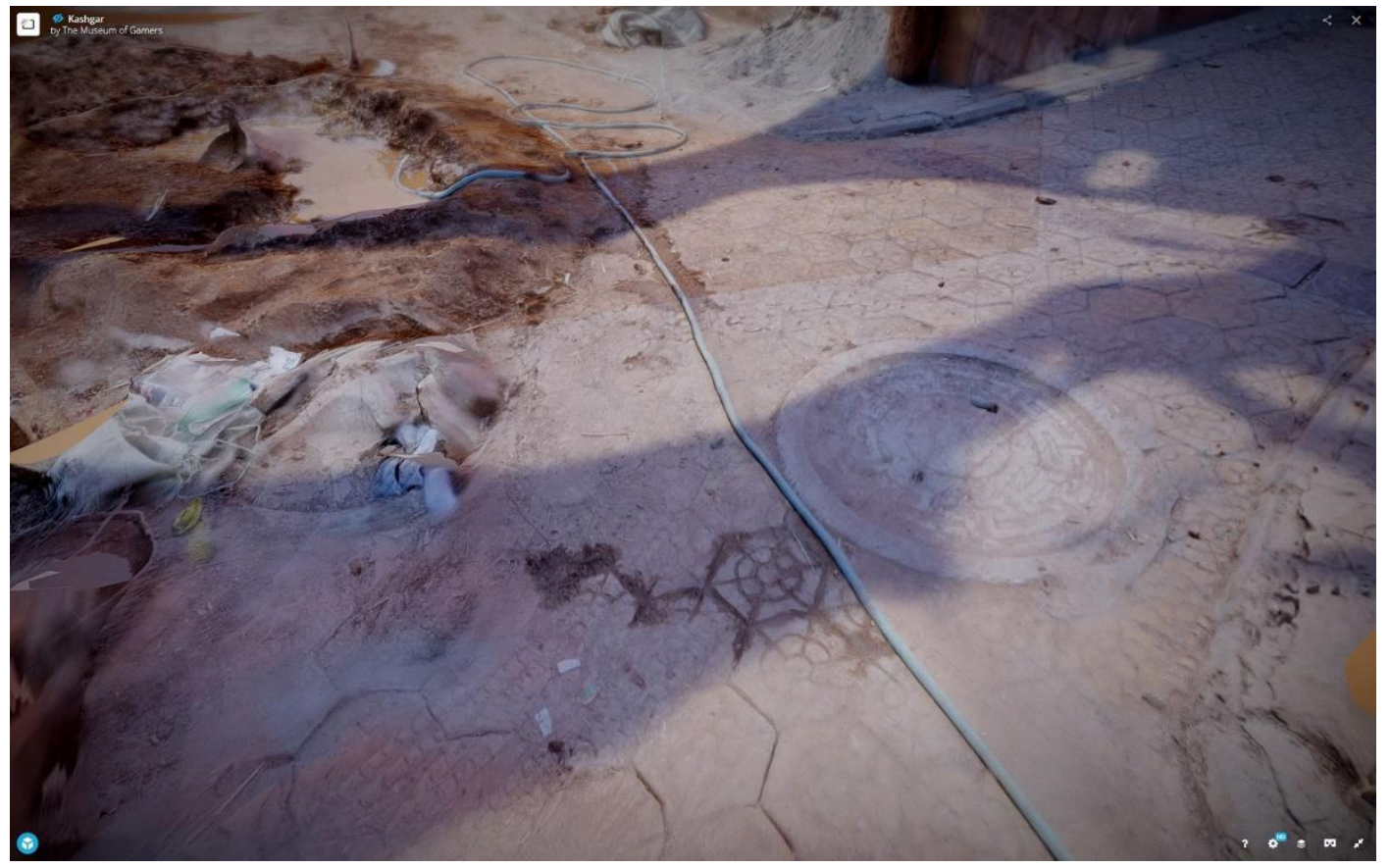

Fig. 153. A detail of the virtual model of a junction of narrow lanes in Köziciyerbişi (Gaotai).

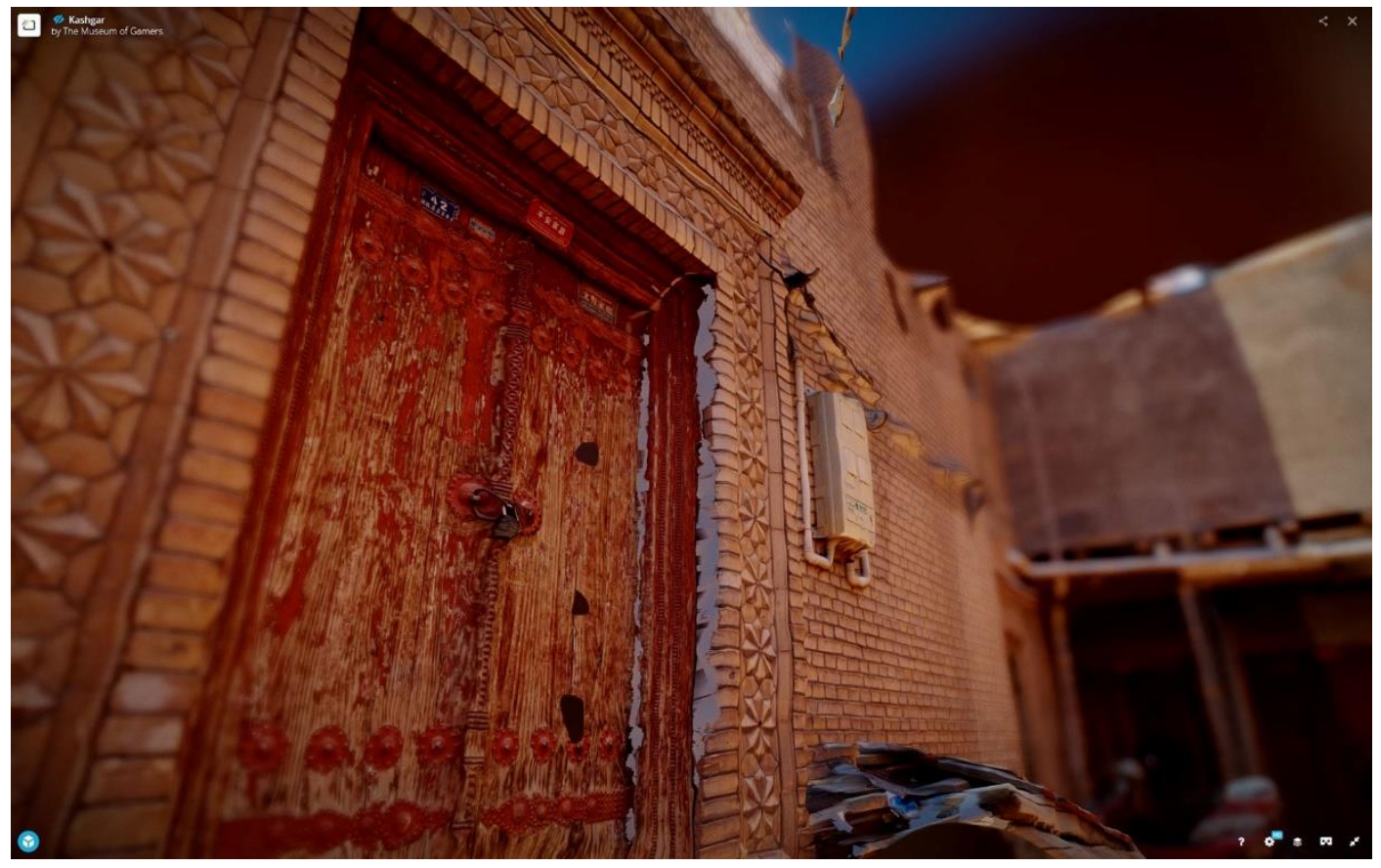

Fig. 154. A view of the virtual model of a timber door at the end of a cull-de-sac in Köziciyerbişi (Gaotai). 


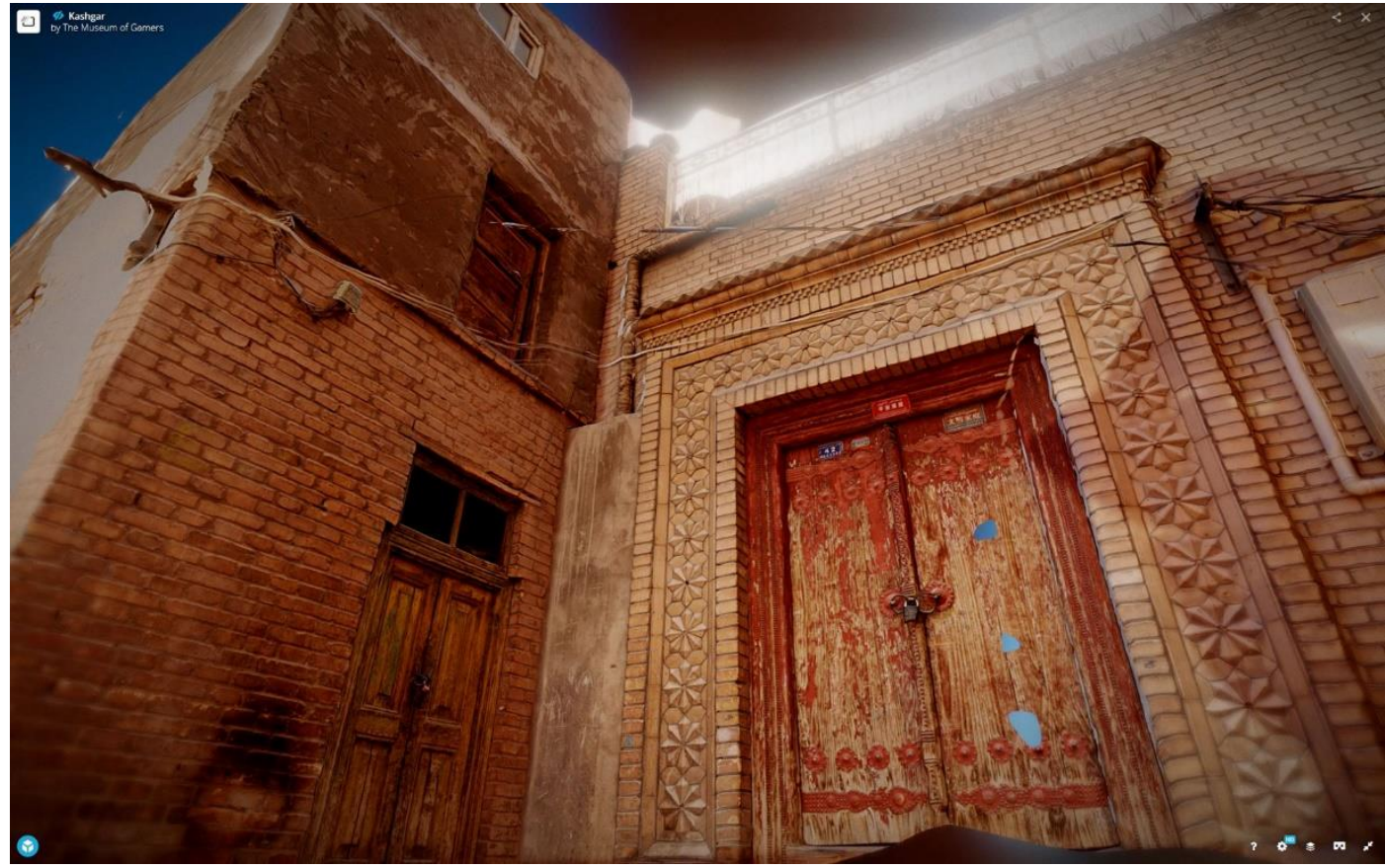

Fig. 155. A view of the virtual model of house extension at the end of a cull-de-sac in Köziciyerbişi (Gaotai).

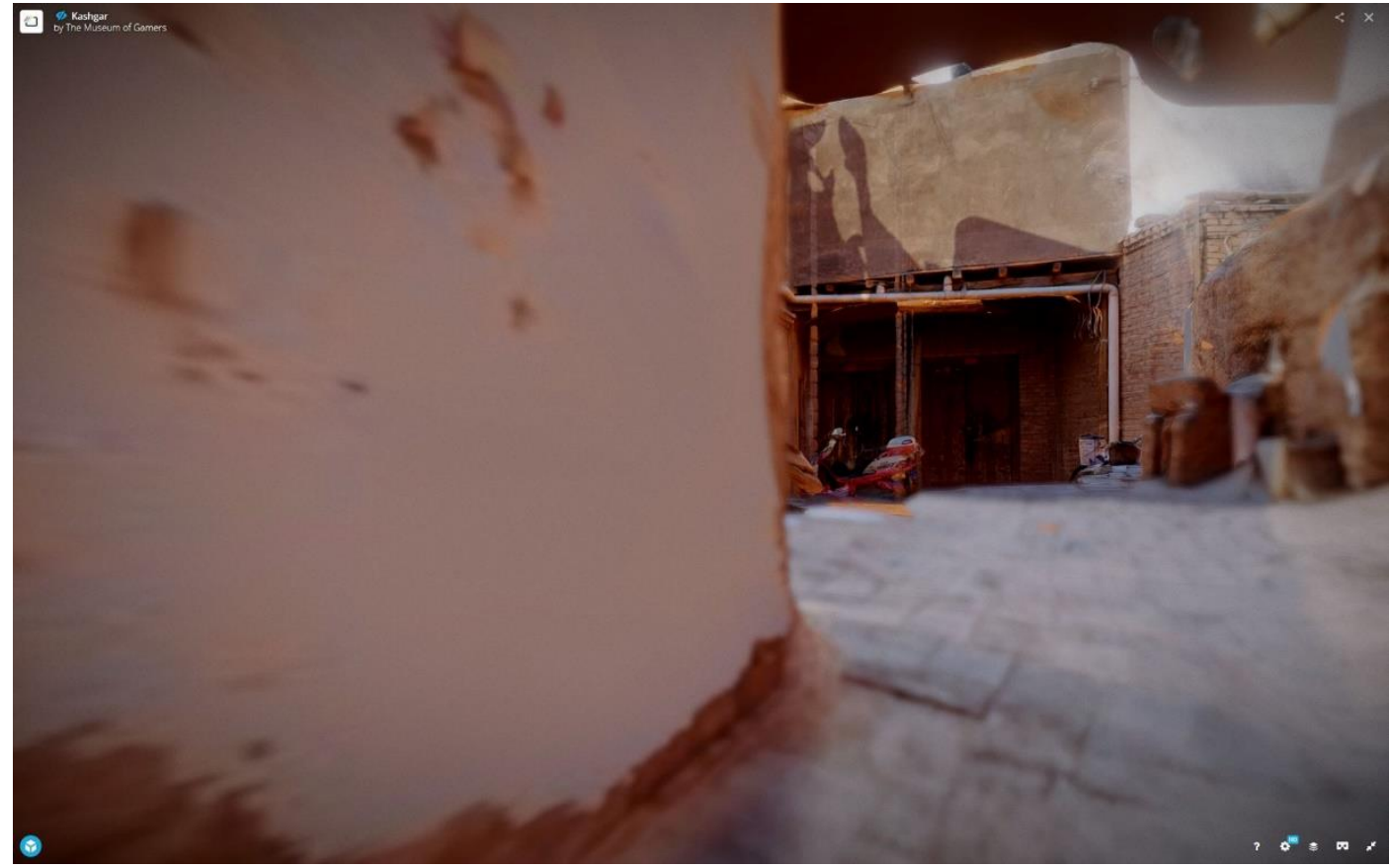

Fig. 156. A view of the virtual model of a cull-de-sac in Köziciyerbişi (Gaotai). 
332

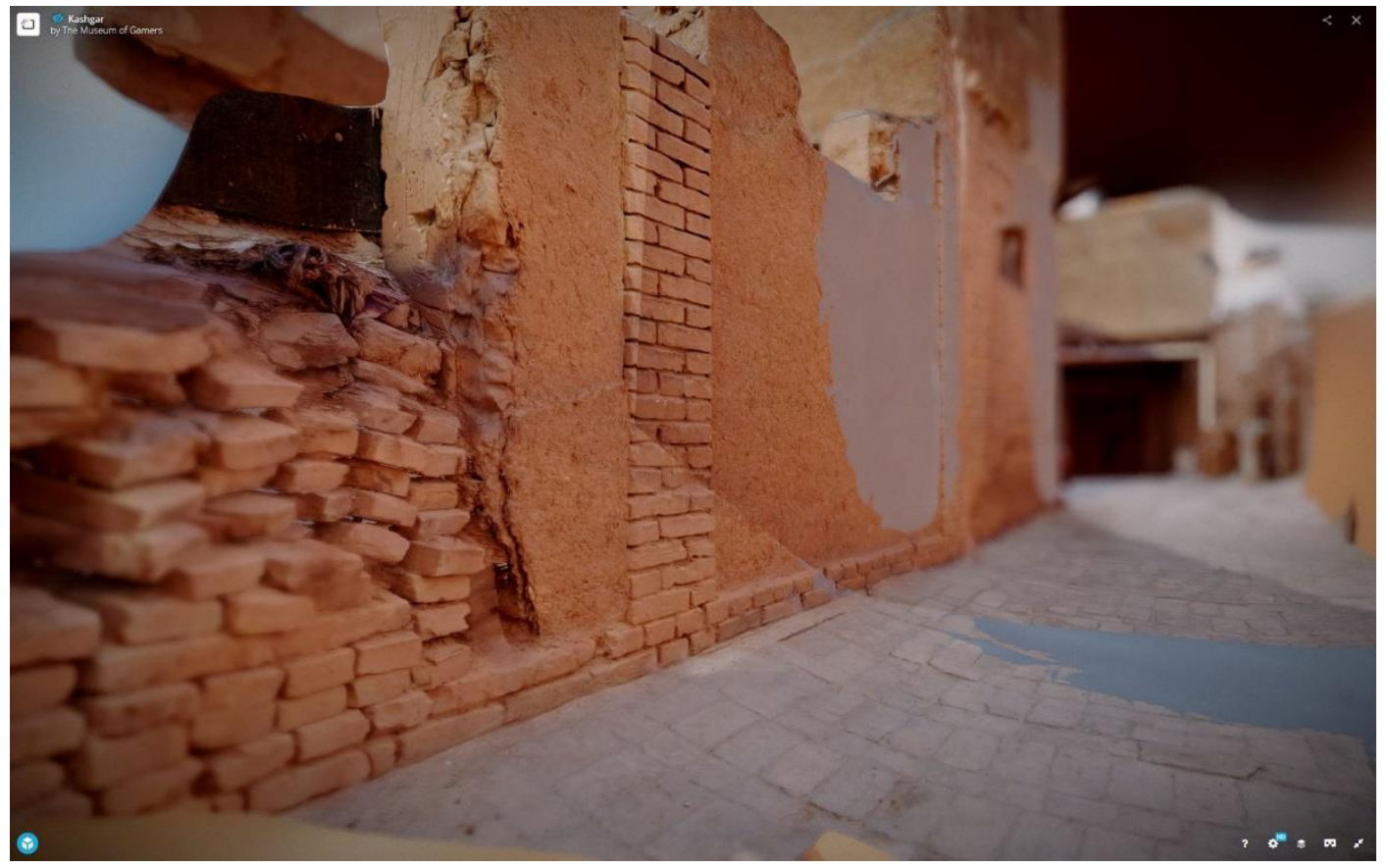

Fig. 157. A detail of the virtual model of brick walls in Köziciyerbişi (Gaotai).

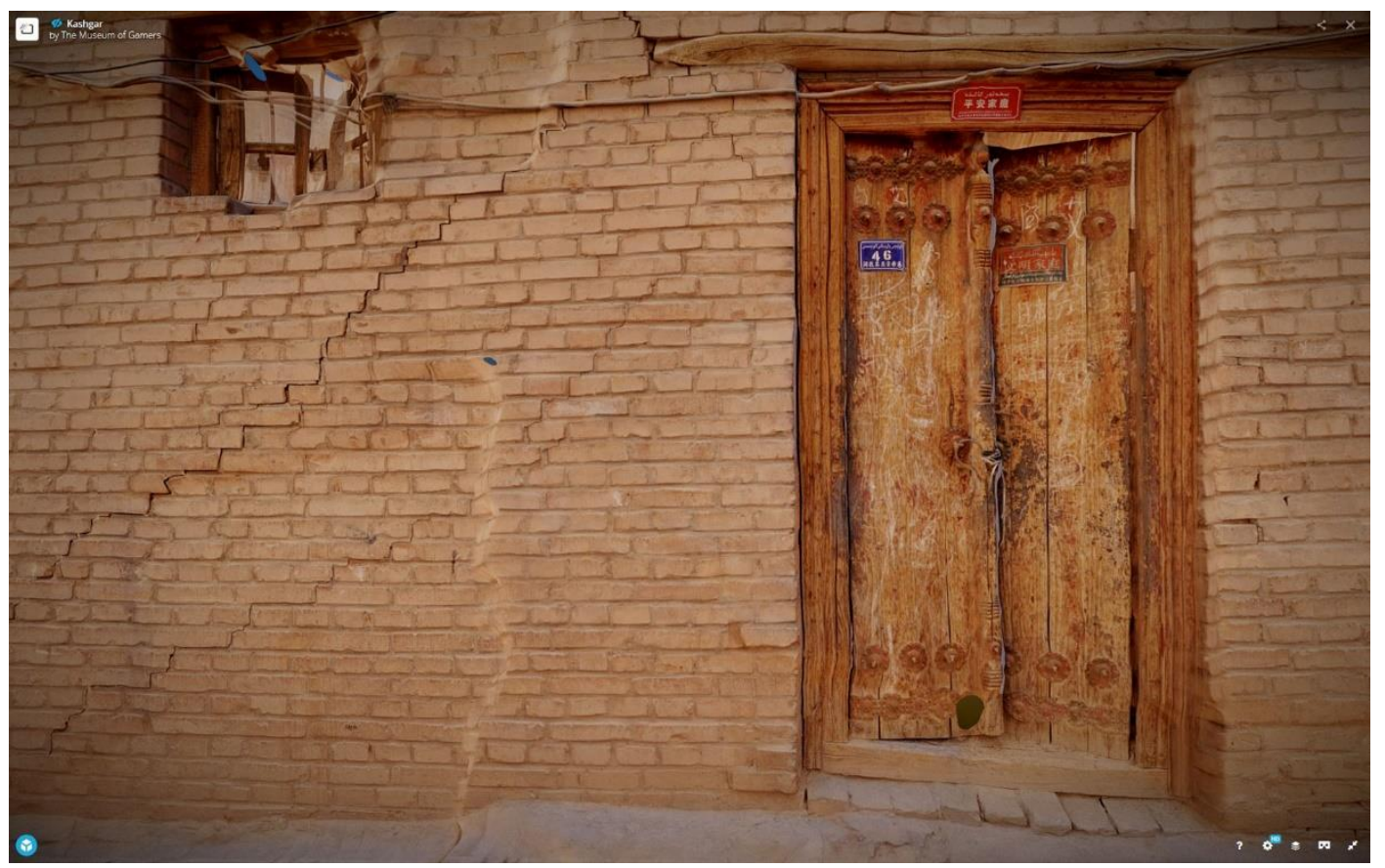

Fig. 158. A view of the virtual model of a brick wall with a crack in Köziciyerbişi (Gaotai). 


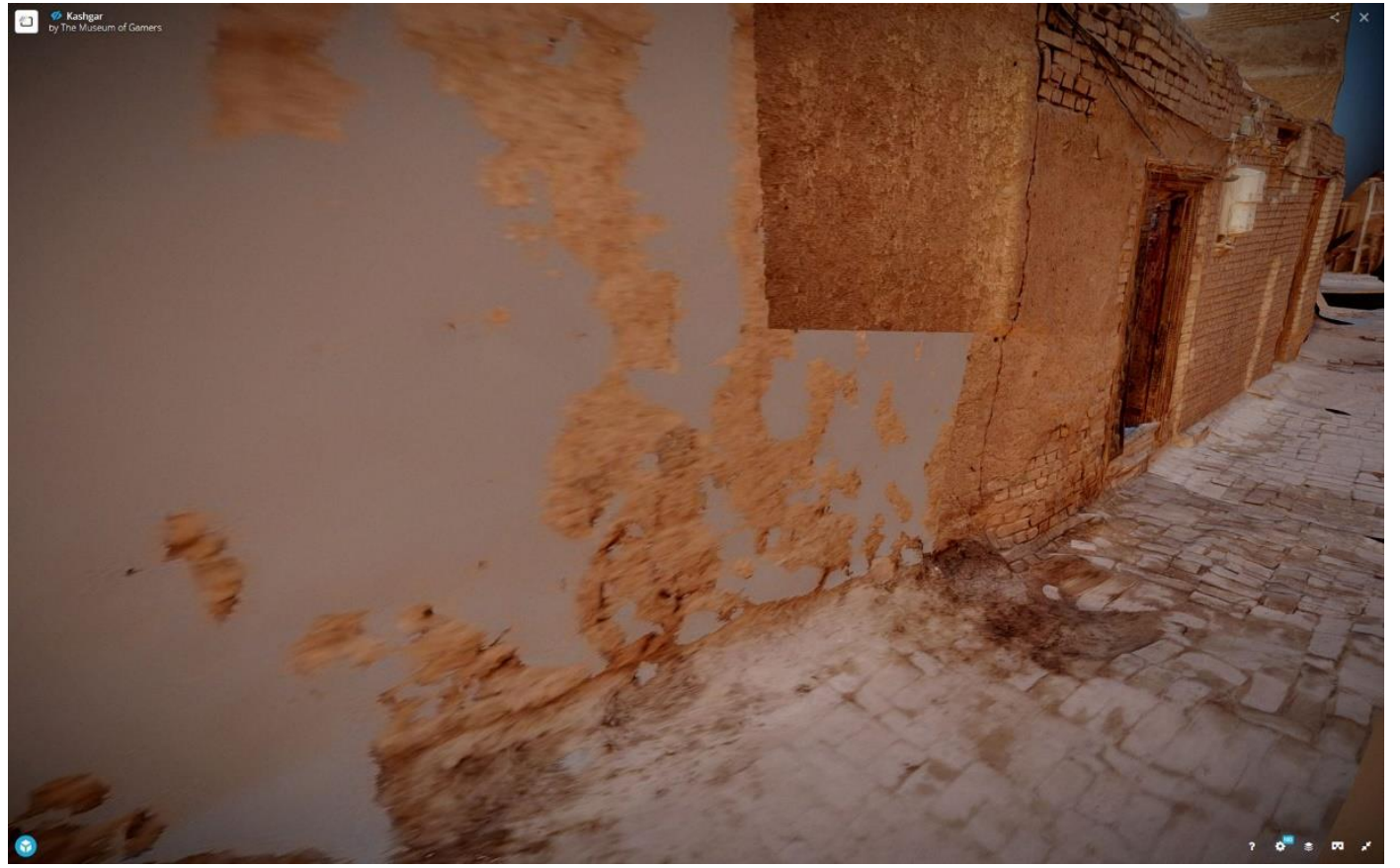

Fig. 159. A view of the virtual model of a mud-brick wall with glitches in Köziciyerbişi (Gaotai).

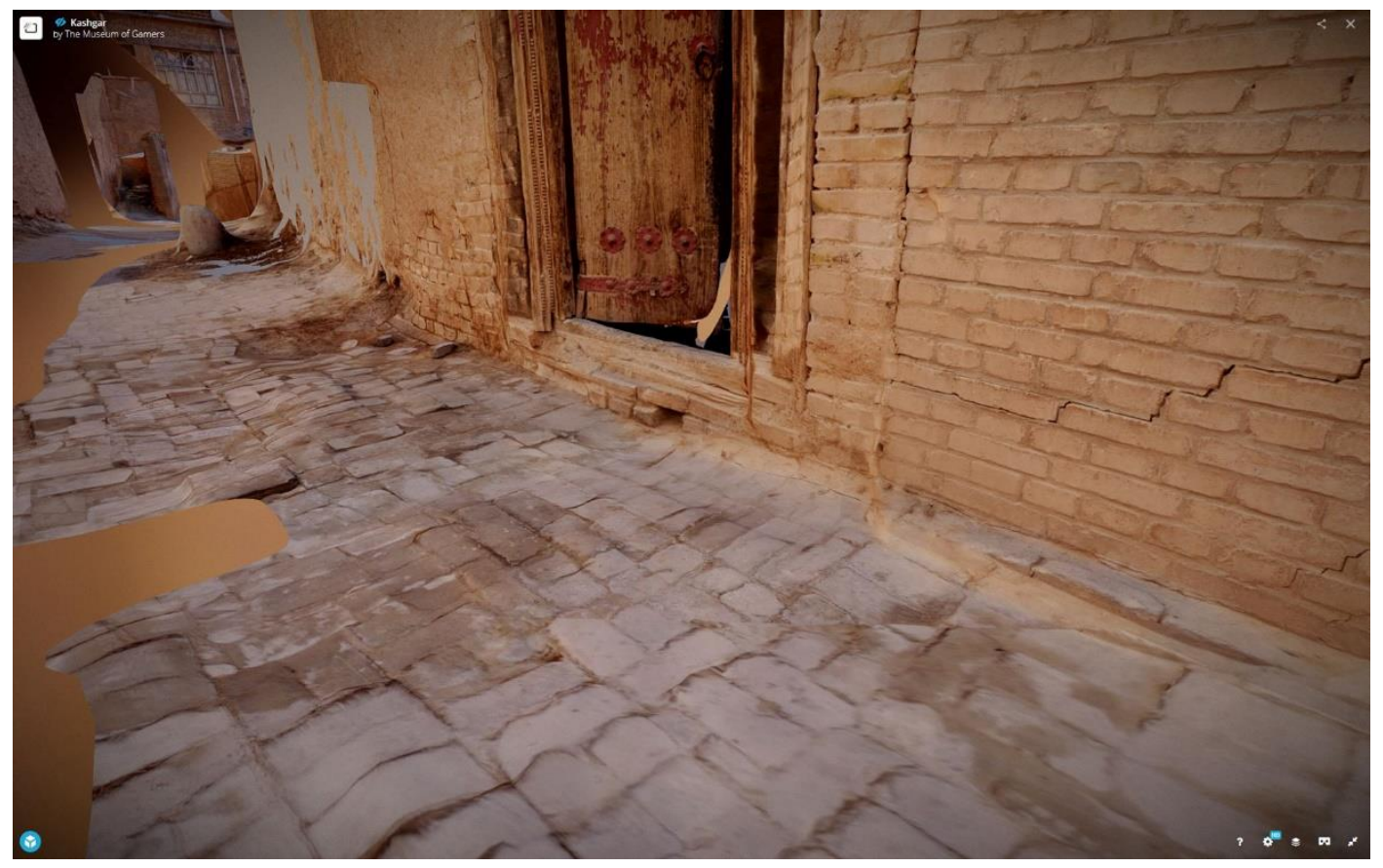

Fig. 160. A detail of the virtual model of rectangular cobblestones in Köziciyerbişi (Gaotai). 


\section{Appendix D}

Table 66. The result of the FP-growth algorithm that searched associations between one million records of artificial participants with the transaction record of the experiment participant whose itemset is $\{$ Metal Door, Timber Wall Structure, Brick Wall $\}$. Mud-Brick Wall=RC; Timber Wall

Structure $=$ TWS; Timber Structure $=$ TS; Metal Door $=M D ;$ Rec Cobblestone $=R C ;$ Hex

Cobblestone $=H C ;$ Red Door $=R D ;$ Blue Door $=B D ;$ Mosque Gate $=M G ;$ Brick Wall $=B W$.

\begin{tabular}{|c|c|c|c|c|}
\hline Size & $\begin{array}{l}\text { Itemsets in the synthetic data found } \\
\text { associated with }\{M D, T W S, B W\}\end{array}$ & Support & Confidence & Lift \\
\hline \multirow[t]{7}{*}{ 1-itemsets } & (2) & 0.028437 & 0.418955 & 1.078894 \\
\hline & $T S$ & 0.028420 & 0.418705 & 1.080406 \\
\hline & $M B W$ & 0.028339 & 0.417511 & 1.077560 \\
\hline & $H C$ & 0.028282 & 0.416672 & 1.073124 \\
\hline & $B D$ & 0.028223 & 0.415802 & 1.072905 \\
\hline & $M G$ & 0.028193 & 0.415360 & 1.072756 \\
\hline & $R D$ & 0.028030 & 0.412959 & 1.067844 \\
\hline \multirow[t]{21}{*}{ 2-itemsets } & $H C, T S$ & 0.011334 & 0.166981 & 1.042608 \\
\hline & $R C, T S$ & 0.011259 & 0.165876 & 1.035166 \\
\hline & $R C, M B W$ & 0.011225 & 0.165375 & 1.034545 \\
\hline & $R C, H C$ & 0.011222 & 0.165331 & 1.030966 \\
\hline & $R C, B D$ & 0.011222 & 0.165331 & 1.032640 \\
\hline & $T S, M G$ & 0.011190 & 0.164859 & 1.033978 \\
\hline & $T S, M B W$ & 0.011182 & 0.164742 & 1.033148 \\
\hline & $H C, B D$ & 0.011164 & 0.164476 & 1.028003 \\
\hline & $H C, R D$ & 0.011145 & 0.164196 & 1.026286 \\
\hline & $M B W, M G$ & 0.011141 & 0.164138 & 1.029353 \\
\hline & $H C, M B W$ & 0.011099 & 0.163519 & 1.022529 \\
\hline & $B D, M G$ & 0.011092 & 0.163416 & 1.021220 \\
\hline & $B D, M B W$ & 0.011090 & 0.163386 & 1.022231 \\
\hline & $R C, M G$ & 0.011086 & 0.163327 & 1.023225 \\
\hline & $T S, R D$ & 0.011070 & 0.163092 & 1.021845 \\
\hline & $B D, T S$ & 0.011070 & 0.163092 & 1.021378 \\
\hline & $R C, R D$ & 0.011065 & 0.163018 & 1.022421 \\
\hline & $M B W, R D$ & 0.011042 & 0.162679 & 1.024169 \\
\hline & $M B W, R D$ & 0.011039 & 0.162635 & 1.021306 \\
\hline & $B D, R D$ & 0.011012 & 0.162237 & 1.016848 \\
\hline & $H C, M G$ & 0.011001 & 0.162075 & 1.015934 \\
\hline \multirow[t]{11}{*}{3 -itemsets } & $R C, H C, T S$ & 0.004128 & 0.060817 & 0.887629 \\
\hline & $R C, T S, M B W$ & 0.004088 & 0.060227 & 0.886715 \\
\hline & $H C, T S, R D$ & 0.004073 & 0.060006 & 0.881334 \\
\hline & $R C, H C, B D$ & 0.004054 & 0.059727 & 0.874768 \\
\hline & $R C, H C, R D$ & 0.004048 & 0.059638 & 0.876117 \\
\hline & $T S, M B W, M G$ & 0.004036 & 0.059461 & 0.880231 \\
\hline & $H C, T S, M B W$ & 0.004022 & 0.059255 & 0.872939 \\
\hline & $R C, B D, M G$ & 0.004018 & 0.059196 & 0.869701 \\
\hline & $B D, T S, M G$ & 0.004018 & 0.059196 & 0.871429 \\
\hline & $H C, T S, M G$ & 0.004016 & 0.059167 & 0.871047 \\
\hline & $R C, B D, M B W$ & 0.004013 & 0.059123 & 0.868759 \\
\hline
\end{tabular}




\begin{tabular}{|c|c|c|c|c|}
\hline \multirow[t]{25}{*}{ Size } & $\begin{array}{l}\text { Itemsets in the synthetic data found } \\
\text { associated with }\{M D, T W S, B W\}\end{array}$ & Support & Confidence & Lift \\
\hline & $H C, B D, T S$ & 0.004010 & 0.059078 & 0.869861 \\
\hline & $T S, M G, R D$ & 0.003994 & 0.058843 & 0.868423 \\
\hline & $H C, M B W, R D$ & 0.003993 & 0.058828 & 0.870595 \\
\hline & $R C, B D, T S$ & 0.003991 & 0.058798 & 0.864161 \\
\hline & $B D, M B W, M G$ & 0.003988 & 0.058754 & 0.862358 \\
\hline & $R C, M B W, M G$ & 0.003987 & 0.058739 & 0.864935 \\
\hline & $R C, B D, R D$ & 0.003985 & 0.058710 & 0.868298 \\
\hline & $R C, H C, M B W$ & 0.003984 & 0.058695 & 0.863013 \\
\hline & $R C, T S, R D$ & 0.003971 & 0.058504 & 0.861134 \\
\hline & $H C, M G, R D$ & 0.003970 & 0.058489 & 0.865747 \\
\hline & $R C, H C, M G$ & 0.003968 & 0.058460 & 0.862375 \\
\hline & $H C, B D, R D$ & 0.003966 & 0.058430 & 0.859569 \\
\hline & $B D, T S, M B W$ & 0.003964 & 0.058401 & 0.859262 \\
\hline & $M B W, M G, R D$ & 0.003961 & 0.058356 & 0.866748 \\
\hline & $T S, M B W, R D$ & 0.003951 & 0.058209 & 0.861209 \\
\hline & $H C, B D, M G$ & 0.003947 & 0.058150 & 0.857571 \\
\hline & $B D, M G, R D$ & 0.003942 & 0.058076 & 0.852712 \\
\hline & $R C, T S, M G$ & 0.003938 & 0.058018 & 0.852936 \\
\hline & $R C, M G, R D$ & 0.003934 & 0.057959 & 0.859270 \\
\hline & $H C, M B W, M G$ & 0.003929 & 0.057885 & 0.855123 \\
\hline & $B D, M B W, R D$ & 0.003913 & 0.057649 & 0.852850 \\
\hline & $H C, B D, M B W$ & 0.003908 & 0.057576 & 0.844923 \\
\hline & $R C, M B W, R D$ & 0.003899 & 0.057443 & 0.853017 \\
\hline & $B D, T S, R D$ & 0.003896 & 0.057399 & 0.845057 \\
\hline \multirow[t]{32}{*}{ 4-itemsets } & $R C, H C, T S, R D$ & 0.001294 & 0.019064 & 0.668426 \\
\hline & $B D, T S, M B W, M G$ & 0.001285 & 0.018932 & 0.668063 \\
\hline & $R C, H C, T S, M B W$ & 0.001274 & 0.018770 & 0.661597 \\
\hline & $R C, H C, B D, T S$ & 0.001270 & 0.018711 & 0.656742 \\
\hline & $T S, M B W, M G, R D$ & 0.001261 & 0.018578 & 0.663524 \\
\hline & $H C, B D, T S, M G$ & 0.001259 & 0.018549 & 0.658333 \\
\hline & $R C, B D, M G, R D$ & 0.001257 & 0.018519 & 0.655287 \\
\hline & $R C, H C, B D, M G$ & 0.001257 & 0.018519 & 0.654754 \\
\hline & $R C, H C, B D, R D$ & 0.001255 & 0.018490 & 0.654569 \\
\hline & $R C, B D, M B W, M G$ & 0.001253 & 0.018460 & 0.649045 \\
\hline & $H C, B D, T S, R D$ & 0.001251 & 0.018431 & 0.651214 \\
\hline & $H C, T S, M G, R D$ & 0.001248 & 0.018386 & 0.651287 \\
\hline & $R C, B D, T S, M B W$ & 0.001248 & 0.018386 & 0.647639 \\
\hline & $R C, H C, M G, R D$ & 0.001241 & 0.018283 & 0.651673 \\
\hline & $R C, B D, T S, M G$ & 0.001238 & 0.018239 & 0.643129 \\
\hline & $H C, T S, M B W, M G$ & 0.001228 & 0.018092 & 0.642419 \\
\hline & $R C, T S, M B W, M G$ & 0.001228 & 0.018092 & 0.637642 \\
\hline & $H C, M B W, M G, R D$ & 0.001227 & 0.018077 & 0.646418 \\
\hline & $R C, H C, T S, M G$ & 0.001227 & 0.018077 & 0.636450 \\
\hline & $R C, H C, B D, M B W$ & 0.001221 & 0.017989 & 0.632358 \\
\hline & $R C, H C, M B W, R D$ & 0.001219 & 0.017959 & 0.640554 \\
\hline & $H C, B D, M G, R D$ & 0.001219 & 0.017959 & 0.634691 \\
\hline & $R C, T S, M G, R D$ & 0.001218 & 0.017944 & 0.634350 \\
\hline & $B D, M B W, M G, R D$ & 0.001212 & 0.017856 & 0.631604 \\
\hline & $H C, B D, T S, M B W$ & 0.001211 & 0.017841 & 0.629059 \\
\hline & $R C, T S, M B W, R D$ & 0.001202 & 0.017709 & 0.631148 \\
\hline & $R C, B D, M B W, R D$ & 0.001202 & 0.017709 & 0.635087 \\
\hline & $R C, M B W, M G, R D$ & 0.001198 & 0.017650 & 0.633019 \\
\hline & $B D, T S, M G, R D$ & 0.001198 & 0.017650 & 0.621889 \\
\hline & $R C, H C, M B W, M G$ & 0.001195 & 0.017606 & 0.624402 \\
\hline & $H C, T S, M B W, R D$ & 0.001195 & 0.017606 & 0.626937 \\
\hline & $B D, T S, M B W, R D$ & 0.001193 & 0.017576 & 0.622562 \\
\hline
\end{tabular}




\begin{tabular}{|c|c|c|c|c|}
\hline Size & $\begin{array}{l}\text { Itemsets in the synthetic data found } \\
\text { associated with }\{M D, T W S, B W\}\end{array}$ & Support & Confidence & Lift \\
\hline & $H C, B D, M B W, M G$ & 0.001188 & 0.017503 & 0.617046 \\
\hline & $H C, B D, M B W, R D$ & 0.001176 & 0.017326 & 0.612931 \\
\hline & $R C, B D, T S, R D$ & 0.001171 & 0.017252 & 0.610670 \\
\hline \multirow[t]{21}{*}{ 5-itemsets } & $R C, H C, B D, M G, R D$ & 0.000315 & 0.004641 & 0.418054 \\
\hline & $R C, B D, T S, M B W, M G$ & 0.000315 & 0.004641 & 0.412737 \\
\hline & $H C, B D, T S, M B W, M G$ & 0.000315 & 0.004641 & 0.416217 \\
\hline & $R C, H C, B D, T S, M G$ & 0.000312 & 0.004597 & 0.414520 \\
\hline & $R C, B D, M B W, M G, R D$ & 0.000310 & 0.004567 & 0.412570 \\
\hline & $R C, H C, B D, T S, M B W$ & 0.000308 & 0.004538 & 0.403817 \\
\hline & $H C, B D, T S, M G, R D$ & 0.000306 & 0.004508 & 0.404325 \\
\hline & $R C, H C, T S, M G, R D$ & 0.000302 & 0.004449 & 0.398861 \\
\hline & $R C, B D, T S, M G, R D$ & 0.000301 & 0.004435 & 0.396686 \\
\hline & $R C, H C, B D, T S, R D$ & 0.000301 & 0.004435 & 0.396474 \\
\hline & $R C, H C, B D, M B W, M G$ & 0.000295 & 0.004346 & 0.386876 \\
\hline & $B D, T S, M B W, M G, R D$ & 0.000293 & 0.004317 & 0.387739 \\
\hline & $R C, H C, T S, M B W, R D$ & 0.000293 & 0.004317 & 0.392213 \\
\hline & $R C, T S, M B W, M G, R D$ & 0.000290 & 0.004272 & 0.386651 \\
\hline & $R C, H C, M B W, M G, R D$ & 0.000289 & 0.004258 & 0.389228 \\
\hline & $H C, B D, M B W, M G, R D$ & 0.000281 & 0.004140 & 0.371059 \\
\hline & $H C, T S, M B W, M G, R D$ & 0.000279 & 0.004110 & 0.374596 \\
\hline & $H C, B D, T S, M B W, R D$ & 0.000279 & 0.004110 & 0.370476 \\
\hline & $R C, B D, T S, M B W, R D$ & 0.000276 & 0.004066 & 0.368319 \\
\hline & $R C, H C, B D, M B W, R D$ & 0.000273 & 0.004022 & 0.365806 \\
\hline & $R C, H C, T S, M B W, M G$ & 0.000272 & 0.004007 & 0.359658 \\
\hline \multirow[t]{7}{*}{ 6-itemsets } & $R C, H C, B D, T S, M G, R D$ & 0.000054 & 0.000796 & 0.200093 \\
\hline & $R C, B D, T S, M B W, M G, R D$ & 0.000051 & 0.000751 & 0.186908 \\
\hline & $R C, H C, B D, T S, M B W, M G$ & 0.000049 & 0.000722 & 0.179133 \\
\hline & $R C, H C, B D, T S, M B W, R D$ & 0.000043 & 0.000634 & 0.160952 \\
\hline & $R C, H C, B D, M B W, M G, R D$ & 0.000041 & 0.000604 & 0.151389 \\
\hline & $H C, B D, T S, M B W, M G, R D$ & 0.000040 & 0.000589 & 0.148553 \\
\hline & $R C, H C, T S, M B W, M G, R D$ & 0.000035 & 0.000516 & 0.130609 \\
\hline 7-itemsets & Null & Null & Null & Null \\
\hline
\end{tabular}


